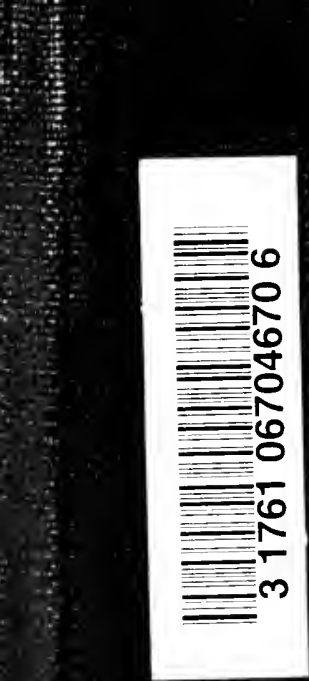




$$
\text { . }
$$





\section{Digitized by the Internet Archive in 2007 with funding from Microsoft Corporation}






\section{VENEREAL DISEASES}

THEIR CLINICAL ASPECT AND TREATMENT 
THE PRACTITIONER'S MANUAL OF VENEREAL DISEASES

With Modern Methods of Diagnosis and Treatment. By A. MAGIAN, M.D. Demy 8vo., 224 pp., Illustrated, Ios. 6d. net.

"The best small book on venereal diseases we have had the pleasure of reviewing."

The Practitioner.

"It is essentially a practical textbook . . . the views set forth should meet with general acceptance." -British Medical Journal.

PREVENTION OF VENEREAL DISEA SE

By Sir ARChDall Reid, K.B.E., with an Introduction by Sir BRYAN DONKIN, M.D. Crown 8vo.

LONDON : WILLIAM HEINEMANN

(MEDICAL BOOKS), LTD. 


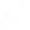




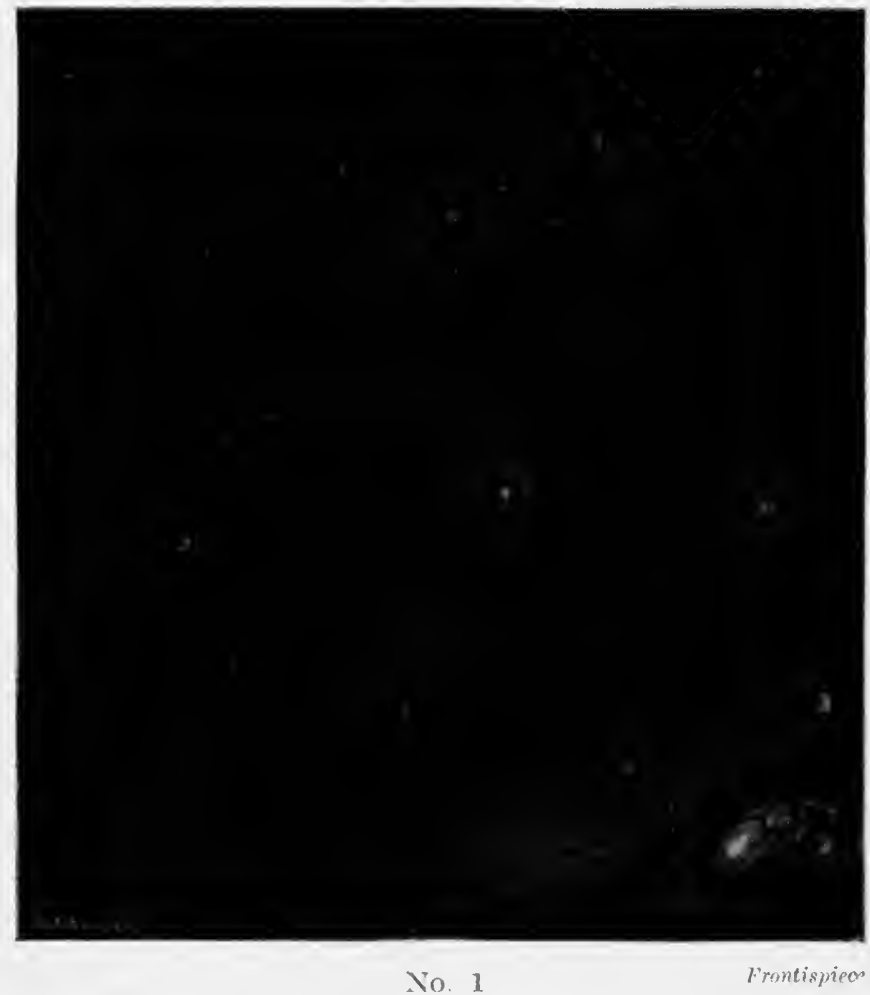

Plate I 


\section{PLATE I}

No. 1.-Early Generalised Syphilitic Eruption affecting a Negro from St. Lucia

THE typical arrangement of the macules with the papules developing in the centre is well shown. In life the papules had a silvery sheen. 


\title{
VENEREAL DISEASES
}

\author{
THEIR CLINICAL ASPECT AND \\ TREATMENT
}

\section{WITH AN ATLAS OF 106 COLOUR AND 21 HALF.TONE ILLUSTRATIONS}

BY

\section{J. E. R. McDONAGH, F.R.C.S. SURGEON, LONDON LOCK HOSPITAL, J.ATE HUNTERIAN PKOFESSOR, ROYAL COLLEGE
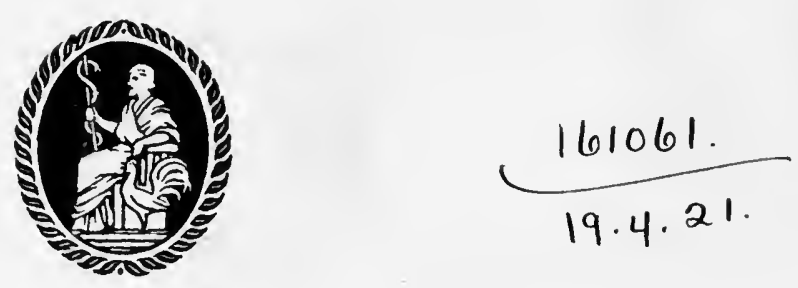

\section{LONDON}




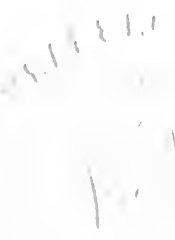

LONDON: WILLIAM HEINEMANN (MEDICAL BOOKS), LTD. 1920 


\section{CONTENTS}

CHA PTER

I. History of SyPHILIS

iII. The Stages of Syphils

III. SyphILIS OF THE SkIN

IV. Syphilis of the Lympho- and Hemopoletic Systems - - 41

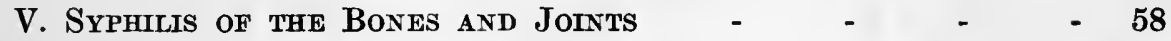

VI. Syphilis of the Mouth and Throat - $\quad-\quad-\quad-65$

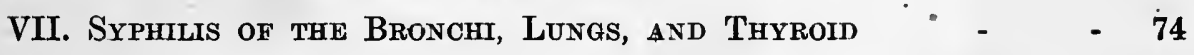

VIII. Syphilis of the Genito-URINary Tract - $\quad-\quad-\quad-\quad 79$

IX. Syphilis of the Abdominal Viscera $\quad-\quad-\quad-\quad-\quad-\quad 87$

X. Syphilis of the Eyes ANd Ears - $\quad$ - $\quad$ - $\quad-\quad-100$

XI. The Biology of Syphilis of the Nervous System - $\quad-109$

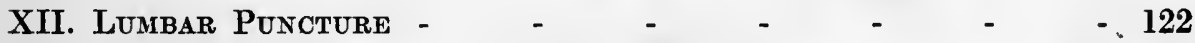

(XIII. The Crinical Aspect of Syphilss of the Nervous System - 128

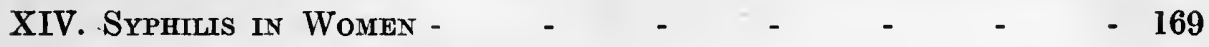

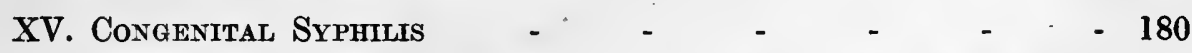

XVI. Drugs used in the Treatment of Syphilis and the Methods

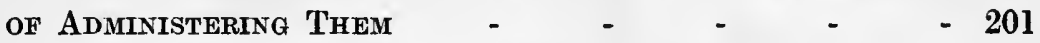

XVII. Toxic Symptoms of Arsenic and Mercury $\quad$ - $\quad$ - $\quad$ - 228

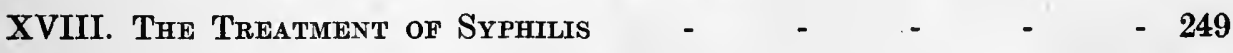

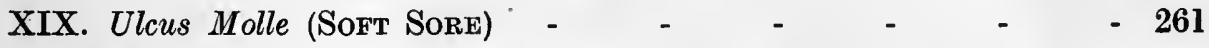

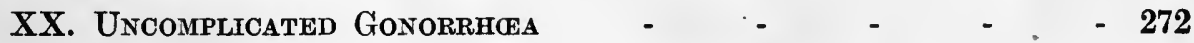

XXI. Compiacations of Urethritis Gonorrhoica, due to Direct Exten-

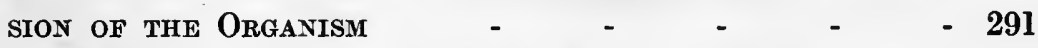

XXII. Complications of Gonorrhea due to a Spread by Metastasis

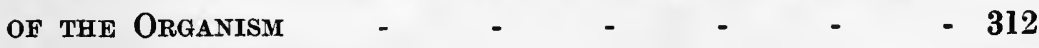

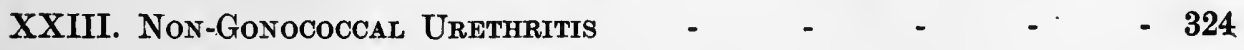


CBAPTER

XXIV. Gonorrhoal Diseases of the Eyes and Other Sense Organs

XXV. Gonococcal Rashes

- 332

XXVI. GoNorrhcea IN WoMen

- 336

XXVII. The Chemotherapeutic and Vaccine Treatment of GonorRHEA

XXVIII. Phimosis and Paraphimosis

- 344

XXIX. Subsidiary Venereal Diseases: Balanitis, Condyloma Acuminatum, Molluscum Contagiosum, Herpes Genitalis, Granuloma Inguinale, Induratio Penis Plastica, AND Pediculosis Pubis

XXX. Sexual Neurasthenta

- 368

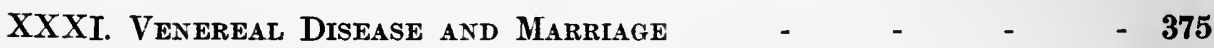

XXXII. Venergal Disease and Public Health - $\quad$ - $\quad$ - 383

XXXIIJ. Syphims as a Poison and its Relationship to Malignant

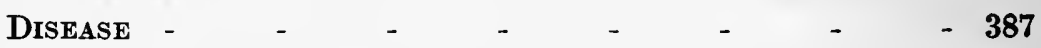

XXXIV. The Bridge Between the Clinical and the Pathological

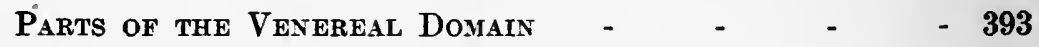

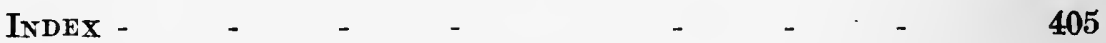




\section{LIST OF ILLUSTRATIONS}

plate No.

FACING PAG

I. 1. Early Generalized Syphilitic Eruption affecting a Negro from St. Lucla - - - Frontispioce

II. 2. A Keloid on the Site of a Primary Sore on the Mons

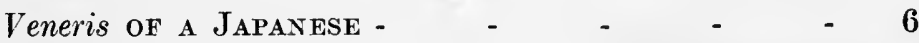

II. 3. A Localized Syphiloma Which developed iN a Lymphatic

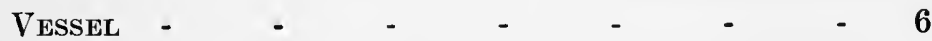

III. 4. Typical Papulo-grosive Chancres on the Skin of the Penis -

III. 5. Three Papulo-grosive Chancres on the Prepuce - 10

IV. 6. Papulo-erosive Chancres $\quad$ - $\quad$ - $\quad$ - $\quad$ - 10

IV. 7. Axother Type of Papulo-erosive Chancre, showing THE WELL-MARKED INDURATION - $\quad$ - $\quad-10$

V. 8. Two Papulo-erosive Chancres on the Skin of the Penis 12

V. 9. A Papulo-erosive Chancre on the Glans Penis assoCIATED With Condylomala Acuminata - $\quad$ - 12

VI. 10. Papulo-erosive Chancre in the Corona $\quad$ - $\quad$ - 12

VI. 11. Papulo-erosive Chancre on the Skin of the Penis - 12

VII. 12. Papulo-erosive Chancre on the Under Surface of the PREPUCE

VII. 13. Papulo-erosive Chancre in Corona $\quad-\quad$ - $\quad-14$

VIII. 14. A Chancre on the Frenum -

VIII. 15. Three Papulo-erosive Chancres on the Skin of the

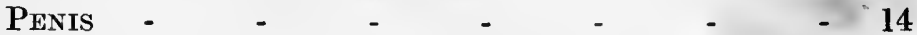

IX. 16. A Chancre in One of the Furrows of the Prepuce - 16

IX. 17. A Chancre of the Franum - $\quad$ - $\quad$ - $\quad$ - 16

X. 18. Chancres on Scabetic Lesions - $\quad$ - 16

X. 19. A Typical Papulo-erosive Chancre of the Type which is liable to be followed by Severe Vascular aNd

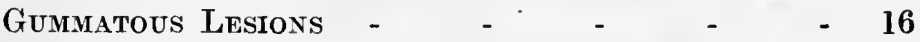

XI. 20. A Very Early Intra-Urethral Chancre at' the Lower Pole of the Meatus - $\quad$ - $\quad$ - $\quad$ - $\quad$ - $\quad$ - 18

XI. 21. An Intra-urethral Chancre on the Left Wall of the Meatus . 
Plate No.

XI. 21. Axother Type of Ixtra-Urethral Chancre exhibitixg the Typical Ixfiltrative Edeya of the Prepuce -

XII. 23. AN Ixtra-urethral Chaxcre and Balanitis Produced By the Discharge Therefrom

XII. 24. A Recurrent Intra-urethral Syphiloma, showing the Typical Violaceous Tint of the Surrounding Tissue

XIII. 25. A Hidden Chaxcre which has produced Enormous Noy-inflamiatory CEdema and Cosstriction of the Prepdce Sufficient to cause Eversion of its IXTERIOR LAMELLA

XIII. 26. A Hidden Chancre causing Inversion of the Prepuce -

XIV. 27. A Papulo-ulcerative Chancre on the Upper Surface OF THE Prepuce

XIV. 28. A Typical Graxulatixg Chancre Fron which no SpIRoCHETA COULD BE OBTAINED

$\mathrm{XV}$

29. Papulo-ulcerative Chaxcres

20

XVI. 30. A ChaNcRe Which BeCAME Secondarily INfected

XVI. 31. A Chancre on the Skin of the Penis which became SEPTIC

XVII. 32. Ax Ulcerating Primary Sore with such Dense Infiltrative GEema of the Pexis as to suggest Maliganant Disease

XVII. 32. A Primary Sore on the Skin of the Penis, which has BECONe SECONDARILY INFeCted With ViNCeNT'S OrgaNisus

XVIII. 34. Papular Syphilide by Direct Extension - $\quad 22$

XVIII. 35. Papular Syphilide by Direct Extexsion - - 22

XIX. 36. A Recurrent Chancre on the Glans Penis on the Same Site as the Original Sore, and a Fresh Chancre on the Skix of the PeNis

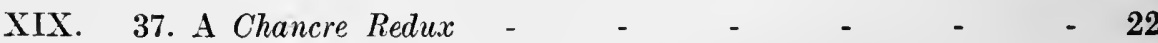

XX. 38. Ax Uncommon but Typical Vexous Syphilitic Eruption -

XX. 39. A Recurrext Papulo-orbicular Syphilide of a Type Commonly seen is Patients who have been Insufficiently Treated with Arseno-Benzene -

XXI. 40. The Typical Early Syphilitic Eruption showing the Papules developisg in the Centre of the Macules -

XXI. 41. A Recurrest Coryubose Syphilide which appeared ox the Back Eighteen Months after the Patient CONTRACTED the Disease -

XXI. 42. Ax Uyusual Fory of the Early Syphilitic Eruption -

XXII. 43. A Typical Polymorphic Syphilitic Eruption of the Early Generalisation Stage 
PLATE

so.

FACING PAGE

XXII.

44. A Typical Follicular Syphilitic Eruption

26

XXIII. 45. A Typical Corymbose Syphilide from the Back of a

Patient who had had a Chancre Two Months Before

XXIII. 46. A Case of Syphilitic Leucoderina ox the Skin of the

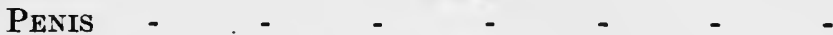

26

XXIV. 47. A Photograph showing the Typical appearance of EARLY SyPhilitic Alopecia -

XXIV. 48. A Photograph of a Frambersiform Syphilide which DeVeloped before the Primary Sore had Conpletely HEaled -

XXV. 49. AN Early Syphilitic Eruption closely resemblisg Seborrhoca Corporis -

XXVI. 50. An Early Macular Syphilide exhibitixg Several Tixy Hanorrhagic Points ix' Each Lesion - - 30

XXVI. 51. An Early Syphilitic Eruption simulating Chicken-Pox (VARICELLA)

XXVII. 52. A CASE of Seborrhoca Corporis

XXVII. 53. AN EARLy Syphilitic Eruption Sivulatixg Seborhoea Corporis, with the Difference that the Lesions are NOT FOLLICULAR

XXVIII. 54. Recurrent Palmar Syphilide affecting One Palm only

XXVIII. 55. Recurrent Palmar Syphilide - $\quad$ - $\quad$ - $\quad$ - 32

XXIX. 56, 57, 58. Recurrent Papulo-Ulcerative Syphilides $\quad 32$

XXX. 59. Recurrent Condylomata Lata _ - $\quad$ - $\quad$ - $\quad$ - 34

XXXI. 60. A Picture to Show the Typical Violaceous Tint Which is oftex Left Behixd by Early Syphilitic Genital Lesioxs

XXXI. 61. A Recurrent Sy philide ox the Site of the Primary Sore

XXXIII.

68. A Recurrent Papulo-orbicular Sýphllide oN the

$$
\text { Penis aNd Scrotul - } \quad \text { - } \quad \text { - } \quad \text { - }
$$

XXXIV. 69. A Typical Recurrent Papular Syphilide which appeared while the Patient was under Treatient - 38

XXXV. 70. A Recurrext Papulo-pustular Syphilide 
PLATE

XXXVII.

$$
\text { so }
$$

73. Lichen Planus i.jmited to the Skin of the Penis

74. A Case of Granuloma Annulare on the BaCK of the LefT HaNd WHICH HaD BEEN MistakeN by SOME FoR

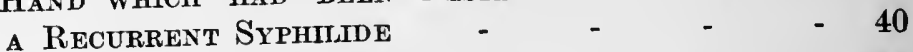

XXXIX. 75. RecURRent Syphintic Lymphangitis - $\quad$ - $\quad 42$

XL. 76. An Interesting Case showing a Healed Chancre on the Prepuce, a Recurrent Papulo-orbicular Syphilide on the Scrotum, and Bilateral SyPhimitic Suppurative Adenitis - $\quad$ - $\quad$ - $\quad$ - 44

XLI. 77. Gummatous Adenitis - $\quad-\quad-\quad-\quad-\quad 46$

XLII. 78. A Primary Sore oN the Upper LIP - $\quad-\quad$ - 66

XliI. 79. A Healing Primary Sore of the Thumb - 66

XLiII. 80. A Typical Case of Malignant Syphilis - - 69

XLIV. 81. The So-called Snail-Track Appearance of Mucous Patches on the Palate $\quad$ - $\quad$ - $\quad$ - $\quad$ - 70

XliV. 82. Gummatous Ulceration following a Parenchymatous Glossitis affecting the Anterior Two-ThiRds of

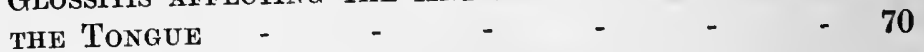

XLV. 83. Gummatous Glossitis Before Intramine $\quad-\quad-72$

XLV. 84. Gummatous Glossitis after Intramine $\quad-\quad 72$ Barker's Lumbar-Puncture Needle - - page 122 Section of the Spinal Cord within its Membranes page 126

XlVi. 85. A Case of Syphiltic Chronic Axterior Poliomyelitis

(Remitting Spinal Amyotrophy) - $\quad-\quad$ - 132

XlVII. 86, 87, 88. Typical Physiognomy of a Congenital SyphiLITIC NOW AN ADULT -

XlVIII. 89, 90, 91, 92. Various Aspects of Congenital Syphilitic

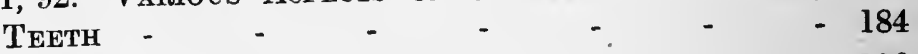

XliX. 93. The So-called Olympic Forehead - $\quad$ - 186

XIJIX. 94. The So-called Natiform Skull - $\quad$ - 186

XliX. 95. The So-Called Sabre-Tible $\quad-\quad-\quad-\quad-186$ The Author's Double-barRelled Syrifge - page 204 The Author's Three-way Syringe - - page 207

L. 96. An Iodide Rash with a Recurrent Lesion on the Fore-

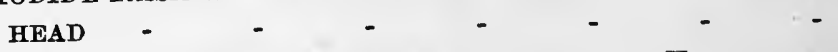

LI. 97. A Bullous Erythema Multiforme wHICH at First was of the Iris Type caused by Arsenic - -

Ll. 98. The Tongue from the Same Case, showing the Combination of Metallic Stomatitis and RecurRent Syphilitic Mucous Papules - - - -

LI. 99. Two NaIls frow Axother, but Typical Case of Arsenical DERMATITIS AFTER THE RASH HAD DISAPPEARED - 238 
PLATE No.

LII. 100. A Healed Vincent's Angina Lesion whith occurRed AFter a Course of Arseno-Benzene and Mercury

LII. 101. Vincent's Angina of the Left Tonsil which appeared One Week after the Completion of a Course of Arseno-Benzene and Mercury

LIII. 102, 103. A Severe Case of Gangrenous Stomatitis ProDUCED BY MERCURY - $\quad$ - $\quad$ -

LIV. 104. A Single Ulcus Molle on the Skin of the Penis - $\quad 260$

LIV. 105. A Single Ulcus Molle on the Skin of the Penis - $\quad 260$

LV. 106. A Spreading Ulcus Molle aS a Result of CIr-

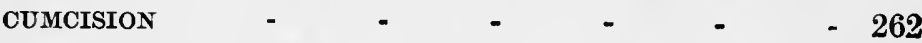

LV. 107. A Spreadivg Ulcus Molle on the Glans Penis - 262

LVI. 108. A Chronic Spreading Ulcus Molle Easily Diagnosed BY THE RAISED UNDERMINED AND IRREGULAR

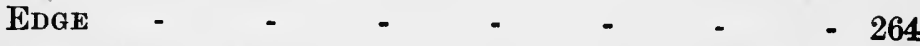

LVI. 109. An Ulcus Molle CAUSED by the Boat-Shaped Form of DUCREY's BACILLUS - $\quad$ - $\quad$ - $\quad$ - $\quad$ - 264

LVII. 110. A Typical CASE of Ulcus Molle Elevatum - - 266

LVIII. 111. A RecurRent Ulcus Molle with a Commencing Ulcus Molle Serpiginosum - $\quad$ - $\quad$ - $\quad$ - $\quad$ - 268

LVIII. 112. AN Early CaSe of Ulcus Molle Serpiginosum - $\quad$ - 268

LIX. 113. A CASE OF Ulcus Molle Serpiginosum IN THE LEFT GROIN - $\quad-\quad$ - $\quad$ - $\quad$ - $\quad$ - $\quad$ - 270

LX. 114. Part of the Scar Resulting from an Ulcus Molle Serpiginosum - $\quad$ - $\quad$ - $\quad$ - $\quad$ - $\quad$ - 270

Bougie a Boule in Urethra, Demonstrating Stricture, and Showing Mode of Irrigation - - - 281

LXI. 115. A Case of Balanitis Circinata which is often Associated WITH GonorRheal Rheumatism, AND IS FREquentLy THe Precursor of Keratodermia Blennorrhagica

LXI. 116. A CASE OF Keratociermia Blennorrhagica WHICH AFFECTED the Penis Only

LXII. 117. A CASE of Keratodermia Blennorrhagica

LXIII. 118. Soles of the Feet in a CaSe of Keratodermia Blennor-

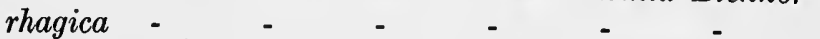

LXIII. 119. Changes NaIls Undergo in a CASE of Keratodermia

LXIV. 120. Condylomata Acuminata SURroundrat the Anus - 358

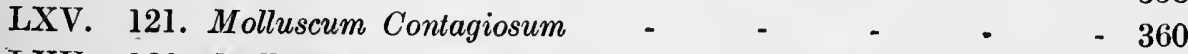

LXV. 122. Molluscum Contagiosum, WHICH HAS BECOMe SEPTIC $\quad-360$

LXVI. 123. Herpes Genitalis showing the Vesicular and Grouped Lesions - 
plate 124 . A Single Herpetic Lesion which has passed the VesiraCiNo PAGE

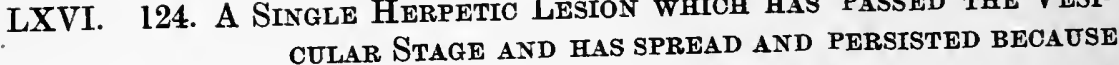
it was Irritated by Local Applcications iN the Belief that it was a Chancre - $\quad-\quad 362$

LXVII. 125. Macula Corulece on the Flanks, CAUSEd by the Bite of the Pediculus Pubis on Its Way fron the Genttals TO THE AXILLE -

LXVIII. 126. An Ordinary Case of Scabies - $\quad$ - $\quad$ - 366 LXVIII. 127. A Case of Long-Standing Scabies, in which Some of the Lesions have simulated Chancees - $\quad$ - $\quad 366$ 


\section{VENEREAL DISEASES \\ THEIR CLINICAL ASPECT AND TREATMENT}

\section{CHAPTER I \\ HISTORY OF SYPHILIS}

Is 1863 Dabry published a translation of Chinese manuseripts about 4,500 years old. In those manuscripts, he considered that syphilis and its treatment were mentioned. Modern Japanese authorities deny the accuracy of Dabry's conclusions, and maintain that the disease described is not syphilis. The Japanese, by reason of their geographical situation, are in a very good position to have excellent opportunities of thoroughly understanding Chinese literature and modes of thought; we therefore must accept their judgment in this matter against that of Dabry.

Human bones have been discovered at Solutre; they appear to be of Asiatic origin, and to have been buried in prehistoric times. Among those bones was found a woman's tibia with nodes, and Virchow and Parrot considered these nodes to be of syphilitic origin. Among Peruvian bones, probably buried before the Spanish Conquest, traces of bone disease of a child's skull were considered by Parrot to be due to syphilis. Other bones of Mexican and Central American origin show signs of syphilitic disease, but in these cases the date of burial cannot be determined.

In India, about A.D. 500, were written the works on which is based the modern system of Ayurvedic medicine. It has been suggested that there are references to syphilis in those works. Those who are best qualified to judge are of opinion that those references, which are of the very vaguest nature, may relate to almost any skin disease, except, possibly, syphilis.

In Leviticus, chapter xiii., there is a lengthy disquisition on the differential diagnosis of leprosy. It has been suggested that syphilis is really intended. This seems to be very unlikely, for the existence of leprosy at this period is well established. Again, the methods of clinical examination were such that very few priests would have escaped syphilitic infection, and they do not seem to have been so infected.

During the period of the decline of Roman greatness, the Romans, rich and poor alike, led lives of unspeakable sexual filthiness. Had syphilis been 
in existence, its incidence and treatment would have caused widespread remark, and some record of it must have come down to us.

From the above notes it will be seen that the evidence of the early existence of syphilis in the Old World is quite unsatisfactory. The evidence afforded by the bone remains may well be disregarded, for it must be remembered that these bones have been macerated by centuries of exposure. Also, the necrotic changes or the enlargements of these bones may well have been caused by rarefying osteitis or by new growths.

Throughout the written records of man's history, so far as they are yet known, there is no definite reference to the syphilitic syndrome until we reach the last four years of the fifteenth century.

Syphilis is the only disease which has a written history beginning with a definite date, and the history dates from the return of Christopher Columbus from his discovery of America. The discoverer reached Barcelona, on his return, in 1493. In the following year Charles VIII. of France invaded Italy, and laid siege to Naples. The campaign was little more than a military promenade for Charles's forces, who easily brushed aside all resistance. Charles set up a Court at Naples, with the intention of maintaining it there. So rapidly, however, did the French forces fall victims to disease that the Neapolitans drove them out in utter disorder in 1495. The disorganisation of Charles's troops was so great that they were scattered into numerous bands, and made their way back to their different homes just as chance allowed.

Now, at the time of this campaign, there was in Europe no race more covetous of military honour than were the Spaniards. The crew of Columbus would certainly include some of the most reckless and enterprising of that warlike race. They had reached Spain shortly before this campaign; therefore it is highly probable that some of the crew of Columbus actually made the campaign. Also, on their return from their voyage, after the fearful hardships which they had endured, it is not likely that they practised exaggerated continence. There would, therefore, be every chance for the disease spreading directly or indirectly from these men. The result of the dispersal of Charles's army was a fearful epidemic of syphilis, and the progress of this epidemic can be traced from contemporary history.

At once both medical and lay writers began to describe a disease of the genitals which was accompanied by constitutional effects, and which was an entirely new disease to them. That syphilis was a new disease is shown by the fact that none of the afflicted nations had a name for it. The Italians called it the Spanish disease or the French disease, and not unjustly, for it was probably introduced by Spaniards serving under Charles VIII. The French called it the Italian disease, because Charles's defeated troops brought it back with them from their Italian campaign. The English called it the French disease, because it reached England through France, and the Scots and Turks gave it also the name of the French disease. 
Andrew Boorde, 1575, says: "In English Morbus Gallicus is called the French pocks, but when that I was young they were named the Spanish pocks." The Hindus and the Japanese called syphilis the Portuguese disease. From this varied nomenclature we may trace the path of the disease. Any doubt of the origin of syphilis is removed by the knowledge that the first Spaniards to recognise this new disease called it the disease of Española, and Española was the name then given to Hayti.

A Portuguese physician, Ruy Dias de Isla, was practising in Barcelona in 1493, the year of the return of Columbus. He leaves on record his conviction that syphilis came from Hayti, and that it appeared first in the crew of Columbus.

It has been suggested that Spanish authors might wish to shift the blame on to the West Indians, as partial excuse for Spanish barbarities to natives. This argument will not stand, for Las Casas (1550) devoted his life to the betterment of the natives, and he mentions "Las Bubas, in Italy called the French disease," as being introduced into Spain by the crew of Columbus or by Indians whom he brought back with him. Also, natives of Hayti, on being questioned, said that syphilis had been common among them for as long as their traditions went back.

From all these definite reports, it appears clearly to be established that syphilis was introduced into the Old World from Hayti by the crew of Columbus. The diabolically humorous genealogy of syphilis in Voltaire's Candide goes back to the fountain-head, and, though not strictly scientific, is well worth reading.

The name syphilis is first mentioned by an Italian physician, Frascator, who in 1530 published a poem, Syphilis sive Morbus Gallicus. Using the quasi-classical poetical machinery which was then fashionable, he describes how the disease was inflicted by the pagan gods on an offender. He probably manufactured the word from $\sigma u ́ v$ and $\phi \iota \lambda$ ós.

Syphilis spread over the whole known world with most horrifying rapidity. The epidemic was noted not only because of the novelty of the disease, but also because of its fearful severity. Within half a century the disease had taken on the modified character with which we are now familiar, and since that time it has been everywhere endemic.

The spread of the epidemic was as follows: Italy and Spain, 1494; France, Germany, Switzerland, 1495; Holland and Greece, 1496; England and Scotland, 1497; Hungary and Russia, 1497; India, 1498; Japan, 1569.

Among the other accusations against Cardinal Wolsey when he was indicted before the House of Lords in 1529 was that of infecting Henry VIII., by breathing on him. Whether this expresses the medical ideas of the day does not appear clear; most probably it was the happy invention of some ingenious lawyer who wished to stand well with the King.

The rapid spread of syphilis among hitherto uninfected races has been lately well exemplified in the case of the African nations, to whom syphilis, 
along with the other blessings of civilisation, was introduced, and among whom the disease spread as extensively as it did in Europe at the beginning of the sixteenth century.

In 1514 Jean de Vigo, writing on Le Mal Français, said that treatment by mercury had given good results. Later on, guaiacum was extensively used. It was at this time that the idea was born that syphilis and gonorrhoa were one and the same disease; and it is most interesting to note that P. Haschard in 1554 recognised that the two diseases were entirely different, for he pointed out that mercury should be used in the treatment of syphilis but not in the treatment of gonorrhœa. Haschard's work was either largely unknown or disbelieved, for many still considered the diseases to be identical.

In 1767 John Hunter inoculated himself with what he supposed to be gonorrhœal pus, and he developed syphilis. This telling experiment silenced all opposition, and the identity of the two diseases was everywhere accepted. In 1786 Hunter published his "Treatise on the Venereal Disease," in which he advocated the use of mercurial treatment. It is astonishing to find that he disbelieved in the hereditary transmission of syphilis, noted by Paracelsus in 1526.

In 1792 Bell inoculated two physicians with gonorrhœal pus, and both developed gonorrhœa, but not syphilis. The magic of Hunter's name, however, was so strong that this piece of evidence was not considered to be conclusive of the dual nature of the diseases.

In 1811 Hernandez inoculated seventeen convicts with gonorrhœal pus. They all developed gonorrhœa, and none of them developed syphilis.

Between 1831 and 1838 Ricord inoculated 667 persons with gonorrhœal pus, and not one of them developed syphilis. This was conclusive, andNeisser's demonstration of the gonococcus in 1879 merely served to make assurance doubly sure.

Williams and Wallace introduced potassium iodide as an antisyphilitic drug, 1830-1835, and Ricord followed them, without knowledge of their discovery.

Ricord for some time thought that secondary syphilitic lesions were not contagious; but fuller experience caused him to alter his opinion-in 1859thus confirming the experience of Wallace, who, in 1835, infected healthy persons by inoculation from secondary lesions.

Ricord did not recognise the difference between simple and syphilitic chancres, but Rollet, in 1852, showed the difference, from examinations of infecting and infected persons. Rollet showed the existence of mixed chancre, caused by double infection, and Ducrey, 1889, discovered the bacillus of soft chancres. It must be added to Ricord's credit that, strongly as he held the view that the two sores had the same ætiology, he afterwards altered his opinion when proof had been brought to the contrary, and his remark, L'homme est absurd qui ne change pas, is one that might well be reiterated by some of his present-day successors. 
Hunter seems to have failed to recognise syphilis of the nervous system, although Van Swieten noticed it in 1772. About a century later, much work on syphilis of the nervous system was done, particularly by Fournier, who, in $\mathbf{1 8 7 5}$, discovered the syphilitic origin of tabes and general paralysis.

An English contemporary of Fournier, Hutchinson, added much to our knowledge of syphilis, so much so that Ricord, Fournier, and Hutchinson can well be named the fathers of clinical syphilology. A sudden change took place at the beginning of the twentieth century which has tended to supplant for clinical, pathological syphilology. In 1905 Schaudinn and Hoffmann discovered the Spirochota pallida, alleging it to be the cause of syphilis. In 1907 Wassermann's non-specific complement fixation test saw daylight, and in 1909 we had Ehrlich's discovery of salvarsan. These three discoveries may be aptly called "the German syphilitic trinity." These discoveries have advanced our knowledge considerably; but, on the other hand, they have made clinical diagnosis-the highest attainment in medicine -take a back seat, with rather disastrous results. The German syphilitic trinity has cast such a spell over the medical world, with the exception perhaps of France, that it almost amounts to heresy to suggest that all is not as is stated. As the following pages will indicate, I believe that the Spirochoeta pallida is only the adult male of a coccidial protozoon, that the C.F.T.* is merely a physical reaction dependent upon the increased number and size of the protein colloidal particles in the serum, and that salvarsan only destroys the parasite indirectly by increasing the oxidising action of the host's protective substance. As proofs for these views are beyond the scope of this, a clinical volume, and as the work is being confirmed, a special pathological volume will be published as soon as possible.

* Throughout the book C.F.T. will stand for complement fixation test, and C.s.F. for cerebro-spinal fluid. 


\section{CHAPTER II}

\section{THE STAGES OF SYPHILIS}

\section{The Primary Stage.}

AfTER a patient has exposed himself to infection, one or more spores gain access to the skin, in which they develop. When sufficient cycles, which comprise the life-history of the Leucocytozoon syphilidis, have been completed, the patient develops a sore. As the host cannot immediately form protective bodies, there is no limit to the number of sores which may be present, the number depending upon the different points of entrance of the organism. The sore is also auto-inoculable up to the eleventh day, therefore multiple sores may be produced from the initial sore. In the latter case, the secondary sores are smaller, less developed, and less indurated than the primary sore.

In 24.0 per cent. of cases of syphilis (5,000 cases examined) there is more than one primary sore, the most I have ever seen being eighteen.

When the spore has entered the body, it seeks out a connective-tissue cell, or an endothelial cell, to the protoplasm of which it gains access. Therefore, the connective-tissue cell and the endothelial cell are the first cells to be attacked in syphilis; consequently, the brunt of the attack, and the resistance offered, will affect these cells, with the result that there will be marked multiplication of them.

Connective-tissue cells later become fibrous tissue, hence the partial explanation of the so-called induration of chancres. The induration is purely relative, since a sore may appear before the connective-tissue cells have had time to form fibrous tissue, or a sore may develop in loose tissue, in which, if fibrous tissue did form, it would be scarcely noticed; or the endothelial cell may be the main cell attacked, in whioh case there is practically no proliferation of the fixed connective-tissue cells. Furthermore, in those cases in which only the asexual stage is perpetuated, the endothelial cells are the cells most involved; hence the type of sore formed is never indurated.

As a sore may appear before there is any induration, it follows that the incubation period of syphilis must vary somewhat, as it does-from eight days to six weeks, or more, the average being thirty-four days (300 cases examined). As the connective-tissue cells and the endothelial cells are first attacked, there will be no great call upon leucocytes, and as, in my opinion, phagocytosis plays no part at all in protozoal infections, there will be no pus and 


$$
\text { 1. . } 1 \text { i. I : }
$$

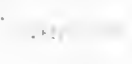

...

-

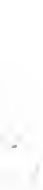

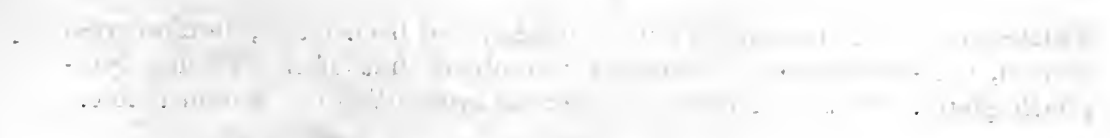

. 


\section{PLATE I1}

No. 2.-A Keloid on tile Site of a Prinary Sore on the Mons Veneris of a JAPANESE

No. 3.-A Locairsed Syphirona Which DEVEloped in A

LyMPIIATIC VESSEL

ThE lesion shelled out after a dorsal incision had been made, and, on cross section, the microscopic appearance resembled that of a syphilitic lymphatic gland. Phases of the Leucocytozoon syphilidis were demonstrable. 
No. 2
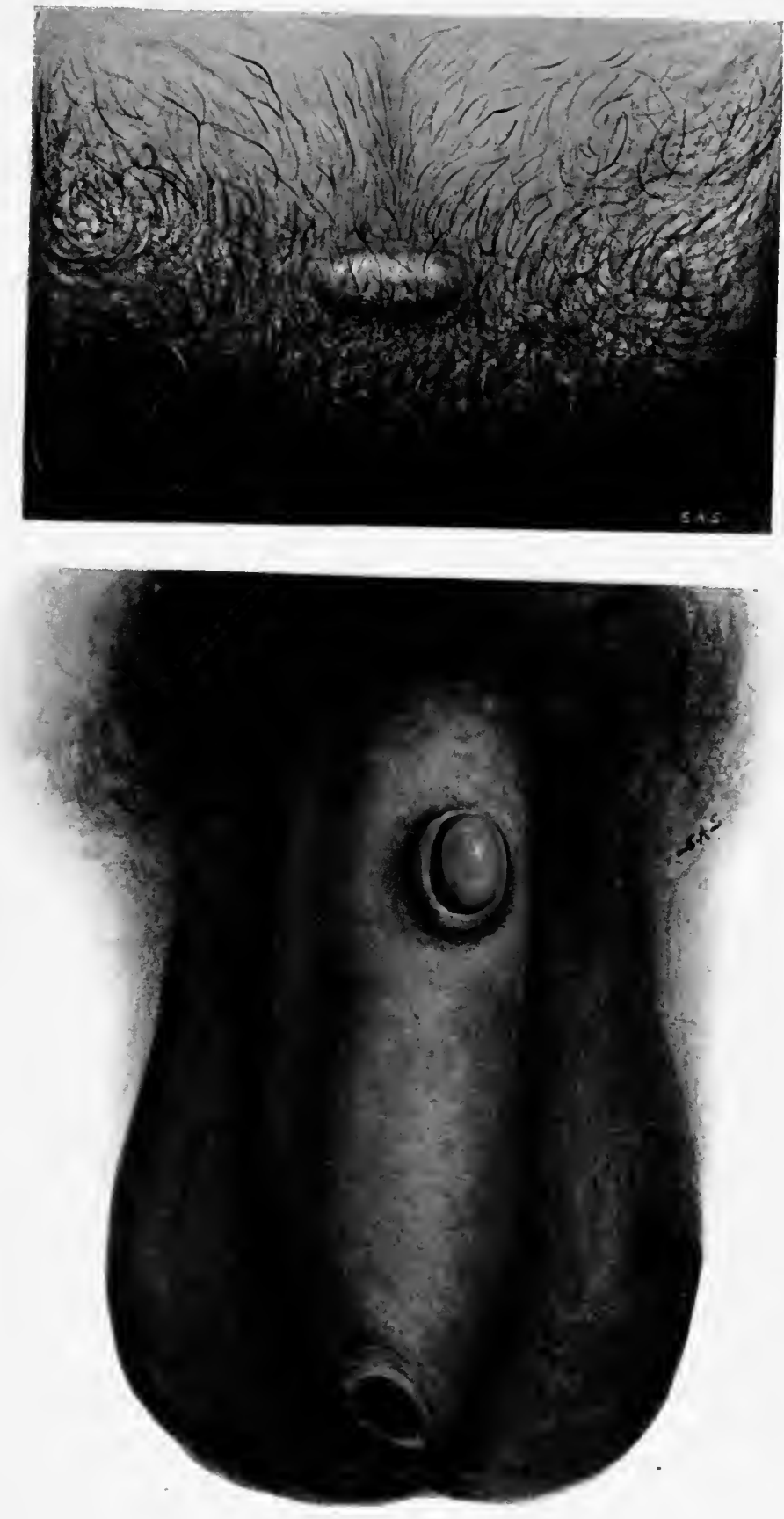

No. 3

Plate II 

no necrosis. Therefore, if a sore occurs, it will be an erosion, and not an ulcer. Occasionally a chancre is to be met with in which the surface epithelium never breaks. In the sore formed by the asexual development of the Leucocytozoon syphilidis, owing to the manner in which the endothelial cell is attacked, and its consequent multiplication, vessels become occluded. This occlusion results in necrosis of the skin above, hence such a primary sore is an ulcer from the start, and often a granulating one.

As a primary sore most usually affects regions which are swarming with saprophytic organisms, it may easily become infected, or even phagedænic; then, instead of a simple erosion, there may be a big ulcer.

If the saprophytic organisms attack the sore early enough, and if they are particularly active, they may succeed in annihilating the leucocytozoon. This explains why some phagedænic sores are not followed later by further evidences of syphilis. In any case, the onset of phagedæna alters the sequence of the disease, usually by delaying the outbreak of the rash, sore throat, etc. Owing to the secondary infection sometimes poisoning the patient, a phagedænic chancre may be followed by malignant syphilis.

In these observations $I$ see the indication for removing a primary sore, when possible, or for rubbing a mercurio-intramine ointment into those that cannot be so handled. As cauterisation may mask the diagnosis in a doubtful case, and be the cause of a secondary infection, owing to the necrosis produced, this measure should not be adopted.

In comparing the primary sore of human syphilis with that of experimental syphilis, one is at once struck by the great tendency to ulcerate exhibited by the latter. Moreover, the experimental sore is always clinically the same, while the sore of human syphilis may show numerous clinical varieties.

A human chancre is generally an erosion and not an ulceration (the asexual sore excepted); the experimental sore is always an ulceration.

From this simple clinical difference between ordinary and experimental syphilis, and from the light which has been thrown upon the causative agent of the disease, it may be assumed that, as the lesions of experimental syphilis never vary, only one portion of the life-cycle of the specific organism develops. Since spirochætæ are found in greater abundance in experimental chancres than in ordinary chancres, and since the long angulated forms are frequently to be met with, it is possible that only the extracellular development of the male body occurs.

The spirochætæ obtained from experimental chancres are typical of those grown in culture, and those I have found in the brain from cases of degenerative encephalitis. Therefore, it will now be seen that my remark, "What is happening in the test animal's body may be no more than is taking place in a culture tube," is possibly near the truth. In rabbits, after the primary sore has existed for a little time, the inguinal lymphatic glands become enlarged and the virus becomes generalised in the system. According to Graetz 
and Delbanco it is extremely rare for any histological changes to be found in the inguinal lymphatic glands, and often spirochætæ cannot be demonstrated. To prove that the virus is undoubtedly harbouring in them, it was found necessary to inoculate another animal with part of the lymphatic gland.

Comparing this with human syphilis, again great differences arise. Broadly speaking, the smaller the lymphatic glands the more marked are the histological changes found therein. Many of the small lymphatic glands which I have examined are crowded with giant cells and small areas of necrosis, exactly resembling a tubercular lymphatic gland. The cells in the small lymphatic glands are mainly endothelial cells: a few lymphocytes may be found, and only a very small number of plasma cells, showing that the resistance of the host is feeble, and that his last line of support has been attacked.

The large glands also show marked changes; but, instead of consisting mainly of endothelial cells, they are crammed with plasma cells. It is not difficult to find spirochætæ in the inguinal glands removed from human syphilis.

Tissue which, when inoculated into animals, gives rise to lesions from which spirochætæ can be obtained, need not necessarily contain spirochætæ, etc. Therefore, when the organisms are found in the fresh lesion, it is no proof that the tissue which gave rise to that lesion harboured spirochætæ. All that can be said is that the tissue in question contained spores, which were capable of giving rise to spirochætæ when inoculated. As I have frequently been able to demonstrate, spores of the Leucocytozoon syphilidis do not give rise to inflammation, and therefore the histological structure of any tissue in which they are present may remain unaltered. The phase which causes the greatest histological change is that of the Spirochoeta pallida, the adult male form.

It must be noted how frequently animals which have been inoculated with syphilitic material fail to develop even an initial lesion. It is doubtful whether a single human being would escape under similar circumstances. The quantity of material that is usually required to infect the animal should also be taken into consideration; it would be equivalent to giving a human being a few ounces of the infective material.

Therefore it is not surprising that an infection arises, or, rather, what is taken for an infection.

\section{Other Stages.}

At a varying interval after the sore a lymphangitis and enlargement of the lymphatic glands occur, and this is part of the host's protective machine, and is for the purpose of elaborating lymphocytes to attack the infection. The enlargement of the lymphatic glands bears no ratio to the severity of the disease. On the contrary, it bears a ratio to the protective capacity of the host; hence big lymphatic glands are not the best to choose in which to hunt. 
for organisms-it is in the small lymphatic glands that most are to be found.

Lymphatic glands should be looked upon as the base on the field of operation, and therefore should not be removed, as has of late been advised.

Another point in favour of my view that phagedæna kills the syphilitic organism is the fact that most phagedænic chancres are not accompanied either by a lymphangitis or by an enlargement of the nearest chain of lymphatic glands; oddly enough, not even the secondary infection causes them to become enlarged. The reason of this is, that when a chancre becomes phagedænic the secondary infection is not a staphylococcic or streptococcic infection, but an infection due to the fusiform bacillus and the Gram-negative spirochæta, which live in symbiosis. The glandular enlargement following this infection is minimal.

From the nearest chain of lymphatic glands the organisms spread along the lymphatics, until other chains and, ultimately, all the lymphatic glands in the body are infected. While the lymphatic extension is proceeding, the organisms are also pervading every nook and crevice in the body by means of the bloodstream. In this way the generalisation symptoms arise.

The symptoms will eventually disappear without treatment; but their disappearance is hastened by the administration of mercury, which works slowly, or of arseno-benzene, which works almost instantaneously.

The action of treatment is primarily to destroy the spirochætæ, which are mainly responsible for the symptoms. The other phases are destroyed secondarily and indirectly. As the spirochætæ are mainly responsible for the lesions, the protective mechanism of the host will be especially directed against these bodies, but their death does not mean that the spores are destroyed.

If no treatment be given, or if mercury alone be prescribed, the spirochætæ will vanish for a time. The spores, as they seek fresh hunting-ground, will spread peripherally, so that when the symptoms recur the lesions will be in the form of circles, or segments of circles. When no symptoms are visible the patient is said to be in the latent stage, and when symptoms reappear again he is said to be in the recurrent stage.

If arseno-benzene is prescribed the spirochætæ are destroyed at once; the spores are crippled temporarily in situ, so that when they start their life-cycle again it will take place in the same positions; hence the reason why recurrences after arseno-benzene simulate the lesions for which the drug was given.

The early lesions of syphilis are more infectious than the recurrent ones. Therefore, insufficient use of arseno-benzene, or the failure to supplement its administration with mercury, iodine, and sulphur, may do more harm than good, in that the infectious period may be thereby lengthened.

I have seen ten cases in which the wife was infected by her husband, 


\section{CLINICAL ASPECT AND TREATMENT OF VENEREAL DISEASES}

who had been told that he was cured, after he had had a course of arseno-benzene injections.

In view of what has been stated, and for reasons which will be given later, I feel that I am justified in advising, as has been my practice for over seven years, several injections of arseno-benzene, given as close upon one another as possible, to be followed by one to two years' treatment with mercury, iodine, and sulphur.

The longer the spores are present in any one spot the more chronic inflammatory changes will the local vessels exhibit. Hence, should a lesion occur, it will lead to still further trouble, even to obliterative endarteritis, which will result in necrosis of the skin over the area fed by the affected artery, and the formation of a gumma.

A gumma occurs mechanically, and the necrosis is not due directly to the specific organisms. In the necrosis, saprophytic organisms flourish, and they at once kill the leucocytozoon, with the result that the secretion therefrom is, to all intents and purposes, non-infectious. The specific organism lives in the tissue surrounding the necrosis.

As the endothelial cell is most frequently the cell upon which the Leucocytozoon syphilidis is parasitic, it will be readily understood why vascular lesions are so common in syphilis.

The biology of syphilis in women will be considered in another chapter, and likewise the biology of syphilis of the central nervous system, and congenital syphilis.

As a recurrent lesion appearing many years after infection may simulate a lesion which is most commonly seen soon after infection, it appears to me best to drop the division into primary, secondary, and tertiary stages, and to adopt the following nomenclature:

Primary Stage-CFrom the first appearance of the sore till the C.F.T. becomes positive.

Generalisation Stage.-From the moment the blood becomes positive till the organisms, after spreading over the whole body, become dormant. This stage lasts for roughly two years.

Latent Stage.-When the organisms are dormant.

Recurrent Stage.-When the organisms become active again and produce lesions. 


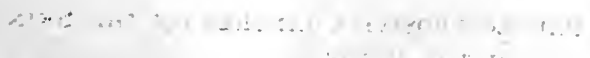

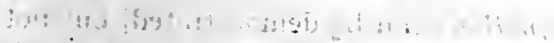

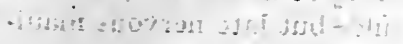

$$
\therefore 2 \quad \therefore \cdots
$$

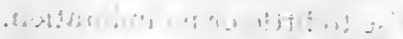


PLATE III

No. 4.-Typical Papulo-enosive Cilancres on tile Skin of thif. Penis

TiE phases of the Leucocytozoon syphilidis can be demonstrated, but not readily. Tlue generalisation stage is usually mild, but late nervous manifestations are common.

No. 5.-Three Papulo-erosive Chancres on the. Preplce, OF THE SAME NATURE AS THE ABOVE

The sores are true erosions and give rise to little or no induration. 
No. 4
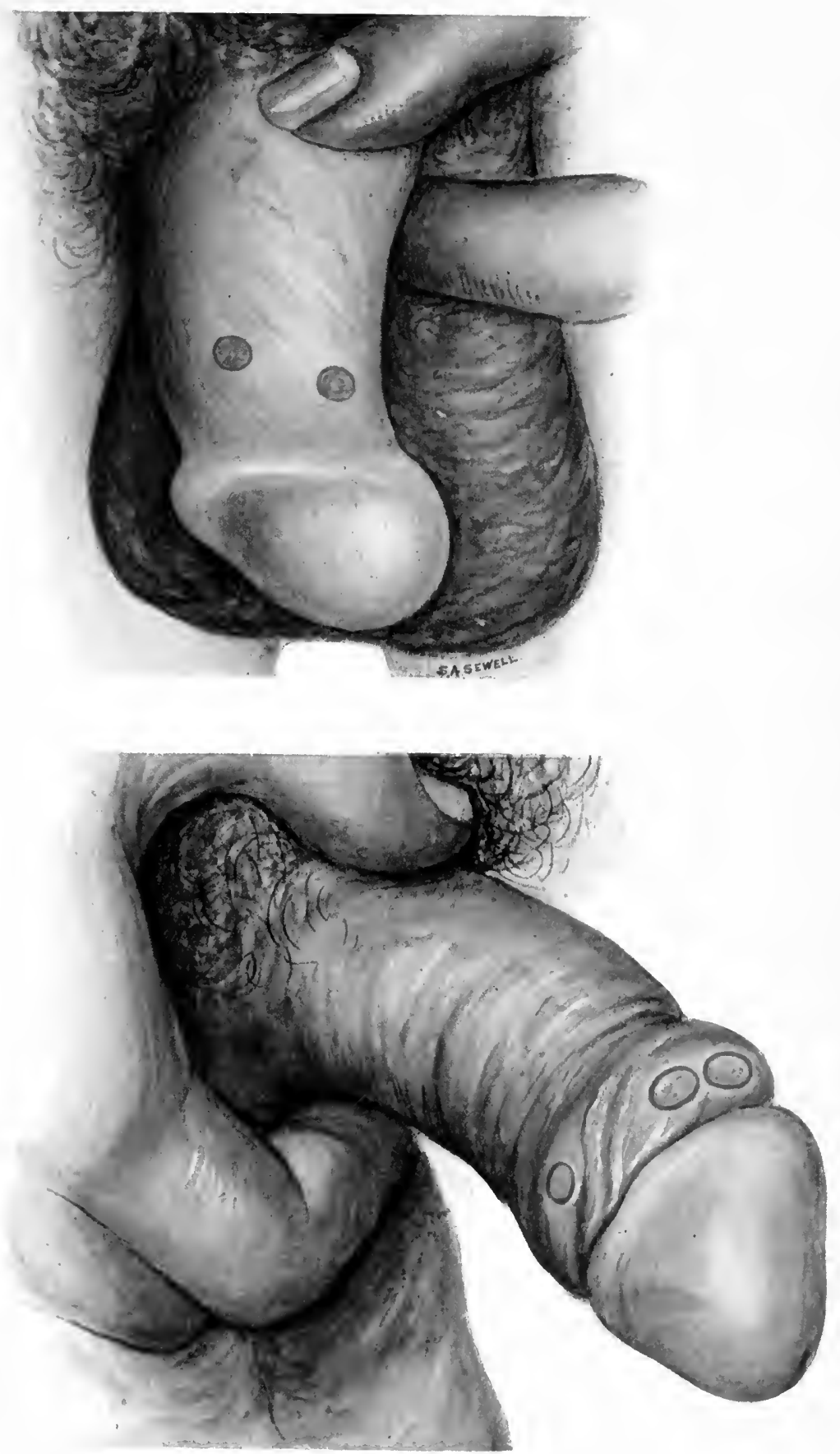

No. 5

Prate III 



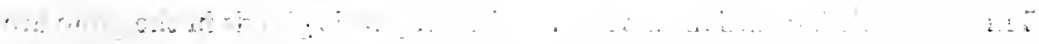

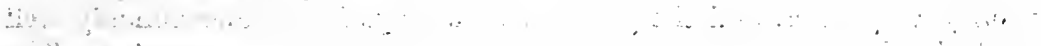

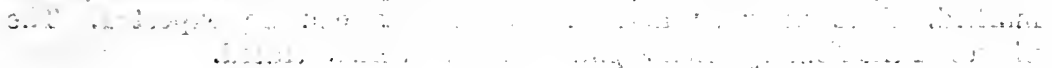

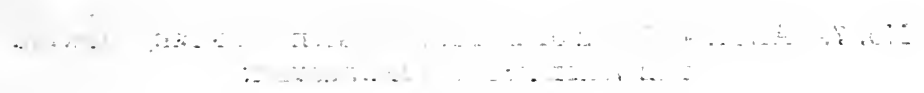

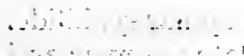$$
\therefore+\cdots, \cdots, \cdots
$$$$
\therefore \quad \cdots \quad+\quad \cdot \quad \cdot
$$ 


\section{PLATE IV}

\section{No. 6.-Papulo-erosive Chancres}

THERE is little induration as a rule. The lymphatic glands in the groin are usually very hard, and the generalisation stage is not uncommonly well marked. Late vascular and nervous lesions can be expected. The phases of the Leucocytozoon syphilidis can be demonstrated.

No. 7.-Anotier Type of Papulo-erosive Chanche, siowing THE WELL-MARKED INDURATION

THis type is, as a rule, followed by a stubborn generalised papular syphilide. Recurrences are frequent, irrespective of treatment, and late vascular and nervous lesions are not uncommon, especially if the treatment be spasmodic and with metals only. The phases of the Leucocytozoon syphilidis can be demonstrated. 
No. 6
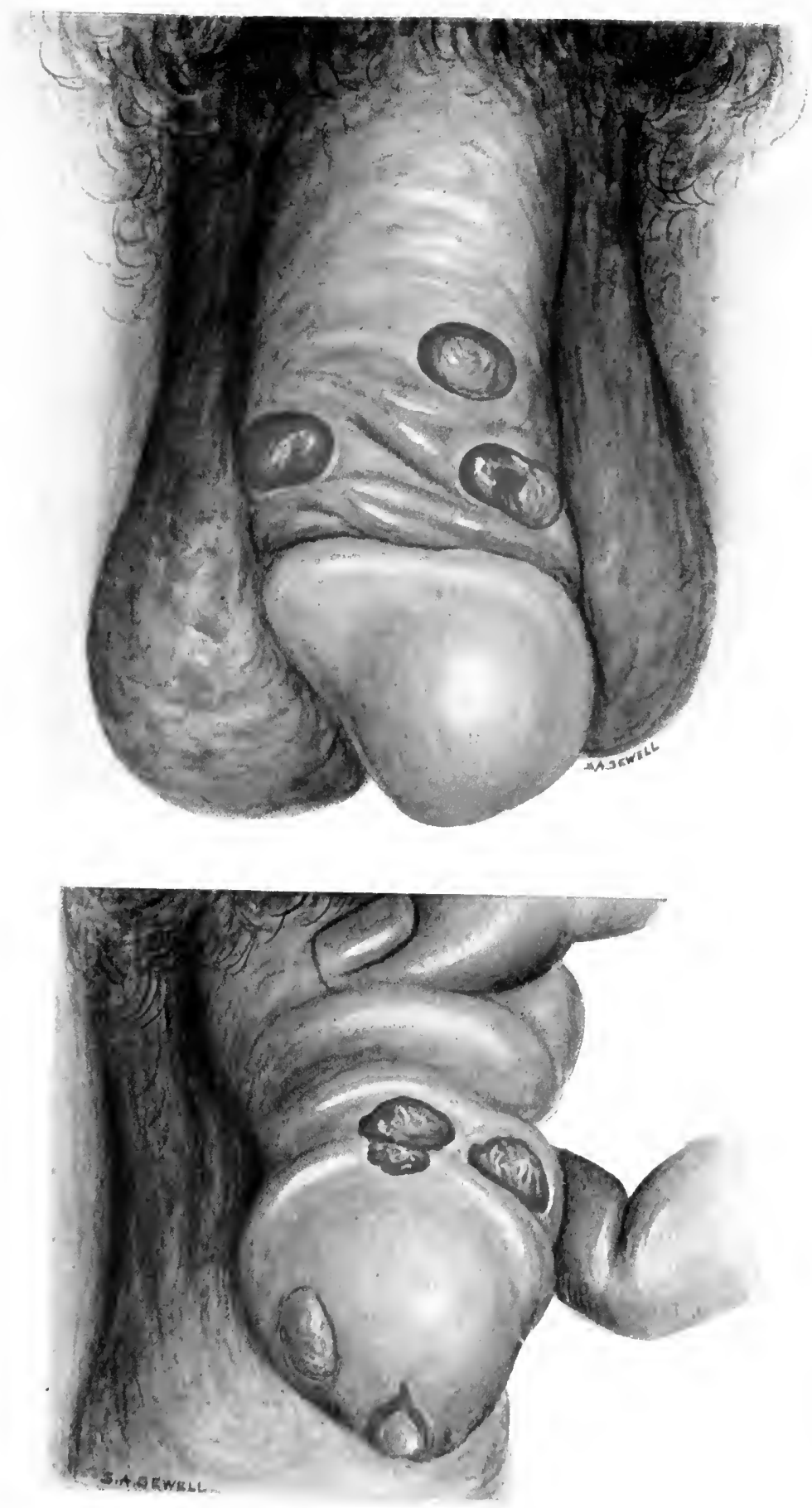

No. 7

Prate IV 



\section{CHAPTER III \\ SYPHILIS OF THE SKIN}

\section{History.}

THERE is nothing particularly interesting in the history of syphilis of the skin. The diagnostic EEdema indurativum received little or no attention till about 1850, while Syphilis d'emblêe appears to have been well known since the time of Fallopius, who died at Padua in 1562. Extragenital chancres were known to Paracelsus in 1528, and Torella, in 1497, described the transmission of syphilis between sucklings and nurses, and through kissing. The lancet used for blood-letting was frequently responsible for conveying infection, and all know the stir in the last century vaccinal syphilis caused in the medical and daily press. The signs and symptoms of the generalisation stage, which received first the name of "second infection," were not clearly outlined until about 1770. The various skin eruptions received at different times all the fancy names which the older scientists were renowned for coining. The so-called "raw ham colour" of syphilis undoubtedly dates from Fallopius, who first referred to it as Porcinoe carnis salito color. The Corona veneris dates from Rondelet, who described it first as Papula carnosce in fronte. Alopecia was known to the earliest writers. Leucoderma syphiliticum was first described in Astruc's handbook (1736) as Cutis maculoe ephelidibus similis, and for years afterwards the condition was known as venereal ephelides; it was not until 1881 that the true nature of this condition was recognised.

\section{General.}

A written description of the cutaneous syphilitic lesions may give a little help in diagnosing them when seen. It must, however, be remembered that the only way in which anyone can obtain a good clinical knowledge of the skin manifestations, is by careful study of as many cases as possible. This especially applies to the chancre.

Everyone is agreed that if syphilis is to be lessened, diagnosis of the initial lesion at the earliest possible moment is essential. I feel very strongly that the best diagnosis is a clinical and not a bacteriological one. Therefore, it behoves the whole medical profession to make themselves au fait with the clinical methods of diagnosing early syphilis, and to see that the future generation is thoroughly taught such methods. 


\section{Chancre.}

A primary sore may occur on any part of the body, but, for the sake of convenience, primary sores may be divided into genital and extragenital sores. In most countries the syphilitic infection is genital in origin, but in some districts, where the people are very poor, uncleanly, and many live together in one room, the infection is more often extragenital. In certain parts of South-Eastern Europe, the ratio between extragenital and genital sores may be as high as 20 to 1 .

Four main points are usually sought for in diagnosing a sore. (1) The sore must be single; (2) it must be indurated; (3) it must not appear for from four to six weeks after connection; and (4) the lymphatic glands in the groin must be enlarged and hard.

Let us take each of these points in turn, and see how far they are of value in assisting one to make a diagnosis in a difficult case. In 24 per cent. of cases of syphilis there is more than one primary sore when the infection is a genital one. When extragenital, the sore is more commonly single. A soft sore, which appears in the minds of most to be the only sore which has to be distinguished from a primary sore, is also sometimes single, especially if it be seen early or is of the elevatum type.

Induration, when present, may be valuable as a proof of syphilis, but its absence by no means negatives syphilis. Induration is, in part, a process of healing; consequently, if patients are to be urged to seek advice the moment they notice a sore, the value to be placed upon induration as a diagnostic sign will be considerably diminished. A chancre, as a rule, becomes most indurated when it is about to disappear; consequently, if the sore is indurated when the patient seeks advice, the chancres are greatly in favour of the generalisation stage having already started. Many sores heal and vanish without ever becoming indurated.

The sore must not appear for from four to six weeks after connection. Those who wish to diagnose a sore correctly will be well advised never to ask how soon after connection a sore appeared. In the first place, the period of incubation varies from eight to sixty days; in the second place, many men have connection once a week, and the chances are that they will blame the woman with whom they last had intercourse.

The lymphatic glands in the groin must be enlarged and hard. In about 5 per cent. of cases no palpable change in the lymphatic glands can be ascertained. In many cases it is impossible to distinguish an enlargement due to syphilis from an enlargement caused by any other venereal disease; for instance, in about 90 per cent. of all cases of acute gonorrhoea the inguinal lymphatic glands are enlarged. Hardness, if present, is characteristic of syphilis, but it means that the patient has passed into the generalisation stage-indeed, almost any change in the lymphatic glands signifies that the 


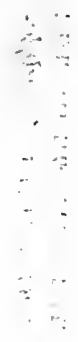




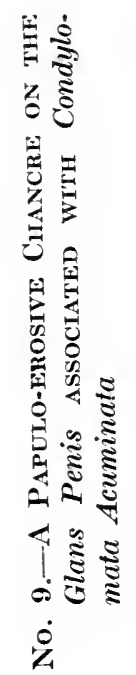

ह

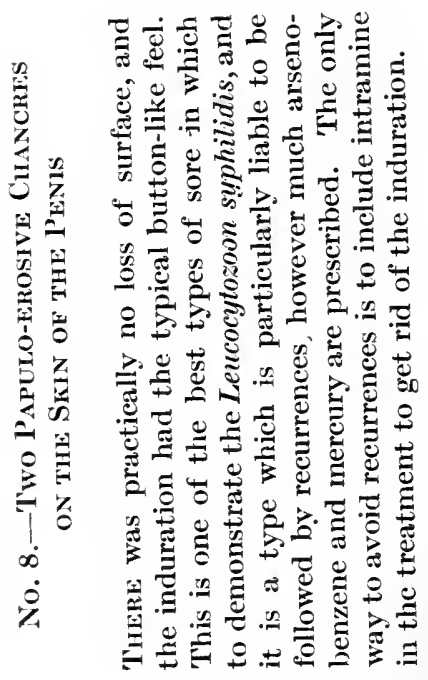




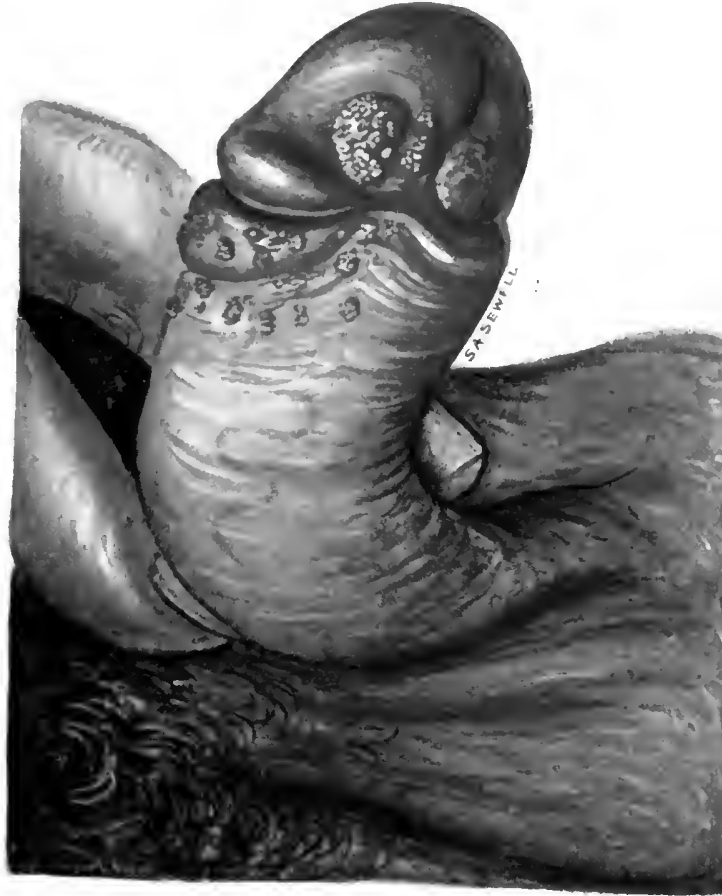

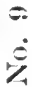

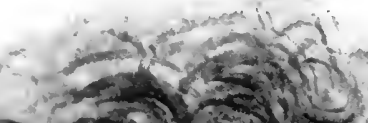

$\stackrel{5}{5}$
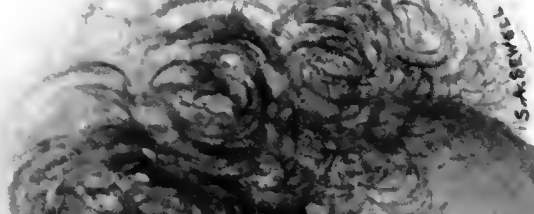

(a) $x^{2}+x^{2}$

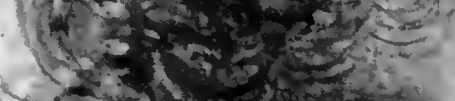

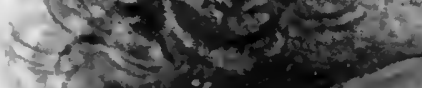
.

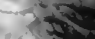
(3) 3

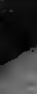

.

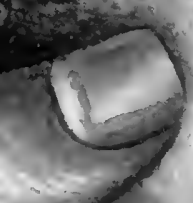

$y^{2}$

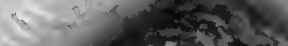

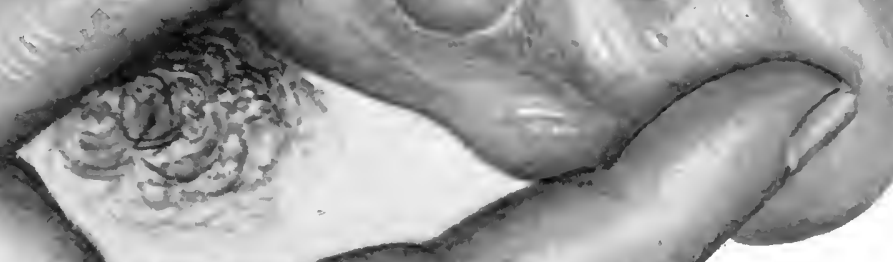





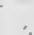

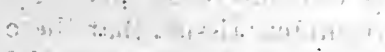
$\therefore+\ldots+2 .+2$

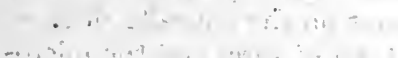
… $\because \quad \therefore, \cdots$ $\because \quad \because \quad \%$ $\therefore \quad 2193 \cdots+\cdots !+\cdots$ ari $\cdots+a$ $\because \vdots: i^{\circ}$

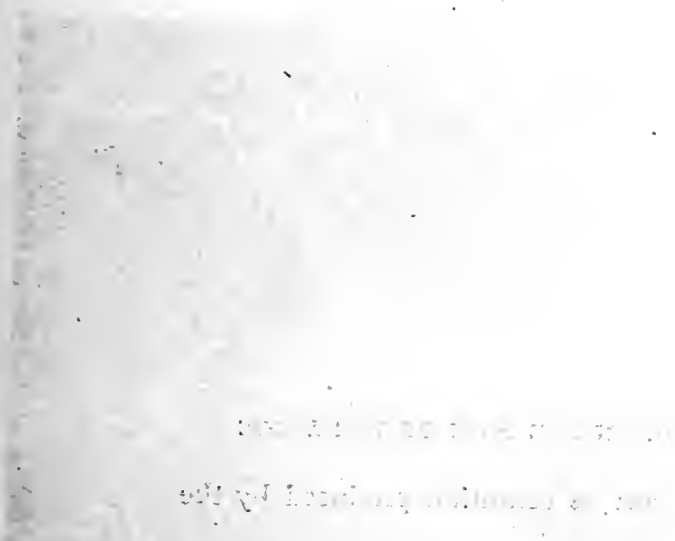




\section{PLATE VI}

\section{No. 10.-Papulo-trosive Chaxcre in tile Corona}

IT should be noted that the lesion is sharply circumscribed, perfectly regular in outline, that it is raised above the surrounding tissue, that there is very little loss of surface, and that there is not a trace of circumferential inflammation. The patient had two similar sores on the opposite side.

Spirochaetae can always be found in this type of sore, and the microscopic appearance of the lesion is characteristic. There is a marked hyperplasia of the connective-tissuc cells; the walls of the vessels are thickened, and the endothelial cells are increased in number. There are few leucocytes, and those present arc nearly all plasma cells ; the others are lymphocytes, but there are no polymorphonuclear leucocytes. The section is crowded with all the phases of the Leucocytozoon syphilidis. This is the type of sore which gives rise to the severest cases of nervous sypliilis.

No. 11.-Papulo-erosive Cilancre on the Skin of the Penis

Showisg the anaemic ring round the sore, a condition produced by the induration. 
No. 10
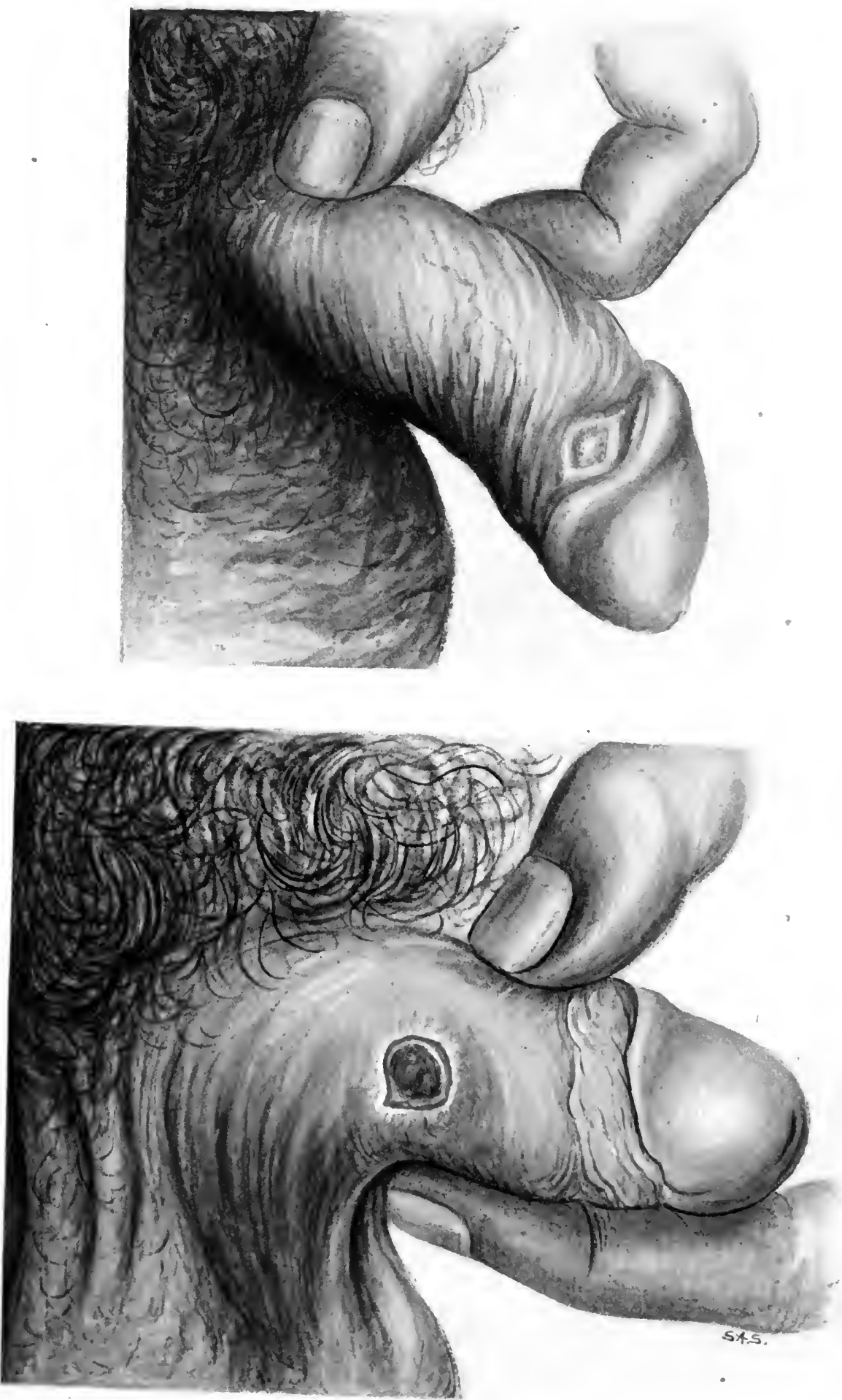

No. 11 
disease has become generalised. If cases are to be diagnosed much earlier than is now the case, palpation of the inguinal regions will give little or no clue as to the nature of the sore.

If there is still any doubt resort is had to a bacteriological examination. If the Spirochoeta pallida is found it is a proof that the sore is syphilitic; but if the organism is not found it is no proof that the sore is not syphilitic. In many syphilitic sores very few spirochætæ exist, and therefore they may easily be missed; in a few syphilitic sores the spirochætal stage is never reached; therefore, however careful the search may be, no spirochætæ will be found. In quite a large percentage of cases when the sore is in the corona, and the patient has a phimosis, it is impossible to reach the sore, far less to obtain a scraping from it.

If any observer would examine bacteriologically 100 consecutive doubtful sores, which, to a trained clinical eye, were considered to be syphilitic, le would be very much surprised and chagrined at the comparatively large percentage of cases in which he failed to find the Spirochoeta pallida; in fact, he would be fortunate to find it in 40 per cent. It is in just this type of sore that an inexperienced clinician is most dependent upon the finding of the bacteriologist.

There is only one certain way of diagnosing a sore, and that is by looking at it. I will endeavour in the following lines to put into words the mental pictures which I have of the various sores; but I must warn the reader that were these pictures ever so faithfully portrayed, they would not adequately take the place of a few visits to a hospital for venereal diseases.

The spore of the Leucocytozoon syphilidis is the infective agent of syphilis. When the spore gains entrance to a new host, it develops at the expense of the endothelial and connective-tissue cells. At first it produces little or no change in the host's protective cells, consequently; the incubation period of a syphilitic sore is a comparatively long one; but, naturally, it is dependent upon how the parasite develops, as its modes of development are various. If the Leucocytozoon syphilidis develops into male and female bodies, and it does so in most cases, the host will increase the production of his protective cells, as the male and female bodies cause the greatest reaction. The more protective cells that are formed, the more swollen will the lesion be, the epithelium is raised, and the tissue beneath the corium is depressed. Such a lesion is a papule. As more sexual bodies are formed, and more plasma cells and lymphocytes are turned out, the connective-tissue cells rapidly increase, and so do the endothelial cells. Now, every one of these facts must have an influence upon the blood or lymphatic supply to the epithelium directly above, with the result that any external friction will be just sufficient to injure it further, so that it is rubbed off. The lesion is now an erosion.

If the proliferation of the connective-tissue and endothelial cells persists, the fluid or nutritive supply to the tissue above will be cut off, with the result that it will necrose. The lesion is now an ulcer. 


\section{CLINICAL ASPECT AND TREATMENT OF VENEREAL DISEASES}

When necrosis occurs, a secondary infection is very liable to supervene, especially if the sore is nicely tucked away under a tight foreskin. The secondary infection is usually caused by the Gram-negative spirochæta and the Gram-positive fusiform bacillus, two organisms which will live in symbiosis, and which flourish well under anaerobic conditions. The lesion is now a phagedænic ulcer.

The process may stop at any one of the stages just mentioned. A primary sore may never be more than a papule. Most primary sores are simple erosions, but many primary sores are markedly indurated before any ulceration occurs.

With the exception of the sore that becomes secondarily infected, there is no increase of polymorphonuclear leucocytes, because phagocytosis plays no part in the destruction of the syphilitic parasite; hence there is no pus, and the necrosis which occurs is a mechanical necrosis, and is not due to the presence of several polymorphonuclear leucocytes, which cause necrosis owing to their proteolytic action. Therefore, the necrosis of a syphilitic sore, not secondarily infected, is a dry necrosis.

As the loss of surface of a syphilitic sore occurs more or less mechanically, it will naturally follow that the loss of surface will exactly correspond with the area of the cellular infiltration in the corium which is, by its pressure, shutting off the nutrition from the tissue above; consequently, the loss of surface will be circular, and circumscribed. As the host soon begins to protect himself against the parasite, and as there are no phagocytic cells present which have a proteolytic action, it will follow that the sore will neither increase in size, nor will there be any ragged edge, nor will the edge be raised or undermined, and there will be no circumferential inflammation. As the sore is an erosion the surface is smooth, red in colour, and raw-like, and one from which serum is being continuously pressed out.

Connective-tissue cells can proliferate more abundantly in some areas than in others; moreover, there are areas in which, even if the connectivetissue cells did proliferate abundantly, it would be difficult to feel them, owing to the looseness of the tissue. Hence, induration, when present, is most marked when the sore is in the corona; when in the skin of the penis it seldom gives rise to more than a feeling as of parchment, or as if a button were lodged in the skin. It often happens that sores on the glans penis never become indurated, and the induration of intra-urethral chancres is, as a rule, felt in one diameter only. There may be almost a circle of induration around the corona; and yet be no loss of surface, and, throughout the course of the button-like nodules in the skin of the penis, the surface epithelium may remain intact.

If there is a sore on the under-surface of the prepuce, when the skin is retracted, instead of the usual folds being seen the mucous membrane is drawn out tightly where the sore is, and it rolls back as one big piece. 'Any stretching of the part where there is induration formed or forming, will 


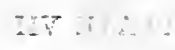

$$
\therefore \quad+5 \cdot \cdots: \cdots
$$

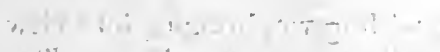

$$
\begin{aligned}
& \therefore \mathrm{L}^{\circ}
\end{aligned}
$$

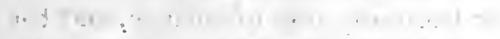

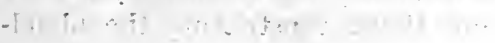




\section{PLATE VII}

No. 12.-Papulo-erosive Chaycre on the Under Surface OF THE FREPUCE

IT will be noticed that the prepuce has been withdrawn, bringing into view a white or swollen area, over which the folds of the prepuce are absent. The lesion felt like a button, there had never been any loss of surface, and the appearance is white owing to the fibrous tissue constricting the bloodvessels when put on the stretch.

\section{No. 13.-Papulo-grosive Chancre in Corona}

IT will be noticed that the epithelium has only just been eroded in the centre of the lesion and that the edges are white owing to the fibrous tissue formation, which has produced induration. 
No. 12
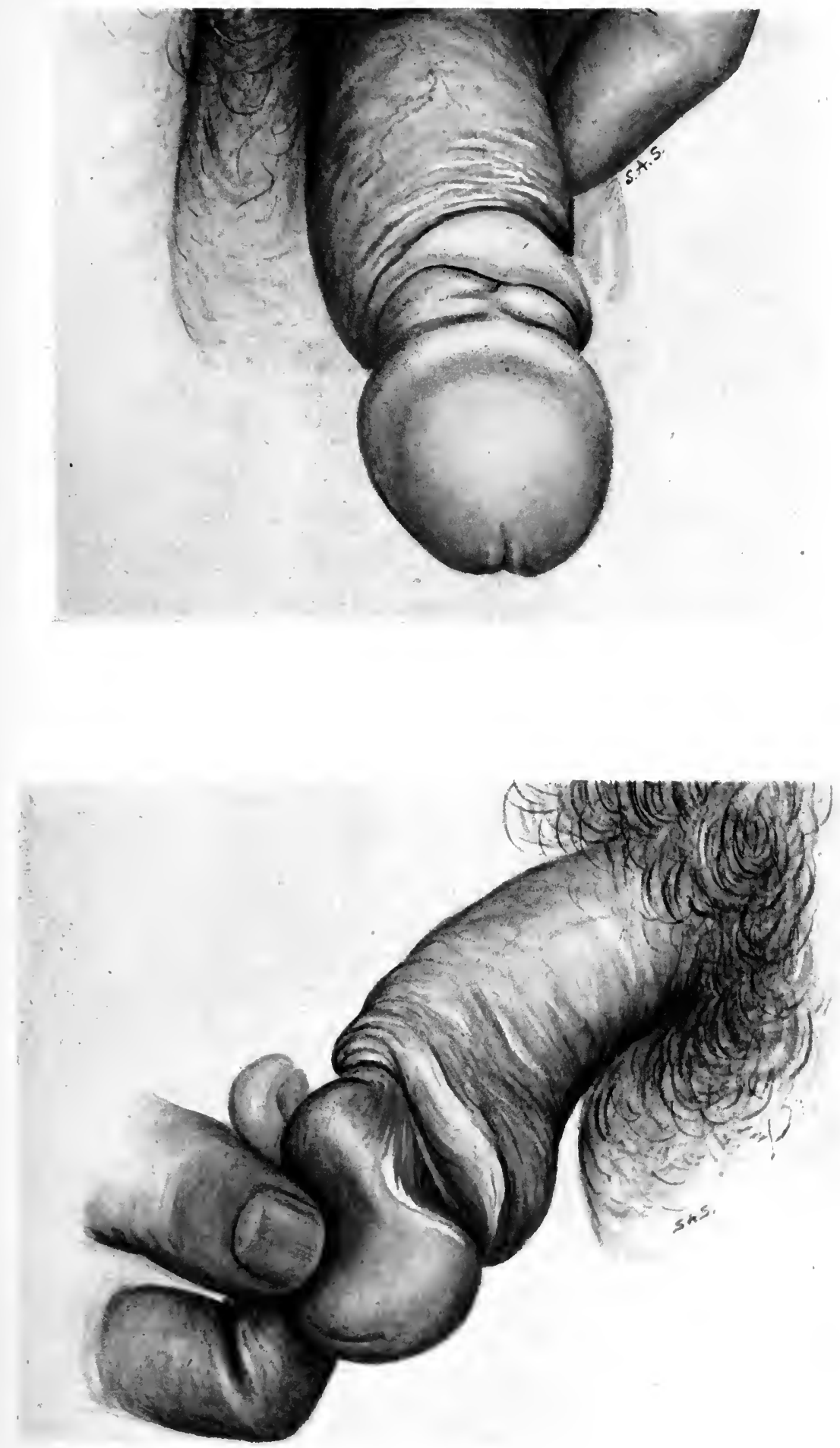

No. 13

Plate VII 



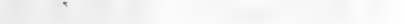




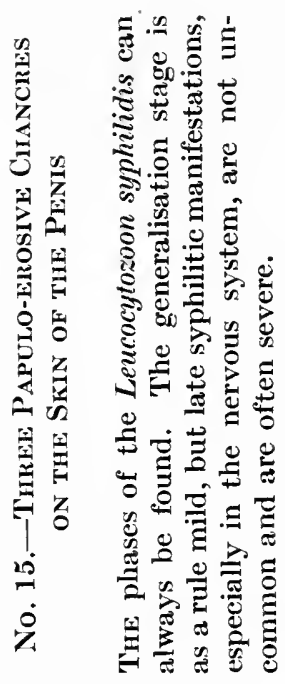

$\exists$

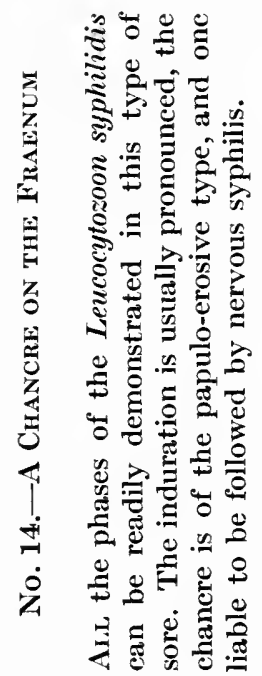



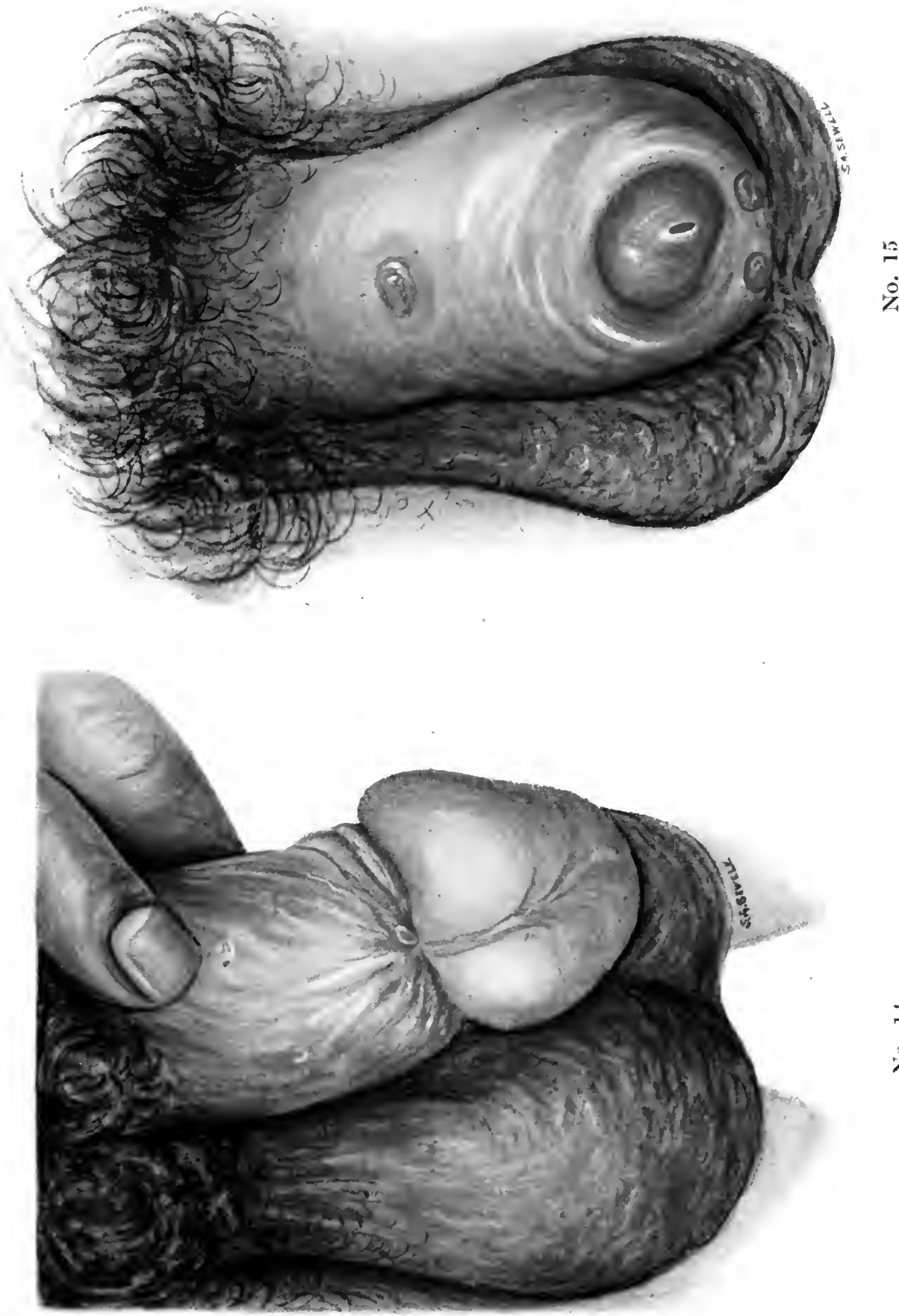

$\stackrel{2}{8}$

$\Xi$ 

produce a white colour, and the area left by a recently healed sore is always bluish.

In some sores, the Leucocytozoon syphilidis develops aberrantly, and does not give rise to sexual bodies. As to the percentage of such sores, I have as yet no knowledge, but I believe them to be not very uncommon. If the asexual stage be compared with the sexual stage, it will be noticed that the development of the former is more intracellular than that of the latter, and the phases formed are less motile, therefore there will be a greater call upon the endothelial and connective-tissue cells, and the process will be more localised. The more the endothelial cells and connective-tissue cells are attacked the greater the loss of nutrition to the tissue above, and the greater the likelihood of early necrosis. When one set of endothelial cells is finished with, the organisms will radiate peripherally, and will develop at the expense of another set. Such a manœuvre will result in the base of the ulcer being uneven. As the peripheral radiation may be incomplete, so far as the whole circumference is concerned, the lesion will not necessarily be beautifully circular, as in the type of chancre above described. A spread of the organism can more easily take place when it develops asexually, because the stimulation of the host's protective substances is practically nil. The ulcer is extremely chronic, not readily influenced by treatment, and the organisms in the end are most probably overcome by the host's local protective mechanism only. This type of sore is characterised by having a raised edge, but it is not undermined, and there is no surrounding inflammation. The reason why the edge is raised is perfectly simple. Practically every chancre at first is a papule,' whether the organism develops sexually or asexually; since, in the asexual development, the process is more localised, the destruction of tissue will occur in the centre of the papule only, and as the organism spreads and the necrosis gets bigger the protective cells will still outline the infected area, and, where protective cells are massed, there will be a swelling, consequently the edge of this type of chancre is always raised. Moreover, where there is necrosis, polymorphonuclear leucocytes congregate, consequently a collection of these will increase the size of the area affected.

In the other type of chancre, in which the nutritive supply is not wholly cut off in any one spot, but only diminished over the whole area of the infiltration, it will follow that the erosion will correspond exactly with the infiltrated area, hence the edges will not be raised, but the whole sore may be.

In other sores, possibly, the Spirochoeta pallida develops as it does in inoculation chancres. If the conditions under which a chancre is existing be altered, not only may the characters of the sore itself change, but a change in the life-cycle of the organism occurs also, with the predominance of one phase, which was barely in evidence before. Moisture will cause a chancre to ulcerate or to hypertrophy, and will cause the presence of spirochætæ in abundance, when before they could not be found. I mention this point about moisture, because it may be such a stimulus for the formation of spiro- 


\section{CLINICAL ASPECT AND TREATMENT OF VENEREAL DISEASES}

chætæ as to render the action of treatment upon them innocuous. I have recently seen two cases in which the chancre became hypertrophic, something like a condyloma, which went on developing, and from which myriads of spirochætæ could be obtained in spite of the patients being under the influence of arseno-benzene. Even after nine intravenous injections the lesions were worse than when the treatment began, and they only healed when a drying powder was applied.

To sum up: A primary sore is always at first a papule; it may remain so, but it usually becomes an erosion. Induration then commences, ulceration may follow, and the sore may become secondarily infected. The syphilitic sore is sharply circumscribed and non-inflammatory-i.e., when compared with inflammation as caused by pus-producing organisms.

Sometimes a primary sore may be an ulces from the start.

\section{Types of Chancres.}

Erosive Chancre.-This is the most common, and is very frequently multiple. It is often to be met with on the skin of the penis, and here it might be mentioned that if a patient has a sore or sores on the skin of his penis, the chances are 90 to 1 that they are syphilitic. The erosive chancres may encircle the corona, and when they occur on the glans penis they are the most difficult sores to diagnose, unless a similar case has been seen before. The erosive chancres on the glans penis are multiple, beautifully circumscribed and circular; only the most superficial part of the epithelium is rubbed off; there is no induration; and, however close the sores may be to one another, there is no tendency to coalesce.

The button chancre in the skin of the penis is an erosive chancre, in which either the surface epithelium has never been rubbed off, or it has healed over again, before the patient sceks advice.

The erosive chancre is the easiest chancre to diagnose, and is the one from which the Spirochoeta pallida is the most easily obtained.

In this type it is not at all uncommon to meet with two contiguous chancres -i.e., one on the under-surface of the prepuce, and the other on the glans penis, in exactly corresponding positions.

There is a type of erosive chancre which is always multiple, and which at first sight is difficult to distinguish from a case of balanitis; this type is found in the corona as well as on the gans penis and the inner lamella of the prepuce. One lesion is always bigger than the others, and not infrequently irregular in its outline. The lesions begin to heal quickly under peroxide and powder, but soon come to a standstill, thereby differing from a simple balanitis. When examining these sores microscopically, the observer must beware of confusing the Spirochata balanitidis with the Spirochata pallida. A sore belonging to this group is one in which the sore is single and irregular in outline. It shows no sign of healing under ordinary treat- 

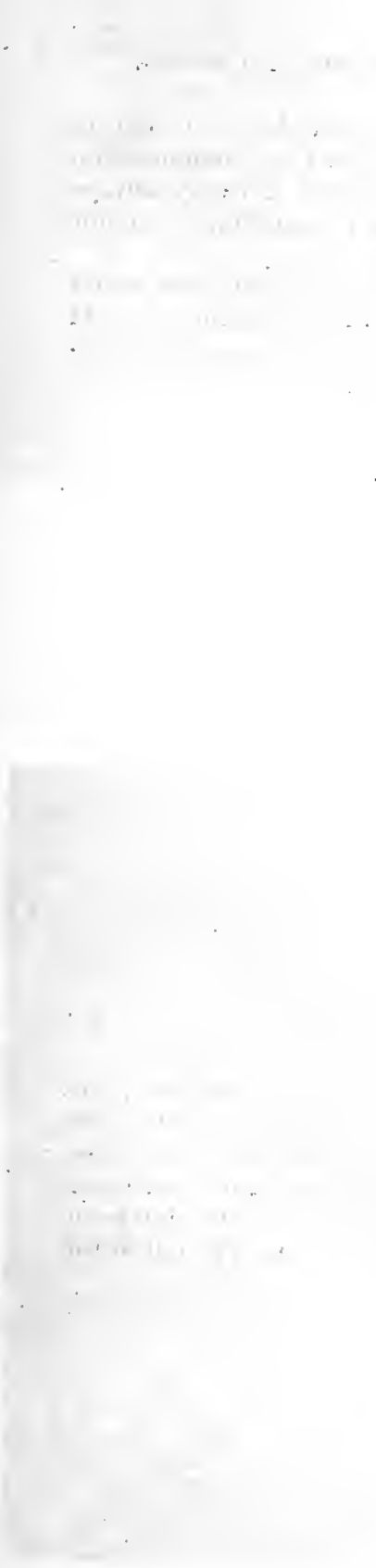
PI.ATE IX

No. 16.-A Chancre in One of the Furrows of the Prepuce

It will be noticed that only one furrow is ulcerated, that the sore is sharply circumscribed, red on the surface, and not surrounded by inflammation. When a primary sore occurs on the tip of the prepuce it nearly always produces a non-inflammatory oedema of the whole of the prepuce ; a point which is well brought out in the painting.

If the sore had been a soft sore, it is tolerably certain that there would have been a sore in every furrow, and that a condition of phimosis would have been produced owing to the acute inflammatory oedema on the foreskin which always accompanies a pyogenic infection.

\section{No. 17.-A Chr.ncre on the Franencu}

IT will be noticed that the sore is slightly ulcerated and covered with pus. The sore is really a papulo-erosive chancre, in which slight ulceration has occurred, probably owing to the fact that the forcskin was tight, and therefore the conditions in the region of the sore were practically anaerobic The most characteristic point and the most important diagnostic sign is the non-inflammatory oedema of the prepuce, produced by the left half of the sore. 
No. 16
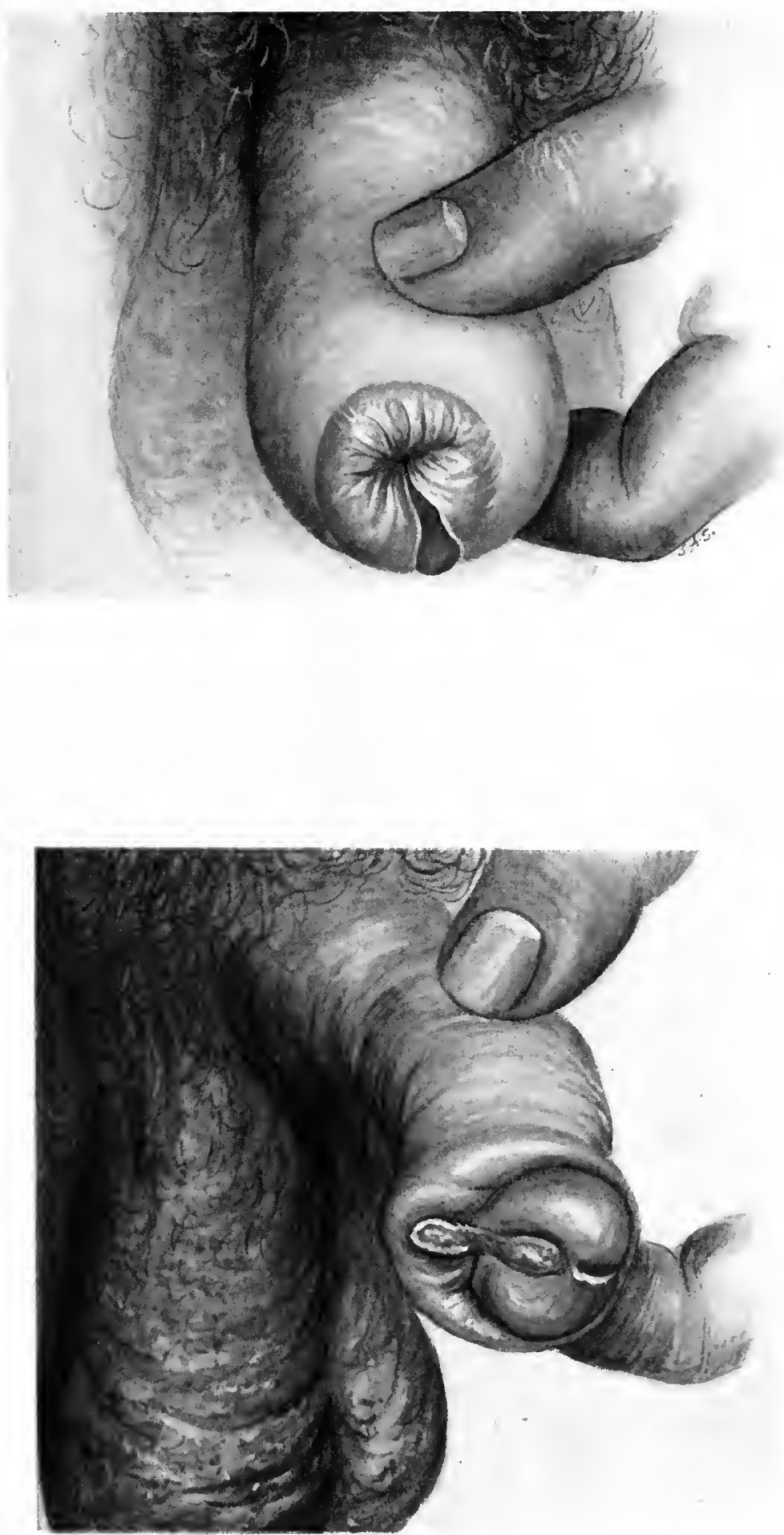

No. 17

Plate IX 



\section{PLATE X}

\section{No. 18.-Chancres on Scabetic Lesions}

Thus patient had scabies and developed primary sores in twelve of the burrows. The six sores not depicted were on the under surface of the penis and on the scrotum.

No. 19.-A Typical Papulo-erosive Chancre of the Type which is liable to be followed by Severe Vascular and GumMATOUS LESIONS 
No. 18
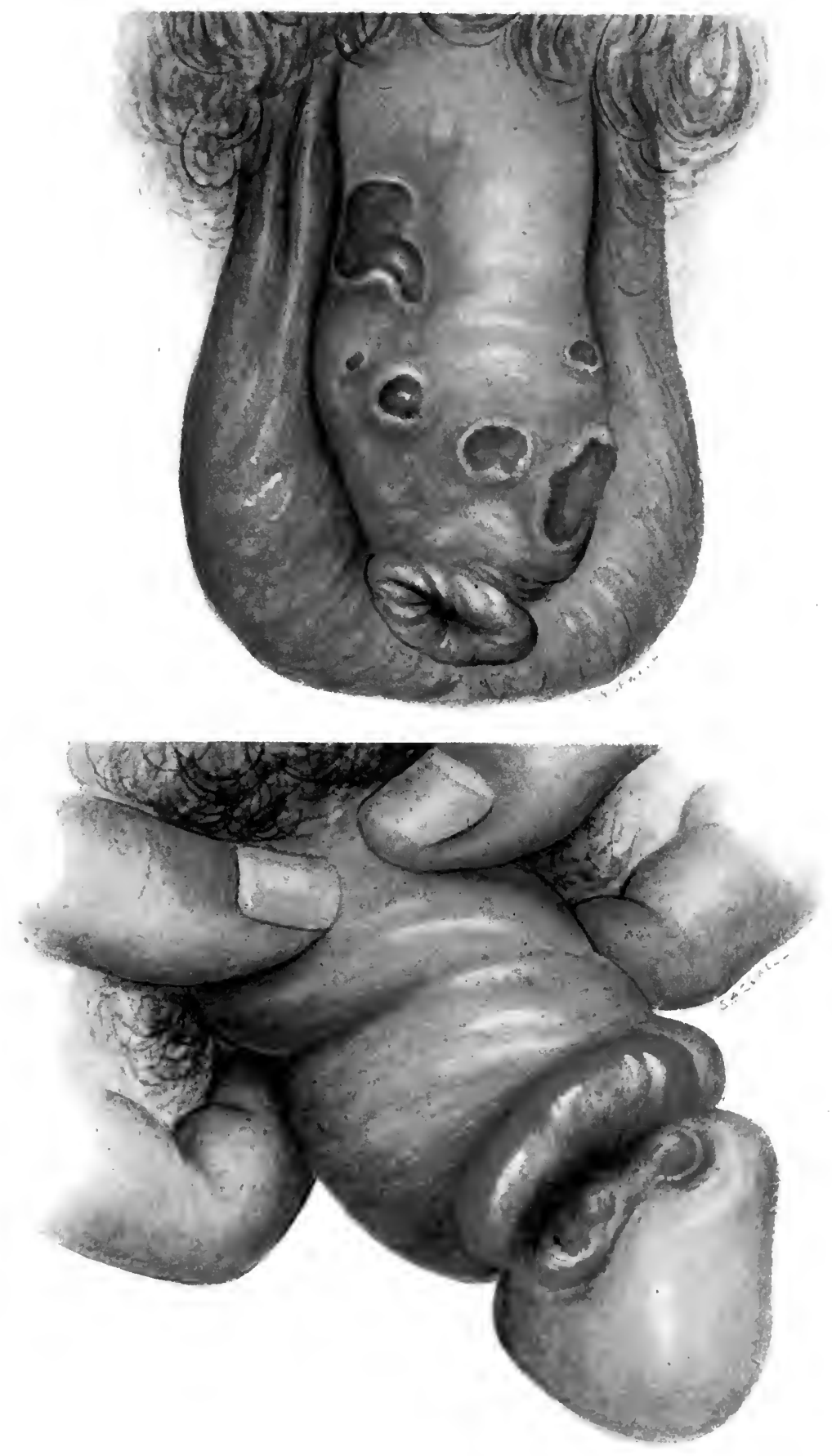

No. 19

Plate X 

ment, but loses its irregular outline and becomes a typical erosive chancre. The probable explanation of these sores is that an initial trauma occurred, which was not sufficient to cause a naked-eye lesion, but which became one when the growth of the organisms aggravated the original damage done. Sometimes an erosive chancre develops in a furrow caused by the folds of skin in a retracted. pre $\epsilon_{1}$ uce, in which case the lesion is longitudinal and not circular. These sores are usually covered by a crust, which must be removed before an attempt at a diagnosis is made. A typical erosive chancre is red, raw-like, and shiny on the surface from dried lymph, or it may be yellow and covered with a spurious membrane. Serum can be readily pressed out from both varieties.

If the term "papulo-erosive chancre" is applied to all the types of sores described under the heading erosive chancre, the term "papulo-ulcerative chancre " can be applied to those now about to be described. It is from the papulo-erosive chancre that the worst cases of syphilis arise.

The papulo-ulcerative chancre may be single or multiple, but the chief point about it is that induration is very frequently absent. Under this heading should not be included those erosive chancres which later become ulcerative, because the ulceration is purely secondary to the fibrous tissue contraction; in other words, the induration causes necrosis by shutting off the nutritive supply from the tissue above.

In the true papulo-ulcerative chancre, ulceration occurs early, and is due to the organism itself. There is no doubt that the Leucocytozoon syphilidis develops in a different way in the papulo-erosive chancre to that in which it develops in the papulo-ulcerative chancre. In the one, certain phases predominate; in the other, other phases. At present I am unable to give more exact details than this, as I have not examined a sufficient number of sores from this point of view.

Apart from the difference in the development of the organism, another very important factor has to be dealt with, and that is, the protective response of the host. In some chancres a connective-tissue celled hyperplasia, with only a few lymphocytes and plasma cells, is the histological picture given; while in others the tissue is crowded out with plasma cells. Whether the varied development of the organism, and the varied protective response of the host, are interdependent, has yet to be ascertained; but suffice it to say at present that an exhaustive histological and bacteriological study of the various kinds of chancre would throw a considerable light upon the future course of the disease in each case, and would aid one in making a prognosis, and in gauging more accurately the amount of treatment required. I have been trying for some time to reach the same goal by clinical means-i.e., to see if there is any connection between the kind of sore and the future course run by the disease. Again, I have to say that up to the present I have not :studied sufficient cases for a sufficiently long period of time to be able to make any authoritative statement, except in so far that, generally speaking, 


\section{CLINICAL ASPECT AND TREATMENT OF VENEREAL DISEASES}

the future course is more severe after papulo-erosive than after papuloulcerative chancres. It is not at all uncommon for the papulo-ulcerative chancre to be followed only by buccal lesions in the generalisation stage. Severe rashes most frequently follow the papulo-erosive indurated chancres. Late nervous lesions are particularly apt to follow the small multiple discrete papulo-erosive chancres. Early nervous lesions are most frequently met. with in those cases in which the elosive chancre was indurated. Papuloulcerative chancres, on the other hand, may be followed by lesions which have the same character-namely, ulcerative. Although a careful study over many years may yield profit, one will always be handicapped by altered circumstances in the patient modifying the development of the organism.

The remark made about the severity of the case is also borne out by my histological and bacteriological examinations of chancres and lymphatic glands removed from the set draining the primary sore. Many more organisms are found in the papulo-erosive than in the papulo-ulcerative chancres, and many more organisms are found in the small and hard lymphatic glands than in the swollen and soft ones. As regards the host's protective response, the state of affairs is exactly reversed-that is to say, in the papulo ulcerative chancres, and in the large lymphatic glands, the greatest numbers of lymphocytes and plasma cells are to be met with. In the erosive chancres, and in the small and hard lymphatic glands, connective-tissue cells and endothelia] cells predominate.

Of the papulo-ulcerative chancres there are several kinds. Each kind may receive a different name; but it must be understood that many are only different stages of the same sore, and they vary according to the degree of the ulceration.

Simple Papulo-Ulcerative Chancre.-This chancre is often very small; it is frequently missed, and in many of the so-called cases of Syphilis d'embléei.e., in which no primary sore could be found-there was one present of this nature. This kind of sore is very often tucked away in the angle fo med by the frænum with the corona.

In the larger sores, the ulceration may or may not be covered with a crust. The crust must always be removed before a diagnosis is made. In an ulcer which has a crust, the base is covered with pus, and the margin of the ulcer is red and inflamed owing to the proteolytic action of the crust. If the crust be removed, as a rule it will be found to cover the whole area of the sore, not only the centre, as is the case in an ulcer of pyogenic origin. The base is smooth, the edges are not raised, they are never undermined, and, in most cases the circumference is perfectly regular.

This type of chancre may occur anywhere on the penis, but it is commonly to be found on the frænum, or on the corona, just by the frænum. Occasionally there are two sores on the corona, one on either side of the frænum, and they may coalesce and ulcerate through the frænum. 

PLATE XI

No. 20.-A Very EARLY INtra-Urethral Chanche at the Lower Polf of THE MEaTus

No. 21.-AN INTRA-URETHRAL Chancre on the Left Wall. of the Meatus

Note the gaping orifice caused by the induration.

No. 22.-Another Type of Intra-Urethral Chancre fximbiting tue Typical Infiltrative Oedena of the Prepuce

INTRA-URETHRAL chancres are particularly liable to be followed by early constitutional disturbances, such as fever, headaches, and pains in the bones and joints, probably owing to the fact that the organisms become generalised quickly, as the primary lesion is on a mucous membrane. The course run by the disease is apt to be severe. 
No. 20

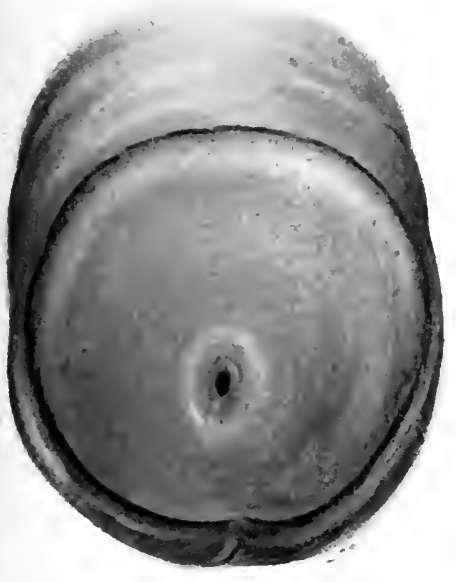

No. 21

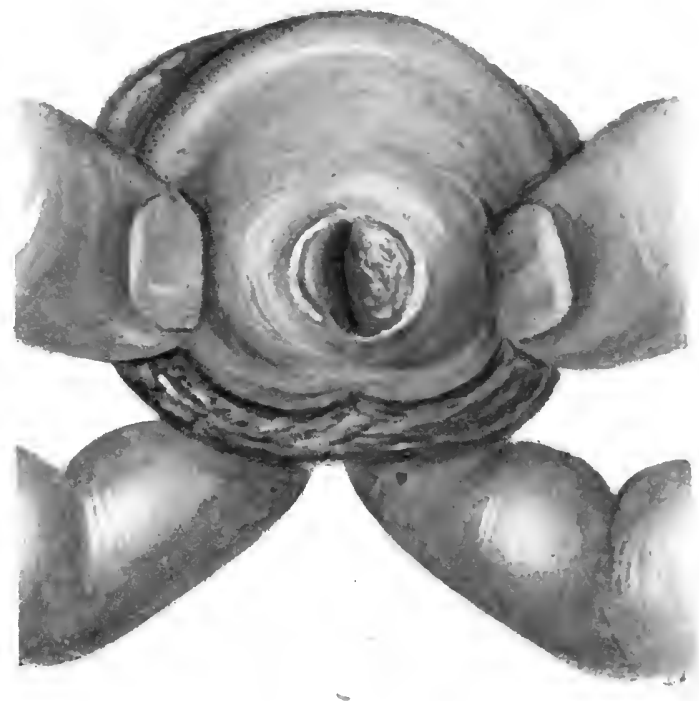

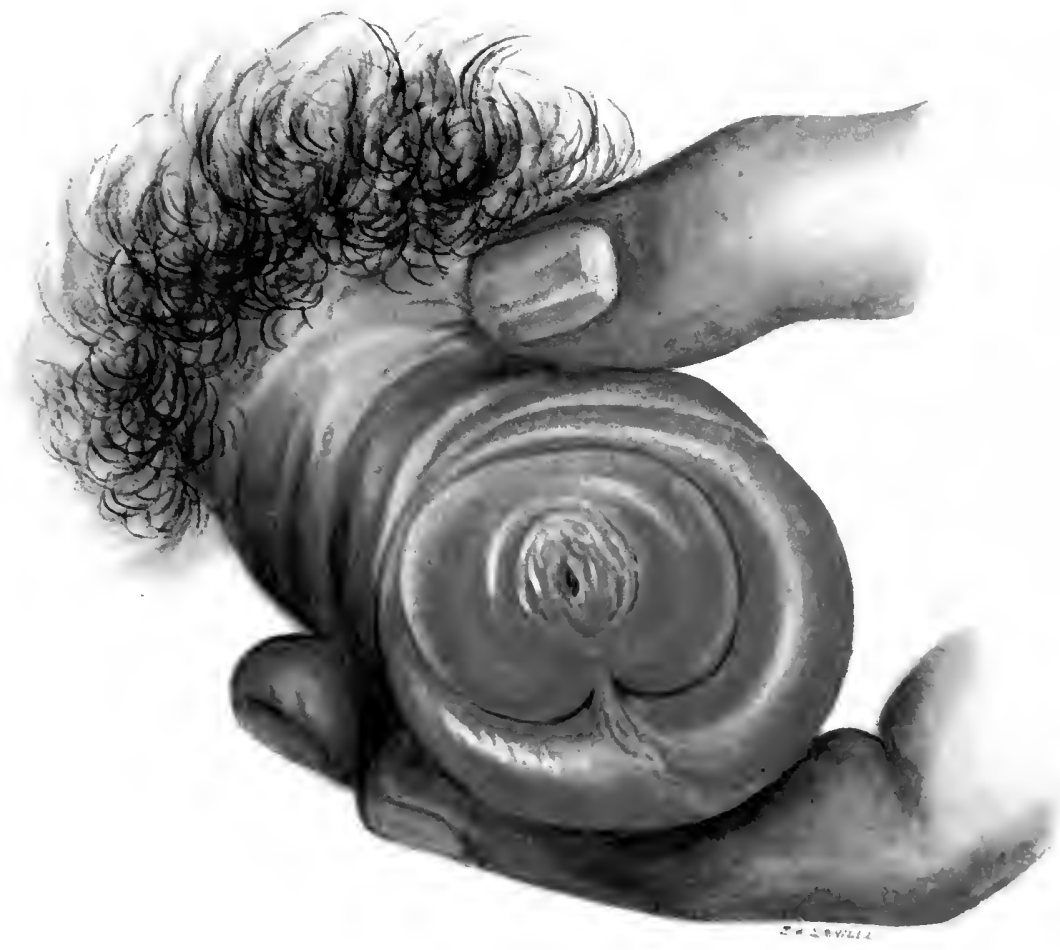

No. 22

Plate XI 


PLATE XII

No. 23.-An Intra-urethral Chancre and Balanitis produced RY THE DISCHARGE THEREFroN

No. 24.-A Recurrent Intra-urethral Syphiloma showing tim: Typical Violaceous Tint of tue Surrounding Tissue 
No. 23
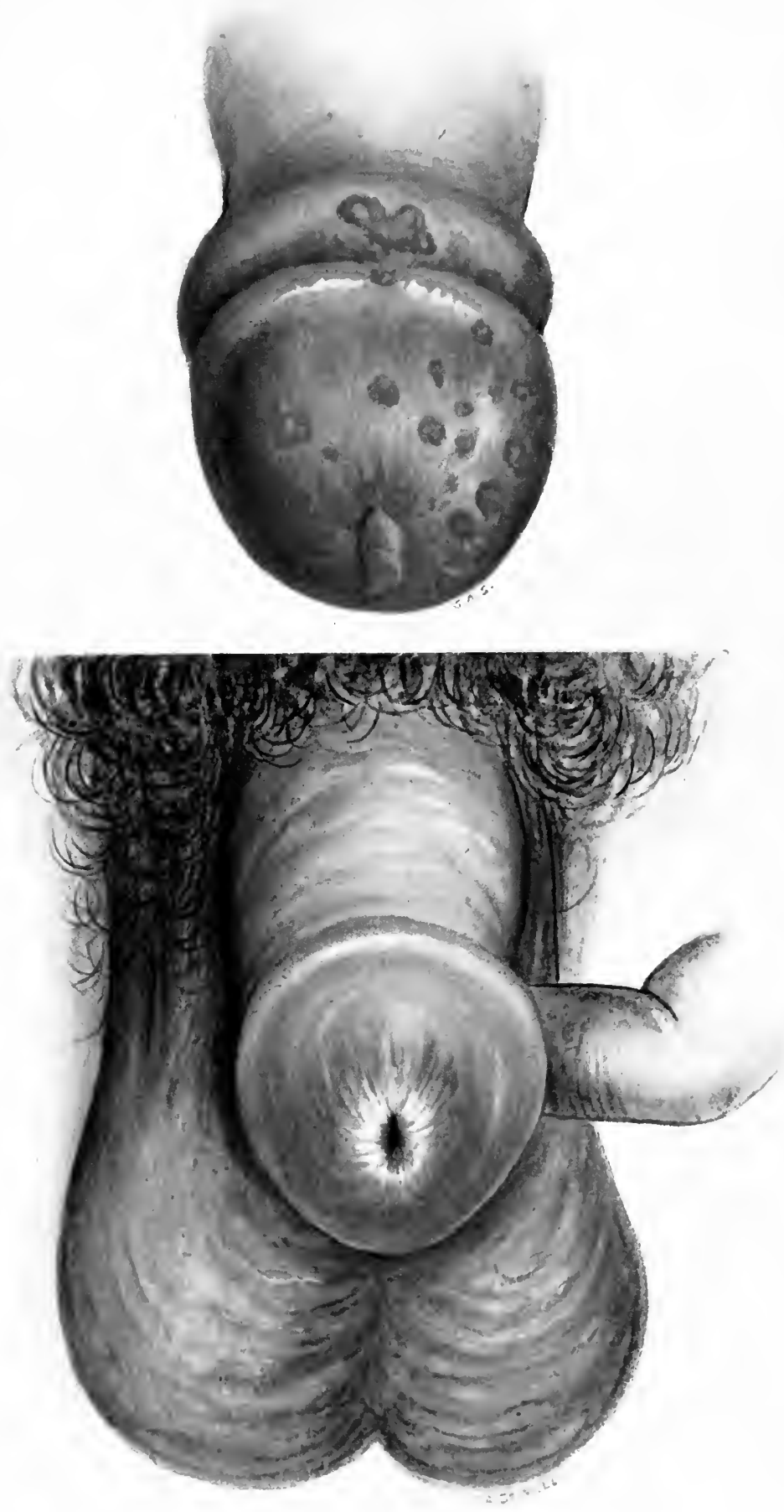

No. 24

Plate Xí 


$$
\text { . }
$$




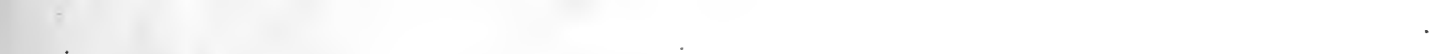

.

-1
-1
-1




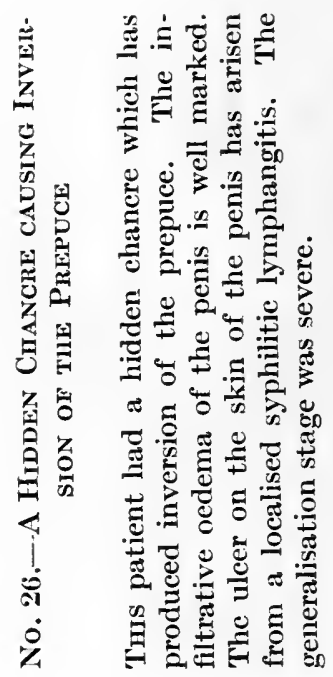

$\Xi$

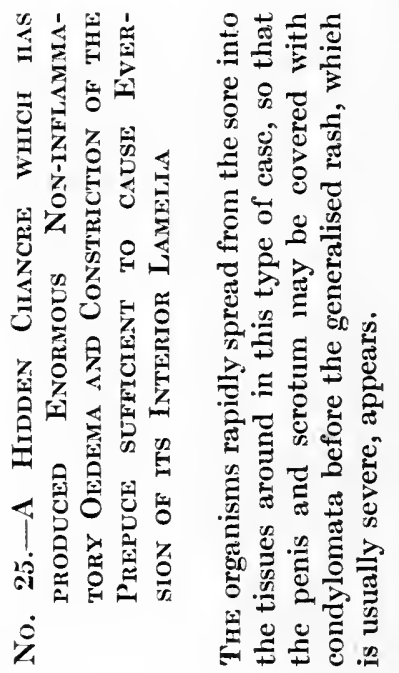



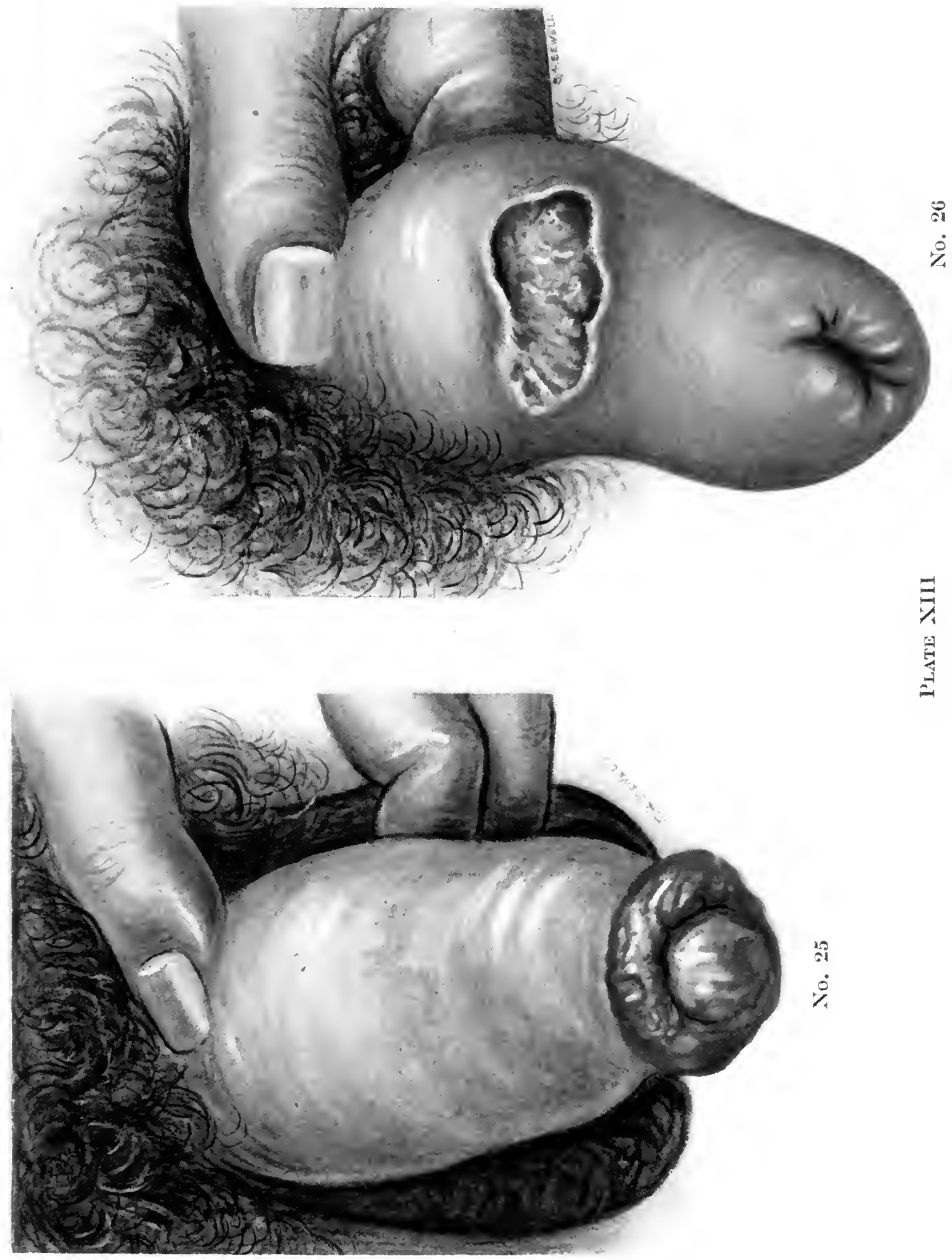

Ecthymatous Chancre.-This chancre is most frequently found on the skin. It is usually single, sharply circumscribed, raised, and crusted on the surface. Considering the appearance of the sore, the amount of surrounding inflammation is less than might be expected. Some of the large sores have a granulating-looking surface, and take a long time to heal. The future course run by the disease is, as a rule, milder than that following any other chancre, except the chancre due to the asexual development of the organism. In most granulating chancres it is difficult if not impossible to find the Spirochata pallida.

Phagedænic Chancre.-This is only a further stage of the preceding, in which a secondary infection has supervened. The secondary infection is usually due to the symbiotic Gram-negative spirochæta and Gram-positive fusiform bacillus.

Pseudo-Membranous Chancre.-These chancres are usually multiple; they are about the size of a threepenny piece, and always sharply circumscribed; the surface may be flush with the surrounding skin, or even raised above it; the base is yellow; nothing can be rubbed off; it is surrounded by a sharply circumscribed red ring; and the sore is generally slightly indurated. A favourite localisation is on the under-surface of the prepuce, and when the prepuce is withdrawn the folds are missed in the areas where the sores are situated.

Hypertrophic Chancre.-This chancre is almost invariably single, and is most frequently met with on the pubis. It presents no difficulty in diagnosis. A chancre in this region is sometimes an ulcerative one, usually of fairly large dimensions, and frequently sores are to be met with which combine the two types. Very rarely multiple chancres are to be met with on the pubis. The lesion is a follicular one, due to infection of a hair follicle. I saw a case with seven such sores; in the centre of each was a tiny pustule through which projected a hair.

Herpetic Chancre.-A group of small and ulcerative chancres not at all unlike Herpes preputialis. The sores sometimes become confluent to form a single chancre.

Regarding a chancre from the point of view of its site will allow of the mention of several little diagnostic points.

Intra-urethral Chancre.- It is very rare for a primary sore to be so wholly urethral that it cannot be seen with the naked eye. In every case but three I have seen-and the urethra is not at all an uncommon site-one pole of the sore has always involved the glans penis. The sore quickly causes a narrowing of the orifice, and it gives rise to an induration which is best felt by pressing the glans between the forefinger and thumb, from above downwards. When palpated from side to side, the induration is frequently missed. If the patient has a tight foreskin, and the orifice of the urethra cannot be seen, the chances are that the phimosis has been largely produced by the syphilitic lymphangitis, in which case the glans penis feels hard all over. 


\section{CLINICAL ASPECT AND TREATMENT OF VENEREAL DISEASES}

There is usually a urethral discharge, which is serous and occasionally bloodstained in nature. The discharge is occasionally profuse enough to be mistaken for gonorrhoa. The first sign of a relapse may be threads in the urine.

Lymphadenitis is, as a rule, an early sign, and the patient enters the generalisation stage quickly. I have seen many urethral sores in which the patient ran a sharp evening temperature, probably owing to the fact that the urethra is lined by a mucous membrane through which the organisms can easily gain access into the circulation and so set up, before the patient can protect himself, a widespread generalised infection.

Primary Sore in Corona, with Phimosis.-These cases can usually be diagnosed at the first glance. The prepuce is swollen on the side on which the sore is situated, and the swelling is non-inflammatory. When it is palpated, the induration is at once obvious. Sores which are complicated by phimosis are usually of the papulo-erosive type, in which induration is a prominent feature, and the phimosis is often produced by the widespread indurative syphilitic infiltration of the lymphatics, and, possibly, of the smaller veins and arterioles. This syphilitic infiltration imparts a blue to violet coloration to the part, and this is so pathognomonic of syphilis that once seen it will never be forgotten. I have seen a chancre develop into a malignant epithelioma; likewise a gumma on the site of an old primary sore.

\section{Recurrent Chancres.}

Owing to the frequency of recurrent chancres in cases which receive only one or two courses of arseno-benzene and mercury, and the mistake that is often made by the unwary in considering them as cases of re-infection, it is necessary to describe the lesion here. The sore usually appears about the twelfth week after the completion of the course, but it may show itself while the patient is still under treatment, or not until some years have elapsed. The longest interval so far has been eight years.

The sore may be single, or there may he more than one; it usually occurs on the site of the original sore or in close proximity to it, although it may be met with in any part of the body.

CASE 1.--A patient had a primary sore on the penis, general adenitis, but no rash, for which he received eight injections of arseno-benzene and nine of mercury. Fourteen weeks later he returned to hospital with a sore on his shoulder-blade. The sore was absolutely indistinguishable from a primary sore. Spirochætæ were obtained from it, and the C.F.T. was negative.

That the sore appears with the usual incubation period after a connection means nothing, because many cases occur when there has been no connection, and because any irritant may be the cause of a sore, which will appear after the usual incubation period. Indeed, a recurrent chancre sometimes occurs independently of an irritant. In some cases of a recurrent chancre spirochætæ are found, and in others they are not. If present, it can generally be assumed 


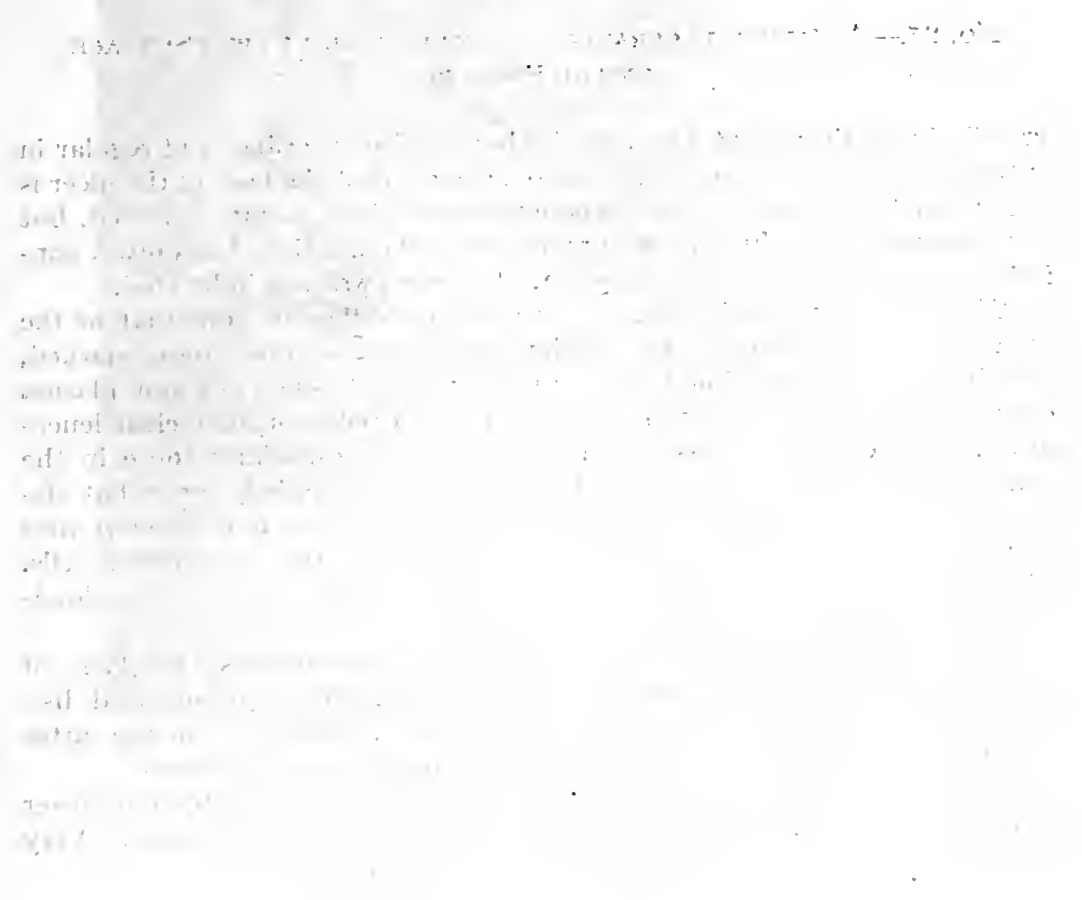




\section{No. 27.-A Papulo-ulcerative Chancre on the Upper Surface of tile Prepuce}

IT will be noticed that the sore is sharply circumscribed and regular in outline, that there is a moderate loss of surface, that the base of the ulcer is not covered with pus, and that the edges are raised, slightly everted, but not undermined. There is no surrounding inflammation, but only a noninflammatory oedema of the foreskin. The sore was not indurated.

The histology of this type of sore is quite different from that of the papulo-erosive chancre. The cellular infiltration is much more marked, and instead of consisting mainly of connective-tissue cells and plasma cells, it is made up mostly of lymphocytes and polymorphonuclear leucocytes. The connective-tissue cells are increased, especially those in the walls of the blood-vessels, but they are hypertrophied, somewhat degenerated, non-pyroninophile, and therefore they do not develop into fibrous tissue. There is a slight hyperplasia of the endothelial cells. The what may be called oedematous condition of the walls of the bloodvessels is characteristic of this kind of sore.

The lymphatic gland enlargement which accompanies this type of sore may be very pronounced, but the lymphatic glands are not hard, like those which accompany the papulo-erosive sore. However, in the latter case the glands are often not enlarged, and always remain discrete.

This type of sore is an indication of the host's high protective power against the infection, hence the disease usually runs a mild course. Very often no signs of the generalisation of the virus appear.

\section{No. 28.-A Typical, Granulating Sypilitic Chancre, fron Which no Spirochatetae could be obTained}

THis type of sore is resistant to arseno-benzene and mercury, and only heals up quickly when intramine is prescribed. Spirochaetae are seldom found, and the course the disease runs is usually mild, and vascular and nervous lesions are rare sequelae. 

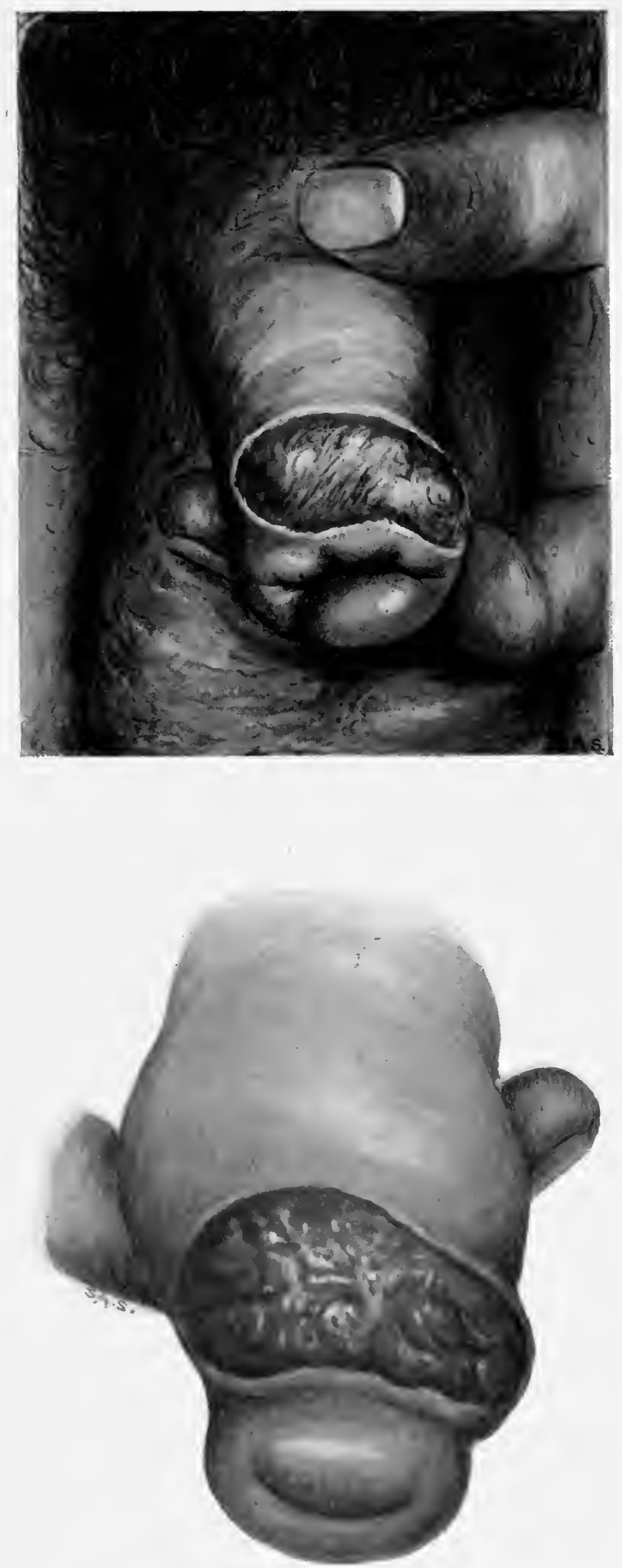

No. 28

Plate XIV 
. 


$$
\text { - }
$$

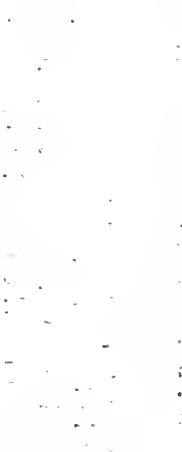

-
$\therefore$
$\therefore$
$\therefore$

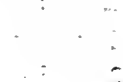




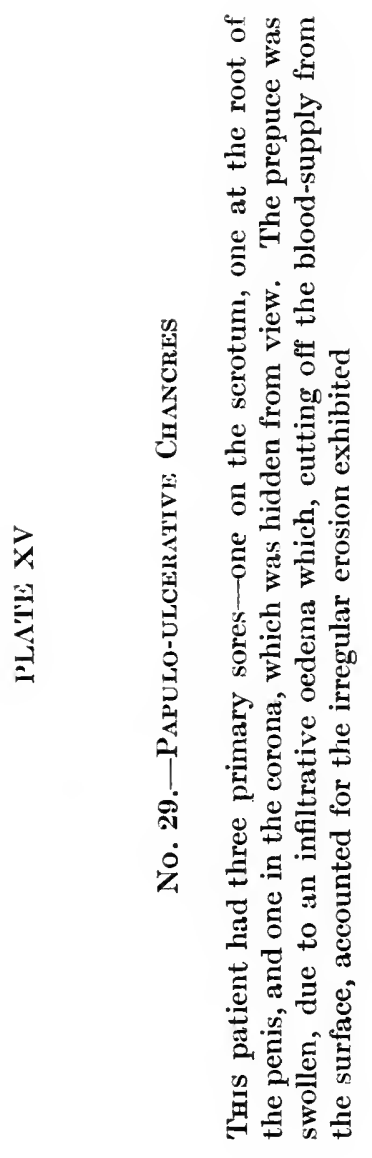




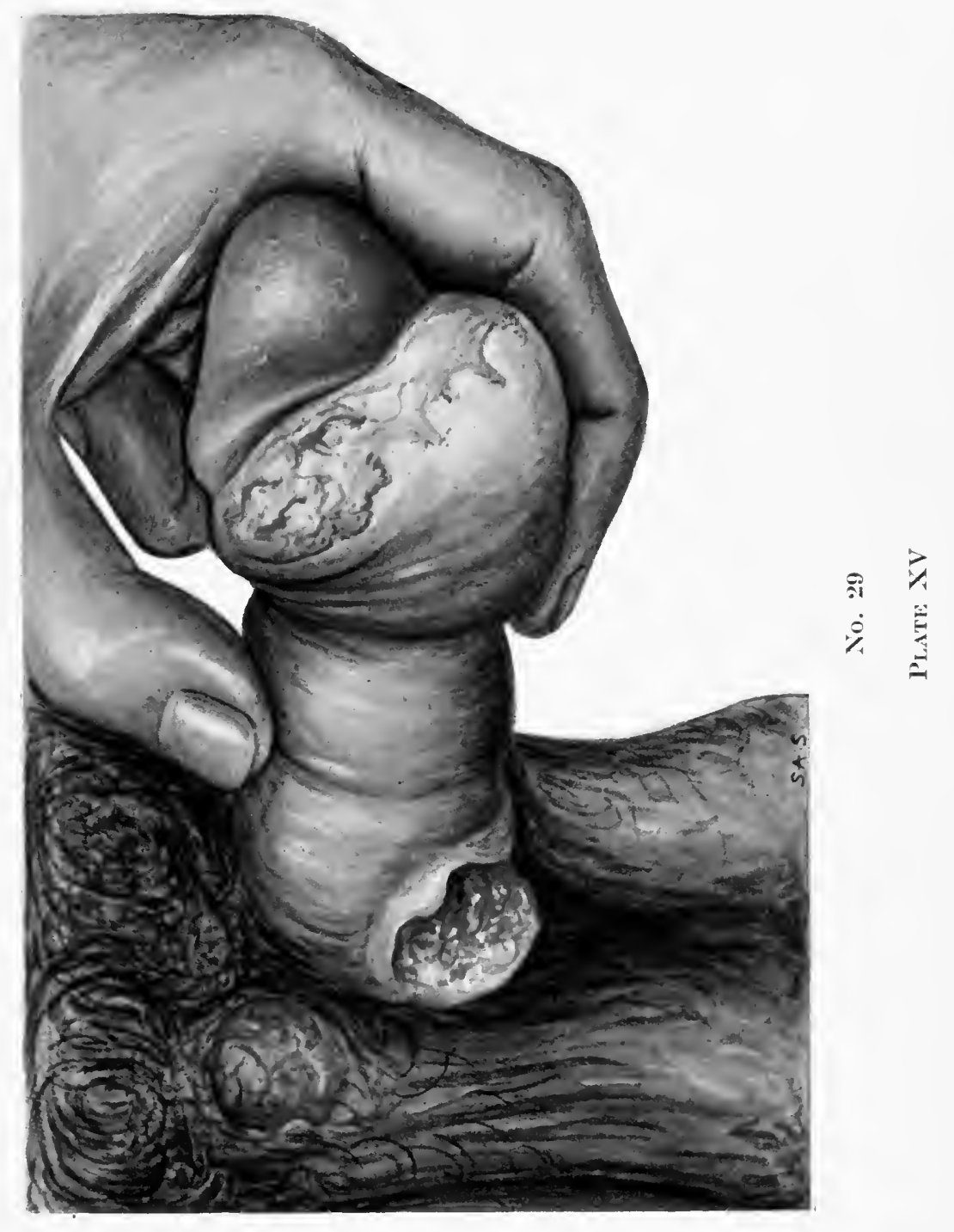






\section{PLATE XVI}

No. 30.-A Chancre which becane Secondarily Infected

Ture generalisation stage was normal because the secondary infection had occurred late.

No. 31.-A Chancre on the Skin of the Penis which became Septic

THE generalisation stage was severe, as all the lesions which appeared became septic - in fact, a case of malignant syphilis. The patient was cxtremely ill and intolerant of metals. If arseno-benzene is prescribed in this type of case, it is best to inject intravenously $0 \cdot 1 \mathrm{grm}$. per diem until twelve doses have been administered. 
No. 30
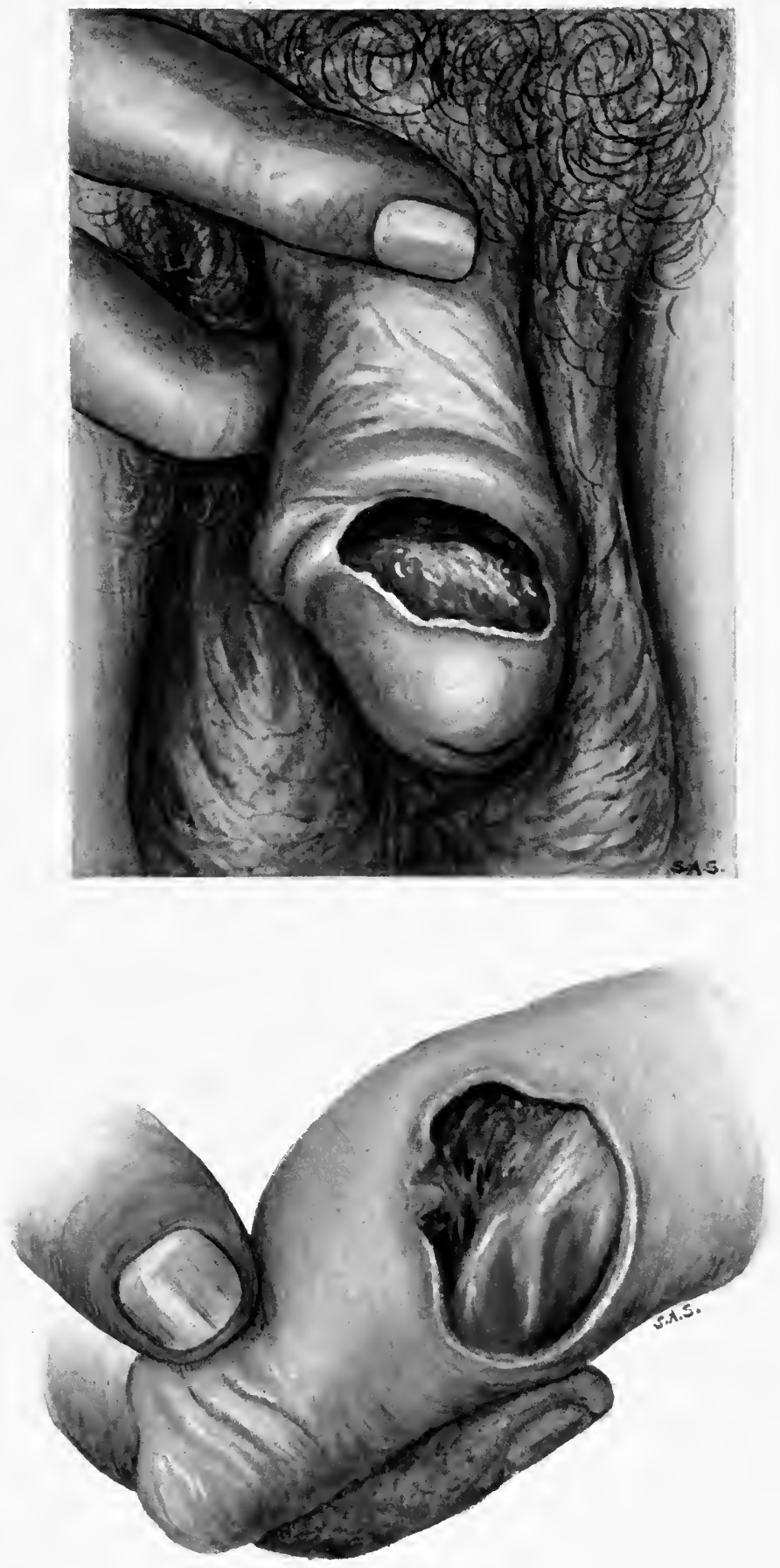

No. 31

Plate XVi 

that treatment of the original infection was begun very early, whilst, if not present, the chances are that the treatment was not begun until the patient had had the sore for some time, or until he had just entered the generalisation stage. Therefore the presence of spirochætæ, even if found in abundance, cannot be held as a criterion of a true re-infection.

The reason why the recurrent sore is on or near the original sore is because intensive arsenical treatment does not kill the spores of the organism of syphilis, and spores both remain locally and spread peripherally. A recurrent chancre not infrequently has satellite erosions around it from which spirochætæ cannot be obtained.

One other point is that, as a rule, the inguinal adenitis following a recurrent chancre is not so pronounced as that accompanying the original sore. If the host has not had to form a protective substance for an extended period, or until he has become used to doing so, as he does in all chronic infections, even beyond the time when all the organisms he is combating have been vanquished, a condition we call immunity, and as intensive treatment has the effect of reducing the host's resistance, it stands to reason that, should the spores re-awake, the host's response will be the same as when he originally became infected, with the result that the recurrence will simulate a chancre.

The next grade is when the host has formed a resisting substance over

longer period than in the instance just cited. In this grade the recurrent chancre bears the following characteristics: it is, as a rule, raised; the lesion has a swollen appearance; the surface tends to be more that of an ulcer than of an erosion; it is more purulent; there is more circumferential inflammation and less induration. If the patient had entered the generalisation stage before treatment was begun, the recurrence seldom simulates a chancre, but it may sometimes do so; in fact, $I$ have seen a recurrent chancre on the neck following an irritation six weeks previously, and I have also seen a recurrent chancre on the penis, while on the scrotum the patient had a recurrent orbiculopapular syphilide. These were two of the cases which made me wonder whether what I had diagnosed as re-infections were really so.

It is absolutely impossible at present, in some cases, to say whether one has to deal with true re-infection or a recurrent chancre; but, going solely by my clinical experience, I should say that over 95 per cent. of the published cases of re-infection, following arseno-benzene within six months of the cessation of treatment, are really recurrences.

Should a recurrence occur in the next grade-that is to say, when treatment was not begun until the patient has entered the generalisation stageit may be an exact replica of the lesions the patient originally presented; it may be a neuro-recurrence, localised or general, or the lesions may be destructive and simulate gummata. The severest lesions, in both the systemic and the nervous parts of the body, have always been in those cases which have been treated most drastically with arsenic and mercury.

The case above referred to, which presented a recurrent chancre on the 
penis and a recurrent orbicular syphilide on the scrotum, suggests that there may be various degrees of immunity produced in different parts of the same body. Another interesting point about the recurrent chancre is that it responds to treatment much more slowly than did the original sore. This strongly supports my views: (1) that the frequency of recurrent chancres is due to the use of too much metal, because recurrent chancres heal better with intramine than with arseno-benzene; (2) that metals destroy the host's protective substance; (3) that metals do not directly attack the parasites, but only indirectly through the protective substance. A recurrent chancre may lead to a generalised infection, as the following most interesting case shows:

CASE 2.-In 1914 the patient had a papulo-erosive chancre on the penis, for which he received seven injections of galyl. In 1915 a recurrent chancre appeared on the same site as the original sore, and only healed when eleven injections of kharsivan and nine of mercury had been given. In 1916 the patient developed another recurrent chancre close to the original site, which vanished at once with intramine. The patient received two injections of intramine and three of galyl. He was advised to continue treatment with intramuscular injections of mercury, but failed to do so. In March, 1918, the patient had another recurrent chancre which was followed in eight weeks by a generalised maculo-papular rash, mucous papules in the mouth, and a general adenitis.

It must not be forgotten that there is such a condition as a super-infection - that is to say, a second infection-supervening on one already in being. A super-infection is probably determined by the amount of virus conveyed into the system at the time of infection.

The following is what I take to be a typical case of super-infection:

CASE 3.-A patient had syphilis nine years ago, and for the last two years had been under mild treatment for degenerative myelitis. After the usual incubation period the patient developed a typical intra-urethral chancre. The induration was characteristic, there was marked inguinal adenitis, and spirochætæ were demonstrable.

Gummata are very apt to occur on the site of primary sores, and might, on cursory examination, be mistaken for the initial lesion of a super-infection. Gummata cause deep destruction of tissue, which is foreign to an initial lesion. Gummata themselves are not indurated, but the tissues around them often are. Gummata do not produce adenitis.

Auto-reinfection must now be considered-that is to say, when a patient has had generalised syphilis, and later develops a recurrent chancre, which is followed some weeks later by a fresh generalisation of the virus. The following is a case I exhibited some years ago:

CASE 4. - The patient had a ringed chancre on the corona, the induration of which never entirely disappeared. This sore was followed by a mild rash, which 


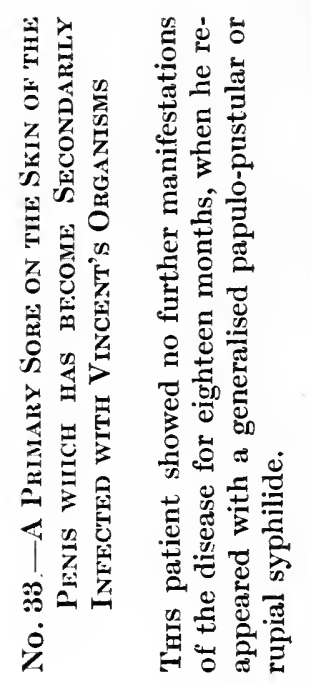

紊

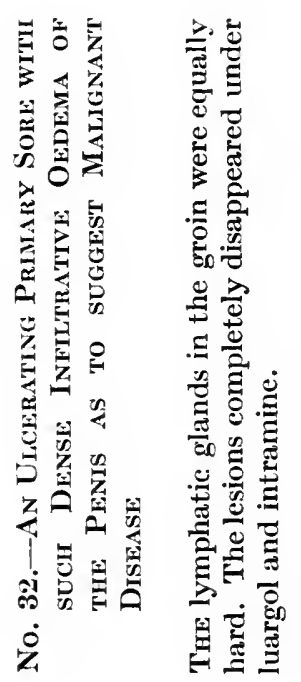




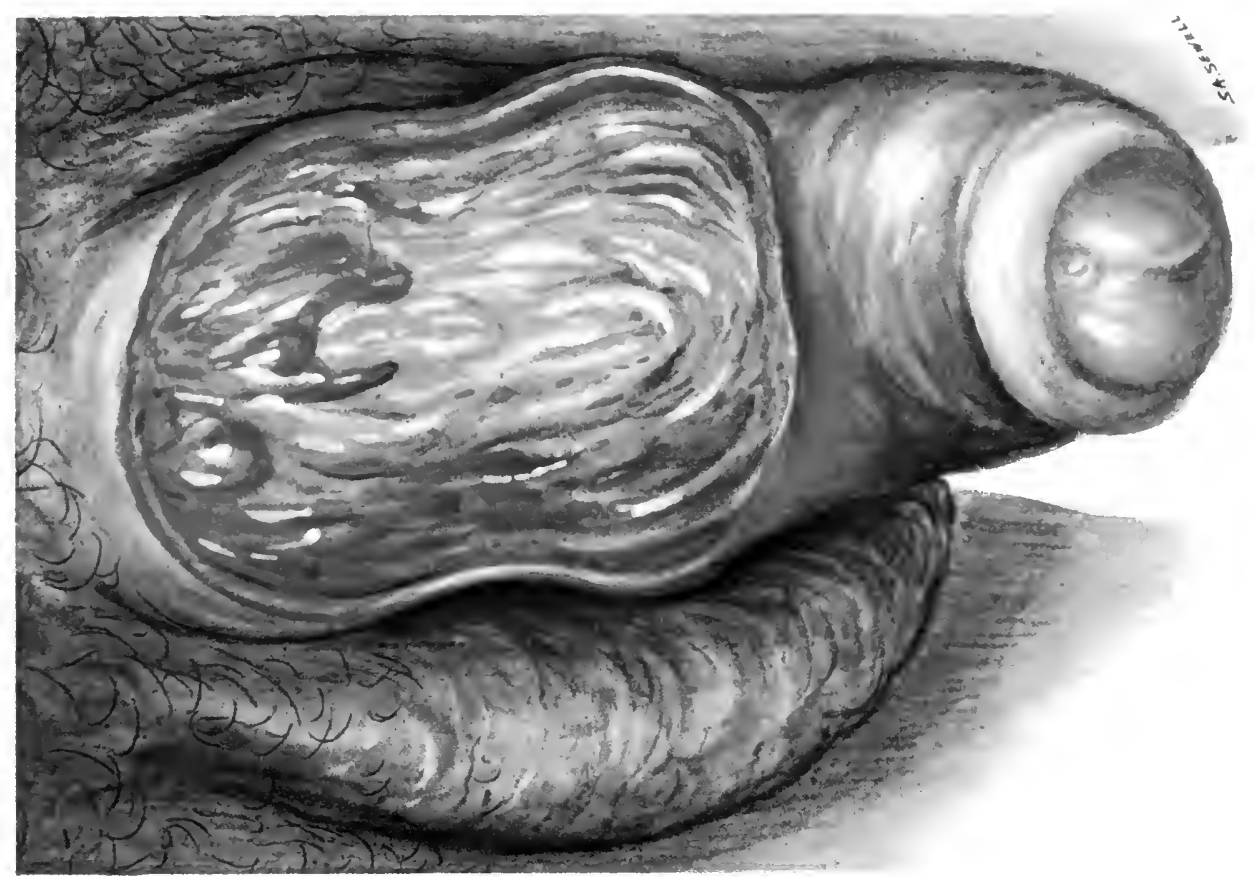

苟

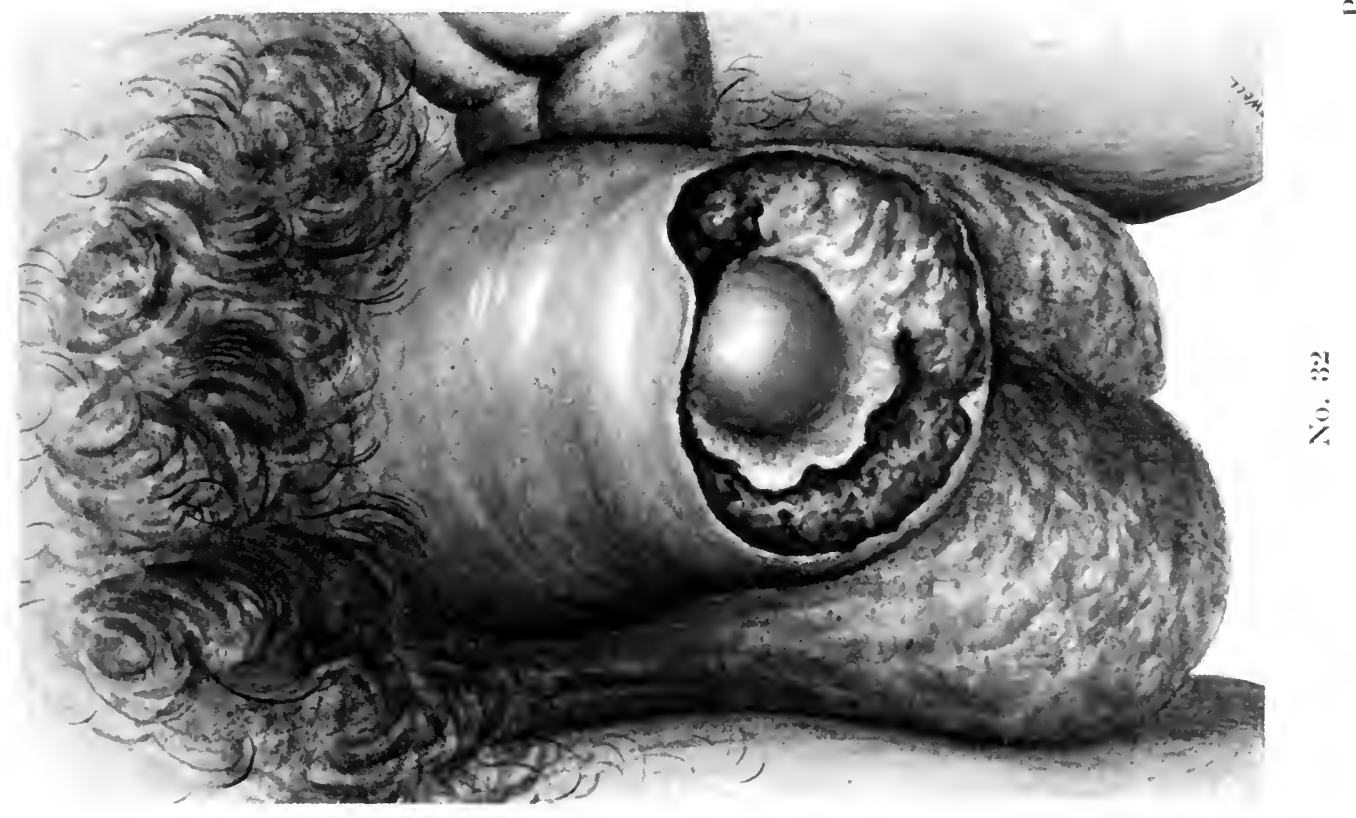





\section{PLATE XVIII}

\section{No. 34.-Papular Syphilide by Direct Extension}

Tuis is a painting to show the diffuse papular eruption on the genitals which arises by a direct extension of the organisms from the primary sore. In this case the primary sore was intra-urethral. The patient had no other signs of syphilis, and it is usual for this rash, localised to the genitals, to appcar some time before the generalised rash manifests itself.

No. 35.-Papular Sypinlide by Direct Extension

A CASE showing an intra-urethral chancre and a well-marked papular syphilide, due not to a blood infection but to a local extension and development of the organisms from the sore, probably along the superficial lynphatics. The roseola on the trunk was just beginning to appear. It is not uncommon to find even condylomata on the genitals before the generalised rash has fully developed. 

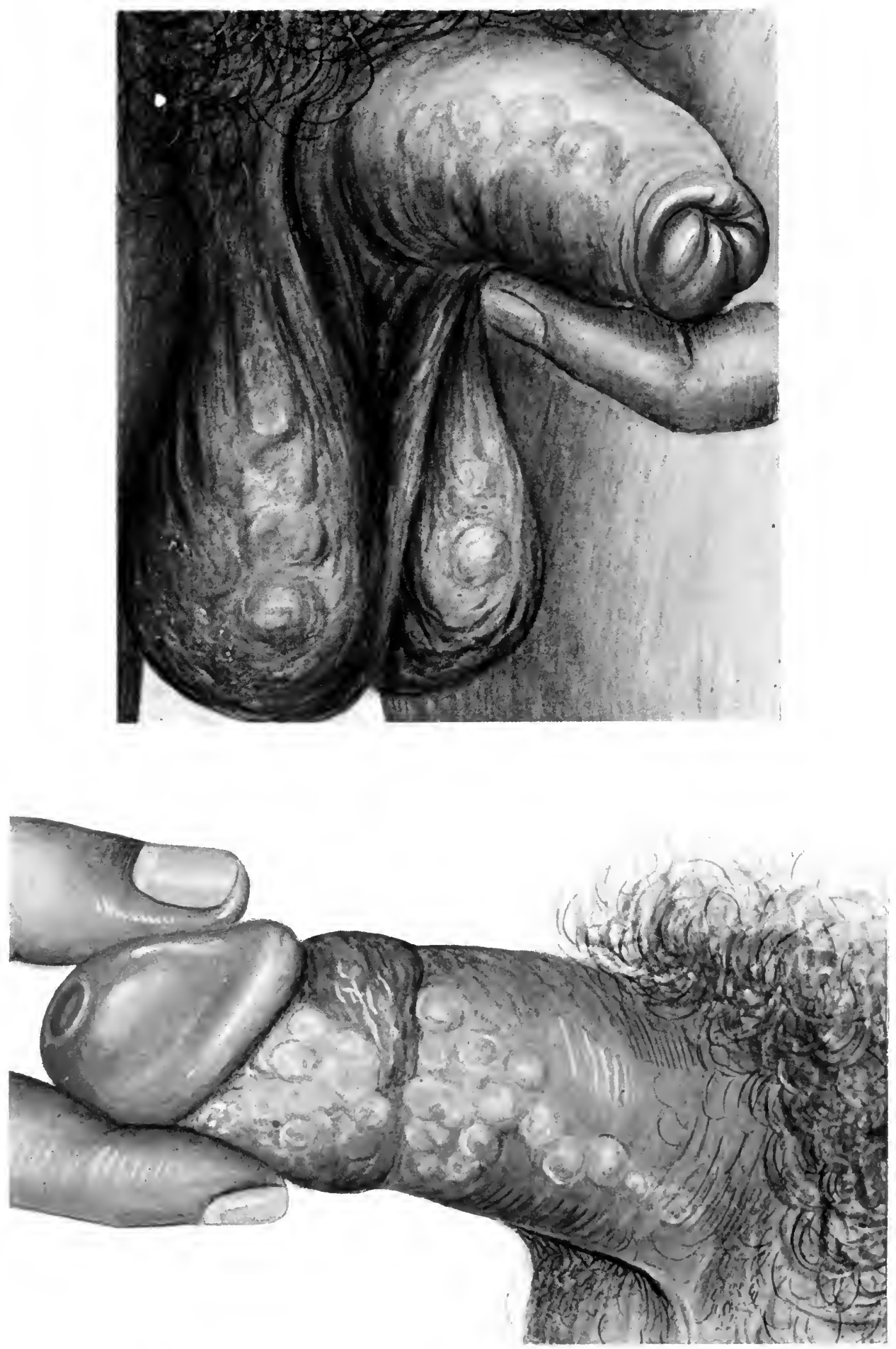

No 35. 


PLATE XIX

No. 36.-A Recurrent Chancre on the Glans Penis on the same Site as the Original Sore, and a Fresil Chancre on the Skin of the Penis

Tires two chancres appeared while the patient was under treatment with intramuscular injections of mercury and two and a half months after a full course of arseno-benzene and mercury. From both sores spirochaetae were obtained, and there was a recurrent inguinal adenitis. Neither sore healed till intramine was injected. The sore on the skin of the penis was probably lymphatic in origin.

No. 37.-A Chancre Redux

THE sore which is depicted with a large collar of induration which is more apparent than real, but typical of recurrent chancres, reappeared on the same site as the original sore and twelve weeks after the completion of the course of treatment. The treatment for the first sore consisted of eleven injections of arseno-benzene and thirteen injections of mercury. The second sore was uninfluenced by six injections each of arseno-benzene and of mercury, and only healed when intramine was prescribed. 
No. 36
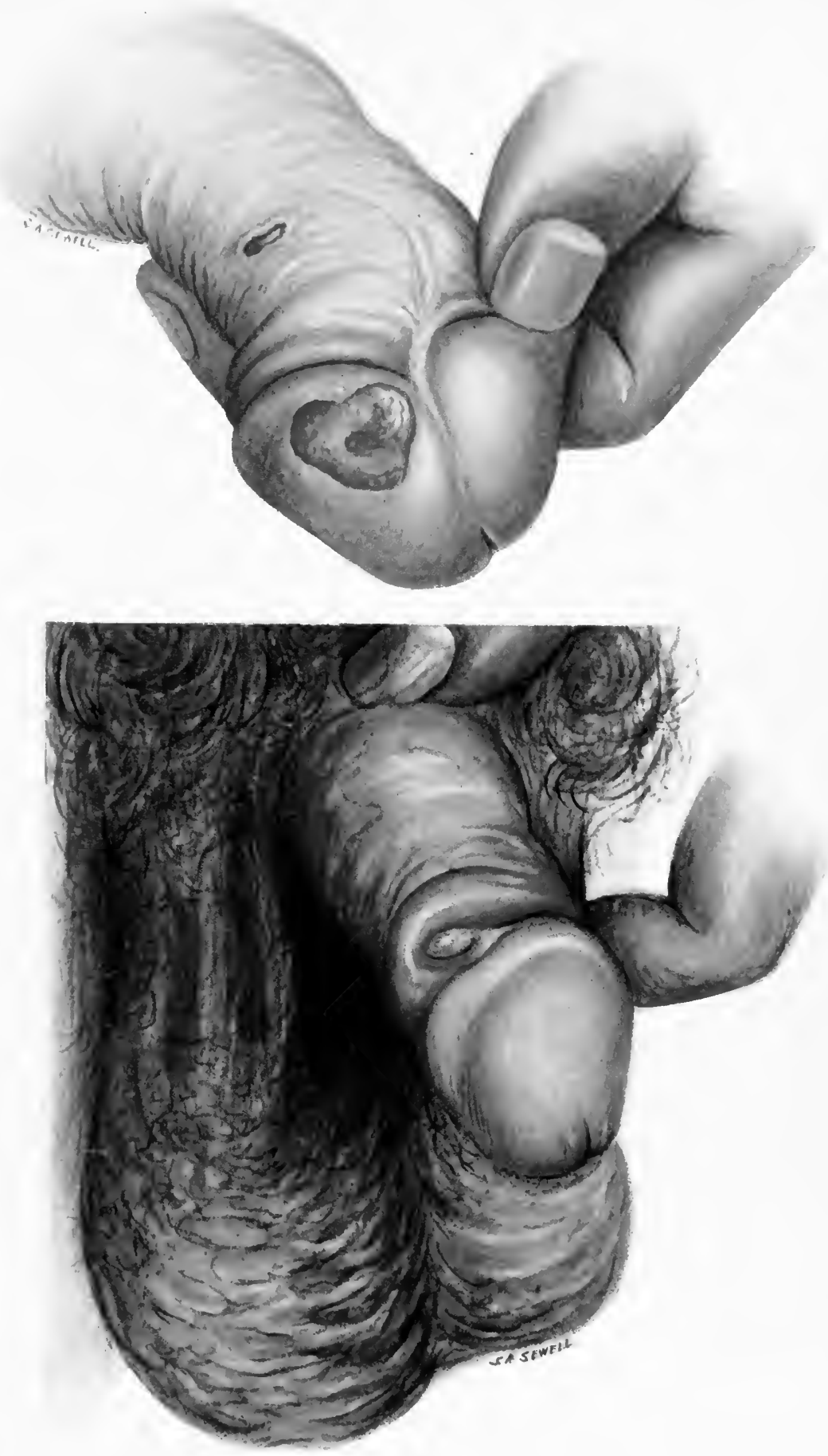

No. 37

Plate XIX 
rapidly disappeared under treatment (mercury only). Six years later a chancre developed on part of the indurated corona. A few weeks later general adenitis, a maculo-papular rash, and a sore throat supervened.

In closing this part of the subiect I particularly wish to emphasise the point that, not only is it useless to prescribe chemotherapeutic agents for a patient who has exposed himself to the risk of infection with the object of aborting that infection, but it is actually detrimental, as, should the patient become infected, his lesions will not so readily respond to the treatment.

\section{Extragenital Chancres.}

Extragenital chancres may occur anywhere, and they are generally very easy to diagnose. The sore is nearly always single, but occasionally erosive chancres of the anus and lips are multiple, in which case the sores are contiguous. The sores have, as a rule, been present for some time before the patient seeks advice; hence an enlargement of the lymphatic glands, draining the infected site, is practically never missed. However big the sore may be, and many of the extragenital chancres are of the ulcerative and hypertrophic type, the amount of surrounding inflammation is always minimal, and this at once excludes a pyogenic lesion.

If the sore is on a mucous membrane, as all intrabuccal sores are, the glandular enlargement is always very pronounced, and however closely a sore on the tonsil, for instance, may simulate or suggest a chancre, syphilis can be at once excluded if the lymphatic glands are not enlarged in the submaxillary regions and in all the triangles of the neck. In the case of a pyogenic infection, and in Vincent's angina, the enlargement of the lymphatic glands is negligible compared with a syphilitic enlargement, and, moreover, they are only enlarged in the submaxillary region on the side affected. A useful point to remember is that syphilitic adenitis is painless.

A common extragenital chancre is the digital, and, as a rule, it is situated in the margin between the nail and the skin. There is nearly always a swelling of the epitrochlear lymphatic gland, which not infrequently goes on to suppuration. Suppuration in the region of the inner side of the elbow should always raise the suspicion of a digital chancre, as I have seen three cases operated upon for supposed cellulitis when the real nature of the disease was missed.

It should not be forgotten that in cases of anal infection the lymphatic glands to be enlarged are the inguinal. It is important to remember this, as the diagnosis of Syphilis d'emblée has been made when no sore on the genitals could be found.

\section{Syphilis d'Emblée.}

Syphilis d'emblée is the term applied to cases of acquired syphilis, in which no primary lesion exists. The possibility of this is often questioned-possible it certainly is, and cases are from time to time met with; but the condition is often hard to prove, as some syphilitic sores are so small, and others heal 


\section{CLINICAL ASPECT AND TREATMENT OF VENEREAL DISEASES}

quickly without leaving any mark behind them. I have had two cases of Syphilis d'emblée in which the infection was a direct one into the blood-stream. The first sign and symptom was the rash, and neither patient had any enlargement of his lymphatic glands. One of the patients was a medical man and the other a medical student, and both were looking after syphilitic cases when the rash appeared. The medical man remembered pricking his finger while giving a mercurial injection, but no sore developed at the site. I have just seen a case in which a bulla containing myriads of spirochætæ was the primary lesion marking the site of a prick on the finger.

\section{Differential Diagnosis.}

A chancre may be confounded with a traumatic lesion, soft sore, Herpes genitalis, aphthous ulcer, gonococcal abscess, and Balanitis erosiva et gangrenosa.

A traumatic lesion is irregular in outline; it is an ulcer in parts, and an erosion in parts; at one pole it often is difficult to say where the trauma ends and the healthy skin begins; the lesion soon becomes infected with pyogenic cocci, hence it is surrounded by an area of inflammation; unless soon healed, pseudo-induration may occur; and the sore is usually situated on the frænum. Furthermore, the lesion is painful, which a primary sore practically never is.

Soft Sore.-The incubation period of a soft sore is, as a rule, as many days as that of a syphilitic sore is weeks. It is an ulcer almost from the start, at first quite superficial, and, later, deep. The circumference is irregular, although sharply circumscribed, because one pole of the ulcer tends to heal while the other pole is spreading. Most ulcers have a zone of protective cells surrounding them, which causes the edge to be raised. If the cause of the ulcer is an organism which produces pus-and nearly all organisms which do form pus spread very quickly in the superficial part of the lesion, as they prefer aerobic conditions-it will be readily understood how easily the raised edge can be undermined. Ducrey's bacillus, the organism which causes the soft sore, fulfils all these conditions, and, as it is a pus-producing organism, every lesion is surrounded by an area of inflammation, which is naturally most pronounced at the spreading part of the ulcer, where the edge is also most undermined. The base of a soft sore is not smooth like that of a syphilitic sore, and resembles almost exactly a map in relief.

Some soft sores are very chronic, and they may become pseudo-indurated, but they, nevertheless, still retain the characteristics above mentioned. The adenitis is generally a late, and not an early, manifestation.

If a primary sore is about to develop on a soft sore-i.e., if the patient has a mixed infection, an occurrence frequently met with in text-books, but not in practice-the base of the ulcer, not, as a rule, the whole of it at one time, becomes raised, the distinct raised and undermined edge vanishes; the ulcer looks more like an erosion, and the loss of surface is often raised above the level of the surrounding skin, or is flush with it. Induration is a late phenomenon, and only becomes manifest when the chancre begins to heal. 


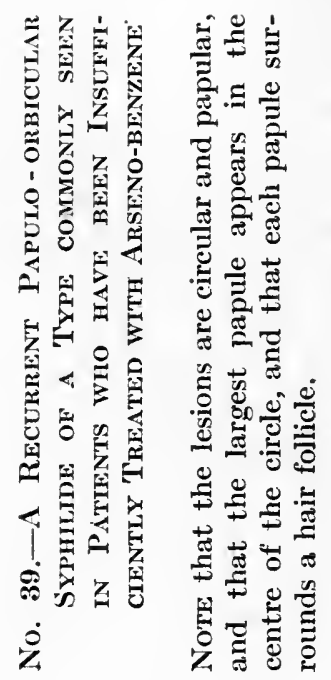

章

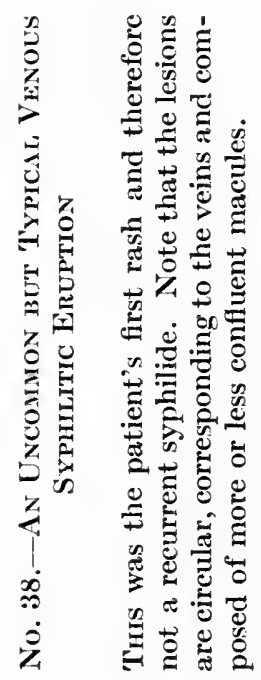




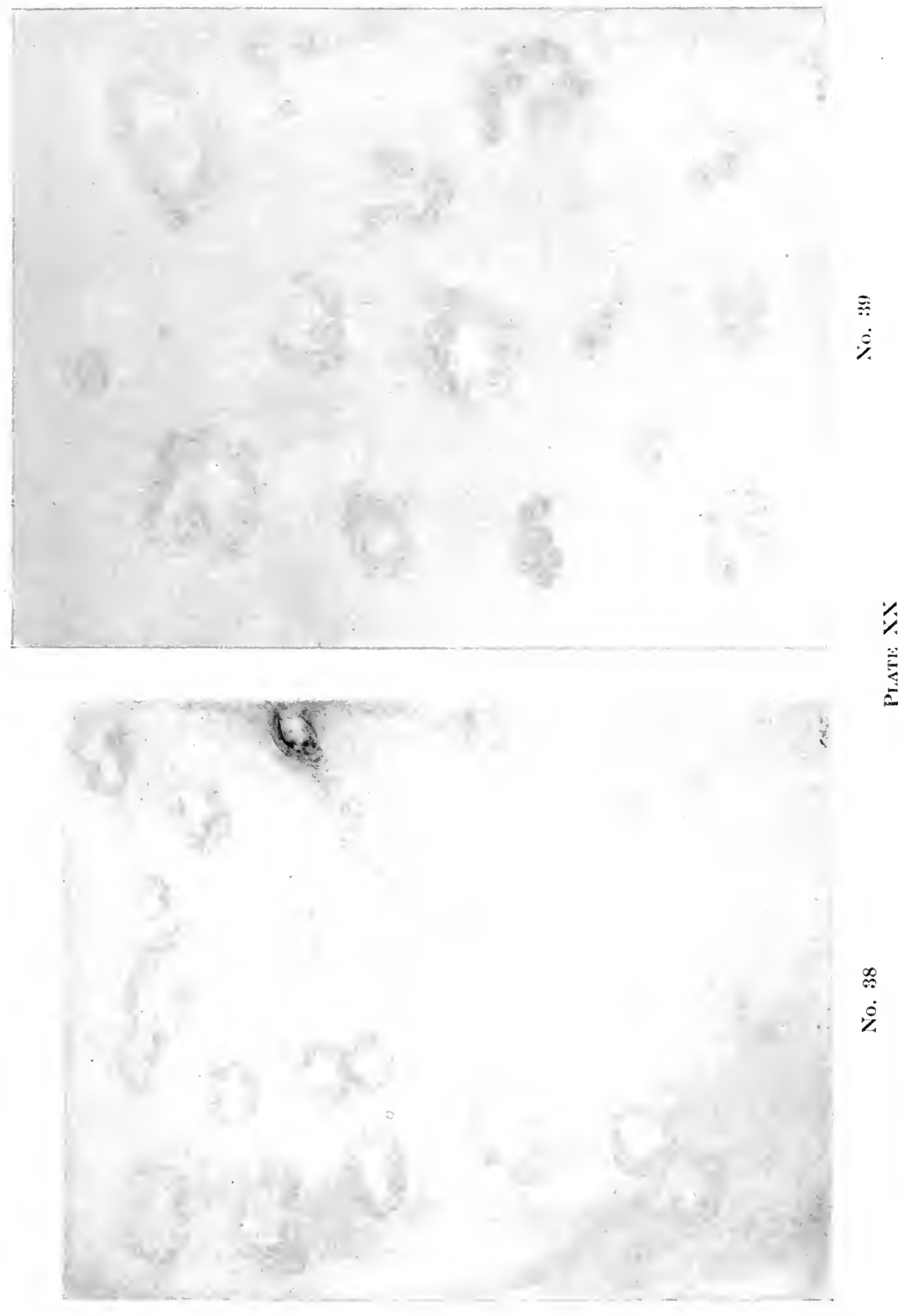




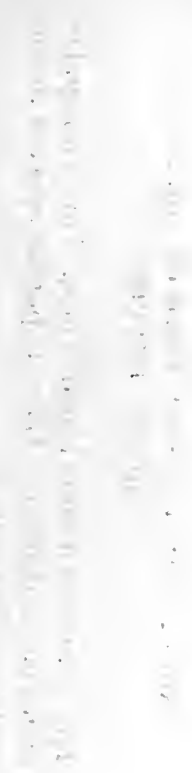



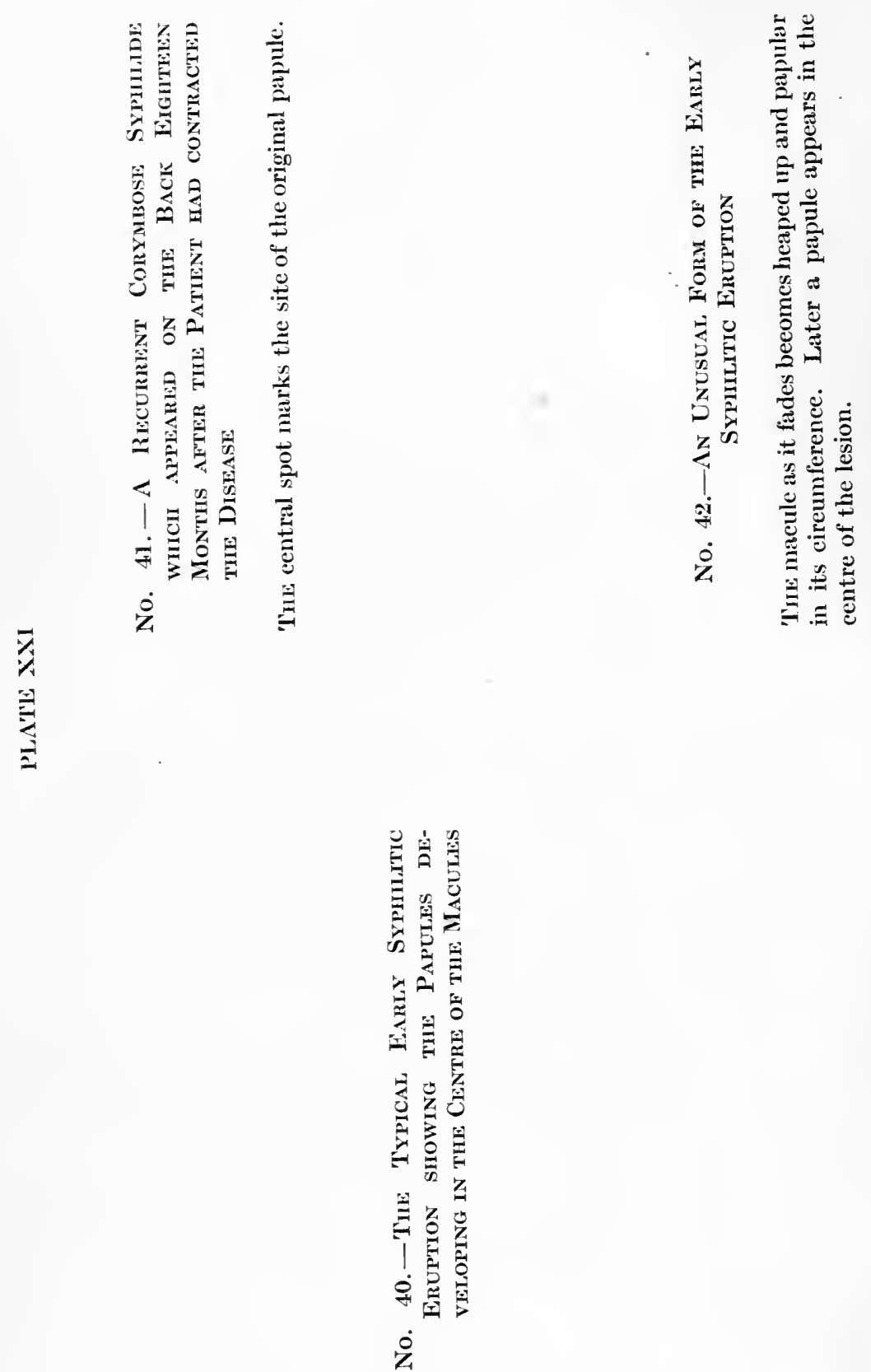


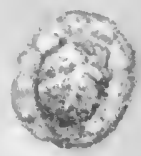


. 
Herpes genitalis is primarily a vesicular eruption of nervous origin, and is very apt to recur. The lesions are grouped. They may quickly become crateriform ulcers, and, if treated with caustics, pseudo-induration is certain to supervene. As a rule, herpes is only confounded with syphilis when, under energetic treatment, the lesions get worse instead of better. Herpes is primarily a lesion due to irritation, therefore it follows that the longer the irritation is kept up, the more chronic the lesions will become. The herpes lesion may be single and very like a simple erosive chancre the papular stage of which is not discernible, but this single lesion is similar to the multiple lesions in being very painful. The pain is a very useful diagnostic sign. The single herpes lesion has also primarily a vesicular stage.

An aphthous ulcer is also due to irritation, and is likewise of nervous origin. It occurs quickly, and before the patient is aware that anything is wrong a characteristic ulcer or ulcers, as they are frequently multiple, have developed.

The lesion is small, sharply circumscribed, generally circular; the base of the ulcer is smooth, yellow, and depressed; the edge is not raised, but is marked by a narrow red inflammatory ring, which is sharply cut off from the healthy skin just external to it. The ulcer is frequently crateriform.

Gonococcal Abscess.-Tiny abscesses, which occasionally ulcerate, may be mistaken for chancres, especially in women. A gonococcal abscess is like a furuncle, but it ulcerates rapidly and more readily, otherwise it is not characteristic.

Balanitis erosiva et gangrenosa is a condition which consists of several lesions, which spread extremely rapidly, and cause more destruction in twentyfour hours than a syphilitic lesion would do in a month. The incubation period is short; the organisms causing the trouble will only develop under anaerobic conditions, therefore the lesions will begin to heal in a few hours if left exposed to the air and frequently bathed with hydrogen peroxide. The two characteristic features of this condition are the pain and smell.

\section{Skin Eruptions of the Generalisation Stage.}

It has been customary to divide the course of syphilis into three stagesi.e., primary, secondary, and tertiary.

The primary stage only included the chancre.

The secondary stage began when the headaches appeared, and when the patient developed a rash and sore throat.

The tertiary stage began after the second year.

Symptoms of the secondary stage may be met with years after the infection. There is a common opinion that secondary syphilis is unimportant compared with tertiary syphilis. From these facts it seems to me to be wiser to abandon the above nomenclature and to adopt the following:

The stage of the initial lesion-i.e., the chancre. 


\section{CLINICAL ASPECT AND TREATMENT OF VENEREAL DISEASES}

The stage of the generalisation of the virus. All symptoms arising while the organisms are pervading every nook and crevice in the body would be included in this stage. The latent stage-i.e., the period during which the patient has no symptoms, or, in other words, the period during which the organisms are presumably dormant.

The recurrent stage, or the stage in which symptoms recur after the virus has become generalised. Gummata may be considered as a variety of this stage.

For the moment, we are concerned with the stage of the generalisation of the virus.

The organisms reach the skin by the blood-stream, and they begin to develop in the uppermost layers of the corium. The macule is the first indication of the local development of the parasites, the roseola being, in my opinion, the first indication that the organisms have reached the skin, and it is of the nature of a toxic rash. When the organisms first reach the skin they act as foreign bodies. The host will make an attempt at defence, which is signalled by a dilatation of the capillaries. This dilatation allows more blood to come to the skin, with the result that it gets redder, the so-called roseola.

Later, owing to the fact that the organisms are developing in areas, and not equally all over the skin, the generalised roseola gives place to the more localised macule.

The macule varies in size, from that of a threepenny-piece to that of a half-crown. Although more or less circular in outline its circumference cannot be accurately mapped out, as it is difficult to say where the macnle ends and where the healthy skin begins. This is due to the fact that the macule does not accurately correspond with the area in which the organisms are developing. The macule is mainly a toxic phenomenon, like the roseola. Later, in the centre of a macule, a papule appears, and, from this time onwards, the macule gradually fades, and finally vanishes. The papule slowly increases in size, but scarcely ever covers the entire area of the macule.

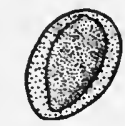

MACUle.

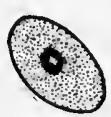

Papule developing in Centre of Macule.

In some of the larger macules, a tiny little papule appears in the region of each hair follicle.

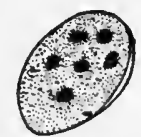

Follicular SyPHILIDE.

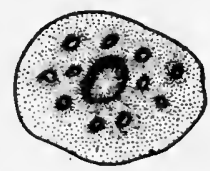

CORYMbose Syphilidf. 



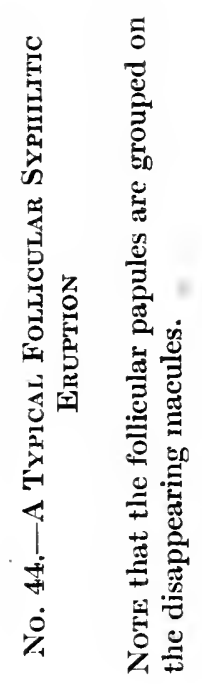

齐

空

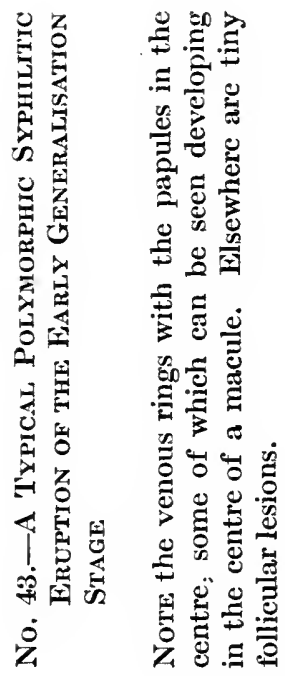




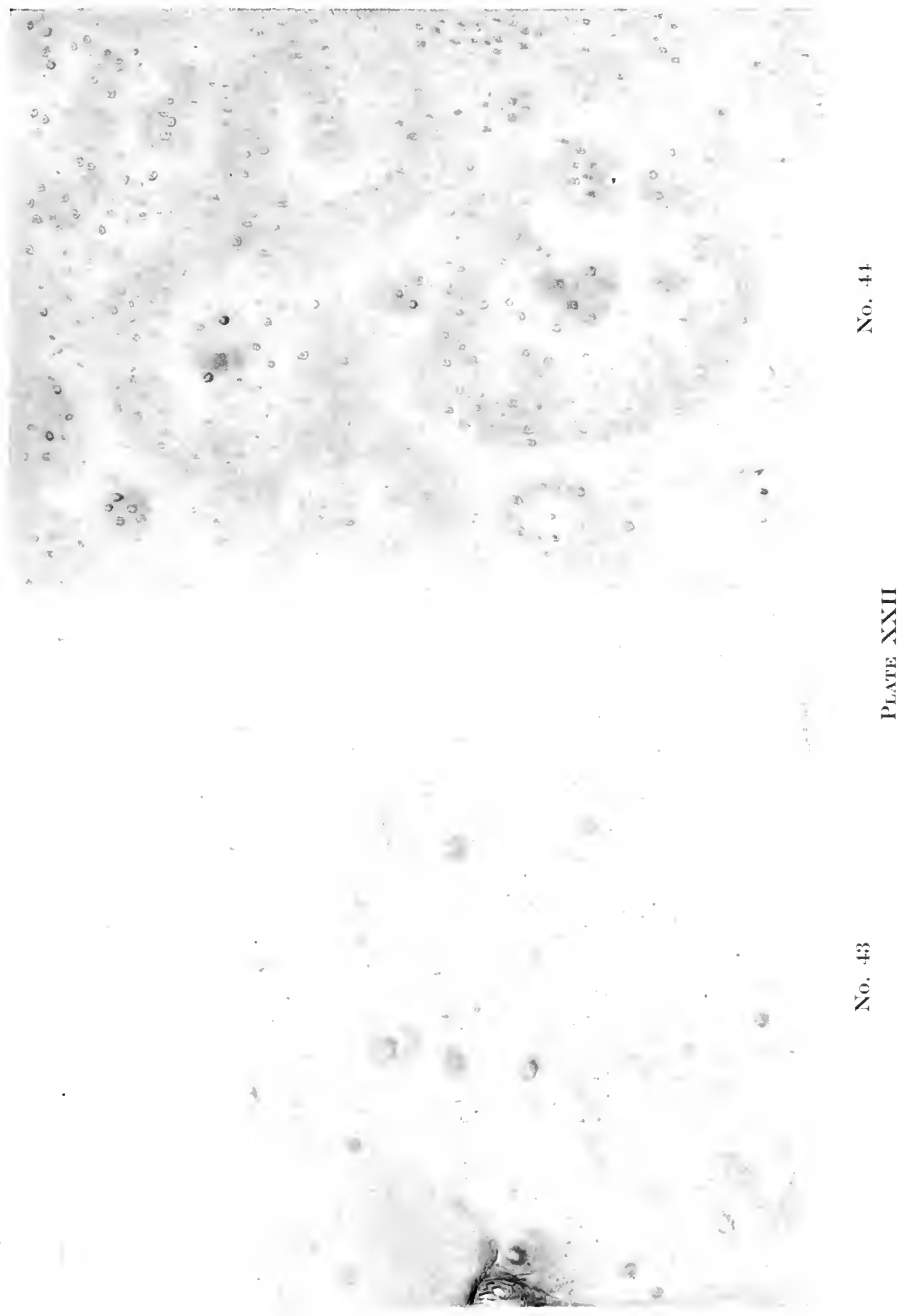






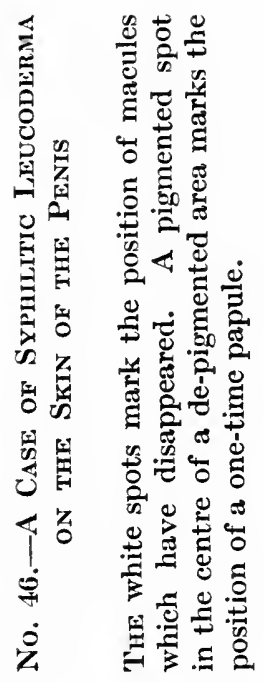

$\exists$

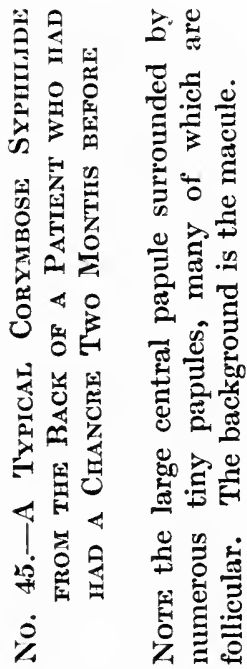



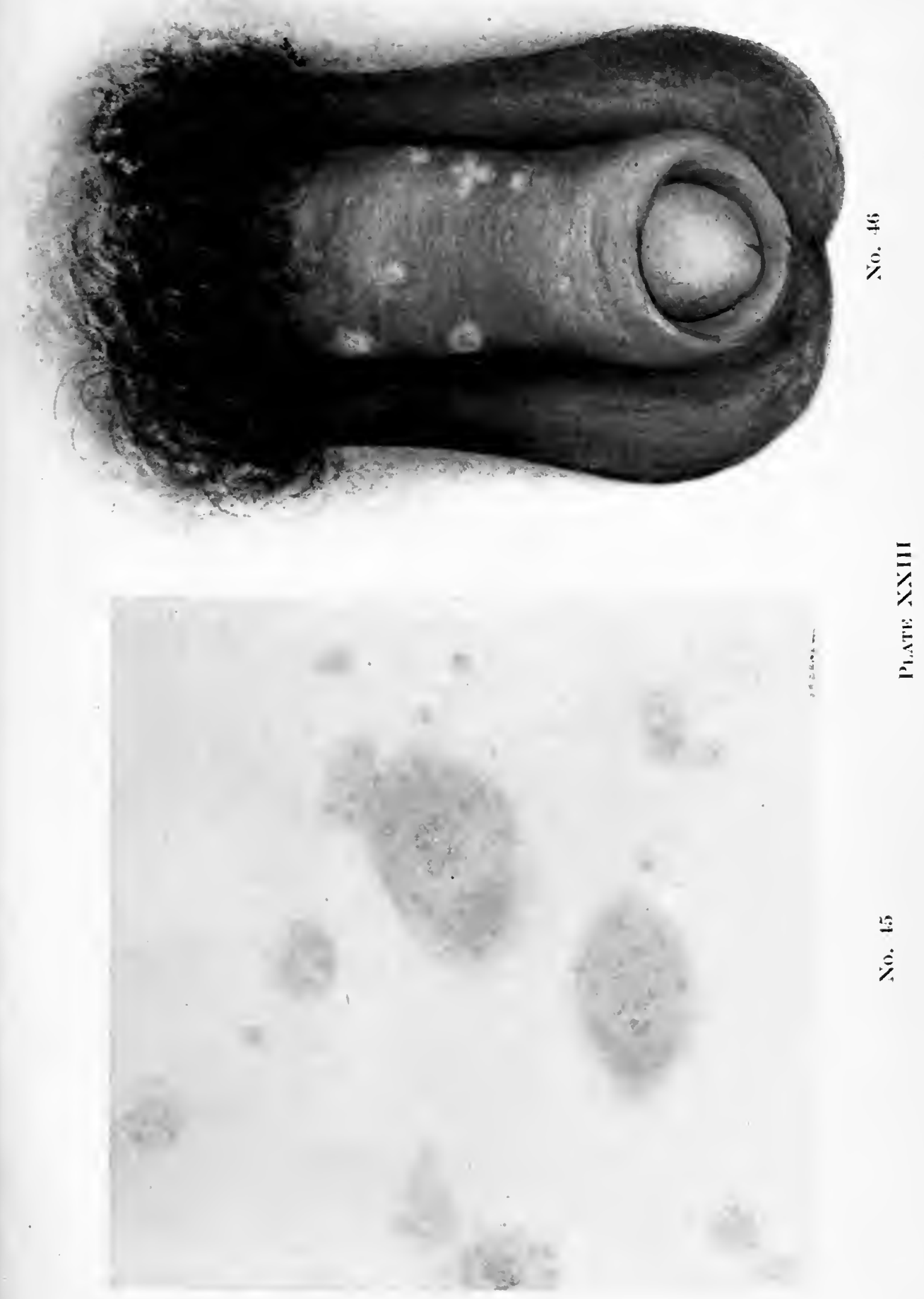


\section{$\checkmark$}


Occasionally it happens in the follicular syphilide that the central papule develops in the ordinary way. This lesion receives the name of corymbose syphilide.

As a rule, the follicular and corymbose syphilides are most marked on the back, especially along a transverse line drawn through the centre of both scapulæ.

When a papule begins to disappear it develops scales, and, as the central part of a papule is the oldest part, retrogression will commence in the centre, in the same way as the papule develops in the centre of a macule. The result is that the scales will be found only in the middle of the papule. When the whole papule has begun to retrogress, naturally its whole area will be covered with scales, but there are bound to be some papules elsewhere on the body, and in them only the central part has scales.

Owing to this unequal development, a macule may exist near a scaly papule, and it is this polymorphic character of the first syphilitic rash which is so diagnostic. The polymorphism is, I think, another point in favour of my life-cycle view, because it would be difficult to explain why lesions in various stages should be met with when the spirochætæ are evenly distributed over the skin at the same time. The explanation is simple on the basis of my life-cycle view, because so many phases have to be perfected before the end phases are in sufficient number to give rise to symptoms; and, in the process of formation of these phases, so many factors may arise to hinder their development. This, to my mind, also explains why recurrent lesions may be seen exhibiting the characters of a chancre in one part of the body, a papular syphilide in another, and a gumma in the third. The degree and nature of the resistance proffered by the host vary in different areas, as I have seen cases of co-existing papular syphilides and gummata in which the former were untouched by intramine, and the latter healed at once; and other cases in which the former disappeared at once under arseno-benzene, while the latter remained resistant. A papule need not necessarily become scaly, it may degenerate in the centre instead, with the result that a pustule forms. Some of these pustular lesions are not unlike varicella; but the distinction between the two becomes simple when the anatomy of the two lesions is considered.

In varicella, the organism develops in the epithelial cells. These cells degenerate, and, owing to the presence of the lymph between them, a vesicle is formed. As the degeneration extends to the corium, and leucocytes can obtain entrance to the vesicle, it is quickly turned into a pustule.

As the organism develops in the epithelium, the surrounding toxic or areal inflammation will be minimal, with the result that, when the vesicle is first formed, it will cover practically the whole lesion. Since the vesicle forms in the epithelium, it follows that the roof is very thin and insecure. The syphilitic organism, on the other hand, develops in the corium. Degeneration of the corium leads to a pustule at once, therefore no vesicular lesions 
will be found. The degeneration occurs primarily in the centre only, therefore the pustule will not cover the whole area taken up by the papular lesion. The degeneration commences in the corium, therefore the roof of the pustule will not be thin and insecure.

The whole papule may degenerate, and then usually a crust forms. Owing to the strong proteolytic action of crusts, degeneration of the subjacent tissue may continue. The underlying tissue may be digested in the centre only, or at the periphery. If the former, there will be a heaping up of the crust in the centre, so that ultimately it will resemble a limpet shell.

If the latter, the lesion will increase in circumference, but not in depth.

To either, or both, the term rupial syphilide is frequently given.

Occasionally a group of papules may never disappear. I have such a case in mind, a medical man, who consulted almost everybody, and underwent every kind of treatment imaginable. I removed one of these persistent papules, and histologically it contained nothing else but typical connectivetissue cells-in other words, it was a soft fibroma.

Another very typical early skin eruption is the so-called seborrhœic syphilide.

The lesions may be the first to appear, and, on the whole, they are commoner in women than in men. They are circular, the edge is often raised, the centre is yellowish, and the whole lesion looks as if it were covered with very fine fatty scales, which come more into evidence when the lesion is rubbed.

The lesions invariably occur on the face, and some are always situated at the corners of the nose and mouth.

In a few cases, instead of a macule and papule developing as above described, the first lesion may be a circular erythematous one, corresponding to the venous circle which surrounds each area of skin, and which is seen so well in the condition called livido. This circular lesion must not be confounded with a recurrent syphilide. The lesion is irregular in outline, and continuous, while the recurrent lesion is, if larger, regular in outline, and if of the same size, not continuous, as the circle is made up of a number of papules.

A papule in a moist area may become oedematous and hypertrophic, and then it receives the name of Condyloma latum. Condylomata lata are most usually seen about the genitals and the anus, but they may also occur in the umbilicus, axillæ, eyelids, and between the toes. A papulo-pustular lesion may become œedematous and hypertrophic, when it receives the name of frambcesiform syphilide. The scalp is the most commonly affected part, but I have seen them on the palms of the hands.

Two important clinical observations may be mentioned here, (1) that patients with a well-marked papular rash are particularly liable to develop recurrent mucous papules in the mouth throughout their lifetime, and irrespective of treatment; (2) that patients with a severe generalised eruption are particularly liable to develop severe syphilitic meningitis. 


\section{$-$}




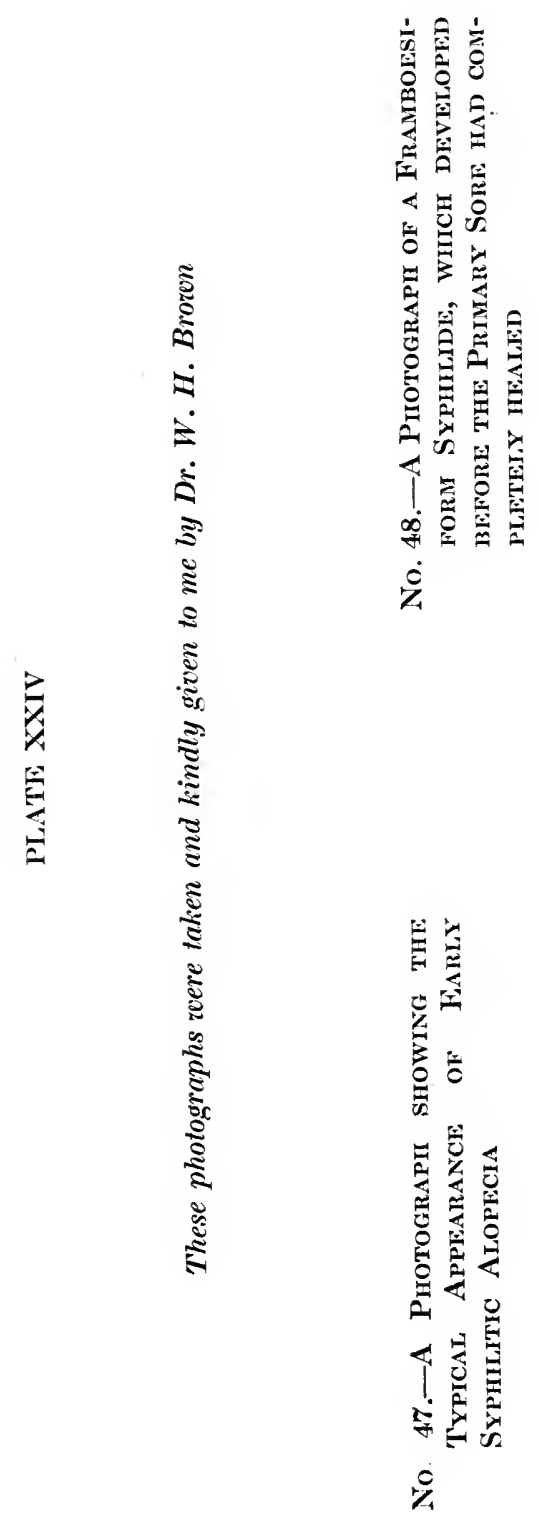




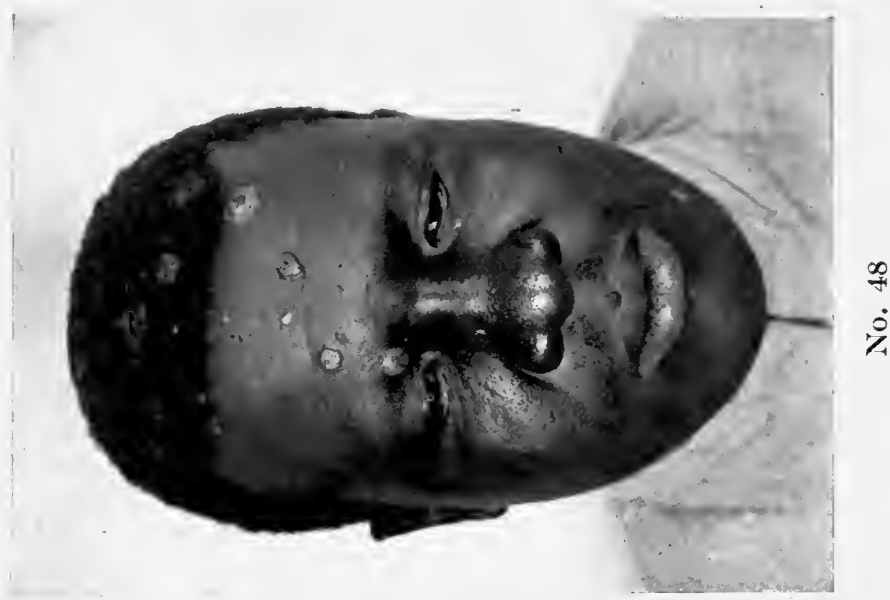

稌

客

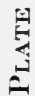

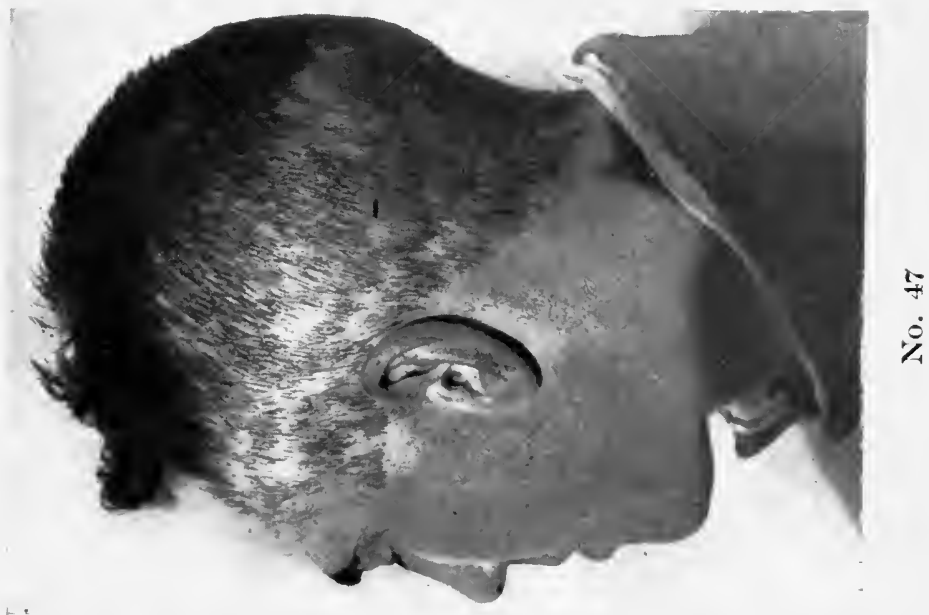





\section{.}




\section{PLATE XXV}

No. 49.-An Early Sypinditic Eruption closely resembling

Seborrhoea Corporis

Tins type of rash is more common in women than in men, the lesions are oily as in seborrhoea, and as a rule they affect the face only. In practically every instance some lesions will be found in the naso-facial folds and some in close proximity to th e mucous membranc of the lips. As in this case, the lesions may form the so-called Corona veneris. 


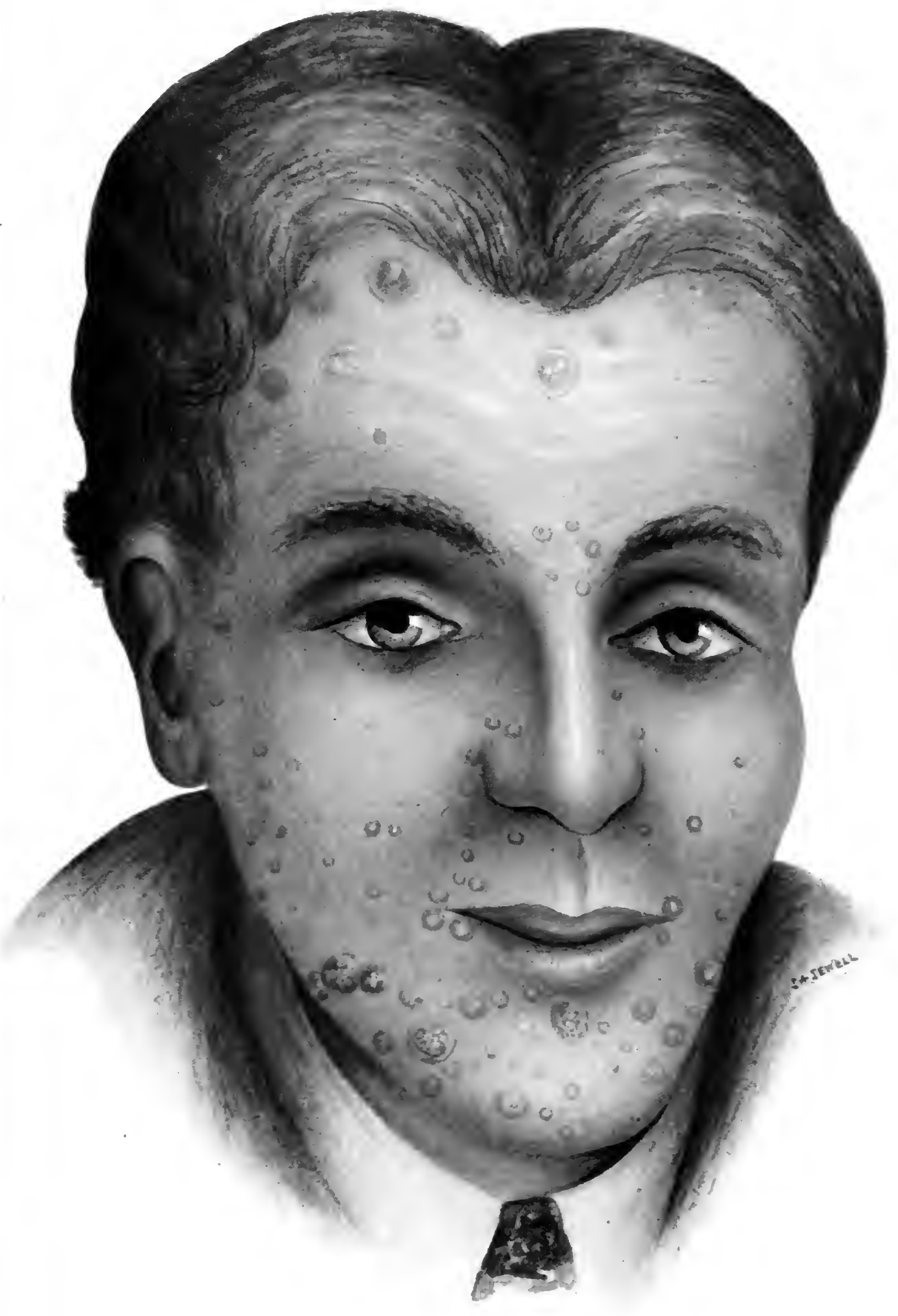

N๊. 49

Plate Xxy 

The syphilitic alopecia is the result of a maculo-papular eruption of the scalp, and occurs in the following way: Where the true macule occurs there is little or no loss of hair. Where the true papule occurs there is complete loss of hair, and in the area between the two the loss of hair is less. This accounts for the irregularity of the loss of hair which is characteristic of syphilitic alopecia.

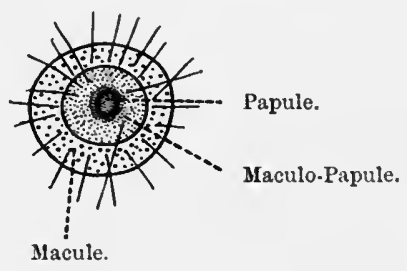

Typical Alopecia areata may occur in early syphilis, but this is an atrophic phenomenon, and probably in part due to the toxic action of arsenic and mercury. A similar atrophic condition may affect the nails. Another characteristic lesion, for which both the macule and the papule are responsible, is the socalled Leucoderma syphiliticum.

The lesions may appear singly, or in rosettes. They are roughly circular in outline, about the size of a sixpenny piece, and are depigmented.

Occasionally, in the depigmented areas, a hyperpigmented spot may be observed. The former corresponds to the macule, and the latter to the papule.

Leucoderma syphiliticum is most common in women, because the skin is more delicate, and therefore the lesion can be more easily seen. For the same reason it is more apparent in brunettes than in blondes.

It is most frequently seen on the neck and the folds of the axillæ, but it may affect practically the whole trunk. It disappears in course of time, but is uninfluenced by treatment. Vitiligo in syphilis is discussed in Chapter IX. Naturally, the clinical appearance of papules will vary according to their localisation. The so-called mucous patches are papules occurring on mucous membranes. Striking lesions of the nails may occur, according to the position of the papule and the manner in which it develops.

A papule may occur far back at the base, and injure the bed of the nail, with the result that the development of the nail is impaired. A characteristic doughy feeling of the base ensues, and the nail is atrophied and soft.

A papule may break through the centre of the nail, or develop at the sides, and, provided that it remains dry, it not infrequently causes a brown to black discoloration of the nail.

All the lesions so far described are discrete, but there is a diffuse syphilitic lesion to which sufficient attention has not been drawn; but, nevertheless, it is very characteristic of the disease. This lesion is a diffuse papular infiltra tion of the skin of the penis, and it arises by a direct spread of the organisms from the chancre (Plate XVIII.). This lesion is not an uncommon one, and it 


\section{0}

may well have retrogressed before the generalised eruption puts in its first appearance. It is not at all uncommon for the first and the most developed lesions to appear in the neighbourhood of the primary sore. Occasionally, the maculo-papules on the trunk may lead to true atrophy of the skin. The atrophic lesion corresponds with the area covered by the maculo-papule. The skin is wrinkled; it looks as if it were raised; it feels very soft and thin; and a pit is generally to be ascertained on pressure. Atrophic lesions are most frequently met with on the shoulders, especially over the scapulæ behind. Naturally, the lesions never alter. Papulo-ulcerative lesions may occasionally heal with keloid formation, which remains indefinitely or disappears spontaneously.

\section{Recurrent Syphilitic Eruptions.}

All recurrent syphilides tend to appear in circles or in segments of circles, owing to the peripheral spread of the organisms from the region in which they were when their development gave rise to the papule. The lesion may appear as a single circle, the so-called orbicular syphilide, or concentric circles may appear, the so-called annular syphilide.
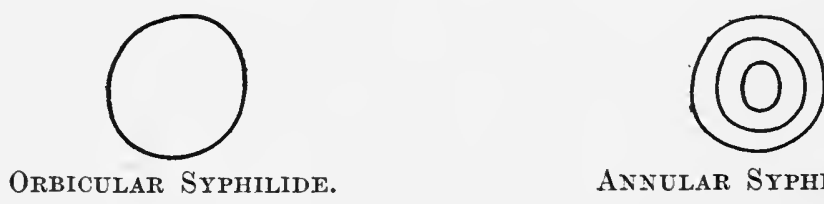

ANNulaR SyPhilide.

The commonest recurrent lesions are not so regular as the two just mentioned; either the whole circle is made up of distinct papules or only a segment of it is apparent.
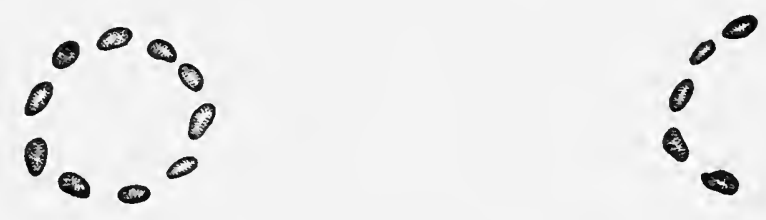

Not infrequently the original papule itself recurs, as, often in the middle of the circular lesions, a papule is seen.
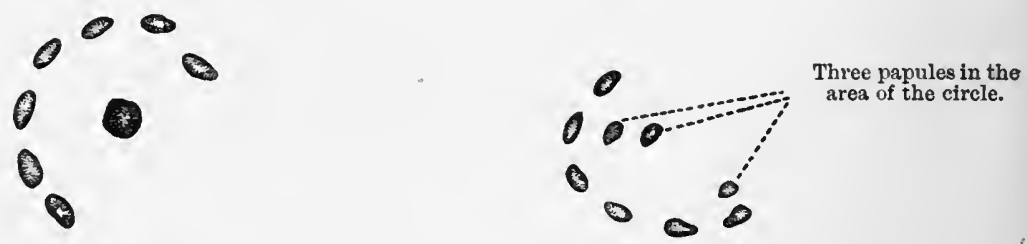

It can be easily understood that, as the papules in the circumference of the circle mark the limit of extension of the organisms from the centre, some 

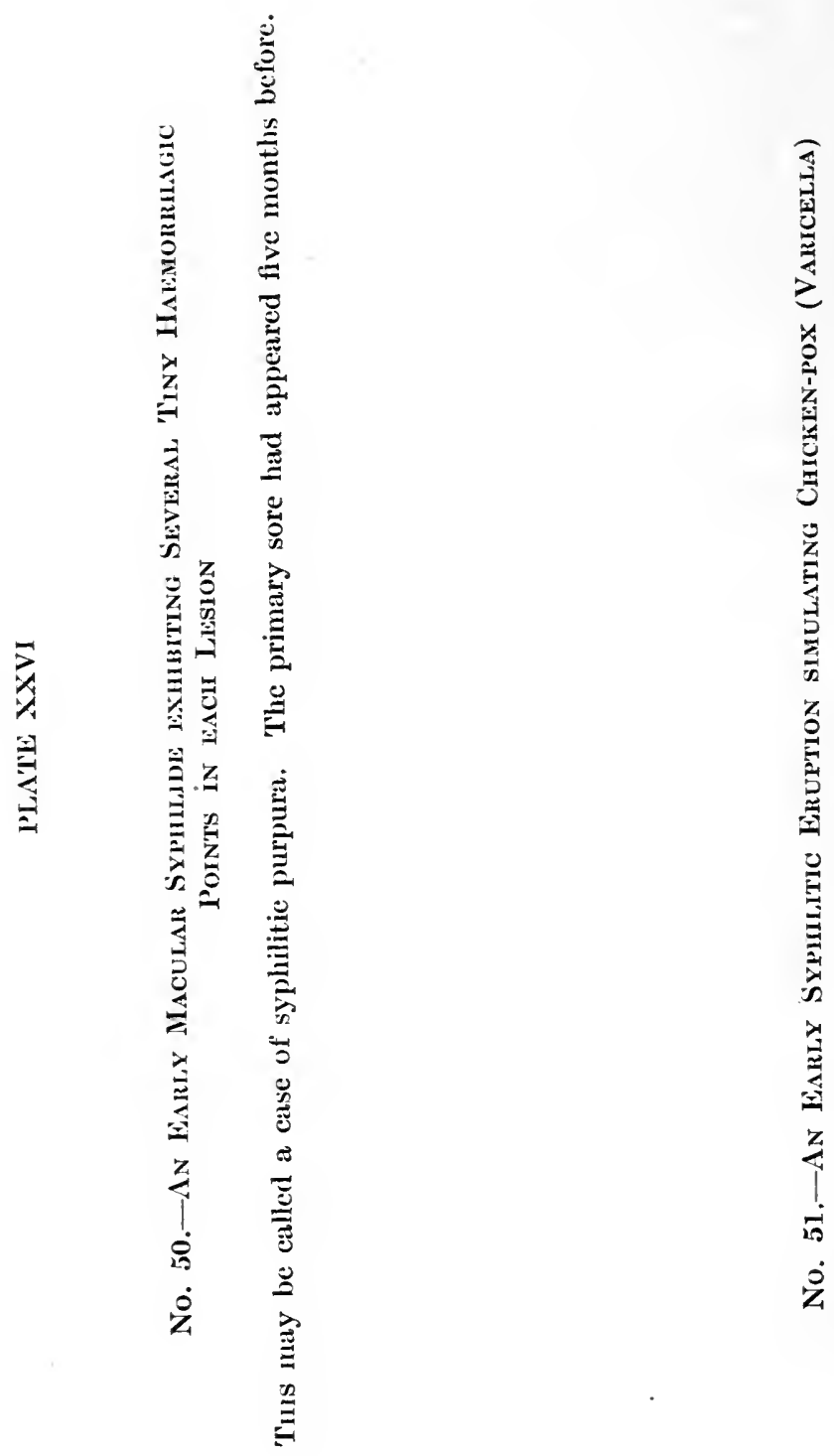

范

烦

年 

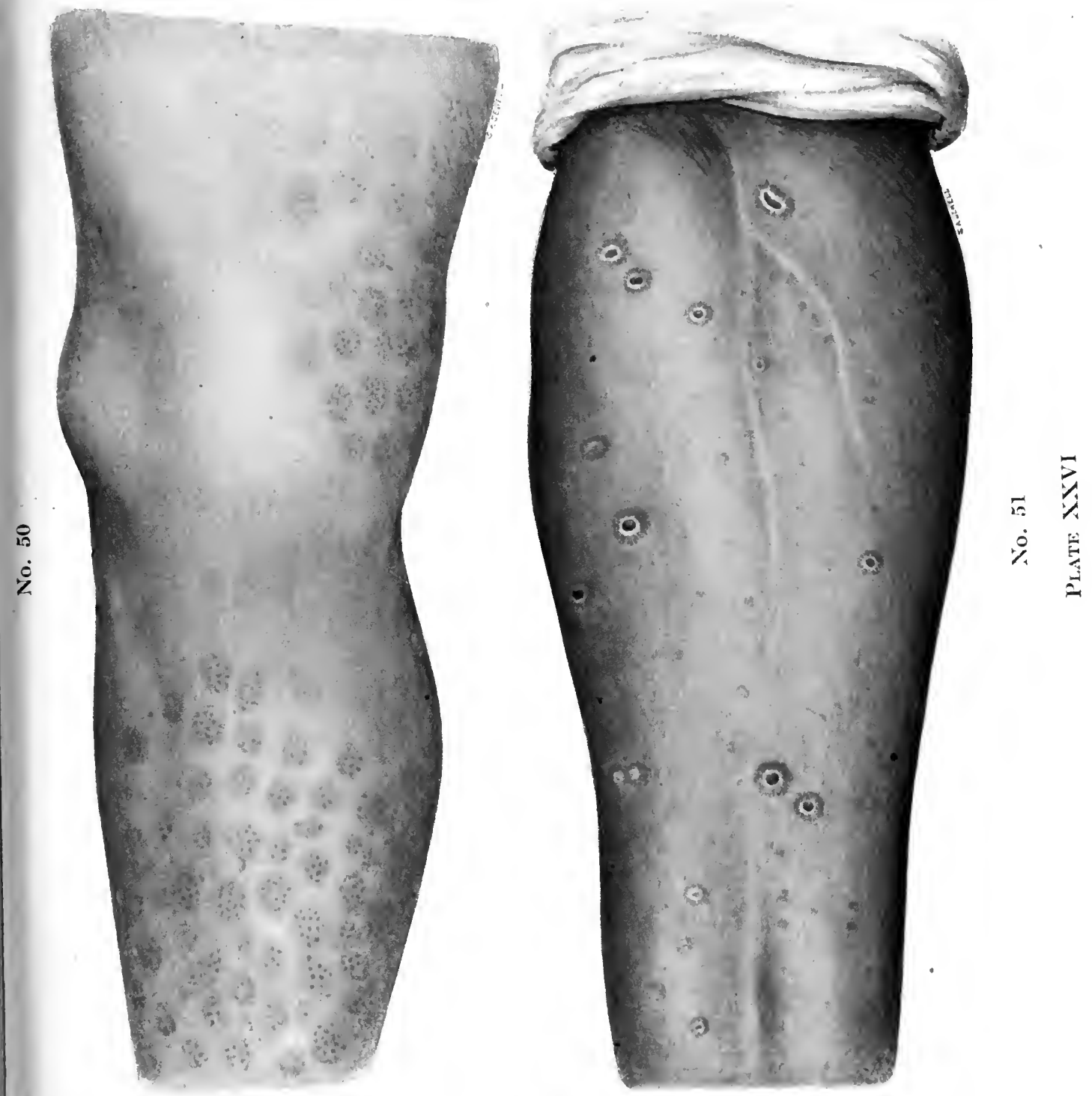



\section{PLATE XXVII}

No. 52.-A CASE of Seborrhoea Corporis

Note the primary lesion with a hair in the centre.

No. 53.-An Early Syphilitic Eruption Simulating Seborrhoea

Corporis, with the Diffenence that tine Lesions are not Follideut.ar 
No. 52
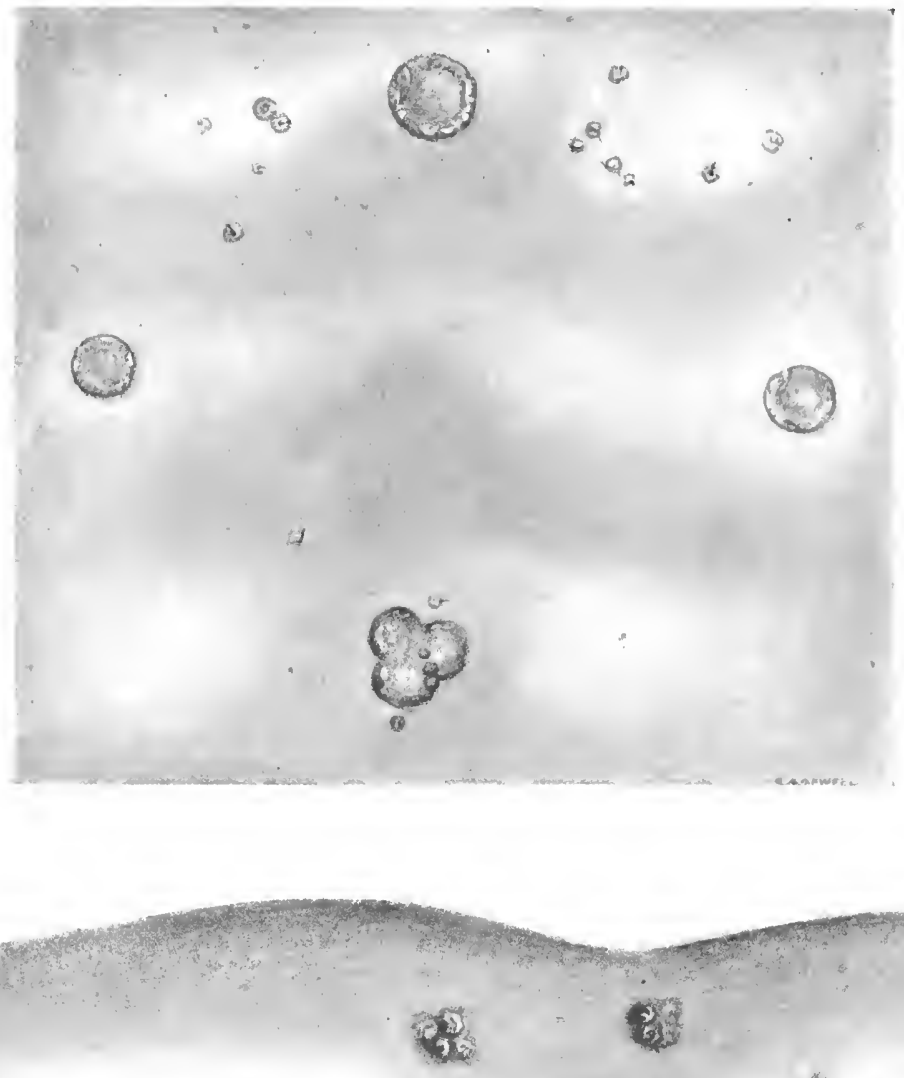

z Q6

9

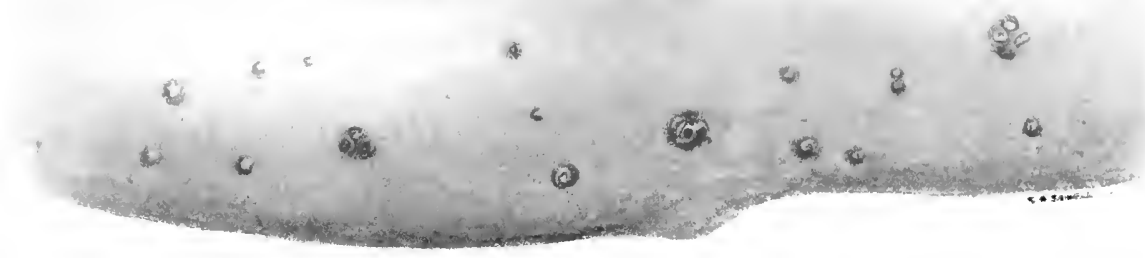

No. 53

Plate XXVII 

of the organisms may have remained half way, for instance. This will result in papules being found in the recurrent lesion at any part of the area of the circle.

Many circular lesions may occur close together, and the tout ensemble may look something like a maze; but, if carefully studied, the circular arrangement of each individual lesion can be easily made out.

As the original papules may undergo certain transformations, so also may the recurrent papules. Some may be larger than others; in some, necrosis may occur, with the result that a scab develops. On removal of the scab, if the lesion is an early one, a small ulcer may be met with underneath; but most frequently the ulcer beneath has scarred, and the floor is covered with well-formed epithelium. This latter condition is rather characteristic of syphilis.

Another almost pathognomonic feature of the scabbed recurrent papule is, that when the scab is removed, the healed floor is uneven-divided into several loculi-and each of these loculi is separated by a tiny bridge of normal skin.

Presumably each loculus has been a distinct lesion, but, when each ulcerated, a common scab formed for all. These loculated scarred lesions are frequently to be seen on the face, in the naso-facial and oro-facial grooves. Especially when they occur in the region of the mouth, almost certainly one or more lesicns will be met with, one pole of which will be touching the mucous membrane of the lower lip. A recurrent syphilide affecting a lip is apt to be accompanied by a hard œdema of the lip, which is characteristic.

One is frequently called upon to differentiate the facial scars which have resulted from Lupus vulgaris and from syphilis.

The tubercular scar is, as a rule, irregular in outline, sharply circumscribed, and only very slightly depressed below the surface of the surrounding skin. It is, moreover, even and shiny on the surface, and not infrequently infiltrated with telangiectases, especially if the lesion has been treated with X-rays.

The syphilitic scar, on the other hand, is not one flat scar, but an area made up of several little ones. Each little scar is deeply depressed, not. shiny, and usually quite white. If the organisms, instead of spreading from a papule here and a papule there, spread from those papules covering one large area of the skin; should they in time develop their life-cycles, the lesion formed will be either a very large circle, or a segment of a very large circle. This is the so-called serpiginous syphilide. Characteristic of the serpiginous syphilide is the red-purple discoloration of the skin in the circle. There is a special kind of recurrent syphilide which $I$ have never seen described, and which occurs in the form of large erythematous plaques. The patient looks very ill, he is emaciated, and exudes a peculiar but typical odour. If the lesion occurs on the face it takes the butterfly-pattern so typical of Lupus erythematosus. When the face is involved the patient is usually a sufferer 


\section{CLINICAL ASPECT AND TREATMENT OF VENEREAL DISEASES}

also from syphilitic rhinitis. The skin lesions rapidly disappear under treatment, but the rhinitis appears to be little benefited.

The last recurrent skin lesion to be described is the gumma, and the way in which a gumma is formed is, in my opinion, the following:

The distribution of the blood-supply in the skin is not unlike that in the liver-i.e., the skin is divided into circular areas roughly about the size of a shilling. There is a venous ring, so to speak, in the circumference of each circle, and, when congested, it gives rise to circular purple patches with a white centre, to which the name of livido is given. In the centre of each circular area an artery runs, and it gives off branches as radii.

It is along this central artery that the organisms reach the skin, and it is in the walls of vessels that the organisms develop. Should the recurrent papule be of a more pronounced character than those just described-i.e., should the development of the organisms be on a larger scale-there will be a corresponding increase of connective-tissue cells. As these will be formed in the walls of the central artery, they may be sufficient to occlude its lumen. Occlusion of the lumen would result in the loss of blood-supply to the circular area affected; consequently, the skin corresponding to this area would necrose, an ulcer would be formed, or, in other words, a gumma.

In support of the view just enunciated, are the pathognomonic signs that, however many gummata form in a certain area of skin, each will remain separate, they will not coalesce, and each will be approximately the same size, and the scar resulting from the ulceration will have the same diameter as the ulcer had. A point of extreme diagnostic importance, and one that should always be borne in mind, is the fact that the Leucocytozoon syphilidis is not a pus-producing organism, therefore the lcsions resulting from its development have, as a rule, no circumferential signs of inflammation.

A recurrent syphilitic eruption which requires special mention is the Lichen syphiliticus. This is a grouped papular syphilide, in which the lesions are usually deep brown in colour, infiltrated, some follicular, and all extremely resistant to treatment. In some of the groups the central papule is larger than the rest. As a rule, it is a late recurrent syphilide, and only responds to colloidal iodine and intramine.

It sometimes happens that the recurrent rash is generalised, and indistinguishable from the first eruption. Such a rash may or may not be preceded by a sore indistinguishable from a chancre. The chancre-like sore may either appear on the same site as the original sore, or elsewhere.

If the recurrent rash is generalised, and is not preceded by a chancrelike sore, it means that, when the organisms first reached the skin, either the treatment or the host's resistance, or both, were sufficiently powerful to prevent the organisms from spreading peripherally; hence, when they became active again, they did so in the original areas in which their further progress was stopped. Since the arseno-benzene era such recurrences are not uncommonly seen, but before the advent of this drug they were distinctly rare. 


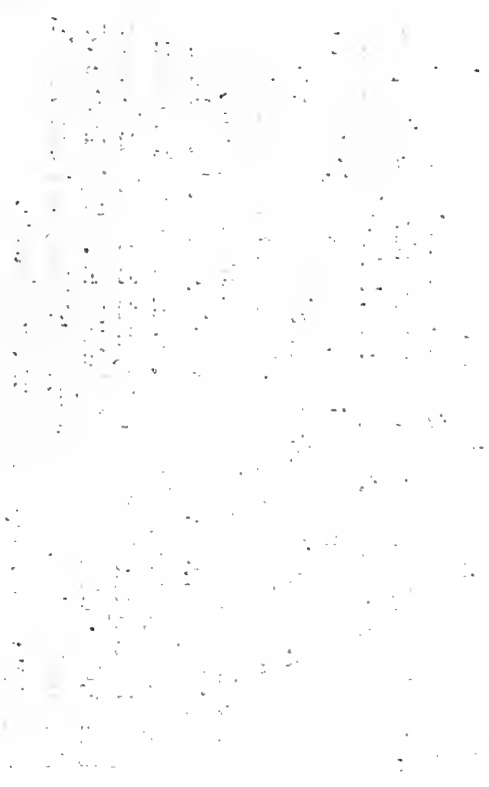


言
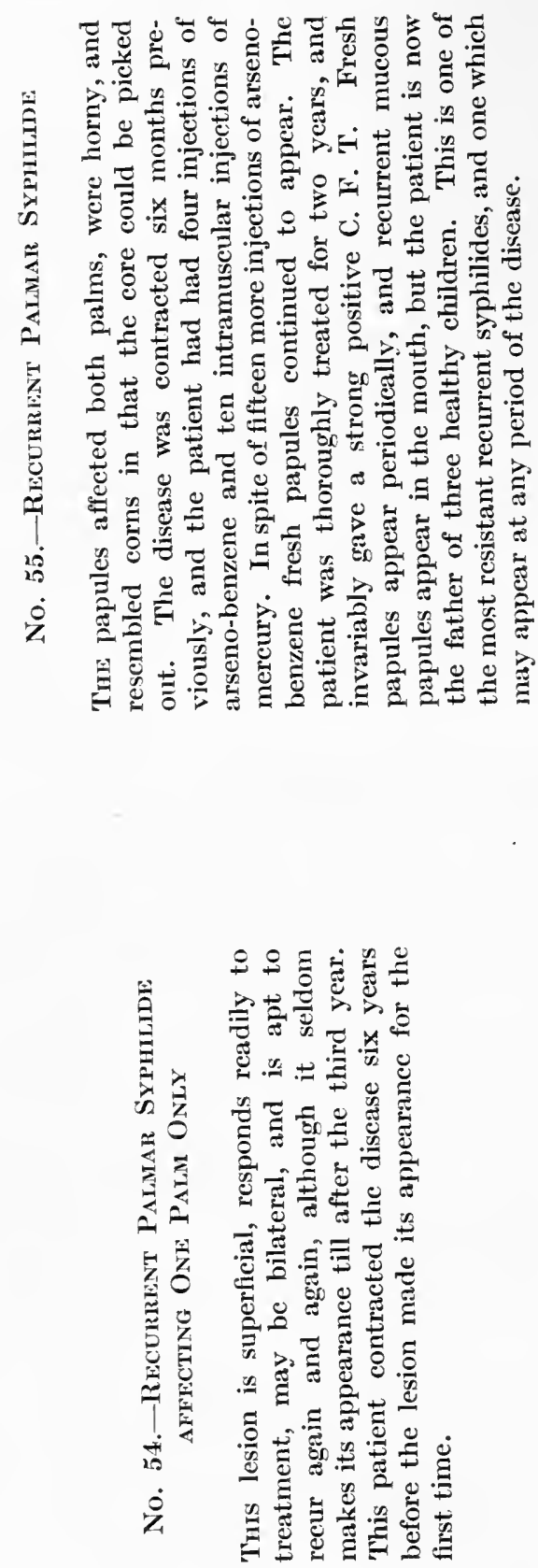


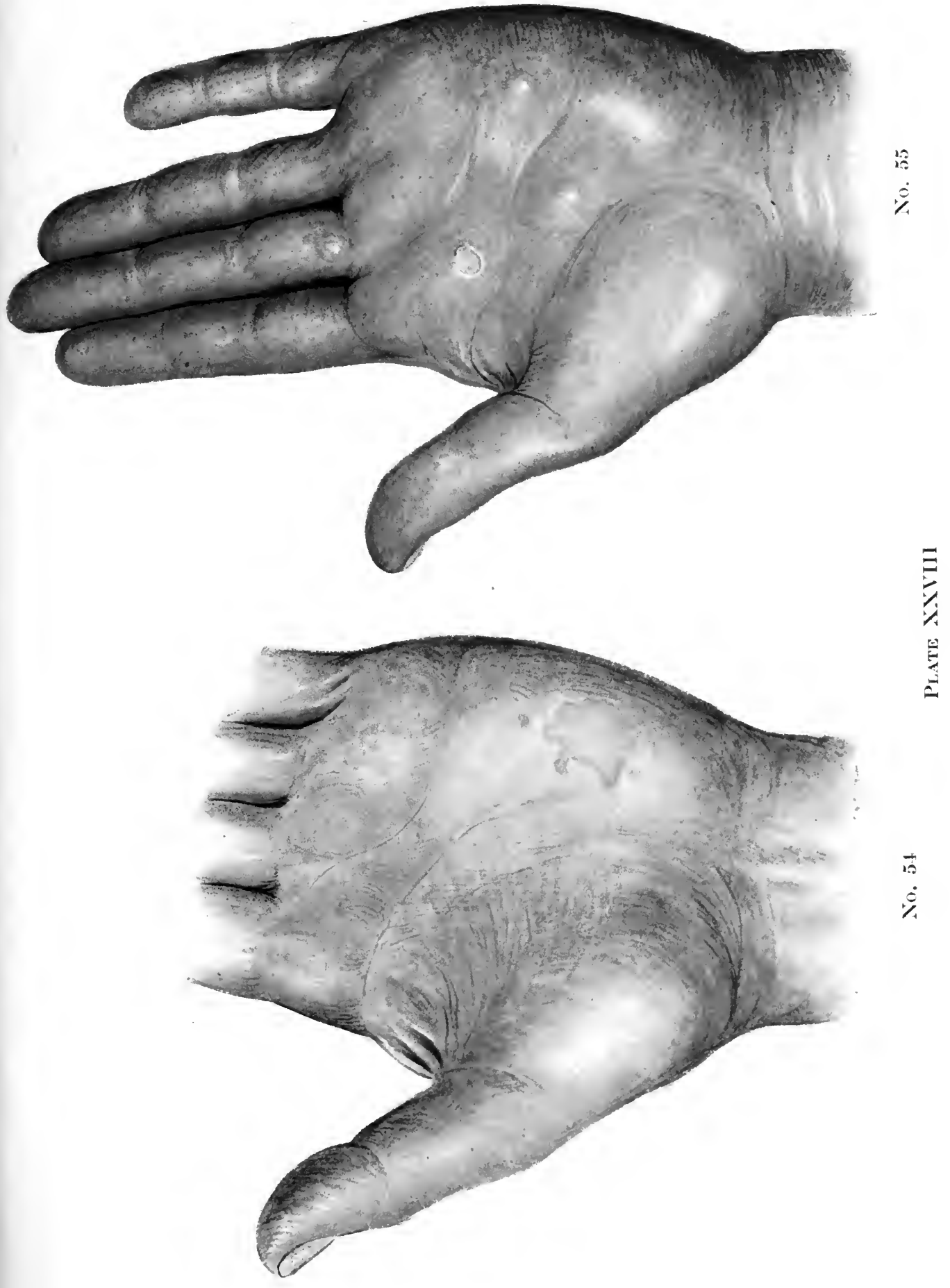





\section{$\bullet$}




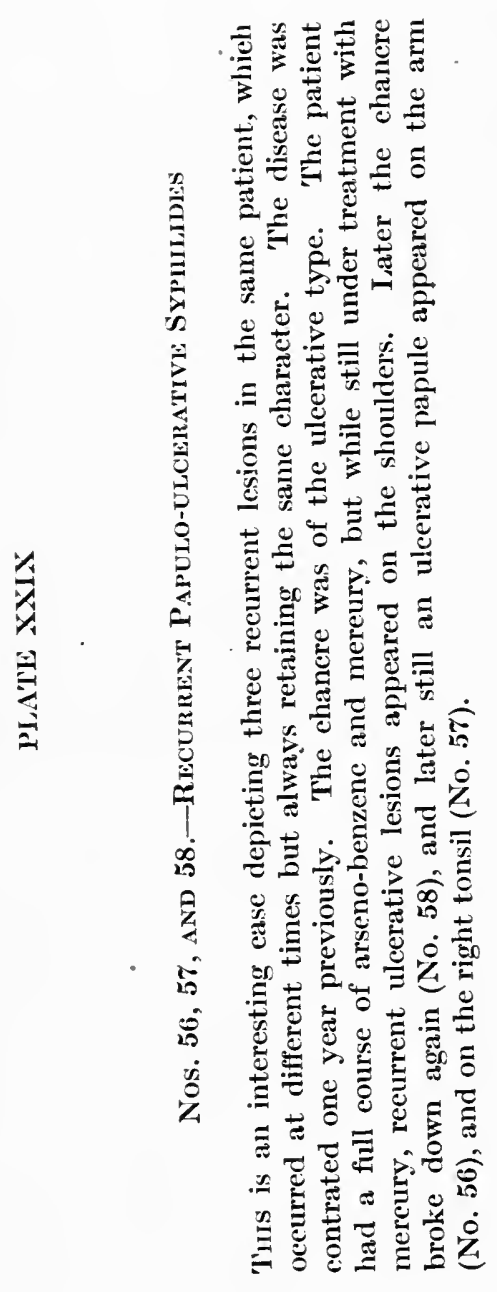



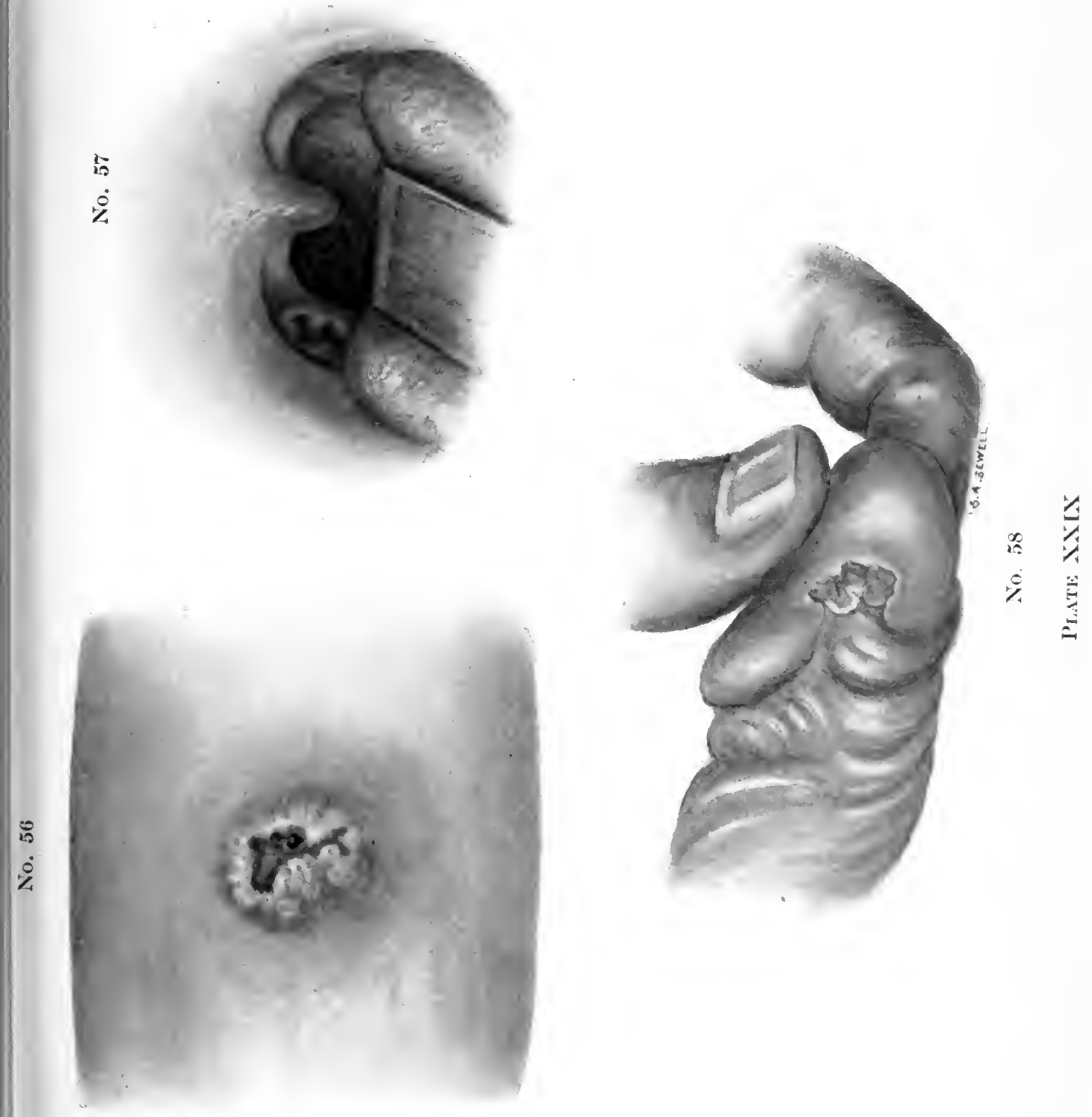
I had one remarkable case before arseno-benzene was in use which might be recorded here.

CASE 5.-A man, aged 54, consulted me, complaining of a rash and a sore throat. The rash was a typical maculo-papular syphilide, and its distribution was widespread. There were multiple lesions in the mouth, which were mucous papules. Every lesion was discrete; there was no attempt at the formation of circles, there was no general enlargement of the lymphatic glands, and the patient had not recently had a sore.

This patient had contracted syphilis twenty-three years previously, and beyond the early symptoms, which simulated those of which he now complained, he had always been in the best of health, and had never had a recurrence.

Originally the patient was treated for about three years with mercury internally.

A recurrent rash preceded by a sore is a case of auto-reinfection, which has already been described.

When a patient presents himself for examination, suffering from a rash the diagnosis of which is not at first sight obvious, the observer should never fail to examine the palms, as syphilitic lesions on this part of the body are so typical. The early palmar syphilides are papular and almost invariably scaly, while the late are distinguished by being circular. If an early palmar syphilide is seen before the scaly stage has been reached, it is most likely to be mistaken for a case of Erythema multiforme; but a further examination of the patient will at once clear the diagnosis. The scaly lesions are sometimes just like corns, and in my experience these cases respond to treatment very badly. The recurrent circinate lesions are apt to be mistaken for ringworm; but in the latter condition characteristic vesicles and pustules are to be found in the circumference. There is a diffuse form of squamous syphilide, which not at all uncommonly affects the soles as well as the palms, which is frequently mistaken for eczema. If the lesion affects one palm or one sole, then it is syphilitic, and if both palms or soles, then it is eczema. Eczema, again, generally involves the fingers, which is not the case with syphilis. The edge is sharply demarcated in syphilis, but not in eczema. There is a syphilitic lesion which may affect the palms, but more frequently the soles, especially the heels and margins of the feet-namely, the verrucose syphilide, which is seldom described, and still less seldom diagnosed correctly. I have seen a verrucose syphilide affect the ankle, and it was accompanied by a solid œdema of the leg, due probably to an invasion of streptococci in the cracks and ulcers which are liable to occur between the warty growths. The lesion just described is most likely to be mistaken for the verrucose tuberculide. Another very favourite locality for a recurrent syphilide is the scrotum; a circinate lesion occurring here and on the forehead at the margin of the hair, the situation of the well-known Corona veneris in the early stages, is practically pathognomonic of syphilis. 


\section{CLINICAL ASPECT AND TREATMENT OF VENEREAL DISEASES}

Syphilis affects the nails in characteristic ways, and the lesions can be divided into two groups:

1. Onychia sicca syphilitica.

2. Paronychia or Perionychia syphilitica.

\section{Onychia Sicca Syphilitica.}

The various forms of dry onychia which have been described may be classified as follows:

1. A brittle condition of the nails, so that the free ends tend to split and break, and the nail border becomes notched or serrated. All the nails may be involved, but especially those of the hands. Fournier ${ }^{1}$ has described this condition as more common in women, and as not of rare occurrence, and he has given to it the name of Onyxis craquelé.

2. A pitting of the dorsal surface of the nail, the pits being arranged more or less in a linear series from the root forwards. The pits begin as whitish points in the nail, which can be dug out with the point of a knife, and which eventually leave roughened, blackened holes.

A combination of these two types is not infrequent.

3. Fournier, ${ }^{1}$ Batut, ${ }^{2}$ Sabouraud ${ }^{3}$ and other writers, mention partial or complete separation of the nails during the course of syphilis without any other local symptoms, and in a painless or "silent" manner. Sometimes the new nail is of normal formation; sometimes it may be of the type of Onychia sicca.

CASE 6.-The patient was a man, aged 56 years, who had, when first seen, a squamous syphilide upon the scrotum, flat condylomata, and shallow ulcerations on the tongue, and the condition of the nails seen in the photograph (No.63). The proximal part of the nail was thickened, opaque, ridged and pitted, and friable. The distal part, from which it was sharply marked off, had its normal transparency and polish. Six months later the dystrophied nail had grown forwards, and had entirely replaced the healthy nail, so that the whole nail was.then roughened, pitted, opaque, and brittle (No. 64). The subsequent history is not known.

CASE 7.-The patient contracted syphilis in 1906 . He was treated with pills on and off during four years, and also had sixteen injections of mercury. When the patient first came under observation, in January, 1911, he had a psoriasiform syphilide affecting both palms. All the nails on the hands, but not those on the feet, were affected in the following manner: They were more brittle than natural, and covered with fine longitudinal ridges. The central part of each nail was raised and convex, and with marked transverse grooving, some of the grooves being pigmented. On some of the nails the transverse grooving extended from top to bottom. In others, the grooves, which had grown forwards from the lunula, were limited to the anterior part of the nail. The condition began two years after infection in the thumb of the right hand, and soon afterwards the other nails became affected.

Although some of the lesions belonging to this group may be due to a direct involvement of the nail matrix by the disease, it is more likely that they 


\section{$\therefore x$}

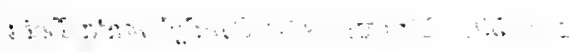

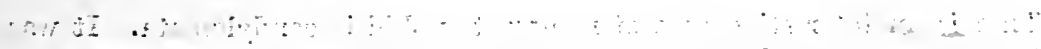

?

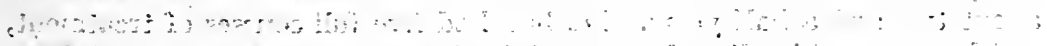

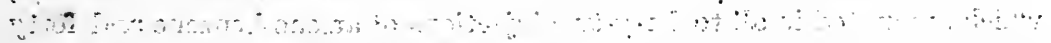
$\therefore$ a - 
PLATE XXX

No. 59.-Recurrent Condylomata Lata

THus is an interesting case of recurrent syphilitic condylomata. It was the patient's fourth recurrence of the same nature in the course of about two and a half years. He had had five full courses of treatment, which amounted in all to forty-two injections of arseno-benzene and forty injections of mercury. After each course his blood became negative and each time he reappeared his C. s. F. was found to be more pathological. 

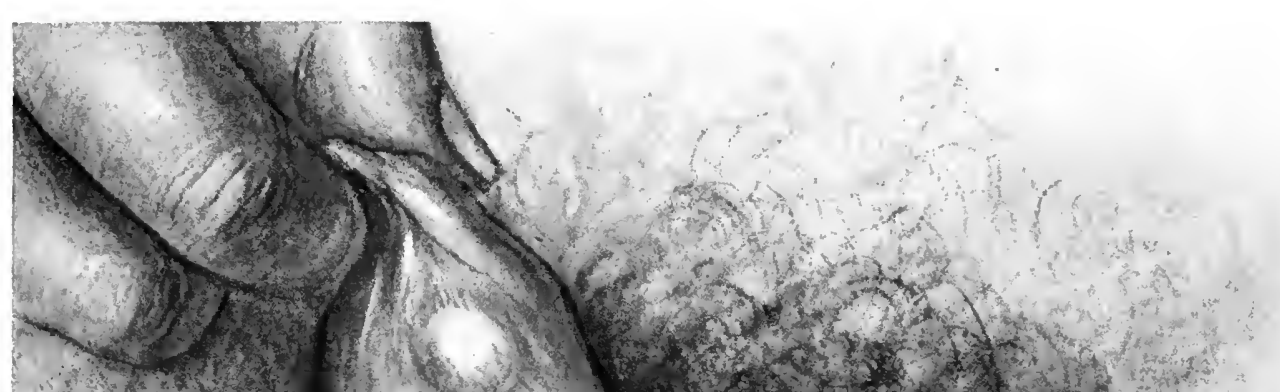

as andertats

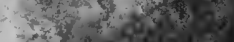

wines

Mes $+x+20$

$\therefore+3 x^{6}+2$

(f) $35 x^{2}+4$

- 150400

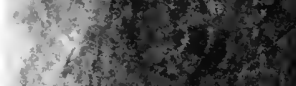

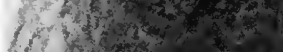

$(1$

(jin

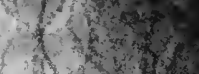

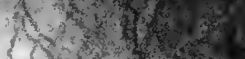

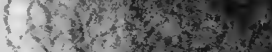

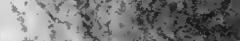

$+3 x^{2}+4$

i $1 y^{2}+x^{2}$

ment

$=x^{2}$

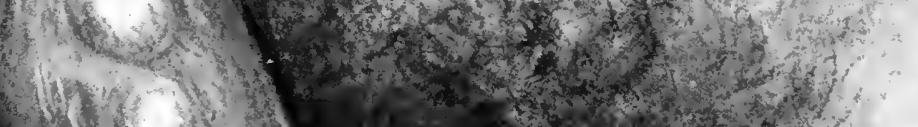
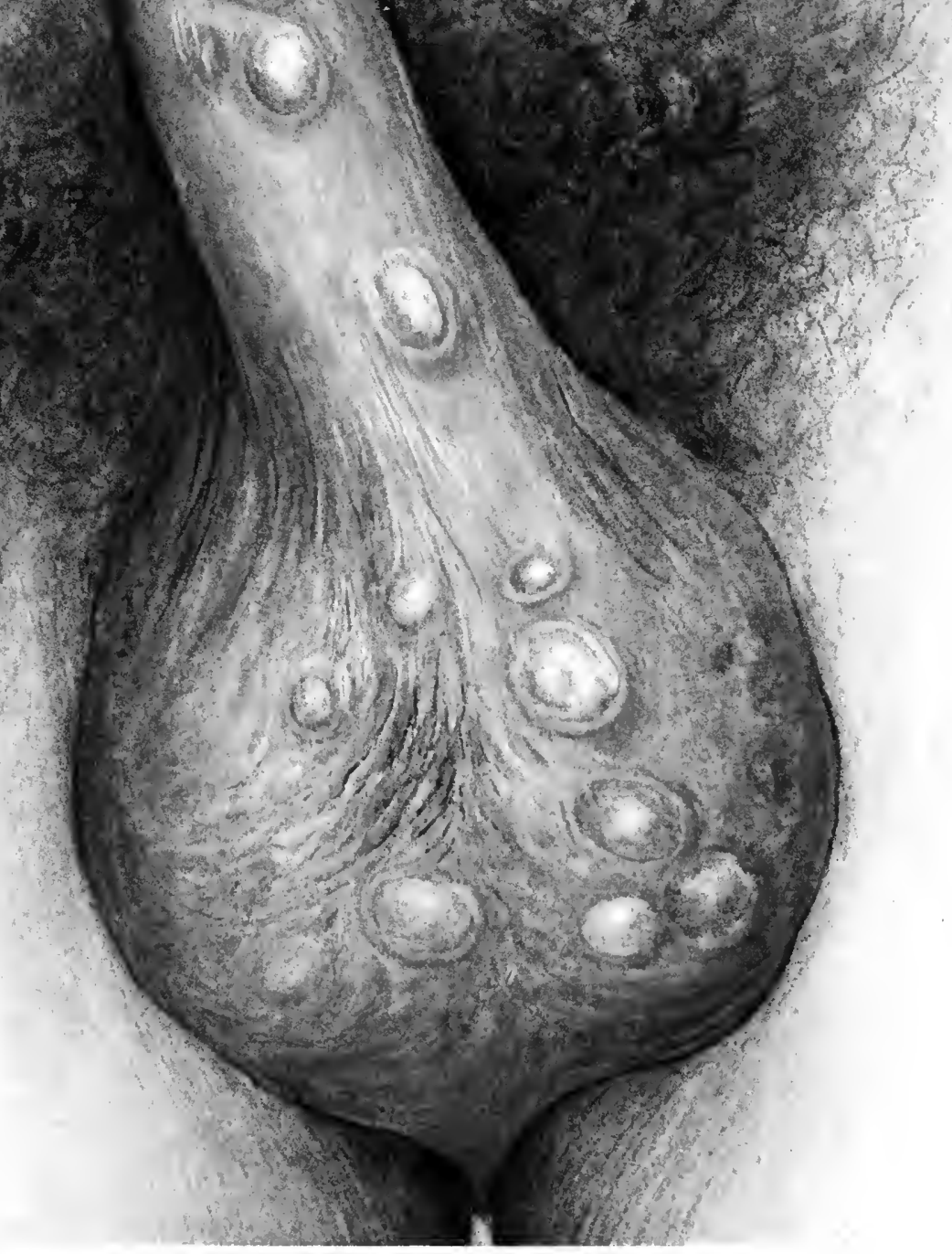

No. ก!

PLATE XXN 



\section{PLATE XXXI}

No. 60.-A Picture to siow the Typical Violaceous Tint whici is often left behind by Early Syphilitic Genital Lesions

THe condition is due to a syphilitic infiltration of the superficial vessels and lymphatics. The primary sore in this case was near the fraenum.

No. 61.-A Recurrent Syimilide on the Site of the Primary Sore

The patient had received no treatment for his disease and fifty-seven years had elapsed between the disappearance of the chancre and the appearance of the lesion depicted. In the interval no other manifestations had occurred. 
No. 60
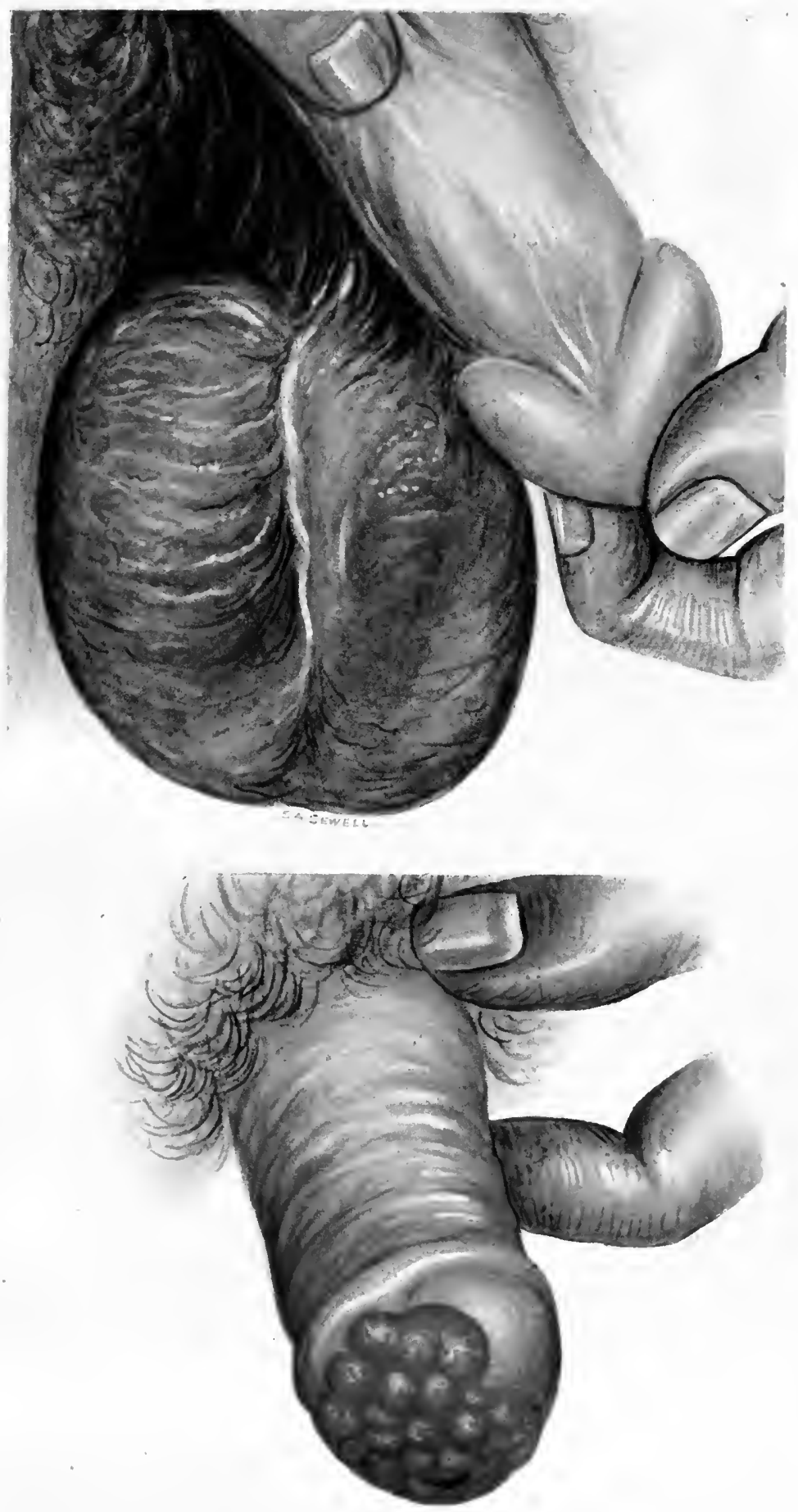

No. 61

Plate XXXI 

are atrophic in character, and occur because the patient has the disease in his system, and so they resemble syphilitic Alopecia areata. The lesions do not alter under treatment. I have seen cases of syphilitic Onychia sicca associated with acro-vasomotor disturbances.

\section{Paronychia or Perionychia.}

The second form of syphilitic nail in which the nail lesions are obviously secondary to local inflammatory disturbance includes two varieties:

(a) Papular or pustular lesions on the nail-bed (the "isolated papule" of Heller. ${ }^{4}$

(b) Papulo-crusted or ulcerative lesions around the nail, and involving also the matrix and nail-bed-Perionychia, Paronychia desquamativa, Syphilis ulcerosa unguium, Paronychia pustulosa, etc.

\section{(a) Papule on the Nail-Bed.}

Each of these varieties is said to be more common than Onychia sicca. Heller describes the first under the name of "the isolated papule of the nailbed." He says it occurs at the time of the exanthem. A pea to bean sized patch appears under the normally transparent nail. At first the patch is intensely red, later yellow, and without pustulation. The nail-plate gets thinner at this spot, and finally crumbles away, leaving a gap in the nail. He states that it is always limited to one finger. Finger ${ }^{5}$ says that the papule on the nail-bed often accompanies palmar or plantar psoriasiform syphilides, and that it may sometimes be half on the skin and half on the nail-bed. The following case is a striking example of the syphilitic papule on the nail-bed. It differs, however, from Heller's type in that nearly all the finger-nails were involved.

CASE 8.-Patient was a male, aged 23 years. The nail changes appeared, about four months after infection, as part of a general papular eruption, which involved the palms and soles. The lesions of the nails persisted after the general eruption had disappeared. They were the result of the formation of papules on the nail-bed, the nail-plate immediately over the papule becoming secondarily affected. At the time when the photographs (No. 64) were taken all the nails of the hands were affected; the little finger of the right hand $(R)$ and the ring-finger of the left (L) showed early papules beneath the lateral margin of the nail. The thumb of the right hand showed complete perforation of the nail-plate subsequent to the formation of a papule at the central part. There was some discharge of pus from the more advanced nail-bed lesions. A marked feature in this case, which can be observed in the photograph, was the deep pigmentation of the nails over the papules, a pigmentation to which attention has been especially called by Vörner, and which has been shown to be due to changes in the horny substance, due to exposure to the air and not to a deposit of true pigment.

The photograph (No. 65) is an example of another case of papule formation on the bed of the nail. In this case the nail has not been eaten away, but it 


\section{6}

has been separated from its bed at its anterior part by the formation of the papule there. Patient was a male, aged 37 years, and the nail trouble had commenced as part of a secondary generalised papular eruption six months after infection.

\section{(b) Paronychia or Perionychia.}

The other forms of syphilitic nails associated with inflammatory disturbances are those known as Perionychia, Paronychia desquamativa, and Syphilis ulcerosa unguium. Several nails may be affected, but often only one is involved; and, commonly, that of the thumb, of the first finger, or of the great toe, probably because these are most exposed to injury, which acts as a determining cause. There is at first redness and swelling of the parts around the nail, with some pain, though generally less than is the case with acute septic paronychiæ. If the case is not under treatment the inflammation may extend beneath the nail to the matrix and nail-bed, and the skin around the nail gives way, forming a discharging horseshoe-shaped ulcer. The nail blackens and falls, cxposing an unhealthy looking ulcer. If the inflammation of the matrix has not been severe, a normal nail may grow under treatment, but often the nail is afterwards deformed, or no new nail may be produced.

The photograph (No. 66) shows a syphilitic perionychia in the early stage, at which it was arrested by treatment.

There is another syphilitic affection of the nails which has not been previously described, and of which I have seen four cases. The two cases about to be mentioned were published by Adamson and myself some years a.go. ${ }^{6}$

CASE 9.-A man, aged 25 years, developed syphilis when twenty-one years of age. The chancre was followed by slight generalised manifestations, which disappeared under a six months' course of mercury given internally in the form of grey pills. Internal treatment was discontinued owing to the fact that the tongue became very sore. It was considered at that time to be due to a mercurial stomatitis, but as the tongue continued to get worse, the patient received, a year later, ten mercurial injections of grey oil, which had no influence in improving the glossitis. Consequently mercury was no longer prescribed, and from that time until the patient came under observation, in the beginning of November, 1910, he had received no antisyphilitic treatment. On examination, the tongue was smooth and shiny on the surface; there were no papillæ; and the whole of the upper surface of the tongue was covered with patches of leucoplakia, in between which were scarred fissures. On palpation the tongue was soft, except in a few places where the fissures were, indicating also some interstitial glossitis. There were no leucoplakic patches on the mucous membrane on the under surface of the tongue, nor elsewhere in the mouth. Except for a peculiar condition of the nails the patient showed no other evidence of syphilis. The patient was accustomed to smoke, but not excessively. There was no evidence of gastro-intestinal disturbance. C.F.T. positive.

Nails.-The nails of both hands were malformed, except that of the ring-finger on the right hand, which was normal. The nails on the feet were unaffected. The affected nails were short and irregular at their free ends, as if they had been bitten. The nail-plates were abnormally thin, so that they felt soft along their whole length. They were somewhat concave in the centre and irregular on the surface, due to 


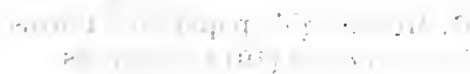

$\therefore ! \cdots$

$\because \cdots+\cdots$

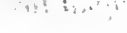


PL.ATE XXXII

From Dr. H. G. Adamson and Mr. J. E. R. MeDonagh's paper on " Forms of Srphilitic Nails," British Journal of Dermatology (1911), xxiii, 68

No. 62.-Onychia Sicca Syphilitica

No. 63.-The Sine Case as Above Six Months Later

No. 64.-Syphilitic Papule Formlatiox ox the Nall-hed, from a Patiest of Mr. A. Shili.itoe's 


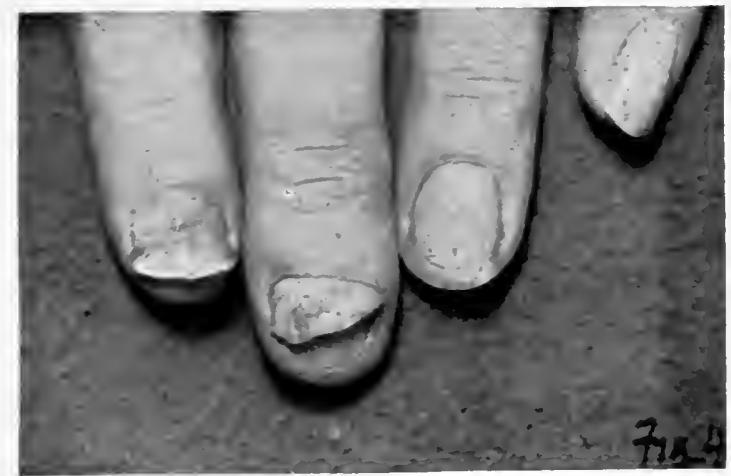

No. 62

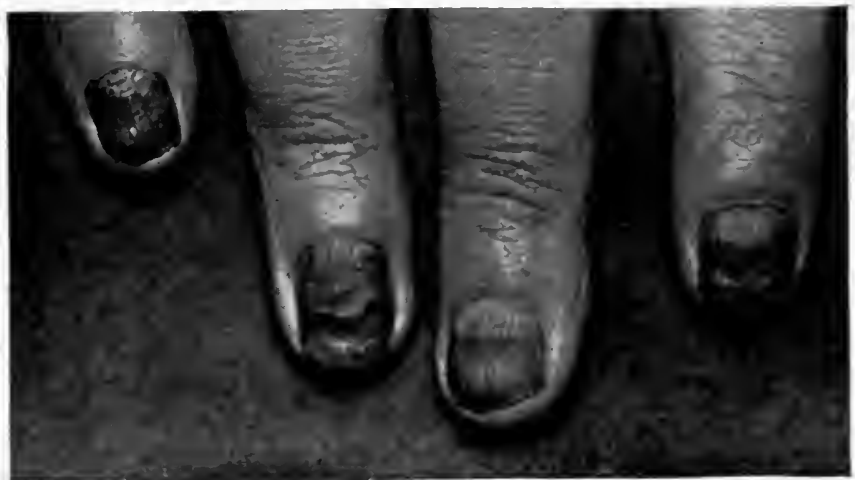

No. 63
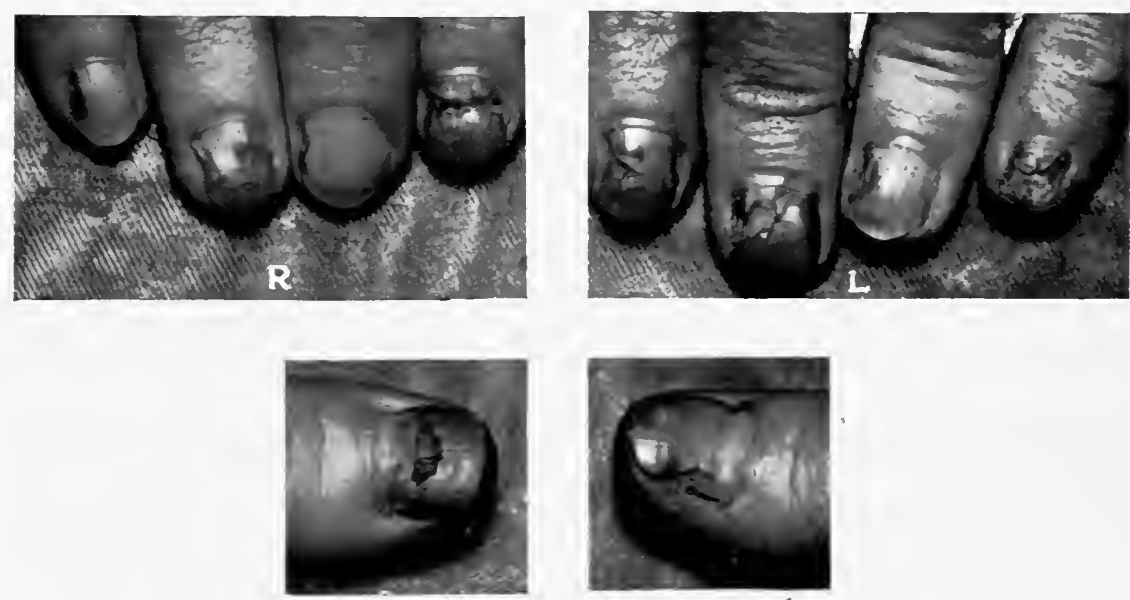

No. 61

Plate XXXII 



$$
\text { . }
$$




\section{PLATE XXXIII}

From Dr. H. G. Adamson and Mr. J. E. R. MeDonagh's paper on "Forms of Syphilitic Nails," British Journal of Dermatology (1911), xxiii, 68

No. 65.- Separation of the Anterior Part of the Nall as the Result of a Papulo-pustule of the Nall-bed

No. 66.--Paronychia Syphilitica

No. 67.-ATrophic SYPHILTIC ONYCHL

THE nails are remarkably thin and soft, ridged longitudinally, and split at their free cnds. The paronychial area is soft and baggy. 


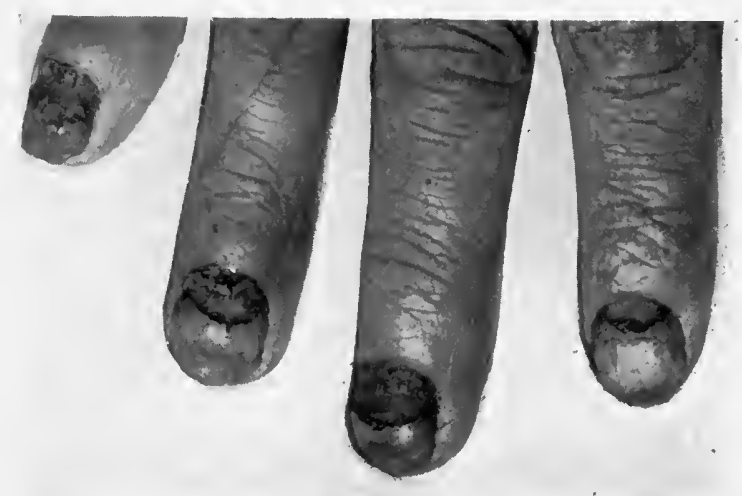

No. 65

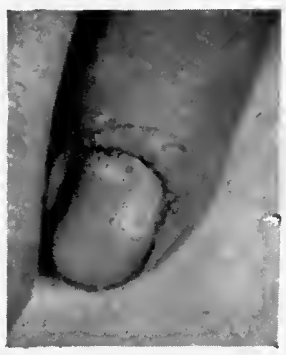

No. 66

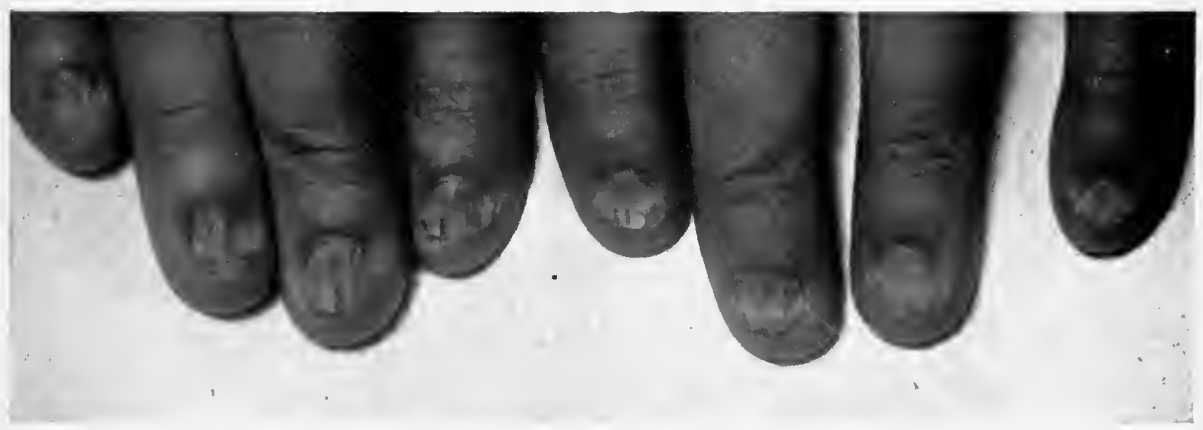

No. 67

Plate XXXIII 

parallel longitudinal ridges. There was no lunula, but in that position the nail was extremely soft. The skin over the root of the nail appeared raised and had the feel of a sac, which could be flattened out on pressure. This was no doubt due to the fact that the roots of the nails were soft and gave no support to the skin above them. Only when the nails first became bad was there any pain. The pain was situated at the bases of the nails, the parts around which were at that time inflamed. The onychia commenced about six months after the infection, and, according to the patient's history, one nail after the other, except the one mentioned above on the right hand, became sore and soft at its base, and within a short time the nail fell off, the nail taking its place having the characteristics as above described.

On November 11 th the patient received $0.6 \mathrm{grm}$. of " 606 " intramuscularly; within a few days the pain in the tongue had vanished, so that the patient could eat or smoke in comfort. When next seen, on January 1lth, 1911, the tongue had greatly improved; the surface was still shiny, and the leucoplakic patches had not disappeared, but the consistency was softer. The C.F.T. was negative. In the nails there was no appreciable change.

CASE 10.-A man, aged 31 years, seen at the beginning of November, 1910. had a chancre on the lip two years ago, which was followed by the usual symptoms, Treatment was commenced with forty inunctions, when the rash was diagnosed as being syphilitic; pills were then taken for eighteen months, and followed by fifteen injections of grey oil. The patient now sought advice for a nasal discharge of three months' duration, caused by a perforation of the nasal septum, the edges of which were ulcerated and the ulcer spreading. There was much discharge, so that the patient's pillow was soiled every morning. There was a scar on the left side of the neck caused by Lupus vulgaris, which had been cured by " light treatment " five years previously. Except for the nasal trouble and the condition of nails, which will be presently described, there were no other manifestations of syphilis. C.F.T. positive.

Nails.-Every nail, both on the hand and feet, was shortened, soft to the touch, jagged at the free edges, ridged longitudinally, and concave in the centre. The nails were extremely thinned but retained their normal translucency, having much the consistency of a soft quill. The root of the nail was particularly soft, and offered no resistance to pressure, the skin orer it being, as it were, puffed out and wanting the support of the nail, a feature which can be seen very well in the photograph (No. 67). The patient had always had bad nails, as he had been in the habit of biting them. The nail condition started about six months after infection, with red, painful swelling at the base, affecting the fingers one after the other. The old nail soon became shed, and the new one taking its place had the appearance as above described, but never grew beyond a certain length. Although the base of the nail was soft and the skin over it raised, there was no pain at the time of examination. On November 15th patient received $0.6 \mathrm{grm}$. of " 606 " intramuscularly. Within ten days the ulceration in the nose had healed and the discharge had ceased. When seen again on January 11th, 1911, the nasal condition had completely healed; the nails remained unaltered. C.F.T. negative.

Before closing this chapter, a few more words might be said with advantage regarding the differential diagnosis of syphilitic rashes of other origin, which may be confounded with them.

Mention has already been made of varicella and lupus; but before mentioning other diseases, certain toxic erythemata must be considered, as there is a causal relationship between some well-known types and syphilis. 
Syphilis may cause typical Erythema nodosum and Erythema multiforme.

The two types of toxic erythemata just mentioned are indistinguishable from those types produced by other causes, and they invariably occur early in the generalisation stage, indeed sometimes before the true syphilitic rash makes its appearance. Syphilis may also not infrequently be a cause of urticaria, and very rarely of purpura. A case of syphilitic urticaria associated with Alopecia areata recently came under my care. The patient had also the early signs and symptoms of degenerative myelitis.

In late syphilis, although the patient may not exhibit any typical lesions, it is not at all uncommon for him to complain of dryness and itching of the skin, and tendency for the skin to crack in places, especially between the fingers and toes. Occasionally the skin may even become atrophic. One of the most troublesome fissures is that affecting the nasal orifice at the tip. These symptoms arise from want of oxygen which results from the increased reducing action of the protein colloidal particles, hence the reason why they respond so quickly to colloidal iodine and intramine.

I have seen cases of Acne rosacea caused by syphilis, and Herpes zoster appear as a sign of syphilitic meningitis, involving the posterior root ganglia. I have seen Herpes zoster usher in a severe syphilitic nervons lesion, as well as appear after definite nervous symptoms had been present, independent of treatment. Herpes zoster may also be caused by arsenic and mercury. There is a recurrent zoniform syphilide which is apt to be mistaken for herpes at first sight, but the lesion; have scabs on them which, when removed, reveal the typical syphilitic scarring. Syphilis may cause Mycosis fungoides or what I have preferred to call aleucæmic cutaneous lymphocytomata, a better name, since all grades of cases may be met with, from the comparatively innocent to the rapidly malignant. The case may be either of the typical tumour formation type or nlcerative from the start, as in the following case:

CASE 11.-A man, aged 47 years, having had syphilis over twenty years before, sought advice for a skin lesion he had had for the last three years.

The patient had a rash which extended over the upper half of the left side of the chest, the left shoulder, and down the left arm to about the lower third. The rash was typical of Dermatitis atrophicans, the skin rolled and looked thin, like cigarette-paper, and the vessels were clearly discernible underneath. The periphery of the lesion simulated exactly the localised patches of erythrodermia which precede the condition called Lymphogranulomatosis cutis. The itching was intense, and the skin trouble was undoubtedly spreading. On the arm, where the skin had not become atrophic, was a very slowly spreading ulcer, which simulated closely a rodent ulcer. All treatment was unavailing. The patient was not clear as to whether the ulcer had been preceded by a swelling in the skin or not.

The other skin diseases, which are apt to be confused with syphilis, are Pityriasis rosea, Urticaria pigmentosa, Scabies, Psoriasis, Erythema induratum, fungus diseases, such as sporotrichosis and blastomycosis, certain drug erup- 


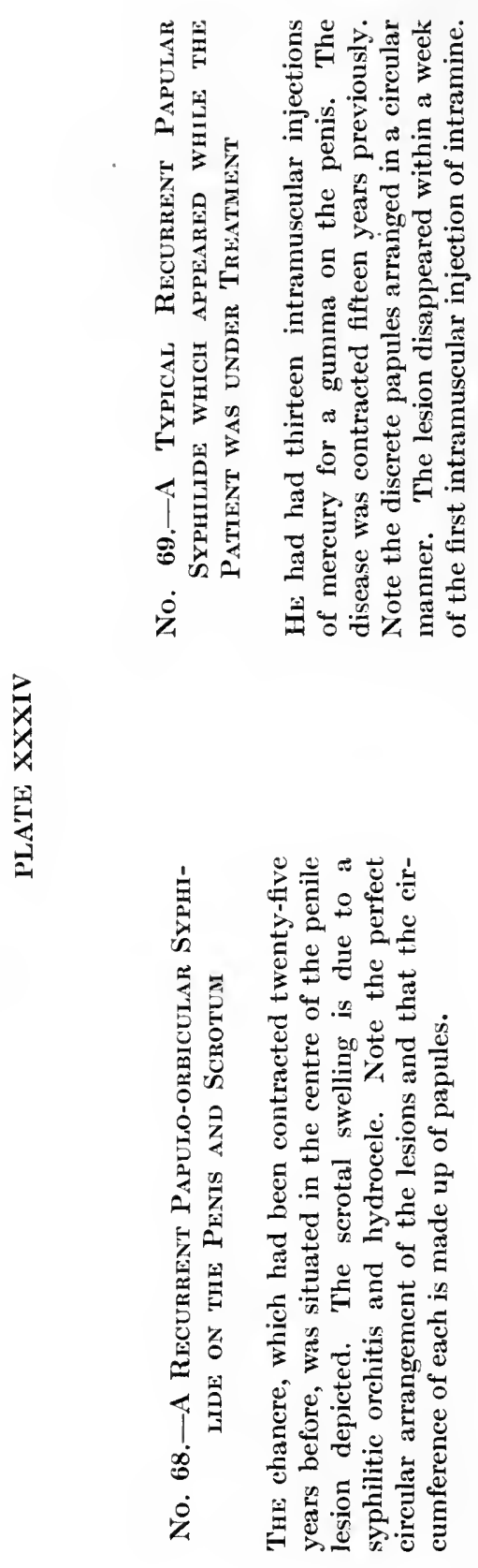




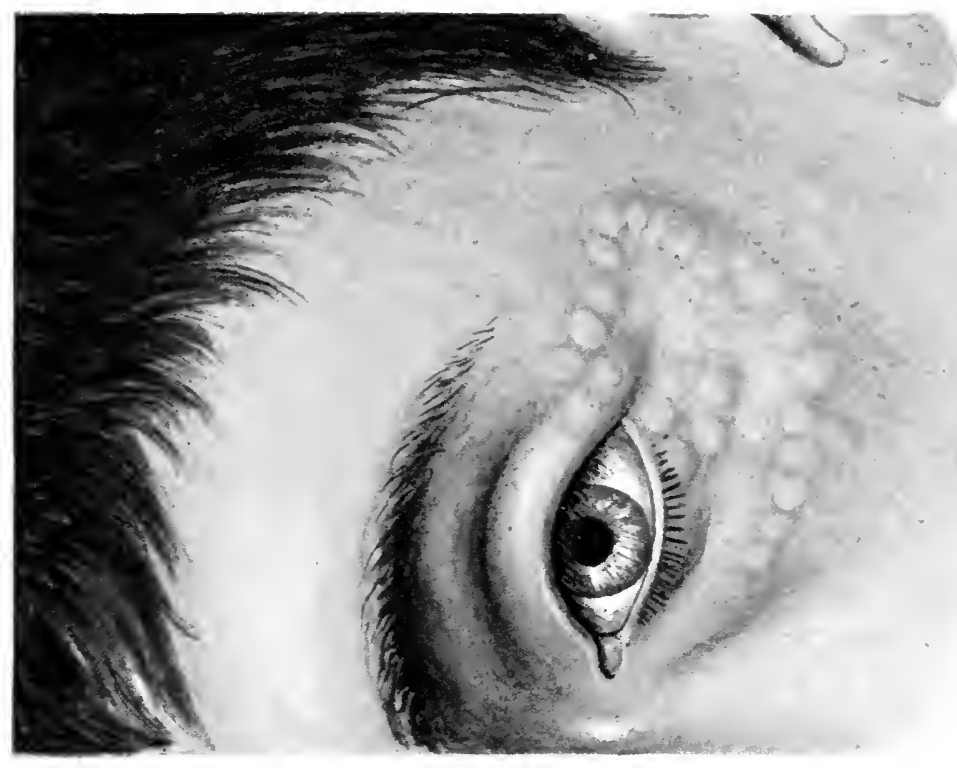

8

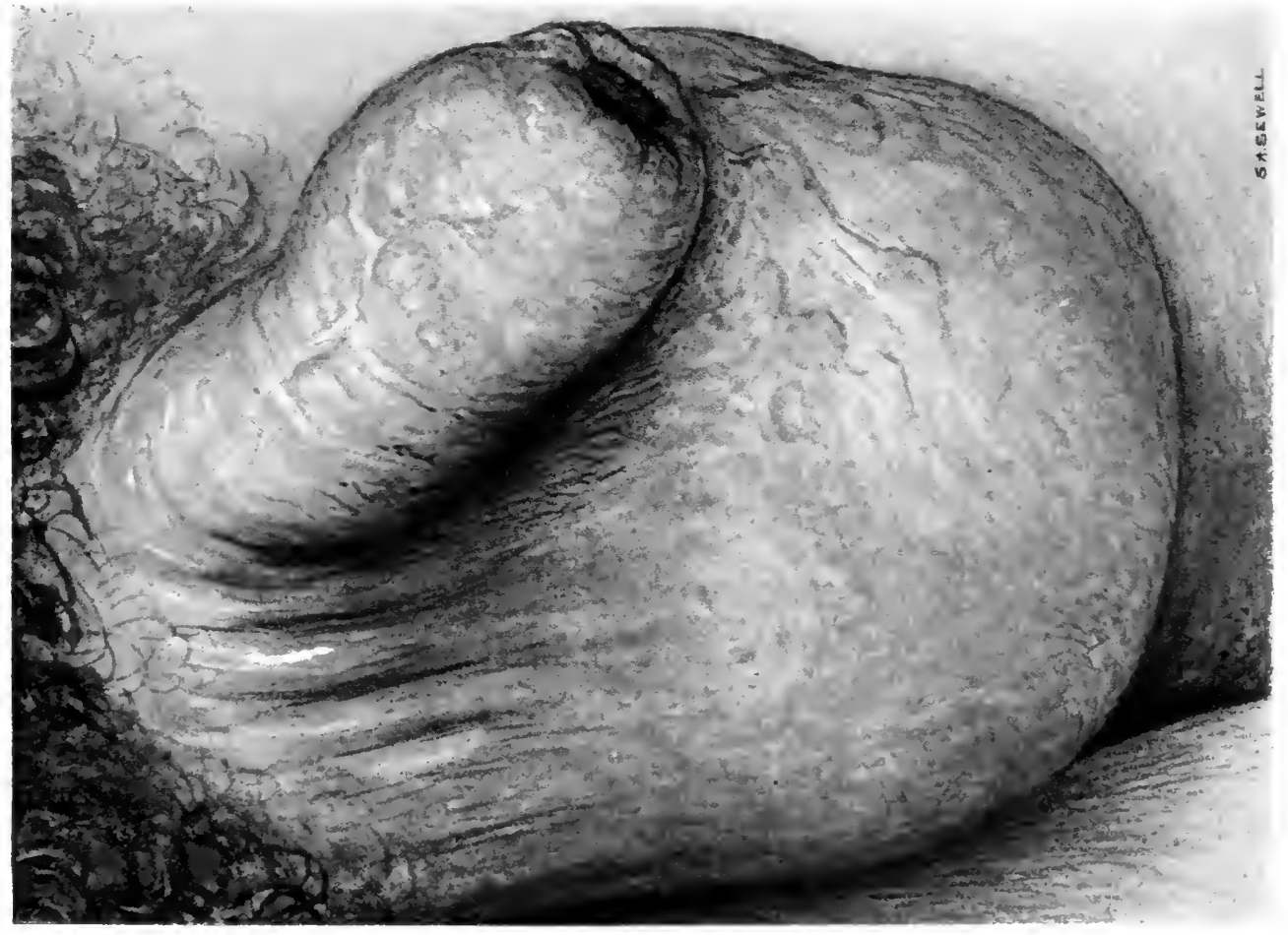

$\stackrel{0}{0}$ 

$\because: \cdots,+, \cdots: \quad \cdots, \cdots, \cdots$

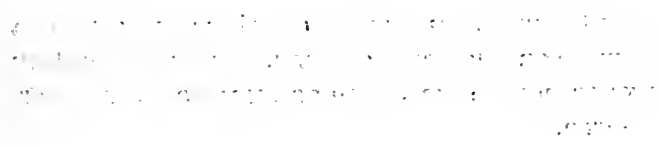




\section{PLATE XXXV}

No. 70.--A Recurrent Papulo-pustular Syphilide

TiIs is a late manifestation, but typical in that the lesions appear on a dusky and inflamed base, form deep ulcers and scars, which ultimately become quite white, and however near several lesions may be to another, they show no tendency to coalcsce. 


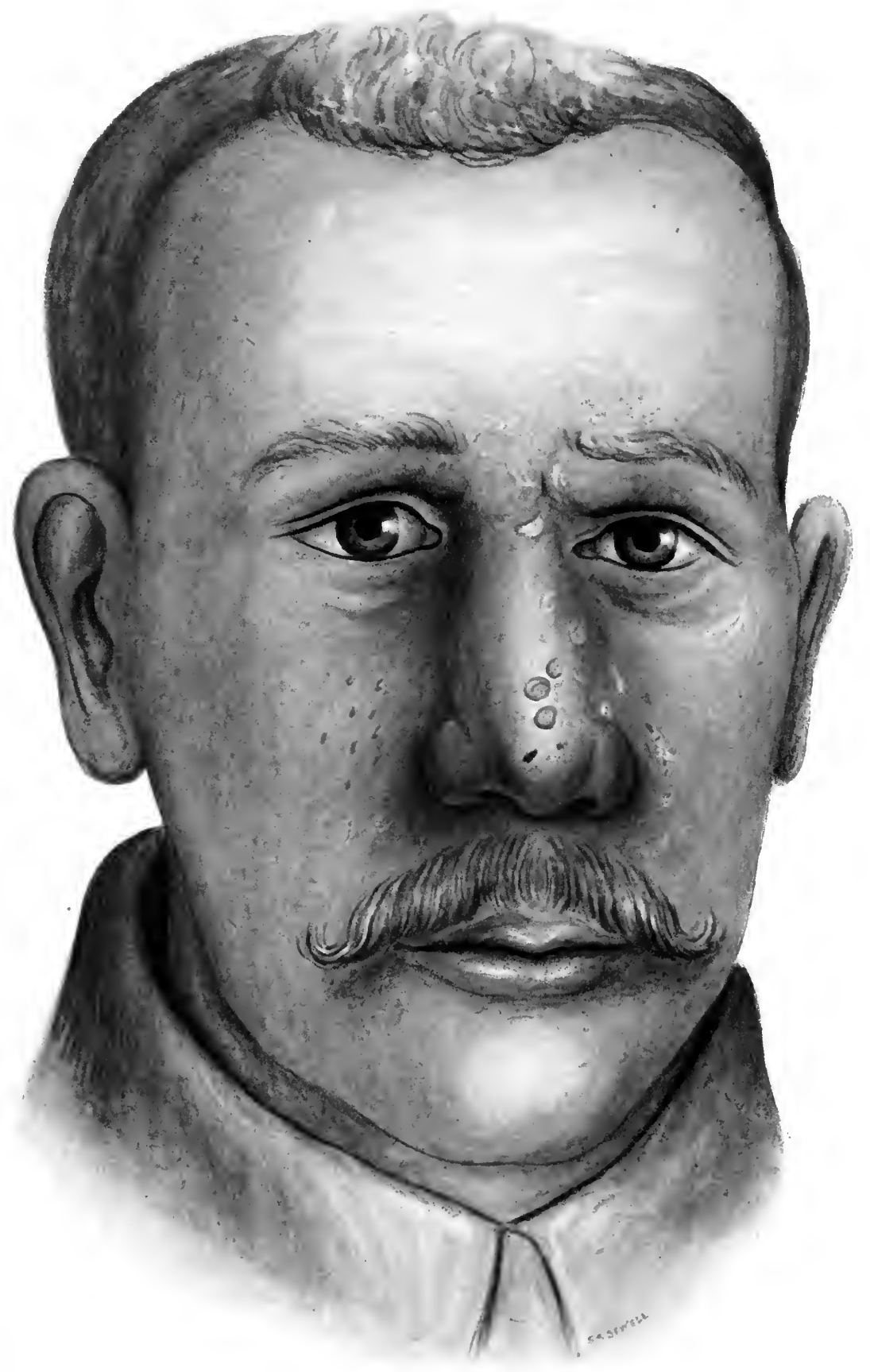

No, 70

Plate XXNY 




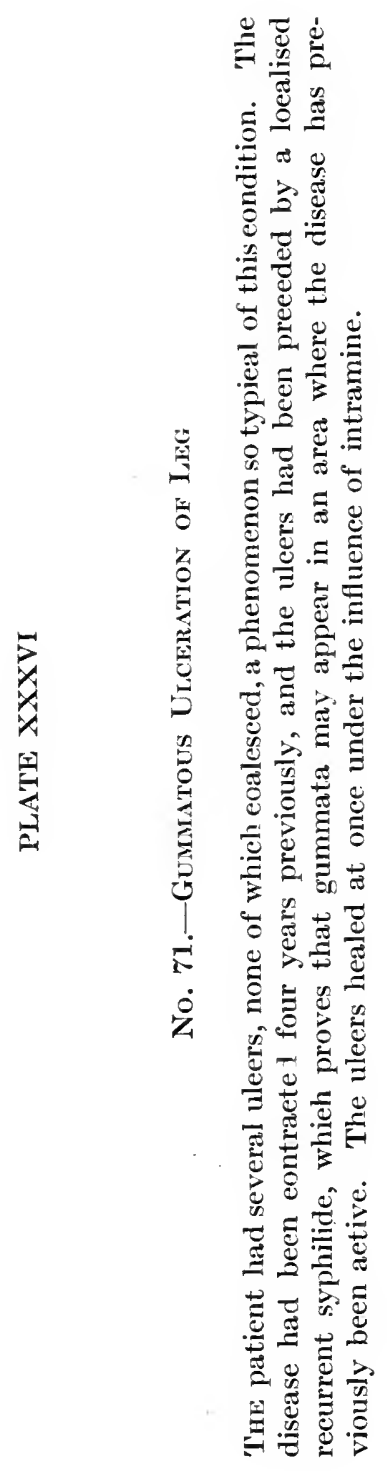




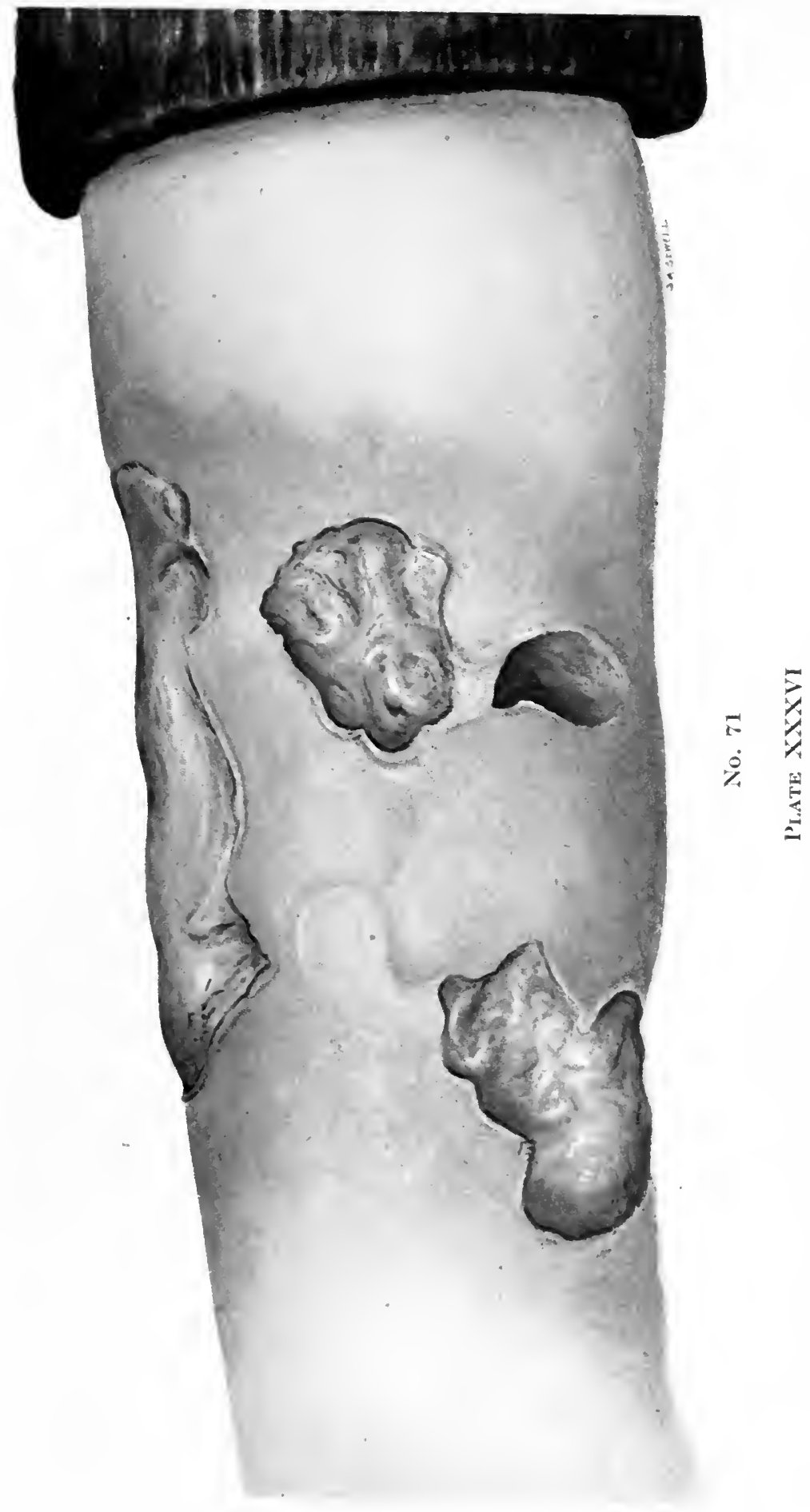


tions, especially the one caused by iodides, leprosy, and various rare tuberculides.

Pityriasis Rosea.-This rash is, generally speaking, limited to the vest area; it covers this area in a few days after the appearance of the initial patch, which is sometimes called the herald patch, and is scaly almost from the commencement.

The lesions are bigger than syphilitic lesions, they are not so papular, those on the back run in the direction of the ribs, a point which often raises confusion with syphilis, but no trouble should ever arise in differentiating the two diseases, if it be borne in mind that a syphilitic lesion does not scale until it retrogresses, while a lesion of Pityriasis rosea is scaly from the beginning.

A lesion of Pityriasis rosea is red in the circumference, yellow in the centre, the scales are most evident at the junction of these boundaries, and the scales have their loose ends inwards.

When a syphilitic lesion scales, the scales form in the centre, they are more apparent and not so sebaceous as those just described, and, moreover, they are more adherent.

Urticaria Pigmentosa.-As adults arc not infrequently affected, and as the characteristic pruritus may be absent and the dermatographia not very apparent, without a histological examination, to show the mast-cells, it may be almost impossible to differentiate between an early papular syphilide, when the lesions are sparse and discrete, and Urticaria pigmentosa.

Scabies.-A very favourite location for scabies lesions is on the penis, where they form papules, which at first sight might suggest syphilis. When examined, the papules are found not to be infiltrated, and burrows, and even the acarus itself may be found. Itching, and finding similar lesions elsewhere -namely, on the buttocks, wrists, and in between the fingers-clinches the diagnosis.

Psoriasis.-From a naked-eye examination of the lesions it may in some cases be absolutely impossible to distinguish between psoriasis and syphilis. In such cases one has to rely upon history, and to note whether the scales when removed reveal bleeding-points, a phenomenon which is typical of psoriasis only.

Many patients with psoriasis have either had the complaint for years, or have had recurrences of it, and often another member of the family is subject to the disease. A squamo-papular syphilide, resembling psoriasis, is a recurrent syphilide, therefore every lesion should be thoroughly examined, to see if any of them are circular or gyrate in form, because, if so, they are certainly syphilitic.

An examination of the scalp should always be undertaken, since psoriasis very commonly affects the scalp, while a squamo-papular syphilide does so rarely.

Psoriasis may affect the penis only, and so may Lichen planus, in which case the lesions are usually on the glans. The type of Lichen planus which affects 
the glans penis is the circinate form, but, from its smallness and its perfect regular outline, it ought never to be mistaken for syphilis.

Erythema Induratum.-This condition is a tuberculide, and practically affects only girls between the ages of fifteen and twenty-four. The lesions usually affect both legs, and are situated on the posterior aspects. The initial lesion is a red patch, this becomes a purple-coloured papule, and later the centre breaks down to form a deep crateriform ulcer. The only syphilitic lesion it could possibly be confounded with would be a gumma; but, as I have already stated, a gumma is an ulcer the size of which exactly corresponds to the area affected, hence the term crateriform could never be applied to it.

Sporotrichosis, blastomycosis, and Oriental sore can only satisfactorily be diagnosed by demonstrating the specific organism, either in culture, film, or section, made from the lesion in question.

An iodide rash is most likely to be confused with a papulo-pustular syphilide, which is an early syphilitic eruption, hence other signs and symptoms of the disease will generally be found on a further examination. An iodide rash disappears very quickly on suspension of the drug.

Difficult tuberculides are best diagnosed by the way in which they react to tuberculin.

During the past few years I have come across rashes accompanying Vincent's angina which have been very difficult to distinguish frcm syphilis. I remember three cases where the patient presented himself with a widespread maculo-papular eruption, which alone was identical with the early maculopapular syphilide. There was no general adenitis, and on further examination all three patients had typical membranous anginal lesions on the tonsils. Rheumatic cutaneous lesions may sometimes closely resemble a generalised syphilitic eruption, but, as the lesions are most pronounced around the joints, and as the patient may have had the same trouble before, a correct diagnosis can usually be quickly arrived at. With rheumatic dermatitis the patient generally complains of joint pains, which are usually much more severe than those met with in early syphilis. Furthermcre, a patient who complains of pains in early syphilis usually refers most of the pain along the course of the long bones.

If every reader will bear in mind the few points mentioned in this chapter, and then confirm them on clinical material, I think he will soon agree with me in stating that, of all skin diseases, the various syphilitic eruptions are the easiest to diagnose.

1. Fournier (1873): Syphilis chez la Femme. Delahaye, Paris.

2. Batut (1894): Gaz. Hebdomadaire, iv., 163.

3. Sabouraud (1905): Dermat. Topographique Régionale. Masson et Cie., Paris.

4. Heller (1900): Die Krankheiten der Nägel. A. Hirschwald, Berlin.

5. FINGER (1908): Lehrbuch der Hand u. Geschlechtskrankheiten. F. Deuticke, Wien.

6. Adamson and McDonagir (1911): Brit. Journ. of Dermat., xxiii., 68.

7. McDonagh (1914): Brit. Journ. of Dermat., xxvi., 283, 337. 


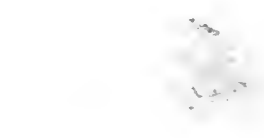

$$
4
$$

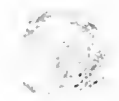


PLATE XXXVII

No. 72.-Lichen Planus Limiten to the Glans Penis

Note the perfectly clean-cut square papules in the circumference of the lesion. It is interesting io remark that this patient was a profound sexual neurasthenic.

No. 73.-Lichen Planus limited to the Skin of the Penis

Note again the square papules and the violaceous tint of the lesions. 
No. 72
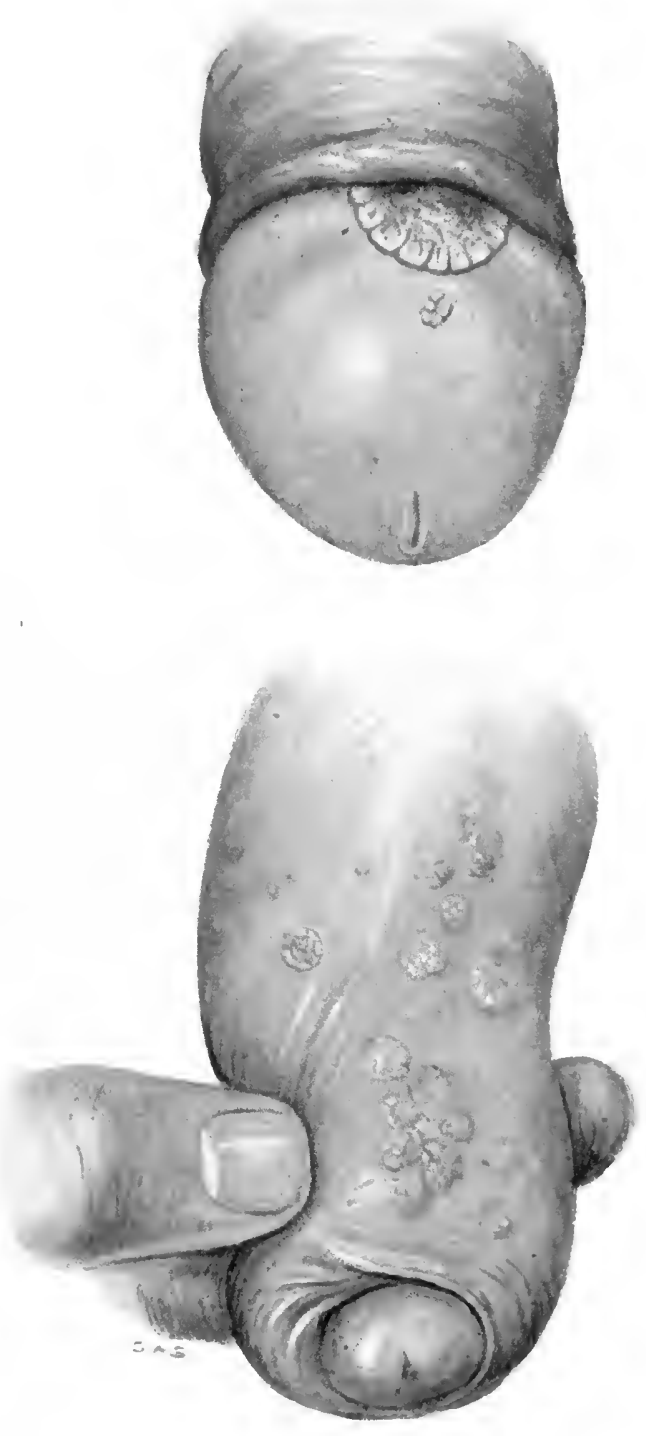

No. 73

Plate XXXVil 



\section{PIATE XXXVIII}

No. 74.-A Case of Granuloma Annulare on the back of the Left Hand Whicil HAD BEen Mistaken by some for a Recurrent Syphilidf.

THE patient had had frequent attacks before. The primary Jesion was always a papule which spread peripherally, and in the early stage the circumference of the lesion was cut up into square-shaped papules not unlike those of Lichen planus - points which are by no means characteristic of a recurrent syphilitic lesion. 


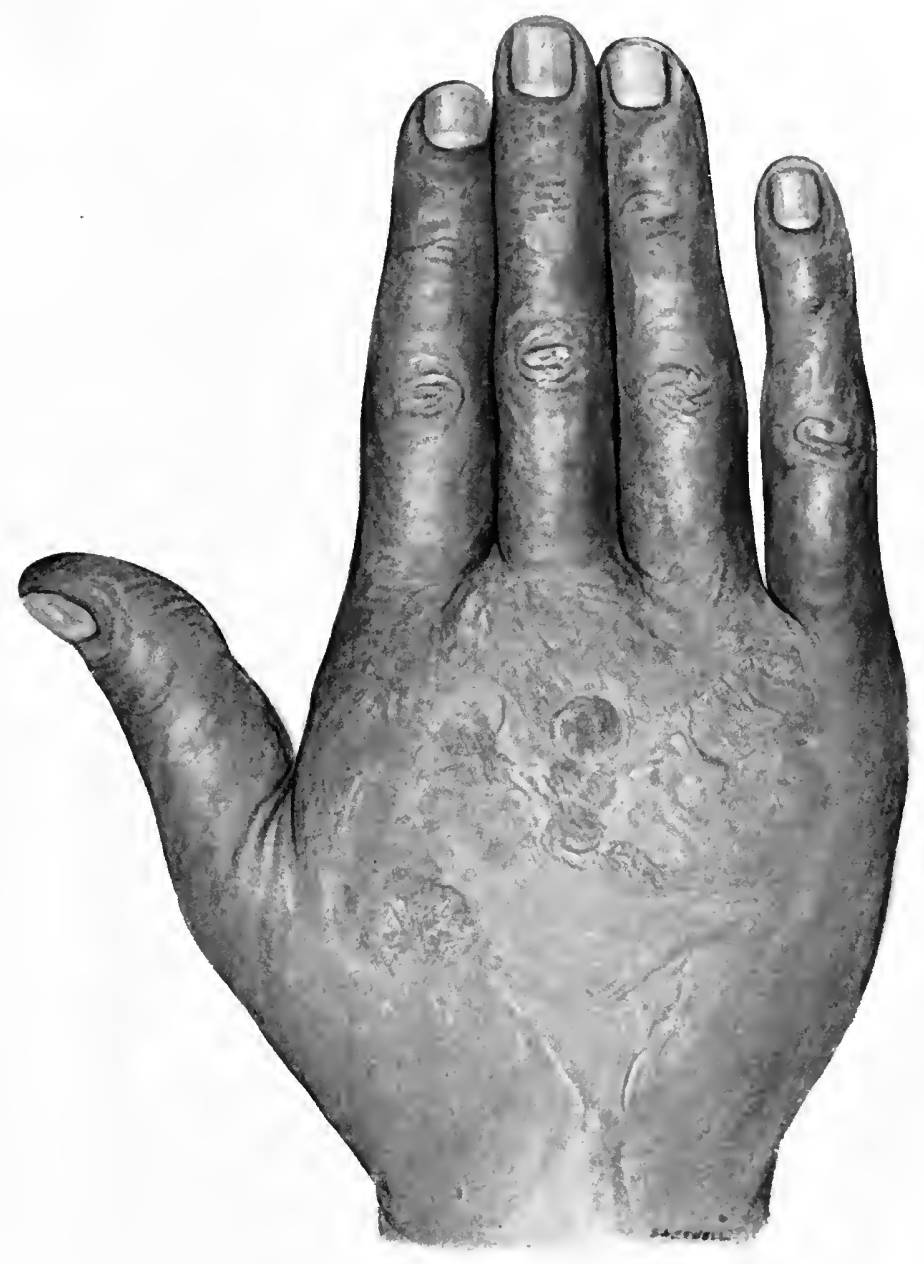

No. 74

Plate XXXVIII 


\section{SYPHILIS OF THE LYMPHO- AND H出MOPOIETIC SYSTEMS}

\section{History.}

ALthough adenitis was familiar to the older syphilologists, it took many years before the connection between adenitis and syphilis was understocd, and even in Ricord's time inflammation of the lymphatic glands was only considered to be syphilitic so long as there was no suppuration. The lymphangitis on the dorsum of the penis was observed by Torella in 1497, and described later by John Hunter and Benjamin Bell, and shown to be an affection of the lymphatics by Bassereau in 1852. Discussion concerning the infectivity of the blood was rife at the end of the eighteenth century. John Hunter maintained that the blood could not carry the contagion, because if it did one would expect to see an ulcer arise in any part of the skin which had been pricked. It was not until the middle of the nineteenth century that the blood was proved to carry the infection. Except for the condition of Chlorose syphilitique described by Ricord, and the various experiments which were undertaken to show the presence of mercury in the blood, the history of this part of the subject is void. Cardiac syphilis was seldom mentioned; it was looked upon as a fatal complication, and was generally considered to be due to hydrargyrosis. The Italian authors in the middle of the eighteenth century were the first to give good descriptions of syphilis of the heart and aneurysms. The frequent implication of the vessels of the brain by syphilis was not only admirably described, but considered pathognomonic of the disease by Morgagni. From the clinical and naked eye pathological standpoints there is little of the present day knowledge of cardio-vascular syphilis which was not known to and described by Morgagni, in spite of which his work remained in oblivion for about a century.

\section{Lymphangitis.}

The organisms from the site of infection soon reach the local lymphatics, and spread alorg them into the nearest chain of lymphatic glands.

The lymphangitis may be marked enough to cause occlusion of the vessel when a hard cord may be felt running along the dorsum of the penis.

The lymphangitis may be more marked in some areas than in others 
along its course, which on palpation may feel like a chain of beads. One bulging only may be present, or several. Occasionally, one of the beads develops more than the others, comes to the surface, and simulates an abscess. Plate II. shows a case where a small incision was made and the bead shelled out like a pea from its pod. Cross-section of this bead gave the microscopic picture not unlike that of a lymphatic gland. I have known a superficial lymphangitis to ulcerate, and bear the characters of a chancre, as a recurrent lesion in a case which had been well treated with arseno-benzene.

Lymphatics, other than those running along the dorsum of the penis, may be affected in the same way. If the lymphangitis is widespread, cedema of the whole skin of the penis may result.

Edema of the skin of the penis is most marked when the sore is in the corona. The sore may often be hidden because the foreskin cannot be drawn back; but a hidden chancre can always be diagnosed if it be remembered that such an œdema is non-inflammatory-i.e., not red and painful-as it is in soft sore and in gonococcal infections.

During the stage of the generalisation of the virus all the lymphatic glands become implicated, but the set which is always most enlarged is that draining the site of the primary sore. This point will often enable one to localise a sore which has escaped notice during the first examination.

Owing to the richness of the lymphatic vessels draining the mucous membrane of the mouth, any sore in this region is always accompanied by an enormous enlargement of the lymphatic glands in the neck.

As lymphatic's cross the middle line of the body, the enlargement of the glands on the opposite side to that on which the sore is situated may be the greater. Syphilitic lymphatic glands are hard and discrete, or enlarged, matted together, and soft. The degree of enlargement varies enormously in the different cases. As a rule, it may be said that the greater the enlargement the better the protective capacity of the host against the parasite, and vice versâ.

It must always be remembered that cocci usually accompany the syphilitic parasites along the lymphatics into the glands, with the result that they may at any time multiply and cause acute inflammation. Acute inflammation causes the lymphatic glands to become glued together, and one or more of them may suppurate, and simulate the bubo so common in the soft sore infection. Generally speaking, the only lymphatic glands which suppurate in syphilis are those draining the site of the initial lesion, and the lymphatic glands in the neck. The reason why the lymphatic glands in the neck not infrequently suppurate is because of the lymphangitis which results from the early mucous membrane lesions in the mouth, and as the mouth is exposed to the air, and always crowded with pus-producing organisms, some of these find easy entrance into the lymphatic glands, in which they can cause suppuration.

Suppuration of these lymphatic glands, although most common during 


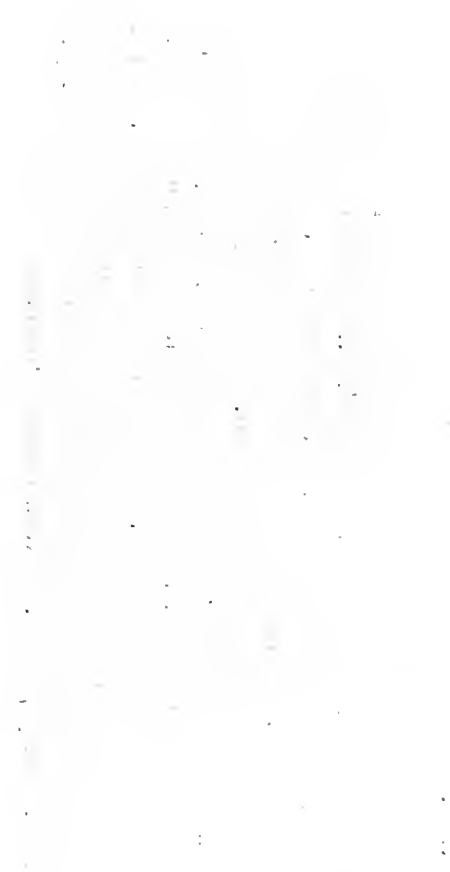

. 


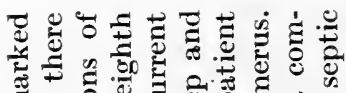

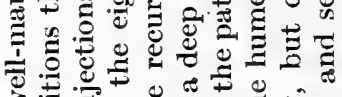

月

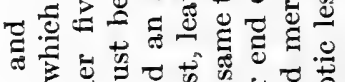

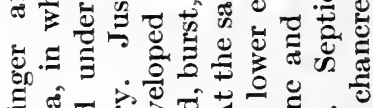

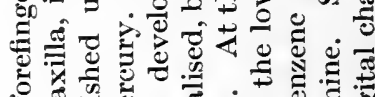




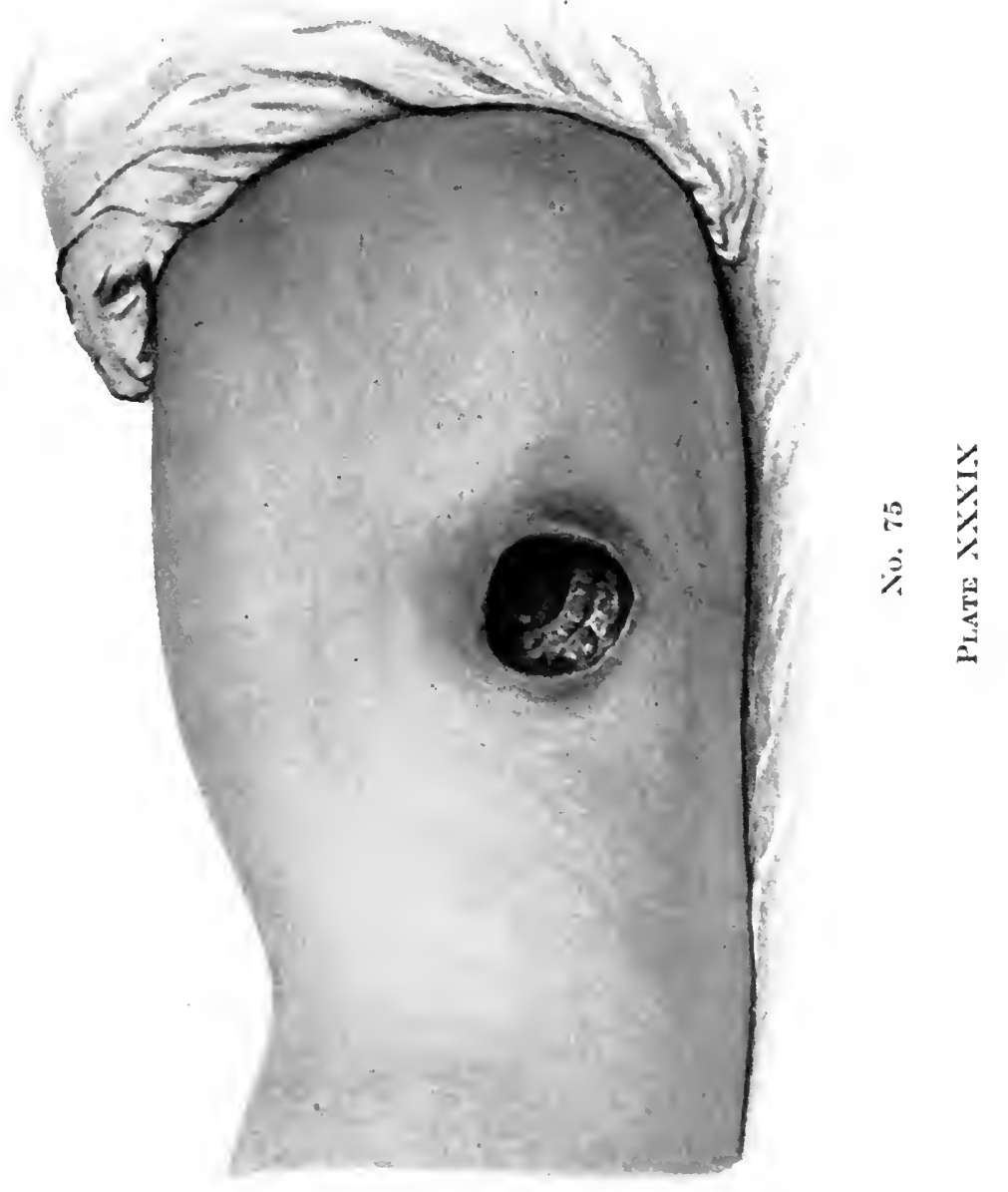


, 
the acute stage of the disease, may commence long after all the syphilitic symptoms have vanished. The lymphatic glands may also be the seat of recurrent syphilitic lesions, and gummata not infrequently affect the cervical set. Just recently I have had a case of multiple abscesses in both axillæ which were due to syphilitic lymphangitis and lymphadenitis. The following is rather an interesting case:

CASE 12.-The patient had syphilis six years ago. A gumma developed in 1912, and another in 1914. In January, 1916, the patient came, complaining of an ulcer on the site of the old chancre, and a large swelling in the left groin. The ulcer was a typical gumma, and the swelling a gummatous lymphadenitis.

The diffuse papular syphilitic eruption which is so commonly seen on the penis, in the neighbourhood of the primary sore, and is usually well marked before any signs of a rash have occurred elsewhere, doubtless arises from a spread of the organisms, from the site of infection, along the lymphatics, as well as along the vessels.

Discussion has been rife during the last few years as to the rôle played by syphilis in the rtiolcgy of Elephantiasis nostras. Before giving an outline of this discussion I will cite two cases which have been under my care:

CASE 13.-A man who contracted syphilis in 1882. His first recurrence was a papular syphilide of the right palm, in 1890 . The next recurrence was a serpiginous syphilide on the left half of the scrotum, and another on the gluteal region on the same side, in 1900 .

A year later, the scrotum, first on the affected side, and in time as a whole, began to enlarge.

On examination (1911) the syphilides had disappeared, the scrotum was $28 \frac{1}{2}$ inches in circumference, and was somewhat eczematous on the surface. The swelling appeared to be in the skin, which was hard, barely œdematous, and retained its rugose appearance. The testicles could not bc felt. The patient had also chronic superficial glossitis. As a résult of thirteen intramuscular injections of grey oil the circumference of the scrotum was reduced to $13 \frac{1}{2}$ inches, the skin became softer, the swelling of the penis disappeared, and the testicles, which appeared normal, could be felt underneath. I saw this patient again, two years later, and his scrotum was then nearly normal in size.

CASE 14.-A man, aged 24, contracted syphilis two and a half years before I saw him, and he consulted me for a uniform swelling of the right leg. Seven months after infection the right leg swelled, and in time the swelling disappeared. Twice since then the leg has swelled again, and on each occasion the swelling was preceded by malaise and a rise of temperature. The swelling always disappeared, but left the leg somewhat bigger each time. I saw the patient when the leg swelled for the fourth time, and after the feverish attack had vanished. The leg was swollen; from the knee to the ankle it was white, very painful, and the œedema was hard; on the shin and on the calf was a rash which could be best described as a mixture of Erythema nodosum and purpura, amongst which were some definite syphilitic papules. Under antisyphilitic treatment the leg became very much better, and smaller than it had been since the first attack; but, nevertheless, there was still some hard odema left. 


\section{CLINICAL ASPECT AND TREATMENT OF VENEREAL DISEASES}

A histological examination of the skin from the calf showed inflammation around the vessels, with a marked dilatation of the lymphatics. The lymphatics had their single layer of endothelial cells, but only in places was there any pericellular inflammation, and even where found it did not consist of more than a few stray cells.

It was impossible to say whether the perivascular inflammation was of a syphilitic nature or not.

In my last book ${ }^{16} \mathrm{I}$ described Case 13 as one of true syphilitic elephantiasis, but when I had investigated the next case, and read an interesting article by Elliot ${ }^{5}$ on the subject, I came to the conclusion that Elephantiasis nostras, although it may occur in syphilis, and perhaps be secondarily due to that disease, is really primarily due to a streptococcal infection, as criginally pointed out by Sabouraud. ${ }^{6}$ The opinion is steadily gaining ground that even those cases of elephantiasis which are supposed to be due to filariasis are primarily caused by the streptococcus of Fehleissen. ${ }^{7,8}$. The recurrent attacks of fever, followed by œdema, in Case 14, strongly support the view that a streptococcal infection was the real cause of the elephantiasis. It is easy to see that cases of elephantiasis following gummata, periostitis, etc., may readily be due to a secondary infection, the open skin being a portal of entry for the streptococci; but it was not so easy to see how those cases in which there was no abrasion could be due to a secondary infection, until Sabouraud definitely proved that many cases occur spontaneously, and that an abrasion is not necessary. Sabouraud also pointed out that it was almost impossible to culture the organism from the lesion, unless the attempt was made during the acute inflammatory period.

The reason why elephantiasis was commonly held to be due to lymphatic origin was owing to the fact that the early histological reports laid so much stress upon the lymphangitis. Cohnheim was one of the first to show that lymphatic obstruction alone could not produce stasis and odema. Unna came to the opinion, from his histological studies, that the cause should be sought in the virus and not in the lymphatics, and it is evident in Case 14 that the bloodvessels were primarily attacked. Although perhaps not absolutely germane to the subject in hand, I think some advantage may be gained in discussing further at this juncture the rôle played by streptccceci in syphilis. A primary sore may become secondarily infected with streptococci, or through a primary sore streptococci may gain entrance to the bedy. Although the generalisation of the syphilitic virus may be delayed thereby, there is no doubt that, when symptoms do appear, they are particularly severe and septic, and they do not always respond readily to antisyphilitic treatment. In some cases, antisyphilitic treatment, especially mercury, makes the patient worse, and this is particularly noticeable in cases of malignant syphilis, which are usually due, in my opinion, to a streptococcal infection, which invades the body viâ the tonsils. The severity of an attack of scarlet fever and measles is in many cases due to a superadded streptococcal infection, which seems to show a predilection for the middle-ear and mastoid 



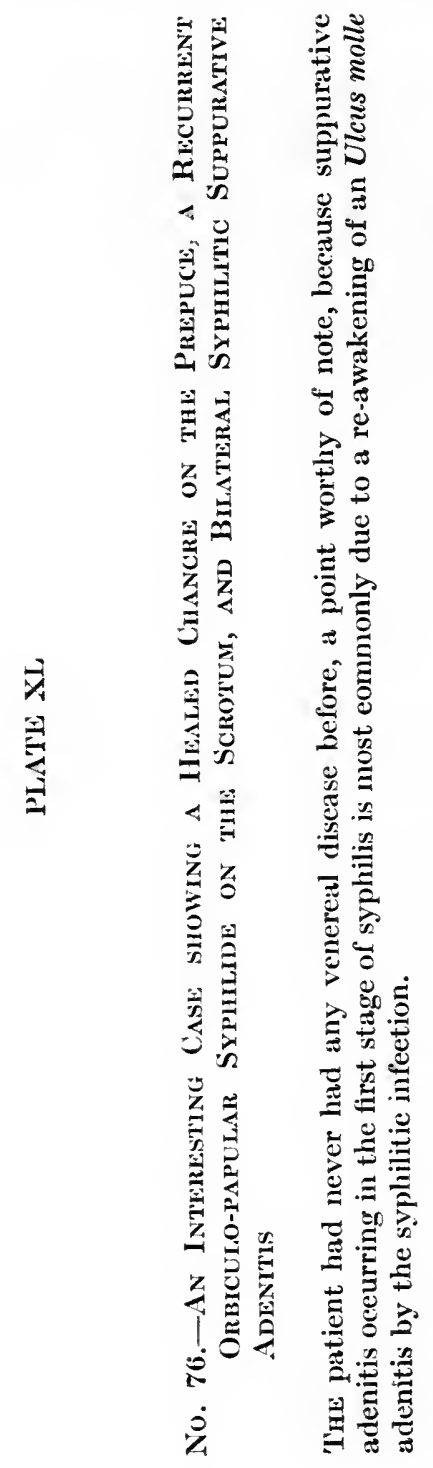




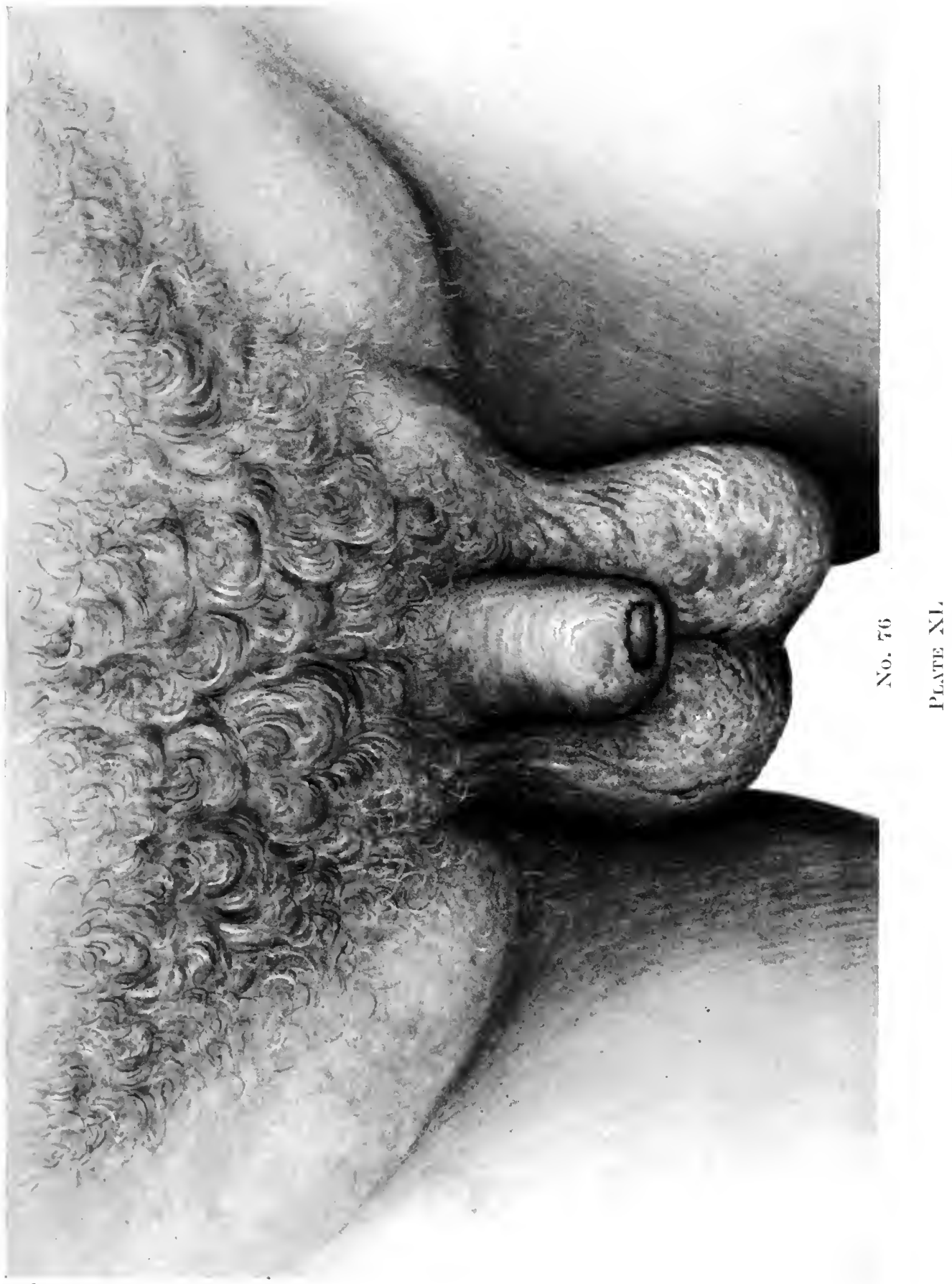



region. Many gummata refuse to heal under syphilitic remedies until antiseptics are applied. Even in cases of degenerative encephalitis and myelitis, a secondary infection may aggravate the condition and even kill the patient. These few points should be borne in mind, because the judicious use of tonics and antiseptics may, in refractory cases of syphilis, prove more availing than all the antisyphilitic treatment in the world. I know not the exact reason for the following phenomenon, but all who deal with syphilis must have noticed how frequently arsenical injections have stirred up a latent coccal infection-furunculosis, etc. I have seen diphtheria, measles, and scarlet fever develop while the patient was still under the influence of salvarsan.

\section{Phlebitis.}

A neglected chapter in the clinical story of syphilis is phlebitis. Veins are infected with the organism of syphilis much more frequently than is supposed to be the case. In my experience, the veins most often affected are those of the upper and lower extremities, especially those of the latter.

Syphilitic phlebitis of both arms, or, more commonly, of only one, causes congestion in the fingers. The congestion may come and go, with the result that the diagnosis of Raynaud's disease is usually made. When the legs are affected-and, again, it is usually only one leg that is involvedit is nearly always the internal saphenous vein that is thrombosed. As the condition is typical of the infection which caused it, and as the descriptions of it are so scanty, it may be well to describe two typical cases, which I have had under my care.

CASE 15.-A woman had a sore in July, the eruption commenced in September, when treatment was started. In December, patient complained of severe pain on the inner side of the thigh. There was nothing to be seen; but, on palpation, one could feel, in the line of the internal saphenous vein, about $1 \frac{1}{2}$ inches above the knee, a hard, tender, spindle-shaped swelling, roughly 1 inch in length.

As time went on this swelling came gradually nearer to the surface, and finally ulcerated. Papules developed along the course of the vein, both above and below the ulcer.

"Nodules" of phlebitis were also found in both legs, and some of them had come to the surface and ulcerated.

CASE 16.-A man, aged 36, no history of syphilis, sought advice for acute pain and swelling of his right leg. He had several attacks of this pain and swelling, and occasionally the whole foot became quite blue. No diagnosis was at this time made. While these periodic swellings of the leg were taking place the patient, on two occasions, had a pulmonary embolus, and on each occasion very nearly lost his life. The patient frequently complained of very bad headaches, and often felt sick and giddy. The next step in the case was the appearance of very painful red nodules in the skin. These nodules were much longer than they were broad; they were surrounded by inflammation, and they commenced in the internal saphenous vein and gradually descended along all its branches, until the toes were reached. Under appropriate antisyphilitic treatment the patient made a good recovery. 
Twice since the above was written the pationt has had a recurrence, the last being in the form of thrombosis of the right popliteal vein, which quickly subsided under colloidal iodine and intramine.

An interesting point in connection with syphilitic phlebitis is the fact that the process always descends, and does not ascend as in cases of venous thrombosis. This is doubtless due to the fact that the lesion starts in the vessels which supply the vein with blood, and not in the intima, due to some damage of the endothelium, as happens in venous thrombosis. In the upper extremities the phlebitis begins in the middle part of the flexor surface of the arm, descends to the elbow, then turns round and runs down towards the fingers along the extensor surface of the forearm. Much the same course is followed in the lower extremity.

Venous lesions may occasionally be the first signs of the generalisation of the virus, and they are therefore possibly in part toxic in origin.

Such lesions may give rise to symptoms and signs which closely simulate two well-known clinical conditions: (a) Erythema nodosum; (b) Erythema multiforme.

Neither of these can be distinguished from the same clinical condition produced by other causes. Erythema nodosum syphiliticum simulates exactly the clinical condition which so frequently accompanies rheumatic fever, and it occurs in the same situation-namely, on the anterior surfaces of both legs. The same applies to Erythema multiforme syphiliticum, which affects most commonly the dorsum of the hands. Plate XX. shows a case where the generalised eruption was venous in origin, each lesion being mapped out as in a case of livido.

\section{Arteritis.}

Since the leucocytozoon pervades every nook and crevice in the body, by means of the blood-stream, and since the organism has a predilection for the walls of vessels, in which to carry out its life-cycle, it is not to be wondered at that syphilitic arterial lesions are common. Any lesion in the wall of an artery is liable to lead to an endarteritis, which is certain to occlude the vessel, if it be small, hence the blood-supply to the area fed by this vessel will be cut off.

There are some arteries which are more frequently involved than others. The arteries which make up the circle of Willis are those an affection of which gives rise to symptoms often within a few months of the time of infection. The commonest cerebral arterial lesion is one which gives rise to a hemiplegia. The hemiplegia is usually unilateral. An early syphilitic monoplegia is very rare. Later in the course of the disease the anterior artery of the cord becomes involved, and gives rise to a paraplegia. Later still the affected arteries undergo a lipoid degeneration, with the result that the vessel gives way, and the patient ultimately dies of hæmorrhage. 


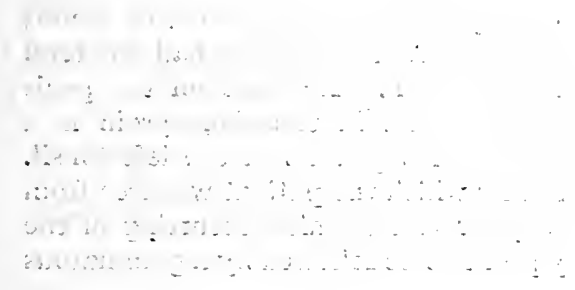




\section{PI.ATE XLI}

\section{No. 77.-Gummatous Adenitis}

THIs patient contracted syphilis twenty-eight years before the lesions depicted appeared. At the time of infection the patient had bilateral suppurative adenitis, which required operation. The sore on the penis is a gumma on the site of the chancre, and in the right groin is a gummatous adenitis. The paticnt also had a gumma of the left tonsil. The interest lies in the long period during which the patient was free from symptoms, and in the fact that a suppurative adenitis occurring in the early stages of syphilis is particularly prone to be followed by a gummatous adenitis in the late stage. 


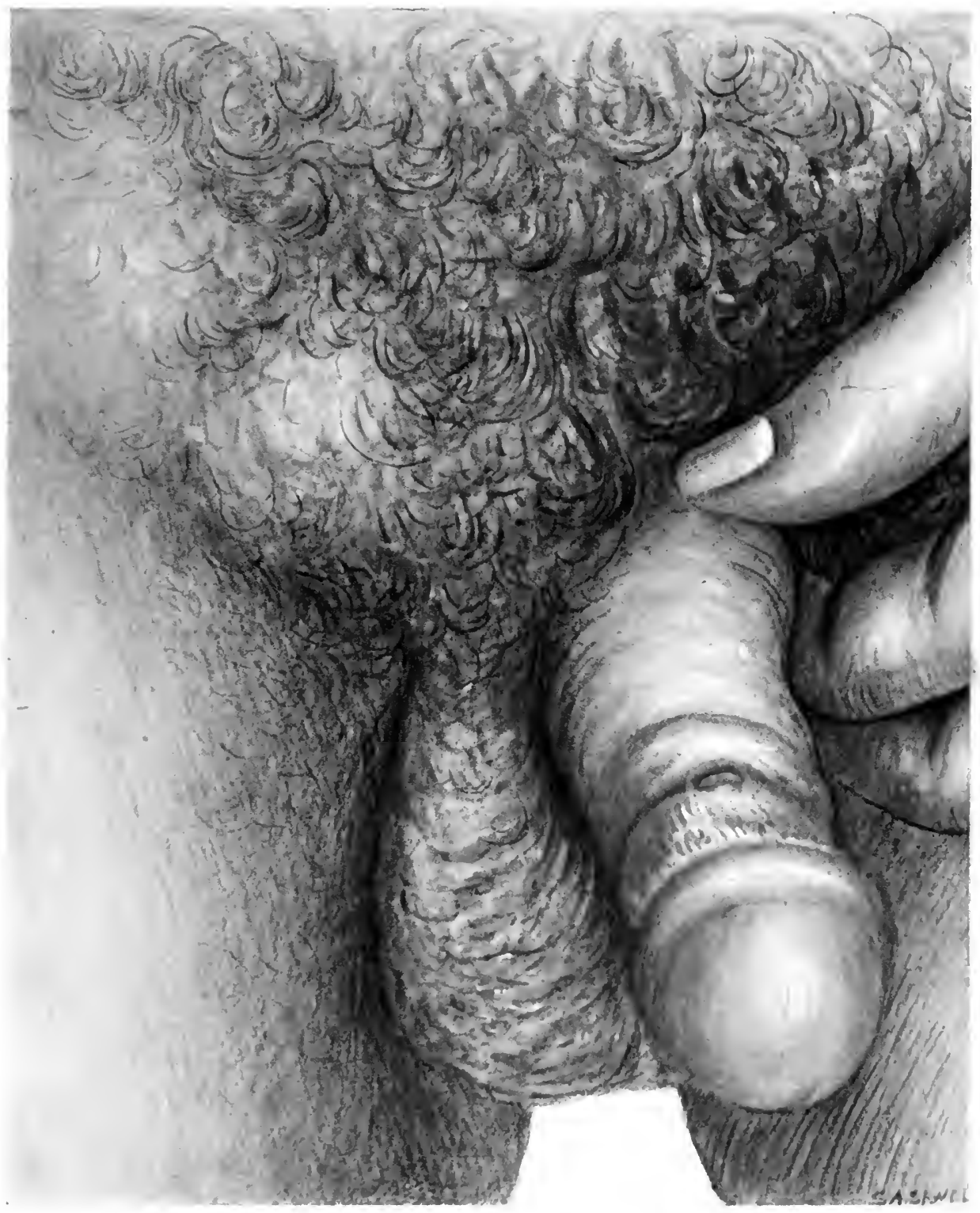

No. 78

Platte XI I 
The cerebral vessels, again, are those most commonly affected, and the arch of the aorta is a frequent victim.

The main feature differentiating syphilitic arteritis from other forms of arteritis is the marked localisation of the trouble. The whole of the arch of the aorta may be diseased, and yet the descending and abdominal aorta may be natural. Syphilitic aortitis is markedly different from that form met with in general arterio-sclerosis resulting from other causes.

Lipoid degeneration is a pathognomonic feature of syphilitic aortitis, hence the calcareous plates so commonly seen in the other forms are absent.

Late syphilitic lesions are characterised by the lipoid degeneration undergone by the cells in the affected area. The lipoid formed by these cells is not a true lipoid, but a compound containing globulin. This lipoid-globulin compound only differs from the normal protein particles of the cells and of the serum in that the particles are richer in carboxyl-groups. Owing to certain physical properties possessed by such particles the C.F.T. is apt to be particularly positive in these arterial cases.

The increase of carboxyl-groups in the lipoid-protein particles takes place to a certain extent at the expense of some of the ions attached to them. Some of the salts most easily displaced are those of calcium; hence the explanation of the absence of the calcareous plates in syphilitic aortitis, and of the fact that sera of late cases of syphilis do not clot so readily as the sera of early cases.

Andrewes $^{1}$ has done some very interesting and important work in this connection. He incinerated several diseased aortæ, estimated the calcium in the ash, and found that the calcium content of syphilitic aortæ was far and away below that of aortæ diseased from other causes.

In some cases of syphilitic aortitis, Andrewes also estimated the calcium content of the aorta away from the syphilitic lesion, and found that not infrequently the calcium content was very much raised.

This work of Andrewes proves that the syphilitic process is quite localised, and that the vessels elsewhere may show the signs of ordinary arterio-sclerosis. Syphilitic aortitis may lead to an aneurysm; but all aneurysms are not due to syphilis, as is evidenced by the fact that animals may succumb to the condition. An aneurysm need not necessarily be a late syphilitic lesion, as I have seen two cases of popliteal aneurysm, of which one occurred four years, and the other five years, after infection. I remember another case in which a bilateral popliteal aneurysm occurred seven years after infection; and I have notes of a case of an aneurysm of the arch of the aorta in a congenital syphilitic girl aged 15. A point to remember is that aneurysms mav be multiple, as in the following case:

CASE 17.-A man, aged 52, who had that sort of sallow and worried look that is not uncommonly seen in bad syphilitic arterial cases, consulted me for a swelling in the femoral region of the right thigh. The swelling was a typical aneurysm of the femoral artery about the size of a hen's egg, and projecting above the surface 
of the skin. The patient also complained of pains in the chest, which were most marked on the left side and in the substernal region. Occasionally the pains radiated to the shoulder and head, especially after any exercise. The patient had equal pupils and pulses, and had none of the symptoms which are usually met with in aortitis of the ascending and transverse portions of the thoracic aorta. The left ventricle was hypertrophied, the second sound over the aortic area was accentuated, and abnormal sounds were to be heard at the left sternal border, which could be traced downwards. An X-ray examination showed that the patient had an aneurysm of the descending thoracic aorta, and possibly a dilatation of the ascending aorta. Under antisyphilitic treatment the femoral aneurysm improved enormously, till it could only just be felt; but in the chest it was difficult to say whether any improvement had taken place or not.

Aortitis of the descending aorta is very rare, compared with the frequency with which the ascending and the transverse portions are affected. Occasionally the abdominal aorta is exclusively involved.

In syphilitic aortitis there are some more or less characteristic features. The commonest form is that which goes by the name of Döhle-Malmsten's aortitis. It is also frequently called the Mesaortitis productiva of Chiari, and syphilitic aortic sclerosis of Benda. The process begins in the ascending aorta just above the valves, and spreads along the arch. This form of aortitis is characterised by pleats and furrows, the latter being scars, which run longitudinally; here and there are tiny sacculations, which may lead to small or big aneurysms. The intima is hard, it has a shiny mother-of-pearl appearance, it is not ulcerated, and in it there are no calcareous deposits. The real difference between this syphilitic aortitis and atheromatous aortitis is that in the former there is proliferation of the intima and in the latter destruction.

The proliferations of the intima are sharply defined, pale and hyaline in appearance; they are also soft and smooth to the touch, as they contain no calcium. In most cases the aortic valves are secondarily involved; more rarely the coronaries may become occluded, and even such-big vessels as the innominate and left carotid. A very large number of cases never proceed to aneurysmal dilatation, but remain localised, to do no more than cause a slight aortic insufficiency. This is the reason why the condition so frequently remains undiagnosed until revealed at autopsy.

A point of interest is the frequent association of syphilitic degenerative nerve lesions with aortitis. In my experience intravenous injections are not contra-indicated in cases of aneurysmal dilatation of the aorta, and therefore the antisyphilitic treatment should in all cases be given, because, although it may produce no change in the aorta itself, it does undoubtedly help to remove the troublesome symptoms of which the patient complains-the sternal pain, for instance.

Syphilitic endarteritis only occurs in those arteries which are supplied with Vasa vasorum, and, as even the small intracranial arteries are rich in these nutrient vessels, the explanation is forthcoming as to why the intracranial vessels should be so frequently involved. 
It was generally thought that intracranial aneurysms were of syphilitic origin, but Fearnsides ${ }^{15}$ has conclusively shown that such is not the case, the cause being usually an infective embolism.

Syphilis may cause Arteritis obliterans, especially of the intracranial vessels; but when the vessels of the extremities are affected, it is necessary to distinguish it from the Thrombo-angiitis obliterans, which usually affects Hebrews, and seems to be connected in some way with nicotine poisoning.

The following is an interesting case of progressive syphilitic Arteritis obliterans :

CASE 18.-The patient, a Gentile, aged 40 , contracted syphilis about ten years before the symptoms about to be described appeared. In 1912-that is to say, five years before I first saw him-the patient complained of swelling, pain, and redness of the right arm, the typical erythromelalgic symptom-group. The swelling disappeared, the arm became white, and the index and middle fingers became quite numb. Later still the whole arm wasted, the fingers became glossy, and no pulsation was perceptible in any of the arteries of the right upper extremity.

In January, 1915, the patient's condition was diagnosed as syphilis, and this was confirmed by a positive C.F.T. In view of this, an intravenous injection of salvarsan was given, with the result that the patient got sudden excruciating pain in the right arm, and from that moment wasting, glossy fingers, etc., gradually set in. In consequence of such a severe disturbance, no more intravenous injections were given, and instead the patient was treated with mercurial inunctions and potassium iodide internally, without very much improvement. Whether the disturbance was of the nature of reactionary inflammation or not I cannot say.

On December 26th, 1916, the patient went suddenly blind in the left eye for about an hour.

For the last year the patient has complained of a feeling of tightness below the right calf after walking; cramps and blanching of the toes are frequent, and the right leg is generally smaller than the left.

When the patient gets into a very hot bath, both feet first become white and then red, but the right foot takes some time longer to do so than the left foot. This anomaly was also noticed in the initial stages of the arm trouble. The patient also has frequent attacks of giddiness.

On examination, the heart was normal; no nervous lesion was ascertainable; the optic discs were normal; livido was very well marked on the thighs when the patient undressed; no pulsation could be felt in the right arm, but it was perceptible in the posterior tibial and the Dorsalis pedis of the right leg. On the sole of the right foot was a cord of contracted plantar fascia opposite the second toe. Two years before the arm became affected there was a cord of contracted palmar fascia opposite the middle finger, and this was suggested at the time as likely to lead to Dupuytren's contraction, but it has since disappeared.

I could find no evidence of Induratio penis plastica, and there was no family history of Dupuytren's contraction. The father died of jaundice, aged 36, and one brother, aged 44, is a great victim to gout. There was no sugar in the urine, but a trace of protein. I examined the blood again, and found that the C.F.T. was positive. The symptoms of this case are so like those of Thrombo-angiitis obliterans (non-syphilitic Arteritis obliterans of Hebrews), about which so much has been written by Parkes Weber, ${ }^{9}, 10,11$ that at first I hesitated in labelling it as 
syphilitic, in spite of the positive C.F.T., but as the condition improved considerably under colloidal iodine and intramine, and as we know that syphilis is in some cases particularly prone to pick out fibrous tissue, I think justification exists for considering the above as a case of syphilitic Arteritis obliterans.

A new light has recently been thrown upon the rtiology of Thromboangiitis obliterans by Meyer, ${ }^{14}$ who proposes to label the condition "Glycophilia," owing to the readiness with which hyperglycæmia is produced after the ingestion of glucose. The concentration of sugar in the blood of fasting normal individuals after the injection of $100.0 \mathrm{grm}$. of glucose does not rise above 0.15 per cent. at the end of an hour, and by the end of the second hour the concentration of blood-sugar has again returned to the normal. If the $0 \cdot 15$ per cent. is increased, then hyperglycæmia exists, and it may be associated with glycosuria. In some of the cases studied by Meyer, the blood-sugar after glucose reached as high as 0.248 per cent., and in none was the normal level reached after two hours. This undoubtedly points to some derangement of the carbohydrate metabolism. The thrombi Meyer regards as being a conglomeration of red blood-corpuscles due to erythrocytosis and stasis, the latter being a sequence of the increased viscosity of the blood. 'This point is confirmed by the aggravation of the lesion after the first injection of arseno-benzene, because one of the first effects of injecting a protected colloid is to increase the viscosity.

The question now arises as to the cause of the altered carbohydrate metabolism, one which particularly interests the syphilologist, as glycosuria is not uncommonly caused by syphilis, and is most prone to occur in those cases showing evidence elsewhere of a syphilitic fibrosis. Possibly the primary lesion is a syphilitic involvcment of the adrenals, about which more will be found in Chapter IX.

Another branch of vascular syphilis veiled in obscurity, and allied to the condition just mentioned, is the so-called vaso-motor affection producing a modified Raynaud's disease, or lesser marked peripheral vascular disturbances. Whether the lesion is a central one or due to inflammation in the walls of the vessels affecting the vaso-motor nerves therein is not known, in spite of the condition being comparatively common. The following are two typical cases:

CASE 19.-A woman, aged 31, contracted syphilis at 18. She was treated with mercury internally for two and a half years. The patient was sent to me as a case of syphilitic onychia. On examination, eight of the nails of the hands-feet were normal-had the typical rough, brown, furrowed degenerative condition, which had begun a year previously. The circulation of the hands was very bad: sometimes they were red, at other times blue, and occasionally quite white. The changes were not always uniform, as when some of the fingers were blue others would be white, etc. Although this vaso-motor phenomenon was present all the year round, it was naturally worse in the winter, and it had been present for two years. The patient had three children, all showing minor signs of congenital syphilis. Under treatment the nails did not improve, but the circulatory disturbance vanished, and she has been free of trouble for four years. 
CASE 20.-A patient had eight injections of arseno-benzene for an early generalised syphilitic eruption. Six months later, while under treatment with mercury, he complained of pain and coldness of his hands. Although it was mid-summer, both hands, but especially the left, evidenced the same changes as described in the previous case, from which it otherwise differed, in that the symptoms did not disappear as the treatment was continued.

A point not yet settled is the exact influence syphilis has in the production of arterial hypertension. The prevailing view is that this disease is without effect on the blood-pressure. Cautley, in 1901, emphasised the absence of high blood-pressure as well as cardiac hypertrophy in syphilis, and Clifford Allbutt ${ }^{13}$ is equally emphatic in his belief that there is no important relation between syphilis and arterial hypertension. There is no doubt that, considering how readily syphilis attacks the arterics, it is a fact that syphilitic aortitis with aneurysm, etc., may occur without hypertension, but as we do not understand the forces at work responsible for the increase of bloodpressure, it is scarcely right to say that syphilis may not set them going.

Having registered the blood-pressure of a very large number of late syphilitics, I have come across three cases which presented the same phenomena. In all three the blood-pressure was about 220 , no arterial disease was ascertainable, there was pronounced proteinuria, with no other signs of nephritis except slight œdema of the ankles apparent at night, after a hard day's work, and the serum gave a particularly strong positive C.F.T. Examined ultra-microscopically, the serum in all the cases was remarkable for the large lipoid-globulin colloidal particles it contained. The C.F.T. tends to be markedly positive when the particles are large, and it would be expected that millions of large particles circulating in the body would increase the viscosity of the blood. When the particles are particularly numerous and particularly large, the glomerular filter appears to allow some of them to pass into the urine. That the protein found in the urine in these cases is largely a filtration, and not dependent upon a nephritis, regarded purely as an inflammatory lesion, appears to me to be most probable, for the following reasons: (1) The patients are in robust health, in spite of having passed large quantities of protein in the urine for years, which could scarcely have been the case if the inflammation of the kidneys had been proportionately severe. (2) The protein particles in the urine are large, refractive, and in many respects resemble those in the serum. (3) Antisyphilitic drugs are well borne, and as they make the protein particles smaller the proteinuria decreases. From these few points it would appear that syphilis can produce hypertension, and that the first force at work is an altered viscosity of the blood, brought about by the increase in number and size of the protein colloidal particles circulating in the serum. The second force would appear to be a faulty filtration of the glomeruli, dependent upon an altered state of the colloidal particles forming the filtering membrane. The third force is probably a general fibrosis of the vessels, with possibly a specific aortitis, about which 


\section{2}

\section{CLINICAL ASPECT AND TREATMENT OF VENEREAL DISEASES}

more cannot yet be said, as no studied case has come to post-mortem. Finally, we must consider a mesenchymatous syphilitic inflammation of the suprarenals, allowing an excess of the internal secretion getting into the circulation and thereby causing the rise of blood-pressure.

\section{Cardiac Syphilis.}

Our knowledge of syphilis of the heart is vague, and this is probably the reason why little or no mention is made thereof in textbooks. This has naturally led to the belief that cardiac syphilis is rare; consequently, we were not a little startled when Warthin ${ }^{19}$ stated that microscopic lesions were discernible in 88 per cent. of the autopsy cases on adults examined, while the aorta was involved in only 78 per cent., a finding contrary to the prevailing idea heretofore. In a disease like syphilis, once the organisms become generalised, it is only natural to expect that every organ becomes invaded; but invasion does not necessarily mean the production of symptoms which are sufficiently pronounced to be diagnosed by clinical means. Clinically, involvement of the heart in early syphilis is rare, but when it does occur it does not differ from myocarditis produced by other organisms. The patient complains of precordial pain and cardiac embarrassment. The pulse is accelerated, and the temperature is usually raised. There is an enlargement of cardiac dulness, and possibly a systolic murmur at the base. The only cases I have seen have been those in which the above-mentioned symptoms became particularly pronounced, as a result of inflammatory reaction after the first or second injection of arseno-benzene. Even in late syphilis cardiac involvement is rare, probably because the symptoms presented do not differ from those produced by other causes more frequently met with. In fact, it is almost impossible to say for certain whether the precordial pain, palpitation, dyspnœa, and tachycardia complained of by patients who one knows to have had syphilis are really due to the disease or not. Gummata of the heart may exist and heal without ever giving rise to symptoms, and it seems to be only when situated in a position such as His's bundle that the symptom complex produced is sufficiently pathognomonic to exclude any other cause but syphilis. A patient with such a lesion usually seeks advice for periodic attacks of giddiness, with maybe temporary loss of consciousness. StokesAdams symptoms may occasionally be met with, and usually the pulse-rate is slow.

A slow pulse-i.e., between 30 and 50 in a young subject-should always make the observer suspect a syphilitic cardiac lesion. A physiological brachycardia is extremely rare; the best-known instance is that of Napoleon, who is said to have always had a normal pulse-rate of 40 .

Except perhaps as a pathological curiosity, I do not believe there is such a condition as a primary syphilitic endocarditis. We are all familiar with sclerosis and retraction of the aortic valves producing incompetence, secondary 
to a syphilitic aortitis, a familiarity which has led to its being said too often that syphilis is a frequent cause of aortic regurgitation. Without aortitis, I should say that syphilis was never the cause of aortic insufficiency. During the years I have been studying this disease I have come across one case of mitral disease and three cases of aortic disease in which the question of syphilis as an ætiological factor arose. The one mitral case had had rheumatic fever, and died of malignant endocarditis, which from a post-mortem examination proved to be obviously non-syphilitic. The three aortic cases presented such obvious murmurs of both stenosis and regurgitation, and affected soldiers who had been subjected to great strain, and as the condition in one case was undoubtedly aggravated by a course of arseno-benzene and mercury, I think the strain in all was the cause, and not syphilis. The coronary arteries are generally considered to be frequently involved in cases of syphilis, an opinion not borne out from the few cases I have had the opportunity of examining. Anginal attacks are also in my opinion not so frequently met with in cases of syphilitic aortitis as in cases of general arterio-sclerosis due to other causes.

It should not be forgotten that the cardiac sympathetic nerves are not at all infrequently involved in cases of degenerative myelitis, and, if the symptoms produced are at all severe, the physician's attention may be drawn to the heart only, with the result that the true nature of the trouble is overlooked. To cite a case:

CASE 21.-A man, aged 37, who had contracted syphilis eight years previously, came to me complaining of attacks of shortness of breath, giddiness, and a feeling as if he were going to faint. He had previously suffered from very bad attacks of coughing, for which a throat specialist had snipped off a piece of his uvula. The patient still had attacks of coughing, and he often felt very sick.

On careful examination, one could plainly see that it was a typical case of degenerative myelitis, as many of the other cardinal symptoms were present.

As time went on the sickness developed into typical gastric crises, and the cardiac condition became very much worse. The patient would suddenly swoon away and become quite unconscious; his complexion would become blue, and then ashen grey, and for a short interval his pulse could not be fclt. Antisyphilitic treatment aggravated the cardiac crises very much indeed.

\section{Blood-Changes.}

We have now to discuss the blood-changes which usually occur in syphilis. The true blood-changes affect only the leucocytes, since it is only in those cases in which syphilis has caused an anæmia that changes are to be found in the red blood-corpuscles, and then the changes are typical of ordinary secondary anæmia. Syphilitic anæmia, owing to the use of salvarsan, is not now often met with, and, in many of those cases in which it did occur, it was often aggravated by, if not actually caused by, the mercurial treatment. Hence the reason for stating that changes in the red blood-corpuscles, such as are 


\section{4}

CLINICAL ASPECT AND TREATMENT OF VENEREAL DISEASES

to be sometimes met with in syphilis, are generally only secondary in nature, and not changes actually produced by the syphilitic organism itself.

The changes in the leucocytes are very interesting. In early syphilis the total number of leucocytes is increased. Before treatment is commenced, the relative increase of the polymorphonuclear leucocytes is greater than that of the lymphocytes; a ratio between the two exists, and it is dependent upon the severity or lightness of the case. In the severe cases the relative increase of the neutrophile leucocytes is very much greater than that of the lymphocytes-indeed, the total number of the lymphocytes may be diminished. In light cases the percentage of lymphocytes may approximate to the number given by the polymorphonuclears, and may even exceed it.

Treatment very quickly diminishes the percentage of the neutrophiles, and increases the percentage of the lymphocytes. Arseno-benzene is much more powerful in this respect than mercury.

The lymphocyte count may approximate, in early cases of syphilis, to 60 per cent. Occasionally a small rise in the eosinophiles is to be met with, but there appears to be no relationship between the eosinophile count and the kind of case. According to Hazen, ${ }^{4}$ from whose pioneer work in this field most of these details are taken, there is no alteration in the large mononuclears or basophiles. Curiously enough, the total increase of leucocytes is greater in the negro, and the relative lymphocyte count is higher also than in the white man.

In severe cases the lymphocyte count is low in comparison with the lymphocyte count in the mild cases; and in those cases which are going to do well under treatment, the lymphocyte count becomes, as a rule, very much higher than in those cases which are not going to do well. Hence, from a lymphccyte chart a prognosis can be made.

Mercury administered to normal men causes a rise in the absolute and relative lymphocyte count, but a slight fall in the total leucocyte count; therefore the main decrease is in the relative neutrophile count.

In the second and third years after treatment the total leucocyte count is only slightly raised, and the main increase affects the lymphocytes. In the late stages of the disease the same picture is to be found, and, in cases in which treatment is prescribed, the absolute and relative lymphocyte count increases. As a rule, after the second year, whether the patient is under treatment or not, there is no increase in the eosinophile count.

Another interesting point which Hazen brings out is that males show a slightly greater increase in the total count than do females, and that females show a higher lymphocyte count than do males.

No relationship exists between the lymphocyte count and the degree of enlargement of the lymphatic glands, a point which supports my view that the lymphocytes manufactured in the lymphatic glands remain in the glands, and that those which reach the circulation emanate from the bone-marrow.

From these few remarks on the blood-picture to be met with in syphilis, 
the reader will at once observe how important the lymphocytes are, and how relatively unimportant are the polymorphonuclear leucocytes, a point which clearly shows that phagocytosis does not play a great part in bringing about the destruction of the syphilitic parasite. Probably one of the reasons why syphilis is not such a severe disease in women as it is in men is due to the higher lymphocytosis in the former-after all, it is from the lymphocytes that the protective substances of the host originate. The reagin in the C.F.T. comes from the lymphocytes. Considering how chronic a disease syphilis is, and what a call it makes upon the lymphocytes of the host it attacks, it is not to be wondered at that the manufacture of the lymphocytes becomes abnormal, and that various kinds of lymphocytomata arise, although the syphilitic parasite may have been driven out of the system.

Syphilis, though rarely, may be the underlying cause of pernicious anæmia. The only case I have seen was a woman who had an enlarged spleen, but none of the ordinary symptoms or other signs of what is commonly known as splenic anæmia or Banti's disease. The patient had had several miscarriages, and had given birth to a congenital syphilitic infant. As the patient had very bad teeth, which so commonly accompanies pernicious anæmia, and is probably responsible therefor, as held by Hunter, it is not easy to estimate the rôle syphilis played, especially as pernicious anæmia of certainly nonspecific origin may be temporarily benefited by injections of arseno-benzene.

\section{Syphilitic Lymphocytomata.}

Most interesting is the rôle syphilis plays in the rtiology of the various lymphocytomata. ${ }^{16,17,18}$

Syphilis is undoubtedly a potent factor in the cause of intermediate aleucæmic lymphocytomata, affecting the skin (Mycosis fungoides), lymphatic glands (Hodgkin's disease), and other organs (Miculicz' disease). The type is always aleucæmic, but intermediate, because the cases are, as a rule, neither purely inflammatory nor purely malignant. In some cases they are ultimately malignant, which opens the interesting subject of the connection between syphilis and sarcoma. The following is one of the most illuminating cases I have ever had:

CASE 22.-A man, aged 39 years, contracted syphilis fifteen years ago, for which he was treated with mercury internally for three years. Five years after the infection diffuse swellings appeared in the neck, and mainly affected the salivary glands and the lymphatic glands around. In spite of the most vigorous antisyphilitic treatment, the glands remained unmoved. During the ten years before I saw him, the patient had frequent attacks of œdema over various parts of the body, and they not infrequently commenced either in or around the lachrymal glands, which spread to the neck, necessitating frequent tracheotomy, and, finally, a permanent tracheotomy tube had to be worn. Around the enlarged glands the œedema was chronic. At frequent intervals the patient developed swellings in his skin, and they ultimately burst, leaving small holes, which were extremely deep, not at all 


\section{CLINICAL ASPECT AND TREATMENT OF VENEREAL DISEASES}

like gummata. Another complaint was an almost incessant tinnitus. None of these symptoms was influenced in the least by mercurial inunctions or injections, or by iodides administered internally. The C.F.T. was one of the strongest I had ever come across. The blood-count showed a diminution of lymphocytes, a slight increase of the large mononuclears, and an increase of eosinophile leucocytes, up to 21 per cent. All the tuberculin tests were negative. A piece of lymphatic gland was removed for microscopic examination, and revealed the following points:

Capsule thickened; follicles, on the whole, well maintained; where other follicles should have been, was a cellular infiltration made up of alternating lymphocytes and plasma cells, but characterised by some endothelial cells with young lymphocytes in their protoplasm, and also a number of endothelial cells whose nuclei were undergoing division like Sternberg's multinucleated giant cells. Throughout the cellular infiltration were several eosinophile cells.

Such a histological picture is certainly not that of syphilis, but one of what would have been called lymphogranulomatosis or Hodgkin's disease.

When treated with salvarsan, an ulcerated nodule on the chest healed at once, the glands went down to almost their normal size, the attacks of œdema became very much less frequent, and the tinnitus was diminished. This was doubtless a case of mixed Hodgkin's and Miculicz disease of syphilitic origin, with Mycosis fungoides as cutaneous lesions.

CASE 23.-A boy, aged 21 years, contracted syphilis in November, 1913. I saw him first in January, 1914, when he showed the remains of a chancre on his penis and a papular rash, but he was covered from head to foot with what appeared to be a diffuse pigmented erythrodermia, upon which were innumerable urticarial and prurigo-like lesions, some of which had pus in them, owing to a secondary infection caused by the intense itching accompanying the eruption.

It was difficult to distinguish the syphilitic papules from the rest.

The glandular enlargement was very marked, especially the two inguinal sets, and they certainly were more enlarged than one would expect to see in an early syphilitic infection.

Thinking the syphilis might have given rise to an intermediary form of the aleucæmic lymphocytomata of the lymphatic glands, with a secondary prurigolike eruption, I did a blood-count with the following striking result:

\begin{tabular}{|c|c|c|c|c|c|c|}
\hline White blood-c & rpuscles & & & & $\cdots$ & 26,000 per c.mm. \\
\hline Red & ," & . & . & • & . & $4,960,000 \quad$, \\
\hline Hæmoglobin & -. & & & . & . & 90 per cent. \\
\hline Colour index & $\cdots$ & $\cdots$ & $\ldots$ & $\ldots$ & $\ldots$ & 0.9 \\
\hline
\end{tabular}

Differential count of whites:

\begin{tabular}{|c|c|c|c|c|c|}
\hline Polymorphonuclears & . & . & . & $\begin{array}{l}\text { Per Cent. } \\
\ldots \quad 65.0\end{array}$ & $\begin{array}{c}\text { Per C.Mm. } \\
16,900\end{array}$ \\
\hline Lymphocytes & . & . . & .. & $12 \cdot 0$ & 3,120 \\
\hline Large mononuclears & . & . & .. & $2 \cdot 2$ & 572 \\
\hline Eosinophiles & .. & . & .. & . $20 \cdot 0$ & 5,200 \\
\hline Basophiles .. & .. & .. & $\ldots$ & $0 \cdot 8$ & 208 \\
\hline
\end{tabular}

This shows a polymorphonuclear leucocytosis with a marked eosinophilia, not a blood-count one would expect to get in the early generalisation stage of syphilis.

After several weekly injections of neo-salvarsan and a few intramuscular injections of grey oil, the blood-count was done again. In the meantime the syphilitic symptoms had vanished, the prurigo-like eruption had improved, but by no means 
gone, and some of the individual lesions had increased somewhat in size. The glandular enlargement, though not so marked, still persisted:

\begin{tabular}{|c|c|c|c|c|c|c|}
\hline White blood. & rpuscles & & & & . & 12,000 per c.mm. \\
\hline Red &, & $\cdots$ & $\cdots$ & . & . & $5,680,000 \quad$, \\
\hline Hæmoglobin & .. & & & . & . & 95 per cent. \\
\hline Colour index & . & $\ldots$ & $\ldots$ & $\ldots$ & $\ldots$ & $0 \cdot 8$ \\
\hline
\end{tabular}

Differential count of whites:

\begin{tabular}{|c|c|c|c|c|c|}
\hline ais & & & & Per Cent. & Per $0 . M m$ \\
\hline Polymorphonuclears & $\cdots$ & $\cdots$ & .. & $\ldots 60 \cdot 0$ & 7,200 \\
\hline Lymphocytes & . & . & . & $23 \cdot 0$ & 2,760 \\
\hline Large mononuclears & . & . & . & $4 \cdot 0$ & 480 \\
\hline Eosinophiles & . & - & . & $11 \cdot 4$ & 1,368 \\
\hline Basophiles & $\ldots$ & .. & $\ldots$ & $1 \cdot 6$ & 192 \\
\hline
\end{tabular}

The above count shows a slight polymorphonuclear leucocytosis with still a marked eosinophilia.

The C.F.T. was still markedly positive. Unfortunately I could not remove a gland for microscopic examination, but the outbreak of the prurigo-like eruption accompanied by a great enlargement of the lymphatic glands early in the stage of generalisation of the syphilitic virus, together with a blood-count as above, and the way in which the whole picture altered under specific treatment, strongly suggests not only that the prurigo-like eruption was a symptom of an aleucæmic lymphocytoma, but also that syphilis was the cause thereof.

I have seen typical cases of intermediary aleucæmic lymphocytoma of the lymphatic glands in the neck and mediastinum, which ultimately caused the patient's death, which I feel convinced were caused by syphilis. It is interesting to note that Melchior describes similar cases. ${ }^{18}$

Owing to the light thrown upon the rtiology of malignant disease by a study of syphilis as a producer of inflammation, I propose to devote a special chapter to this subject, where the important connection will be more fully dealt with (vide Chapter XXXIII.).

1. ANDREwes (1914): Local Government Board-Rep. of the Med. Officer.

2. Parkes Weber (1909): Trans. Med. Soc. of London, xxxii., 370.

3. OSLER (1900): Johns Hopkins Hosp. Bull., xi., 41.

4. Hazzen (1913): Journ. Cutan. Diseases, xxxi., 618.

5. ElLiot (1917): Journ. Cut. Dis., xxxv., 17.

6. Sabouradd (1892): Ann. de dermat. et de syph., iii., 592.

7. P'ROUT (1908): Brit. Med. Journ., ii., 1364.

8. ShattucK (1910): Boston Med. and Surg. Journ., ii., 107.

9. Parkes Weber (1916): Proc. Roy. Soc. of Med., x., 1 .

10. Parkes Weber (1917): Proc. Roy. Soc. of Med., x., 18.

11. Parkes Weber (1916): Quart. Journ. of Med., ix., 289.

12. Sinkowitz ANd Gottlieb (1917): Journ. Amer. Med. Assoc., lxviii., 961.

13. Allbutr (1915): Diseases of the Arteries, including Angina Pectoris. Macmillan, London.

14. Meyer (1918): Journ. Amer. Med. Assoc., Ixxi., 1268.

15. Fearnsides (1916): Brain, xxxix., 224.

16. MCDonagh (1915): Biology and Treatment of Venereal Diseases. Harrison and Sons, London.

17. McDonagh (1914): Brit. Journ. of Dermatol., xxii., 283, 337.

18. MelChior (1917): Ugeskrift fur Laeger, lxxix., 2143.

19. Warthin (1914): Trans. Assoc. Amer. Phys., 416. 


\section{CHAPTER V \\ SYPEILIS OF THE BONES AND JOINTS}

\section{History.}

THE history of bone syphilis is interesting, because this branch of the subject was more prominent than any other, owing to the uncertainty which existed between syphilis and mercury as being the principal cause. Considering syphilis was unknown in Europe prior to 1493, the treatment thereof with mercury must have caught on extraordinarily rapidly, because in 1519 Ulrich v. Hutten, the greatest satirical writer of the time, and himself not a physician, says he did not suffer any less from bone syphilis after having had eleven courses of inunctions in six years than his father and friends, who had had no mercurial treatment. Half-way through the sixteenth century, many authors stated that mercury was to be found with regularity in the bones of those who had been treated with it. Every early writer on syphilis appears to have been struck by the pain met with in bone affections; in fact, the so-called Dolores osteocopi was almost regarded as a distinct disease. The bones most frequently implicated were the tibiæ, sternum, and clavicles. All the varieties of syphilitic arthritis recognised to-day were well known to Paracelsus, as the following interesting quotation from Proksch's ${ }^{1}$ article shows: The joints appear als sey dieser Sack foller Eyter etwann als voller Eyerklar, etwann gleich als ein Wassergriff, vund begeren doch nicht auffbrechen. Hunter disbelieved in syphilitic arthritis, an opinion which was shared by Ricord for many years, although it must be said that he never went further than saying that the arthritis was not characteristic. This shows the tremendous influence Hunter's teaching had, and what a veil it cast over the clinical experience handed down to us from those who preceded him.

\section{General.}

In discussing syphilis of the bones, lesions of the periosteum are, of course, included; and for the sake of making the description of the subject easier, the lesions of the periosteum will not be, as they usually are, considered separately from those of the bones.

Although, perhaps, as much attention has been paid to syphilis of the bones as to that of any of the other organs, none of us knows for certain whether, in the majority of the cases which we call periostitis, the disease really started 
in the periosteum, or in the bone itself. Many observers hold to-day that there is no such thing as primary syphilitic periostitis. Others are of the opinion that syphilitic disease of the bones always begins in the periosteum, and that those severe cases of osteitis and osteo-myelitis, which were much more often seen years ago than they are now, were due to the mercury which had been prescribed for the disease.

There is no doubt at all that the injudicious use of mercury, which was practised only a comparatively short time back, mainly in the form of fumigations, was partly responsible for a large amount of bone trouble. This planted the seeds of suspicion, which have blossomed forth into that mistrust of mercury which is possessed by many lay people to-day.

The view that was, and is still, held is that mercury caused bone trouble by collecting in loco; but animal experiments, and the fact that those who work in quicksilver mines are no more prone to bone diseases than those who follow other trades, all militate against current opinion. The fact that the patient has syphilis is an important factor. Secondary anæmia in syphilis is common; mercury often aggravates this secondary anæmia. Fatty degeneration is a frequent result of syphilitic inflammation. Fatty degeneration causes a diminution of calcium; so does the prolonged and injudicious use of mercury. Therefore, although one may still hold the view that mercury was the cause of many of the fine specimens of bone diseases which adorn our museums, it must be said that it was not entirely responsible.

In connection with the above, it may be of interest to draw attention to a recent article by Kolmer and Mastin, ${ }^{2}$ who found globules of mercury in an aortic aneurysm, and in the vertebræ which had been eroded by it.

The periosteum is continuous with the interstitial tissue which interlaces the parenchyma of the bone. The organism of syphilis develops in the interstitial tissue, and not in the parenchyma. Therefore, it will at once be seen that syphilitic osteitis cannot be separated from syphilitic periostitis. It may be said, with a great degree of certainty, that a syphilitic periostitis cannot occur without an osteitis, and vice versâ.

Therefore, instead of making two divisions, it appears to me to be wiser to talk about syphilitic osteo-periostitis, as the signs and symptoms produced by the two are identical.

\section{Syphilitic Osteo-periostitis.}

Any bone in the body may be affected. Of the long bones, the tibia, humerus, ribs, and clavicle, are the most commonly involved, and of the flat bones the nasal, frontal, and parietal.

There is a great difference in the course run between syphilitic osteoperiostitis of the flat and of the long bones.

In the case of the long bones, the process is generally dry throughouti.e., there is no liquefaction of tissue, and no pus formation. Therefore the process is one which can scarcely be called gummatous. The skin over the 
bones often is not even inflamed. Such a process invariably ends in new bone formation, and this corresponds in size with the area affected. The new bone formed is very hard, may be eburnated, usually smooth on the surface, always sharply circumscribed, and is, as a rule, surrounded by a groove. Should the case be one of true gummatous osteo-periostitis, there will be destruction of bone, of the nature of a rarefying osteitis, in the region affected; but around and in between the gummatous areas, osteophytic growths are always to be found.

In gummatous osteo-periostitis, the overlying tissues are inflamed, œdematous, and there is often marked ulceration of the skin. Many of the ulcers are fistulæ, which reach down to the bone. Osteo-periostitis of the flat bones is, almost invariably, of a gummatous nature. The patient frequently seeks advice for swellings of the forehead and scalp. These swellings may or may not be inflamed; they usually fluctuate, and the diagnosis of lipomata, sebaceous cysts, or cold abscesses, is usually made.

On incising them extremely little pus comes away, and as such a procedure may allow coccogenic bacteria to gain entrance, an acute osteo-myelitis of the diploe may result; therefore, on no condition whatever should an exploratory incision be made. Only quite recently there was a death at the Lock Hospital from a pyogenic infection, which had supervened upon a gummatous osteo-myelitis of the diploe. The Dura mater was covered with pus.

There is always a destruction of bone in these cases, and it may remain limited to the outer plate, or it may reach right through to the Dura mater, which practically always remains intact.

In by far the greater percentage of cases, the osteo-periostitis affects the external plate and the pericranium, but neither the internal plate nor the periosteum.

A hypertrophy of bone, and the formation of osteophytic growths, is practically never seen in the skull-bone lesions, except in the region of the orbital ridges of the frontal bones. The fact that osteo-periostitis of the long bones run a different course from that of the flat bones depends upon bloodsupply.

The arterial blood-supply to the scalp is extraordinarily rich, hence gumma formation, and the spread of the process is therefore favourable; and, finally, arterial blood can cause bone atrophy. Inflammation of the extremities, where the arterial blood-supply is not so good, favours venous congestion, and venous congestion is a potent factor in the causation of bone hypertrophy. Syphilitic osteo-periostitis, such as that just described, may occur as early as a few months after infection, or may not occur for several years. Early gummatous osteo-periostitis affects the flat bones more frequently than the long bones. In the case of the nasal bones, it is highly probable that the initial trouble arises in the submucous tissue and involves the periosteum and bone later, since it practically never happens that the skin surface of the bones or the skin itself is attacked. 
During the stage of the generalisation of the virus, there may be an inflammation of the periosteum of the long bones, and this often causes severe pain; but, as a rule, nothing is to be seen or felt. Even in the later stages of syphilis, when no swelling can be seen, a commencing osteo-periostitis may be accompanied with such violent pain as to lead one, if in a hurry, to make a diagnosis of neuritis. Local tenderness on pressure and the distribution of the pain soon suggest the seat of the trouble. However severe bone pain may be, it very quickly responds to treatment, while the pain of neuritis is often temporarily aggravated.

Another pitfall for the unwary is the diagnosis of a syphilitic osteo-periostitis as a sarcoma. Quite recently I have had three such cases. On two, an exploratory incision was made, and the third was advised to have an operation, but refused. The mistake has even led to the removal of limbs.

Syphilis does not only affect the bone and periosteum, it may also affect the medulla. As a rule, from a clinical examination alone, a syphilitic osteomyelitis cannot be diagnosed from a syphilitic osteo-periostitis. In a large number of cases, when one is in a position to examine the bone, it is found in a condition of panosteitis-i.e., the periosteum, the bone itself, and the medulla are all affected.

Some of those cases with chronic odema, ulceration, and fistula formation of one leg, are really cases of syphilitic osteo-myelitis, or, better to say, panosteitis.

Only an X-ray photograph can indicate, during life, whether the medulla is involved or not. If the medulla is affected, the inflammation is of the gummatous type, with the result that the medullary surface of the true bone shows marked signs of rarefying osteitis. The abscess formation so common in tubercle is not met with in syphilis. One of the best ways of distinguishing a syphilitic osteo-myelitis from a tubercular one is to remember that lipoid or fatty degeneration is a typical feature of a syphilitic process, when there is destruction of tissue. Consequently, a fresh or a well-prepared specimen of osteo-myelitis looks yellow and full of fat, while a tubercular osteo-myelitis has a white or more waxy appearance.

Cases of spontaneous fracture have becn recorded following syphilitic osteo-myelitis. This must not be confounded with fracture of a normal bone, as may occur when there is inco-ordination; for instance, I have seen two cases of fracture of the patella in degenerative myelitis.

Syphilitic bone lesions show rather clearly the influence which irritation or continued trauma has upon their origin.

The clavicles are most prone to develop osteo-periostitis at the place where the braces touch the skin. Shoemakers are liable to osteo-periostitis of the sternum; and it is highly probable that the reason why the tibix are so commonly affected is because of their prominent position, and because of the fact that the anterior surface is not guarded by muscle.

Syphilis may attack the vertebræ, and when it does so it affects the base 
of the skull and the cervical vertebræ more frequently than other parts of the vertebral axis. As a rule, syphilitic osteitis of the vertebræ is not recognised until some neural complications supervene, and then it is liable to be overlooked, as the symptoms pointing to nerve involvement may be severe. The ninth, tenth, and eleventh cranial nerves are particularly prone to be pressed upon by the exudative processes as they pass through the jugular foramen, with the result that the patient may lose his sensation about the pharynx, larynx, and oesophagus. Paroxysms of coughing, inability to swallow, and regurgitation of food may be the symptoms encountered in a severe case.

The following case is representative of a mild form of syphilis of the cervical vertebræ:

CASE 24.-Man, aged 52, contracted syphilis nineteen years ago. For several weeks patient had complained of pain in the neck, which was aggravated by movement. The painful area covered the whole neck, and over this area there was diminished tactile sensation. The neck was kept more or less rigid, owing to the pain movement caused. X-ray examination showed erosion of the bodies of the upper cervical vertebræ, with pronounced osteophytic growths, which are rather characteristic of syphilis of the spine. The patient improved under treatment.

\section{Syphilitic Arthritis.}

From simple inflammation of a joint, up to its complete destruction or ankylosis, the various clinical types met with may be caused by syphilis, and are, per se, indistinguishable from the same conditions produced by other causes.

The inflammation may commence in the synovial membrane, in the capsule, or in the articular surfaces of the bones. Slight inflammation of the synovial membrane and capsule may produce no other sign or symptom than pain. When the inflammation commences in the synovial membrane, as a rule fluid is excreted into the joint, and there is one clinical condition which is sometimes caused by syphilis in which, without warning or pain, the joint suddenly and very quickly becomes distended with fluid. The condition is called Hydrops articuli, and the knee-joint is almost invariably the joint affected. In some of these cases the fluid disappears as quickly as it came, and then is liable to recur without any provocation. In those cases in which the inflammation is very acute, and in which one or more joints are affected, the patient usually looks extremely ill, emaciated, and anæmic. There is always marked wasting of the muscles above and below the affected joint. To my mind, it is very odd that patients with a syphilitic arthritisand still more so is it the case with a gonococcal arthritis-should generally look so desperately ill and lose so much weight. It is absolutely impossible to differentiate between a syphilitic and a gonococcal arthritis. If the patient has a discharge, and signs of an old prostatitis can be detected by a rectal examination, the diagnosis is often obvious; but, not infrequently, a patient 
may have a severe gonococcal polyarthritis after all signs of gonorrhœa have disappeared from his urinary tract.

A mono-articular gonoccocal arthritis ends in an Arthritis deformans. A syphilitic arthritis does not do so.

There is still much division in opinion as to whether syphilis can be a cause of Arthritis deformans. Personally, I think it is very doubtful, and in every case of Arthritis deformans which I have seen in syphilitics the patient had had gonorrhœa and gonococcal arthritis, which, in my opinion, was the cause of the Arthritis deformans. I recently had two cases of Arthritis deformans affecting both hip-joints.

Both these cases had had gonorrhœa and syphilis, and in both the C.F.T. was positive. In the one case the process was early in both joints, therefore some improvement could be expected from treatment. Arseno-benzene, mercury, and iodides had no influence whatever. The patient began to improve only when gonococcal vaccines had been administered.

In the other case, one hip showed all the classical signs of advanced Arthritis deformans, while the process in the other had only just commenced. Here, again, benefit resulted from gonococcal vaccines, and not from antisyphilitic treatment.

That familiar condition so commonly seen in tubercular patients, to which the name of Tumor albus has been given, may also be met with as a late manifestation of syphilis, and, from an examination of the joint alone, the two diseases cannot be differentiated. This fact has led some clinicians (who cannot make a mistake) to diagnose many cases of Tumor albus as a mixture of tubercle and syphilis.

In all these very difficult cases the best means of arriving at a correct diagnosis is to give an injection of arseno-benzene. The treatment test is far superior to any other, as I have frequently seen patients with symptoms which might not be syphilitic give a positive C.F.T., while the lesions were really due to causes other than syphilis. It should always be remembered that a patient who has had syphilis is just as prone to contract other diseases as a patient who has not been so infected.

Syphilitic lesions of bursæ and tendons are more or less curiosities, and, as they have no distinguishing features, it does not appear necessary to discuss them further.

Before closing this chapter I should like to make a few remarks re syphilitic rheumatism and fibrositis. General muscular pains may be complained of in the very early stages, but true syphilitic fibrositis is, in my experience, always a late manifestation. Cases of metallic fibrositis are here excluded.

CASE 25. - The patient, a man, aged 58, contracted syphilis thirty-one years ago. For the past ten years he had not been really well, but for the last three years he had suffered, in various parts of the body, from rheumatism, which had been gradually getting worse. When I saw the patient he could hardly walk, and he had what appeared to be typical lumbago. He was also very deeply depressed, 


\section{CLINICAL ASPECT AND TREATMENT OF VENEREAL DISEASES}

and had no appetite. I happened to examine his serum with the ultra-microscope, and I have seldom seen one in which the colloidal particles were so big. Under colloidal iodine, intramine, and colloidal mercury injected intravenously, the patient became rejuvenated.

It is probable that the symptoms in this case were not due to the direct presence of the syphilitic organism in the parts affected, but to the diminished oxidation of the tissues, which would result from the colloidal particles in the serum being very big. The injection of colloids breaks up these particles into several small emulsoid particles, whereby their oxidising action is increased. This is the probable explanation of the sense of rude health which syphilitics, and especially old cases, experience after treatment with the various colloids.

1. Proksch (1910): Handbuch der Geschlechtskrankheiten, i., 1. A Hölder, Wien u. Leipzig.

2. Kolmer and Mastin (1918): Journ. Amer. Med. Assoc., 1xx., 1290. 


\section{CHAPTER VI \\ SYPHILIS OF THE MOUTH AND THROAT}

\section{History.}

THe buccal lesions best known to the older syphilologists were ulcers, because, as long ago as 1554, various types of obturators for palate defects were described. Musitano in 1689 was the first to report cases in which the primary sore was situated on the tonsils, tongue, and gums. A lesion not uncommonly met with at the present day, although seldom if ever described, is aphonia, occurring some years after the infection without pain or obvious macroscopic disturbance of the throat. This aphonia was recognised and called angina by Barbantini in 1821, who was struck by the absence of pain and nakedeye trouble of the throat. Wallace in $\mathbf{1 8 3 5}$ drew attention to the close resemblance between the papules in the mouth, which he called condylomata, with those occurring in other moist regions of the body. Syphilis of the nose was always well known, owing to the deformities ulceration was apt to produce. Possibly the scorn and derision which was hurled at the unfortunate victims was the start of the horror with which this disease was regarded by people later. There is every reason to suppose that Shakespeare had syphilis in his mind when he wrote, "Down with the nose, down with it flat, take the bridge quite away," spoken by Timon to the mistresses of Alcibiades ("Timon of Athens," Act IV., Scene 3). In Ambroise Paré's time artificial noses of gold, silver, and papier machê were made, and as some of the protheses even had beards, the ulceration in those days must have been particularly extensive. Scarring leading to stenosis of the larynx first received attention at the hands of Morgagni, and cases were frequently described afterwards.

\section{Syphilis of the Mouth.}

A chancre may occur anywhere, but there is one type of chancre to which special reference should be made, because of the way in which it simulates a large-spored ringworm lesion. It is called the hypertrophic chancre, and it may be situated at the corners of the mouth or on the lips, where the skin and mucous membrane join. The differential diagnosis is simple, if it always be remembered that chancres in the oral region are always accompanied by very marked enlargement of the lymphatic glands. Chancres are most common on the lips, then on the tonsils, then on the tongue, and lastly on the gums 
and other buccal mucous membrane. Prominence is frequently given to differential diagnosis between a labial chancre and a labial epithelioma, stress being laid on the lip involved. As a matter of fact, chancres may occur on either lip, and practically never present any difficulty in diagnosis. A point to bear in mind with cephalic chancres is that treatment requires to be particularly energetic and carefully prescribed, because they are more frequently followed by nervous manifestations than genital chancres. As nervous syphilis may be aggravated by oral sepsis, and as oral sepsis is prone to occur when the chancre is situated on the lips or in the mouth, iodine and intramine must play a large part in the treatment, as metals are so liable to cause oral sepsis.

The so-called mucous papules are nothing more nor less than syphilitic papules. Although mucous papules may occur in all cases of syphilis, they are most commonly found in those cases the chancres of which are of the papulo-erosive type. Small indurated chancres, which frequently escape recognition by the possessor thereof owing to the very slight loss of their surface tissue, are very prone to lead to recurrent mucous papules in the mouth for years and years after the infection, and in spite of all treatment. I have seen recurrent buccal mucous patches appear as long as thirty-five years after infection, and as soon as a month after a prolonged course of arseno-benzene. In fact, I have seen them arise while the patient was under treatment with arseno-benzene.

Mucous papules may develop into ulcers, just as the precursor of a rupial lesion may be a dry papule. In both cases, the chancre has usually been of the gangrenous kind, or one which has been secondarily infected. The secondary coccal infection of the chancre gives rise to a sapræmia, or even to a septicæmia, with the result that any of the syphilitic lesions which appear later assume an ulcerative character. Ulcerative lesions of the tonsils again allow cocci to gain entrance to the blood-stream, and this frequently leads to what has been termed malignant syphilis. In most of the cases of malignant syphilis I have seen the buccal lesions have been the worst, and apparently those which caused the malignancy, which is really, of course, a septicæmia, not due to the syphilitic organism, but to staphylococci, streptococci, etc. This is the reason why antisyphilitic treatment often proves unavailing, and in some cases is actually injurious. In spite of our present-day advances, the old-fashioned Decoctum Zittmannii is still an excellent form of treatment in most of these cases. As malignant syphilis is due to a coccal infection, and as manganese is more or less a specific for such an infection, it is wisest in all such cases to use trimine in preference to arseno-benzene or mercury. Boils, etc., occurring while a patient is undergoing treatment with arsenic or mercury will immediately disappear with trimine, and it is not necessary to stop the antisyphilitic treatment at any time. It is extraordinary how rapid the destruction of tissue may be in some cases of malignant syphilis, the whole of the superior maxilla necrosing in a very short time. The proof that malignant syphilis is really a septic infection is forthcoming in the fact 


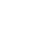




\section{PLATE XLII}

No. 78.-A Primary Sore on the Upper Lip

Note the typical infiltrative oedema of the surrounding mucous membrane. The submaxillary lymphatic adenitis was, as is usual, very pronounced in this case. Macrocheilia, as a recurrent syphilitic manifestation, appeared three years later.

No. 79.-A Healugg Primary Sore on the Thumb

ONE week after the thumb became sore a chancre began to develop on the Glans penis. The generalisation stage in this patient was particularly severe. Suppurative lymphatic adenitis more commonly follows extragenital than genital chancres. 
No. 78
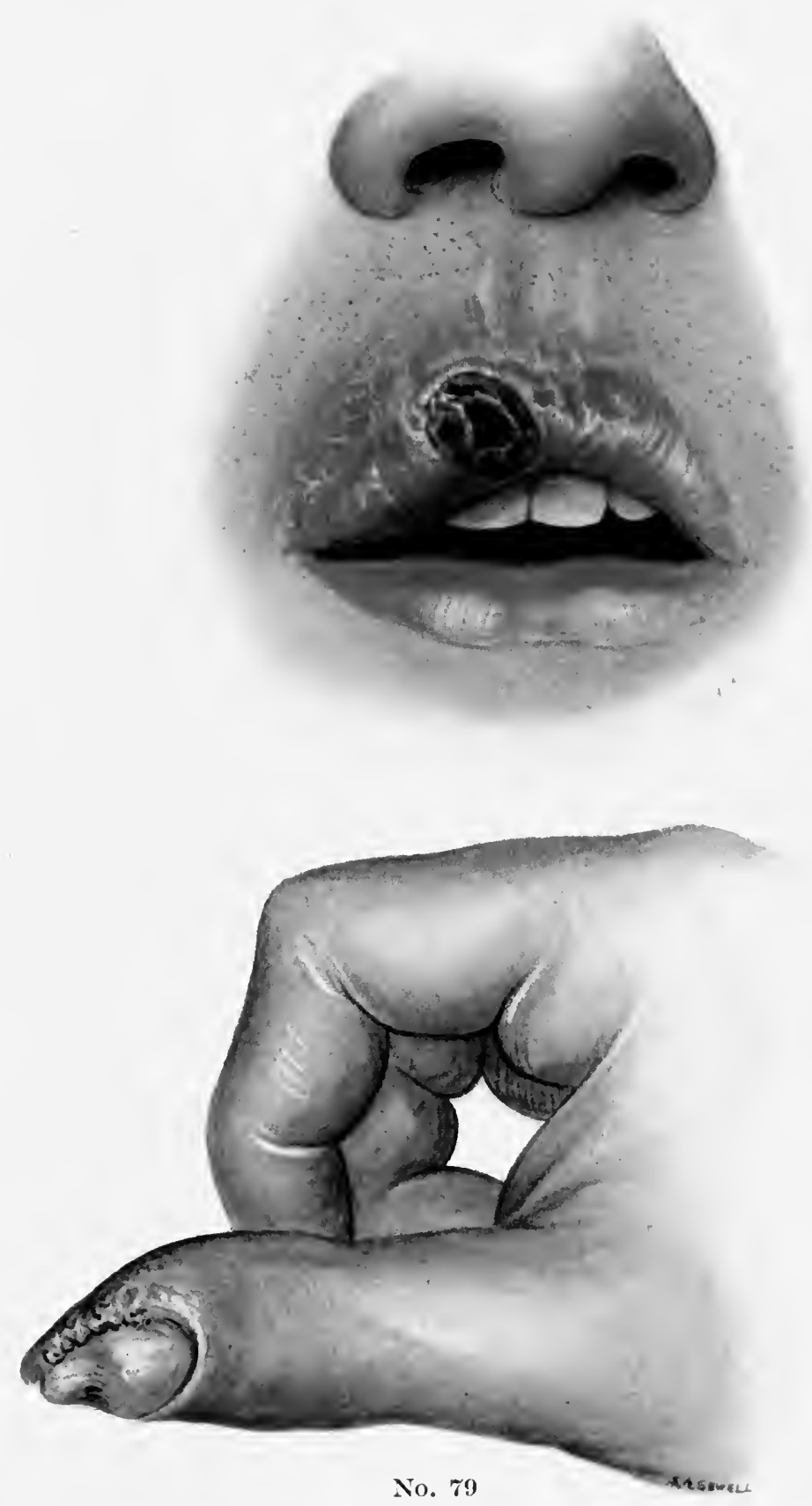

Pi.ate XIII 

that some cases commence with a tonsillitis, which is followed later by rheumatic purpura and other so-called rheumatic exanthemata. If it is desired to prescribe arseno-benzene in cases of malignant syphilis, the best plan to adopt is to inject $0 \cdot 1 \mathrm{grm}$. intravenously, daily for twelve days.

Oral sepsis is very largely due to injudicious use of mercury and arsenic, as all metals, even including lead, bismuth, and manganese, are liable to cause gingivitis, which forms a ready breeding-ground for organisms, causing Pyorrhoea alveolaris and Vincent's angina. When arseno-benzene and mercury are used simultaneously, the danger of oral sepsis is increased. If intramine were employed in every case of syphilis, gingivitis would never be seen, and the effect of this drug on metallic oral sepsis is really marvellous, as the following cases show:

CASE 26.-The patient had a primary sore on the skin of the penis, general adenitis, and was more or less covered with a follicular syphilitic eruption. I injected intravenously $10.0 \mathrm{c.cm}$. of a 5.0 per cent. emulsion of colloidal mercury. In the quantity injected there was 0.5 gramme of colloidal mercury, the action of which would be very much greater than 0.5 gramme of ordinary mercury. On the following day the patient had severe abdominal pain and diarrhœa. The diarrhœa increased, and blood was passed per rectum. This continued for a week, during which time not a drop of urine was passed. On the second day severe stomatitis set in, which became gangrenous a few days later. The patient also had hæmorrhagic conjunctivitis, Herpes oris, looked and was desperately ill, and I had fears that he might die. This was a typical case of acute mercurial poisoning, and the mercury was acting as a poison in virtue of its oxidising power. This being the case, it struck me that the administration of an equally powerful reducing agent might cure the condition. I injected $100.0 \mathrm{c.cm}$. of colloidal iodine intravenously, and $5.0 \mathrm{c.cm}$. of intramine intramuscularly. Within forty-eight hours the diarrhoea ceased, and the patient passed nearly his full amount of urine. The condition improved so much from day to day that, with no other treatment whatever, he was perfectly well eighteen days later, when there was no albumin in the urine and every syphilitic symptom had vanished.

CASE 27.-A patient was being treated with arseno-benzene and mercury simultaneously. A few days after the third intramuscular injection of grey oil, stomatitis set in. When I saw him a week later he had commencing gangrenous stomatitis, had scarcely been able to open his mouth for a week, and consequently had had no food. The patient was in severe pain, and in the ordinary course of events would have died, as the urine was full of protein. In the morning I injected $5.0 \mathrm{c.cm}$. of intramine intramuscularly, and the same evening the patient was able to sufficiently open his mouth to take a meal. Four days later the intramine was repeated, and a fortnight from the time he was admitted he was discharged perfectly well, and with no trace of protein in the urine.

Oral sepsis has loomed large in syphilitic soldiers, partly because they have to go through a routine treatment and are not treated individually, and partly because their diet contains too much cooked protein (bullybeef) and too few fresh vegetables. My own opinion is that oral sepsis is largely responsible for the frequent and early relapses one sees in the army, 
19 per cent. relapsing within three months of the cessation of a full military course. Apart from this, oral sepsis must have a very bad influence on the future course run by the disease, because a concomitant infection so often produces a recurrence in an otherwise latent case. The aggravating effect which stomatitis as well as cystitis have on lightning pains in degenerative myelitis probably explains why the pains are sometimes relieved by dilatation of the urethra, there being often latent gonorrhœa in such cases.

A patient who has had syphilis, especially if he has been treated with mercury internally, is very liable to develop attacks of Herpes oris and aphthous ulcers. Fear of a recurrence of symptoms usually accompanies each outbreak, and as these ulcers are so frequently confounded with mucous papules, it would be well to draw attention to their differentiation. Mucous papules, like all syphilitic lesions, are non-inflammatory-that is to say, a mucous papule is sharply circumscribed, and has no inflammatory ring surrounding it. A mucous papule is white and raised above the surface. Herpes starts as a crop of vesicles, which quickly become small ulcers. Often the vesicular stage is not observed. Each lesion has a yellow base, which is depressed beneath the surface, and each lesion is surrounded by a marked inflammatory ring. An aphthous ulcer has exactly the same appearance as the ulcerative stage of the herpetic lesion, and it is highly probable that the two terms are only different names for the same condition.

The ulcers are painful; the mucous papules are painless. The former may appear in twenty-four hours; the latter do not appear under several days. Therefore, there should never be any difficulty in differentiating between the two conditions.

A very common site for an aphthous ulcer is the space posterior to the last tooth on either side of the lower jaw. When this heals, the surface has a white irregular appearance, which suggests leucoplakia. Similar white patches are frequently seen in the cheeks, and indeed on any part of the mucous membrane in the mouth, in patients who have taken mercury internally.

Aphthous ulceration in the neighbourhood of the last molar tooth is not at all uncommon in patients who have been well treated with the arsenical preparations. Metals are very prone to cause gingivitis, Herpes oris, etc., and mercury is not the only offender, as I always thought. If a patient has mercurial gingivitis, an injection of arseno-benzene makes it infinitely worse, while an injection of intramine causes its immediate disappearance; indeed, the moment this sulphur compound has been injected intravenously, the patient notices his gums to be less tender. Therefore, gingivitis must be in large part due to the blood being too acid, because metals increase the hydrogenion concentration of the serum. In connection with this point, it is interesting to note that Herpes oris may be frequently caused by increased gastric acidity.

Leucoplakia is regarded by many as being pathognomonic of syphilis. Leucoplakia is merely an attempt of the mucous membrane to form a Stratum corneum, as it will aways try to do, whatever be the nature of the irritant. 
Leucoplakia of the tongue is common in syphilis, and is usually met with in those cases which have received no treatment, and in those which have been treated with mercury internally.

Smoking will cause leucoplakia in a non-syphilitic subject, so will psoriasis, Lichen planus, etc.

Leucoplakia may be followed later by carcinoma; but it is not the leucoplakia which predisposes the organ to undergo the cancerous change; it is merely the persistence of the irritant which primarily led to the production of the leucoplakia. Leucoplakia is a manifestation of the protective mechanism of the cells against the agent which is irritating them.

If the epithelial cells persist indefinitely in undergoing changes of a protective nature, the cells will ultimately become parasitic upon the host which gave rise to them-i.e., they will become cancerous; but that does not mean to say that the leucoplakia is the cause of the cancer, an opinion which is very widely held.

A leucoplakic tongue may remain as innocent as a normal tongue, or may quickly become cancerous. Which of these results will supervene will depend, not upon the degree of the leucoplakia, but upon the nature of the irritant which gave rise to the leucoplakia.

The changes which the tongue undergoes in syphilis are both interesting and important, owing to the frequency with which they end in cancer.

The changes about to be described, broadly speaking, are to be seen in those cases of syphilis which are not treated at all, and in those which are treated with mercury internally, or too energetically with metals generally.

Absence of treatment does not prevent the tongue from becoming infected directly with the organisms of syphilis. Oral administration of mercury frequently causes indigestion, and indigestion is liable to cause inflammation in any part of the mouth, especially in the tongue. Other factors also come into play-namely, bad teeth, smoking, tobacco chewing, alcohol, etc. All these are liable to increase, or to keep up, any inflammatory changes which have been initiated by syphilis.

The first change to be noted is a swelling of the base of the tongue, and this frequently leads to the patient seeing the circumvallate papillæ for the first time in his life. After recovering from the shock, he runs up to his doctor for advice as to what to do for the sores or spots at the back of his tongue. This swelling soon extends to the anterior part of the tongue, when the tongue appears to be too big for the mouth, and its edges clearly show the irregularity caused by the pressure of the teeth.

Swelling indicates parenchymatous inflammation. If the inflammation persists, fibrous tissue formation is the result. Fibrous tissue contracts, and hence the tongue becomes smaller than it normally was.

Fibrous tissue contraction indicates loss of blood-supply to the surface, hence the papillæ vanish, the dorsum of the tongue takes on a smooth, glazed, 
shiny appearance, and then the epithelium begins to protect itself by forming horny tissue.

The inflammation may not be evenly marked all over the tongue. In some places it may be worse than in others, hence the tongue may be atrophic in some parts and hypertrophic in others. This irregularity leads to fissure formation, and as fissures occur where the fibrous tissue is densest and the blood-supply is poorest, it can be easily understood how readily ulceration, and even cancer, may arise. Warts are very liable to occur in the atrophic areas, and the risk of these becoming malignant is great. Cauterisation will stimulate their malignant tendencies; therefore, on no condition whatever should caustics ever be applied to a tongue, or, indeed, to any other mucous membrane. The warts should be locally excised. There is no necessity to remove half the tongue, as, in these atrophic tongues which become malignant, the process is so slow and localised, and it practically never gives rise to a metastasis.

When an ulcer becomes malignant it is a different question. Removal of half or the whole of the tongue and the lymphatic glands is usually the most expedient course to adopt.

A curious point about chronic syphilitic glossitis is that arseno-benzene may make the condition worse, while, on the other hand, there is no syphilitic lesion in which the beneficial effects of intramine are so pronounced.

The following cases are particularly illustrative of what has just been said :

CASE 28.-A man, 70 years of age, had had syphilitic glossitis for twenty-five years. Eighteen months before he was given intramine he had two intravenous injections of arseno-benzene, and these were followed by three courses of mercury and iodides, with practically no improvement in the clinical condition. When an intramuscular injection of intramine was prescribed $(2 \mathrm{grm}$.$) , the tongue exhibited$ the following features: There was an ulcer in the centre about the size of a sixpence, with a lateral fissure running from it on both sides. Just posterior to the ulcer a lard wart was situated. The movements of the tongue were impeded, and very painful. In a few weeks after the injection of intramine the ulcer had completely healed, the fissures and wart had vanished, the natural movements of the tongue and its suppleness had returned, and, in the words of the patient, "My tongue has never been so well as long as I can remember."

Over three years have elapsed since the intramine was injected, and, in spite of the fact that no other treatment has been prescribed, the patient has never once gone back.

CASE 29.-The patient contracted syphilis seven years ago, for which he was treated irregularly. One year ago the tongue became sore, swollen, and ulcerative, and recently the movements have been restricted.

On the third day after one intramuscular injection of intramine, the tongue began to improve. Eleven days later the swelling had vanished, the ulcers had healed, all pain had disappeared, and the movements were perfectly free.

CASE 30.-The patient contracted syphilis fifteen years ago, for which he was treated with mercury internally on and off for three years. Two years ago the 
PLATE XLIV

No. 81.-The so-Called Snall-track Appearance of Mucous Patches on the Paidate

No. 82.-Gumantous Clecration following a Parenchymatous

Glossiris affecting the Anterior Two-tminds of tme Tonguf:

This type of case frequently fails to respond to arseno-benzene and mercury; indeed, metals may aggravate the condition. Intramine is particularly indicated. 
No. 81
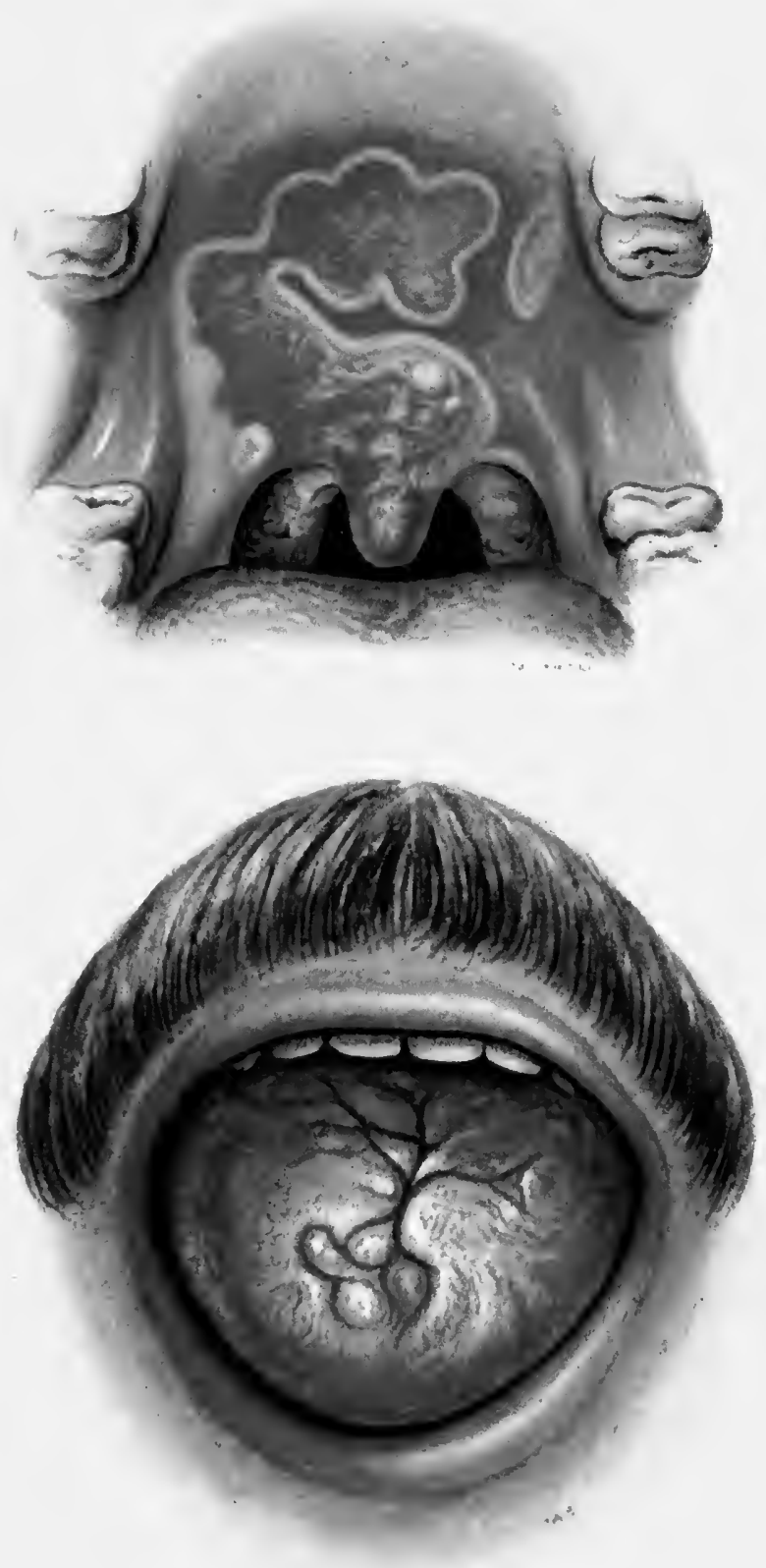

No. 82

Plate XIIV 

tongue became sore, and when I saw the patient the tongue was swollen, deeply fissured, and covered with leucoplakia. The movements were both restricted and painful. When the tongue condition was diagnosed as syphilis, the patient was treated with salvarsan and intramuscular injections of mercury, but with no improvement. The same day on which I administered the intramine the patient noticed a difference; in three days he could not tell from the feel that he had a sore tongue; and in ten days, not only had the swelling and fissures vanished, but the leucoplakia had disappeared.

CASE 31.-Syphilis thirty years ago; two primary sores; treated for one year with mercury internally. Ten years ago the patient's feet swelled at night, and he was found to have protein in the urine. Three years ago gummatous ulcers on the tongue appeared. In consideration of the albuminuria, the patient was treated with mercury and iodides, and advised against having arseno-benzene, with the result that he became gradually worse. When I saw him he had a gummatous ulcer on the tongue, and one on the inside of the left cheek; there was a considerable amount of protein in the urine, a portion of which was globulin. Another interesting feature about the case was a periodical rise of temperature, which would last for a few days. Considering the healthy appearance of the patient, I felt sure that the proteinuria was due to syphilis. I gave the patient $2.5 \mathrm{c} . \mathrm{cm}$. of intramine, and two days later the mouth had so much improved that the patient could smoke a cigar, which he had not done for years. A week later the intramine was repeated, by which time the ulcers in the mouth had completely healed. The amount of protein in the urine had also very considerably diminished. Two injections of galyl were then given, but before the second had been injected, all the swelling of the legs had completely disappeared, so had the puffiness in the eyes, and the patient declared he had never felt so well for years.

\section{Tonsil.}

A primary sore may have its seat on the tonsil, when it invariably gives rise to a difficulty in diagnosis.

A primary sore has to be distinguished from a well-marked case of follicular tonsillitis, a gumma of the tonsil, and from Vincent's angina. Over and over again these four conditions have been confused with one another, although the differential diagnosis between them should never present any difficulties.

A chancre is either a superficial lesion, circumscribed, and not surrounded by an inflammatory area, or, if secondarily infected, is a deep ulcer surrounded by a minimum of inflammation. Whether the chancre of the tonsil is secondarily infected or not, the enlargement of the lymphatic glands is always very much more marked than is the case with follicular tonsillitis. Follicular tonsillitis is very painful, a chancre of the tonsil is not. Follicular tonsillitis is ushered in with fever, rigor, etc., and throughout its course the patient feels very ill, and almost invariably small areas of folliculitis will be observed on the opposite tonsil. A chancre of the tonsil begins insidiously; the patient does not feel ill, and the act of swallowing usually causes nothing more than a mere feeling of slight discomfort, and the lesion is single.

A gumma causes more destruction of tissue, for its size, than a chancre, and it is unaccompanied by an enlargement of the lymphatic glands. 


\section{CLINICAL ASPECT AND TREATMENT OF VENEREAL DISEASES}

Vincent's angina is usually mistaken for a chancre, or more often for a gumma.

The course of Vincent's angina should suffice to distinguish it from any other condition. The patient suddenly feels very ill, the temperature shoots up, and the patient may have a rigor; in two or three days there is a deep ulcer in the tonsil, extreme pain on swallowing, and only a very slight enlargement of one or two lymphatic glands. The rapidity with which the ulcer develops is the pathognomonic sign of Vincent's angina, and a point which absolutely excludes syphilis is an affection of the opposite tonsil.

A film made from a case of Vincent's angina will also quickly settle the diagnosis, since the condition is caused by two organisms which live in symbiosis-namely, an irregularly coiled Gram-negative spirochæta and a Gram-positive fusiform bacillus.

Owing to the great increase of Vincent's angina in the last few years, and to the fact that a generalised rash may accompany it which closely simulates syphilis, the condition deserves, from our point of view, more attention. Metals predispose to the trouble, and in practically every case the commencement is a gingivitis around the incisors of both jaws. One of the first symptoms is a swelling of the mucous membrane posterior to the central incisors of the upper jaw. The membrane which is sometimes found on an angina lesion is easily removable, thereby affording a difference to the diphtheritic membrane. In fact, it is often necessary to remove the membrane before applying a local remedy if a quick cure is to be obtained. As Vincent's organism quickly dies in the presence of oxygen, no local remedy is better than hydrogen peroxide. The rash above referred to is a generalised one, almost indistinguishable from an early maculo-papular syphilide.

The lesions are not so infiltrated, and should they become squamous they do so much earlier than is the case in syphilis. The cases I have seen which presented a rash felt perfectly well and had no general adenitis. The most interesting case I saw was one in which the throat lesion was of the membranous type, and which was followed, sixteen days later, by a rash simulating chicken-pox. The C.F.T. is sometimes positive, and the whole condition rapidly disappears under arseno-benzene. If Vincent's angina has been produced or aggravated by mercury and arsenic, then it is necessary to prescribe colloidal iodine and intramine.

\section{Palate and Nose.}

Syphilitic periostitis and its sequelæ are so absolutely pathognomonic of syphilis that only a passing word is necessary.

Syphilitic periostitis of the hard palate often leads to ulceration and perforation of the bone, and it is not at all an uncommon symptom in both acquired and congenital syphilis.

In acquired syphilis, so far as the nose is concerned, a perichondritis is 


\section{PLATE XLV}

No. 83. -SYPhilitic Glossitis before INTRAMINE

Syphilis was contracted forty years ago, and the tongue became bad ten years ago. The points to note are the ulcer and that the tongue cannot be protruded.

No. 84.-Syphilitic Glossitis after INTramine

The tongue from the above patient taken one week after he had had $2.5 \mathrm{c.cm}$. intramine injected into each buttock, no other treatment having been prescribed. Note that the ulcer has healed and that the tongue can be protruded. 
No. 83
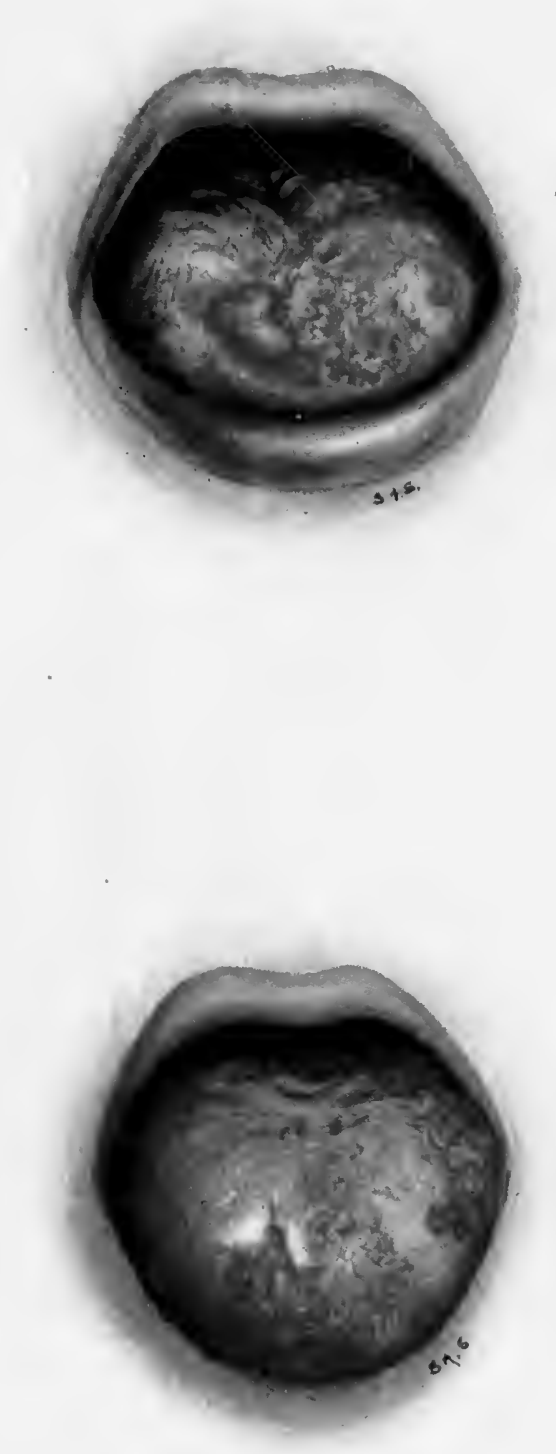

No. 84

Plate: XI/V 
1 
more common than a periostitis, and this almost invariably results in a perforation of the nasal septum.

The following is an interesting case, as it shows what can be achieved in severe cases if oxidising agents are combined with reducing agents:

CASE 32.-The patient came to me in the condition about to be described, having already had three injections of galyl. On examination, the patient was found to have several ulcers scattered about the body, with one large one practically covering the whole deltoid region of one arm. The nasal septum was perforated, and there was an acute periostitis of the left nasal bone, which was on the point of bursting through the skin. The patient was first given an intravenous injection of colloidal iodine, with the result that all the ulcers discharged to such an extent that the dressings had to be changed several times a day. Two days later, and again two days after that, intravenous injections of ferrivine were prescribed. Five days later, by which time a great improvement had been wrought, galyl was injected. Three days later, and again a week later, intramine was injected, and followed at weekly intervals by two more injections of galyl. After the sccond injection of intramine, every lesion had vanished, and the nasal gumma did not perforate the skin. Syphilitic ozæna is not uncommonly met with in the later stages of the disease, and it is a condition which responds well to intramine, while often remaining refractory to arseno-benzene and mercury.

\section{Syphilis of the Pharynx and Larynx.}

There is nothing peculiar about the mucous papules affecting the pharynx or larynx, except that the change of voice brought about by the lesions may persist unless the lesions are got rid of rapidly. Ulceration of these parts is only characteristic in so far as the scar tissue formed is particularly pronounced and apt to lead to stenosis. I have seen the soft palate become adherent to the posterior wall of the pharynx, and completely shut off the throat from the posterior nares, and the larynx become so stenosed as to necessitate wearing for always a tracheotomy tube, because no remedial agents will dissolve the scar tissue. There is a peculiar and infrequent condition which gives rise to hoarseness or to almost complete aphonia, occurring between the sixth and tenth year after infection, which sets in either suddenly or gradually. The patient has no pain, and on examination nothing abnormal may be found, or the mucous membrane may appear slightly granular and paler than normal. It is possible that the primary lesion is a local arterio-sclerosis, because, unless treatment be given early, the loss of voice may become permanent. Neither arseno-benzene nor mercury benefit the condition-in fact, they may aggravate it-while one or two intramuscular injections of intramine suffice to cause its complete disappearance. I have known œdema of the glottis to occur in syphilis, as well as a suppurative œdema in the deep tissues of the neck, but as they occurred in patients who were taking iodide of potassium, it is possible that the drug was responsible. It is well to remember that mucous papules in early syphilis may cause middle-ear deafness by blocking the Eustachian tubes. 


\section{CHAPTER VII \\ SYPHILIS OF THE BRONCHI, LUNGS, AND THYROID}

\section{History.}

ALtнобgr gummata of the lungs were familiar to the syphilologists at the beginning of the sixteenth century, Morgagni was the first to draw attention to pulmonary syphilis by the publication of four cases. As a commentary to one of his cases Morgagni added: Certe ad luem illam non raro phthisim tandem se adjungere, tum medicinam, tum anatomen exercentes non ignorant. In this connection it is interesting to note that Astruc prior to Morgagni had made the following remark: Tuberculis vel gummatis in pulmonum substantia latentibus sive cruda sint, sive suppurata. Horne, in 1775 , speaks of "syphilitic phthisis," but even now, as then, the difficulty existed of differentiating between tuberculosis and pulmonary syphilis. The cases which cleared up under treatment were regarded as being syphilitic in origin. Owing to Laënnec's statement that every case of pulmonary phthisis was due to tuberculosis, the mention of syphilis as a cause appeared no more in the textbooks till comparatively recently. Morgagni also was familiar with syphilitic pleuritis. Virchow and others did much to clear up the anatomico-pathological aspect of syphilitic pulmonitis and pleurisy. Syphilis of the thyroid was not recognised till our time, because Lancereaux, only such a short time ago as 1868 , wrote that syphilis of the thyroid was unknown.

\section{Bronchi.}

A syphilitic bronchial catarrh is not very uncommon, but it is more frequently seen as a recurrent syphilitic manifestation than as an early symptom of the disease. The chronic ulcerative syphilitic bronchitis may occur alone, but it is more often associated with a similar condition in the trachea, larynx, or pharynx.

This late syphilitic bronchitis, as a rule, affects only one or other of the chief bronchi, and the most usual situation for the ulcers is in the neighbourhood of the bifurcation.

The ulceration causes a thickening and raising up of the mucous membrane, and these two changes together give rise to violent fits of coughing, and, at the end of a fit, there may be a hæmorrhage.

No signs are to be found in the lungs. An X-ray examination of the chest. gives no clue to the condition, and the diagnosis is rendered extraordinarily 
difficult. I have seen only one case, and the symptoms were coughing and hæmorrhage. The patient first of all complained of a peculiar feeling in the throat, which led to slight attacks of coughing. Later, these attacks became more frequent, and lasted longer, until ultimately every attack resulted in a severe hæmorrhage.

Repeatedly the patient would experience difficulties in breathing, and his complexion would assume the colour associated with venous congestion.

Antisyphilitic treatment stopped the symptoms at once; but in the course of two years the patient had three very severe relapses, which were checked immediately by further treatment. Ultimately the ulcers doubtless scarred over, as the patient has been free of any trouble for over two years. Considering the severity of the hæmorrhage in this case, the ulcers must have developed backwards, and eroded a small bronchial vessel.

Cases have been reported in which the scarring resulting from the healed ulcers has given rise to stenosis. Purulent mediastinitis and lobar pneumonia may also be the end of a case of gummatous bronchitis. Symptoms of an ulcerative bronchitis may be produced by a syphilitic process, starting in the bronchial lymphatic glands, which become adherent to the bronchi, and may even ulcerate through.

\section{Lungs.}

Syphilitic affection of the lungs is, in my opinion, commoner than is thought to be the case; but, unfortunately, its diagnosis is fraught with difficulties, owing to the lesions being sometimes indistinguishable from those produced by tuberculosis. There is still a great difference of opinion as to the influence of syphilis on the incidence of tuberculosis of the lungs, and vice versî.

I think there can be no doubt that individuals whose resistance against tuberculosis is lowered are more prone to develop this disease if they also suffer from syphilis. In support of this view may be mentioned the not infrequent occurrence of tuberculous adenopathy in congenital syphilitics, and the very high incidence of pulmonary tuberculosis in the black races in those individuals who have syphilis. If a patient has tuberculosis and syphilis, the latter disease aggravates the former, but the former appears to have no influence upon the course run by the latter.

Treatment of syphilis has its usual effect on the disease, whether tubercle is present or not, but it is seldom that the tuberculosis is much influenced by it-indeed, mercury, if pushed, may make the tuberculosis worse. From this it would appear that there is little ground for assuming that a lesion can be caused by an association of tubercle and syphilis.

In recent years one has not infrequently heard the diagnosis made that a certain orchitis or an arthritis is due to a combination of syphilis and tubercle. Such a diagnosis shows that the observer does not know whether it is tubercle or syphilis, and, to save himself from making a mistake, he says the lesion is due to a combination of the two diseases. 


\section{CLINICAL ASPECT AND TREATMENT OF VENEREAL DISEASES}

When the differential diagnosis of a lesion between tubercle or syphilis arises, it can be taken for certain that the lesion is due to either one, but never to a combination of both.

As the changes produced in the lung by tubercle and by syphilis are much alike, it may be impossible to differentiate between them.

Broadly speaking, the area affected by syphilis is greater than that affected by tubercle, and the symptoms produced are relatively greater in tubercle than in syphilis.

Syphilis affects the middle and lower lobes more often than the upper lobe; but a true syphilitic apical lesion may be met with. So far I have had three cases under my care. One of these used to get periodically very severe attacks of hæmoptysis; but tuberculosis could easily be excluded, because the patient always felt well, he never looked ill, he never lost weight, and antisyphilitic treatment benefited him. I have recently had a case which improved under treatment, and was kept in check for five years, but which finally terminated fatally with hæmoptysis. Syphilitic lesions are more bronchiectatic than tubercular ones, hence one of the best diagnostic means is the X-rays. Arseno-benzene has a rapid action upon syphilitic lung lesions; therefore, if there is any difference in the $\mathrm{X}$-ray picture before and after treatment, one can be practically certain that the condition was syphilitic.

As is well known, pulmonary tuberculosis is frequently preceded by pleurisy. This is not the case in syphilis-not that syphilis does not affect the pleura, but, in practically all cases of syphilitic pleuritis, the pleuritis is only part of a widespread pulmonary affection.

As in other organs, so in the lungs syphilitic gummata may be encountered. Pulmonary gummata are usually found in proximity to the bronchi, and there are almost invariably nodules in the pleura.

In other cases of pulmonary syphilis, fibrous tissue formation, sometimes sufficient to lead to extensive pulmonary fibrosis, and shrinkage, is a marked feature. In these cases, the fibrous tissue bands appear to enter the lung from the hilus along with the bronchi and bloodvessels. In these cases the lymphatic glands in the hilus are often enlarged, and there may be sufficient periglandular inflammation to cause a mediastinitis. It is probably owing to the frequency with which fibrous tissue bands occur that pulmonary syphilis is sometimes bronchiectatic. In early cases of syphilitic bronchiectasis treatment undoubtedly does good, but in late cases, owing to the severe secondary bacillary septic infection, antisyphilitic treatment may prove unavailing. I have had one case which died without being benefited in the least by treatment. I will cite the case in detail, because some of the symptoms produced were undoubtedly due to the lymphadenitis in the hilus.

CASE 33.-The patient was 59 years of age, never remembered having had syphilis, although an ulcer had destroyed his frænum, therefore he had received no treatment. He looked much older than he was; his complexion was sallow; he was very much troubled by a profuse muco-purulent expectoration, and he had 
an abductor paresis of the left vocal cord. The sputum was frequently examined for tubercle bacilli, but none were ever found, therefore the blood was tested serologically, and a positive C.F.T. obtained.

There were scattered areas of dulness to percussion, and marked crepitations about the scapular region on the left side, but there was no pyrexia. The mediastinal lymphadenitis, which was most probably the cause of the paresis of the recurrent laryngeal nerve, was diagnosed by the $\mathrm{X}$-rays. Under intravenous injections of colloidal iodine, colloidal mercury, and intramine, the patient made a temporary improvement; but the expectoration never ceased, and he ultimately died.

The following is a good case of pulmonary syphilis which I had under my care:

CASE 34.-Patient, a man now aged 47, contracted syphilis ten years ago, and ten months ago he began to be troubled with a cough. The cough was dry and hard; it was not worse at any one time of the day, and there was no expectoration. Friction sounds were discernible almost all over the lung area, and those who examined him said that there were changes in the lung which were undoubtedly due to tubercle. The patient went away, did what he was told, and became much worse.

Seven months later I saw him. He looked as if he had fever, but was not emaciated, and I learned that he had lost no weight; he had never had an hæmoptysis, and there was no expectoration. Friction sounds could he heard over both lungs, and on the left side were signs of both thickened pleura and patches of consolidation. There was no family history of tubercle; the patient had spent his life in British East Africa, where tubercle amongst the white population is almost unknown. He looked too well to have such widespread tuberculous lung symptoms, and, as there had never been any expectoration or loss of weight, I considered the trouble must be due to syphilis.

After the first injection of neo-salvarsan the cough vanished, and by the time the fourth had been given, practically all the physical signs had disappeared, except for some impaired resonance over left lung behind.

\section{Thyroid.}

Although syphilis of the thyroid is admittedly rare, nevertheless several cases have been described, and probably more would have been described had the condition been in any way characteristic. I have seen only two cases myself, and they were gummata of the isthmus, and both in women; but on searching the literature, I find that a general swelling of the thyroid in early syphilis has been described by many observers. 'Mauriac ${ }^{1}$ cites a case in which the gland became so large that it compressed the trachea and larynx. Engel-Reimers ${ }^{2}$ observed enlargement of the thyroid 86 times in 152 female patients, and 44 times in 98 male patients. He states that it appears very early in the disease, is uninfluenced by treatment, but disappears gradually in a year or two, as does the swelling of the lymphatic glands. Lockwood $^{3}$ reported five cases of early involvement of the thyroid, and, cddly enough, all of these cases occurred in women.

Several cases of gummata are on record, and both Kohler ${ }^{4}$ and Pospelow ${ }^{5}$ 


\section{CLINICAL ASPECT AND TREATMENT OF VENEREAL DISEASES}

described swellings of the thyroid, with myxœdema, the signs and symptoms of which cleared up with antisyphilitic treatment.

Gummatous infiltration of the thyroid may lead to such severe dyspnca and difficulty in swallowing, owing to the infiltration spreading to the trachea and œsophagus, that the diagnosis of malignant disease has been made more than once. There is also no doubt that the symptom complex known as Graves' disease can be produced by syphilis.

1. Maurtac (1890): Syph. Primitive et Syph. Secondaire, 474.

2. ENGEL-REIMERS (1891): Jahrbuch d. Hamburg. Stadtkrankenanstalt, ii.,430.

3. Lockw0oD (1895): St. Bart.'s Hosp. Reports, xxxi., 232.

4. KOHLER (1893): Berl. klin. Woch., xxix., 743.

5. Pospelow (1894): Monatsschrift f. prakt. Dermatol., xix., 125. 


\section{CHAPTER VIII}

\section{SYPHILIS OF THE GENITO-URINARY TRACT}

History.

Gummata of the kidney were known to the clder syphilologists, and Paracelsus appears to have been the first to mention nephritis-a type which at the present day is extremely seldom met with. Paracelsus writes: So derselben frantzösischen Leuten rot Vrin lieff vund wenig. Morgagni described seven cases of renal syphilis, each having its own particular changes. Rayer, in 1840, pointed out that Bright's nephritis could occur in a syphilitic, but neither he nor any of his contemporaries were at all clear as to the connection between the two. Even the advances made by Beer, Lancereaux, and Cornil did little to clear the matter up, which was only to be expected considering how meagre is our knowledge of the subject to-day. Ulceration of the ureters were described by Ferrier, Dodoens, and Morgagni (four cases). Sparmann, in 1733 , reported a case of ulceration of the bladder which extended through the wall of the bladder into the large intestine. Morgagni described five cases of vesical ulceration, and Ricord two cases. Lallemand reported two cases which healed with mercurial pills. From the earliest time chancres of the urethra were known, and ulceration with scar tissue leading to stenosis of this canal appears to have been by no means infrequent. Syphilitic orchitis was common, and Paracelsus attempted to draw distinctions between it and swellings of the testicle produced by other causes. Owing to the fact that it was known in the time of the ancient Greeks that gonorrhœa frequently caused the testicle to swell, it took many years before physicians felt themselves competent to differentiate a gonorrhœal from a syphilitic swelling. In fact, it was not until 1793 that the ground was cleared by the admirable work of Benjamin Bell, which, unfortunately, quickly fell into oblivion owing to Hunter's teaching. Astley Cooper in 1830 put the matter straight again, although he had a tremendous opposition to face, which was finally overcome when Ricord, Colles, and others, confirmed his work.

\section{Kidneys.}

The kidney may be affected in the early, as well as in the late stages of syphilis. From analogy with late syphilitic lesions in other organs, and considering that cases of late syphilitic nephritis are more apt to be met with in the post-mortem room than cases of early syphilitic nephritis, a fairly clear 
picture can be given; but as regards the implication of the kidneys in the early stages of the disease, we know little. In many cases of early syphilis a trace of protein may be demonstrated in the urine. Provided the patient has had no treatment, no attention need be paid thereto, as it rapidly disappears, provided metals are not used in excess. Excluding the foregoing, I arbitrarily divide syphilitic proteinuria into three classes, (1) those cases in which metals, especially mercury, are the cause; (2) syphilitic parenchymatous nephritis; (3) those cases in which there is excessive filtration from the blood. Group 3 has never been previously described as such, because in the literature ${ }^{5,}, 6,7,8,9,10,11,12$ it is designated as Nephritis acuta syphilitica proecox, which in my opinion is incorrect. As it is a new division I will describe it first. The patient appears remarkably well; the generalised rash may be coming out, or it may be fully developed, being usually of the densely infiltrated papular type. There is at first no œdema, although the urine may go solid on boiling. The amount of urine passed in the twenty-four hours is normal, and may even be raised. The protein consists of albumin, globulin, and lipoid-globulin particles, the last two being largely in excess of the first. If a Nicol prism is attached to the microscope, beautiful double-refractive (lipoid-globulin) particles will be seen. With the ultra-microscope, the particles look like those seen in a syphilitic serum. There are few or no casts in the urine, and no red or white cells. Although the protein may be at first increased when mercury or arseno-benzene are prescribed, it rapidly disappears, especially under the latter drug as its administration is continued.

If no treatment is given, the protein excreted diminishes; but œdema and other signs of nephritis set in, owing, no doubt, to a true glomerulitis having been caused.

Once the kidney has been damaged in this way, although the subjective signs may be made completely to disappear by treatment, the objective signs are seldom more than lessened. Up to the present I have encountered five cases, three of which cleared up entirely with arseno-benzene and mercury. The other two had a somewhat eventful career, so I will narrate them in full here.

CASE 35.-A man, aged 35, presented himself for life insurance, but he was refused because he had so-called albumin in his urine. He was referred to a doctor, who treated him carefully for eighteen months as regards rest, diet, etc., with the result that the protein did not diminish, and, except that the patient felt weak, he presented no other signs or symptoms of nephritis. He was then sent to me with a sore on his lip, a typical mucous papule, and, on further examination, I discovered he had contracted syphilis about two years previously. The patient looked well, there was no odema, the blood-pressure was not raised, and the urine contained a considerable amount of lipoid-globulin and globulin particles, but no casts. The following treatment was then given:

24.5.17.-Intravenous injection of colloidal iodine, $100 \cdot 0 \mathrm{c.cm}$.

25.5.17. - Intravenous injection of galyl, $40 \cdot 0 \mathrm{c.gr}$.

29.5.17.-Intravenous injection of galyl, $40 \cdot 0$ c.gr. Proteinuria less. 
31.5.17.-Intravenous injection of colloidal mercury, $10 \cdot 0 \mathrm{c.cm}$. of a 1 per cent. emulsion. This increased the amount of protein, and caused the appearance of blood.

2.6.17. Intravenous injection of intramine, $100.0 \mathrm{c.cm}$. of a 0.1 per cent. emulsion.

4.6.17.-Intravenous injection of colloidal iodine, $100 \cdot 0 \mathrm{c.cm}$.

5.6.17. - Intravenous injection of intramine, $100.0 \mathrm{c} . \mathrm{cm}$. of a 0.1 per cent. emulsion. Examination of urine now showed diminished protein, and no red cells. All the syphilitic symptoms had vanished, and the patient stated that he had never felt so well.

CASE 36.-Patient, a woman, aged 34, contracted syphilis when she was twenty. Early in the disease she developed a severe attack of so-called syphilitic nephritis, and was given by her medical attendants three months to live, consequently no antisyphilitic treatment was prescribed. The patient had no obvious syphilitic recurrence, but was sent to me to have her blood tested in 1914, as she was anxious to have a child. The patient looked very well; she had never had any antisyphilitic treatment, nor had she adhered to the diet prescribed when she was so ill. The ankles swelled in the evening if she had been on her legs much during the day; but she never complained of headaches, and her blood-pressure was not raised. The C.F.T. was positive, and the urine contained a fair amount of protein, and a few casts. Under six intravenous injections of neo-salvarsan the protein was reduced to a trace, and was not increased by the internal administration of mercury, which was continued for a year.

These two cases show that, even if the condition is left untreated, the patient's health is not seriously affected, as it certainly would have been if it had been a true nephritis. I have just seen a case of degenerative encephalitis affecting a man aged 36, who fourteen years ago had such severe hyperproteinæmia and proteinuria that the skin of the abdomen, back, and thighs is covered with atrophic striæ resulting from the ascites and œdema. The protein in the urine disappeared under treatment, and the urine has been normal since. Arsenic is, except in a few cases, non-injurious, but mercury has to be always cautiously prescribed. If mercury aggravates the condition, then iodine and intramine will repair the damage done. Although a nephritis (glomerulitis) may result primarily, I believe the trouble to be a filtration of the excess of globulin in the blood through the glomerular membrane, the colloidal particles of which have become in some way altered as to allow the protein particles to pass through. If correct, one is not justified in calling such a filtration a nephritis, because the same phenomenon appears when the blood can no longer hold the sugar it contains. In the latter case a hyperglycæmia occurs before sugar is demonstrable in the urine.

True parenchymatous syphilitic nephritis is fortunately very rare, as a fatal termination is not unlikely. The patient has all the symptoms of acute nephritis, œdema, etc., the albumin in the urine is in excess of the globulin, and there may be no double-refractive particles. There are many casts, and usually red and white corpuscles-in fact, there may be a large quantity of blood. Antisyphilitic treatment generally makes the patient 
worse, especially mercury, so he has to be treated like any other case of acute nephritis. The amount of urine passed is diminished, and although I have only seen two cases, one of which died, the symptoms in both did not appear until the patient had had the disease some months. This is probably mainly a tubular nephritis. If we had only the opportunity of examining the kidneys in all cases of early syphilitic nephritis, we should doubtless find that there are intermediary and connecting types between the two just described. Mercurial nephritis is a tubular nephritis, resembling in many points the type aforementioned. Casts are found in the urine, the white cells usually exceed the red, and a somewhat characteristic feature is the presence of calcium salts in excess. All metals seem to replace calcium in corpore, and to cause it to deposit in an insoluble form in the kidney substance; hence the explanation of calcification which is to be found in the kidneys of animals which have been killed by toxic doses of arsenic, bismuth, etc. Metals appear to attack in the main the tubules, hence the reason why their use is contra-indicated in syphilitic tubular nephritis. Metals, furthermore, may cause fatty degeneration, and even complete necrosis of the tubular cells. Further details regarding arsenical nephritis will be found in Chapter XVII.

Even in late syphilis there appear to be the glomerular and the tubular types of nephritis, as a marked difference exists in the form of protein excreted, as well as in the way the cases behave to treatment. I will cite what I take to be cases of the glomerular and the tubular types respectively.

CASE 37.-Syphilis thirty years ago; two primary sores; treated for.one year with mercury internally. Ten years ago the patient's feet swelled at night, and he was found to have albumin in the urine. Three years ago gummatous ulcers on the tongue appeared. In consideration of the albuminuria, the patient was treated with mercury and iodides, and advised against having "606," with the result that the patient became gradually worse. When I saw him, he had a gummatous ulcer on the tongue, and one on the inside of the left cheek; there was a considerable amount of protein in the urine, most of which was globulin. Another interesting feature about the case was a periodical rise of temperature, which would last for a few days. Considering the healthy appearance of the patient, the slight. œdema, and the time the trouble had been present, I felt sure that the proteinuria was in the main due to a syphilitic glomerulitis, which had probably begun as a simple filtration. I gave the patient $2.5 \mathrm{c.cm}$. of intramine. A week later the intramine was repeated, by which time the amount of protein in the urine had very considerably diminished. Two injections of galyl were then given, but before the second had been injected, all the œedema had completely disappeared, and the patient declared that he had never felt so well for years. Three years have elapsed since the above report was written, during which time the patient has enjoyed the best of health, in spite of the blood-pressure being as high as $220 \mathrm{~mm}$. of $\mathrm{Hg}$. Owing to the fact that the urine has a normal diastase content and shows no white or red cells and no casts, I take this case to be one of glomerular nephritis.

CASE 38.-The patient was a medical man, who was never aware he had ever had syphilis, but admitted having had gonorrhœa fifteen years ago. He came to me suffering from Hydrops articuli of the right knee-joint. The C.F.T. was positive. There was proteinuria, which consisted mainly of albumin. The urine 
also contained some white cells, casts, and a few broken-down red corpuscles. The first injection of arseno-benzene made the patient desperately ill, the albumin in the urine was increased, and blood appeared. Mercury afterwards had the same effect, unless given in minute doses internally. Iodine could only be tolerated in small doses. The patient looked nephritic, and was always tired, and, in view of the fact that anti-syphilitic treatment was badly tolerated, I considered this a case of late tubular nephritis.

The cases of glomerular and tubular nephritis may be classed as cases of parenchymatous nephritis, or large white kidney. If allowed to progress they begin to exhibit marked interstitial changes. The connective tissue contracts, causes degeneration of the kidney cells, and the end is an irregularly shaped contracted kidney. On the other hand, syphilis may primarily affect the connective tissue-mesenchymatous nephritis-but in my experience this is more common in congenital than in acquired syphilis.

Although I have drawn a distinction between glomerular and tubular nephritis, the reader must not consider-and especially is this true of the late syphilitic cases-that the differentiation is complete. In most cases of the glomerular type, for instance, some inflammation of the tubules co-exists, but the glomeruli appear to be in some cases the structures primarily and particularly involved. Furthermore, in most cases there are mesenchymatous changes, and it is by no means settled whether such changes are primary or secondary in nature, and we cannot say how the mesenchymatous changes alone influence the signs and symptoms.

In a large out-patient department, the most convenient way to test for protein in the urine is with a few drops of a saturated solution of salicylsulphonic acid. When the quantity of protein is small, only a cloud will be seen, but when large, a precipitate will be formed. A rough idea of the quantity can be gauged by the rapidity with which a precipitate forms. If it is desired to estimate the kinds of protein, a twenty-four hours' specimen should be taken and treated with a saturated solution of ammonium sulphate, which precipitates all the globulin and lipoid-globulin. If the urine is then filtered, the albumin can be estimated by precipitation with a saturated solution of magnesium sulphate.

Amyloid disease may supervene upon some of the acute cases, but this complication is more often written about than seen.

Gummata of the kidney are nearly always multiple, and they vary much in size. A large gumma may simulate tuberculous disease, but as often as not the condition gives rise to no trouble, and is not diagnosed during life. One of the most interesting points is the association of syphilis, particularly congenital syphilis, with paroxysmal hæmoglobinuria-interesting because this condition seems to be peculiar to protozoal infections, occurring, as it does, in malaria and in the piroplasma disease of horses. 


\section{Bladder.}

It is only recently that attention has been paid to syphilis of the bladder, but in the short time a number of cases have been collected.

Naturally, the symptoms will not differ from those produced by other factors which cause cystitis. In the stage of generalisation of the virus mucous papules may occur, but they seldom give rise to symptoms unless they ulcerate. There may be a slight frequency of micturition, and an excess of a mucinoid substance in the urine. This mucinoid substance quickly becomes precipitated as clouds when the urine is allowed to stand. It is soluble in acetic acid, and it reduces Fehling's solution. It is found in most cases of cystitis, whatever be the origin of the inflammation. In the late stages of syphilis, gummatous ulceration and papillomatous growths have been described.1, 2, 3, 4 Whenever ulceration of the bladder is diagnosed by a cystoscopic examination, syphilis should always be considered, since the progress is good if syphilis is the cause, as the cases respond at once to treatment. I have only seen one undoubted case of gummatous cystitis, and the signs and symptoms were indistinguishable from tubercular cystitis. The diagnosis was only cleared up by treatment.

It is really in syphilis of the nervous system that the bladder comes most to the fore, and, as vesical trouble is frequently the first symptom of a nervous lesion, I think it would be advisable to deal somewhat fully with the connection. here.

Micturition disorders are very common. Incontinence is a familiar symptom, but that frequency of micturition occurs in a large majority of the degenerative lesions as an early symptom is not so well recognised. In some cases of degenerative myelitis an obstruction to micturition is met with, and in these cases it is often difficult to pass a catheter into the bladder. Dilatation improves these cases temporarily, as the following case shows:

CASE 39.-The patient contracted syphilis twenty-one years ago. Signs and symptoms of degenerative myelitis were first recognised twelve years ago, and even to-day they have not materially progressed. The patient has the Argyll-Robertson pupil, slight Rombergism, no sexual desire, no knee-jerks, and is a martyr to lightning pains. The patient has had every possible treatment, including eight intraspinal injections. As he had a difficulty in passing his water I dilated the urethra, with the result that he had the longest interval of freedom from pains that he had had for twelve years. The pains have since returned.

The reader will have noticed in the case just reported, and in several others which I could cite, that the patient had lost all sexual desire. So common is this symptom, and so frequently is it a precursor by years of a supervening degenerative lesion in the cord, that too much stress cannot be put upon its importance. The loss of sexual power is in some ways like the Argyll-Robertson pupil-that is to say, it may remain the only sign of a degenerative myelitis, or it may be associated with miotic pupils. I have 
just seen a case of a man who has had three perforating ulcers of the foot and loss of sexual desire for seven years, and he presents no other signs or symptoms of degeneration; so it must not be necessarily inferred that if a patient has lost all sexual power that he is going to develop typical Locomotor afaxia. It is extremely seldom, in my experience, that treatment restores the sexual function, but it frequently causes nocturnal emissions when it is first prescribed.

I have seen one case of hæmaturia in a tabetic which occurred at the end of micturition, probably due to some vaso-motor disturbance. According to Caulk and Greditzer, ${ }^{13}$ uræmia commonly occurs in cases of syphilis of the central nervous system. These two observers also lay stress upon the presence of prostatic hypertrophy in tabetics, and warn against prostatectomy in such cases, as the prognosis is so bad; and in the same article it is alleged that the bladder gives a definite cystoscopic picture in many cases of tabes.

The most constant and striking finding is the appearance of the internal sphincter. Inspection is simple, and usually painless, owing to the relaxation and insensitiveness of the part, with the result that the verumontanum and the opening of the various ducts may readily be observed. The trigone is usually elevated, and laterally at its tips it fans out into trabeculæ. There is also usually a general vesical trabeculation. It should be more generally recognised that the bladder trouble is often the start of severe nervous involvement, and more attention should be paid to the treatment of this organ, since it may mean so much to the comfort of the patient. Urinary antiseptics should be prescribed; the patient should be taught to pass his water at regular intervals, and the bladder should never be allorred to get too full. Any residual urine, if present, should be drawn off periodically, the greatest attention always to be paid to asepsis. Presumably tha appearance of the inside of the bladder is due to weakness or paralysis of the internal sphincter and to the atonic condition of the unstriped muscular tissue.

\section{Testicles.}

Early syphilitic epididymitis is not at all an uncommon symptom of the disease. The epididymitis may be unilateral or bilateral, and may be well marked before the rash has even made its appearance. This is an important point to remember, because $I$ once saw a case in which a testicle was removed for a supposed tubercular epididymitis, the generalised papular rash not making its appearance till the patient was convalescing from the operation. In every case I have seen, it has been only the caput major that has been affected. The feel alone of the epididymis will suggest syphilis at once. The affected pole in syphilis feels uneven, as a bunch of grapes would feel through a soft bag. In the other infections which cause epididymitis the organ is evenly enlarged, and feels more or less smooth and hard on the surface. I had one case in which the epididymis became adherent to skin, broke through, and produced a condition to which the name of Hernia testis is sometimes 
given. In the lata stages of the disease the commonest lesion is a gumma of the testis, but occasionally a gumma may be limited to the epididymis, in which case the caput minor, or body, is the portion most frequently affected. A short time ago I saw a case of a man, aged 45, who never remembered having had syphilis, and who came up for advice for pain in his "testicle." The caput minor was enlarged, hard, and slightly tender, and the whole of the vas deferens was markedly thickened. Had the patient been younger and delicate looking, no one would have hesitated in diagnosing the condition as tubercular epididymitis.

However, the fact that the condition cleared up under antisyphilitic treatment clearly showed that the affection was syphilitic.

Hydrocele may result from any injury to the epididymis or testis, and is not specially prone to occur in syphilitic affections of these organs.

Occasionally syphilitic epididymitis may involve the cord as well, as I have had a case under treatment in which the funiculitis reached into the inguinal canal.

\section{Prostate and Urethra.}

Syphilitic prostatitis is a pathological curiosity, and the urethra, with the surrounding tissues, is not frequently affected. I have known dilatation of the urethra for gonorrkœa in a syphilitic subject to set up a syphiloma which spread to the cavernous and spongy bodies, and such a lesion to arise independently of an injury. The lesion causes a general hardness, and gives rise to a peculiar urethral discharge, which consists mainly of mucin and desquamated epithelium. The trouble rapidly subsides under treatment, and does not lead to the permanent formation of hard bands or nodules. Syphilis is not a cause of Induratio penis plastica. I have known cases in which a syphilitic urethritis had produced a discharge sufficiently severe and purulent in nature to be diagnosed and treated as of gonococcal origin.

1. Lévy Bing et Durveux (1913): Annales des Malad. Vénér., i., 242.

2. Asch (1911): Zeitschr. f. Urologie, v., 504.

3. Pereschiwkin (1911): Zeitschr. f. Urologie, v., 732.

4. DRE Yer (1913): Dermat. Zeitschr., xx., 477, 591.

5. KARv ONEN (1900): Dermat. Zcitschr., vii., 37.

6. Fournier (1906): Traité de la Syphilis. J. Ruefi, Paris.

7. Hoffmand (1913): Deutsch. med. Woch., xxxix., 353.

8. Munk (1913): Zeitschr. f. innere Med., lxxviii., 19.

9. MacDonald (1915): Lancet, ii., 702.

10. HaHN (1912): Deutsch. med. Woch., xxxviii., 759.

11. Nauta (1914): Ann. d. Mal. Ven., lx., 352.

12. Nicholas Et Montot (1914): Ann. de Derm. et de Syph., 391.

13. CaulK and Greditzer (1917): Amer. Journ. of Syph., i., 42. 


\section{SYPHILIS OF THE ABDOMINAL VISCERA}

\section{History.}

From the literature it would appear that ulceration of the csophagus was more often encountered than to-day, and possibly some of the worst cases were aggravated, if not actually caused, by over-treatment with mercury. Syphilis of the stomach was known from the first, and Fernel, in 1548, recognised that it could be affected in the early stages of the disease. According to Proksch, syphilitic ulceration of the stomach was first described by Schenck v. Grafenberg in 1594, since when several cases have been recorded; but no differentiation was made between syphilitic ulcers and those produced by other causes until the present day. Syphilitic ulceration of the intestines, leading to diarrhœa, etc., was well known in the sixteenth century, and by many rightly prescribed to the injudicious use of mercury. Both Morgagni and Monteggia described undoubted cases of syphilitic ulceration, and Cazenave, in 1843, wrote that from post-mortem examinations he had come across multiple ulcers in the ileo-cæcal region, some of which closely resembled Hunterian chancres. Cullerier, in 1854, gave an excellent description of syphilitic ulceration of the large intestine. Ulcerating condylomata were probably common in the old days, and as condylomata were usually confused with hæmorrhoids, no clear picture of syphilitic affections of the anus is given until 1689 by Musitano. Abercromby, in 1691, was the first to report clearly a case of syphilitic ulceration of the rectum. Morgagni reported cases of ulcers of the vagina perforating the rectum, and similar cases were described by others who followed him.

The older syphilographers looked upon the liver as the original seat of the disease, and it was not until the circulation of the blood was discovered that this idea was given up. Nevertheless, the view was responsible for the general belief that in every case of syphilis the liver was involved. When Hunter stated that he had never seen a case of visceral syphilis, all the preexisting knowledge of this part of the subject faded away. Although Bell, Monteggia, and Swediaur attempted to draw attention to visceral syphilis, it was left to Ricord and his pupils to place the subject again in its proper light. Syphilis of the pancreas was only occasionally referred to, while syphilis of the spleen appears to have been better known. 


\section{General.}

One of the least studied chapters of syphilis is that dealing with syphilis of the abdominal viscera. This omission is largely due to the fact that the correct diagnosis is frequently not made until the patient has reached the post-mortem room. Some cases escape detection because they recover from an abdominal section which has been performed for an inoperable malignant growth; while others, because they have obvious signs and symptoms elsewhere, receive treatment without a thorough examination having been made, with the result that all the symptoms disappear, the undetected as well as the apparent.

\section{Esophagus and Stomach.}

Syphilis of the oesophagus is undoubtedly rare, and the only case I have seen was in a healthy-looking young woman, who was operated upon for a supposed carcinoma of the cardiac end of the stomach. The operation revealed a diffuse induration of the cardiac ends of the œsophagus and stomach, and the abdominal wound was closed, with the idea that the lesion was cancerous. The patient was given some potassium iodide, and from that time onward made an uninterrupted recovery. Cases have been described of syphilitic stenosis of the œesophagus.

Syphilis of the stomach is a great deal more common than is generally supposed, and those who are anxious to learn more about syphilis of this organ than can be dealt with here should refer to Chiari's ${ }^{6}$ classic contribution upon this subject, and to articles written by Smithies, ${ }^{8}$ Myer, ${ }^{9}$ Tuohy, ${ }^{10}$ Cronin, ${ }^{11}$ and others. ${ }^{12,13,14}$ A catarrh in the generalisation stage can sometimes be ascertained if every patient be thoroughly examined. The diagnosis is admittedly difficult, owing to the fact that anæmia may be primarily responsible for the indigestion, hyperacidity, sickness, and loss of appetite, these being the main symptoms complained of. If mercury has been given internally it is impossible to say how far it is responsible for the gastric trouble.

In the later stages of syphilis the disease may produce a diffuse infiltration of the walls of the stomach. I have seen such a case, which recovered under antisyphilitic treatment, after the diagnosis of sarcoma had been made at an abdominal section.

The infiltration may affect the pylorus; indeed, it is frequently limited to this portion of the stomach.

CASE 40.-A medical man, aged 36, came to me for some injections of salvarsan because he had had syphilis. He informed me that he had a swelling over the pyloric end of the stomach, and it was quite distinct on palpation, and had aroused the suspicion of carcinoma, in the minds of some of the surgeons whom he had consulted. He had been strongly advised to have a gastro-jejunostomy performed, but, knowing that he had had syphilis, he thought he would try salvarsan first. After a few injections, the swelling completely disappeared. 
Since then I have seen three other cases of a swelling in the pyloric region, in young and apparently healthy individuals, in all of whom the symptoms and swelling vanished under salvarsan. It is extremely probable that many of the cures, in young middle-aged persons, which have resulted from short circuiting, are not due to the operation, but to the spontaneous disappearance of the syphilitic lesion, as some of the late symptoms of syphilis are occasionally wont to do.

Syphilis may also cause ulceration of the stomach; there may be a single ulcer, or several; they may be of any size, and occur in any part of the stomach. Such cases, again, are not, as a rule, diagnosed until after death, when it is seen that the syphilitic ulcer is different from the usual type of gastric ulcer.

A syphilitic ulcer is sharply circumscribed; there is no surrounding inflammation; it is never terraced, and, as a rule, the edge is raised and thick.

As a rule, syphilitic ulcers of the stomach heal and leave a scar; the scar causes contraction, and usually the patient suffers from chronic indigestion. In some cases a typical hour-glass constriction may be formed. Naturally, if the case has remained undiagnosed for several years, and adhesions have formed with neighbouring organs, treatment cannot do much good; therefore, no response to treatment does not necessarily exclude old syphilis.

In most cases of gastric ulcer syphilis should be borne in mind, because, if syphilis is the cause, the cases recover rapidly under appropriate treatment. Hæmorrhage is not a common symptom, for the simple reason that the ulcer is usually a necrosis of that area which was fed by a vessel which had become occluded by inflammation. Perforation practically never occurs, as the outer portion of the wall and the peritoneum remain unaffected, and because the ulcer practically always starts in the submucous tissue and erodes only the mucous surface.

A great deal of work on the chemical analysis of the gastric contents in cases of syphilis of the stomach still remains to be done. My experience in this direction is very limited, but, from the few cxaminations which have been made, a typical feature seems to be the decrease in the hydrochloric acid content. In some cases a marked excess of mucus may be noted.

Hausmann, ${ }^{1}$ who has made a special study of syphilis of the abdominal organs, mentions the following points in the differential diagnosis between syphilis and other diseases of the stomach:

1. A normal or increased $\mathrm{HCl}$-content excludes gumma of the stomach, gummatous ulceration, syphilitic hyperplasia, and shrunken stomach.

2. Nocturnal gastric pains, with anacidity, is in favour of syphilis of the stomach or of a syphilitic retro-peritoneal tumour, but it does not exclude ordinary pyloric stenosis.

3. Characteristic symptoms of gastric ulcer, associated with anacidity, favours the diagnosis of gummatous ulcer.

4. A palpable pyloric swelling, with anacidity and absence of the symptoms of stenosis, and a negative blood-test in the stomach secretion and in the 
fæces, points to there being a diffuse gummatous infiltration of the pylorus.

5. In all cases of shrunken stomach, the diagnosis of syphilis must be seriously borne in -mind.

$\mathrm{McNiel}^{7}$ reports an interesting case of syphilitic gastric ulceration in which a fistulous opening between the stomach and the small intestine occurred. In this case occult blood was demonstrated in the gastric contents and stools. I mention this case, because blood, even amounting to tarry stools and extreme emaciation, which are so suggestive of cancer, may be met with in syphilis.

Recent work has added two more tests which should be undertaken in doubtful cases of gastric ulceration: one is the formol index, and the other is the roentgenoscopic test. The formol index, as estimated by the formaldehyde titration method suggested by Sörensen and Schiff, averaged for 35 cases reported by Smithies $^{8} 9 \cdot 6$. The minimum was 6 and the maximum 14 . In a series of 87 cases of gastric cancer investigated by the same author, the average formol index was 21 .

The following notes on the roentgenological study of gastric syphilis are taken from articles by Carman ${ }^{15}$ and Eusterman. ${ }^{16}$ In the ulcerative type hour-glass contraction can easily be recognised. Absence of a niche, accessory pocket, or typical incisura-classic signs of a simple gastric ulcer-is practically pathognomonic of a syphilitic ulcer, because, as already stated, a syphilitic ulcer usually only involves the mucous and submucous tissue. X-rays may reveal a distorted stomach, altered peristalsis, and an obstructed pylorus, in a patient who is not ill in proportion to the extent of disease shown, in which case syphilis is the more probable diagnosis than cancer. A shrunken stomach is readily detected by the X-rays. Another point of diagnostic importance is that, in syphilitic gastritis, extensive pathological changes and altered chemistry may be met with in a case which has only run a brief clinical course. Of the 23 cases reported by Eusterman, ${ }^{16}$ the average duration of symptoms was three years, while in benign ulceration of the stomach the average was twelve and a half years.

In every case in which pains in the stomach are complained of, the possibility of these pains being the gastric crises of degenerative myelitis should always be considered. I know of five patients who have had a gastro-jejunostomy performed, when their symptoms were really those of degenerative myelitis; one of these was operated upon twice, and I have been fortunate enough to save three others from suffering a similar fate. It should also not be forgotten that gastric pains may be the only symptom of which a patient with syphilitic aortitis complains.

The following is one of the most interesting cases of gastric syphilis I have come across :

CASE 41.-A man, aged 29, had a papulo-erosive chancre in July, 1915. He had one injection of salvarsan in September, another in December, and a third in January, 1916, during which time mercury was taken internally. In April, 
1916, a recurrent maculo-papular rash appeared, which was indistinguishable from the original eruption, also Hydrops articuli of both knees, and rheumatic pains in both shoulders and elbows. The patient had nine injections of galyl, and took mercury internally for a year. In November, 1917, patient complained of gastric pains, which became persistent and aggravated by food. With careful dieting and rest in bed he did not improve, so the gastric contents were analysed with the following result: Absence of free hydrochloric acid; total acidity 25; lactic acid present; occult blood present in both the stomach contents and in the stools. In January, 1918, the stomach trouble was in statu quo; but a syphilitic orchitis appeared, and various ulcerative papular syphilitic lesions on the trunk. The moment the patient received colloidal iodine and intramine, all the signs and symptoms began to vanish, and three days after the second injection of intramine the patient was able to eat what he wished without discomfort.

\section{Syphilis of the Intestines.}

In the acute stage of syphilis, there may be a catarrh of the duodenum, which is nearly always associated with a catarrh of the bile ducts, without and within the liver, the result being that the patient becomes jaundiced.

Doubtless a catarrh of the rest of the intestinal tract occurs, but its diagnosis would be a matter of extreme difficulty. The Condylomata lata which one so commonly sees around the anus may extend up the anal canal, but, as a rule, they give rise to no symptoms unless they ulcerate, and then give rise to a stricture, a sequela which is very rare. Syphilis of the intestines is more common in the congenital than in the acquired form, hence, in acquired syphilis, one would expect to find the intestines affected in the late stages, which is the case. The commonest syphilitic lesion of the gut is a diffuse syphilitic infiltration of the wall. Occasionally the infiltration is localised and annular, and then it quickly gives rise to narrowing of the lumen and to stenosis. Such cases, when operated upon, are almost invariably regarded as carcinomata. If the stenosis is of long standing, naturally the mucous membrane ulcerates, but, in all these cases of syphilitic infiltration of the gut wall, the mucous membrane is intact, and only becomes secondarily involved. The diffuse form is usually diagnosed as sarcoma.

Syphilis may affect the tissue subjacent to the peritoneum. The peritoneum becomes secondarily involved, and adhesions are formed between the affected area and the surrounding structures.

I. have seen a case of this sort, involving the under-surface of the liver, the duodenum, and the pancreas. I also have notes of another case, in which the pyloric end of the stomach was bound down to the viscera around by adhesions.

This is probably the pathology of the so-called syphilitic stricture of the rectum.

Syphilitic stricture of the rectum is more common in women than in men, and, in some cases, the whole pelvis seems to be filled with a dense mass of infiltrated tissue. It is the contraction of this tissue which leads to the 
narrowing of the lumen of the bowel, and here again any ulceration of the mucous membrane is the result, and not the cause. Gummatous ulceration of the bowel is never diagnosed except post-mortem-i.e., when it affects any part of the bowel other than the rectum.

The gummata are almost invariably multiple. They are sharply circumscribed, have raised edges, and practically no surrounding inflammation. They do not, as a rule, affect the Peyer's patches, and they often heal spontaneously.

Gummatous ulceration of the rectum may lead to stricture, and an important point to remember in these cases is that if the rectal stricture is due to gummatous ulceration-i.e., if it is a primary lesion of the mucous membrane-the chances are that there are gummata higher up in the gut. If there are gummata higher up in the gut, there may also be a stricture higher up.

Unfortunately, these cases do not give rise to symptoms until the syphilitic lesion has healed, and its place has been taken by contracting fibrous tissue; therefore, surgical interference is usually called for.

\section{Syphilis of the Liver.}

Syphilitic jaundice was first described by Paracelsus in 1585, and has been frequently referred to since; but, in my experience, it is far from a common manifestation. I have only seen one case in the last twelve years. Jaundice in early syphilis may be mild or severe. The mild form is by far the more common, and differs little from ordinary catarrhal jaundice. The mild form usually occurs while the rash is developing: its onset is often rapid, and the patient does not usually complain of gastro-intestinal disturbance. The stools may be normal or clay-coloured, and in most cases a uniform swelling of the liver is found. The jaundice readily responds to treatment, and the powerful oxidising agents now in use should be quickly prescribed, because jaundice is not a contra-indication to their use, and their early employment may save the onset of Icterus gravis syphiliticus. Observers are not at one as to the pathology of syphilitic jaundice. Quoting from an interesting article written by Wile and Karshner, ${ }^{17}$ the French writers in general regard it as the result of swelling of the portal lymph vessels. Engel-Reimers ${ }^{18}$ was able to show at the post-mortem examination in three cases that it was an obstructive jaundice caused by specific adenitis of the portal lymphatic glands. Mauriac ${ }^{19}$ believes that the jaundice is due to an active hepatitis, with hyperæmia and stopping up of the biliary ducts. Fournier ${ }^{20}$ was of the opinion that the jaundice was due to the syphilitic toxine. Talamon ${ }^{21}$ described an interesting case in a girl, aged 17, in whom fear brought about an emotional jaundice within eighteen hours, followed in three weeks by death from acute yellow atrophy. Doubtless the fear in this case rendered the liver more susceptible to the syphilitic poison.

The severe form of jaundice may supervene on the mild form, or a foudroyant course may be run from start to finish. The danger of this form 
is that, unless the patient is treated energetically at once, death from acute yellow atrophy is more than likely to be the result. Once acute yellow atrophy has set in, a fatal termination is practically certain; therefore, one need not hesitate to prescribe the powerful oxidising agents now in use, for, should one be fortunate in catching an early case, they, and nothing else, can save life. I know of a case which was thus successfully treated.

The pathology of Icterus gravis syphiliticus has been carefully worked out by Buschke and Zernik, ${ }^{22}$ who concluded that it was due to a syphilotoxic parenchymatous hepatitis, and was not an obstructive jaundice.

Engel-Reimers, ${ }^{18}$ and others, report cases in which pregnancy complicated the condition. This is no doubt the reason why the majority of cases reported have been in women.

There is no doubt that both jaundice and acute yellow atrophy have been frequently caused by the arseno-benzene preparations. The pathology of these cases is clear. It is a parenchymatous inflammation of the liver cells, which first swell, then undergo fatty degeneration, until complete necrosis takes place in the fatal cases.

Milian ${ }^{24}$ regards these cases of acute yellow atrophy occurring after arsenic as actually due to syphilis, the lesion corresponding to a Herxheimer reaction. As five of the cases I have seen had never more than a primary sore, and had not entered the generalisation stage, it would be impossible for a Herxheimer reaction to occur; therefore, in these cases at any rate, I feel convinced that the arsenic was to blame, and not the disease.

Intramine is a specific for arsenical jaundice. For further details of arsenical atrophy the reader is referred to Chapter XVII.

The late syphilitic lesions of the liver are very easy to understand, if it be remembered that the disease begins in the connective tissue, either between the acini or between the cells themselves.

The interstitial or indurative hepatitis may be diffuse or localised. If localised, it may be single or multiple.

The diffuse form gives rise to a hypertrophic cirrhosis, which cannot be distinguished from a similar condition produced by other causes. As the fibrous tissue contracts, the blood-supply to the parenchyma cells will be diminished, and therefore many of the latter will degenerate. The liver gets smaller and smaller-the so-called atrophic cirrhosis.

The localised form varies enormously in size. Usually the parenchyma cells completely degenerate, and the lesion becomes a gumma.

This is also the pathology of the multiple localised lesions. Those on the surface, owing to the contraction of the fibrous tissue, often have a marked depression in their centre.

It sometimes happens that only one lobe is affected. Say, for sake of argument, that it is the left, then the right lobe will hypertrophy-a compensatory hypertrophy.

Since the right lobe is more easily palpated than the left, a mistake may 


\section{CLINICAL ASPECT AND TREATMENT OF VENEREAL DISEASES}

easily be made in diagnosing a compensatory hypertrophic right lobe as hypertrophic cirrhosis. Most cases of syphilis of the liver run a symptomless course, and are not diagnosed until after death, a circumstance which gives the clinician the idea that syphilis of the liver is rarer than it really is.

Boardman ${ }^{23}$ draws attention to the so-called heparlobatum, which appears to be more common in negroes and in women. In this condition the liver is separated into numerous small lobules by scars and bands formed of fibrous tissue caused by syphilitic inflammatory changes, resulting in an over-production of fibrous tissue. This is doubtless mesenchymatous hepatitis, akin to what is described below as affecting the spleen. Amyloid disease is said to result from syphilis, although I can find no detailed account thereof in the literature.

If Glisson's capsule is affected, and there is a marked peri-hepatitis, pain is a very common symptom. The pain is independent of taking food, and it is increased on deep breathing and coughing.

Pain is a good sign, as it shows that the peri-hepatitis is acute, and therefore much is to be expected from treatment.

Vomiting is a common symptom; but possibly the most important symptom is intermittent fever.

This intermittent fever is often the only symptom which the patient develops, and for which he seeks advice, so that many clinicians have described a condition to which they have given the name of tertiary syphilitic fever. ${ }^{2}$

I had one case which had been diagnosed for months as malaria. Typhoid fever, malignant endocarditis, febrile cholelithiasis, pulmonary tuberculosis (as the spitting up of blood sometimes occurs), and lymphadenoma may easily be mistaken for late syphilitic fever.

I had another interesting case, in which the febrile attacks were accompanied by hæmoglobinuria.

Late syphilitic fever is best diagnosed by the rapidity with which it disappears under antisyphilitic treatment.

In practically all the cases of tertiary syphilitic fever the spleen is enlarged as well as the liver, and one of the distinguishing features between syphilitic cirrhosis and alcoholic cirrhosis is that, in the former, there is more often an enlargement of the spleen than ascites, while in the latter the reverse is the case.

Since the above was written I have been fortunate in seeing other cases of syphilitic fever. In all the cases the patient had had the disease for some years, therefore the fever was a late manifestation. In most of the cases the fever was intermittent, often peculiarly so; for instance, one patient, a woman, almost daily for several weeks had an attack of fever from 3 p.m. to 7 p.m.; the temperature sometimes rose as high as $104^{\circ} \mathrm{F}$., and usually at the end of the attack the patient sweated profusely. In the minority of the cases the fever was continuous for some weeks, with periodical exacerba- 
tions. One case was a medical man, who had continuous fever for five weeks, during which time he became emaciated and had night sweats, just like a patient with advanced pulmonary tuberculosis. All the cases rapidly recovered under antisyphilitic treatment. It must not be forgotten that a continuous high evening temperature may be the first sign of generalised syphilis. I remember being called to see a case which had been diagnosed as everything, until the patient drew attention to a sore at the meatal orifice of the penis. This patient had evening fever to $103^{\circ} \mathrm{F}$. every day for three weeks, and, in addition, he complained of severe joint and bone pains.

Ascites is not a common complication of syphilitic cirrhosis.

Alimentary galactosuria is, according to Bauer, ${ }^{3}$ a frequent sign of syphilitic hepatitis; and so also is urobilinuria; and the occurrence of both of these points to damage of the parenchyma cells.

\section{Syphilis of the Pancreas.}

What has been stated on the interstitial nature of the syphilitic process in the liver applies also to the pancreas. In most cases of syphilitic disease of the pancreas it is only the head of the organ which is involved.

Syphilitic pancreatitis usually leads to the formation of adhesions which involve all the neighbouring structures, so the clinical picture is often a varied one. The following is a good example of a case of syphilitic disease of the head of the pancreas:

CASE 42.-Patient was a medical man, aged 35, who contracted syphilis three years before onset of present trouble. Treatment had been more or less continuous; the notes are best given as he wrote them out for me:

"In January, 1910, I first commenced to experience attacks of pains in the epigastrium. The early attacks took the form of a dull ache across the epigastrium, commencing about midday, after having been at work for two or three hours. Pain would gradually become worse, till I was forced to lie down, which would almost immediately relieve me of further pain. Minor attacks of this pain were very frequent, and on a few occasions severe attacks were experienced, which necessitated my lying up for a week. The pain was always of a dull, aching character, never localised, but indefinite, and extending across the epigastrium. It was seldom accompanied by any other symptoms, except occasionally by a mild nausea and a peculiar chilly feeling.

"The attacks were in no way connected with food. During January, February, and March, 1911, the attacks became so bad that in April I left for home. The attacks occurred daily, and were now almost invariably associated with nausea. In May, jaundice developed, which lasted three weeks, so a diagnosis was then made of catarrhal inflammation of the gall-bladder and duodenum. X-rays showed nothing abnormal. Pains continued throughout June and July, when I consulted Mr. who diagnosed duodenal ulcer.

"In July I was obliged to return to duty, but as the trouble persisted I came home again in November, 1911, and was operated upon January, 1912, by Mr. No ulcer of the stomach or duodenum was found, and the appendix appeared normal. The gall-bladder was attached to all the surrounding structures by adhesions, and 
there was a typical chronic pancreatitis, which had previously been suggested as a resultof Cammidge's tests. Following the operation I remained free of symptoms till March, 1912, when they all returned, and three months later they were worse than they had ever been before."

In September, 1912, I saw the patient, and, as the C.F.T. was positive, I gave several injections of neo-salvarsan, and followed up the treatment with mercury and iodides. After the first injection the symptoms vanished, and patient has been perfectly well since.

Intermittent glycosuria may be the only symptom of a syphilitic affection of the pancreas, but it must be remembered that not every case of syphilitic glycosuria is of pancreatic origin; for instance, it may be a symptom of an intracranial lesion. It appears probable that glycosuria may also occur in syphilis from mere increased permeability of the glomerular membrane, produced by the altered physico-chemical state the protein colloidal particles in the serum undergo; but, unfortunately, not enough is known of the carbohydrate metabolism to go further into this interesting problem.

A thorough examination of the fæces and urine is necessary in all cases of suspected implication of the pancreas. Warthin ${ }^{25}$ has microscopically examined the pancreas in a large number of autopsies in cases of apparently latent syphilis, and he found no one of them showing a normal pancreas. All evidenced changes in the form of a diffuse or patchy fibrosis, with active inflammatory foci corresponding to the localisation of the spirochætæ.

\section{Syphilis of the Spleen.}

An enlargement of the spleen in congenital syphilis is a rery well-known fact, but that the spleen is often enlarged in early stages of acquired syphilis few have noted. The spleen is nothing more nor less than a huge lymphatic gland. A general enlargement of the lymphatic glands is one of the commonest and earliest signs of syphilis; therefore, if one thinks, it would be only too reasonable to expect a hyperplasia of the spleen in the early stage. In early syphilis the spleen may come as far as two fingers'-breadth below the costal margin. In the majority of cases of syphilis it is palpable, and it may be felt before the rash appears. Like adenitis, the enlargement may be hard or soft, and it does not readily disappear under treatment.

Gummata, of course, may affect the spleen, but they are seldom diagnosed during life. In late syphilis an enlargement of the spleen may be of a mesenchymatous or parenchymatous nature. Syphilitic mesenchymatous splenitis may or may not be associated with cirrhosis and ascites. As a rule, it is the occurrence of fluid in the abdomen which draws one's attention to the splenic condition. Syphilitic parenchymatous splenitis is a semi-malignant disease akin to Mycosis fungoides, Miculicz's disease, and Hodgkin's disease. The spleen is the only organ involved, unless during the last stages the bonemarrow, in attempting to come to the rescue, produces a leucæmia. Ordinarily the condition is aleucæmic, the patient is anæmic, and the spleen may reach as far down as the pelvis. 


\section{Suprarenals.}

Considering that spirochætæ are more readily demonstrable in the suprarenals than in any other organ in congenital syphilis, and are possibly one of the causes of those curious blood-cysts which kill the infant affected, it has often struck me as peculiar that in acquired syphilis our attention is practically never drawn to these glands. Unfortunately, the rôle these glands play in the body alone, and in conjunction with the other endocrine glands, is far from being understood; add to this the fact that the clinical symptoms produced by an affection of these glands are variable, and focus one's attention on either the nervous system, vascular system, or pancreas, we can readily understand that next to nothing of syphilis of the adrenals is known. We are still further hampered by the facts that an involvement occurs in the late stages of the disease, and that the symptoms produced are chronic. As our knowledge increases, I feel confident that it will be necessary to devote a special chapter in textbooks on venereal diseases to syphilis of the ductless glands. As far as my experience goes, syphilis causes a parenchymatous and a mesenchymatous inflammation of the adrenals, the latter being more common than the former. A parenchymatous inflammation may give rise to a symptom complex which we call Addison's disease. A mesenchymatous inflammation gives rise to symptoms referable to (1) the nervous system; (2) the vascular system; (3) other organs. Although the cases may be roughly divided into these groups, it is not at all uncommon to come across one having symptoms referable to two or all. Vitiligo, occurring with or without alopecia, may be the first symptom; later, nervous symptoms appcar, which are almost invariably those produced by an implication of the regetative nervous system. This is also just the type of case in which syphilitic aortitis occurs as well. Apart from the rôle mesenchymatous syphilitic inflammation of the suprarenals plays in the causation of aortitis, there appears to me to be little doubt but that syphilitic thrombo-angiitis, hyperglycæmia, and high blood-pressure are due thereto. Hyperglycæmia is not at all uncommon in late syphilis, and only when the blood can hold no more sugar is it filtered through the kidneys to become demonstrable in the urine. This is the explanation of transitory, intermittent, or periodic glycosuria. It has yet to be ascertained the rôle the pancreas plays in these cases; and still less is known of how the thyroid and other ductless glands behave when the suprarenals are thus attacked. The following is one of the most interesting cases of transitory glycosuria I have come across:

CASE 43.-The patient, a man, aged 49, contracted syphilis eighteen years ago, for which he was well treated. In 1917 he developed multiple cutaneous gummata, for which he received six injections of galyl with some mercury and iodides. In January, 1919, he consulted me about a recurrent gonococeal urethritis and epididymitis, which cleared up with trimine and intramine. The day following the injection of intramine the patient began to pass enormous quantities of urine, which 
I found a few days later was full of sugar. With a little rest and dieting the sugar completely disappeared in a month, during which period the patient lost over two stone in weight. During the bout of glycosuria all the deep pigmentation around the scars of the old gummata completely vanished. The C.F.T. of the blood was negative, in spite of which in June, 1919, a gumma occurred on the glans penis. Whether the intramine stirred up a dormant syphilitic lesion or not is impossible to say, although it is suggestive that it did so, and that the lesion was in the suprarenals owing to the very rapid depigmentation of the hyperpigmented areas.

In one of the three cases of increased blood-pressure which I have seen, although sugar was never demonstrable in the urine, it could be easily made to appear, and there was ample evidence that the patient was hyperglycæmic. The connection between hyperglycæmia, thrombo-angiitis, and high bloodpressure is so interesting, and to have the riddle solved would be so valuable, that not a stone should be left unturned to get a true picture of how the suprarenals are affected in syphilis.

\section{Syphilis of the Peritoneum, ete.}

Retro-peritoneal and mesenteric swellings may sometimes be of syphilitic origin, and the site of the trouble is usually in the lymphatic glands. The swelling may sometimes reach an enormous size. An analogous condition occurs in the mediastinum, and a glandular swelling in this situation is usually mistaken for an aneursym.

There is an extremely interesting condition which is sometimes met with in syphilis, in which the patient has a pseudo-chylous ascites.

Though not a true syphilitic disease of the peritoneum, it may occur in cases in which no trouble of an abdominal organ can be traced, although it is usually secondary to a lesion of an abdominal viscus.

The chemical and physical properties of the fluid from cases of chylous and pseudo-chylous ascites have been so ably worked out by Mackenzie Wallis and Schölberg, ${ }^{4,5}$ that anyone who is interested in this subject should refer to them.

Our attention has been recently drawn to syphilitic peritonitis in an able article by Letulle. ${ }^{26}$ This author describes a diffuse peritonitis, affecting even the epiploices associated with ascites and cirrhosis of the liver. The infiltrated patches of peritoneum have a pathognomonic mother-of-pearl or enamelled appearance.

Although both the visceral and parietal peritoneum may be involved, the inflammatory process appears to show preference for the intestinal peritoneum. The Appendices epiploica are less numerous than in health, thickened, whitish, and hard, reminding one in severe cases of the sausage known under the name of boudin blanc. The intestine is reduced in length, and, in the large gut, the peritoneum covering it may be so thickened as to obliterate the characteristic features-longitudinal muscular bands, etc.

Letulle considers that the ascites is due to the peritonitis, and that the 
condition is curable. He further suggests that every case of hepatic cirrhosis should be treated with antisyphilitic remedies, as syphilis underlies most of the cases, whether alcohol plays a causative rôle or not.

1. Hausmann (1913): Die luet. Erkrankungen der Bauchorgane, C. Marhold, Halle a. S.

2. Parkes Weber (1907): Lancet, i., 728.

3. BaUer (1910): Lues und innere Medizin.

4. Mackenzie Wallis ANd Schölberg (1910): The Quarterly Journ. of Med., iii., 301.

5. Mackenzie Wallis and Schölberg (1911): The Quarterly Journ. of Med., iv., 153.

6. Chiari (1891): Internat. Beitr. z. Wiss. Med., ii., 211.

7. MCNieL (1917): Amer. Journ. of Syph., i., 91.

8. Smithies (1917): Amer. Journ. of Syph., i., 100.

9. MYER (1912): Interstate Med. Journ., xix., 974.

10. Тиону (1914): Interstate Med. Journ., xxi., 1036.

11. Crovin (1914): Interstate Med. Journ., xxi., 1019.

12. Downes AND LE' WALD (1915): Journ. Amer. Med. Assuc., lxiv., 1824.

13. Brugsch U. SchNeIder (1915): Berl. klin. Woch., lii., 601.

14. Clark (1916): Brazil-Medico, xxx., 89.

15. Carman (1917): Amer. Journ. of Syph., i., 111.

16. Eustermax (1916): Amer. Journ. Med. Sci., clii., 21.

17. Wile and Karshner (1917): Journ. Amer. Med. Assoc., lxviii., 1311.

18. ENGEL-RETMERS (1892): Monatschr. f. prakt. Dermat., xv., 475.

19. Mauriac (1888): Gaz. Hebd. de Méd., Xxv., 564.

20. Fournier (1906): Traité de la Syphilis, J. Rueff, Paris.

21. Talamon (1897): Med. Mod., viii., 97.

22. Buschke U. Zernik (1911): Areh. f. Dermat u. Syph., cvi., 121.

23. Boardman (1917): Boston Med. and Surg. Journal, elxxvii., 72.

24. Milian (1914): Bull. de la Soc. Franc. de Derm. et Syph., xxv., 365.

25. Warthin (1914): Amer. Journ. of Med. Science, cxlvii., 607.

26. Letulde (1918): La Presse Médicale, lii., 477. 


\section{CHAPTER $\mathrm{X}$ \\ SYPHILIS OF THE EYES AND EARS}

\section{History.}

Most of the references to eye trouble deal with ulceration affecting the eye either directly or by extension from caries of the skull bones, lesions with which we at the present day are not familiar. Needless to say, there was always confusion between syphilitic and gonorrhœal lesions. Syphilitic iritis, as we now know it, was not described till the beginning of the sixteenth century, and for more than fifty years it was considered by many to be due to mercury. William Lawrence did most to give us a clear conception of syphilitic iritis, cyclitis, etc. As the ophthalmoscope was not discovered till 1851, our knowledge of choroiditis, retinitis, etc., is necessarily of recent origin. With the exception of descriptions of condylomata causing deafness through obstruction of the meatus or Eustachian tubes, we find no account of nerve deafness which can yet be considered historical, although we know of certain well-known personages who were victims. For instance, there is little doubt that Beethoven's deafness was of nervous origin, and due to congenital syphilis.

\section{Eyes.}

The commonest early syphilitic eye symptom is iritis. Syphilitic iritis does not materially differ from iritis produced by other causes, but it has frequently to be distinguished from gonococcal, or the so-called rheumatic iritis. As a rule, syphilitic iritis is not so acute as the gonococcal form, but when one is confronted with a case of iritis this difference is of little value. It should be remembered that syphilitic iritis is generally a symptom of early syphilis. Gonococcal iritis is more prone to develop during a recurrent attack of gonorrhœa than during the initial infection. Gonococcal iritis may arise months after the patient has ceased to think of a discharge-i.e., during the latent stage of the disease. Gonococcal iritis is often accompanied by gonorrhœal rheumatism, arthritis, and neuritis. Gonococcal iritis is recurrent, or even periodical, in one or other eye. Once a patient has had gonococcal iritis, he is always prone to develop it again, although there may be no exacerbation of gonococcal symptoms elsewhere. Early syphilitic iritis may affect both eyes, and, as a rule, they are affected simultaneously, and it seldom recurs, unless a gumma develops in the affected eye. 
The following case will be of interest:

CASE 44.-Patient contracted syphilis in 1914, and developed bilateral iritis. As he was in the wilds of British East Africa he could only get mercury pills, which he took for two years. Late in 1916 he developed gummata in both irides, and on examination synechiæ were found, which had resulted from the first attack of iritis. Under colloidal iodine and intramine the lesions immediately disappeared.

Early syphilitic iritis, although it usually clears up rapidly under treatment, is one of the conditions which may prove very intractable, as the following case shows:

CASE 45.-The patient was admitted with iritis of the right eye, the infection having been contracted six months previously. After eight injections of arsenobenzene and nine of mercury the eye was worse, and showed a well-marked keratoiritis. C.F.T. negative. Sub-conjunctival injections of salvarsanised serum were employed, and iodides prescribed internally, without much improvement. Further injections of grey oil made the condition worse, and the eye did not clear up until the patient had had several injections of a soluble preparation of mercury (benzoate).

Late syphilitic iritis attacks one eye only; it is insidious in its onset, never very acute, and not very amenable to treatment, except to iodine and sulphur. This form of iritis is frequently accompanied with scleritis, and occasionally with choroiditis.

As gonococcal iritis is usually more acute than syphilitic iritis, and is less amenable to treatment, it will follow that synechiæ are more liable to be found in the former than in the latter infection.

True gummata of the iris and ciliary body may occur, but they are rare.

I have seen one case of syphilitic dacryo-cystitis, but it is probable that the initial site of the lesion was in the periosteum of the lachrymal bone, and this brought about a narrowing of the nasal duct and sac, owing to the swelling which had been caused. Choroiditis is, on the other hand, a common symptom of late syphilis. Almost invariably the condition is bilateral, but one eye may be worse than the other; and, as a rule, the symptoms are only subjective, with the result that the patient does not seek advice until the condition is far advanced.

The patient complains of seeing large floating specks; if asked to look at parallel straight lines, they appear curved; and the patient may have noticed dark spots in his visual field. The vitreous is usually crowded with opacities, which often blur the patient's vision.

If the case comes under observation during the acute stage, treatment will be of benefit; but in many cases the progress is very insidious, and even the most drastic form of treatment cannot arrest it. Nevertheless, treatment must be persisted in, as I have had cases in which the lesion appears to have been brought to a standstill. Naturally, the scars left by the disseminated patches cannot be altered by treatment. Sub-conjunctival medication is practised, but I cannot admit to having seen much advantage from it. 


\section{CLINICAL ASPECT AND TREATMENT OF VENEREAL DISEASES}

Interstitial keratitis is a very common symptom in congenital syphilis, and, oddly enough, it may not show itself until the patient has become an adult. I once saw a case of congenital syphilis in which the patient developed a most acute bilateral interstitial keratitis at the age of 36 . Many textbooks state that interstitial keratitis is never seen in acquired syphilis. I have certainly had three cases under my care, and in all of them there were other signs of a recently acquired infection. The lesion may be unilateral or bilateral.

Syphilitic retinitis is usually asscciated with, and secondary to, choroiditis. Isolated syphilitic retinitis is very rare. I have seen only one case, and that was in a patient who also had a syphilitic myelitis. In spite of the most vigorous treatment, he suffered from hæmorrhages into his vitreous at periodical intervals. The right eye was the eye always affected, and, oddly enough, in all the cases which have been described the lesion has been unilateral. The first hæmorrhage occurred a little more than a year after infection. As the case is of somewhat unusual interest, a fuller report would not be out of place.

CASE 46.-August, 1910.--Primary sore. Commenced treatment at once.

February, 1911.-Complete paraplegia, which ended in partial recovery after three injections of salvarsan, mercurial inunctions, and potassium iodide.

October, 1911.-Syphilitic retinitis; hæmorrhage into the right eye. Patient then had six intravenous injections of salvarsan and mercurial inunctions.

April, 1912.-Gumma on calf of left leg, for which patient was treated with mercurial injections and iodides internally.

August, 1912.-Hæmorrhage into right eye.

September, 1912.-Hæmorrhage into right eye.

November, 1912.-Hæmorrhage into right eye. Patient then had seven intravenous injections of salvarsan, and one year's mercurial treatment.

December, 1913.-Hæmorrhage into right eye.

March, 1914.-Hæmorrhage into right eye.

November, 1914.-Hæmorrhage into right eye. The C.F.T. has been negative, in spite of the recurrent hæmorrhages, for the last two years.

Syphilitic optic neuritis is not a very uncommon symptom in early syphilis, and, like most early syphilitic lesions of the eye, it is usually unilateral. Optic neuritis in syphilis has come into great prominence lately, owing to the fact that blindness resulted from the use of the earlier arsenical preparations. There is no doubt that cranial nerve lesions did increase in frequency when salvarsan first came into use; but we have since learnt that that was due in the main to the fact that salvarsan was not supplemented by mercury, or to the fact that not enough salvarsan had been given. If a case of optic neuritis is recognised early, adequate treatment with salvarsan and mercury will quickly cure it. When the body is infected with syphilis the organisms invade every part of it, and, as I have shown in Chapter XI., meningeal and nervous lesions are prevented from arising, owing to the antibodies circulating in the systemic part. Should the production of these antibodies be checked-as occurs when salvarsan is given, but not in that quantity which 
will sterilise the meninges-it allows the organisms to develop in the meninges. If they develop around or in nerves-nerves which have to pass through bony canals-it will follow that the pressure caused by the meningitis will inflame the nerve, and so lead to its atrophy.

Atoxyl amblyopia is due to a direct degeneration of the optic nerve itself, and is not secondary to a meningitis.

Although the optic neuritis occurring after treatment is usually accompanied by meningitis, to which it is undoubtedly secondary, there is no doubt that the lesion may be a primary one in the nerve itself, as evidenced by the C.s.F. being normal. The spread of the infection in these cases is probably via the lymphatics or arteries, as the incidence is higher when the chancre is situated on the face, lips, or in the mouth. These cases do not do so well under treatment, and it is probable that the arsenic has acted injuriously by lowering the resistance of the nerve tissue, because this type of case does not necessarily follow what would ordinarily be termed inadequate treatment.

In spite of the above, arseno-benzene cannot be said to cause blindness. I have given several thousands of injections, and have seen only eight cases of optic neuritis follow its use.

When meningitis is the root of the mischief, the cases do very well under further administration of mercury, iodides, and sulphur. I have seen three cases of optic neuritis in early syphilis in patients who had never received any treatment. In one case the eye became quite blind before anything could be done, and subsequently it had to be removed.

Late syphilitic optic neuritis is usually bilateral, and is due to some intracranial mischief.

It may be primary or secondary. If the former, one eye is always affected before the other, and it is practically never diagnosed until supervening atrophy has advanced sufficiently to cause the patient to seek advice, and too far to be remedied by treatment. These cases of so-called primary optic atrophy may be the only symptoms of a degenerative lesion, and remain so till the end. On the other hand, they may be followed by degenerative myelitis or encephalitis. A widespread degenerative lesion may be followed by optic atrophy, in which case the subjective symptoms, such as ataxia, etc., may venish when total blindness ensues. If lightning pains only were complained of, total blindness does not mitigate them; and that signs of inco-ordination vanish is due to the influence mind has upon them. Secondary optic neuritis is due either to a basal meningitis or to a meningitis over the frontal regions of the brain, and, if diagnosed quickly enough, it responds well to treatment. For a fuller account of these cases the reader is referred to the chapter on nervous diseases (Chapter XIII.).

Ophthalmoplegia, or multiple ocular paralyses, may be complete or partial, and may affect one or both eyes. If the external muscles are affected, the term Ophthalmoplegia externa is used, in contradistinction to paralysis of all the intra-ocular muscles-Ophthalmoplegia interna. 


\section{CLINICAL ASPECT AND TREATMENT OF VENEREAL DISEASES}

Of all the causes of the various forms of ophthalmoplegia, syphilis is probably the most frequent. The paralysis may be either sudden or gradual. If sudden, and if the patient is over 50, the paralysis, which, as a rule, affects one muscle only, is due to an arterio-sclerotic lesion of syphilitic origin, and the chances are that, sooner or later, the patient will succumb to a hemiplegia. If gradual, one muscle may be at first affected; but in most cases, sooner or later, other muscles become involved, and degenerative myelitis, or, more rarely, encephalitis, generally ensues.

Ophthalmoplegia interna, although a common symptom in degenerative myelitis-and, indeed, it may be the first symptom-may never be followed by other nervous manifestations. In most textbooks the reader will find it stated that pin-point pupils, which react to neither light nor to accommodation, always signify that a widespread degenerative lesion will ensue later; while the occurrence of late syphilitic nerve lesions, such as those just described, following upon paralysis of the external muscles, is barely mentioned.

Provided pin-point pupils (the reflexes of which have disappeared) are the only signs which the patient has, the chances are that further degenerative changes in the central nervous system will not set in. I have been able to collect eleven such cases. In three, the patients had had iridoplegia for over thirty years, and in seven in which I did a lumbar puncture the C.s.F. was normal. In cases where other symptoms are present, they barely exceed lightning pains and loss of sexual power.

Nearly every case of degenerative external ophthalmoplegia which I have seen, whether it disappears at first under treatment or not, has since developed further degenerative changes of the nervous system. The following case is a typical example:

CASE 47.-A patient contracted syphilis in 1904, and he was treated for it for three years with mercury internally. In 1910 patient complained of double vision. He had ptosis of the left eye and paralysis of the external rectus. Under treatment the eye symptoms disappeared. Two years later the classical symptoms of degenerative myelitis supervened, after the ocular trouble had reappeared.

The following is also an instructive case:

CASE 48.--Five years ago patient had an attack of double vision, which got well of its own accord. Three years ago the double vision recurred, and the patient had very severe attacks of vomiting. The patient was unaware that he had ever had syphilis.

I thought the stomach pains were gastric crises, but the patient had no other symptoms of degenerative myelitis. Under the most vigorous antisyphilitic treatment the stomach symptoms vanished, but the ophthalmoplegia extended until the patient had almost complete bilateral ophthalmoplegia and ptosis. The gastric crises returned, and one by one the other symptoms of degenerative myelitis began to reveal themselves.

In no complicated case of syphilis should an examination of the fundus be omitted. The white streaks along the vessels, and even spreading peri- 
pherally here and there, producing degenerative changes in the retina, at first sight not unlike the Retinitis pigmentosa sine pigmento, may give the observer more information about the other vessels in the body than any other test. Finally, the absence of the oculo-cardiac reflex may often clinch the diagnosis of a difficult case of nervous syphilis.

\section{Ears.}

In early syphilis, deafness is not an infrequent symptom. If the deafness is bilateral, and the patient has a bad throat, it will almost certainly be due to mucous papules in the Eustachian tubes. If unilateral, the deafness will almost certainly be due to nerve trouble, excluding, of course, first mucous papules in the external auditory meatus. The nerve trouble is primarily a meningitis, and is analogous to that described in connection with the optic nerve. The trunk of the eighth nerve may be affected, or only its cochlear or vestibular branches, or both. Syphilitic disease of this cranial nerve (neuro-recurrence) is more often bilateral than is the case in any of the other cranial nerves.

If the cochlear branch alone is affected the patient complains of deafness, which may come on suddenly, but more often gradually. Its course may be short, or the deafness may get worse so slowly that the patient's attention is scarcely drawn to it. When the vestibular nerve is involved, the patient complains of tinnitus, giddiness, and vomiting; the vomiting is irrespective of food, and is usually worse on getting up in the morning, owing to the change of posture causing a disturbance in the semicircular canals. In the early stage nystagmus is present.

The following case is typical of a lesion of the trunk of the auditory nerve:

CASE 49.-L. M., female, aged 35, came to the hospital with psoriasiform syphilides on her legs. Two years before the patient contracted syphilis, and was treated for eight weeks with inunctions in the General Hospital, Yarmouth, where she developed double iritis. Two months after leaving hospital condylomata appeared around the anus and between the toes. Since then the patient had not been treated. It was noticed that she did not seem to hear very well, and, on inquiry, she stated that she had been deaf in the right ear for six months. The deafness had come on gradually, and was slowly getting worse, and, at the same time as it commenced, noises in the ear were experienced, and the patient was much troubled with attacks of giddiness, which prevented her from going out. The patient always had the feeling as if she were going to fall forwards, and she actually did so on two occasions. The giddiness and vomiting were always worse on getting up in the morning, and the latter occurred during the day, quite irrespective of food. These symptoms, except the giddiness, were increasing in severity. When I first saw her, she had slight nystagmus, which later disappeared.

Examination of the ears was kindly undertaken for me by Mr. S. R. Scott. The left external meatus showed old stenosis, but there was no defect of hearing on this side, and electrical reactions were normal. There was a marked reaction to the caloric test in one minute at $115^{\circ} \mathrm{F}$., the patient falling to the right. On the right 


\section{OLINICAL ASPECT AND TREATMENT OF VENEREAL DISEASES}

side hearing was diminished; no artificial Romberg's sign or nystagmus was produced by syringing for three minutes with water at $118^{\circ} \mathrm{F}$; t the electrical reactions of the vestibular nerve were sluggish, but had not quite disappeared. All pointed to a neuritis of the trunk of the eighth nerve on the right side, most probably of syphilitic origin. This case is instructive, since the patient had never had " 606 ," would not have complained of her nerve condition had her attention not been drawn to it, and had never connected it with her disease. Under mercurial injections all syinptoms referable to the vestibular branch cleared up, but the deafness remained much about the same, and, like so many of these cases, was much less one day than another.

I also saw a man who became gradually deaf in one ear four months after the appearance of the sore, and who had had no treatment at all. His deafness almost completely disappeared three months after two intravenous injections of salvarsan and eight intramuscular injections of grey oil.

The cochlear branch is not infrequently implicated alone, much more commonly so than the vestibular.

If the neuritis first sets in after the patient has had the disease three or more years it is usually progressive, and responds badly to treatment. The following cases illustrate the truth of this statement:

CASE 50.-Syphilis contracted 1912. Patient took mercury internally for two and a half years. In 1915 he became gradually deaf in the right ear, which did not improve under treatment. A year later the left ear became involved, and, in spite of all treatment, the patient is now practically stone deaf. In 1918 he developed irido-scleritis of the right eye.

CASE 51.-The patient contracted syphilis fourteen years ago. The rash was a severe one, and the patient particularly remembers having had very bad headaches at the time. On two occasions patient has had gummata, and once he developed syphilitic arthritis of the right sterno-clavicular joint. For some years patient has been gradually getting deaf, first in one ear and then in the other. When the deafness started the patient complained of severe headaches and giddiness, which after a time disappeared. The patient is now practically stone deaf in both ears. Two months ago the patient suddenly lost his power of speech. On examination the pupils were unequal, right $>$ left; reflexes normal; disc shows signs of old optic neuritis; arm and leg reflexes increased, and memory gone. Treatment in this case made no alteration in the hearing.

In other cases of late syphilitic deafness nothing else may be discovered on thorough examination of the patient, and in my experience treatment in these cases is always unavailing. Late syphilitic deafness may be due to a re-awakening of the neuritis of the auditory nerve; it may also be due to a primary atrophy akin to optic atrophy, and in some of these cases the patient presents other symptoms and signs of degenerative myelitis. Auditory crises in degenerative myelitis are not unknown. Late syphilitic deafness may also be due to old Otitis media, or to inflammatory trouble, which commences in the bony labyrinth.

Apart from a neuritis of the eighth and second cranial nerves, of which the former is more frequent than the latter, the other cranial nerves are involved 
in the following order of frequency: seventh, third, fourth, fifth, and sixth. I have seen cases of facial palsy in which the nerve affection was almost the first symptom of the generalisation of the virus. All recovered completely after salvarsan and mercury.

For the past few years I have paid great attention to syphilis of the cranial nerves, and in particular to the auditory nerve, since it is the one most commonly involved. In untreated cases of syphilis, if patients in the generalisation stages are submitted to the new ear tests, which have in the main been elaborated by Bárány, who has recently received the Nobel prize for his work, many cases will be found in which the eighth nerve is involved without there being any clinical symptoms.

The main tests deal with the static portion of the ear-that is to say, that portion which consists of the utricle, saccule, and the three semicircular canals-since it is easy enough to test the organ of hearing. These tests are three in number, although it is really only necessary to do the first two, and they are as follows:

1. Revolving a person in a turning chair.

2. Douching the ears with either hot or cold water.

3. Applying the galvanic current to the ear.

These tests have the effect of producing in normal persons nystagmus and vertigo. The eyes are drawn in the direction of the endolymph movement, and are then quickly jerked back in the opposite direction by impulses from the cerebrum. The vertigo is in a direction opposite to the endolymph movement. The nystagmus and vertigo are due to the nerve paths from the ear to the eyes on the one hand, and from the ear to the cerebrum on the other hand being intact. If the auditory nerve is attacked by a syphilitic lesion, then the nerve paths are totally or partially blocked, according to the degree of the inflammation, with the result that nystagmus and vertigo do not occur.

When the auditory ncrve is affected in early syphilis, the neuritis is secondary to a meningitis; consequently, if the patient is put under treatment at the earliest possible opportunity no permanent damage to the nerve may result. When salvarsan first came in, we were struck by the increase in the number of cases which developed paresis of certain cranial nerves, a paresis which would disappear if more arseno-benzene or other antisyphilitic remedies were prescribed. At first one wondered whether this increase was more apparent than real. Experience has shown me that the increase was really greater, but it was magnified by two factors:

1. The neuritis occurred sooner after infection.

2. The course run by the neuritis was quicker, with the result that the clinical symptoms were more pronounced.

When it became customary to prescribe more than one or two injections of salvarsan, the incidence of neuro-recurrences fell rapidly, but as so few 


\section{CLINICAL ASPECT AND TREATMENT OF VENEREAL DISEASES}

injections, even when followed up with mercury, did not cure syphilis, the number of injections prescribed was increased.

This has resulted, not in increasing neuro-recurrences, which are due to a more or less localised meningitis, but in an increase of cases of generalised meningitis, in many of which the symptoms are too slight to cause the patient to seek advice. Even in some of these generalised cases the cranial nerves may be involved, and the following is an interesting case, as, in addition, it shows the usual period at which the symptoms manifest themselves after treatment.

CASE 52.-The patient, a man, aged 32, had a primary sore on the prepuce, and had just entered the generalisation stage when treatment was commenced, in June, 1916. The treatment consisted of eleven intravenous injections of kharsivan and seven intramuscular injections of mercury, which ended in August, 1916. Two months later the patient developed bad headaches, unsteadiness of gait, and became very depressed, which resulted in his being sent home as a case of shell-shock. Soon after his arrival in England he became deaf in the left ear, and had double vision. The pupils were unequal, the patient had bilateral choked dises, paresis of the left auditory and oculo-motor nerves, due to basilar meningitis. The reflexes of the upper extremity were exaggerated; in the lower extremities they were unequal and diminished. Romberg's sign was present, and the patient had pains down one leg, and had lost all sexual desire, due to a meningo-myelitis. An examination of the C.s.F. revealed the following: Pressure raised; positive lymphocytosis, 650 cells per c.mm.; albumin and globulin increased; C F.T. positive. From October till the above examination was made in January, 1917, the C.F.T. of the blood was negative (tested three times). Under colloidal iodine, colloidal mercury, and intramine, injected intravenously and intraspinally (prepared serum), the patient's condition became quite normal.

The rationale of the increase in nervous syphilis which is due to the free use of the toxic element arsenic is more fully considered in Chapter XIII.

All I need state here is that, since I have employed reducing agents along with oxidising agents in every case of syphilis, I have not seen a single case of recurrent cerebro-spinal syphilis.

While on the subject of syphilis of the cranial nerves, I should like to mention an ear symptom which commonly occurs when the facial nerve is affected. The patient complains of odd noises in his head on the side affected, and he likens them to vibrations. This is due to paresis of the nerve supplying the stapedius muscle. I have had four cases of unilateral facial paralysis. All set in suddenly, from six to twelve weeks after the sore was first noticed, and in two the stapedial nerve was affected. The vibrations complained of were not incessant, but came on in attacks, which gradually lessened as the patient got better; but in both cases the patients had reminders, even two years later.

Ménière's symptom complex-deafness, giddiness, tinnitus, and vomiting -although an uncommon sequence of syphilis, is met with occasionally as a late syphilitic manifestation; but opinions differ as to whether treatment improves the condition or not, as it is by no means easy to be sure whether syphilis is the cause, even if the patient has had the disease. 


\section{CHAPTER XI}

\section{THE BIOLOGY OF SYPHILIS OF THE NERVOUS SYSTEM}

\section{History.}

To my mind there is no more important subject in medicine than syphilis of the nervous system, the chief reason being that nervous syphilis is largely on the increase. Unless this increase is checked, and this can be done, in time syphilis may well have to be regarded, in the main, as a nervous disease. It is only in our own time that the subject has been understood, not because the ancient writers on syphilis were ignorant of nervous syphilis, but because the cases we now see did not occur in those days. If the ancient literature on nervous syphilis be referred to, the reader will find that in most of the cases described there was necrosis of the cranial bones, to which the dural and cerebral infections were secondary. There was, no doubt, an epoch in the life-history of syphilis when the bones were the main structures attacked, this being the case when syphilis invaded Europe, and when mercury was used to a point of intoxication. Jean Astruc, a French physician, early in the eighteenth century, appears to have been the first writer to regard headaches, vertigo, epilepsy, and paralysis as being in some cases caused by syphilis. Primary syphilitic meningitis was first described by Lallemand in 1834. Although not many mentions are made of spinal syphilis, in nearly all the lesion is regarded as being secondary to vertebral caries. Considering that syphilitic necrosis of the cranium and vertebræ are among the rarest of presentday lesions, I think we are justified in assuming, in addition to the frequent reference to those lesions in the old writings on syphilis, that a change has taken place in the course run by the disease. As we proceed, I shall attempt to suggest various reasons for this change. In glancing over the history of syphilis, one of the most striking facts is that the observations made by Morgagni on syphilis of the intracranial vessels remained unrecognised until Heubner, in 1874, brought them to light, and made more of his own, which hold the field to-day. There must be very few who are unfamiliar with what is known as Heubner's arteritis. The relationship between syphilis and general paralysis was first pointed out by Esmarch and Jersen in 1857, but it has taken half a century to have this relationship accurately defined. KrafftEbing proved that general paresis was due to syphilis when he vaccinated nine paretics with the exudate from chancres, and found that none reacted. Fournier, in 1875, was the first to point out that tabes was due to syphilis, 


\section{CLINICAL ASPECT AND TREATMENT OF VENEREAL DISEASES}

an opinion which has only been accepted in its entirety to-day. At one time there was no more debatable subject, as some of the leading neurologists were strong opponents to Fournier's view. In France, Charcot combated it to the last, while Gowers in England, and Erb in Germany, upheld it. It is interesting to note that syphilophobia, which is so common to-day and so difficult to handle, was often a worry to the older syphilologists. John Freind, in 1725, wrote, "that an honest practitioner generally finds it more difficult to cure the imaginary evil than the real one." John Freind was a medical practitioner in London, a Member of Parliament, and Physician-inOrdinary to Queen Caroline. From these few lines the reader can see that our knowledge of syphilis of the nervous system is of quite recent date, which would not have been the case had it been in bygone times of common occurrence. Owing to the fact that this increase of nervous syphilis is denied by many, we must consider first the evidence in support of the statement; and, second, the causes which have brought about the increase.

\section{Evidence as to Increase of Nervous Syphilis.}

Between 1892 and 1901, Nonne had in his private practice 5,500 persons with nervous diseases, from which number syphilis was diagnosed 85 times. The hospital material during the same ten years amounted to 72,180 patients, and syphilis was diagnosed 282 times. From 1903-07, out of 5,649 nervous patients seen in his private practice, Nonne diagnosed nervous syphilis 88 times. During the same period, Nonne treated 9,936 cases at the Eppendorf Hospital, and made the diagnosis of nervous syphilis 104 times. From 1907 onwards Nonne states there has been a steady increase of nervous syphilis in both private and hospital patients, which has been most notable since the advent of arseno-benzene. The above figures show an increase of two and a half times the amount of nervous syphilis in hospital patients, in only a few years. This was due doubtless in part to the better treatment the poorer classes received, because, in Nonne's hospital lists, cases of tabes and G.P.I. were not included. Naunyn, several years ago, came to the conclusion that specific disease of the nervous system appears most frequently in the first year after infection, an opinion now shared by many, although the student's idea of nervous syphilis is summed up even to-day in the words tabes and G.P.I. Quoting from Ball's translation of Nonne's book, "One may say in general that brain lues can develop in any stage of syphilis, and also that the tertiary stage is by no means the favourite one."

Between April, 1908, and April, 1909, in a large Continental venereal clinic, I saw only three cases of cerebro-spinal syphilis (meningitis). Between August, 1914, and March, 1917, I saw eighty-seven cases of cerebro-spinal syphilis, which developed clinical symptoms or pathological changes in the cerebro-spinal fluid within a year from the date of infection, and usually within three months after a course of several injections of an arsenical product 
had been prescribed. Most of the patients were private patients who had been treated with arseno-benzene and mercury, but neither sufficiently nor correctly. According to statistics of Althaus, taken from the lunatic asylums of England during the years $1838-40,12 \cdot 6$ per cent. of the patients admitted were paretics; between 1869-91, the number from the same institutions rose to 18.2 per cent. In the Deggendorf Hospital, during the years 1867-74, the percentage of paretics in men was $9 \cdot 3$, and in women, $5 \cdot 2$; while from 1885-90, the percentage of cases in men was $23 \cdot 2$, and $9 \cdot 3$ in women. Professor Wimmer wrote me saying that there had been an increase of general paralysis in Copenhagen during the past twenty-one years. Owing to the difference of opinion which was held regarding the cause of tabes, no accurate figures are handy to show whether the condition has increased or not. My own opinion is that both general paralysis and tabes have become more frequent, that they are more likely to occur in patients who have been treated than in patients who have had no treatment, and that the onset occurs earlier now than used to be the case.

Summing up the few points available, I think it may be taken as suggestive that nervous syphilis is on the increase; that the increase is most marked in the meningeal cases; and that some relationship exists between this increase and treatment.

Before considering the influence of treatment, there are some generalisations which require to be discussed:

Neurotropic Action of Organism and Neuropathic Disposition of Patient.For many years past, owing to the bustle of life, the nervous system has been subjected to a greater strain, becoming a locus minoris resistentice. Two facts may be adduced as illustrations: (1) the more common occurrence of degenerative encephalitis in those who use their brains; (2) the sudden onset of a degenerative lesion in those who considered themselves perfectly well, brought on by war strain. Life in the trenches, being continuously under fire, has frequently precipitated degenerative myelitis. I had a patient in the merchant service who became almost blind from double optic atrophy (tabetic) within three days of being thrown into the sea after his boat had been torpedoed. I had another interesting case of a man who developed cervical degenerative myelitis within fourteen days after hitting his neck on the hatchway while his ship was being shelled. The next point to consider is the nerrotropic action of the organism-that is to say, has the syphilitic organism developed a greater liking for nerve tissue, or are there special strains of organisms which show an affinity for nerve tissue?

If the organism had in general developed a greater liking for nerve tissue, I think we should have seen a greater uniformity in the lesions produced. No two cases of nervous syphilis are exactly the same, and the structures involved frequently vary in different areas in the same individual, being meningeal in one part, purely nervous in another, and vascular only in the third. 
If we take poisons which have a neurotropic action, the clinical picture given and the area involved are nearly always the same. The diphtheria toxine has a predilection for certain nerves; tubercle and alcohol seldom affect the cord. Ergot favours the posterior columns. Lead picks out special peripheral nerves, such as the musculo-spiral; and, oddly enough, that musculospiral belonging to the arm which does the most work is most frequently affected, a neuropathic phenomenon. It is true that if we go still further into the matter we find that the neurotropic action becomes less specific; but, nevertheless, there is a sufficient group-specificity to which syphilis has not yet approached. Arsenic and mercury both cause peripheral neuritis and I have seen two cases in which the latter, as a succinimide, produced musculo-spiral paralysis indistinguishable from that caused by lead.

In favour of the neurotropic action of the organism, is the fact that cases have been described in which two or more individuals-not blood relationshave been infected from the same source, and have developed a degenerative lesion later.

If there was anything in the neurotropic action of a certain strain of the specific organism, I am certain many more cases would have been described. Think of the thousands of cases of degenerative myelitis and encephalitis there have been, so that the few described, as pointing to a neurotropic action of the organism, may safely be regarded as coincidences. In my experience, up to date, I have only come across two examples.

Weygandt and Jakob ${ }^{1}$ proved experimentally that if they infected rabbits with a neurotropic strain of organism - that is, a strain that had already produced nervous symptoms - no more developed nervous lesions than rabbits which had been infected with a non-neurotropic strain. Against the neurotropic action is the fact that parasyphilitic affections of the nervous system are rare, and in many cases unknown, in spite of the fact that syphilis is very common in Turkey, Persia, Egypt, Algiers, Abyssinia, China, and in certain parts of Africa, where often more than $70^{\circ} 0$ per cent. of the natives have syphilis. Moreover, the natives who have contracted the disease have been infected by white men, many of whom develop a degenerative lesion later.

The reason why these natives do not suffer from late nervous lesions is, in my opinion, due to the fact that they are so badly treated that a period seldom arises in which the antibodies in the systemic part are absent; therefore, what organisms there are in the nervous part are kept at bay.

The Stage in Syphilis at which the Nervous System becomes involved.-It is very generally assumed that infection of the central nervous system by the organism which is the cause of syphilis takes place at a late stage of the disease.

This assumption has probably been made because of the known clinical fact that nervous lesions appear in the so-called tertiary stage of the disease, frequently at a considerable interval (sometimes many years) after the primary 
infection. There is, however, no doubt that infection of the central nervous system, including the bloodvessels and meninges, may occur at an early stage of the disease, and personal observations, which will be considered here, incline me to believe that every syphilitic nerve lesion arises directly from the presence of the organism, which reached the nervous system during the stage of the general infection.

From my own researches, I have no doubt that the leucocytozoon reaches the central nervous system during the stage of generalisation only, and that in from 60 to 70 per cent. of all cases of syphilis it is, moreover, the direct cause of the syphilitic nervous lesions. By this last statement I mean that every nerve lesion is due to the presence of the organism itself, and not to its toxine alone.

It is not in every case of syphilis that the organism becomes generalised, because, as I have already explained, the Leucocytozoon syphilidis may develop aberrantly-that is to say, only the asexual stage may be perfected. When the organism develops abnormally, the disease remains more or less localised to the site of infection. Naturally, in these cases the nervous system is spared. Every clinician is fully aware that, so far as the systemic portion of the body is concerned-i.e., in contradistinction to the nervous portion-the patient may develop no signs or symptoms during the generalisation stage, and that, in a few cases, the C.F.T. may even be negative throughout the early part of this period. The same may happen in the nervous portion. Organisms may reach the nervous system, and not give rise to signs or symptoms, and the C.s.F. may show no pathological change.

Therefore, when one states that there is evidence that the syphilitic organism invades the central nervous system in from 60 to 70 per cent. of all cases of syphilis, it does not mean that in 30 per cent. of cases it remains free.

Many nervous lesions, as we know, are purely arterial in origin, such as hemiplegia and paraplegia. Now, pure arterial lesions of the nervous system, in the early part of their career at all events, may produce no pathological changes in the C.s.F.

The parts of the nervous system first involved are the meninges and the bloodvessels. It has just been shown that an involvement of the bloodvessels cannot be detected beforehand; therefore, it is only when the meninges are infected that we can be sure that the organisms have reached the nervous system.

Unless a sufficient number of organisms reach the meninges, and unless they produce actual inflammation in the meninges, the latter being analogous to the rash in the systemic portion, it will naturally follow that there will be no signs or symptoms of a nervous infection, and the C.s.F. will be normal. From this the reader will see that, because in 30 to 40 per cent. of all cases of syphilis no sign or symptom of a nervous infection is ascertainable, it is no proof that the organisms have not invaded the nervous system.

When it is stated that in 60 to 70 per cent. of all cases of syphilis the 


\section{CLINICAL ASPECT AND TREATMENT OF VENEREAL DISEASES}

Leucocytozoon syphilidis reaches the nervous system, it means that, in 60 to 70 per cent. of all cases of early generalised syphilis, either the patient has signs or symptoms of a meningitis, or pathological changes are to be found in the C.s.F. These are the figures I have arrived at from a careful examination of a very large number of cases. Only those cases have been considered which were in the generalisation stage, and in which no treatment had been prescribed.

The reader might now say to himself, What proof is there that, in the 30 to 40 per cent. of cases in which there is no sign or symptom of a syphilitic invasion in the generalisation stage, the organisms do not reach the nervous system at a later date? The proof that the nervous system is invaded at about the same time as the systemic portion of the body, and that any late lesion which arises dates from this invasion, and from this invasion only, is forthcoming in the study of the relationship which exists between the first maculo-papular rash and the recurrent papular lesions and gummata. Furthermore, and this fact proves my point, in many of these 30 to 40 per cent. of cases, signs and symptoms of nervous lesions make their first appearance when the arseno-benzene treatment is stopped, and in many it is only then that pathological changes in the C.s.F. are to be found. Clinical manifestations of nervous involvement may sometimes be detected, while the C.s.F. is normal. Arseno-benzene sterilises the systemic portion of the body, but not the nervous portion; therefore, it would be impossible for an invasion to take place from an area in which there were no organisms.

From what has been stated, it may be reasonably assumed that, broadly speaking, the nervous portion is invaded by the syphilitic organism as often as is the systemic portion. This may not be absolutely true, but if the chances of the nervous portion being infected are considered to be as great as those of the systemic portion, it will make the physician much more careful. It will make the physician take more pains to examine thoroughly every case of syphilis; it will also make him cautious as to the amount and kind of treatment that he is about to prescribe; and it will urge him to place more value upon an examination of the patient, and of the C.s.F. after the patient has received treatment, than he has been accustomed to do heretofore.

We have now to consider the clinical evidence in support of the early nervous infection of syphilis (1) before, and (2) after treatment.

The clinical evidence before treatment is commenced is usually slight but unmistakable, and the symptoms are usually of this nature: Inequality of pupils; irregularity of the pupil reflexes; disturbances in the cranial nerves; altered elbow and radial reflexes; altered abdominal and cremaster reflexes; increase of the knee-jerks and ankle-jerks on one or both sides; occasionally a loss of reflexes; but at least a difference in the reflexes on the two sides; faint alterations in cutaneous sensations; and paræsthesiæ.

The clinical evidence after treatment is, as a rule, more pronounced, and naturally it largely depends upon the nature of that treatment. 
The following few cases will give some idea of the varied manifestations to be met with:

\section{Before Treatment.}

CASE 53.-A man, aged 27, consulted me for a rash, which was a papular syphilide. The primary sore had just healed. The pupils were unequal, and acted feebly to light, but well to accommodation. All the other reflexes, with the exception of the cremaster reflex on the right side, could not be obtained. An examination of the C.s.F. revealed a positive lymphocytosis, an excess of albumin and globulin, and a positive C.F.T.

I have had two cases in which the patient had developed typical symptoms of degenerative myelitis (tabes) within a year and a half of contracting the disease.

CASE 54.-A man, aged 23, with very severe generalised syphilis of six months' standing, had marked symptoms of an acute meningo-encephalitis. The pupils were unequal, all the reflexes in the body were much exaggerated, the skin was hyperæsthetic, and the patient complained of violent headaches and sleeplessness.

Examination of C s.F.: Pressure raised; marked lymphocytosis; excess of albumin and globulin; C.F.T. positive.

It not infrequently happens that the cases with the severest cutaneous manifestations develop meningo-encephalitis and meningo-myelitis.

CASE 55.-A man, aged 31, had a chancre nine months previously, and had had the usual symptoms of generalised syphilis, for which he had received no treatment. He then came up for advice, complaining of very bad headaches. As the patient appeared rather strange, he was admitted into hospital. The next day the patient had a fit, and was unconscious for two days. A lumbar puncture was done, relieving the condition only slightly. When the patient regained consciousness he became very strange in his habits, talked gibberish incessantly, and became very restless and excitable. A week later, after the excitable condition had given way to a more morose state, the patient became unconscious again, and died. Post-mortem it was found that the patient had an acute meningo-encephalitis and myelitis. There were some punctate hæmorrhages in the cortex of the brain, and these were especially marked in the pons. Microscopically, the cortical vessels and those of the meninges were dilated and surrounded by a lymphocytic infiltration.

I have seen another case similar to this, and the condition is exactly analogous to the hæmorrhagic encephalitis which follows arseno-benzeneindeed, it is the same condition, only not quite so acute.

Perhaps the most interesting of all the cases of early syphilitic involvement of the nervous system which I have seen is the following:

CASE 56.-A man, now aged 45, contracted syphilis in 1907. Less than three months after the appearance of the sore he developed basal meningitis, with symptoms of bulbar paralysis. He improved slightly under treatment; but when I saw him again, in 1916, he had diplopia, left facial paresis, difficulty in deglutition (ninth nerve) frequent fainting attacks (tenth nerve), and speech defect owing to restricted movements of the tongue (twelfth nerve). There was no optic neuritis; pupils reacted to 


\section{CLINICAL ASPECT AND TREATMENT OF VENEREAL DISEASES}

light and accommodation. All reflexes were much exaggerated, but there was no ankle-clonus or Babinski. There was no alteration of cutaneous sensibility, and no muscular atrophy.

\section{After-Treatment.}

CASE 57.-A man, aged 26, came up for advice concerning further treatment, having contracted syphilis one year previously, for which he had been treated with salvarsan and mercury. Patient had no symptoms of a nervous affection before treatment began, and the C.s.F. was normal.

The only symptoms of which the patient complained were full sensations in the head, which came on every third or fourth day, and lasted for a whole day. Occasionally the patient had acute pain in his left shoulder.

The pupils were unequal, and reacted feebly to light, and there was slight nystagmus. The elbow reflexes could not be obtained, and the wrist reflexes were feeble. The abdominal reflexes were difficult to obtain, and so were the cremaster reflexes. The knee-jerks were quite absent, and all tendon sensations had vanished. Romberg's sign was present.

The C.s.F. on examination gave the following tests: Positive lymphocytosis; Nonne-Apelt reaction, very faintly positive, heavy precipitate with salicyl-sulphonic acid, showing excess of protein, which was mainly albumin.

CASE 58.-A patient, who had had syphilis for nine months, came up for advice because he felt run down and could not sleep well. He had previously been treated with salvarsan and mercury, and he had no signs or symptoms of a nervous affection before treatment was begun.

Pupils were dilated, and did not react to light; all the reflexes were very brisk, but uneven, the abdominal reflexes on one side being more pronounced than on the opposite side. No ankle-clonus, but there was a bilateral Babinski's phenomenon. Tactile sensation increased. There was a positive lymphocytosis of the C.s.F., an excess of albumin and globulin, and a positive C.F.T.

CASE 59.-A patient, a boy, aged 17, contracted syphilis in October, and came under care the following January, being covered from head to foot with a diffuse papulo-erythematous eruption. After eight weekly intravenous injections of neo-salvarsan, the C.F.T. became negative, then mercurial injections were carried on until April. Three weeks after treatment was stopped the patient began to complain of headaches, insomnia, and loss of appetite. These symptoms became gradually worse, and he came up for advice again in June, by which time he had lost $2 \frac{1}{2}$ stone in weight. The boy looked pale and emaciated, the pupils were slightly unequal, and the reflexes were on the plus side, and there was a general hyperæsthesia, otherwise nothing abnormal was discovered; C.F.T. was negative. Fearing cerebro-spinal syphilis, a lumbar puncture was performed, with the following extraordinary result:

Cells : 450 per c.mm.; 68.0 per cent. lymphocytes; 27.0 per cent. endothelial cells; 5.0 per cent. polymorphonuclears.

Nonne-Apelt reaction positive.

C.F.T. positive in all dilutions-i.e., from 10.0 to $500 \cdot 0$ per cent.

\section{Influence of Treatment upon the Incidence of Syphilitic Nervous Affections.}

Curiously enough, the influence of treatment upon the incidence of syphilitic nervous affections has never heretofore been considered. A more important chapter in syphilis than this does not, in my opinion, exist. I trust it will 
not be long before the profession at large takes the matter into very careful consideration, as I have no doubt myself but that nervous symptoms are very rapidly on the increase, and, as this view is a rather disquieting one, it would be well to go as fully as possible into this question.

Two facts must, first of all, be borne in mind: one is, that the organisms reach the nervous system at about the same time as the so-called secondary rash appears, and that all future trouble dates from this invasion; the other is, that such an invasion occurs in at least 70 per cent. of all cases. As there is little communication between the blood and the C.s.F., the body, for sake of argument, may be divided into $(a)$ the systemic portion; $(b)$ the nervous portion.

In the blood, or, rather, in the serum, the natural protective substances of the host circulate. These natural protective substances are lipoid-globulin colloidal particles, and have their origin in lymphocytes, which are again mainly manufactured in the lymphatic glands. Broadly speaking, there is no limit to the production of these protective substances.

Although the blood from the systemic portion circulates in the nervous portion, the individual cells of the latter are not bathed with it in the same way as is the case in the former. When there is a nervous lesion, the protective substances circulating in the blood do not readily enter the C.s.F., in which circulates the main supply of protective substances to nerve tissue. Protective substances can enter the blood directly only from the C.s.F., and the C.s.F. is the fluid supply, so to speak, of nervous tissue. . The reagin which circulates in the C.s.F. can enter the blood-stream, and this is the partial explanation of the positive C.F.T., which is obtained in the blood, in cases of degenerative encephalitis and degenerative myelitis.

Hence, if there is a lesion of nerve tissue proper-i.e., apart from the meninges - the main supply of protective substances reaches it viâ the C.s.F. Those circulating in the blood are, nevertheless, useful. The protective substances in the C.s.F. come partly from the epithelial cells of the choroid plexuses, the supply of which bears no relative proportion to that from the lymphatic glands; partly from the blood by ultra-filtration; and partly they come from the lymphocytes, which constitute the lymphocytosis; and these in their turn come from the lymphatic vessels of the meninges and nerve tissue.

With and without treatment, the rule is for all the early symptoms of syphilis to disappear spontaneously. Treatment naturally aids their disappearance, as it increases the production and efficacy of the protective substances, or, as they may be called for short, antibodies.

Owing to the comparative weakness of the protective substances in the C.s.F., the host is partly dependent upon those circulating in his blood to overthrow the organisms in his central nervous system.

As substances cannot reach the C.s.F. viâ the blood-stream in any large quantity, it will be easily understood that treatment given by the mouth, 


\section{CLINICAL ASPECT AND TREATMENT OF VENEREAL DISEASES}

skin, muscle, or vein, is only going to reach the nervous tissue in infinitesimal doses. Therefore, the sterilisation of the systemic portion will be achieved long before that of the nervous portion.

If the systemic portion is sterilised early in the disease the production of antibodies is checked, hence an important supply to the nervous part is cut off at a time when it is most needed. The result is that the organisms may get the upper hand, and may give rise to serious symptoms.

From what has just been stated, it will readily be seen what a close connection there is between treatment and the occurrence of nervous lesions.

For the sake of clearness, treatment may be divided into four heads, and every patient will be regarded as a possible candidate for a nervous affection.

1. Treatment by Mercury Alone.-Sterilisation of the system by mercury only is a long process, hence it will be a matter of years before the supply of systemic antibodies to the nervous part is cut off. The supply to the nervous part may prevent the spores of the Leucocytozoon syphilidis from developing into their gametal forms, but it will not prevent them from extending. The spores will extend from the meninges into the cord and brain. Should the supply of antibodies be cut off when the spores have reached the cord and brain, the spores will develop in situ, and will cause degenerative myelitis and degenerative encephalitis. The more thorough and the more drastic the mercurial treatment is, the quicker and more perfect the stoppage of the supply of antibodies to the nervous system will be; therefore, the greater likelihood of degenerative myelitis and encephalitis arising, and the more meningeal in character the early symptoms will be.

As I have frequently stated, it is not the untreated cases which are more liable to develop a degenerative affection; it is those which have been well treated with mercury. In more than 75 per cent. of the cases of nerve degeneration of which $I$ have notes, the mercurial treatment has been severer than either Fournier or Hutchinson would have advised in their time.

2. Spasmodic or Inadequate Treatment by Arseno-benzene, supplemented or not with Mercury.-Although one, two, or three injections of arseno-benzene will not, strictly speaking, sterilise the system, the sterilisation being only pro tempore, they will stop the manufacture of antibodies. The spores may be still in the meninges, or may be on the point of entering the nerve tissue, hence the symptoms will be mainly meningeal. In the majority of cases these symptoms are so slight that, unless an exhaustive examination be made, nothing is detected.

3. Several Injections of Arseno-benzene, given as closely after One Another as Possible, and followed by Mercury for Two Years. - Such a treatment is able in some cases not only to sterilise the systemic part, but to sterilise the nervous part as well.

If the sterilisation of the latter is not complete, the check on the production of antibodies has been so quick and sudden that the spores will develop rapidly and early. Early development of the spores means that the development 
will take place in the meninges. Rapid development signifies that the symptoms will be severe, and therefore noticeable.

Consequently, these cases are purely meningeal, easily diagnosed, and, being meningeal, can be cured (with reserve) by further drastic treatment.

4. Several Injections of Arseno-benzene, with One or Two Injections of Intramine, and followed by Mercury and Iodine for Two Years.-This treatment has up to the present given the best results, but has not been in use sufficiently long to say whether nervous syphilis will be relegated to the past or not. I have only seen one case so treated in which nervous manifestations appeared, and that was a unilateral optic neuritis and a neuro-recurrence of meningeal origin.

These views are not hypothetical, but views which I have been forced to hold from my clinical experience of several hundreds of cases.

There are several other factors which come into play in the causation of nervous lesions, such as the resistance of the host, the protective power of the C.s.F., the virulence of the infection, and the interval which is allowed to elapse between the commencement of the generalisation of the virus and the inauguration of the treatment. Also, it must not be forgotten that spontaneous cure plays a great part in syphilitic nervous affections.

To make the subject still clearer, a brief discussion of these various points will not be out of place.

The resistance of the host plays an important rôle in this respect. The reader has doubtless often heard the remark made that, in many cases of degenerative encephalitis and myelitis, either no history of syphilis could be obtained, or that the primary stage and stage of the generalisation were slight. The remark is perfectly true, and its explanation is, in my opinion, the following:

When such a patient is infected, the generalisation of the virus takes place, but symptoms do not appear owing to the patient's natural protective power being above the normal. As times goes on, this naturally high protective power will succeed in annihilating all the organisms in the systemic portion, with the result that the formation of antibodies will be checked.

The organisms have meanwhile been resting in the nervous portion, where they do not come under the influence of this naturally increased protective power to the same extent, consequently they have sufficient life in them to spread. A peripheral spread of the organisms in the nervous portion always means a spread into nerve tissue proper, and the further the spread into nerve tissue, the less the influence the natural protective capacity of the host has upon them. Deduct from this the power systemic antibodies would have, as by this time they will have ceased to exist, and there is very little to check a widespread and active development of the organisms. The C.s.F. is not itself strong enough to vanquish the organisms, as by this time one has to counterbalance its action with the wonderful medium nerve cells form for the organisms to develop upon, hence degenerative encephalitis and 
myelitis result. Here I should like to point out a very important clinical observation. A patient who, during the latent stage, gives apersistently positive C.F.T. of the blood, stands little chance of getting a degenerative nerve lesion; while a patient who during this stage gives a persistently negative C.F.T. is more likely to develop a degenerative nerve lesion.

Therefore, I have for some time made the rule not to treat a patient who persistently gives a positive C.F.T. during the latent stage-i.e., provided his previous treatment has been adequate-because I regard such a reaction as an indication of his protective capacity, which I am only likely to damage by treatment.

Cases which have very bad symptoms during the generalisation stage are very prone to develop acute meningo-encephalitis and myelitis. Severe symptoms in the systemic portion call forth an abundance of antibodies, and the greater the call upon antibodies the more persistent their production, and not only that, the production continues in spite of the destruction of the organisms. Severe symptoms in the nervous portion call forth an abundance of antibodies, which raise the protective capacity of the C.s.F. The result is that, once the active organisms-i.e., those which cause the symptoms in hand-are vanquished, the spores are unable to spread, owing to the powerful protective bodies circulating in both the blood and in the C.s.F., consequently degenerative encephalitis and myelitis do not follow.

From what has already been stated, the reader can easily argue out for himself the influence which the interval which is allowed to elapse between the commencement of the generalisation of the virus and the inauguration of the treatment has upon the incidence of syphilitic nervous manifestations.

Broadly speaking, the earlier treatment is commenced in the generalisation stage, the greater the likelihood of the nervous lesions being meningeal in character, and therefore being earlier in appearing. The later treatment is commenced, the greater the likelihood of the nervous lesions being degenerative, and therefore being later in appearing. Naturally, the kind of treatment, the kind of case, and all the other points which have been discussed, will exert their influence in each individual case.

Spontaneous cure is a factor always to be reckoned with, although it is one which does not lend itself to discussion. Spontaneous cure naturally depends upon the protective capacity of the antibodies which circulate in the blood-stream and in the C.s.F., hence one would expect spontaneous cure most readily to follow true cases of meningitis. This we know clinically to be the case.

It is a well-known fact that many cases of degenerative myelitis are spontaneously cured, but it has not previously been pointed out that it is in the meningeal form that a spontaneous cure is most likely to occur.

The same with degenerative encephalitis. It is in the degenerative meningo-encephalitis that periods of quiescence are most common, while, in the ameningeal form, death frequently terminates the first attack. 
The reason is obvious. In the meningeal lesions the organisms are not living upon a particularly luxuriant medium, and they are more open to attack from the protective bodies both in the blood and in the C.s.F.

In the ameningeal lesions the organisms are living upon a particularly luxuriant medium, and they are only open to attack from the protective bodies in the C.s.F.

The moral of this nervous discussion is clearly that no treatment, however perfect it may be, which is begun only after the commencement of the generalisation of the Leucocytozoon syphilidis, is an absolute guarantee of a cure. For a cure to be guaranteed, the treatment must be prescribed before the organisms have reached the nervous part. This necessitates an early diagnosis of the primary lesion, and brings me to say once more that patients should be urged to come up for treatment sooner than they are now accustomed to do; that there should be sufficient men scattered about the United Kingdom who know what a sore is when they see one, as the reader will have learnt ere this that a bacteriological examination of a sore is not quite so satisfactory as it is frequently stated to be.

At one time I examined the C.s.F. after treatment of all my cases of early syphilis, and prescribed a series of injections of salvarsanised serum if $I$ found it positive; but as time went on I had to alter my procedure, because I found (1) that a negative examination did not prove that the nervous system had not been attacked; (2) that intraspinal injections did not preclude the later onset of a degenerative lesion; (3) that intravenous injections with intramuscular injections of intramine and the intermittent treatment afterwards gave better results. I have also found that a pathological C.s.F. may be a sign of the patient's protective power against the spread of the organisms, as a destruction of it has so frequently precipitated a degenerative lesion. The experience I have gained may be summed up as follows: The best way to avoid a nervous infection is to prescribe treatment before the disease becomes generalised. The best way to prevent symptoms of nervous syphilis arising is to pay more attention to the intermittent treatment than to getting a negative C.F.T. after so many injections of arseno-benzene, and then stopping treatment. Much nervous syphilis can be prevented by the routine use of intramine, which prevents arsenic and mercury from exercising a toxic influence on nerve tissue, thereby rendering it a locus minoris resistentioe for the syphilitic organism. This is proved by the fact that I have seen only one neuro-recurrence occur in cases so treated since 1915. After the conclusion of the intermittent treatment it is best to examine the patient clinically, and not to worry about the blood or the C.s.F., because it is impossible to say in any individual case whether, if one or both are positive, implication or protection is signified. It is wisest clinically to overhaul the patient annually for a few years, and to prescribe treatment only should signs or symptoms be discerned.

1 Weygandt u. Jakob (1914), Dermat. Wochenschr. Festschrift, 150. 


\section{CHAPTER XII \\ LUMBAR PUNCTURE}

For drawing off the C.s.F., I prefer Barker's needle to any other, as it is neither fine enough to be broken, nor thick enough to make too big a hole in the dura. The patient is made to lie on his left side, on a hard couch for preference, so as to avoid a spinal curve. The knees should be well drawn up, the left arm and shoulder pulled down, and the head and neck bent towards the knees. The observer should then see that the back is straight-i.e., that the plane of the back is perpendicular.

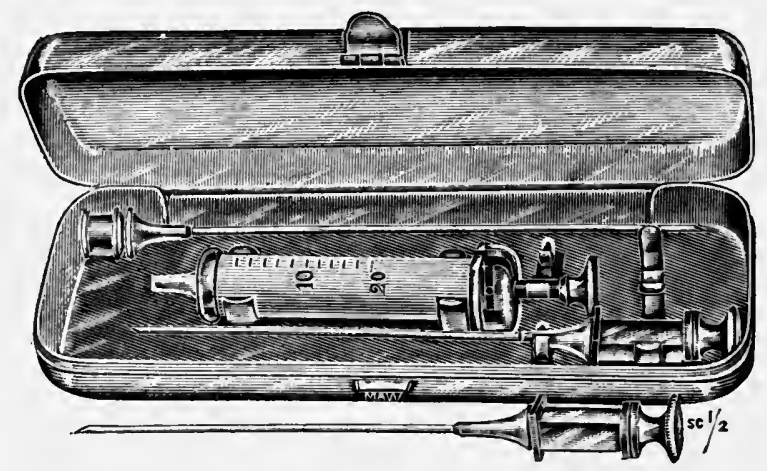

Barker's Lumbar Puncture Needles

The highest point of the right iliac crest is ascertained, and then the intervertebral space found, which lies to the sacral side of a line drawn across the back from this point. The best inter-vertebral space is the one between the fourth and fifth lumbar vertebræ, and it is not in the same position in everybody.

It is scarcely ever on the line from the highest point of the iliac crest, but almost invariably just to the sacral side, or well to the sacral side, of it.

After the injection of a local anæsthetic $(0.5$ per cent. novocain) the forefinger of the left hand is placed on the spine of the fourth lumbar vertebra, and the needle is inserted in the middle line by the side of the finger. The needle is then directed horizontally inwards until the canal has been reached. If the point of the needle impinges on bone, it should be withdrawn, and another direction tried. An experienced observer knows by the feel when he has pierced the dura mater. If the needle has entered the canal too low down, the patient experiences a sharp pain down one of his legs. Occasionally the dura mater becomes attached to the cord higher up in some individuals 
than in others, so it is always wise to pierce the skin near the spine of the fourth lumbar vertebra. Personally, I think it is easier to insert the needle in the middle line than half an inch below, the point that is usually advised, and I much prefer the recumbent to the sitting posture.

However careful or expert one may be, difficulties in tapping the theca may be met with, and the operation is similar in this respect to vene-puncture. Men who have had a very wide experience of giving intravenous injections know only too well that they may be baffled now and again. If the puncture is a dry one, it almost invariably means that the needle has been inserted too low down. Very rarely indeed it may mean that the meninges have become adherent higher up the cord, and so have shut off the continuity of the fluid. It may also result from pushing the needle too far in.

If the C.s.F. is being withdrawn for testing purposes, and a drop of blood comes through the needle, the needle should be withdrawn and the operation postponed for a week or two.

A collodion dressing is all that is required after the needle has been withdrawn. A syphilologist may be called upon to tap the theca for three purposes: (1) for testing; (2) for relieving pressure; (3) for injection.

If the fluid is only being withdrawn for testing purposes, as little should be taken as possible, because, even if the quantity is made up by injecting saline, excruciating headaches, which persist from one to seven days, cannot always be avoided.

Headaches are a little less frequent if saline is injected, and they appear to be less frequent in those cases in which the central nervous system has already been attacked by disease.

If the patient is kept in bed after a lumbar puncture, and the head is allowed to rest on a lower plane than the feet, headaches are rare. The headache usually begins next day, when the patient first gets up, therefore it is advisable, when possible, for the patient to remain in bed for two days. I have known the headache come on immediately, and be delayed for a few days, and to last as long as a fortnight. The headache may be accompanied with nausea, vomiting, giddiness, and mental confusion. However severe the headache may be, the lying posture will instantaneously relieve it.

The fact that instant relief is caused by the horizontal position strongly suggests that the headache is due to the removal of the water pad of the brain, which causes the brain to rest unprotected on bone, so irritating the fibres of the fifth and occipital nerves. Dana ${ }^{1}$ suggests that the puncture inhibits the activity of the choroid plexuses, or causes some block in the normal flow of the fluid secreted therefrom. The facts that the horizontal position increases the flow, as does compression of the abdomen, as well as relieving the pain, certainly support the theory just promulgated. Another point, demonstrated by McRobert, ${ }^{2}$ is that the headache following tapping is due to a continuous flow of C.s.F. when the puncture holes in the dura and arachnoid approximate, thereby allowing invagination of the latter through the former, and the invaginated portion to act as a drain, like a piece of gauze 


\section{CLINICAL ASPECT AND TREATMENT OF VENEREAL DISEASES}

in an abscess cavity. In most cases the puncture holes do not approximate, and so, being on a different level, the fluid cannot escape.

If lumbar puncture is done to relieve pressure, the patient is certain to be in bed, and the quantity drawn off does not matter; 30.0 to $40.0 \mathrm{c.cm}$. can be withdrawn comfortably. At any one time the nervous system contains about $70 \mathrm{c.cm}$. of fluid, and about $500 \mathrm{c.cm}$. are formed every twenty-four hours.

When an operation for pressure has to be performed, the case is almost invariably one of pachymeningitis, for which arseno-benzene has been given. Arseno-benzene causes reactionary inflammation; reactionary inflammation in an already thickened dura mater may be just sufficient to cause compression. In the case of pachymeningitis, the symptoms of compression-i.e., loss of consciousness, etc.- usually set in between forty-eight and seventy-two hours after the second or third intravenous injection of arseno-benzene. Lumbar puncture may relieve these cases at once. Since the reactionary inflammation is largely due to a vascular dilatation, injections of adrenalin are very useful.

Although a recurrence of the compression, after subsequent injections of arseno-benzene, is not likely to occur, a subcutaneous injection of adrenalin will certainly minimise the chance, and it may be administered prior to the injection should the observer require more self-confidence.

Pachymeningitis is a late symptom of syphilis; but symptoms like those caused by compression, which usually start with Jacksonian epilepsy, may occur after arseno-benzene in early syphilis. The attack generally sets in about fortyeight hours after the second injection, and is due to a hæmorrhagic encephalitis.

This form of hæmorrhagic encephalitis is a reactionary inflammation of the vessels in the cortex of the brain, which have been affected by syphilis. A lumbar puncture alone in such a case is valueless, so is trephining, the only remedies of any use being adrenalin, colloidal iodine and more arseno-benzene intravenously. Regarding lumbar puncture for injecting purposes vide Chapter XVIII.

\section{Infectivity of the Cerebro-spinal Fluid.}

The question sometimes arises as to the infectivity of the C.s.F. The question must be answered in the affirmative, because several observers ${ }^{3,4,5}$ have succeeded in producing syphilitic lesions in rabbits with the C.s.F. withdrawn from cases of cerebro-spinal syphilis, tabes, and general paralysis, Arzt and $\mathrm{Kerl}^{6}$ on two occasions succeeding in inoculating rabbits with C.s.F. which was normal to the usual tests from two cases of early syphilis. Dohi and Tanake, ${ }^{7}$ in 1905 , and several other investigators ${ }^{8,9}$ since, have been able to demonstrate the Spirochoeta pallida in the C.s.F.

The latest work on the subject comes from Wile, ${ }^{10}$ who inoculated the testes of rabbits with the fluid withdrawn from eight cases of active syphilitic disease of the nervous system. In not a single case was a permanent experimental syphilis, in the sense of a definite testicular nodule, elicited. In all the cases in which spirochætæ were found in the testes they remained for a 
time, and then gradually disappeared. Unless this work is repeated and other investigations made, it is impossible to divine the correct interpretation of this finding, but it certainly suggests that in injecting the C.s.F. only spores were injected, and that the testes did not prove a suitable soil for the development of the life-cycle.

\section{Flow of the Cerebro-spinal Fluid.}

Owing to the introduction of intraspinal medication, it is necessary to discuss the origin and flow of the C.s.F. It is well known that only a minute portion of any drug injected into the blood-stream reaches the C.s.F. It is thought by many that no arseno-benzene gets into the C.s.F. when injected intravenously, but Barbat ${ }^{11}$ showed that if a lumbar puncture was done shortly after the injection, he was able at the end of twenty-four hours to demonstrate 0.25 part of arsenic per million in twenty-three out of the twentysix fluids examined. On the other hand, Rieger and Solomon ${ }^{12}$ show that without auxiliary puncture $42 \cdot 0$ per cent. of the fluids examined revealed 0.20 part of arsenic per million one hour after an intravenous injection. If three hours or more are allowed to elapse before the C.s.F. is examined, no arsenic is found. This shows, then, that although arsenic does reach the C.s.F. when injected intravenously, its presence there may be maintained by drainage of the fluid. What do we find clinically? Drugs injected intramuscularly and intravenously are able to produce as good results in meningeal lesions as when injected intraspinally, provided a sufficient number of injections is prescribed, though the immediate action is not so rapid, but that the immediate action is as rapid if the C.s.F. is drained one to one and a half hours after the intravenous injection. As nothing like so much arsenic could get into the C.s.F. after these two manœuvres as when injected intraspinally, and as equally good therapeutic results may be obtained, especially when the second of the former is practised, it would look as if the C.s.F. was draining away rather than supplying, and that accelerating the drainage created a negative pressure which enabled the blood to give up certain constituents which the central nervous system required.' The C.s.F. is probably, as McClendon ${ }^{13}$ suggests, an ultra-filtrate of the blood, the choroid plexuses acting as the sieve, so to speak. The C.s.F. bathes the nervous tissue, and then receives its waste products to filter through the arachnoid villi into the large venous sinuses. If this be correct, then injecting anything intraspinally is only adding to the waste products which have to be excreted, hence the good therapeutic results obtained are probably not due to what is injected, but to the drainage of the C.s.F., which increases the ultra-filtration from the blood. Ultra-filtration is enhanced by inflammation, and probably the same changes occur in the colloid "membrane" of the choroid plexuses in early syphilis, as was previously described to take place in the glomerular membrane in the kidneys (vide Chapter VIII.) It still further follows that the ideal method of treatment is either to inject directly into the C.s.F. where it is formed 


\section{CLINICAL ASPECT AND TREATMENT OF VENEREAL DISEASES}

-namely, into the lateral ventricles-or into the internal carotid, employing auxiliary drainage in both cases: in the first, to accelerate the circulation; and in the second, the ultra-filtration.

From the light now thrown upon the rational route along which the drugs should travel to reach the nervous system, it is only logical to assume, in view of the important rôle the blood-stream plays in the dissemination of organisms, that the spores of the Leucocytozoon syphilidis should, during the stage of the generalisation of the virus, pass in the ultra-filtration between the blood and the C.s.F. This being the case, the organisms would flow between the arachnoid and the pia, both of which they would be expected to infect, and especially where cisterns, which favoured stagnation, occurred

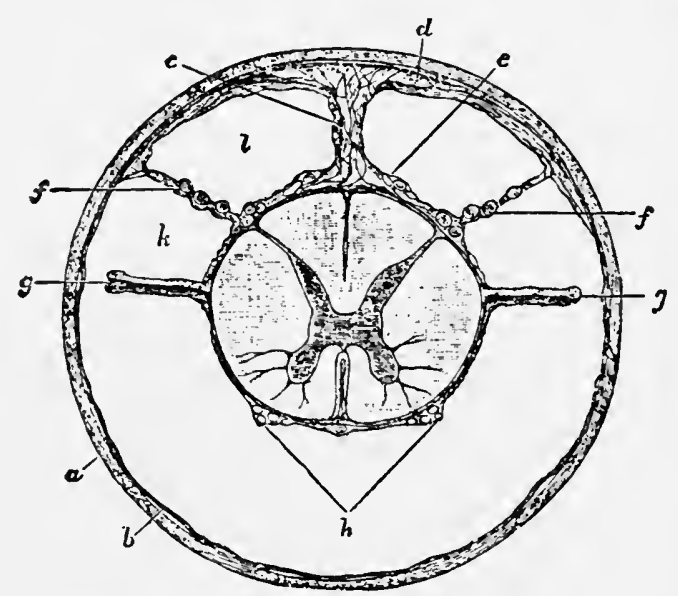

Section of the Spinal Cord within its Membranes (Key and Retzius,

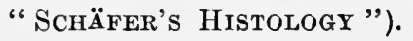

$a$, Dura mater; $b$, arachnoid; $c$, septum of arachnoid; $d, e$, trabeculæ of arachnoid; $f$, bundles of posterior root; $g$, Ligamentum denticulatum; $h$, bundles of anterior root; $k, l$, sub-arachnoid space.

in the course taken by the fluid. Intracranially, we find basal meningitis the most common lesion, and it is on the base where the principal sub-arachnoid spaces exist. One of the cranial nerves which is most frequently involved in syphilis-namely, the second-has a distinct sub-arachnoid space. Intraspinally, the sub-arachnoid spaces are on the postero-lateral surfaces of the cord, the part of the cord which is infected by syphilis more than any other. As there is a prolongation of the arachnoid and pia along the posterior roots, and another which forms the septum posticum, it seems only reasonable to suppose that in many of the syphilitic lesions affecting the cord the primary trouble is in the meninges. Looking at the C.s.F. canal as a whole, four landing-stages are to be met with: (1) optic tract; (2) interpeduncular space; (3) cervical part of cord; (4) lumbar part of cord. In the early stages of syphilis, when the meningeal inflammation is general, these stages are not 
evident; but in the late stages, when the organisms have settled down to work their mischief in certain areas, it would be expected that the areas would be the landing-stages above referred to. What do we find clinically? That in degenerative myelitis the lesion is in the posterior nerve roots either in the lumbar or cervical region, or in both, and frequently in the interpeduncular space affecting the third and sixth nerves, and in the optic tract affecting the second. It seems to me only logical to assume that there is some connection between the eally generalised meningitis and the late so-called primary degeneration. It seems to me immaterial whether the changes met with in optic atrophy, for instance, affect the nerve cells and fibres or the interstitial tissue, because we know that the syphilitic organisms may affect either, and that its presence is not necessarily productive of inflammatory changes in the nature of a response exhibited by the host, for the spore may remain dormant for an indefinite period. The idea that any syphilitic nerve lesion is due to the toxine of the organism, exclusive of the presence of the latter, is, of course, now no longer tenable. The reason why it is so necessary to show that the so-called primary degenerations are really secondary to an inflammatory focus which has occurred before, without necessarily giving rise to symptoms, and is now no longer apparent, is because appropriate treatment at the right time should minimise the chances of degeneration ensuing.

There seems also no doubt that substances from the blood can reach the C.s.F. viâ the meninges; in fact, there appears to be a relationship between the degree of meningitis and the amount of filtration possible. Dujardin ${ }^{14}$ is of the opinion that the substance in the C.s.F. responsible for the positive C.F.T. filters from the blood through the meninges, and, by comparing the positivity of the C.s.F. with the blood, he obtains an index which enables him to ascertain whether he is dealing with a meningeal or degenerative lesion. Another interesting fact is that tapping the canal may lead to the temporary formation of a lymphocytosis, or may increase it in a case of meningitis. This observation supports the view of meningeal filtration, as there is every reason to suppose that the bulk of the cells found in the C.s.F. come from the meninges. So far as syphilis is concerned, it is difficult to say to what degree the lymphogenous route is responsible for disseminating the organism.

1. Dana (1917): Journ. Americ. Med. Assoc., Ixviii., 1017.

2. McRobert (1918): Journ. Americ. Med. Assoc., i., 1350.

3. Uhlenhuth U. Mulzer (1913): Centralblt. f. Bakteriol., lvii., 158.

4. Nichols and Hough (1913): Journ. Americ. Med. Assoc., lx., 108.

5. Hoffmann (1906): Dermat. Zeitschr., 561.

6. ARZT U. KerL (1914): Wien. klin. Woch., xxvii., 785.

7. DoIII U. Tanake (1905): Japan Zeitschr. f. Dermatol., v., 537.

8. Gaucher et Merle (1910): Ann. de mal. Ven., v., 426.

9. Sezari et Paillard (1910): Société de Biologie, lxviii., 295.

10. Wile (1917): Americ. Journ. of Syphilis, i., 84.

11. Barbat (1918): Journ. Americ. Med. Assoc., i., 147.

12. Solomon (1918): Journ. Americ. Med. Assoc., ii., 15.

13. McClendon (1918): Journ. Americ. Med. Assoc., i., 977.

14. Dujardin (1918): Archiv. Médicales Belges, lxxi., 390. 


\section{CHAPTER XIII \\ THE CLINICAL ASPECT OF SYPHILIS OF THE NERVOUS SYSTEM}

\section{Syphilis of the Spinal Column.}

ALTHovar syphilis of the spinal column is rare, there are certain clinical points about it the remembrance of which may save one from making a wrong diagnosis. In syphilis, the cervical part is, almost without exception, the site of election, the dorsal part being very rarely affected, although it is the most common site in tuberculosis. Further, syphilis affects the spinous and transverse processes in preference to the bodies of the vertebræ; in fact, whenever the bodies alone are attacked, it is usually due to backward extension of a gummatous pharyngeal ulcer. The symptom complex caused by syphilitic cervical caries is almost pathognomonic. The patient first of all complains of pain in the neck, which is increased by movement, causing the neck ultimately to become stiff. The area of pain is accurately mapped out by the affected cervical nerves, and over the area there is diminished sensibility to all forms of cutaneous sensation. X-ray examination reveals erosions and bony overgrowths of the affected vertebræ.

\section{Syphilis of the Neuro-vascular System.}

The most striking point about syphilis of the neuro-vascular system is the frequency with which it appears to be infected compared to the systemovascular system. The most common lesion is endarteritis, and the reason why it is so frequent is that even the very small neural arteries are provided with vasa vasorum, which is not the case with arteries of a similar calibre in the extremities. Another striking point is the occurrence, very early in the disease, of hemiplegia and paraplegia, both of which I have seen occur three months after infection. If treatment be commenced at once, and made as drastic as possible, such complete recovery will take place as will defy detection of there having been any trouble upon the closest scrutiny. If, on the other hand, treatment is delayed, the patient may become a cripple from contractures, etc. The later hemiplegia or paraplegia occurs, the latter being much rarer than the former, the graver the prognosis, because, although the clinical signs may disappear on treatment, the occurrence of such a lesion is a warning of a fatal extension occurring sooner or later-in my experience, 
usually within three years. An interesting question arises in connection with early vascular lesions-namely, is a patient who develops hemiplegia or paraplegia early in the course of the disease more liable than another patient, not so affected, to develop a vascular lesion later? The answer is, undoubtedly, Yes. I have seen cases of early recurrent hemiplegia and paraplegia, also several cases of degenerative encephalitis and myelitis, which were preceded by a vascular lesion; and in every case of syphilitic phlebitis I have had, a recurrence has always been a venous one; added to this is the toxic action exhibited by arsenic and mercury on endothelial cells. Another interesting fact about neural endarteritis, likewise explainable on anatomical grounds, is that the softening of brain tissue which follows the vascular lesion is much more common in the regions of the large ganglia, the internal capsule, the pons, and the medulla than in the cortex or the base, although the vessels on the convexity, as well as on the base of the brain, are as frequently implicated. The reason is that in the cortex there exists to a larger degree the possibility of the occurrence of a collateral circulation. Heubner, ${ }^{1}$ in 1870 , and Rumpf, ${ }^{2}$ in 1885, showed that the arteries, which are given off at right angles from the middle cerebral, are end arteries in the sense of Cohnheim, which means that the brain region supplied by them has no possibility of a collateral blood-supply. This also explains why so often endarteritis of the basilar arteries presents the same clinical picture-viz., hemiplegia on the one hand, and symptoms of a pons affection on the other hand.

Fortunately, in cases of endarteritis occurring in the later stages of syphilis, premonitory symptoms may often be observed, and if they be correctly diagnosed, and the appropriate treatment be given, the otherwise certain onset of hemiplegia may be for a long time delayed. The commonest symptoms are headaches and dizziness; transient pareses, accompanied by paræsthesiæ, are also frequently to be met with, and the reflexes are usually much exaggerated, especially on the affected side. The paræsthesia I have most frequently come across is the sensation of cold water trickling in the vessels of the skin. These premonitory symptoms are due to the fact that the lesion at first causes only a partial obstruction, which leads to a diminution of the blood-supply, with a consequent disturbance of the nutrition of the area supplied by the distal portion of the affected vessel.

A pure vascular lesion, even when consequent softening has occurred, produces little or no change in the C.s.F. This is, of course, a helpful sign in those cases where it is difficult to differentiate between degenerative encephalitis (G.P.I.) and endarteritic softening; but as syphilis of the central nervous system attacks various structures in different levels, and as the clinical symptoms presented may only point to the implication of one structure at a certain level, even a combined clinical and pathological examination may fail to reveal the true nature of the case.

Too much emphasis cannot be laid on the protean character of syphilis of the nervous system, and every observer would be well advised in each case 


\section{CLINICAL ASPECT AND TREATMENT OF VENEREAL DISEASES}

to attempt to draw a mental picture of what he has been unable to detect, to form a relief to what he has discerned. If this most important fact had not been overlooked by the older neurologists, I doubt whether we should have had such names as tabes and G.P.I. to-day.

Tabes and G.P.I. are not clinical entities, but merely the end result of a previously undetected lesion, for the most part in another structure of the cord or brain (including meninges and bloodvessels). Broadly speaking, practically no two cases of tabes or G.P.I. are exactly alike, for the reason that they arise in various ways, and are frequently accompanied by other lesions, which are not of a degenerative character. Finally, although the latter is a fatal complication, the former practically never kills, and even in both death very frequently results from a vascular lesion, differing in no way from the uncomplicated late syphilitic endarteritis. These various points are mentioned here to show the influence which syphilis of the vessels has in the causation of the more purely nervous manifestations, a view which is still further strengthened by the frequent occurrence of degenerative encephalitis following early hemiplegia, etc. Under the heading Meningitis a causal relationship will be shown between an early meningeal lesion and a later degenerative one of the nerve tissue proper, but suffice it here to mention once more that a degenerative lesion has nearly always been a nondegenerative one at some period of its history, and that in many cases the primary trouble was a partial or complete endarteritis.

There are a few more points connected with neural endarteritis which require mentioning. A gumma is nothing more nor less than a panarteritis which produces a local cellular infiltration and necrosis, and that in the one case a gumma should form, and in the other a plain endarteritis, depends upon the resistance of the host against the infection, and the predominating phases of the Leucocytozoon syphilidis causing the lesion. Owing to there being more or less certain clinical symptoms suggestive of gummata, these lesions will be considered separately. The general arterio-sclerotic arteritis which leads to encephalomalacia, and which is such a frequent cause of death in late syphilitic diseases of the central nervous system, is a widespread degenerative condition which has resulted from the continuance of the syphilitic organism in the nervous system, as well as from the continued formation of protective substances on the part of the host, and is not a syphilitic process per se. It should not be forgotten that cerebral arteriosclerosis, whether caused by syphilis, alcohol, or any other poison, is a frequent cause of the loss of the pupil light reflex, and even of the accommodation reflex. In addition to this, symptoms of softening may so closely simulate those of syphilitic degenerative encephalitis that, in every case of the lastmentioned lesion, the diagnosis of such should not be made until every other possibility has been excluded. As syphilis of the nervous system produces no symptoms, or combination of such, which may not be produced by other causes, the mistake is apt to be made of diagnosing syphilis as a cause, when 
it is not-tumours of the brain are frequently mistaken for gummata; multiple sclerosis is especially apt to be wrongly labelled as syphilis, etc. On the other hand, as early syphilitic implication of the nervous system may frequently be without symptoms, nervous syphilis is not diagnosed as often as it should be, which is to be regretted, because in this stage it is particularly amenable to treatment. It is such a pity that clinicians should be more au fait with ataxia, etc.-end results-than with the significance of a localised headache and other easily recognisable early diagnostic symptoms. Once a lesion has become degenerative, treatment by drugs is out of the question-in fact, in many cases the free use of metals is only likely to extend the degeneration, as both mercury and arsenic may themselves cause arterio-sclerosis. I mention this because a large number of cases of nervous syphilis which one sees are cases in which the syphilitic process has healed, and the patient is only presenting the symptoms referable to the damage done. The reader will no doubt have noticed that no reference has so far been made to the cerebellum. Syphilis of the cerebellum is not common, probably because it is supplied by two large arteries, both branches from the basilar and vertebral, which afford good opportunities for a collateral circulation. This absence of cerebellar syphilis, to my mind, emphasises the great rôle played by the vessels in the ætiology of nervous syphilis, and diminishes the importance of primary degeneration. The more I see of nervous syphilis the more I am inclined to think that the so-called primary degenerations are really the end results of a previous vascular or meningeal lesion. The arseno-benzene era has brought to the fore a condition called hæmorrhagic encephalitis, which, before 1910, may be said to have been unknown. From examinations made post-mortem, and from clinical observations, I feel sure hæmorrhagic encephalitis is reactionary inflammation of a diffuse syphilitic cerebral arteritis. Clinically, ordinary diffuse syphilitic cerebral arteritis does not produce symptoms, unless there is a similar condition in the meninges or a local arteritis develops so far as to produce a monoplegia, hemiplegia, or triplegia. As time goes on, degeneration of the vessels sets in-not equally all over, because even arterio-sclerosis may be a localised process, and the development of the organisms extends extra-murally. This leads to nerve-cell degeneration, or, in other words, degenerative encephalitis (G.P.I.), which, if this is the case, can scarcely be regarded as a primary degeneration.

On the other hand, a generalised arterio-sclerosis may ensue, instead of an extra-mural development of the organisms, in which case the nerve degeneration and glial hypertrophy typical of degenerative encephalitis do not occur. In spite of this, clinically psychic changes may be produced, which makes a differential diagnosis difficult. When a case presents itself, alcoholic dementia should first be excluded, as being a condition which may most closely simulate degenerative encephalitis. Next, the age of the patient, and the age of the mode of onset of the dementia, and the course it has run, should be considered. If the patient is over 50 , and more than fifteen years 


\section{CLINICAL ASPECT AND TREATMENT OF VENEREAL DISEASES}

have elapsed since the infection, then the condition is probably arteriosclerotic encephalomalacia. In arterio-sclerosis, the psychic changes are not so pronounced as in degenerative encephalitis; the onset is very insidious, the course run is very slow, and throughout the patient's intelligence may not be much impaired. Articulatory speech disturbances are lacking, except in rare cases of arterio-sclerotic bulbar paralysis; headaches are generally severe. The pupil disturbances are not the simple loss of the light reaction alone, but this is combined with loss of accommodation as well; the course is extremely variable, but there is never a complete remission. Although the vascular lesion most commonly occurs in the internal capsule, there are some special forms of associated hemiplegia which are now and again met with in syphilis.

1. Hemiplegia with bulbar paralysis, a condition characterised by a double or single hemiplegia with involvement of the bulbar centres, causing a diffculty in speech, mastication, and swallowing. I recently saw a case of a man who contracted syphilis in 1889 , developed mono-hemiplegia with pseudo-bulbar paralysis in 1900, during which attack he had to be artificially fed, as food placed in his mouth came out through his nose. He completely recovered from this attack, but in 1919 developed double hemiplegia, with a return of the pseudo-bulbar paralysis, which soon proved fatal.

2. Hemiplegia with hemianopsia. In these cases the hemiplegia is slight and transient only. A characteristic feature is persistent anæsthesia, and pains of the extremities and hemianopsia. As a rule the deep reflexes are not exaggerated. In some cases there is paralysis of emotional facial expression on the affected side, without paralysis of the facial muscles. The lesion is in the optic thalamus.

3. Hemiplegia with third nerve paralysis. This condition is not at all uncommon, but is more often due to an extension of the meningeal inflammation into one or both peduncles than to a primary endarteritic lesion therein. The lesion causes a paralysis of the third nerve on the same side, and hemiplegia on the opposite side. Most of the cases I have seen had other signs of a basilar meningitis, and responded well to treatment.

4. Hemiplegia with third, fifth, sixth, or seventh nerve paralysis. This association points to involvement of the pons, and which of the four nerves is affected depends upon the situation of the lesion. The primary lesion is an endarteritis of the basilar artery, which causes a pronounced hemiplegia, with some paresis on the opposite side. There is usually anæsthesia and ataxia on the same side, and spasmodic weeping and laughing are common. If the lesion be high up, oculo-motor palsies occur; and if low down, fifth and seventh nerve palsies on the opposite side. In fatal cases, due to an extension of the process, paralysis of the bulbar centres ensues. There is not much difficulty in diagnosing these cases, and very little satisfaction to be obtained from doing so; the cases which really are difficult, mainly because the patient is never properly 


\section{PLATE XLVI}

No. 85.-A Case of Sypililitic Cironic Anterior Poliomyelitis (Remitring Spinal Amyotropily)

Note the wasting of the muscles of the upper extremities, especially of the left shoulder girdle, and the position in which the fingers are held, consequent upon atrophy of the intrinsic muscles of the hands. The left deltoid and the muscles of the left forearm were particularly wasted. The condition started two years before the picture was drawn and fourteen years after the syphilis was contracted. The wcakness appeared to reach its climax about four months after the onset. Treatment had no effect. An examination of the C. s. F. revealed the following: Pressure, raised; cells, 38 per c.mm. (lymphocytes, 90 per cent.; endothelial cells, 10 per cent.) ; albumin, 0.02 per cent.; globulin, faint trace. No reduction with Fehling. C. F.'T. negative. 


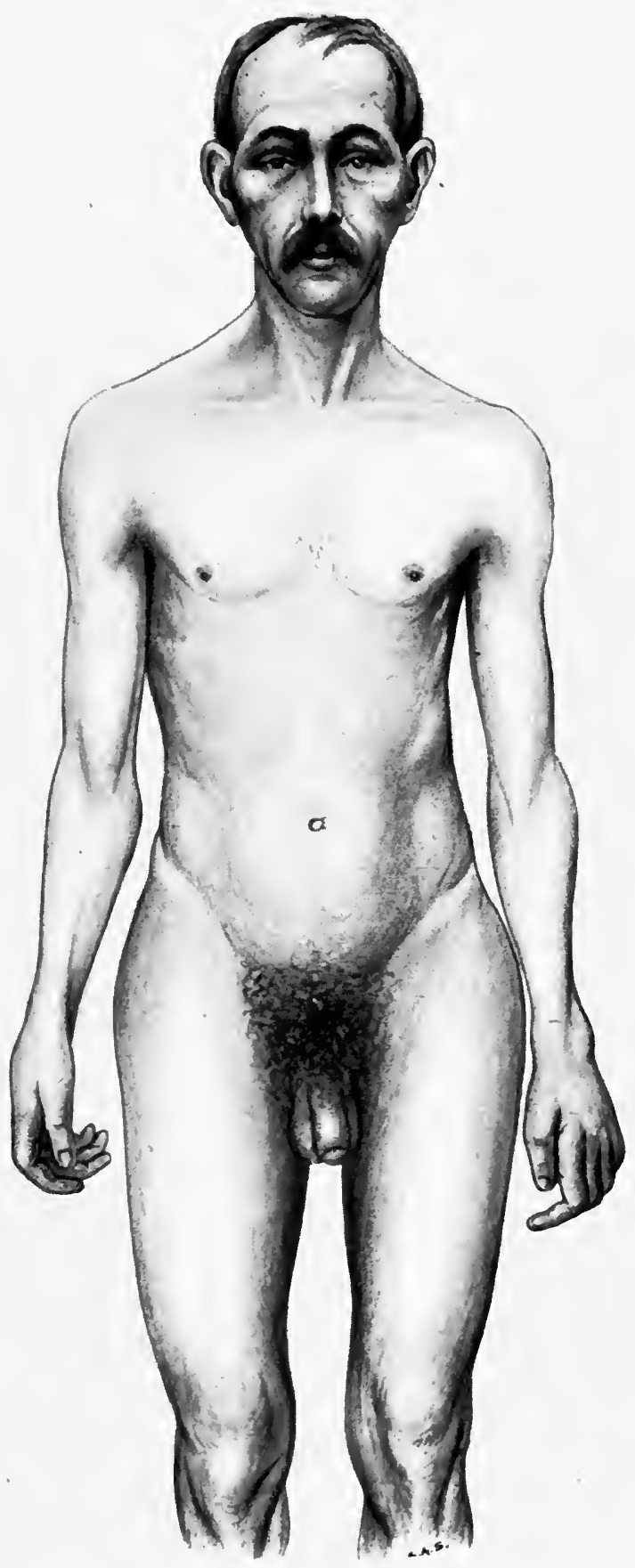

No. 85

Plate Xl.vi 
examined, are those of early syphilitic thrombosis, which, if diagnosed, could be prevented to a certain extent from causing hemiplegia later. Every case of early generalised syphilis ought to be thoroughly examined, as syphilitic thrombosis is not uncommon, and almost invariably exhibits premonitory symptoms. Headaches are common; attacks of giddiness may be complained of; insomnia is characteristic, although the patient may be drowsy during the day-time; and paræsthesiæ are invariable.

Although what has been described as affecting the brain occurs also in the cord, it is not so easy, in the case of the latter, to differentiate a primary myelitis from a meningo-myelitis. The cases of paraplegia which come on quite suddenly and occur in the early stages of the disease are cases of primary myelitis, due to an endarteritis of one of the branches of the anterior spinal artery. Provided treatment is instituted at once, complete recovery ensues, and, in my experience, there is not a very great tendency for the lesion to recur. To show what treatment will do, I need only cite a case of complete paraplegia with sphincter paralyses, in which, because treatment was begun on the second day, the patient was able to play tennis four months later. If treatment is delayed, then permanent damage results. Thrombosis of the anterior spinal artery may occur without producing paraplegia, giving rise only to pains in the back, slight bladder disturbance, and paræsthesiæ in the lower extremities. Syphilis also causes what is known as chronic anterior poliomyelitis, or remitting spinal amyotrophy, a lesion due to obliterative vascular disease. The trouble is insidious in onset and slow in progress; it may respond to treatment at first, but in all cases recurs sooner or later, to end fatally, usually with symptoms of bulbar paralysis. The clinical picture is characteristic. The symptoms of paralysis precede the atrophy; there are slight, or no signs of involvement of the pyramidal tracts, and there is sometimes a slight fibrillary tremor. In all the cases so far described, only the upper extremities were affected. The shoulder-girdle only may be involved; but, in some instances, the muscles of the arms, forearms, and hands become completely paralysed and atrophic. There have been no sensory symptoms in any of the cases so far described. The few cases which came to autopsy showed vascular lesions, with secondary degeneration of the anterior horn cells and of the anterior fundamental column. Curiously enough, at the time of writing, a case turned up in my out-patients at the Lock Hospital, the history of which was as follows:

CASE 60.-A man, aged 43, contracted syphilis in 1902, and took mercury internally for three and a half years. He had no recurrence, and was perfectly fit until he noticed he could not use his left hand well, about the beginning of 1918. Six months later the same condition appeared in his right hand. On examination, the muscles. of both hands were atrophic, and some of the fingers firmly flexed. According to. the patient, the weakness first affected the little finger of the left hand. Both hands, were practically useless. The left deltoid had almost gone, the right not so mueh, and atrophy was well advanced in the muscles of both forearms. The pupils were 


\section{CLINICAL ASPECT AND TREATMENT OF VENEREAL DISEASES}

equal, but reacted sluggishly to light; the other reflexes were normal; there was no affection of the pyramidal tracts nor alteration of cutaneous sensibility, and nothing else abnormal was found.

This is only the second case I have seen, and in both the weakness appeared to affect first the little finger of the left hand.

Chronic anterior poliomyelitis, which is probably always due to syphilis, must not be confounded with the spastic form of progressive muscular atrophy, which is by no means invariably caused by syphilis. Although the wasting may be particularly noticeable in the intrinsic muscles of the hands, all the reflexes are exaggerated, and the gait is usually slightly spastic. In syphilitic progressive muscular atrophy, beside the symptoms just mentioned, which are due to a lesion affecting the pyramidal and lateral tracts, as well as the anterior horn cells, the trouble may start or end in the medulla, when the patient first notices some difficulty in speaking and swallowing, and death in either case is nearly always due to bulbar paralysis. Both conditions are alike, in that there are no sensory changes. The spastic form of progressive muscular atrophy is better known by the name of amyotrophic lateral sclerosis. The only case I have seen of this condition improved at first under treatment, but a few months later the lesion rapidly progressed, and the patient died eighteen months later of hemiplegia with bulbar paralysis.

Syphilitic vascular disease may so affect the anterior horns as to be responsible for the clinical syndrome of Landry's paralysis, as evidenced by cases published by Macnamara ${ }^{7}$ and Sutherland. ${ }^{25}$

It may be very difficult, without an examination of the C.s.F., to differentiate between chronic myelitis and meningo-myelitis. In pure vascular lesions the C.s.F. is more or less normal, while in meningeal lesions there is always an increase of cells and protein. It may be sometimes difficult to distinguish between a syphilitic and a non-syphilitic vascular lesion, such as a meningeal hæmorrhage, for instance. If blood is found in the C.s.F., provided it has not been produced by the operation of lumbar puncture, the lesion is certainly not syphilitic.* If the C.s.F. is coloured yellow, then an almost certain diagnosis of malignant tumours may be made. I have seen this clinch a diagnosis in two cases which were clinically considered to be gummata.

There still requires to be mentioned syphilitic neuro-phlebitis, about which, unfortunately, little is known. I have only come across two cases of pure venous syphilis, the histories of which were as follows:

CASE 61.-The patient had syphilitic phlebitis of the right leg, and it developed three months after infection. In spite of thorough treatment, he developed a recurrent superficial phlebitis in the same leg, which travelled downwards from just below the knee to the foot a year later. The next recurrent lesion was a transient monoplegia and aphasia, which rapidly improved under treatment. The next recurrence was phlebitis of the right popliteal vein. Three years later, the patient was kicked on the left leg, and developed a descending syphilitic phlebitis. Con-

* Erythrocytes may be found in the C.s.F. in cases of hæmorrhagic encephalitis. 
sidering that every lesion was a venous one, I think the assumption that the monoplegia and aphasia were due to phlebitis is justified.

CASE 62.-A woman presented herself with a rash, which I diagnosed as venous syphilis (vide Plate XX.). Four months later she returned with syphilitic phlebitis of the right internal saphenous vein, which was of the descending type, and signs of beginning myelitis-incomplete spastic paraplegia.

Lamy ${ }^{4}$ describes a case of acute myelitis in which pathological changes were to be found in the veins only; also a similar condition was discovered in a case of meningo-myelitis by the same author.

\section{Gummata.}

Gummata may affect the brain or cord, primarily or secondarily due to extension into the nerve tissue proper from the meninges; they are almost invariably multiple; they may be large enough to form tumours, or so small as only to be recognised by aid of the microscope. Gummata of the brain usually affect the surface of the base and then the frontal area, and in most cases are associated with syphilitic meningitis. Miliary microscopic gummata may be found in the brain substance either in or near the cortex, and in the region of the cranial nerve nuclei. These lesions are primarily vascular in origin, and they may give rise to clinical pictures typical of degenerative encephalitis and myelitis respectively. Gummata associated with meningitis do well under treatment, provided no compression or degeneration of nerve tissue has taken place. Miliary gummata occurring in nerve tissue may improve for a time and to a certain extent, but practically always they recur to produce degenerative changes, which are progressive, whatever form the treatment may take. Multiple or solitary gummata may also occur in the spinal cord, either in the white or grey substance; but in most instances there is an associated meningitis, therefore the symptoms produced will be described under meningo-myelitis. Gummata associated with meningitis produce changes in the C.s.F. typical of a meningeal infection, while miliary gummata may give rise to little or no alteration. It is often difficult to differentiate between tumours proper and gummata, especially as the former may be temporarily relieved by iodides, due no doubt to the influence iodine has in reducing the surrounding inflammation. Even an examination of the C.s.F. may fail to give assistance. One sign, if present, is pathognomonic of new growth, and that is xanthochromia of the C.s.F., which is most pronounced in the quickly growing metastatic sarcomata.

\section{Syphilis of the Peripheral Nerves.}

Syphilitic neuritis may be single or multiple. The lesion, when one nerve is affected, is usually to be found in its distal part, while in those cases in which more than one nerve is involved the lesion or lesions are either in the plexus itself, or the case is one of a root neuritis. The so-called syphilitic polyneuritis, which is only met with in the generalisation stage, is a peripheral neuritis, and is probably due partly to the presence of the organisms them- 


\section{6 CLINICAL ASPECT AND TREATMENT OF VENEREAL DISEASES}

selves in the endo- and perineurium, and partly to the toxines which are elaborated by the death of the spirochætæ in situ. That the neuritis is most probably partly a toxic neuritis is proved by the fact that, in nearly all cases of syphilitic polyneuritis, the patient has had mercurial treatment. Because of this fact, it has been frequently maintained that the polyneuritis under discussion was a neuritis due to mercurial poisoning. This is certainly not the case, because if mercury is pushed in such a case, or arseno-benzene is prescribed, all the symptoms disappear.

The most common nerve to be affected, when the neuritis is single, excluding the cranial nerves, is the sciatic nerve; but it must be remembered that neuritis of the sciatic nerve is not infrequently secondary to a syphilitic lesion in the neighbourhood. It may be secondary to a gumma in a muscle, or to a periostitis of the ischium. It depends upon the position of the inflammation, whether the motor part is more involved than the sensory part, or vice versâ.

Treatment in these cases should be begun as quickly as possible, so as to prevent the fibrous tissue contraction from causing degeneration of some of the nerve fibres.

If fibrous tissue contraction and degeneration have set in before treatment is prescribed, it may be a matter of weeks before the patient is relieved, and, even after drastic treatment with arseno-benzene, pains may still persist. In all cases of peripheral neuritis not of syphilitic origin, the administration of arsenic is bound to aggravate the symptoms in increasing ratio as the number of the injections is raised; hence this form of aggravation can be differentiated from that of reactionary inflammation, which only follows the first two or, at most, three injections. In syphilitic neuritis of the sciatic nerve, the sensory fibres are more often affected than the motor fibres. If the neuritis is mainly a motor neuritis, the chances are that the inflammation is in the sciatic plexus, and if only one motor nerve is affected, and it is most often the peroneal, it is to be feared that the lesion may be central, although a gummatous perineuritis must be first excluded.

The brachial plexus is, in my experience, the plexus most frequently affected, and the side I have seen most frequently involved has been the right. It is characteristic for a syphilitic plexus neuritis to be unilateral. When the condition is bilateral, it is almost certain that the patient has a root neuritis, or, in other words, a cervical pachymeningitis.

In the cases of brachial neuritis which I have seen the motor disturbance has always been greater than the sensory disturbance; while in two cases of root neuritis I have had under my care, the sensory signs were more pronounced than the motor signs. In both of my cases of root neuritis, the C.s.F. was markedly pathological, and indicated a meningitis; while in one of my cases of brachial neuritis the C.s.F. was normal. In both the cases of root neuritis referred to, the upper extremities were much more involved than the lower ones. 
Both plexus neuritis and root neuritis do excellently under treatment with arseno-benzene and intramine, but in the case of the latter it is advisable to practise auxiliary tapping of the spinal canal.

Polyneuritis syphilitica is most commonly to be met with in the generalisation stage, while the other forms just described generally occur round about the fourth year and later, seldom earlier. Neuralgias are sometimes due to syphilis, the two commonest being trigeminal and intercostal. There is nothing characteristic about them, and the diagnosis can only be confirmed should the pain disappear with treatment.

\section{Syphilis of the Cranial Nerves.}

As syphilis of the cranial nerves is usually secondary to a meningitis, and as in many of the other cases an involvement of these nerves form a part only of a widespread degenerative condition, a description will be found under the headings of Meningitis, etc., but the importance and difficulty of the subject form a sufficient excuse for any reiteration under a separate heading.

Olfactory Nerve.-As this nerve is seldom examined, an involvement thereof is usually missed. A diminution or destruction of the sense of smell usually points to a frontal meningitis, with or without a basal meningitis.

Optic Nerve.-Lesions of the optic tract are most common in the optic nerve and the optic chiasma. An optic neuritis occurring early in syphilis is almost invariably secondary to a meningitis. The lesion is usually unilateral, and responds readily to treatment, if sufficient be prescribed. The incidence of optic neuritis was increased when arseno-benzene came in, but it was soon recognised to be due to inadequate use of the drug, and not to the toxic action of arsenic. The toxic neuritis which rapidly went on to atrophy, caused by arsenic in such preparations as atoxyl, arsacetin, etc., was invariably bilateral, and it is interesting to note that cats are particularly liable. Toxic neuritis is seldom diagnosed until pronounced atrophy has occurred, while meningitic neuritis is diagnosable at once, because conjunctivitis and photophobia usually call one's attention to examine the affected eye. A bilateral optic neuritis, which occurs in the later stages, is due either to a frontal pachymeningitis, when no other cranial nerves, with the exception of the first, are involved, or to a widespread basal meningitis, when a neuritis of one or more other cranial nerves will almost certainly be found to co-exist. Atrophy only occurs in these cases when the condition has been of long standing, and may always be prevented, providing early and sufficient treatment is prescribed. The ophthalmoscopic changes consist in optic neuritis and choked disc, or in a combination of the two. It should always be borne in mind that there is little or no impairment of the field of vision until atrophy has ensued, therefore casually asking a patient if his sight is affected is useless. We are indebted to Alexander ${ }^{8}$ for pointing out an important difference 


\section{CLINICAL ASPECT AND TREATMENT OF VENEREAL DISEASES}

between secondary and primary optic neuritis. In the former, which is a perineuritis, central vision is first and most affected, because the peripheral fibres go to the macula lutea, the point of sharper vision. In primary parenchymatous neuritis, the central parts are first and most severely affectednamely, the fibres which go to the periphery of the retina. This explains why primary optic atrophy is often far advanced before the patient is aware of any change in his sight, and why in many cases total blindness does not occur. Primary optic atrophy is practically always only one of the signs of a degenerative myelitis. It may be the first symptom, and therefore for a time may occur alone. According to Maloney, ${ }^{8}$ when the disease starts in the optic nerves, the lesions there are usually more severe than if the optic follow spinal lesions. Whether the optic trouble precedes or succeeds the posterior columns' degeneration, one eye is nearly always involved before the other, but the other never escapes. An interesting prognostic observation was made by Pierre Marie, ${ }^{10}$ that, when tabes has lasted several years without optic atrophy, optic atrophy seldom supervenes. The disease affects mainly the intracranial portion of the nerve, which accounts for a normallooking disc being associated with a considerable loss of vision.

Although the atrophy is probably always slow in progress, the diminution and loss of vision may be very sudden, as in the following case:

CASE 63.-An engineer on board a vessel which was torpedoed nearly lost his life, but was picked up out of the water in time; three days later he was practically blind in both eyes, although before the incident he said his vision was normal. On examination, six months later, I found he had right Ophthalmoplegia externa, bilateral optic atrophy, loss of reflexes and of sensibility to pain over certain root areas in the upper and lower limbs.

Unfortunately, syphilitic optic atrophy is irreparable, and in no case have I seen treatment do any good or delay the gradual progress of the trouble. One of the most interesting points in connection with optic atrophy is that the spinal lesions are mild, and appear to improve on the onset of the eye trouble, which was first noted by Benedikt, ${ }^{10}$ in 1881. Déjérine and Martin ${ }^{11}$ concluded that optic atrophy occurring at the beginning of tabes almost always arrests the development of sclerosis of the posterior columns, and at the same time diminishes the sensory symptoms. The reason why a patient with optic atrophy and ataxia becomes less ataxic as the duration of the blindness continues is undoubtedly due to psychic influences, such as a tranquil mental state, which blindness insures. Many are familiar with cases which have been described, in which ataxia first became evident the moment the patient realised he was suffering from Locomotor ataxia. The relationship between the mental state and the symptoms of degenerative myelitis is well portrayed in Maloney's most interesting monograph. According to this lastmentioned observer, the relations of blindness to ataxia may be postulated as follows: 
I. Improvement in ataxia will result from blindness after an adequate interval has elapsed:

(a) When inco-ordinating lesions remain stationary, and the mental capacity for training is not destroyed by cerebral disease.

(b) Even if the structural tabetic lesions leading to inco-ordination extend, provided that the effect of this extension be less than the compensating influence of the blindness upon the mental state.

II. No change in the ataxia will follow loss of vision if the advance of the inco-ordinating lesion just neutralises the beneficial influence of the blindness. Obviously this stationary phase can be but temporary.

III. Increase of ataxia, in spite of the blindness, arises:

(a) When the inco-ordinating lesions advance faster than the coordinating tendency of the blindness grows.

(b) When blindness is accompanied by mental deterioration.

Mott's ${ }^{12}$ statement that 50.0 per cent. of early optic atrophies develop general paralysis is interesting, as affording a posssible explanation of cases of this class. Syphilis is not an uncommon cause of hemianoptic disturbances, and, when so, basal meningitis is usually the cause of the trouble; hence the reason why homonymous hemianopsia is more frequent than heteronymous. Oppenheim ${ }^{13}$ has described an oscillating form of hemianopsia occurring in cases of syphilitic basal meningitis.

Oculomotor Nerve.-Owing to the peculiar blood-supply of the third cranial nerve, a hæmorrhagic lesion is apt to affect a portion of the nerve only. Sudden diplopia in a patient over 50 is almost pathognomonic of a later occurring and fatal vascular lesion. I have observed three cases; in all the diplopia occurred over-night, disappeared under treatment, and was followed by a fatal hemiplegia within three years. Oculomotor paralysis occurring in the early stages of syphilis is always secondary to a basal meningitis. The third nerve may be the only nerve affected, or may be associated with a second nerve palsy, or with a paresis of the fourth, fifth, sixth, seventh, and eighth nerves. When the third nerve alone is involved, or with the second as well, the lesion is generally unilateral, and not at all infrequently associated with polyuria due to an involvement of the pituitary gland in the meningitic process. When the later cranial nerves are implicated as well, the lesion is generally bilateral, one side being worse than the other, and the meningitis is usually of long standing. The third nerve is affected more frequently than any other cranial nerve, next coming the sixth, and the second. Ptosis is one of the most common symptoms of third nerve palsy, and it may be the only symptom. Occurring in the later stages of the disease, ptosis, with or without incomplete Ophthalmoplegia externa, which has come on slowly, no other nerve being involved, is, in my experience, almost certainly to be fol- 
lowed by degenerative myelitis, far less often by degenerative encephalitis later. In nearly every case I have seen, the symptoms have at first disappeared under treatment, to be followed in months or years by a recurrence of a severer nature, which did not respond to treatment, and on examination the patient evidenced disease of the posterior columns. I have kept four cases under observation for several years; in all, the patients developed gastric crises, cutaneous anæsthesia, and loss of reflexes, but never lightning pains nor ataxia. Whether the association of third nerve atrophy with gastric crises in my cases is a coincidence or not I cannot say, as no reference is made to it in the literature. The close connection between third nerve and sympathetic nerve trouble and the possible sympathetic nerve origin of gastric crises may afford some explanation. Owing to the peculiar blood-supply, isolated gummata of the third nerve nuclei are by no means uncommon. However extensive the lesion be, it is rare to find the ophthalmoplegias complete. I have never seen a case of complete Ophthalmoplegia externa, but have observed cases of complete Ophthalmoplegia interna, which has been the only symptom, and was undoubtedly due to an arterio-sclerotic process. The isolated loss of the reaction of the pupil to light and convergence is, in my experience, not followed so frequently by degenerative myelitis and encephalitis as is usually considered to be the case. Two cases observed, which are since deceased, died from a cerebral apoplexy.

On the other hand, complete Ophthalmoplegia interna may be followed by degenerative myelitis so many years later, and the signs and symptoms may be so mild, that it is well to bear in mind the possibility of such a sequela happening.

As some people are born with unequal pupils, and others develop the phenomenon later through various causes, and as conditions other than syphilis may produce an alteration in the reflexes, syphilis should not be too hastily diagnosed, especially when the pupil changes are the sole signs obtainable.

Trochlear Nerve.-Fourth nerve paresis is extremely seldom found alone, and is usually associated with paralysis of the third nerve in gummatous basal meningitis. The same applies to the Trigeminal nerve.

Abducens. - The sixth nerve is frequently involved. Arterial lesions of the pons may cause a disturbance of the sixth nerve, with a paralysis of the seventh on the same side. The sixth nerve may be involved in gummatous basal meningitis. When a paresis of the sixth nerve is the only symptom, then it may practically be assumed that, sooner or later, posterior column degeneration will ensue.

Owing to the rapid way in which some patients are able to suppress the false image resultant on the diplopia, the condition is frequently missed; therefore, in all suspected cases the manœuvre recommended by Mirallié and Desclaux ${ }^{14}$ for the detection of the suppressed image should be performed. Prepare a conical paper tube, $25.0 \mathrm{~cm}$. long, $4.0 \mathrm{~cm}$. in diameter at the base, 
and $1.0 \mathrm{~cm}$. in diameter at the apex; also a dark-coloured circular patch, $3.0 \mathrm{~cm}$. in diameter, in the middle of a large white card. When the card is 6.0 metres distant, if the patient who has a suppressed image places the base of the cone to one eye and looks at the dark central patch, he sees, not one, but two patches. In my experience, the three muscles which are most commonly affected are the internal rectus, external rectus, and superior oblique, in the order named.

Facial Nerve.-Paralysis of the seventh nerve occurring with arterial disease of the basilar is due to a pontine lesion. The most frequent occurrence of facial paralysis is in the early stages of the disease; in fact, I have seen three cases in which there was unilateral paresis of this nerve before the rash had made its appearance. Facial paralysis is not at all uncommon as a neurorecurrence, when it, from an examination of the C.s.F., would appear to be caused by specific meningitis. Although the paralysis does ultimately disappear in these early cases, treatment does not influence it as readily as it does in similar lesions of the second and third nerves. Owing to paralysis of the nerve which supplies the stapedial muscle, the patient is troubled for a long time with a peculiar form of tinnitus.

Gummatous basal meningitis may implicate the seventh nerve, but always in association with other cranial nerves. Transient facial paralysis occurring in the late stages is practically diagnostic of posterior column degeneration. The following case illustrates this point well:

CASE 64.-Patient, a man, aged 46, was admitted with a peripheral unilateral facial paralysis. He gave a history of having had a sore on his penis ten years before; which was undoubtedly syphilitic from the nature of the scar. On a careful examination nothing else abnormal was discovered, except for some patches of vitiligo, which the patient had not noticed. Under specific treatment the paralysis vanished, but at the time I hazarded the opinion that the patient would soon return with a recurrence thereof. Six months later the paralysis of the same nerve reappeared, this time associated with paralysis of the eighth nerve on the same side. No other nervous manifestations were demonstrable, but the lesions did not vanish under treatment. About one and a half years later the patient presented himself with all the signs and symptoms of a degenerative myelitis.

Auditory Nerve.-The eighth nerve is commonly affected in the early stages of syphilis, probably more often than any other cranial nerve. It is a common neuro-recurrence, and in these cases a meningitis is the cause of the trouble. Rapid response to treatment is to be noted; the trunk of the nerve may be affected, or only the vestibular or cochlear portions. Vestibular lesions are more frequent than auditory, and are often missed. Provided middle-ear disease can be excluded, dizziness, swaying, diplopia, and nausea, complained of by a patient in the generalisation stage, should be an immediate summons to apply the three well-known tests to ascertain if the vestibular nerve is affected, because the nystagmus which occurs when the neuritis first appears soon vanishes. 


\section{CLINICAL ASPECT AND TREATMENT OF VENEREAL DISEASES}

1. The water test: The head is maintained upright and the ear is irrigated for twenty seconds with warm water.

2. The galvanic test: One electrode is placed on the tragus of the ear and the other applied to the back or any indifferent site. According as the anode or cathode be placed to the ear, the result differs.

3. The rotary test: The patient is placed in a revolving chair and rotated about ten times in twenty seconds.

In a normal person any one of these procedures produces nystagmus, which vanishes after a few seconds. If nystagmus is not produced and its direction is altered, there is a lesion of the vestibular nerve.

The disturbances in hearing consist in a loss of the high tones, and in a loss or diminution of hearing by bone conduction.

In the later stages of syphilis, the loss of hearing may be due to labyrinthine disease, when it is not an infrequent prodromal symptom of degenerative myelitis. In the cases I have seen, the deafness has been very slow in its progress, and is very liable to be accelerated by treatment with arsenic and mercury.

Although Tinnitus aurium occurs in every case of auditory neuritis, and is much complained of by the patient, it does not follow that in all cases there is an eighth nerve lesion, as it may occur in almost any intracranial trouble, being especially noticeable in cases of arterio-sclerosis.

Glosso-pharyngeal Nerve.-I have never seen the ninth nerve affected, except, and then very rarely, in cases of diffuse basal syphilitic meningitis. Syphilitic implication of the Spinal accessory nerve is unknown to me, but I have seen a case of hemiatrophy of the tongue due to a lesion of the Hypoglossal, which ultimately developed signs and symptoms of posterior column degeneration. The twelfth nerve is also affected in cases of syphilitic bulbar paralysis, either alone or with the ninth, tenth, and eleventh cranial nerves, and more rarely with the seventh and third as well. The lesion is a nuclear one.

\section{Syphilis of the Vegetative Nervous System.}

Under this heading are included the various manifestations exhibited by syphilitic disease of the vagus and the sympathetic. Although the vagus may be involved in syphilitic bulbar paralysis, and abductor paralysis may be caused by a neuritis of the recurrent laryngeal in cases of aortic aneurysm and syphilitic disease of the mediastinum, chief attention will be paid here to the connection between syphilis of the vegetative nervous system and tabes and the part the ductless glands play therein. In the vegetative nerve system there are two groups of nerve fibres which are antagonistic: (1) stimulating, or vagal or autonomic group; (2) inhibitory or sympathetic group. The action of both can be best illustrated in the pupil. Stimulation of the autonomic fibres causes contraction of the sphincter of the iris to a small pupil, while stimulation of the sympathetic fibres produces enlargement of 
the pupil. Therefore a contracted pupil may result from stimulation of the autonomic group (irritation of ciliary ganglion, third nerve), or from paralysis of the dilating fibres of the sympathetic, and vice versâ with a dilated pupil. These changes are most marked in cases of degenerative myelitis. Apart from the loss of the light reflex, while there is response to accommodation (Argyll-Robertson pupil), another important change, because it occurs earlier, is irregularity in the outline of the pupil. At first the irregularity is only to be noticed when the pupil is put into action; later, it is to be seen when the pupil is at rest. Should it be noticed that a previously pin-point pupil has become dilated, the assumption may be made that the autonomic fibres have become paralysed. Owing to the occurrence of plexuses in the intestinal wall, an explanation is afforded for the frequent association of third nerve palsy with gastric crises. There would appear then to be a form of syphilitic degeneration, the symptoms of which for the most part affect the vegetative nervous system. Note again how commonly syphilitic aortitis is associated with a degenerative nervous lesion, and especially with the type of case in which sympathetic nerve lesions predominate. I remember well a case I had of combined cardiac and gastric crises, which started with ptosis and diplopia, exhibited none of the usual symptoms of locomotor, and died of an aneurysm. Another case had, first of all, laryngeal crises, and then combined cardiac and gastric crises, during which the patient's pulse became imperceptible and his colour that of asphyxia, due presumably to stimulation of the vagal fibres. This patient had no ataxia, lightning pains, etc.

In all cases in which a cranial nerve palsy is the first symptom of a widespread degenerative lesion, on careful examination a diminution or loss of the deep reflexes is noticeable, with areas of cutaneous anæsthesia, affecting the ulnar in the upper extremity and the peroneal in the lower. Owing to the fact that the cranial nerve palsy at first responds to treatment, and that it is the third nerve which is most commonly involved, it is reasonable to suppose that the primary lesion is an endarteritic one with vascular degeneration, and that the organisms later develop extramurally, and produce degeneration of nervous tissue, which is irreparable. As in some cases the deep reflexes are at first exaggerated, it seems probable that the primary lesion in the cord is in the meninges, and that the nerve degeneration is secondary, and occurs only when the organisms spread directly into it from the meninges. Further evidence in support of this contention will be adduced when degenerative myelitis is described. Although the crises are due to posterior root and cord lesions, a stimulation of any part of the vegetative nervous system may precipitate a crisis. Pilocarpin will precipitate a gastric crisis because it stimulates the vagus. The importance of bearing this point in mind is great, because, as a rule, more may be done by the general management of a patient suffering from crises than by prescribing specific treatment. Mental stress; physical over-exertion, dietetic errors, etc., are very apt to provoke crises. Abdominal massage and exercises, with careful attention directed to the 


\section{CLINICAL ASPECT AND TREATMENT OF VENEREAL DISEASES}

bowels, will do much to relieve a sufferer with intestinal crises, which doubtless are often started by fæcal stagnation in the colon. Bladder trouble is often due to over-distension having been allowed to occur, with consequent irritation of the sympathetic arc. As bladder symptoms are so common in cases of degenerative myelitis, and cause the patient so much worry, an enormous amount may be done by teaching the patient to pass his water at certain and regular intervals -in other words, to train himself to avoid over-distension. An occasional wash out of the bladder with colloidal silver or iodine is useful, because cystitis is not infrequent. According to Maloney, ${ }^{\circ}$ the destruction of the balance of tone between the vagus and the sympathetic leads to altered cellular activity, a condition which he considers responsible for perforating ulcers, trophic changes in the nails and skin, osteitis, and arthropathy. There is much to support this view, because the removal of the pressure causing a perforating ulcer will often result in the ulcer's healing. It is only natural, in view of the relationship which exists between the vegetative nervous system and the ductless glands, to expect that the activity or non-activity of the latter should exercise an influence on the lesions produced accompanying an infection of the former. Adrenalin stimulates the sympathetic and inhibits the vagus, and so decreases the action of the pancreas and thyroid. Cholin, on the other hand, does the opposite. Atropin paralyses the vagus, hence the reason why this drug is useful in a bad gastric crisis. In this connection it is of interest to state that amyl nitrite will sometimes cut short a crisis. Colloidal palladium (pallamine) may also be of value in cases of crises produced by contraction of unstriped muscular tissue owing to the action this element has in quietening and relaxing unstriped muscle. This action of palladium was suggested to me by King Turner, ${ }^{24}$ who regards it as the possible explanation for the beneficial action of this drug in epilepsy. Colloidal palladium will certainly get a patient in a condition of Status epilepticus out of it, possibly owing to relaxation of the unstriped muscular tissue of the vessel walls inside the cranium. Whether the vitiligo, which is sometimes the first sign of degenerative myelitis, is due to some interaction between the suprarenal and sympathetic or not, is not certain, but anyhow the connection is suggestive. Pituitary and lymphoid extracts have been largely used in cases of syphilitic nervous degenerations, and occasionally they do seem to ameliorate the symptoms.

Although this subject is at present confused, we do know that some of the symptoms found in degenerative myelitis are due to vagal and sympathetic irritation, and that the secretion of the endocrine glands influences that irritation. A rise in the blood-pressure produced by mental effort or physical exertion is said by Cannon to lead to increased secretion of the adrenals. As adrenalin stimulates the sympathetic, we have an explanation as to why strain of any sort is apt to precipitate a crisis. Sweating, again, whether diffuse or localised, is due to vagal irritability, and is not uncommonly a symptom of posterior root degeneration. Knowing it is due to stimulation of the 
vagus enables us to stop it by prescribing atropin, which paralyses the vagus. Blushing, accelerated heart action, alterations in the colour and temperature of a limb, are all symptoms commonly met with in tabes dependent upon stimulation of the vegetative nervous system. Although all these signs and symptoms are more frequently to be met with in cases of degenerative myelitis than in degenerative encephalitis, they may be associated with the latter. A phenomenon which I have seen three times, and which always puzzled me, was pronounced œdema of one lower limb, occurring during an attack of degenerative encephalitis. Doubtless this phenomenon is dependent upon some irritation of the vegetative nervous system. In all three cases, the œdema vanished when the attack passed. In one case, a bad bed-sore occurred, and a most troublesome eczematous condition appeared on the affected foot.

\section{Meningitis.}

Although I propose for descriptive purposes to separate intracranial from spinal meningitis, actually there is no separation, because in practically every case, whether the symptoms be referable to the head or spine only, the whole is in its entirety or irregularly involved.

It is more than probable that, in every case of syphilis in which the organisms become generalised, the meninges do not escape the invasion.

Syphilitic meningitis occurs in the earliest stages, it may be without symptoms; but in the fewer the number of the cases the greater the clinical knowledge of the observer. In most cases the condition is readily diagnosed, but a careful examination may have to be made. Headache, pain in the neck and back, depression, insomnia, and peculiar sensations in the skin, are commonly complained of. Clinically, irregularity in the reflexes, with slight alterations in one or more of the cranial nerves, are generally to be found. The enormous increase of nervous syphilis which has recently occurred is mostly of the meningeal type, the symptoms, almost in clockwork manner, appearing between the twelfth and twenty-fourth week after the last injection of arseno-benzene. Although this is the usual time, I have seen them appear while treatment was being prescribed, and as long as some years after the last injection, when the lesion ceases to differ from an ordinary recurrence in the nervous system. This generalised neuro-recurrence is in part due to the toxic meals arsenic and mercury, for the introduction of intramine has prevented their occurrence, and it is more useful for getting rid of the symptoms than the metals alone. We will admit the action of chemical agents like adrenalin, etc., aggravating degenerative myelitis, and this is a normal constituent of certain of the body cells; while the idea is considered preposterous to blame arsenic and mercury, which are not found normally in any living cell. At one time, owing to the readiness with which the symptoms of the neuro-recurrences vanished under further treatment, I thought such a meningeal involvement would preclude the patient from developing a degenerative 
condition later; but, unfortunately, recent experience has taught me otherwise. Not only is that type of degeneration met with, due to the gradual extension of the organisms into the nerve tissue, owing to the fusion of the brain and cord with meninges-a compression degeneraticr-but what is krown as primary degeneration also occurs. The neuro-recurrerces when salvarsan first came in were of the mono-neuritic type, but when it became customary to give several injections, instead of a single cranial nerve lesion, the symptoms were those of generalised menirgitis, of which the following case is a good example:

CASE 65.- The patient, a man, aged 29, was treated with ten intravenous injections of kharsivan and nine intramuscular injections of mercury, for an early maculopapular rash. When discharged the blood was negative. Two months later the patient complained of bad headaches and insomnia; he was unsteady in his movements, and those around him were aware that he had completely changed. It was stated that on two or three occasions he had fits. He was diagnosed and treated for some time as a case of epidemic cerebro spinal meningitis. When I saw him he was extremely morose, his face was expressionless, and his reaction period was abnormally delayed. The pupils were unequal $R$. $>\mathrm{L}$; the right pupil reacted to accommodation only, and the left was sluggish to light. There was bilateral papillitis and paresis of both auditory nerves. The reflexes were unequal, those in the upper extremity being exaggerated, while those in the lower were diminished. The C.F.T. of the blood was negative. An examination of the C.s.F. revealed the following: Pressure raised; positive lymphocytosis, 1,250 cells per c.mm.; albumin much increased; globulin present; C.F.T. positive. Under colloidal iodine, intramine, arseno-benzene, and auxiliary puncture, the patient's condition so improved as to enable him to go on with his work.

There is a special type of case of generalised meningitis, occurring after treatment with arseno-benzene, which requires special mention, as it is not very uncommon, and is practically never diagnosed correctly. The patient hes terrible headaches, associated, as a rule, with vomiting; then fits eccur, which may follow one another in quick succession, and be Jacksonian in type at first, as later they become general convulsions. The pupils are dilated, and react sluggishly to light, and the vessels on the fundus appear engorged. The other reflexes are unequal, at first exaggerated, and then sluggish. Kernig's sign is present, and the temperature is always raised. If treatment be prescribed at once, the patient recovers; if not, he will probably die and, post-mortem, an acute lymphocytic inflammation of the meninges will be found. In some cases the lymphocytic inflammation is most marked in the brain, when it is only a further stage of what has been already described as hæmorrhagic encephalitis. When the inflammatory process affects the meninges for the most part, the lymphocyte count in the C.s.F. may be as high as 2,000 cells per c.mm., but when it affects the brain chiefly, the cell count may be only slight. I once saw a case of acute lymphocytic meningoencephalitis set in and terminate fatally six months after the patient had had four injections of arseno-benzene in the sixth year of the disease, just because his blood-test was positive. The other cases I have seen have been men 
in the generalisation stage of the disease, who presented severe rashes, and who were inadequately treated with arseno-benzene. This case is quite one of the most illuminating I have had:

CASE 66.-A man, aged 25, contracted syphilis in January, 1916, for which he had one injection of arseno-benzene and mercury internally. In March the patient had very severe headaches, suffered from insomnia, and had three epileptic fits. In June he began to get grandiose delusions, and was completely maniacal a month later. When I first saw him he was restless and excitable; he believed himself to be the Emperor of the World, possessed of untold wealth, and capable of stopping the war if allowed to. Under colloidal iodine, intramine, and further injections of arsenobenzene, the patient completely recovered.

The following case is a typical neuro-recurrence, 'which has since developed degenerative myelitis:

CASE 67.-A man, aged 56, came to me in 1914 with a primary sore on the abdomen, and a profuse lenticular syphilitic eruption, for which he received nine injections of neo-salvarsan, sixteen of mercury and iodides internally. About this time he complained of headaches, insomnia, and polyuria. As he evinced the signs and symptoms of a neuro-recurrence, I advised further treatment with arsenic and mercury, but he would only submit to the latter. In spite of weekly intramuscular injections of mercury and iodides internally, six months later the condition hayd so far progressed that I advised a lumbar puncture to settle the diagnosis. The following was the result: 340 cells per c.mm.; globulin increased; C.F.T. positive in 10.0 per cent. A week later another puncture was made, with the result that the lymphocytosis had increased to 470 cells per c.mm. (note drainage increases cells in C.s.F.). In view of this finding, a series of intraspinal injections were given, and the patient advised to continue treatment with mercury and iodides. The clinical improvement was pronounced, although several of the minor subjective symptoms remained, such as depression, loss of memory, lack of power of concentration, and paræsthesiæ. In 1916 the patient had recurrent mucous papules in the mouth, which rapidly vanished under treatment. In 1918 the patient reappeared with typical posterior column degeneration.

In this connection, it is interesting to note the number of cases of degenerative encephalitis and myelitis which have developed the trouble as the result of war strain, when previously they had signs and symptoms of a simple meningitis.

It is really surprising how frequently inequality of the pupils and irregularity of the other reflexes are observed in patients who have been recently treated with arseno-benzene and mercury. I have watched the pupil changes very carefully, and this is what appears to occur: One pupil becomes a little larger than the other, and reacts sluggishly to light after the reflex has been at one time very jerky and incomplete. These pupil changes may be discernible before the patient complains of anything. Not only does arsenobenzene and mercury, especially if prescribed together, predispose to the occurrence of meningeal lesions, but to vascular lesions as well. I am not referring to the acute hemiplegia and paraplegia which come on within a week after an injection, but to the incomplete lesion which makes its appearance a few months after the completion of the course, and which, as far as I know, has not previously been described. Incomplete hemiplegia and paraplegia may 


\section{CLINICAL ASPECT AND TREATMENT OF VENEREAL DISEASES}

thus occur without any apparent accompanying meningitis. In all, I have seen five cases, three of incomplete hemiplegia, one of incomplete paraplegla, and one of complete hemiplegia, all occurring within six months of the last injection of arseno-benzene while the patient was well under mercury, and all improving with further injections of arseno-benzene. The case with complete hemiplegia developed widespread Lichen syphiliticus a few months later, which responded neither to arseno-benzene nor to mercury, disappearing only under intramine.

Another interesting clinical point which I have observed in cases of neurorecurrences is the not infrequent association with a recurrent syphilitic arthritis or periostitis.

There is not a shadow of doubt but that the continued use of arsenic and mercury is generally injurious, and I feel sure it is due to the action which over-dosage with metals has in depressing the oxidising processes in the body, as the oxygen required by the cells, especially the nerve cells, is used up in oxidising the excess metal to get it out of the system. This is the reason why intramine, a powerful reducing agent, has proved so successful in curing metallic poisoning, and in preventing the severe syphilitic recurrences when properly combined with the metals in use. In all cases treated with intramine since 1915 I have only had one in which a neuro-recurrence occurred, and that was a case of unilateral optic neuritis, which quickly got well under further treatment.

Many of the cases of neuro-recurrences remain undiagnosed, while others are diagnosed as neurasthenia, shell-shock, and epidemic cerebro-spinal meningitis. Some of the cases of cerebro-spinal meningitis, in which no meningococci were demonstrable in the C.s.F., were undoubtedly cases of a meningitic neuro-recurrence, and probably most of the published cases of so-called lymphocytic meningitis were.

The following case is illustrative of what happens if the condition remains undiagnosed:

CASE 68.-A patient had a full course of arseno-benzene and mercury in 1915. Late in 1918 he was brought to me, because his behaviour had been very peculiar. On going into the history I found that, about three months after the completion of the course, he felt very depressed, and complained of bad headaches. The headaches continued, and he complained of pains in the back and lassitude, found that he was losing his memory, and could not take an interest in anything. He consulted many doctors, who said that nothing was wrong with him. Two years later he was observed to be doing extraordinary things, and crying without provocation. On examination I found that his pupils were unequal, and reacted sluggishly to light. His speech was affected, and his face appeared expressionless. The reflexes of all the extremities were increased; he had slight tremors, and a somewhat spastic gait. His memory for recent events was extremely bad; he had lost all sexual desire, and nothing appeared to interest him. The blood C.F.T. was positive, and an examination of the C.s.F. revealed the following: Pressure raised; cells 142 per c.mm.; marked globulin increase; C.F.T. positive in all dilutions. The diagnosis was made of degenerative meningo-encephalitis and myelitis. Under drastic treatment, including intraspinal injections, the patient improved for a time, but soon developed a recur- 
rence, which was indistinguishable from degenerative encephalitis (G.P.I.), during an attack of which he died.

The following case is also an extraordinarily interesting one, as showing the connection between a meningeal neuro-recurrence and degenerative myelitis:

CASE 69.-The patient was in the early generalisation stage of syphilis when he received a full course of treatment with arseno-benzene and mercury. Seven months after the completion of the course he had a typical meningeal neuro-recurrence, the symptoms of which vanished unider another course and a half. Two years later I again saw the patient, and found that he had Argyll-Robertson pupils, increase of reflexes in the upper extremities, loss of reflexes in the lower, sluggish cremaster and abdominal reflexes, and peroneal anæsthesia. The patient had slight rombergism, pains in the legs, and no sexual desire. When the neuro-recurrence occurred, an examination of the C.s.F. revealed the following: Pressure raised; cells 310 per c.mm.; slight globulin increase; C.F.T. positive in $100 \cdot 0$ per cent. When the patient returned with symptoms of degenerative myelitis (tabes), this was the result: Pressure normal; cells 96 per c.mm. ; marked globulin increase; C.F.T. positive in all dilutions.

My own view is that practically every case which has had one course only of arseno-benzene and mercury will relapse sooner or later; and, so far as the relapses affect the nervous systcm, two statements which have hitherto remained unobserved may now be made. Degenerative encephalitis and myelitis will not be relegated, as was hoped, to the past, but, on the contrary, will set in earlier than they did before. As each recurrence (system) receives another course of treatment, not only is the patient no better off than when he was first treated, but with each course the C.s.F. becomes more pathological, as evidenced in the following case:

CASE 70.-A patient in the early generalisation stage received eight injections of arseno-benzene and seven of mercury. A recurrent maculo-papular rash appeared twenty-two weeks later. Examinations: C.F.T., blood negative; C.s.F., 12. cells per c.mm.; no globulin increase; C.F.T., negative in all dilutions. The patient received again eight doses of " 606 " and seven of mercury, and returned to hospital with a similar rash twenty-eight weeks later. Examinations: C.F.T., blood positive; C.s.F., 23 cells per c.mm.; trace of globulin; C.F.T. positive in $1,000.0$ per cent. The patient was then treated with eleven injections of kharsivan and nine of mercury. Forty weeks later the patient reappeared with condylomata on the under-surface of the penis and on the scrotum and around the anus. Examinations: C.F.T., blood positive; C.s.F., 39 cells per c.mm.; globulin increased; C.F.T. positive in 100.0 per cent. Thirty-five weeks later the patient returned again with condylomata, for which he received nine injections of galyl and eight of mercury. Examinations: C.F.T., blood positive; C.s.F., 27 cells per c.mm.; globulin increased; C.F.T. positive in all dilutions.

Even in cases which have no clinical relapse, an examination of the C.s.F. made some time after the last course gives positive reactions with either a negative or positive blood-test. Two questions now arise: (1) What is going to happen to these cases?

(2) How should they be treated?

Not sufficient time has elapsed to answer these questions definitely, which permits one to make generalisations only. As many cases develop a more pathological fluid the more courses are given, it is best, when repeating the course for a clinical recurrence, to supplement it with intermittent treatment 
for at least two years. If the recurrence is a patholcgical one only, either in the blood or C.s.F., or both, treatment should not be prescribed until clinical manifestations appear, because, although sufficient may be given to render the reactions negative, it is apt to precipitate a degenerative nervcus lesion, which is sometimes very rapid in its course. I could cite several cases which were clinically sound, in which treatment was prescribed because the C.s.F. was positive, and they developed a fatally terminating degenerative encephalitis and myelitis within a year of its cessation, in spite of the fluids having been rendered temporarily negative. When symptoms of a meningitis present themselves, drastic treatment, which should be continued for two years, and treatment in which intramine and colloidal iodine figure largely, should be prescribed; but even if this is done, no guarantee can be given that degeneration will be avoided. The best prcedure to adopt is to reduce the likelihood of a neuro-recurrence to a minimum, and this can be done only by continuing the treatment for two years, and by incorporating intramine and colloidal iodine therewith, to prevent the toxic metals from robbing the nervous system of the oxygen which it so badly requires. Recent experience. has shown that intraspinal injections are not necessary, equally good, if not better, results being obtained by draining off the C.s.F. after an intravenous or intramuscular injection of the drug employed has been given. In patients not treated with arseno-benzene, symptoms of meningitis did not, as a rule, make their appearance before the fourth year: the symptcms were often severe, secondary degeneration was not uncommon, and, even when treated with present-day methods, only amelioration of the condition was achieved, because the cases exhibited a strong tendency to relapse, with an extension of the process.

I will now cite two cases of meningo-encephalitis and myelitis which cccurred in the pre-salvarsan cra:

CASE 71.-A man, aged 40 years, developed syphilis eleven years previously, but as he had no other symptoms but the sore, he only took mercury internally for six months. In 1908 gummata developed in the skin, and some of the cranial nerves became involved. Some improvement was obtained after several injections of soamin and potassium iodide internally. In January, 1910, he had diplopia, and was much troubled with headache and vomiting. In March, 1910, he first noticed weakness of the right side of the face, and was treated with mercury and iodide, with only temporary improvement. When seen on September 12th of the same year, the condition was as follows: "There is an internal strabismus, with diplopia, due to paralysis of the right external rectus and weakness of the left external rectus. The movements of the eye dependent on the third nerve are good. The pupils react well, and the vision is good. There is some swelling of both optic discs. There is paralysis of the right side of the face, and both motor and sensory portions of the right fifth nerve are involved. There :s deafness on the right side, but the tuning-fork is audible when placed in contact with the mastoid. There is some unsteadiness in gait, but this would seem to be dependent on the diplopia. Romberg's sign is absent, and there is no weakness of the limbs. The knee-jerks are active and equal; the plantar reflex cannot be obtained, and the abdominal reflexes are difficult to elicit! but they are equal. The articulation is somewhat nasal, but no 
weakness of the palate ean be detected, although there is some difficulty in deglutition. The movements of the tongue are good. It seems probable that there is a circumscribed syphilitic meningitis at the base of the brain, involving the right fifth, sixth, and seventh cranial nerves, and probably also the right eighth and ninth, and the left sixth to a lesser degree. There is no evidence of any affection of the pyramidal tracts."

CASE 72.-A married woman, aged 47, whose infection occurred probably twentyfour years ago, and who, after her second marriage, had five consecutive miscarriages. Nervous symptoms began in 1901, when the left arm and leg were noticed to drag, and there was giddiness, with disturbance of vision-in the patient's words, "things went suddenly black." She was admitted into the Great Northern Hospital for seven weeks. Four years later she complained of partial paralysis of the left arm and leg, cramps in both legs, double vision, and loss of memory for minor events. This time she was admitted into King's College Hospital for sixteen weeks, and left much better. A year later she was readmitted for eleven weeks, because she had occasional attacks of unconsciousness, during which she squinted. During the past year there were further attacks of faintness and giddiness, which have been very much worse for the past three months. Three weeks ago she dropped down unconscious in the street; since then she has complained of continual dizziness and headache, and a marked tendency to fall to the right side when walking. On admission she had double optic neuritis; the right pupil was smaller than the left, but both reacted to light and accommodation. There was paralysis of both external recti, paresis of the lower half of the left side of the face, and deafness on the right side. The patient could not hear a watch held within an inch of the ear, nor when it was placed on the temporal bone; the tongue was protruded to the right; the other cranial nerves were normal. Muscular power and sensation in the arms were good and equal, tendon reflexes: increased, there was inco-ordination. In the legs, muscular power and sensation were good, and equal on both sides. Knee-jerks present, and equal; no clonus; plantars brisk, and flexor; co-ordination impaired; other systems normal.

From the numerous cases I have seen, a few observations have been made which may be of interest to the reader. Frontal and basal meningitis are most apt to produce optic neuritis. Headache is a sign of pachymeningitis, but not of leptomeningitis. The headache may be situated anywhere, but as a rule it is worst over the vertex and at the occiput-in fact, it is often localised to one of these two areas. The pain does not tend to shift about, and not infrequently a tender spot is to be felt when palpating the head. The patient usually knows where it is, as he has frequent cause to touch it when combing and brushing his hair. If the headache does not vanish under treatment, or soon recurs, either the lesion is a vascular one, or one of pachymeningitis, in which the dura has become adherent to the inner table of the skull. A differential diagnosis can only be made by examining the C.s.F. Pain in the neck, especially when associated with a sense of dulness between the scapulæ, which either spreads to the small of the back or not, is frequently met with in cases of spinal meningitis. Pachymeningitis is most common on the vertex, while leptomeningitis is most common on the base. The latter does not, as a rule, give rise to symptoms until the cranial nerves are involved. Should a patient become apathetic, take no interest in his work, and his memory deteriorate, then it may be certain that he has 


\section{CLINICAL ASPECT AND TREATMENT OF VENEREAL DISEASES}

a meningo-encephalitis. Should bladder trouble set in, the knee- and anklejerks disappear, after having been increased, and patches of anæsthesia are demonstrable, then a diagnosis of meningo-myelitis may be made. If this development is watched and controlled by repeated examinations of the C.s.F., it will be noted that the more pronounced the degeneration becomes, the lower becomes the cell count, but the higher the globulin content, and the more positive the C.F.T. I cannot help thinking that some of the cases of so-called G.P.I. and tabes are cases of degeneration secondary to a meningitis which has, as a rule, exhibited no clinical symptoms. The so-called cases of pseudo-tabes are really cases of meningo-myelitis. In support of this contention are the facts that in cases of meningo-myelitis and degenerative myelitis the posterior portion of the cord is most involved because of the meningeal covering of the posterior roots, because of the septum posticum, which is a meningeal prolongation, and because the anastomosis between the vessels is not so free as it is on the anterior surface of the cord. Again, if the lateral columns become involved in cases of meningo-myelitis the symptoms do not disappear under treatment, which points to the myelitis being degenerative. In the brain, localised meningitis may cause Jacksonian epilepsy, monoparalysis and hemiparalysis, cortical speech disturbances, hemianopsia, etc. In the cord, localised meningitis may cause the well-known BrownSéquard syndrome. Mention will now be made of the more or less clinical entities which may be produced by cerebral, basal, and spinal meningitis.

Amnésie Syphilitique.-A name given by Fournier to a progressive dementia which is caused by a chronic specific meningitis of the convexity. Although the patient's reaction period is slow, his gait heavy, and he is slovenly in his ways, he presents none of the typical signs of degeneration, and the condition improves with treatment.

Epilepsy.-The epilepsy may be of the Jacksonian type, or it may be general. In some cases treatment appears to be very efficacious; in others, relapses soon occur; and, as I have seen cases in which degenerative encephalitis has set in later, the possibility of this sequela should be held in mind, especially in those cases which are not benefited by treatment.

Syphilis of the Pituitary Gland.-This gland may be affected by a specific arteritis, or more commonly by a basal meningitis, when polyuria and polydipsia are predominant signs. In my experience, Diabctes insipidus is more common in congenital than in acquired syphilis. A gumma may occur in the gland, and may grow to the size of a hen's egg, as it did in a case which was under Dujardin's care. This patient presented all the signs and symptoms. of acromegaly. Gummatous meningitis affecting the chiasma may be responsible for glycosuria. The headaches caused by an involvement of the pituitary are characteristic enough to suggest a diagnosis before the signs just mentioned are discernible. The headache is very severe, frontal, affecting chiefly both temples, frcm which the pain radiates to the back of both eyes. According to Pardee, ${ }^{18}$ the skin on stroking shows a broad white line due to adrenal insufficiency, which is ascribed to the drain on adrenal function by the exhausted pituitary. 
As Diabetes insipidus occurs more commonly in syphilitic nervous affections than is usually thought to be the case, a few more remarks about this interesting condition are warrantable, for the polyuria, unless excessive, may be missed. A case of Diabetes insipidus passes on an average eight pints of urine a day, but before it reaches this amount it may be ascertained that the patient has a greatly increased tolerance for sugar, and that sodium chloride acts as a powerful diuretic. So far as the urine is concerned, no single constituent is changed as to its proportion to the others, except occasionally when sugar makes its appearance towards the end.

Erb's Syphilitic Spinal Paralysis.-The condition occurs in late syphilis, and is characterised by a slow and gradual development of a spastic paresis of the lower extremities, with spastic gait, motor paralyses, slight muscle tension, slight increase of tendon reflexes, the sensory disturbances being either slight, severe, or absent, and sphincter weakness, bladder especially. The condition is not very uncommon, and, in my expérience, benefits from treatment if diagnosed early, but invariably recurs when it remains uninfluenced, eventually proving fatal from nephritis secondary to the cystitis. The premonitory symptoms of chronic myelitis are characteristic, and they deserve mentioning and remembering. The patient first notices that he tires easily, his legs feel like lead to him, and he is worried because his sexual power has quite or nearly gone. He may complain of pain in the back, and of a sense of constriction around the waist. The reflexes are exaggerated, and an ankle-clonus and extensor response may be elicited. Months may elapse between this stage and the onset of paraplegia, which, in my opinion, may be materially delayed provided treatment is prescribed.

Although this form of myelitis is generally meningeal in origin, my own opinion is that it may sometimes be caused by a vascular lesion, in which degeneration has taken place, of the nature of an arterio-sclerosis, the myelitis being a myelomalacia similar to the encephalomalacia which is the result of cerebral arterio-sclerosis. I have a case at present under observation in probably its earliest stage. The pupils are dilated and unequal, the light reflex has gone, and the accommodation reflex not entirely. The arm and leg reflexes are increased, and there is marked general cutaneous anæsthesia. Spastic paresis is just beginning. There is sphincter weakness of the bladder and complete loss of sexual desire. The C.s.F. is normal. The following case is also probably one of the latter kind:

CASE 73.-A man, aged 56, contracted syphilis twenty-four years ago, and was well until about five years ago, when he found he could not hold his water, or walk any distance without immediately getting tired. All sexual desire had vanished some years previously. When I first saw him his gait was inclined to be spastic, and he had great difficulty in walking. He was obliged to wear a urinal, as he had cystitis, and had lost all control. His memory and power of concentration were excellent; the pupils reacted normally, but all the other reflexes were exaggerated. There was no ankle-clonus, but a questionable extensor response. There was general muscular weakness, affecting even the face muscles, as food tended to drop out of his mouth once he had put it in; but there was no paralysis. There was a slight general hyperæsthesia. 


\section{$15 \pm$ CLINICAL ASPECT AND TREATMENT OF VENEREAL DISEASES}

After an intravenous injection of arseno-benzene he developed a general twitching of his muscles, which became less and less pronounced after each of the succeeding injections (four). In my experience, this kind of reactionary inflammation is only to be met with in late degenerative and general vascular lesions.

The Brown-Séquard Symptom Complex.-This syndrome is not at all unecmmonly met with in syphilis. The symptoms may come on rapidly, but the lesion is really a chronic one, and is not much improved by treatment. The following is a typical case, and one of the finest examples of the condition I have scen:

CASE 74.-The patient, a man, aged 32, who had had treatment for syphilis four years previously at the Lock Hospital, reappeared, complaining of a rash on his trunk. The rash was Herpes zoster bilateralis, corresponding to the distrikution of the eighth and ninth dorsal roots. The patient had severe girdle pains. On examination there was a zone of hyperæsthesia mapped out by the Herpes zoster. Below this zone the sensibility was diminished. There was paresis of the right leg, and tactile hyperæsthesia. There was no paresis of the left leg, but a paralysis of cutaneous sensation. On both sides the deep reflexes were absent. The pupils were unequal, and reacted sluggishly to light.

It is sometimes difficult to differentiate the syndrome caused by specific meningitis from that caused by a tumour. If the symptoms àre atypicaland they are in the above case, because the deep reflexes are, as a rule, exaggerated on the paretic side-if the patient has pupil signs, and if a marked amelioration of the symptoms occurrs as a $\mathrm{r} \in$ sult of treatment, then a certain diagnosis of syphilis may be made. The case just reported improved with treatment, but he was by no means cured, as degeneration in the cord had undoubtedly occurred. Unfortunately, I have lost sight of the case, as it would have been interesting to watch the future development. This symptom complex may also be produced by a purely vascular lesion, as I have recently observed a case of a man who contracted syphilis twenty-two years ago, and who presented himself with the left leg paralysed and hyperæsthetic, while there was no paralysis of the right leg, but only diminished sensation. The knee-jerks were absent, and the C.s.F. was practically normal.

Lateral Selerosis.-The lesion is one affecting the lateral columns and the pyramidal tracts. Clinically, it is characterised by progressive spasticity and increase of the reflexes, without any sensory disturbances or sphincter trouble. I have seen two cases, but neither improved to any great extent under antisyphilitic treatment.

Hypertrophic Cervical Pachymeningitis. - This is merely a meningo-myelitis affecting the cervical more than the other parts of the cord. It is often called Charcot's pachymeningitis, although this observer was unaware that syphilis was usually the cause. The patient first complains of pain and stiffness in the neck. The pains radiate up to the occiput, down the back, and down one or both arms. Later the arms bccome weak, atrophy occurs, with consequent contractures and trophic disturbances, unless treatment be prescribed early enough to check the advance. If untreated, the patient devclops paraplegia later 
and leads a miserable existence owing to the persistence of the pain. The reflexes are exaggerated, and the skin is anæsthetic and hyperæsthetic irregularly. Death results from exhaustion or from some intercurrent trouble.

\section{Degenerative Encephalitis.}

This condition is known as general paralysis of the insane, or Dementia paralytica. From the various brains I have been able to examine, I am of the opinion that the condition may follow and result from previous meningeal and vascular lesions, and that it is not always a primary degenerative condition dependent upon the growth of organisms which have reached the brain by the lymph stream-the route along which the organisms are most wont to travel, according to the work of Orr and Rows. ${ }^{17}$ It is true that, microscopically, the inflammatory changes may be minimal, in which cases a lymphogenous infection is possible; but, on the other hand, the perivascular inflammation may be so pronounced as, at first sight, to suggest the diagnosis of lymphocytic encephalitis. The latter is only a further stage of the hæmorrhagic encephalitis which is apt to occur and end fatally on the third day after the second injection of arseno-benzene. Microscopically, a case of hæmorrhagic encephalitis may exhibit little or no change at all in the brain, and yet we know that it is a vascular lesion. In some cases of degenerative encephalitis, the small round-celled infiltration may reveal central necrosis, and in no way differ from what is commonly called a gumma. Take again the C.s.F. In some cases the cell count is near a hundred; in others, scarcely raised. The globulin may be only slightly increased, or may almost replace the albumin. The C.F.T. may be positive in all dilutions, or it may be negative; the pathological changes may be reduced by treatment, or they may remain uninfluenced; the course run by the disease varies also. Some cases seem to be considerably improved by treatment, while others are undoubtedly made worse. All this is very suggestive that the lesions vary in the different cases. We will first consider the prodromal symptoms, then the symptoms of activity, and finally the prognosis and effects of treatment.

Prodromata.-There may be none; some years before the patient may have had an arterial lesion, such as hemiplegia or a meningitis, which produced epilepsy; but usually, some time before the disease becomes active, the patient becomes irritable, especially with the people he knows best, loses his memory and power of concentration, and is inefficient and slovenly in his habits, although he has an urgent desire to be otherwise. Insomnia is frequent, and the patient is particularly worried about his condition. On the other hand, a mania may be the only symptom. Degenerative myelitis may, of course, precede the brain symptoms (tabo-paralysis).

Symptoms of Activity.-The patient may be terribly excited or extremely depressed; his attitude towards his relatives changes suddenly; he is full of complaints, easily fatigued, and muddles his affairs. He may have grandiose ideas, and be very talkative. Although remission may occur at this juncture, 


\section{CLINICAL ASPECT AND TREATMENT OF VENEREAL DISEASES}

sooner or later a relapse sets in, with each time a more progressive dementia. Early in the disease changes in the handwriting will be found, and tremors, espccially of the tongue and face, are noticeable. The speech may be altered; the pupils are unequal, and react sluggishly to light; and the deep reflexes are exaggerated, and there is an early loss of sexual function. Each succeeding attack is more severe than the one before it, and the patient soon becomes dirty in his habits, and fails to recognise the people around him, or to remember the days or even the month of the year.

Prognosis.-As a rule the disease does not last more than three years. During any attack, hemiplegia or epilepsy may carry the patient off; or he becomes bedridden, and simply dies of exhaustion. Some cases have no remission at all, the disease being galloping at the start, and terminating fatally in a few months. In my experience, treatment may have this result, as in the following case:

CASE 75.-A patient had had syphilis six years previously, for which he had been quite well treated, having had no recurrence. Because of the craze for blood-tests, he had his examined, and, as it turned out positive, he received nine injections of arseno-benzene and eight of mercury. Two months after the cessation of treatment he became suddenly demented, and developed degenerative encephalitis, from which he died, without a remission, eight months later.

Effects of Treatment.-The more cases of degenerative encephalitis I see, the less sure I am of the best advice to give. I have seen some cases respond in an extraordinary manner, but never one which did not end fatally, while, on the other hand, I have seen others made infinitely worse. I have also seen cases do as well without as with treatment. In the prodromal stage treatment is indicated, provided it is drastic and the C.s.F. is drained several times to enable the drug to reach the nervous tissue. When the symptoms are active, treatment should only be advised in the early stage, and during the quiescent period it should be undoubtedly withheld, as I shall never forget. a patient who, apparently normal, committed suicide three days after an injection of arseno-benzene.

The following case is instructive:

CASE 76.-A man, aged 39, contracted syphilis in 1896. He had several primary sores, and the usual symptoms of the generalisation stage. He was treated with mercurial inunctions for six months, and then developed a right-sided hemiplegia. The mercurial inunctions were continued, in spite of the hemiplegia, with the result that in three days the patient could talk again, and in a few more days the power in the arm and leg returned. Mercurial treatment was continued for over three years. The patient consulted me in July, 1914, because he felt so fearfully depressed. The depression bordered almost on suicidal mania. The pupils were unequal, right $>$ left. The left pupil did not react to light, but it did to accommodation, while the right pupil reacted to both. All the reflexes were exaggerated, and the patient had tremors. It should be said that the depression had set in only three months before $\mathbf{I}$ saw him.

Examination of the C.s.F.: Pressure not raised; lymphocytes, 12 per c.mm.; phase 1 feebly positive; C.F.T. positive in $1,000 \cdot 0$ per cent. only.

If any case appeared suitable for treatment, this one certainly did, so I gave 
eight intraspinal injections of salvarsanised serum, the number required to render the C.s.F. absolutely normal. The patient appeared to improve somewhat, and the reflexes in the left pupil were undoubtedly brisker than they were to start with, but the faint reaction to light only lasted for a few days. A fortnight later the patient suddenly entered the exaltation stage; a week later he became violent; he then gradually became more and more demented, and had to be interned in an asylum, where he died six months later.

I was able to get the C.s.F. absolutely normal, and yet the case went more and more downhill.

Having been able to follow some of my cases of degenerative encephalitis from start to finish, the following reports of a few cases will probably prove of interest:

CASE 77.-Man, aged 48, contracted syphilis in 1905, and took mercury internally for two and a quarter years; one year later, syphilitic orchitis developed, which was reduced by iodides. In 1915 the patient came to see me with signs and symptoms of posterior column degeneration. I gave him nine intraspinal injections of salvarsanised serum, the number necessary to render the fluid almost negative, and treated him intermittently with mercury and iodides for another eighteen months. In 1917 he developed degenerative encephalitis, as a comparison of his handwriting will show.
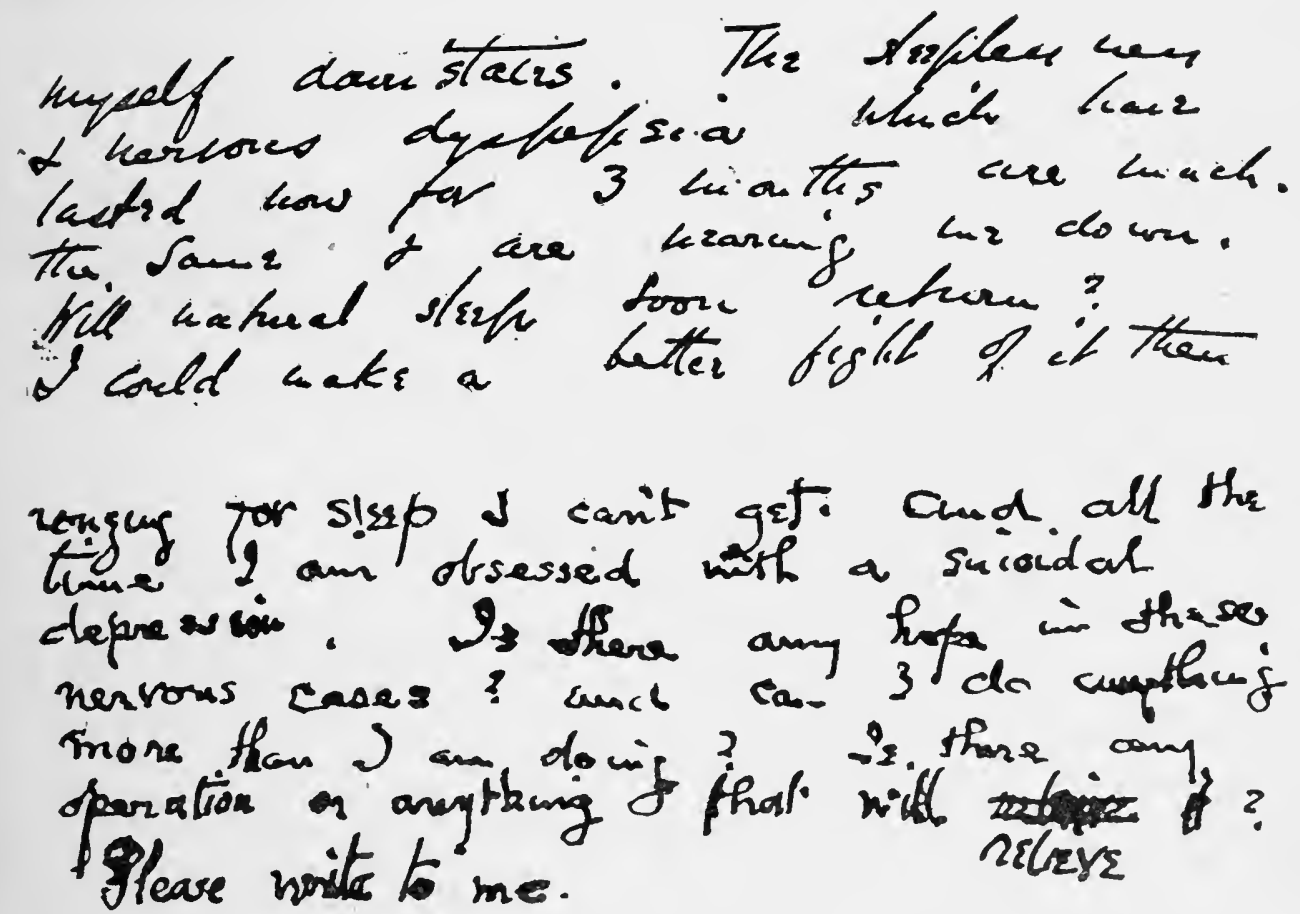

The first sample was written on July 27 th, 1917 , and the second, September 9 th, 1917. From this attack the patient recovered; but ultimately he relapsed, and died in May, 1919. 


\section{CLINICAL ASPECT AND TREATMENT OF VENEREAL DISEASES}

- This patient's greatest friend contracted syphilis from the same woman a month earlier. The friend took mercury internally for thrce years. I c xamined him in 1916, and diagnosed the prodrcmal stage of degenerstive ercephalitis. As the patient was not prepared to have a series of intraspinal irjections, he took mercury and iodides internally. Although he has steadily gc ne downhill, he is, nevertheless, still alive.

Case No 78 was under my care at the Lcck Hospital, and some of the nctes are taken from Spence's ${ }^{21}$ description in the Lancet.

CASE 78.-A man, aged 39. First symptoms were noticed in December, 1914, when he became irritable and uncertain in temper, this being quite unlike his previous invariable good nature. Thenceforward his wife, the informant, observed a progres-
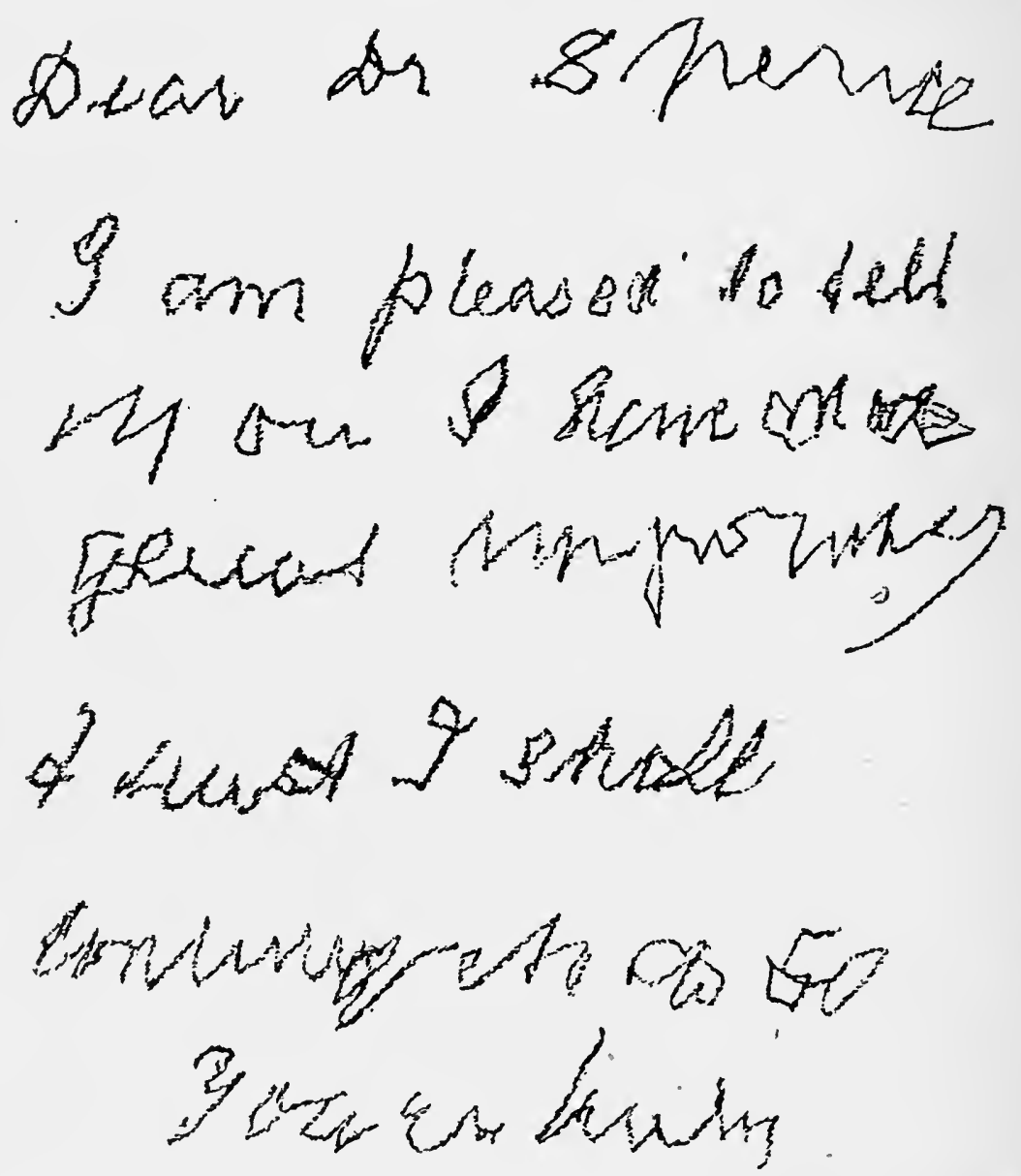

sive change is his disposition: he was always tired, and found increasing difficulty in concentrating his attention upon any task; his handwriting became shaky, and his gait uncertain, his condition at the end of six months being such that he was obliged to relinquish his work. He was then referred to a psychiatrist, who diagnosed general 
paralysis of the insane, a diagnosis subsequently confirmed by a second consultant. After a slight transient improvement, on or about August 10th, 1915, there occurred 8. paralysis of the right upper extremity, and interference with speech, which had now become definitely tremulous and hesitating. He became steadily worse as the weeks passed, displayed marked defects of attention, interest was retarded, and he was frequently confused. Upon admission to the hospital, on January 14th, 1916, he presented a typical picture of paresis of the depressed type. He was disoriented and retarded, any replies finally secured being hesitating, tremulous, and incoherent. He was poorly nourished, filthy, and indecent in habits, occasionally resistive, and required every nursing care. Deep reflexes were minus, pupils unequal, and only very sluggishly responsive to light. On the day following admission he had a slight general convulsion. Three grammes of intramine were given, repeated in one week. Two days following the second injection there was a recognisable and rather sudden improvement; he answered more readily and more accurately, and was cleaner in habits. Three days thereafter he was able to be up in a chair and partially dressed for two hours, was correct in habits and conduct, recognised the call to stool, and returned coherent answers to questions with little retardation. On the following day, however, he relapsed, and was mildly disturbed throughout the night. Again he received intramine, $3.0 \mathrm{grm}$. (January $30 \mathrm{th}$ ), followed in five days by galyl, $40 \mathrm{cgrm}$. Two days subsequently he was better than at any time, conversing pleasantly with his wife and the attendants, and evincing a good realisation of his circumstances. The intramine was repeated on February 10 th, $1 \cdot 0 \mathrm{grm}$., and the galyl three days later. The letter reproduced on the preceding page he wrote and composed himself, at my request, on February 14th, or one month after admission-his first attempt to write in some months. At this time he was sitting up several hours daily, walked with the assistance of his nurse to the toilet rooms, and took a few steps without help, although unsteadily.

In April, 1916, the patient was able to return to work, and this is a specimengof bis handwriting at the time:
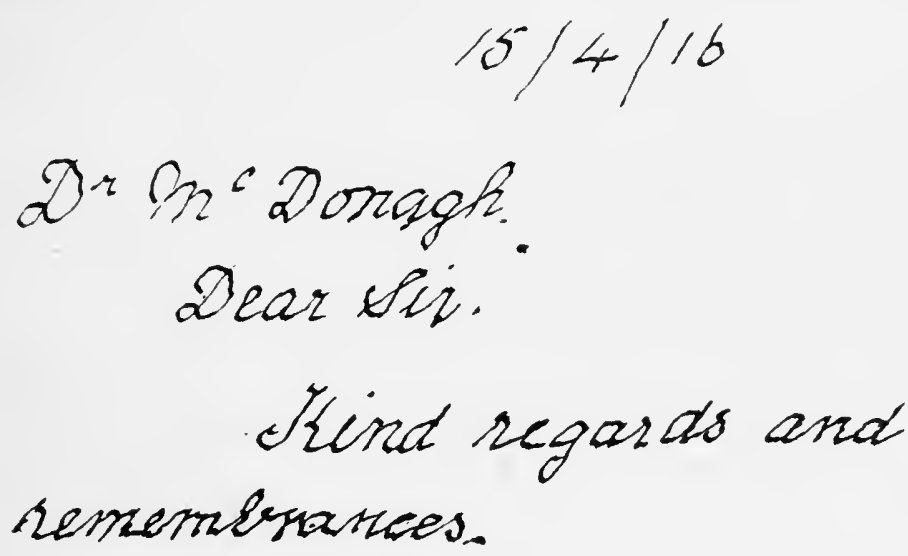

A week after the above was written the patient developed hemiplegia, from which he partially recovered; but in the following October he became again very depressed, and almost helpless, being able neither to read nor write, but he retained full control of his sphincters. As time went on he lost more and more ground, and died in. January, 1917. 
CASE 79.-A man contracted syphilis in 1906, for which he was treated with mercury internally for three years. In 1914 the patient had a series of fits, and suffered badly from hallucinations. After a course of arseno-benzene and mercury he improved sufficiently to return home from the East. I saw him in 1916, when he presented the clinical and pathological signs of degenerative encephalitis. As the fits were bad I gave a course of intraspinal injections, which $I$ followed up with mercury and iodides. The fits disappeared for a time; but a few months later they returned in an aggravated form, each fit lasting for several days, during which period he became moody, aphasic, and quite disoriented. He gradually developed the depressive form of degenerative encephalitis, and died in 1917.

\section{Degenerative Myelitis.}

As the names Locomotor ataxia and Tabes dorsalis are so well known, and as a patient is apt to go rapidly downhill the moment he knows he has this affection, I have thought that the above title is more appropriate. The condition is usually described as being a primary degeneration affecting the posterior roots, ganglia and neurons first, and later the cord and the peripheral nerves. If the affection be always due to a lymphogenous infection, as suggested by Orr and Rows, ${ }^{17}$ then the above is probably correct, but as cases have followed previous arterial and meningeal lesions, one cannot help thinking, in some cases at least, that the degeneration is secondary to such lesions. I have just seen a case in which a partial paraplegia occurring two months after infection was followed by typical posterior column degeneration eighteen months later. The reason that it is the posterior part of the cord which is always involved is presumably because the arteries supplying this part are end arteries, and so do not anastomose freely, and because there is a prolongation of the meninges-called the septum posticum, which is in close connection with the posterior columns, and along which organisms can readily spreadand a continuous meningeal covering of the posterior roots. It is so seldom that an early case comes to autopsy, therefore an opinion as to what has occurred in the past can only be hazarded. Clinically, I have seen cases of spinal meningitis which have become tabetic, although of course it cannot be denied that the latter might have been added or sprung up de novo. I have also watched the C.s.F. change from the reactions commonly associated with meningitis to those typical of a degenerative condition.

As a rule, degenerative myelitis, like its homologue in the brain, makes its first appearance between the fifth and ninth year after infection. Both conditions occur most often in middle life, and much more commonly affect men than women, and it is my opinion that the present-day treatment is in no wise diminishing the incidence-rather, on the contrary, increasing it, and certainly accelerating its onset. Degenerative myelitis occurring in a youth or young adult must make one suspect congenital syphilis. I have seen two such cases in men of 24 and 27 years respectively, in which the signs and symptoms differed in no wise from the acquired form. Although syphilis 
is the only cause, the onset may be hastened by various factors, such as treatment, accidents, etc. Having on more than one occasion seen degenerative myelitis precipitated by treatment which was prescribed because the results of an examination of the C.s.F. were positive in an apparently normal individual, I have for some time advised against the treatment of a clinically sound patient, because, however successful one may be in making the reactions negative, they invariably become positive again, and there is no evidence to show that treatment will prevent degeneration ensuing-on the contrary, there is evidence to show that ill-timed treatment destroys the patient's natural protection against the disease.

One cannot describe prodromata of degenerative myelitis, because there is scarcely a symptom which may not predominate and precede the others. Excepting those cases which begin with a cranial palsy, I should say the earliest symptoms in most cases, and symptoms which so frequently escape diagnosis, are lightning pains, bladder trouble, and loss of sexual desire. The great stimulation of the sexual appetite described by many authors has been very uncommon in the cases I have seen. Although any patient may have any or practically all of the usual symptoms, the cases as they occur clinically can be roughly divided.

Cases with Lightning Pains predominating.-These cases usually run a benign course; there may or may not be associated ataxia, but there is always marked anæsthesia. Trophic changes, such as painless whitlows, perforating ulcers, etc., are not uncommon. The pupils are usually miotic, never react to light, and often not to accommodation.

Cases with Ataxia predominating.-These cases also go on for years, until some intercurrent affection carries the patient off. If the disease itself be the cause of death, then it is due to cystitis with consequent nephritis. In this class, bladder troubles are common. Lightning pains may or may not be troublesome. Crises, with the exception of vesical and rectal crises, are not commonly associated. Spontaneous fractures, especially of the patella, are not infrequent. The pupils are usually typical Argyll-Robertson.

Cases with Cranial Nerve Palsies predominating.-These cases are apt to end quickly, as degenerative encephalitis often supervenes. Lightning pains and ataxia are most usually absent, but crises, especially gastric crises, are common.

Barring occupation, we do not know why one tabetic should develop a certain chain of symptoms and another a different chain. There is no doubt that occupation plays a very important part, as evidenced by the cases reported by Edinger, ${ }^{19}$ Rennie, ${ }^{20}$ and others. The relationship between work and disease was expressed in Edinger's Aufbrauch Krankheiten in exhaustion diseases. If the tabetic patient is a postman, he will complain first of ataxia in the legs; if a tailor, of ataxia in the hands and arms. Possibly the reason why the Argyll-Robertson pupil is such a frequent and early sign of degenerative myelitis is because the pupillary light reflex is so constantly active. Stress would also afford an explanation for the frequency with which sensory 


\section{CLINICAL ASPECT AND TREATMENT OF VENEREAL DISEASES.}

neurons are attacked compared with motor neurons. The action of sensory neurons is incessant, whereas that of motor neurons is intermittent. It is interesting to record that Edinger fatigued rats by placing them on a continuously moving treadmill. He then poisoned them with pyridine, and found the lesions, although disseminated, mainly in the posterior root zones.

A few remarks will now be made concerning the various signs and symptoms which have not previously been described.

1. Lightning Pains and Crises.-At first these are usually described and diagnosed as rheumatic, but their fleeting nature, sharp stabbing and radiating character, and peculiar distribution, at once serve to distinguish them. Before any examination is made there is something peculiar in the facial expression of a tabetic which immediately calls your attention to his condition. The eyes may bulge or be somewhat retracted, the lids are apt to droop (due to paralysis of the cervical sympathetic, which go to the unstriated fibres of the levator palpebrce) and be restricted in their movements, the miotic pupil creates a characteristic appearance, which is especially noticeable when the irides are blue. Whether miosis is more apt to occur in patients whose irides are not deeply pigmented or not I am not certain, as it is probable that the seemingly increased frequency which $I$ have noticed is explained by the more striking appearance produced by miosis with blue irides than with brown irides.

Of the crises, the most common are the gastric, and they are the most important, owing to the frequency with which they give rise to wrong diagnoses. There is practically no intra-abdominal obstructive lesion which a gastric crisis cannot simulate, hence the reason why a useless laparotomy has so often been performed. I have seen five cases which had been so treated; two had been operated upon twice, and one three times, while three others were saved in time from a similar fate. I also remember a patient with laryngeal crises who had an uvulectomy performed before the condition was diagnosed.

An interesting paper by Nuzum ${ }^{22}$ on the needless surgical operations from failure to recognise Tabes dorsalis should be read by all surgeons. Of 1,000 tabetics examined by Nuzum, 8.7 per cent. had been subjected to laparotomy under mistaken diagnoses one or more times. Gastric ulcer was the diagnosis most frequently made; then came gall-bladder disease, with appendicitis a close third.

The symptom which leads the surgeon to operate in practically all the cases is the visceral crises. Now, visceral crises may occur in patients whose knee-jerks are not absent, and who are not tabetic, but I have never yet seen a case in which there were not pupil changes, and in which the C.s.F. was normal; therefore there is no excuse for performing a. needless abdominal section. In a few cases antisyphilitic treatment will, after causing a temporary exacerbation, especially if injections are used, ameliorate the pains; but they are invariably recurrent, and they become more acute; therefore, unless I have good reason for thinking the lesion is active or is associated 
with a meningitis, I rely mainly upon exercises, and upon teaching the patient to regulate his life as far as is possible. In gastric crises, attention to the diet and bowels is of prime importance, and subcutaneous injections of atropin and hyoscine will, in their place, be found useful. Violent neuralgic pains sometimes occur in the face, which at first sight suggests the diagnosis of Tic douloureux.

2. Ataxia.-The manner in which the patient keeps his eyes on the ground and on his feet, and the sharp way in which he brings his foot down on the heel and spreads his legs apart, are characteristic. The tendons become relaxed and lengthened, and may give rise to deformities, such as Genu incurvatum, etc. Here, again, specific treatment is of very little use, while patiently practising exercises will often do wonders, showing in part the influence the mental state has upon the inco-ordination.

3. Reflexes.-The patellar reflex, as well as the ankle reflex, are lost early in the disease, the former disappearing as a rule before the latter. A diminution of these reflexes alone is insufficient evidence upon which to make a diagnosis, but an inequality is important. The following case is instructive in this respect:

CASE 80.-A woman, aged 26, was well treated with arseno-benzene and mercury for syphilis when the disease was contracted. Less than three years after the infection the patient consulted me because she had pains down the left leg. The only features discernible were inequality of the patellar reflexes, although both were brisk, a general whiter appearance of the left leg, which the patient noticed did not get red like the other in a hot bath, and slight peroneal anæsthesia. Examinations: C.F.T. blood positive; C.s.F. cells 92 per c.mm.; globulin increased; C.F.T. positive in all dilutions. Treatment did no good. Four years later the patient had lost both patellar and ankle reflexes, the pains affected both legs, and the anæsthesia had spread and become bilateral.

Since degenerative myelitis may affect any part of the cord-in fact, the lesions are often scattered-and as the loss of knee-jerks point to a lesion in the lumbar area, those cases must not be missed in which the patellar reflexes are present, because the disease is limited to the sacral region. In such cases the Achilles tendon reflexes are lost, and a characteristic, saddlelike area of anæsthesia is discernible, involving the gluteal regions, the perineum, anus, external genitals, and postero-inner surface of both thighs. In addition, there is usually marked bladder disturbance. The loss of the light reflex is an early symptom, while the loss of the arm reflexes appears rather later. The oculo-cardiac reflex is often lost early-i.e., no slowing of the pulse-rate occurs on pressure of the closed eyes.

Anæsthesia.--In the upper extremities the anæsthesia is almost invariably ulnar, while peroneal in the lower. Anæsthesia is common about the region of the second ribs, and when it is present it is usually zonal. In the same way on the face a branch of the fifth nerve may be anæsthetic; usually it is the nasal which supplies the tip of the nose with sensation. Sometimes the supra-orbital region is anæsthetic. Associated with the loss of tactile 
sensibility may be paræsthesiæ, the sensations most frequently complained of being those of insects crawling and those of tingling. Numbness is common, and patients, even when walking on stone, may have the same sensation as if it had been a Turkish carpet.

Palsies.-Most of the cranial nerve palsies have already been referred to, but there are those affecting the larynx which require a short description, as sudden death may result therefrom. The posticus palsy, due to a paralysis of the posterior crico-thyroid muscles, is the most common. Being abductors, a paralysis is apt to cause difficulty in breathing, but fortunately the paralysis is usually only unilateral.

Trophic Disturbances.- (a) Perforating ulcer, occurring anywhere on the foot where pressure is greatest, hence relief of pressure will often cause its disappearance.

(b) Arthropathy, or Charcot's joint, affecting any joint, but most commonly the knees, ankles, and hips. The lesions may be single or multiple; the articular surfaces rapidly get worn away; there is not much inclination to form osteophytes, the joint rapidly becomes distended, and sometimes flail.

(c) Fractures, spontaneous in nature, either due to an osteitis, or to the sudden jerky movement of an uncontrolled and lengthened tendon. Any bone may fracture, but when due to osteitis, it is usually the femur, and when due to the tendon contraction, either the patella or the os calcis. The fractures unite readily, but often throw out an enormous amount of callus. Anæsthesia is typical of arthropathies and fractures, which enables the former to be distinguished from cases of ordinary osteo-arthritis.

(d) Miscellaneous, such as painless whitlows, without lymphangitis and lymphadenitis, alopecia, etc. Degenerative myelitis has frequently associated with it syphilitic aortitis. I have seen one case with gummata of the legs, another develop a gumma of the penis, and several with syphilitic glossitis, otherwise systemic lesions are not commonly found. There are two symptoms requiring mention-namely, Herpes zoster and vitiligo-which are due to inflammation in or on the posterior root ganglia. Herpes zoster may be the first sign, or it may occur at any stage, and is apt to be brought out by injections of arseno-benzene. Vitiligo is also, when present, usually a premonitory sign.

I will now record a few of the most interesting cases of degenerative myelitis I have come across.

CASE 81.-A man contracted syphilis in 1902, and in 1913 developed degenerative myelitis. In 1917 he developed tabo-paresis, and between 1915 and 1917 he put on six stone in weight. As a rule, patients with degenerative lesions of the central nervous system lose weight, but in a few cases, especially in those who become paralytic, weight is put on extremely rapidly.

CASE 82.-A patient contracted syphilis in 1891 and suffered no ill effects till 1908, when he suddenly developed in two days a Charcot's ankle. A few days later he became ataxic in both arms and legs, then everything quietened down until 1916, when he had a fatal attack of hemiplegia. For many years his normal pulse was 118 . 
This case is interesting because of the sudden onset of symptoms, so long after infection. A sudden aggravation of symptoms, which may terminate fatally, may, without warning, follow an intraspinal injection of salvarsanised serum, and, having experienced two such mishaps, which fortunately recovered, although much damaged, I have given up intraspinal injections in favour of intravenous injections with auxiliary tapping. Another case I had unfortunately died a week following the fifth intraspinal injection, the other four having been well borre.

CASE 83.-The patient eontracted syphilis in August, 1914, and reeeived seven injections of arseno-benzene and mereury and iodides for two years. In March, 1916, he found he could not get an erection. In July, 1916, lightning pains made their appearance. On examination in August, 1916, pupils were normal, arm reflexes diminished, no knee- or ankle-jerks. There was bilateral peroneal anæsthesia, but no ulnar anæsthesia.

This case shows the onset of degenerative myelitis within a few months of contracting the disease, in spite of the treatment having been from the start up to date.

CASE 84.-Patient, now aged 53, contracted syphilis when twenty-one years of age. Seven years later lightning pains commenced, but nothing further happened for twenty-five years, when a Charcot's ankle appeared. He had the usual signs of posterior eolumn degeneration, but suffered no more inconvenience than a swollen ankle would naturally eause. The pupils were miotic, and reacted to neither light nor to accommodation.

CASE 85.-Patient had posterior column degeneration, with gastric erises, whieh followed diplopia, as the most troublesome symptom. Between January, 1915, and April, 1915, he had a series of intraspinal injections, which appeared to ameliorate the condition. In July, 1915, patient complained of severe depression and insomnia, associated with bad headaches and lack of vitality. On examination, instead of finding typical Argyll-Robertson pupils, the right had become larger than the left, tremors had appeared, and the knee-jerks had returned. Within a year this patient succumbed to general paralysis.

I could mention many cases in which intraspinal medication appeared to do wonders, but as equally good results may be obtained with other methods, and as I feel sure the onset of degenerative encephalitis in the last case was precipitated by the intraspinal injections, I have thought it wiser to pay more attention to the failures than to the successes, especially as the literature is full of the latter.

\section{Syphilitic Psychoses.}

Some of these cases present great difficulties in diagnosis, because positive changes are found, both in the blood and in the C.s.F., which may not differ from those typical of degenerative encephalitis. Clinically, it may be impossible to differentiate between degenerative encephalitis and manic depressive insanity until the patient has been watched for some time. The following is a case in point: 


\section{CLINICAL ASPECT AND TREATMENT OF VENEREAL DISEASES}

CASE 86.-Nine years ago I was called in to see a man who was extremely excited, and crying like a child; he had no sense of decency, and his personal habits, as could be observed, were filthy in the extreme. The patient was aged 52, and had contracted syphilis fifteen years previously. On inquiry it was found that he had been peculiar for some time, in that he had ideas that everybody was against him, and wanted to bring about his ruin; he had also talked about committing suicide. Quite lately he had become indifferent to the kind of clothes he wore, and dirty in his habits. As far as an examination could be made at the time, the diagnosis of degenerative encephalitis (G.P.I.) was made, and afterwards confirmed by two well-known neurologists. When the patient recovered a little, he was given a course of mercurial inunctions, which considerably improved him. Eighteen months later he again became very excited and boisterous in his conduct; his imagination was particularly rivid; he was full of ideas. This time I was able to make complete clinical and pathological examinations. The pupils were unequal, right >left; reaction to light was extremely sluggish, but to accommodation good. The other reflexes were exaggerated, and the patient had tremors. C.F.T. blood was very positive; C.s.F. showed a lymphocytosis, increase of globulin; C.F.T. very positive in all dilutions. Under two more courses of mercurial inunctions and iodides the patient recovered. Three years later he got a depressive attack again, which left him when the mercurial inunctions were repeated. During this relapse tests were done again, but they showed no change, and likewise was this the case when I saw him again in April, 1919. The last time I saw him he was elated, full of original ideas, witty in his speech, and quite amusing to speak to. He was worried because he could not get any work to do, as nobody would have anything to do with him, probably because of his garrulity and peculiarities. Clinically he still presents all the signs and symptoms of degenerative encephalitis.

This is certainly not the course run by a case of so-called G.P.I., and, to my mind, it is remarkable for the rapid response to treatment and the persistence of such a very strong C.F.T. in both the blood and C.s.F. In both is possibly to be found the fons et origo mali. When the C.F.T. is persistently very positive, the protein colloidal particles are always very large, and when such is the case their power of oxidation is reduced. Owing to the frequent association of depression with a very positive C.F.T., and the immediate relief of the same when a treatment is prescribed which breaks the particles up into smaller ones, thereby increasing their oxidising powers, I would suggest that, in some cases at any rate, the psychoses found in syphilis are due to the want of oxygen from which the nerve cells, which are already below par, suffer.

Another case I had did just as well under arseno-benzene and intramine as the one just described. In this, neither the clinical signs nor the pathological results obtained altered at all under the treatment. In this case the C.F.T. in both the blood and C.s.F. was very positive, and there was also a lymphocytosis. It is probable that some of the reported cases of G.P.I. which have done so well with treatment have really been cases of syphilitic psychosis, and it is also probable that there are many cases similar to the above, only much less marked, due to the same cause, but not presenting symptoms sufficiently clear to be diagnosed. On the other hand, as our 
knowledge of the ætiology of psychoses is limited, we must consider the possibility of the mania in these cases being inherited and associated with, and, perhaps, aggravated by, the syphilitic encephalitis. It is necessary to bear these points in mind, because I had a typical case of manic depressive psychosis, affecting a patient, who developed syphilis after he had had the psychosis for three years. The syphilis never passed the stage of the primary sore; he received nine injections of arseno-benzene, and injections of mercury and iodides internally for eighteen months. Owing to the recurrence of the psychosis he was sent round the world, but he committed suicide four months after his return. I have no doubt but that arsenic and mercury, especially the former, can cause psychoses. In some of the cases which I have in mind, the patient had a neuro-recurrence; therefore the psychosis, which was characterised by mental confusion, defects of orientation, and amnesia, might have been ascribed to the meningitis. In other cases, the patient had a primary sore only, and evidenced no pathological changes in the C.s.F.; therefore the psychosis could only be attributable to the arsenic. Having seen a typical Korsakow's syndrome occur in a case of arsenical neuritis, it is only reasonable to suppose that the profound depression with suicidal tendencies which is sometimes exhibited after a long course of arseno-benzene injections is directly caused by the metal. These views are strengthened by the facts that the psychoses met with in the neuro-recurrences disappear as the meningitis vanishes, and the same with those caused by arsenical poisoning when intramine is administered. I should like to mention here that prolonged arsenical treatment has the power of raising the intracranial pressure, as evidenced by testing the pressure of the C.s.F. as well as increasing the amount of albumin therein. I have recently had occasion to observe that patients who present signs and symptoms of arsenical neuritis in early syphilis are particularly prone to develop syphilitic nervous manifestations years afterwards.

Korsakow's syndrome may also be produced by syphilis without an associated polyneuritis, in which case the infection is of some years' standing, and the reflexes are exaggerated. Very difficult cases are those in which a paranoid psychosis or the symptoms resembling Dementia proecox develop in a syphilitic subject. Some years ago I had a patient, a man aged 32 , who developed symptoms closely resembling those of Dementia proecox, and pathological changes in the C.s.F. were found typical of a cerebro-spinal meningitis. Under intraspinal injections and other antisyphilitic treatment, which were prescribed until the reactions became negative, the patient improved, but the psychosis never disappeared. There was no mental history in this patient's family, and he had always been normal until he contracted syphilis, which was eighteen months before the psychosis appeared. On the other hand, I have seen a true Dementia procox affect a syphilitic subject in whom the C.s.F. was normal, and upon whom antisyphilitic treatment had not the slightest, effect. 


\section{CLINICAL ASPECT AND TREATMENT OF VENEREAL DISEASES}

Wimmer, ${ }^{23}$ in a recent article, warns us about accepting as of syphilitic origin cases of the manic depressive type or the various forms of paranoid psychoses, especially if a history of an inherited constitutional trouble can be elicited. Therefore, even if pathological changes in the C.s.F. are discernible, it does not necessarily follow that the psychosis is of syphilitic origin.

1. Heubser (1870): Archiv d. Heilkunde, xi., 280.

2. Ruмip (1885): Westphal's Archiv, xvi., 410.

3. ERB (1892): Neurolog. Centralblatt, xi., 161.

4. Lamy (1896): Revue Neurol., iv., 481.

5. DANA (1916): Textbook of Nerrous Diseases. J. Wright and Sons, Bristol.

6. Noxie (1916): Syphilis of the Nervous System (Ball's translation). J. Lippincott and Co., London.

7. Macramara (1913): Lancet, ji., 385 .

8. Alexaxder (1889): Syphilis u. Auge. J. Bergmann, Wiesbaden.

9. Maloney (1918): Locomotor Ataxia. Appleton and Co., London.

10. Bexedikt (1881): Wien. med. Presse, xxii., 101.

11. DÉJÉrine et Martix (1889): Séances et Mémoires de la Soc. de Biol., i., 43.

12. Мотт (1903): Archiv. Neurol. Lond. County Asylums, ii. 1.

13. Oppexhein (1887): Berl. klin. Woch., xxiv., 666.

14. Mirallié ett Descladx (1903): Revue Neurol., ji., 649.

15. Caxrov (1915): Bodily Changes in Pain, Hunger, Fear, and Rage. D. Appleton and Co., London.

16. Foursier (1891): Médecine Moderne. Paris ii., 37.

17. ORR AND Rows (1918): Brain, xli., 1.

18. Pardee (1919): Arch. Int. Med., xxiii., 174.

19. Edinger (1904): Deutsche med. Woch., xxx., 1633, 1801, 1921.

20. Rexie (1915): Med. Journ. of Australia, i., 375.

21. Spexce (1916): Lancet, i., 863.

22. Nuzum (1916): Journ. Americ. Med. Assoc., lxvi., 482.

23. Wimmer (1918): Hospitaltidende, lxi., 1169.

24. King Turier (1918): Brit. Med. Journ., ii., 255

25. Sutilerlaxd (1919): Lancet, i., 841. 


\section{CHAPTER XIV \\ SYPHILIS IN WOMEN}

\section{General.}

SYPHIIS in women may be looked upon as the greatest curse of the disease, since a woman who has once given birth to a syphilitic infant may infect, in utero, all her subsequent offspring, although the father of the latter may be a different husband who has never suffered from the disease.

To make matters worse, conceptional syphilis is not recognised until the infant has been seen to settle the diagnosis, owing to the fact that many mothers show no evidence of the disease until after the child-bearing period is over, as the following two cases illustrate:

CASE 87.-A woman, aged 46 years, came up to hospital in March, 1910, complaining of a rash on her right arm. The rash had appeared about Christmas, 1909, and some short time before she had had some sore places on the right leg. The lesion on the arm was a gumma, and the right leg was covered with the scars of gummatous ulceration. This patient was twenty-one years old when she married, and neither before her marriage nor since, until the date above mentioned, had she ever experienced the slightest evidence of syphilis. She had had four miscarriages; eleven children were born, two of whom were still living-the results of the second and fourth pregnancies. All the other children had died within six months after birth, as the result of syphilis. Her last pregnancy had been a miscarriage, immediately after which she developed a bad leg; this period also corresponded with the change of life. The patient had given a strong positive C.F.T. Her second pregnancy resulted in the birth of a son, who had given a negative C.F.T., as had also his wife and child. The fourth pregnancy resulted in the birth of a daughter, who had alsogiven anegative C.F.T. Neither child had shown the least taint of the disease.

CASE 88.-A woman, aged 47 years, had come up to hospital complaining of sores on the calf of the right leg, which were typical gummata. As in the preceding case, the ulcers had appeared just after the " change of life." The patient had been pregnant nine times; the children had mostly been premature. Some had been born alive, others born dead, but not one had lived for more than three weeks.

Since 1910 I have seen numerous similar cases, in all of whom I was able to obtain a positive C.F.T., provided the child-bearing period was over. This led me to rely upon, and to do, the test in every case in which a syphilitic infant had been born, when, to my surprise, I found that many cases of women who were giving birth to syphilitic children themselves gave a negative C.F.T.

A rough rule can be formulated-viz., that if a woman contracts syphilis after she has conceived, the C.F.T. will be positive, because the disease becomes 


\section{0}

generalised, and behaves in the ordinary way; but that if a woman contracts syphilis at the time of conception, the C.F.T. may be negative, because the disease, even if it does become generalised then, does not give rise to symptoms until some later date.

Herein we have the explanation why such patients only develop manifestations after the child-bearing period is over, and why it so frequently happens that the first and last pregnancies result disastrously, while one or more healthy children may be born in the interim. It is interesting to inquire into the rationale of conceptional syphilis.

The germ must, in the first instance, be conveyed by the semen. But does the germ, which travels with the embryo along the Fallopian tube into the uterus, develop after a time into the gamete forms described by me, which I regard as responsible for the symptoms, at the expense of the embryowith, maybe, its death-leave some of the spores behind after its expulsion, to be already there to develop at the expense of the next embryo ? Or does the mother become infected directly, while the symptoms are prevented from manifesting themselves owing to the formation of some chemical substance, possibly in the form of a lipoid-globulin compound from the embryo, which prevents the gametes from developing ?

When the question was discussed after the Spirochota fallida had been discovered, when the Spirochata pallida was held to be responsible for everything syphilitic, only confusion resulted. If my discovery of the Leucocytozoon syphilidis be accepted, and the views accepted that the spore is the infective agent, and that the gametes, the adult sexual forms, are responsible for the symptoms, the alternative need not appear in the above illustration, as both in part may turn out to be correct.

It may be considered that the spores, themselves only potentially harmful, travel in the semen, reach the uterus with the embryo, and find themselves in both the maternal and fœtal portions of the embryo. Those in the fœtal portion, after a period of some weeks, develop into gametes, which may or may not kill the embryo.

Those in the maternal portion find themselves unable to develop owing to a chemical substance emanating from the chorionic cells which circulates in the maternal, but not in the fotal blood, and so they remain dormant for a time. Herein lies the explanation of the phenomenon that a mother may give birth to a markedly syphilitic infant without herself even giving so much as a positive C.F.T.

The theory above put forward will also explain why a woman who has once given birth to a syphilitic child is always liable to do so again, although the father of her later children may be another husband, who has himself never suffered from the disease.

Hence the necessity for treating such a case throughout the whole period of each succeeding pregnancy.

There is no necessity to refer to the lengthy discussion relating to the 
greater frequency of maternal over paternal infection, or vice versâ. A father may be the cause of his first infant's contracting the disease; the mother, ipso facto, becomes likewise affected; her future children may be by another and a healthy man, but they may all be syphilitics. Therefore, maternal syphilis must obviously be more important and more frequent than paternal syphilis.

The information as to the national loss by ante-natal syphilis may be obtained from the oft-culled figures of Hochsinger. ${ }^{1,2}$

This observer, since 1869 , had been able to keep under observation 134 women who showed no signs of syphilis, but had given birth to syphilitic children. These women had given birth 569 times, 253 of the children being born dead-i.e., 44.4 per cent.-and 316 were born alive. Of those born alive 263 were syphilitic, and 53 were without a taint. Of the 263,55 died before the fourth year-i.e., over 20.0 per cent.

Veeder, ${ }^{4}$ in a much more recent article, confirms the figures given by Hochsinger. This observer had under observation 100 syphilitic families. There occurred 331 pregnancies, which resulted as follows: abortions, $30 \cdot 2$ per cent.; still-births, $9 \cdot 3$ per cent.; living births, 60.5 per cent. Thus, 40.0 per cent. of the pregnancies terminated in the death of the fœtus before term. Of the 200 cases born alive, 51 died, and only $\mathbf{3 3}$ of the remainder appeared to be clinically and serologically sound. From these figures, then, only 10.0 per cent. escaped. According to Routh, ${ }^{6}$ about $25^{\circ} 0$ per cent. of stillbirths and abortions are due to syphilis.

These figures are so appalling that it is of the utmost importance for every medical man to have particular regard for the welfare of syphilitic women in seeing that they are properly treated. Care taken at the beginning of pregnancy will always be well repaid, because if thorough treatment is kept up throughout the pregnancy it may be almost guaranteed that a healthy child will be born. I have been obliged to use the word almost, as the following case will show:

CASE 89.-A woman had generalised syphilis and infected her husband at marriage. Both had nine injections each of "914," and were treated intermittently with mercury and iodides for eighteen months, when by accident the wife conceived. When aware of this, I prescribed another nine injections of " 914 " and mercury till term; but during the sixth month the patient became desperately ill, and had to be relieved of a dead syphilitic infant. The patient recovered from the severe hæmorrhage which occurred at the time of operation, but died three weeks later from uncontrollable secondary uterine hæmorrhage.

Speaking generally, congenital syphilis is most likely to occur when both parents have syphilis, or when the mother contracts the disease in the ordinary way, conceptional syphilis being the least dangerous of all.

Reference must now be made to what we have called Colles' law, although Colles originally stated merely that syphilitic children did not infect their mothers. Colles neither enlarged upon this nor attempted to give a reason for the phenomenon. 


\section{CLINICAL ASPECT AND TREATMENT OF VENEREAL DISEASES}

As Colles has been unfortunate in his commentators, ${ }^{5}$ it would be as well to record the observations he made, which read as follows:

"The following fact appears to me very deserving of notice: I have never seen or heard of a single instance in which a syphilitic infant, suckled by its own mother, had produced ulceration of her breasts; whereas very few instances have occurred where a syphilitic infant had not infected a strange hired wet nurse, and who had been previously in good health.

"It is a curious fact that I never witnessed nor even heard of an instance in which a child deriving the infection of syphilis from its parents has caused an ulceration in the breasts of its mother" (New Sydenham Edition of Colles: p. 271).

"One fact well deserving our attention is this: that a child born of a mother who is without any obvious venereal symptoms, and which, without being exposed to any infection subsequent to its birth, shows this disease when a few weeks old; this child will infect the most healthy nurse, whether she suckle it or merely handle and dress it, and yet this child is never known to infect its own mother, even though she suckle it while it has venereal ulcers of the lips and tongue" (New Sydenham Edition of Colles, p. 287).

It is perfectly true that syphilitic children cannot infect their mothers, the reason being that the mothers are invariably syphilitic themselves, in spite of the fact that they may never have had symptoms nor have given even a positive C.F.T. Colles' observation goes strongly to support my view that there are phases in the life-history of the organism of syphilis other than the Spirochoeta pallida.

The observation, moreover, shows that no deductions can be made from a negative C.F.T. where women are concerned.

Profeta's law states that a healthy child born of a syphilitic mother is immune against syphilis, but loses its immunity at puberty. Apparently healthy children may be born' of syphilitic mothers, but yet be syphilitic, although they may never show signs or symptoms of the disease.

On the other hand, absolutely healthy children may be born of syphilitic mothers, but they are not immune against the disease. This possibility should be especially borne in mind to-day, when it is almost always possible -by thoroughly treating a syphilitic mother throughout the whole of her pregnancy - to render the child non-syphilitic at birth. Under such conditions, the mother should never be allowed to suckle the child, because in some cases the mother is not cured by the treatment, and the infective agent may pass through in the milk.

While Colles" "law" still holds good, Profeta's law does not.

Supposing a woman is pregnant, and then becomes infected with syphilis, what are the chances of the fotus becoming infected also? This is a question frequently asked, but extraordinarily difficult to answer. If the fœtus has just reached the uterus, and for a month or two afterwards, abortion is certain to occur. Between the third and sixth month it will be certain to catch the 
disease, die in utero, or be born alive with symptoms, to die shortly afterwards. From the seventh month onwards it usually escapes infection. It is impossible to be more definite than this, because, in the first place, none of us has much opportunity of watching such cases; and, in the second place, the result will be influenced by any treatment the mother undergoes. There is one point which might with advantage be mentioned here-namely, that resolution of a syphilitic fœtal lesion may take place in utero, as I have seen a new-born child with synechiæ which were almost certainly the result of a healed syphilitic iritis.

Another question sometimes asked is: Can syphilis be a cause of sterility ? As regards both sexes the answer is in the affirmative. It is probably more frequently so in the man than in the woman, and in some cases treatment would appear to correct it. On the other hand, one does "occasionally see cases in which syphilis, as well as tubercle, appears to have tended towards proclivity. A woman can infect a man although she has no ascertainable macroscopic lesion, while a man frequently infects a woman without either a macroscopic lesion or a positive C.F.T. I have known a case where a husband infected his wife twelve years after having contracted the disease.

In women the C.F.T. is of little value. If the reaction is positive, then it means to say the patient has had syphilis, and therefore requires to be treated throughout the period of each succeeding pregnancy, whether the last child was born without signs or symptoms, or not. Most syphilitic women give a negative C.F.T. during pregnancy; especially is this the case in conceptional syphilis, whilst in others the test only becomes positive during pregnancy, to revert to negative soon after the child is born. Some time after pregnancy, should another not supervene, or after the child-bearing period is over, the reaction frequently becomes positive. In some cases repeated pregnancies appear to result in a spontaneous cure of the disease. It is not yet realised that the results obtainable in the C.F.T. are cyclicthat is to say, that a negative reaction is just as close to a very strong positive as it is to a weakly positive. Artificial methods employed to make a serum positive, if pushed still further, make the same serum negative.

It is interesting to note, in passing, that not only women, but any syphilitic who is particularly run down and gives a negative C.F.T., may, as the health improves-on taking a tonic only, for instance-give a positive reaction. The importance of this observation will be seen in the treatment of gonorrhœa with chemotherapeutic agents and vaccines.

Another point of some interest is that twins of a syphilitic mother may not be equally affected by the disease; in fact, one may escape infection, as the following case shows:

CASE 90.-A woman became infected at the time of conception, and went full term to be delivered of twins eleven and a half years ago. A girl was born first, and a boy fifteen hours later. The mother has never shown a sign or symptom of syphilis, nor has she ever given a positive C.F.T.; the same with the daughter; while 
the boy is a typical congenital syphilitic in physiognomy, etc., and has always given a positive C.F.T. I have watched the three for several years, and have treated the husband. The mother and girl have had no treatment, while the boy has been treated thoroughly, and improved immensely, especially mentally.

\section{The Primary Sore.}

Many of the points about to be mentioned have already appeared in Chapter III., but as the clinical diagnosis of the initial lesion is one of the most important parts of the struggle against the whole disease, recapitulation is pardonable.

A chancre may occur in any part of the vulva, vagina, or cervix uteri. It always begins as a tiny papule, which in time becomes eroded on the surface.

No value should be attached to history. Asking a patient how soon after connection a sore appeared is often useless, because not infrequently a woman does not know that she has even got a sore. The good old rule that multiple sores are soft sores, single ones syphilitic, has led many astray, because multiple primary sores are more common in women than in men. A single sore on the genitals is usually syphilitic, but not invariably. Induration, when present, is positive, but its absence does not negative syphilis. Many of the multiple primary sores on the external genitals of women never are indurated. Of the greatest value in differential diagnosis is the appearance.

A syphilitic sore is sharply circumscribed, not irregular or undermined at the edge. The erosion is either on a level with the surrounding skin or raised above it. The surface is often dry, especially when on the labium majus; it has a shiny appearance, bleeds easily on friction, and does not discharge freely.

There is none of that surrounding inflammation which is so typical of soft sores and of other infective sores, because the organisms of the latter are pus-producing organisms, while the Leucocytozoon syphilidis is not. This is also the reason why the inflammatory sores are undermined, ragged-edged, and depressed in the centre beneath the circumference-ulcers, in contradistinction to erosions-and ulcers covered with pus, which discharge freely.

\section{Primary Stage.}

1. Erosive Chancre. - This is the most typical of all chancres. It is found most commonly on the labia. There is often a corresponding sore on the opposite side. It varies in size from that of a threepenny to that of a shilling piece, or it may be bigger. It is red, has a shiny surface, the erosion is flush with the surface, and the circumference is sharply circumscribed and regular. Induration is usually present, but a common symptom is the non-inflammatory, hard œdema of the labium on which the sore is situated. The œdema is due to syphilitic lymphangitis.

2. Papulo-pustular Chancre.-This chancre is almost invariably single, and practically only found on the true skin. 
3. Ecthymatous Chancre.-This chancre is also most frequently found on the skin. It is usually single, sharply circumscribed, raised and crusted on the surface. With this chancre there is often some inflammation of the surrounding tissues, owing to the fact that it is a chancre which has become secondarily infected.

4. Ulcerative Chancre.-This may be a sequela of any chancre which becomes secondarily infected. A phagedænic chancre is a further stage of this variety.

5. Pseudo-membranous Chancre.-These chancres are usually multiple: they are about the size of a threepenny-piece, they have a yellow base, which may be flush with the surface or raised above it, and there may be a red inflammatory ring surrounding each lesion. These chancres are usually slightly indurated.

At first sight the chancres look like soft sores, but they can be easily distinguished.

Although the former have a yellow base, no pus comes away from them. They are regular in outline, unlike soft sores. And, again, soft sores always tend to advance in one direction, while healing is taking place at the opposite pole. Furthermore, the surrounding inflammatory zone is much more apparent in the soft sore lesions, and, finally, in soft sores the base is uneven, and not smooth as it is in chancres.

6. Hypertrophic Chancre.-This type of chancre is uncommon, but may sometimes be met with on the labia majora. It is almost invariably single and it closely resembles the hypertrophic large spore ringworm seen on the hairy parts of the face.

7. Lenticular Chancre.-These chancres are invariably multiple. They vary in diameter from 1.0 to $5.0 \mathrm{~mm}$; they are circular, regular in outline, and are merely abrasions.

Most of the types of chancres above described may occur in the vagina and on the cervix uteri. In the vagina they seldom give rise to difficulties in diagnosis, because other venereal infections very rarely attack that tract. Chancres on the cervix are frequently diagnosed wrongly, and then are usually mistaken for erosions, aphthous ulcers, gummata and tuberculous ulcers, and Ulcera mollia.

8. Cervical Chancre.-Chancres of the cervix, which occur in about 1.5 per cent. of cases, nearly always give rise to an indurative œdema of the whole cervix, often giving it a characteristic dark red-purple colour, as if it were venously congested. The lesion may be no more than an erosion, or an ulcer, covered with a diphtheroid membrane, which cannot easily be wiped away.

Erosions can be distinguished from chancres because the cervix is soft and of normal colour; the erosion is bright red, not as a rule sharply circumscribed. In some areas it is difficult to say where erosion ends and normal mucous membrane begins, a feature which is common to traumatic lesions. If an erosion is covered with pus which looks like a membrane, it is easily rubbed off, and this is not the case with a primary sore. 
The follicular and papillary erosions are too distinctive to be confused with any other condition.

In a majority of cases where there is an erosion there is a discharge or exudation from the cervical canal, and this discharge was possibly primarily responsible for the erosion.

Aphthous ulcers are soft, multiple, either flush with the surface or only a little depressed beneath it. They have no undermined edges, such as soft sores frequently have. They have a membranous base, which cannot be rubbed away, and they are almost invariably surrounded by a narrow inflammatory ring.

Gummata are more deeply ulcerated than chancres. Unlike chancres, they tend to spread a little, and, for the degree of ulceration, the surrounding parts are softer and less inflammatory than would be the case if in an ulcerative chancre.

Tubercular ulcers only occur in women who have marked signs of tuberculosis elsewhere. The ulcers are, as a rule, circumscribed, and can generally be diagnosed from other forms of ulceration because, surrounding the ulcers, tubercles are frequently to be seen, and tubercular ulcers are very painful.

Ulcera Mollia.-The sores are soft, multiple, undermined, and surrounded by a red and inflammatory ring. They discharge freely, and the ulcers quickly spread, but, as a rule, in one direction only.

Syphilitic sores of the external genitalia are frequently mistaken for soft sores. This need not be so, for they can be readily distinguished clinically. Failing clinical differentiation, they may be distinguished pathologically, since the spirochæta can be found in the former, and Ducrey's streptobacillus, a Gram-negative organism, in the latter. Syphilitic sores may be confounded with Vulvitis erosiva et gangrenosa, and with the Ulcera pseudo-venerea. A soft sore possesses a feature which is possessed by practically no other ulcer with which we are concerned; it is auto-inoculable.

The Ulcera pseudo-venerea have only very recently been recognised, owing to the work of Lipschütz. ${ }^{3}$ Their occurrence should always be borne in mind, since virgins are most frequently affected; therefore they are not venereal in origin in the sense that syphilis is so.

They may occur "overnight," and are usually ushered in with fever rigors, and local pains. With or without treatment they tend to disappear quickly. In other cases the onset is not so sudden, and the course of the trouble may last several weeks.

The ulcers are soft, deep, usually undermined, and the base is covered with pus. Owing to the close resemblance these ulcers bear to soft sores, a bacteriological examination is usually necessary. The organism always found in Ulcera pseudo-venerea is a Gram-positive bacillus, occurring either alone or in chains, or in threads like a streptothrix. Its ends are square, and no success has so far been achieved in attempts to cultivate it.

Oddly enough, two cases of which I have notes gave a positive Widal's 
reaction. Lipschütz also had a similar experience, although none of the cases developed other symptoms of typhoid. A bacteriological examination will also serve to distinguish these ulcers from the Ulcera gangrenosa, in which the fusiform bacilli and unevenly coiled spirochætæ are found living in symbiosis - the same organisms which cause Vincent's angina.

Gonococeal Ulcers.-These ulcers are more common in women than men; as a rule they are multiple, but they may be single. When multiple they are small and 'generally crateriform. When single it is usually an ulcer situated on the posterior wall of Bartholin's gland resulting from an abscess. The anterior wall is laid open, so if the labium is pressed antero-posteriorly a cavity is exposed. Later the posterior wall becomes perforated. This type of ulcer at first sight is very liable to be diagnosed as a chancre.

\section{Generalised Syphilis.}

The marked difference between the stage of generalisation as it affects men and women is the very much larger proportion of the latter which develop Leucoderma colli. The areas of leucoderma may be discrete or confluent. When confluent, they are frequently arranged in the form of a rosette. These areas occur where macules have been, and, not infrequently, in the centre of the de-pigmented area a hyper-pigmented spot is to be seen, and it occurs where a papule has succeeded the macule.

The condition is more marked in brunettes than in blondes, because it is more easily seen. The condition is probably more common in women than in men, owing to the skin being more delicate and not so red in the former; therefore the contrast is more noticeable. Most women exhibit the condition; it may be limited to the neck and anterior folds of the axillæ, or it may occur over the whole body. Treatment does not alter the condition; it is a chronic one, begins in the early stage of the generalisation of the virus, and only tends to disappear in course of time, which is often a matter of years, usually from one to four years.

\section{Syphilis of the Generative Organs.}

Unfortunately, this branch of syphilis is still more or less veiled in obscurity. A few stray cases of syphilis of the uterus, Fallopian tubes, and ovaries, have been described, but no light has been thrown upon them so far as their differential diagnosis and pathology is concerned.

Recently, some observers have attempted to gauge the relative frequency of syphilis as the cause of chronic metritis by the C.F.T. In the first place, the percentage of positive results obtained was too great to allow much im. portance to be attached to them; and, in the second place, because the reaction is positive, it does not necessarily follow that the menorrhagia and metrorrhagia of which the patient is complaining are significant of a syphilitic metritis. Such symptoms are common in women who have had children, but who have never suffered from a venereal disease.

They are extremely common in women who have had gonorrhœa, and 
it is absolutely impossible from either a clinical or a pathological examination to diagnose a gonococcal from a syphilitic metritis. That syphilitic metritis cannot be very common is seen from the fact that if a series of cases with symptoms of chronic metritis giving a positive C.F.T. be treated with arsenobenzene and mercury, in only a small minority do the symptoms vanish.

Diffuse syphilitic pelvic cellulitis does undoubtedly occur. It is apt to cause stricture of the rectum, and it is almost invariably diagnosed as malignant disease.

\section{Difference between Syphilis in Men and Women.}

It is a well-known fact that syphilis in women is not nearly such a serious disease-i.e., so far as the individual herself is concerned-as it is in men., There is no difference in the nature and severity of the primary lesions in the two sexes. The early manifestations of the generalisation stage are also much the same, but the later manifestations are very much milder in women than in men. Recurrences are fewer and milder in the female sex, and arterial lesions are, comparatively speaking, rare. Gummata, on the other hand, may be equally frequent and severe in both sexes. Late nerve lesions are much less common in women than in men; but early meningeal lesions, which so frequently occur after a course of arseno-benzene injections, affect women and men equally.

There is no doubt that in women who are bearing children the symptoms of syphilis are even milder still. From the facts just brought forward, one is tempted to conclude that the serum of women contains more natural protective substances than the serum of men does, and that these protective substances are still further increased during pregnancy. Routh ${ }^{6}$ goes somewhat further than this, and considers that the protective substances are chorionic ferments. Owing to the fact that the incidence of gummata in both sexes is about equal, and since gummata, as a rule, do not break out until after the ages of 45 to 50 have been reached, it would appear that women lose their increase of natural protective substances after the menopause.

The protective substance against syphilis, as well as against any other infection, microbic or otherwise (vide placenta), is contained in the lipoidglobulin colloidal particles of the serum; but in each infection some of these particles have a stereo-chemical molecular configuration homologous to that of the infective agent, this accounting for specificity. There appears. to be no limit to the range of the specificity.

The specificity does not lie in the lipoid-globulin particles as lipoid-globulin, but in the foundation upon which the particles are built up.

A lipoid-globulin particle may be likened to a house which is built of bricks. The house is always the same, but the bricks with which it is built vary. These bricks are the amino-acids, polypeptides, etc., which go to build up the particle, which is the house. Specificity is caused by the variation in the bricks. We know that the range of specificity is unlimited; therefore the variation in the bricks is not so much the alteration of one brick as a change in the combination of several. 
This being the case, it is possible for different infections to prcaluce partly similar combinations of the groups which go to make up the lipoid-globulin particles. The combination may be the same up to a point, and then the last brick or two may make all the difference.

If this be the case, it may so happen that some of the groups which go to make up the syphilitic lipoid-globulin particles are also the same as some of those which go to make up the lipoid-globulin particles of the sera of women. In other words, there is a common combination of the groups which constitute the lipoidglobulin particles of syphilitic sera and the sera of women up to the menopause, and that the similarity becomes the closer in women who are pregnant.

That this is not pure theory is proved by the fact that the sera of syphilitic non-pregnant women give a positive Abderhalden's reaction with placental extract.

Abderhalden's reaction is, in my opinion, a physical reaction, which depends upon the adsorption of particles possessing homologous stereo-chemical molecular configurations. When such an adsorption occurs the particles are precipitated, and when dialysed they break up. The hydrolysis results in an increase of dialysable amino-acids, which give the positive ninhydrin reaction.

Sera of syphilitic non-pregnant women behave, then, in the same way as the sera of pregnant women. Placental extract contains the analytic products of placental protein, and these products represent some of the bricks.

For adsorption to take place between placental extract and the sera of both pregnant women and syphilitic non-pregnant women, the stereo-chemical molecular configuration of the placental extract particles must have its homologue in the serum.

This proves that some of the bricks which are responsible for the specificity of the sera of pregnant women towards placental extract are likewise responsible for the specificity to be met with in a syphilitic serum. Therefore, that syphilis is a less severe disease in women is probably owing to the facts (1) that sexual differences render the sera of women more like syphilitic sera; (2) that the protective substance is augmented by the chorionic protein colloidal particles during pregnancy, which would account for conceptional syphilis being milder than an infection contracted in the ordinary way, because the chorionic particles are able to act on the site of infection, from which the organisms may never have left to have become generalised; (3) that greater protection is required against protozoa than against bacteria, and against human cells than against protozoa; therefore the chorionic particles would be expected to be particularly actively opposed to the development of the Leucocytozoon syphilidis.

1. HOCISINGER (1910): Wien. klin. Wooh., xxiii., 881, 932.

2. Hochsinger (1898): Studien über die hereditäre Syphilis. A. Hölder, Leipzig.

3. Lipschürz (1913):-Archiv f. Derm. u. Syph., exiv., 363.

4. Veeder and Jeans (1916) Amer. Journ. Dis. of Children, xi., 177.

5. AbNer Post (1916): Amer. Journ. of Cutan. Dis., xxxiv., 589.

6. Routh (1918): Lancet, i., 45. 


\section{CHAPTER XV \\ CONGENITAL SYPHILIS}

\section{History.}

Congenital syphilis was known to both physicians and to the public within a few years of the disease coming to Europe. Paracelsus, in 1528, recognised conceptional syphilis, that children could be born healthy of parents who had the disease, that congenital syphilis might not produce symptoms until the child had reached adult age, and that congenital syphilitics could pass on the disease to their children. Paracelsus was also familiar with the symptoms, and indeed knew more about congenital syphilis, than many of the better-known syphilologists did who long after followed him. The term "born half-baked," which was often applied to congenital syphilitics, originated with Fallopius. Ambrose Paré made the observation that congenital was more difficult to treat than acquired syphilis. Hunter denied the existence of congenital syphilis, an opinion which was not universally held owing to the fact that at that time there was an institute for syphilitic children, pregnant women, and nurses in Paris (Vaugirard) which gave ample opportunities for substantiating the truths enunciated by Paracelsus. In spite of work done by the physicians of this institute, it is doubtful whether congenital syphilis was generally recognised until the monographs of Diday (1854) and Jonathan Hutchinson (1863) appeared.

The immunity of women who had given birth to syphilitic children was first recognised by Benjamin Bell, established by Colles and Baumes, and confirmed by Ricord and Diday.

\section{General.}

Syphilitic infection causes abortion, miscarriage, or still-birth; or the birth before or at full time of a live child showing signs of the disease, either at birth or at some subsequent period.

Congenital syphilis resembles the acquired form, the chief difference being that it is a general infection from the beginning, while the acquired variety commences with a local lesion or chancre. It is a more serious form of the disease than is the acquired form. In the former, the tissues affected are undeveloped, and therefore fall a more easy prey to the poison, the mortality being high, while death, as the result of acquired syphilis, is the exception. 
In the severer form, death takes place in utero, and the macerated fotus is expelled two or three weeks later.

A history of such an abortion occurring in each successive pregnancy is characteristic of syphilis, but habitual abortion of a non-macerated fœetus, within the first four months of pregnancy, is not proof (or evidence) of syphilis.

Frequently, after a series of abortions, a seven or eight months' child is born alive. Premature labours likewise not uncommonly run in succession, until, finally, a full-term and possibly non-syphilitic child is born. It is not at all uncommon to find the first few and the last few pregnancies disastrous, one or two healthy children being born in between.

Many syphilitic children, though born alive, die a few hours or a few days after birth, section usually showing marked syphilitic changes in the internal organs.

Such children come into the world thin and marasmic, with dry, lax, and wrinkled skin, an old and haggard facial expression, and a weak and scarcely audible voice. Arrest of development may occur at any period of extrauterine life; growth is stunted; there is lateness in dentition, speech, and walking; frequently there is deficiency of intelligence, and also a delay in the changes of puberty.

The degrees to which errors of development, such as hare-lip, cleft-palate, club-foot, Spina bifida, and hydrocephalus, are dependent upon syphilis must at present remain unsettled; but in a certain proportion of cases a definite relationship seems to be suggested.

The danger of producing a syphilitic child is greatest during the first year after the contraction of the disease; is great during the first four years, but is largely influenced by treatment; it then diminishes.

Some rough rules can also be formulated concerning the incidence of infection in cases where the mother contracts the disease while pregnant. A syphilitic infection of the mother occurring before the fœtus is five months old will infect the child; during the fifth and sixth months the chances of infection and escape therefrom are equal; after the sixth month the fotus will not be infected.

If the parents are properly treated, healthy children can be produced.

Although of extremely rare occurrence, cases have been recorded in which the disease was handed down to the third generation-i.e., cases of congenital syphilitics propagating syphilis. I have so far only come across two instances. It is practically certain that such cases were not at all uncommon when syphilis invaded Europe as an epidemic.

CASE 91.-A woman, aged 37, brought to see me two of her children suffering from ringworm. The mother had signs of old bilateral interstitial keratitis, fissures at the angles of the mouth, and well-marked Hutchinson's teeth. She had had seven children, no abortions or miscarriages. I examined the seven children, and found that onlys two had any symptoms of syphilis; the others were perfectly healthy. These two died later, at the ages of 8 and 9 , of degenerative encephalitis, while the other children 
remained perfectly healthy, but they are said to be extremely backward at school. The mother's C.F.T. was positive, the father's was negative; the two affected children gave a positive reaction, two of the others gave a doubtful reaction, which two years later had become negative, and the other three gave a negative reaction throughout.

There is no doubt that a child may suffer because his parents and grandparents have had syphilis, without himself showing any symptoms or giving a positive C.F.T. Mental deficiency is a common result, and it is probable that this is the way syphilis plays a rôle, if it does so at all, in the causation of congenital malformations.

The stages of congenital syphilis may be divided into three: (1) generalisation stage; (2) late stage, Syphilis congenita tarda ; (3) latent stage.

Symptoms of the generalisation stage may appear for the first time in utero. If the child is born healthy and is going to develop generalised symptoms, in practically 100 per cent. they will appear before the sixth month, in 80 per cent. before the end of the second month, and most likely during the first month. Lesions appearing after the sixth month are usually single, and resemble in this respect the recurrent syphilitic manifestations in acquired syphilis. Symptoms of Syphilis congenita tarda are most apt to occur about the age of puberty, but their onset may be delayed until the patient has passed the age of 30 . It is my impression that the strain of war has greatly increased the number of cases, as I have been struck by the frequency with which Al men between the ages of 18 and 25 have developed symptoms of congenital syphilis, apparently for the first time. Possibly without the strain of war some of these cases would have remained in the latent stage, and have never developed symptoms. As a rule, cases of Syphilis congenita tarda carry stigmata with them-i.e., facial expression, shape of skull, fissures at the angles of the mouth, and dental changes. On the other hand, the patient may, except for the lesion complained of, be and appear healthy, as far as one can see. The C.F.T. is almost invariably positive when there are symptoms; it is not readily influenced by treatment-indeed, treatment may have no effect upon it in certain cases of late congenital syphilis. In cases which develop symptoms early, the C.F.T. in time becomes negative, and many of these patients are, as far as one can say, cured. Although it is possible for the C.F.T. to be negative during the latent period, and only to become positive when clinical symptoms have appeared, it is more usual for the reaction to have been positive, anyhow, for sometime in those cases which develop symptoms for the first time after having passed the age of puberty. Treatment acts like a charm in early congenital syphilis, provided the symptoms are not very severe, while in late congenital syphilis it may be totally unavailing.

Apart from the wasting and malnutrition which signalise early congenital syphilis, the most frequent symptoms of the generalisation stage are a rash, which almost invariably affects the palms and soles, a rhinitis, and an enlarged spleen. 
PLATE XLVII

Nos. 86, 87, and 88.-Typical Physiognomy of a Congenital Syphii.ltic, showing absence of the Nasal Bridge and Scars around THE MoUTH

The patient had also corneal leucomata, which resulted from a previous attack of bilateral interstitial keratitis. The teeth are typical in that they are irregular, the upper incisors do not meet the lower, and one of the upper incisors shows the absence of the middle tubercle. 


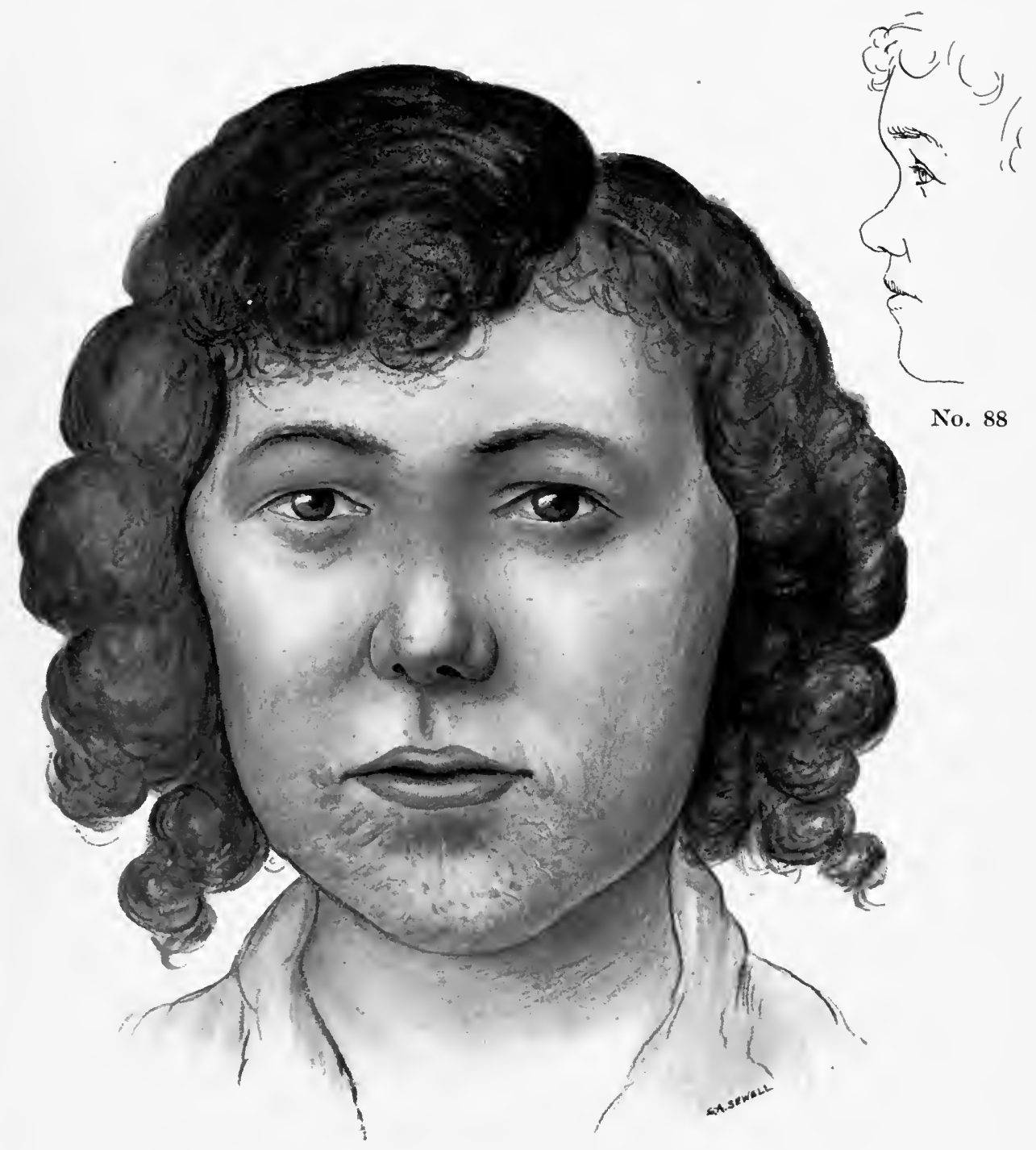

No. 86

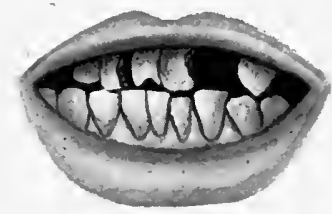

No. 87

Plate XlviI 
Epiphysitis is common, and so are choroiditis and orchitis. If the symptoms are very severe, and the patient very wasted, treatment does not do much good, and a very large number of cases die. Between the ages of 5 and 12 nervous manifestations are perhaps the commonest. From 12 onwards the most frequent lesions are interstitial keratitis, multiple arthritis, and gummata, in any part of the body. I do not wish to assert that congenital syphilis is prone to lead to malignant disease, but it is an odd fact that I have come across three cases of congenital syphilis which died between the ages of 17 and 20 from sarcoma of the sternum. One of the cases had undoubtedly had a syphilitic periostitis of this bone, but in the other two I could get no history. In every case of supposed congenital syphilis met with the possibility of it being of the acquired form should always be excluded.

\section{Skin.}

The skin lesions of congenital syphilis, like those of the acquired form, consist of macular, papular, and pustular rashes. In both cases the rash affects the whole body, but in congenital syphilis it shows a marked predilection for the palms of the hands and soles of the feet; so characteristic do some authors regard this, that they state that papules found in these situations can be safely regarded as syphilitic, and that their absence is evidence that an eruption is non-syphilitic. In spite of the commonly entertained opinion to the contrary, a rash on the buttocks is not absolutely diagnostic of syphilis, being more usually due to the use of dirty napkins $;{ }^{6}$ adjacent surfaces that rub against the buttocks-the backs of the thighs, the calves of the legs, and the heels-are similarly affected.

The non-syphilitic erythemata are always of a bright red inflammatory colour, while the rashes of congenital syphilis have a marked brownish tint, and are often very pronounced in the flexures, where, owing to continuous friction, the horny layer is rubbed off and the surface eroded.

The presence of ulcers does not necessarily indicate syphilis, for the erythemata may become ulcerated; and sometimes deep, punched-out ulcers, resembling gummata, are found-the so-called ecthyma of vacciniform dermatitis. To avoid a wrong diagnosis one should always bear in mind Kaposi's axiom that a polymorphic skin eruption on a baby is diagnostic of congenital syphilis; for example, the presence of a macular rash on the face or other parts of the body, and of a papular rash on the palms and soles, will go far to establish the syphilitic nature of a doubtful rash on the buttocks.

Seborrhœic dermatitis is frequently mistaken for a syphilitic rash, but in the former the scalp is invariably dry and scurfy, and the mother is usually suffering from seborrhœa.

A congenital syphilitic roseola is practically unknown. The commonest congenital syphilide consists of macules distributed over the whole body, and well marked on the face and head. On the face, the eruption is not infrequently orbicular. 


\section{CLINICAL ASPECT AND TREATMENT OF VENEREAL DISEASES}

The papular syphilide affects chiefly the genitals, anus, palms of the hands, and scles of the feet, at the same time as the macular syphilide is present. on the trunk. The papules may coalesce, and the surface may become scaly or eroded; the erosions or rhagades occurring at the corners of the mouth leave, after healing, those radial scars so suggestive of congenital syphilis. Linear scars are also found along the lower lip-a point of some importance, since rhagades at the corners may occur after any acute illness. Fissures are not infrequently found beside the nose and around the anus. Peeling of the palms is a useful diagnostic sign.

Condylomata are found around the anus, and more rarely in the mouth; they do not usually make their appearance until the child is some months old, often a year or more; as a rule, the rash has disappeared and their presence denotes absence or inadequacy of treatment.

The pustular syphilide is the Pemphigus syphiliticus neonatorum of the older writers; it is seldom found alone, for it is almost invariably accompanied by a maculo-papular rash. The typical papules on the palms and soles confirm the diagnosis, and serve to distinguish the syphilitic pemphigus from the streptococcal variety of Pemphigus neonatorum. When found alone, however, it is usually limited to the palms and soles.

Syphilitic pemphigus is generally present at birth, although it may develop later, but seldom after the first few days.

The streptococcal pemphigus always appears after birth, and is really a form of impetigo, impetigo being usually found in some other member of the family; it does not, as a rule, attack the palms and soles. Further, a child with syphilitic pemphigus is always wasted, and looks to be at death's door, while a child with streptococcal pemphigus may look fat and cheerful. The former disease generally ends fatally, while the latter responds readily to weak antiseptic baths.

Gummata are occasionally seen, but do not in any way differ from the acquired form. Cases have also been described of symmetrical gangrene, with and without paroxysmal hæmoglobinuria, occurring in children after two years of age.

It is still open to question whether the cases of purpura which have been described as occurring in congenital syphilis, usually between the ages of 5 and 10 years, are really specific in nature.

The nails are not exempt, and syphilitic onychia is by no means uncommon. The matrix becomes inflamed, and the nail over it loses its gloss and becomes irregular on the surface; the whole nail is gradually shed, and, unless mercurial treatment is given, the new nail will likewise suffer. The bullæ of pemphigus may affect the matrix, the nail being raised off its bed and undermined by sero-pus.

The following is an interesting case, because the nail condition, which was a typical syphilitic onychia, did not appear until the patient was 22 years of age: 


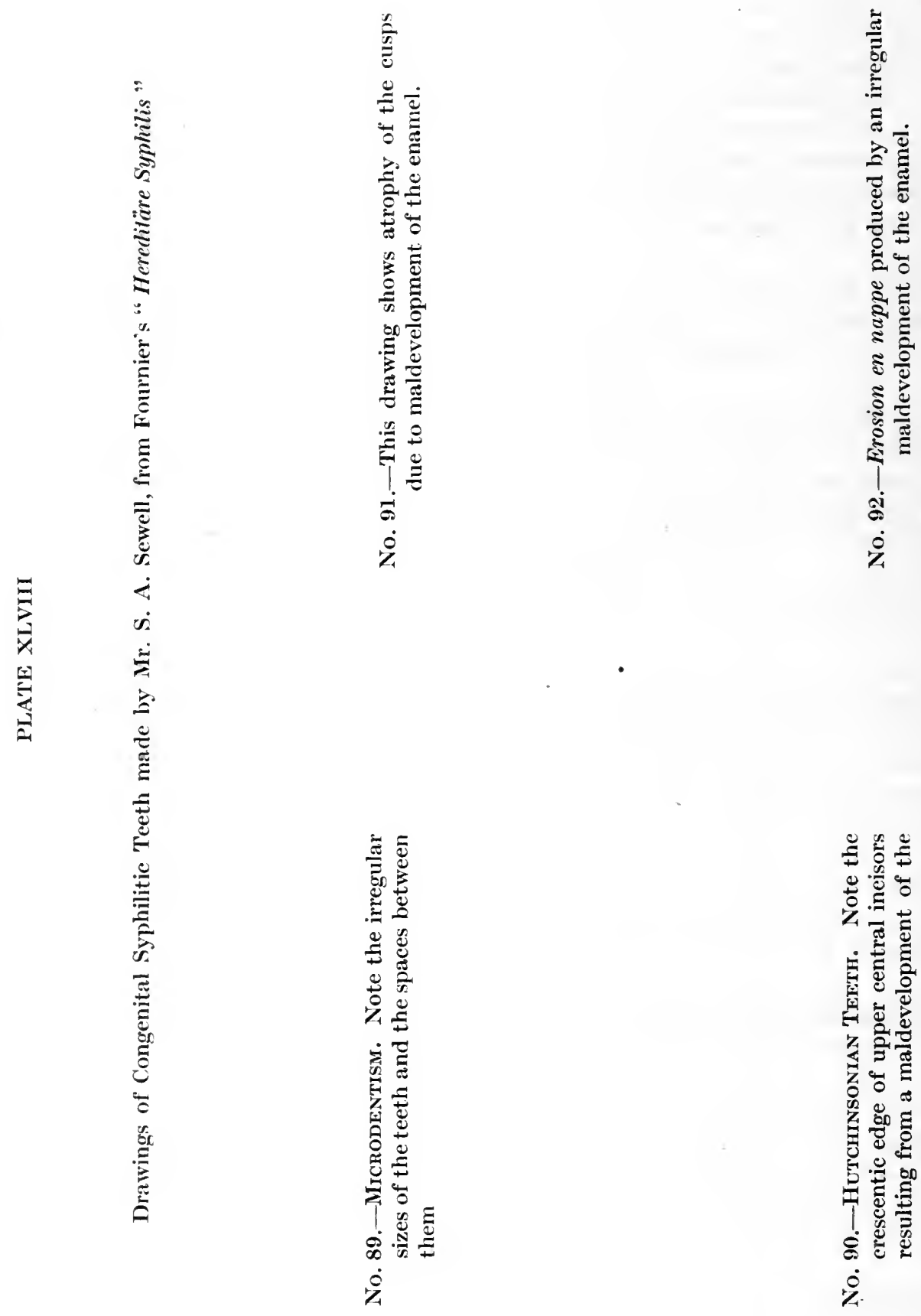

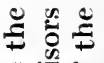

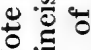
乙.

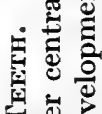

$\Rightarrow$

学矛

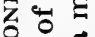

沄范

.

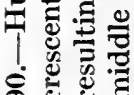

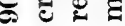

으. 

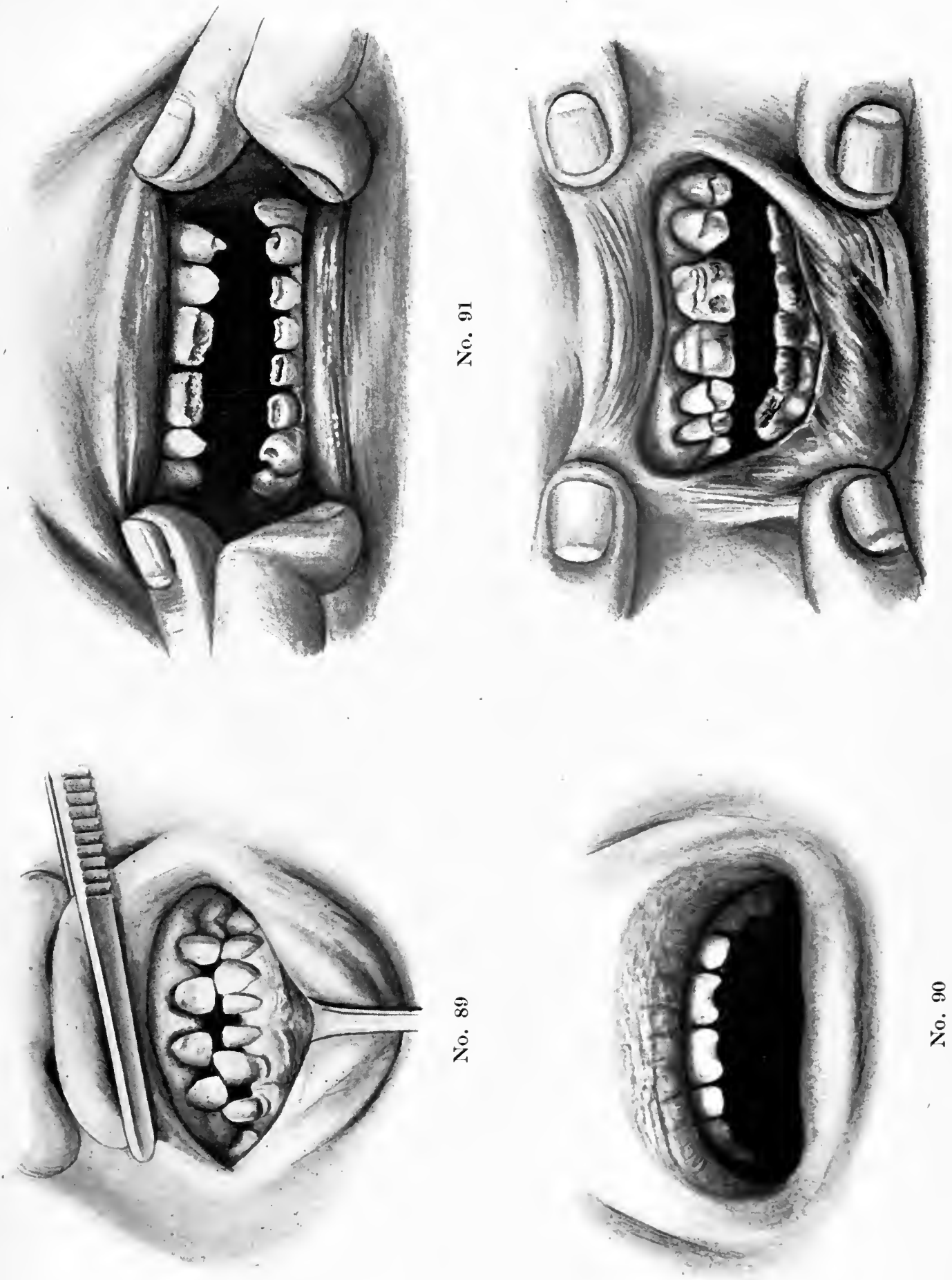
CASE 92.-The-patient, a girl, aged 22, the second child of a mother who died in a lunatic asylum, consulted me for an eye trouble. The cranium was typical of congenital syphilis, but the teeth were normal. The patient had syphilitic onychia of both index fingers and the left thumb, which had been present for two months. It must have been a papular onychia, as only a part of the nail was affected, and because che condition responded to treatment. The patient also had bilateral interstitial keratitis, with a sub-luxation of the left lens up and in.

Diffuse Defluvium capillitii is not uncommon in congenital syphilis. Some children are born without any hair on the head, but such an occurrence is distinctly rare.

The lanugo hair often persists longer than usual; when it disappears, the scalp is left bald or sparsely covered, since, owing to the malnutrition, the new hair does not grow.

The alopecia may affect the eyebrows also. Indeed, thinning of the eyebrows in an infant a few months old is very suggestive of syphilis.

Gummata are late lesions, and differ in no way from the same lesions in the acquired form.

\section{Teeth.}

Contrary to current opinion, the primary teeth are occasionally affected. Still $^{4}$ recorded a case of the primary central incisors resembling in every particular the so-called Hutchinson's teeth.

The permanent teeth show marked and characteristic changes, especially the incisor teeth of the upper jaw, which are shorter and smaller than normal teeth; consequently, there are gaps between the teeth, and when the mouth is closed the teeth of the upper and lower jaws do not meet; the edges are not parallel, but conical and wedge-shaped. The free border is thin and crescentic, a central notch being caused by lack of development of the middle tubercle. The low central incisors not rarely present changes. They may resemble the incisors of the upper jaw, but more often they are rounded, and, in their upper parts, deficient in enamel, and therefore thin and rough. The canines may occasionally be notched, and the molars are not infrequently dome-shaped, owing to the mal-development of their tubercles.

Owing to the prominence given in the literature to the so-called Hutchinson's teeth, a typical example of which is by no means frequently met with, the slighter changes are nearly always overlooked; so I propose to mention the latter in more detail than is done in the outline just given. Teeth which are otherwise normal, but widely interspaced, are strongly suggestive of syphilis. Partial loss of enamel, giving each tooth affected a pitted or a ridged surface, is characteristic. Complete loss of enamel in all the teeth is, in my experience, never met with in syphilis. Teeth which frequently show a partial loss of enamel are the first permanent molars, presumably because their development occurs at a time when the disease in the fœtus is most 


\section{6

active. A little outgrowth on the inner margin, which resembles a supernumerary cusp, is frequently met with, a condition first described by Sabouraud, and named "humpy molar" by Roberts." The last-named observer states that this rudimentary cusp is sometimes seen on the second permanent molars and on the molars of the deciduous group. Early decay of the first and second sets of teeth is common in congenital syphilis, but not characteristic of the disease. Microdentism is not uncommon, and Roberts regards the retention of deciduous teeth long after the period when they are usually replaced, as a significant sign.

\section{Bones and Joints.}

Bone affections in congenital syphilis are usually late signs; but changes in utero do occur-e.g., gummata resulting in spontaneous fractures. Further, there is a characteristic bone lesion of early syphilis, often found at birth, called by its discoverer-Wegner-Osteo-chondritis syphilitica. This is, in the main, an epiphysial disease which affects the long bones and ribs, and is found in almost every case of congenital syphilis, although it may not be sufficiently pronounced to be diagnosed during life. Post-mortem, it is perhaps the most valuable sign in a doubtful case. It is frequently present at birth, but cannot, as a rule, be diagnosed by external signs until some months later. It reaches its acme at the age when rickets are common, making a differential diagnosis extremely difficult. The incidence of this condition is as follows: It is most marked in the lower epiphysis of the femur; next, in the lower epiphyses of the tibia, ulna, and radius, upper epiphyses of the tibia, femur, and fibula; and least in the lower end of the humerus. The increased growth leads to an enlargement of the epiphyses; as a sequel, the bone involved may be shortened or lengthened, or the epiphysis may be separated from the diaphysis. The separated epiphysis usually unites again with the shaft, and no permanent disfigurement ensues.

Separation of an epiphysis gives rise to a chain of symptoms to which the term pseudo-paralysis is frequently applied. In such a case, if the affected limb be raised and dropped, it falls as if paralysed; spontaneous movements are impossible, but muscular action persists; pressure and movement are painful. It always occurs in the first few weeks of life.

According to Schmidt, the pathological changes which result in a separation of the epiphysis are, first, an increase in width of the medulla, in which the cartilage cells disappear and become absorbed by the bloodvessels and the medullary tissue; and, subsequently, a growth of granulation tissue between the diaphysis and the epiphysis. It was held that this granulation tissue originated from the bone-marrow of the diaphysis, but Schmidt showed it to be a richly cellular connective tissue, containing an extraordinary number of bloodvessels, which are shut off from the diaphysis, but communicate with the vessels of the perichondrium, from which they originate. 
. 


\section{PLATE XLIX}

Drawings of Congenital Syphilitic Bonc Lesions made by Mr. S. A. Sewell from Fournier's "Hereditäre Syphilis"

No. 93.-The so-called Olympic forehead, produced by the osteitis, which causes the frontal eminences and a submucous periostitis of the nasal bones, thereby destroying the bridge of the nose.
No. 94.-The so-called natiform skull, produced by an osteo-pericranitis of the frontal and parietal eminences. Note the large patent anterior fontanelle.

No. 95.-Whis drawing shows the sabrc-tibiae due to osteo-periostitis, with gummatous ulceration of the skin. Note also the loss of the plantar arches. 

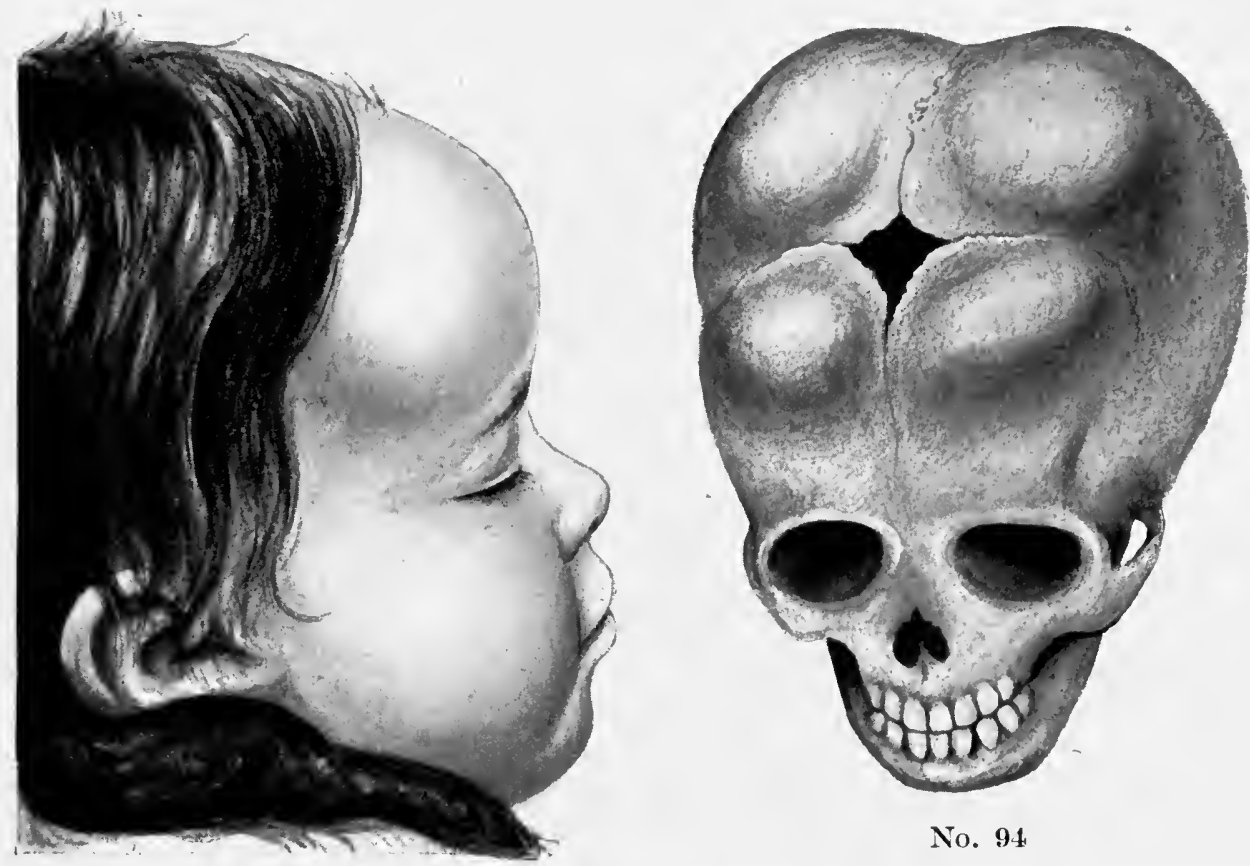

No. 94

No. 93
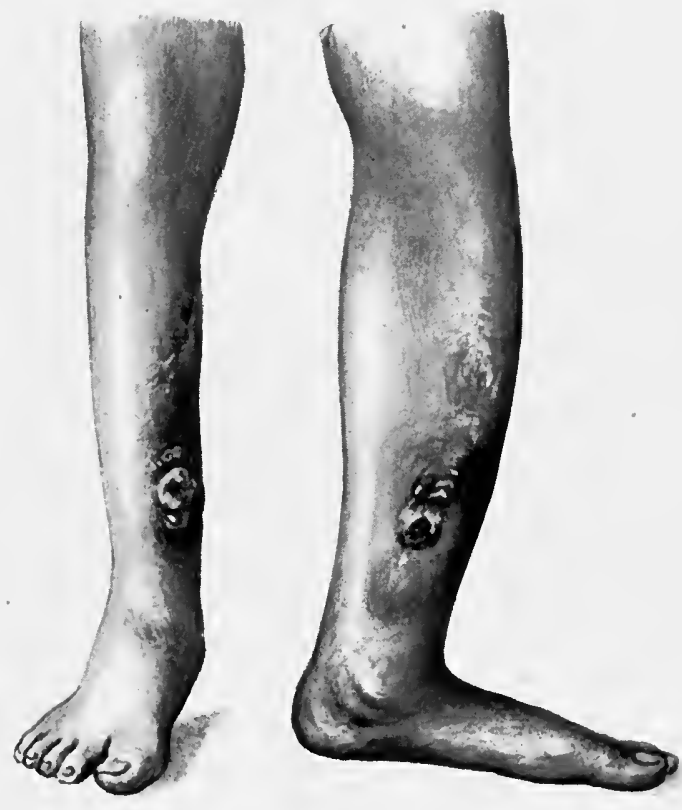

No. 95

Plate XLIX 

In consequence of the syphilitic infection, this connective tissue increases in growth and takes on the character of granulation tissue. Thereby the canals are widened. In this connective-tissue growth there is an attempt at bone formation, from the cartilage cells included in it. The process increases towards the diaphysis, so that in time the cartilage which should next ossify is completely destroyed, being pushed, so to speak, into the marrow of the diaphysis, to become the prey of the bloodvessels and marrow cells therein; further, the granulation tissue forms a barrier which prevents ossification of the cartilage cells above; and, in consequence, separation of the epiphysis follows.

The most characteristic feature to the naked eye, in a longitudinal section of the diseased epiphysis, is the appearance of a yellow line, often zigzag, between the epiphysis and the diaphysis.

Besides the changes described above, there is usually some thickening of the periosteum, and osteophytic growths. Osteo-periostitis is a manifestation of late congenital syphilis, and shows itself in various ways. For instance, in the fingers and toes the phalanges are enlarged in a spindle-shaped manner, the result of an ossifying periostitis of the shafts-Dactylitis syphilitica.

The same condition may also affect long bones, most frequently the tibiæ, especially on their anterior surfaces. New bone forms, as the result of the inflammation, and either gives rise to a spindle-shaped swelling or to multiple swellings-nodes. Bone trouble, even when not clinically evident, causes severe pains, which are undoubtedly the cause of the attacks of screaming, restlessness, and irritability at night, a chain of symptoms so commonly met with in congenital syphilitics.

Ossifying periostitis or pericranitis affects both the parietal and frontal eminences, causing prominences known as Parrot's nodes, and giving a natiform or "hot-cross-bun" appearance to the calvarium.

Syphilitic inflammation, if secondarily infected with septic organisms, may result in necrosis and caries of bones, especially in the case of the hard palate, jaws, nasal bones, and cranium, and may lead to perforation. Perforation of the palate is especially diagnostic of syphilis, in both the acquired and congenital forms.

True gummata may affect any of the bones, as in acquired syphilis, but those occurring on the skull do not usually pierce the pericranium or the dura mater.

Rarefaction of bones-osteoporosis-may also occur, and may lead to multiple fractures. Parrot described a "gelatinous" atrophy which affects the skull-bones, in particular the occipital, giving rise to the softening known as craniotabes. Premature synostosis of the sutures of the skull not infrequently occurs, and is supposed to be a cause of idiocy, or their patency may be abnormally prolonged.

Hydrocephalus occurs in congenital syphilis, but is not common. It is usually due to a lepto- and pachymeningitis. 
Osteo-chondritis syphilitica is an early manifestation, while usually the other bone changes occur much later.

The bone changes above described are, for the greater part, also met with in rickets. So closely do rickets and congenital syphilis simulate one another that formerly they were generally believed to be one and the same diseasean opinion held until it was proved that rickety children could acquire syphilis. There is no doubt that congenital syphilis predisposes to rickets, and, although the two diseases are quite distinct, some of the lesions produced by either are indistinguishable during life.

As a result of osteo-chondritis, hydrarthrosis may supervene.

Occasionally a joint becomes filled with pus; when this occurs, the condition is almost invariably symmetrically bilateral.

A chronic bilateral, painless, symmetrical hydrarthrosis, usually of the large joints, the knees being most commonly affected, is very frequently due to congenital syphilis.

When a child is brought up for consultation with a chronic swollen joint, the diagnosis of tubercle is almost invariably made; but having in the past few years seen quite a number of such cases in which a wrong diagnosis has been made, I must beg the reader to examine the child carefully before making a diagnosis, as not only is syphilis a common cause of arthritis, but in every case the child presents some other picture of congenital syphilis, which makes the diagnosis simple. If syphilis is diagnosed early and the child is young, treatment will considerably benefit the arthritis, which is not the case in tuberculosis, or in syphilis if left for a long time wrongly diagnosed, or in those cases where the lesion makes its first appearance in adult life. The following cases illustrate some of the above points:

CASE 93.-A boy, aged 19, complained of rheumatism and swelling of both knees. He was admitted into hospital and treated as a case of rheumatism, with no improvement for four months. When he had been in the hospital three months he developed keratitis, first in one eye and then in the other. The diagnosis of tubercle was then made, and the patient kept in hospital another three months, until someone suggested the condition might be due to syphilis. When I saw the patient he had double interstitial keratitis, a cranium and teeth typical of congenital syphilis, an arthritis of both knee-joints, with very considerable wasting above and below the joints. Under treatment, with iodine, intramine, and arseno-benzene, he improved rapidly, but only up to a certain point.

CASE 94.-A man, aged 32, received nine intravenous injections of arseno-benzene, and several intramuscular injections of mercury for bilateral keratitis, without the eyes being much, if at all, benefited. Nine months after the treatment he became stone deaf in both ears, for which further treatment with arseno-benzene and mercury did nothing. A year later he developed a large swelling of the right knee-joint, which likewise did not respond to treatment. At the conclusion of the treatment he had an attack of hemipleg:a, which proved fatal. 


\section{Vascular System.}

The heart is rarely affected. Both diffuse myocarditis, secondary to an endophlebitis, and periphlebitis or arteritis of the small vessels, and gummata in the heart muscle, have been described. The gummata are small, multiple, and to the naked eye appear as white spots. Recently Warthin ${ }^{18}$ showed that there exists a special form of diffuse interstitial myocarditis, with nothing in the gross appearance to suggest myocarditis, but with certain fibroblastic epithelioid areas of the heart wall microscopically found crowded with spirochætæ. Warthin considers this an important cause of sudden death in early life, occurring, as a rule, in apparently healthy children, in whom syphilis may never have been suspected clinically.

Changes in the small vessels are all-important, since there is no syphilitic manifestation which is not primarily dependent on such changes. In no way do they differ from those occurring in the acquired form-namely, roundcelled infiltration of the media and adventitia, Endarteritis obliterans, etc. Veins are not uncommonly affected in congenital syphilis. A marked dilatation of the superficial veins is often seen, and there is no doubt that syphilis can be the cause of varicose veins in children and young adults. Aneurysms may be met with in congenital syphilis. I once saw a case of syphilitic aortic aneurysm in a girl aged 15, and I have had a case of syphilitic hemiplegia in a boy aged 8 .

\section{Mucous Membranes.}

Catarrh of the mucous membrane of the nose, causing coryza, the socalled "snuffles," is a very frequent manifestation. It may be the first sign, and it is a persistent one. The probability is that the lesion commences in the submucous tissue. Olceration and involvement of the underlying periosteum and bone not infrequently occur, causing falling-in of the bridge of the nose, and thus producing the sa-called "saddle-nose." It is highly probable that the nasal catarrh has largely to do with the frequency with which congenital syphilitic children suffer from adenoids, and a deformity which only helps to aggravate the condition is the characteristic high-arched palate.

Papules, erosions, and ulcers may also affect the mucous membranes of the lips, cheeks, and tongue; but, with the exception of ulceration of the hard palate and jaw bones, they are more common in acquired than in congenital syphilis.

\section{Lungs.}

One or both lungs, either as a whole or in part, may show the characteristic white pneumonia of Virchow. An affected lung is large, and has impressions of the ribs on its surface; on section, it is white or greyish. The 
chief changes are a growth and desquamation of the alveolar epithelium, with considerable cellular infiltration and hyperplasia of connective tissue. This is the true "white pneumonia," and is generally said to be incompatible with life.

Carpenter ${ }^{4}$ reports a case in which death did not occur until the age of 13 months. Still considers that the condition is consistent with much longer life, and that it is the cause of the fibroid disease of one lung which is by no means an uncommon disease in children.

The following case shows that Still's contention is correct:

CASE 95.-A man, 19 years of age, had had arthritis of the knee-joints, which had been considered to have been due to tuberculosis. He was undersized, had been a weakling all his life, and was brought to me suffering from interstitial keratitis. The patient had had a cough for years, and on examination it was evident that one lung was consolidated. No tubercle bacilli were found in the sputum, and the C.F.T. was strongly positive.

I should not be at all surprised if many of the cases diagnosed as pulmonary tuberculosis were not in reality due to syphilis. The diagnosis is always rendered difficult because congenital syphilitic children are prone to develop tuberculosis. Scrofuloderma, with partial or general adenopathy, is in my experience the most common tubercular lesion. I once saw a case of scrofuloderma which was accompanied by a tubercular keratitis, the patient having previously had syphilitic keratitis.

A second form, usually described as interstitial pneumonia, is, as its name implies, dependent upon a growth of the interalveolar and interlobular connective tissue, which starts from the vessels and bronchi. Owing to this connective-tissue growth the alveoli become compressed. Such a lung is enlarged, pale, or greyish-red, and hard.

The capillaries are often enlarged and tortuous; the alveolar epithelium is swollen, and may show desquamation, but more often it assumes a cubical character, the lung alveoli presenting the appearance of glandular spaces. A combination of the white pneumonia with interstitial overgrowth is the most common appearance in the lungs of syphilitics.

\section{Digestive Tract.}

The intestinal canal may be the seat of ulcers, which are gummatous in nature, and which occur most commonly in the small intestine. Multiple miliary gummata are also met with in the mucous and muscular coats, from the stomach downwards. Bowel lesions are more common in congenital than in acquired syphilis.

Barbier ${ }^{17}$ has recently drawn attention to paroxysmal visceral distu bances in congenital syphilis. Three types are described: (1) paroxysmal vomiting; (2) paroxysmal enteralgia; (3) paroxysmal enuresis. The attacks last from 
a few hours to two or three days, and, when passed, the child, who is usually between the ages of 5 and 10, appears quite normal. There is no periodicity about their recurrence.

\section{Liver.}

The liver is very frequently found diseased in children who are born dead. Jaundice and ascites are rare ccmplications. The liver is usually enlarged. The surface is commonly unaltered, except in the contracted hobnail type of cirrhosis which is found in later childhood.

Hochsinger ${ }^{7}$ describes four varieties of hepatic changes:

1. Diffuse, small, round-celled infiltration of the connective tissue, and involvement of the acini by these cells. The inflammation starts around the small arteries. The macroscopic appearance of the liver is normal.

2. Hyperplasia of the connective tissue, so that the liver is enlarged, hard in consistence, and yellow or yellow-brown in colour. The commencement is a hyperplasia of the connective tissue in the adventitia of the vessels.

3. Miliary gummata (flint-like liver), greyish-yellow nodules about the size of a pin's head, are scattered about, in the parenchyma chiefly, but also in the interacinous connective tissue, and especially around branches of the portal vein.

4. True gummata. A rare condition.

The most typical hepatic condition found in infants is a combination of the first and second of the varieties described by Hochsinger. The liver may be enlarged and hard, but the surface remains smooth, or very slightly granular; there is a diffuse pericellular cirrhosis, the newly formed connective tissue being both cellular and vascular. The liver cells are isolated by this newly formed tissue into small groups of one, two, three, or four cells. The Spirochoeta xallida may be demonstrated in considerable numbers in this form of the disease.

In Hochsinger's third variety there is often marked fibrosis in the neigh-bourhood of the gummata. Large gummata-the true gummata of Hochsinger's fourth variety-are less common in the congenital than in the acquired form of the disease. Amyloid degeneration of the connective-tissue stroma may occur.

Besides the usual forms of Heratitis interstitialis et gummosa, Schiiffel described a condition peculiar to congenital syphilis, and he called this Peripylephlebitis syphilitica. This is characterised by enlargement of the liver, which is of a brown-green colour, and flabby. Throughout the soft parenchyma the larger branches of the portal vein can be felt as hard cords about the thickness of a little finger. Cross-section of a cord shows the lumen of the vein narrowed, the biliary ducts and branches of the hepatic artery shut in and constricted by fibrous tissue. The change depends upon an excessive fibrous tissue increase of Glisson's capsule. 


\section{CLINICAL ASPECT AND TREATMENT OF VENEREAL DISEASES}

The disease affects either of the chief branches of the portal vein, and stops short at the sinus vence portoe.

The umbilical vein is intact. Jaundice, colourless fæces, meteorism, ascites, enlargement of the spleen, and intestinal hæmorrhages are the clinical symptoms.

\section{Pancreas.}

This organ may be affected by a chronic interstitial inflammation leading to enlargement, and also by gummata.

\section{Kidneys.}

Interstitial nephritis is the commonest manifestation of this disease, and it is not infrequently associated with amyloid degeneration. True gummata are very rare.

\section{Suprarenals.}

Virchow and Bärensprung describe the suprarenals as being enlarged, dotted with small, scattered, yellowish-white nodules, and as showing marked fatty changes in the parenchyma. Syphilis may also be the cause of some of those cases in which blood-cysts are found.

\section{Testicles.}

Gummata are extremely rare, but diffuse interstitial inflammation is not uncommon; usually occurring, as it does, within the first few months, it is pathognomonic of syphilis; the epididymis may or may not be affected, and an accompanying hydrocele is rare. In consequence of this connectivetissue hyperplasia, there is a growth and degeneration of the glandular epithelium.

Syphilitic affections of the female sexual organs are extremely rare. Besides the usual interstitial changes, which differ in no way from those observed in other organs, Schukowsky reported an interesting case of Metrorrhagia neonatorum which was caused by an Endarteritis obliterans of the vessels in the fundus of the uterus.

\section{Spleen.}

Enlargement of the spleen is common in cases of congenital syphilis, and it is most obvious when the child is $1 \frac{1}{2}$ or 2 years old. It is difficult in many of these cases to decide whether syphilis or rickets is the proximate cause of the enlargement. Parrot recognised two forms of enlargementone resulting from a chronic hyperæmia due to stasis in the portal circulation, the other due to a true hyperplasia of the connective tissue in the gland and in the capsule. Still ${ }^{4}$ reported two cases of gummata of the spleen, but the condition is extremely rare.

Although Banti himself was against the view that syphilis played a part 
in the ætiology of the disease which goes by his name (splenitis, anæmia, cirrhosis of the liver, and ascites), there can be no doubt now that syphilis is sometimes the cause.

I have had the opportunity of studying two cases of congenital syphilitic splenic anæmia; as both cases presented much the same symptoms, I will describe only one of them in detail :

CASE 96.-A youth, aged 19, had complained for some time of always feeling tired; on occasions he had collapsed from fatigue; and during four months prior to admission he had had three hæmorrhages by the mouth. On examination, the patient was emaciated, anæmic, and his skin had a yellow tinge. There was ascites, and when the fluid was removed the spleen could be felt to come down as far as the umbilicus. The blood showed profound anæmia, and gave a very strong positive C.F.T. During most of the time in hospital the temperature was raised, but it fluctuated considerably. The patient had other evidences of congenital syphilis in shape of cranium and old osteo-periostitis of both tibiæ.

Neither patient improved under antisyphilitic treatment, and both died.

Post-mortem in both cases revealed an enormous splenomegaly. On further examination, the capsule of the spleen was thickened; there was a marked fibrosis, especially around the vessels, with a consequent diminution of the parenchyma, which only showed cellular degenerative changes. In one case there was no cirrhosis of the liver, and in the other it was not very pronounced. In both cases there was endophlebitis of the splenic vein, which had even extended to the portal vein.

It seems a pity to label this condition "Banti's disease," as it is neither a clinical nor a pathological entity. Like other forms of enlargement of the spleen, it may be due to any poison, perhaps more often to tubercle and to syphilis than to any other, and the different picture presented is due to whether the mesenchyme or parenchyme is primarily or secondarily involved.

This mesenchymatous splenitis is due to the extension of a fibrosis starting in a vein in the portal system; therefore the condition will vary according to the path taken by the extension. Herein, presumably, lies the exp anation why in some cases cirrhosis of the liver is present and in others absent.

\section{Thymus.}

This gland is here mentioned, since an affection of it has been described as being diagnostic of congenital syphilis-namely, Dubois' abscesses. These are single or multiple abscesses found in the gland substance. There is very little evidence that they are definitely syphilitic.

\section{Central Nervous System.}

The severe forms of defective development of the central nervous system are characteristic of congenital syphilis-naturally the infant is either born dead, or it dies a few days after birth. It is not at all uncommon for one 


\section{4

cerebral hemisphere to be much smaller than the other, and yet, as far as one can tell, the functional activity of each is the same. A marked asymmetry of the head and face accompanies this deformity. I had such a case quite recently in a boy, aged 11; beyond a high myopia and a positive C.F.T., nothing abnormal could be detected; and the boy's intelligence and knowledge were very much in advance of his years.

Hydrocephalus is sometimes caused by syphilis, and its pathology is not in all cases the same. In some cases it is highly probable that the condition is due to an early syphilitic arteritis. This results in a pronounced exudation of lymph, which, in accumulating, distends the ventricles and the central canal of the cord. On the other hand, and more frequently, the condition is due to a severe involvement of the meninges.

A child may develop hydrocephalus in utero, or not until after birth-if the latter, never later than the first year.

In many cases the condition is associated with nervous symptoms.

A localised gumma, or miliary gummata, may affect any part of the brain and cord, and diffuse gummatous pachymeningitis and leptomeningitis are well known. As in acquired syphilis, the brain is more frequently involved than the cord, except in the true degenerative lesions, when the lesion is generally a mixed one. There is no doubt now that congenital syphilis may cause degenerative myelitis, $\mathrm{n}$ which the symptoms do not differ from those met with in the adult form. Most congenital syphilitic degenerative lesions do not give rise to symptoms until the child is about 8 years old or older. Degenerative lesions are really lesions of late congenital syphilis, and may not manifest themselves until the patient is over 20 years of age; hence it is sometimes quite a difficult matter to be sure, when one is dealing with a degenerative nervous lesion in a patient of 25 years of age, whether the case is one of acquired or of congenital syphilis.

According to Halbau and Dydynski, optic atrophy and sphincter (bladder) paralysis are more frequently met with in the congenital form than in the acquired form of degenerative myelitis, while severe ataxia is rare. Most authorities are now agreed that Friedreich's ataxia is never of syphilitic origin.

Degenerative encephalitis is not at all infrequently met with in congenital syphilis, and the symptoms do not differ from those met with in the acquired form.

As a rule, the patient is over 8 years of age. The course of infantile degenerative encephalitis is, on the average, longer than that met with in adults. According to Alzheimer, the average duration is about four and a half years. In the majority of cases, inferior mental endowment can be dated back to infancy. The course the case runs is usually one of a simple progressive dementia, and the depression and maniacal states so commonly witnessed in the adult form are very frequently lacking.

Actual paralyses are more common in the congenital form, and, as already 
stated, the case is frequently a mixture of degenerative myelitis and encephalitis.

The more thoroughly every congenital syphilitic is examined, the more frequently will nervous symptoms be found, and, as is the case in acquired syphilis, the nervous signs and symptoms may be of almost any nature, both meningeal and arterial. Generally speaking, the nervous diseases of congenital syphilis are more often of ameningeal origin, while in acquired syphilis they are more often of meningeal origin. Although the brain is more frequently involved than the cord, cases of spastic spinal paralysis are undoubtedly met with in congenital syphilis, and sometimes idiocy is associated with the condition. Diminished intelligence, even amounting to idiocy, is no doubt often caused by syphilis, but it is almost impossible to state accurately the rôle which syphilis plays, because many of the cases have never had a syphilitic symptom, nor have given a positive C.F.T. Whether the idiocy is due to the syphilis itself, or occurs only because the parents have had syphilis, is not at present known; but of one thing I am quite sure, and that is that syphilis is far more often blamed in this class of case than it should be. If a patient has an apparently normal childhood, and about the age of 10 his mental capacity begins to show signs of progressive deterioration, then not only may syphilis be considered most probable, but the subsequent development of juvenile paresis prognosticated as well. Such children usually become dishonest, untrustworthy, and uncleanly in their habits.

Spastic spinal paralysis without cerebral symptoms may do well under treatment, although it is liable to recur. The condition is probably analogous to Erb's spinal paralysis, which is met with in acquired syphilis. The spastic spinal paralysis associated with idiocy is often known as Homén's Familial Disease, as a definite clinical picture is presented. The condition begins usually between the ages of 12 and 20 with symptoms of dizziness, heaviness in the head, and disturbance of the general health; then the intelligence and memory begin to fail, the gait becomes uncertain, and speech difficult. Idiocy and spastic paralyses follow, which progress to well-developed contractures. The lesion is a general thickening of the cranium and meninges, with sclerosis of the vessels, and atrophy of the brain in its entirety. The relationship, if any, between syphilis and Little's disease is not yet settled.

Again, as regards epilepsy in children, the greatest difference of opinion exists as to whether syphilis is a potent cause or not. Syphilis should always be considered as a possible cause, for the simple reason that most cases of syphilitic origin do well under treatment; but, according to my own experience, syphilis is far from being a common cause of the condition.

Other lesions which are occasionally met with are cranial nerve palsies, which may be of the true degenerative type or secondary to a basilar meningitis.

That isolated pupil phenomenon, to which I particularly called attention in acquired syphilis, may also be met with in congenital syphilis. Mere 
inequality of pupils should not be invariably diagnosed as syphilitic, since some healthy people are born with unequal pupils.

Almost a characteristic symptom of congenital syphilis, when it occurs, is Diabetes insipidus. The lesion is in the posterior lobe of the pituitary body.

Hypopituitarism is probably more often caused by congenital syphilis than is generally thought, and it is by no means always associated with polydipsia and polyuria. Dystrophia adiposa genitalis-that is to say, stunted growth-obesity, and rudimentary development of the secondary sexual characteristics, may be the symptoms of a syphilitic infection of the anterior lobe of the pituitary gland. Syphilitic inflammation of the pituitary in acquired syphilis is nearly always secondary to a basilar leptomeningitis, but whether this is the pathology of the lesion in congenital syphilis I a,n unable to say. Hypopituitarism in congenital syphilis may be also associated with general paralysis.

The following is an interesting case which $I$ once saw in consultation:

CASE 97.-A girl, 18 years of age, infantile in type, and mentally deficient, had a peculiar nervous trouble. She had bilateral ptosis, was deaf in both ears, and the pupils were unequal. Menstruation began when she was 17, occurred only once then, and for one day; six months later the same occurred again, but since then she had seen nothing. The secondary sexual characters were fairly well developed. There was marked acro-asphyxia of both hands and feet. A twenty-four-hours urine was collected, which amounted to little short of a gallon. Its specific gravity was 1003 , and contained neither protein nor sugar. Both the blood and C.s.F. were positive. Treatment caused a temporary improvement, but the patient died later of degenerative encephalitis. Unfortunately, an autopsy was not permitted.

I had another case not at all unlike the above, which was characterised by bilateral ptosis, acro-asphyxia, and brachycardia, the pulse never exceeding thirty-six beats per minute.

Discussion has always been rife as to whether syphilis plays any rôle in the causation of Mongolian idiocy. Sutherland ${ }^{10}$ was the first to suggest a connection between the two, which has been since corroborated by Stevens. ${ }^{11}$ Out of a total of 25 cases, Sutherland says syphilis was definitely present in 11, and strongly suspected in 3 others. According to Stevens, the C.F.T. with the blood was positive in 10 per cent. of 20 cases, and with the C.s.F. in 25 per cent. Pleocystosis was present in 20 per cent., and the globulin content was increased in 90 per cent. This is strong evidence that congenital syphilis may be a cause of Mongolian idiocy. I have had under my care one undoubted case, which I will cite, as I treated both the father and mother for syphilis.

CASE 98.-The child was an imbecile, with a typical Mongolian physiognomy. The tongue was fissured, and the patient had chronic blepharitis and conjunctivitis. The hands were large, the fingers thick and blunt, and the fingers exhibited the typical shortening of the second phalanges. The feet were thick and clumsy, so that 
the patient, even now at the age of 6 , is unable to walk alone. The child is affectionate, tractable, although silly, and extremely restless. The C.F.T. with the blood was slightly positive, and an examination of the C.s.F. revealed the following: C.F.T. positive; globulin increased; lymphocytes, 14 per c.mm.

Some observers state chorea may be due to syphilis, while others deny any connection. Comby ${ }^{14}$ says he found evidence of syphilis in 7 of 39 cases of chorea, but Koplik ${ }^{15}$ says he found none in a smaller series. Veeder and Jeans ${ }^{16}$ reported 2 cases in which the C.F.T. was positive, and which responded at once to antisyphilitic treatment; therefore I think we must admit that congenital syphilis may be a cause of chorea, although perhaps no more than an exciting one.

\section{Eyes.}

Affections of the eyes are extremely common. Changes in the fundus oculi, choroiditis, and iritis, may occur soon after birth, or they may be strictly congenital. The patches in the choroid are usually irregular in shape and white in colour, with dark, pigmented borders, and they are generally associated with vitreous opacities. Choroiditis may interfere with vision, nystagmus being the first sign which calls attention to the defect.

Hutchinson says that iritis most frequently affects females; is most commonly seen at the age of 5 months, and is often bilateral; that it is not characterised by the inflammatory phenomena which are met with in adult iritis; and that it quickly leads to occlusion of the pupil, but is extremely amenable to mercurial treatment.

The characteristic and diagnostic eye change, in late syphilis, is interstitial keratitis. This usually appears just before puberty, affects one eye first, and then usually becomes bilateral, whether the patient is treated with mercury or not. Opaque areas appear on the deep surface of the cornea, and fine bloodvessels, which come from the conjunctiva and sclerotic, run on and into its substance. The condition may last for months or years, till sight is almost lost; but, however bad the case may be, there is always something to be hoped for from treatment.

Double interstitial keratitis is frequently associated with Hutchinsonian teeth and nodes on the tibiæ-the so-called "syphilitic triad." Any of these eye lesions may appear first in adult life.

I have been thunderstruck by the large number of eye lesions $I$ have recently seen in the army. In one year alone in a small clinic I was able to collect 21 cases of interstitial keratitis, and 4 of choroido-retinitis. All these cases occurred in healthy-looking men between the ages of 18 and 32. In 5 there were no other signs or symptoms of congenital syphilis, and in 3 cases of interstitial keratitis only one eye was involved. In all the C.F.T. was positive. In some of the cases of interstitial keratitis there were the following other eye lesions co-associated: (1) iritis; (2) irido-cyclitis; (3) choroiditis; (4) Reti- 
nitis pigmentosa sine pigmento; (5) optic atrophy. The keratitis cases all did extremely well under the metallic (arseno-benzene and mercury) and non-metallic (iodine and intramine) treatment, provided both were prescribed. If metals only were used, the results were unsatisfactory. Cases which would not improve under arseno-benzene cleared up at once when intramine was injected. In the group were two cases of Retinitis pigmentosa sine pigmento, and one of them was uninfluenced by treatment, as the case had gone too far; while in the other the lesions were undoubtedly improved, and fresh ones prevented from appearing, by the combined use of intramine and arseno-benzene.

Some of the most interesting cases were the following:

CASE 99.-A man, aged 19, gave the history of having had two attacks of interstitial keratitis, the first when 5 year's of age, and the second when 12 . On examination, he had faint leucomata of both corneæ; bilateral retinitis, with pigmentation; partial optic atrophy, with remarkable contraction of the vessels. The C.F.T. was very strongly positive. Treatment proved unavailing.

CASE 100.- A man, aged 23, was admitted with bilateral interstitial keratitis. $\mathrm{He}$ was blind in the right eye, probably from birth, due to choroido-retinitis. The patient was the eighth and last child, and showed no other signs of congenital syphilis. Six of his brothers and sisters had died young; the other remaining child, a girl, also had interstitial keratitis. Four injections of arseno-benzene were prescribed without any improvement, but the moment intramine was injected the trouble immediately began to clear up.

CASE 101.-A man, aged 18, admitted with syphilitic arthritis of the sternoclavicular joint, was treated with two injections of intramine and four of arsenobenzene, which completely cleared up the trouble. After the third injection of arsenobenzene the left eye developed a very acute interstitial keratitis, which quickly vanished when intramine was again prescribed. Three years previously the patient had had interstitial keratitis of the right eye, which had left a pronounced leucoma. Six years before this he had had arthritis of both knee-joints, for which he was under treatment for six months.

\section{Ears.}

Deafness is quite a common symptom of congenital syphilis. Of the deaf mutes, about 5 per cent. are due to syphilis. With snuffles is sometimes associated Eustachian obstruction, which leads to Otitis media, and finally to internal-ear disease. These catarrhal affections develop into conditions which clinically correspond to otosclerosis. Deafness may be a late symptom of congenital syphilis, coming on either gradually or suddenly between the seventh and fourteenth years, or even later. As a rule, these patients have interstitial keratitis, and less often Hutchinson's teeth. Fraser, ${ }^{12}$ who has examined several of these late cases, holds that they are often due to an old syphilitic Otitis media, which either invades the bone of the labyrinth capsule, giving rise to a chronic form of osteomyelitis, which slowly invades the labyrinth and thus produces gradual deafness, or an infection which breaks 
through rapidly into the hollow spaces of the internal ear, causing labyrinthitis, and thus producing sudden deafness.

The following is a typical case:

CASE 102.-A man, aged 52, consulted me with a view to seeing if anything could be done for his deafness. When he was 9 he had a polypus removed from the right ear; at 14, he developed double interstitial keratitis; at 17, the ear trouble began. The deafness varied from day to day, and it was accompanied by Ménière's symptom complex. By the age of 30 he had become stone deaf. Although all methods of treatment had been attempted, none did the slightest good.

A case of exceptional interest is the following:

CASE 103.-A woman died, aged 60, of cerebral hæmorrhage; she was a syphilitic, proved by the children she bore, although she herself had never shown any clinical manifestations. Her first husband died of cerebral hæmorrhage, aged 35, and her second husband died of degenerative encephalitis. By her first husband she had two children, both of whom became stone deaf before they were 40. The two died, between the ages of 45 and 50, of cerebral hæmorrhage (pontine). The younger child had three children. Each child has been operated upon for adenoids; they are stupid, and evidently mentally deranged, and the eldest, who is now 18, is gradually becoming deaf, and gives a positive C.F.T. It will be interesting to see whether the other two become deaf, and if cerebral hæmorrhage ends their careers.

According to Fraser ${ }^{12}$, Hennebert's compression nystagmus is due to a labyrinthitis, which prevents the rotation reaction from being produced. Increase of the air pressure in the external meatus by means of a valveless Politzer bag causes slow movement of the eyes to the same side, while aspiration brings about a slow movement to the opposite side. Hennebert ${ }^{13}$ finds that in these cases the rotation reaction is always absent, while the caloric reaction is reduced. Until Fraser suggested this might be caused by a labyrinthitis, it was held to be due to changes in the nerve endings.

\section{Lymphatic Glands.}

In congenital syphilis the lymphatic glands may become enlarged, but there is never a general enlargement all over the body, as is the case in acquired syphilis; one group becomes enlarged, but not from any ascertainable cause. So rarely are the glands affected that enlargement makes one suspect acquired syphilis.

\section{Diagnosis.}

The diagnosis of congenital syphilis is made too often. A rash on the buttocks seems to be regarded as diagnostic; it is more often due to a simple erythema than not. A macular rash on the face and papules on the palms and soles are, however, pathognomonic. It should not be forgotten that "snuffles" is often due to a simple catarrh, and some flattening of the bridge of the nose is characteristic of all infants. Orchitis and pseudo-paralyses are most important signs, syphilis being the only cause in early life. Much 
weight cannot be laid on enlargement of the liver and spleen. Enlargement of the epiphyses before the first year is diagnostic of syphilis. In a doubtful case, an ophthalmoscopic examination should be made.

In later life, Hutchinson's teeth, interstitial keratitis, and periostitis of the tibiæ are the most important diagnostic signs.

\section{Prognosis.}

Infants born with manifestations of syphilis usually die. Death is the rule in cases of pemphigus. In degenerative nervous cases the termination is almost invariably fatal. Children born healthy, but showing signs in early infancy, if submitted to appropriate treatment for a full period of two or three years, may perhaps escape subsequent manifestations altogether. Children who have late signs have probably often been free in early life, and consequently have had no treatment. In these cases the symptoms often remain uninfluenced by treatment, but ultimately heal up of their own accord. The amount of damage done will depend upon the site affected. Speaking generally, the prognosis is not nearly so unfavourable as is usually thought, and omitting those cases which die in the first year, which amount to roughly 35.0 per cent. the prognosis may be considered as good. Although treating syphilitic women throughout pregnancy results in the birth of apparently healthy children, I have reason to think that many of such children will develop symptoms at adolescence or later; therefore we must expect to see many more cases of Syphilis congenita tarda in the future than we have been accustomed to see in the past. Unfortunately, such children do not give a positive C.F.T. in early life, and treatment does not prevent the incidence of clinical manifestations appearing later.

1. $\mathrm{B}_{\mathrm{AB}}$ (1907): Münch. med. Woch., liv., 2265.

2. Fournier (1886): La Syphilis Héréditaire Tardive. G. Masson, Paris.

3. Nonve (1909): Syph. u. Nervensystem. S. Karger, Berlin.

4. StrLl (1908): "Congenital Syphilis," in D'Arey Power and Murphy's System of Syphilis, i.

5. UxNa U. JANnus (1906 u. 1907): Dermat. Jahresbericht., ii.-iii.

6. Adamson (1907): The Skin Affect. of Childhood. Oxf. Med. Press, London.

7. HochsInger (1904): Studien über die hereditäre Syphilis. A. Hölder, Wien.

8. Hutchrnson (1901): Syphilis. Cassell and Co., London.

9. Roвerts (1918): Journ. Amer. Med. Assoc., Ixx., 372.

10. SUTHERLAND (1899): Practitioner, lxiii., 632.

11. Stevens (1915): Journ. Amer. Med. Assoc., lxiv., 1636.

12. Fraser (1916): Proc. Roy. Soc. of Med., Otol. Sect. ix., 62.

13. Hennebert (1909): La Presse Orolaryng. Belge, No. 5.

14. CoMrby (1915): Arch. d. Med. d. Enf., xviii., 517.

15. Koplik (1915): Arch. Pediat., xxxii., 561.

16. Veeder and Jeans (1914): Amer. Journ. Dis. Child., viii., 283

17. Barbier (1918): Bull. de la Soc. Med. des Hosp., xlii., 174.

18. Warthin (1914): Amer. Journ. Med. Science, exlvii., 667. 


\section{CHAPTER XVI}

\section{DRUGS USED IN THE TREATMENT OF SYPHILIS, AND THE METHODS OF ADMINISTERING THEM}

\section{History.}

MERCURY was used in the treatment of syphilis before the latter was recognised as a distinct disease, merely because it was the drug most employed in skin diseases. The great Arabian physicians were perfectly familiar with mercurial inunctions for scabies hundreds of years before syphilis visited their country, and described the salivation and buccal lesions which followed in its wake. Even in $\mathbf{1 5 0 2}$ we find exact details are given for the use of mercurial inunctions in syphilis, including advice concerning food, outdoor exercise, and the free use of mouth-washes and gargles. Mercurial inunctions remained the favoured treatment throughout, though many changes were made in the modes of administration, some with the object of producing salivation, others to avoid the ill effects from over-treatment, and others to advertise a man or place in order to draw patients to undergo a cure. All of us are familiar with Aachen, which doubtless took its cue from the worldrenowned Montpellier fumigation treatment, which attracted patients from all the world over at the beginning of the eighteenth century. Certainly during, and probably before this period, it was known that inhalation of mercurial vapour was an important part of the inunction treatment, because strict rules were observed about keeping a patient in a closed room while he was being rubbed, and for some time afterwards. It is interesting to note that Welander's mercolint bibs were preceded three centuries by various articles of clothing impregnated with mercury, a method of treatment which is thought originally to have hailed from China. Mercurial plasters, made of linen or leather, were in use for hundreds of years, and were known as Balteum venereum, or Venus's girdle.

Suppositories were first used in 1864, while mercurial pills, known as Pillula barbarossce, were used by Francis I., King of France. Painting ulcers with corrosive sublimate was practised when syphilis invaded Europc, and later sublimate baths was a favourite form of treatment with some. Intramuscular injections date from 1860. Although mercury had been used in almost every conceivable form from the beginning, the drug had its opponents, some of whom were the ablest physicians of the day. The English doctors, from the beginning of the nineteenth century up to thirty years ago, 
and even less, were more than any others against the use of mercury in syphilis. In the middle of the nineteenth century mercury fell into great discredit through Hermann, a Viennese professor, stating that there was no such thing as constitutional syphilis, and that all ist Wirkung des Merkurs. As Boeck was a pupil of Hermann's, we understand why he favoured quinine and arsenic. To-day the use of mercury in syphilis has no opponent, but it is curious that it should be regarded as a specific. With the exception of Ehrlich's theory of chemotherapy, or chemical relationship between drug and parasite, no one has attempted to explain why mercury should be beneficial in syphilis. I have little doubt but that its main action is to split up the big colloidal protein particles, which form the resisting substance, into myriads of tiny particles, the aggregate area of which is vastly larger than that of fewer and larger particles. As the destruction of the parasites is a surface action between the above-mentioned particles and the parasites, it is obvious that an increased area increases the action of the protective substance. Mercury is able to do this in virtue of its high atomic weight, and is only specific in that there are more particles to break up in syphilis than in any other disease.

Williams and Wallace introduced iodine as an antisyphilitic drug and as an antidote to mercury about 1830. For many years iodine was used in the late stages only, and its right place in the treatment of syphilis is only just beginning to be understood. Both arsenic and gold had their adherents from the time of Paracelsus, and of the latter, Pitcairn, in 1713, said its action was superior to that of quicksilver. In view of my theory that the action of metals is to increase the oxidising power of the patient's resisting substance, it is interesting to note that inhalations of oxygen were largely used in the treatment of syphilis by three English physicians-namely, Scott, in 1796, Beddoes, in 1797, and Cruickshank, in 1798.

The use of sulphur in syphilis was known to the oldest syphilologists, and Paracelsus advised natural sulphur baths for hydrargyrosis. For many years sulphur was forgotten, not being brought into prominence again until Aachen flourished. It was not until the introduction of intramine that the action of sulphur and iodine was explained, and now sulphur is one of the most important drugs in the treatment of syphilis. Guaiacum wood had at one time a great rage, as may be gathered from the names it went by-viz., Lignum sanctum, Arbor mirabilis, Spes hominum, etc. Although both Hundt and Paracelsus pointed out dass das Holtz kein fixum morbum heylt, it was years before the uselessness of guaiacum wood was recognised.

Many decoctions were in favour at one time and another, but the two which were most frequently used were the decoctions of sassafras and Zittmann's decoction. Another plant at one time considered specific was the Lobelia syphilitica, a name which it still bears to-day. The benefit following the use of this herb was undoubtedly due to its powerful diuretic action. The use of extracts of animals, such as vipers, lizards, etc., which found most 
favour in Italy, the so-called hunger cures, the Exercitatio corporis, the blood-letting, the Traitement antiphlogistique, are of historical interest only, and play no part in the treatment of the disease to-day.

The drugs we have to consider are preparations of: (1) arsenic; (2) antimony; (3) mercury; (4) iodine; (5) sulphur; and (6) miscellaneous.

\section{Preparations of Arsenic.}

The only preparations of arsenic to be considered are the arseno-benzene compounds. Atoxyl, arsacetin, arseno-phenyl-glycine, orsudan, etc., are now no longer used, owing to their toxic action on nerve tissue. The drugs just mentioned were replaced by salvarsan and neo-salvarsan, preparations which have since been produced in England under the name of kharsivan, in Canada under the name of diarsenol, and in America under the name of arsphenamine. As all these compounds are more or less the same, it is best to call them by the single name-arseno-benzene. The arseno-benzene preparations are of two kinds: (1) soluble in water; (2) insoluble in water. Both may be injected intramuscularly or intravenously, or may be used as suppositories. Speaking broadly, the soluble salt is not so efficacious as the insoluble one, and none of the present-day preparations have such a good therapeutic action as the German salvarsan before its mode of manufacture was altered in 1912. A single intramuscular injection is more efficacious than a single intravenous injection, but as many injections are needed in every case, and as the intramuscular route is painful, to-day intravenous administration finds the greater favour.

\section{Intramuscular Injection of Arseno-Benzene.}

The soluble salt is the better for this purpose, as the bulk of the material to be injected can be reduced to a minimum, and the mixture can be prepared in the syringe. The method suggested by Harrison ${ }^{1}$ is the best, as it is the least painful. Ten drops of water (distilled water is not necessary) are placed in a syringe, and the soluble salt of arseno-benzene is dissolved in it. The quantity is made up to $2.0 \mathrm{c.cm}$. with a melted creo-camph-cream base (Formula 4,631, B. W. and Co., molting at $15^{\circ}$ C.). Although intramuscular injections are best made into the glutoeus medius, they may be made into the trapezius, midway between the vertebral border of the scapula and the spinous processes of the vertebræ, about the level of the angle of the scapula, on the left side in right-handed individuals, and vice versâ. To find the correct spot in the buttock, make the patient stand upright and follow up the gluteal fold until an imaginary muscular oval is formed which corresponds to the glutoeus medius. The injection may be made into any part of the outer and upper quadrant of this oval. A good rule to make when giving an intramuscular injection is to have a two-inch needle, which should be inserted in a straight line for its whole length, separated from the syringe, to avoid failure 


\section{CLINICAL ASPECT AND TREATMENT OF VENEREAL DISEASES}

to detect the penetration of a vessel. If a vessel be entered, the needle should be withdrawn and inserted elsewhere. As many of the preparations which we give intramuscularly contain solid particles, a small pulmonary embolus is apt to result should some of the particles enter a vessel. If this is going to occur, the patient gets a violent fit of coughing, and, in very bad cases, may become asphyxiated and unconscious. As a rule, a coughing fit is all that. happens, from which the patient recovers in about a quarter of an hour. The point of the needle should never touch bone, and, when the material has been injected, the needle should be withdrawn rapidly, to prevent any of the drug. getting into the subcutaneous tissue, whence it is not readily absorbed, and is apt to cause an abscess. In cases where it is necessary to give intramuscular injections, and where great pain is caused thereby, I have introduced a doublebarrelled syringe, whereby an anæsthetic can be injected before or after the main drug, or the needle may be washed out with saline before it is withdrawn. The part injected should be well massaged, the patient instructed

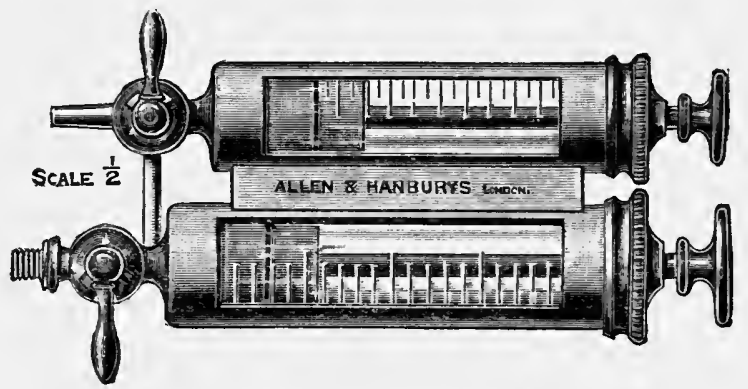

Author's Double-barkelled Syringe, which is Manufactured IN Two Sizes(1) For Intramuscular Medication; (2) For Intravenous Medication.

to take a walk, and hot soda baths should the pain be severe. Heat locally is the best anodyne, but if the pain persists for several days, a belladonna plaster should be applied. Acetyl-salicylic acid is useful prescribed internally, but morphia should be refrained from as far as possible. The local injection of urea and quinine hydrochloride is undoubtedly beneficial, but it increases the risk of abscess formation. Some prefer the deep subcutaneous to the intramuscular route-that is to say, the space between the subcutaneous tissue and the fascia covering the glutous maximus-but as, in my experience, the patient suffers more pain, is more apt to get an abscess, and the drug is not so readily absorbed, this route should not be used. The patient is more apt to get a rise of temperature after an intramuscular than after an intravenous injection-one of the reasons why the former is more efficacious. Constipation is not unusual; therefore a laxative the evening after the injection is advisable. Sometimes the buttock swells-a toxic œedema; it comes on about the third day, diminishes rapidly in size at the end of a week or ten days, after which its diminution is so gradual that a small amount of 
infiltration may be perceptible months and even years after. Infiltration is the bane of arsenical injections, because, in the infiltrated mass, toxic oxides of arsenic form, which, after an accident, may be set free into the system. I have seen an arsenical dermatitis set in eighteen months after a single intramuscular injection had been given. Owing to the fact that one cannot tell how much of a drug has been absorbed when injected intramuscularly, and therefore when it is safe to repeat the injection, or how many injections one is justified in prescribing, so far at least as arseno-benzene is concerned, most will prefer to inject it intravenously. Toxic symptoms are certainly more frequent with intramuscular injections, in spite of the indurations formed. The swelling caused by the toxic œdema is not, as a rule, very painful; it gives the sensation of fluctuation, but under no circumstances should it be opened unless an obvious abscess forms, when it becomes acutely painful. A rash not unlike an urticaria may appear on the buttock injected. Abscesses following intramuscular injections are usually sterile, and are of two kinds: (1) much pus and no induration; (2) little pus and much induration. When there is much pus, the pus should be aspirated. When there is little pus, a small incision should be made, the wound should be dressed with intramine, and intramine should be injected around it intramuscularly. If the lesion is a big one, excision is the best procedure.

\section{Intravenous Injection of Arseno-Benzene.}

The insoluble salt has to be neutralised before use, and is best injected in bulk. A slightly acid solution is more efficacious, but more toxic than a faintly alkaline solution. The soluble salt can be dissolved in $10.0 \mathrm{c.cm}$. of cold tap water, and injected thus in a concentrated form. The following method of preparing a solution of the insoluble salt is that generally in use:

The contents of one tube of arseno-benzene should be slowly dissolved in $50.0 \mathrm{c} . \mathrm{cm}$. of warm physiological 0.9 per cent. saline which has been prepared with freshly distilled water in a $300.0 \mathrm{c.cm}$. graduated glass measure.

When the powder has completely dissolved, after sufficient stirring with a glass rod, $10.0 \mathrm{c.cm}$. of double decinormal* sodium hydrate solution should be added, with the result that a precipitate forms. This precipitate is dissolved by a further addition of sodium hydrate, usually about $10.0 \mathrm{c.cm}$.this may be either more or less, according to the actual acidity of the powder. The $10.0 \mathrm{c} . \mathrm{cm}$. should be added slowly, and the mixture stirred thoroughly. By using a weak solution of sodium hydrate we avoid the risk of making the solution too alkaline, and the exact quantity required is more easily estimated. When the solution is quite cleared by adding the sodium hydrate, the measure should be filled with saline up to $200.0 \mathrm{c.cm}$., and once or twice filtered through muslin or several layers of plain gauze, so as absolutely to exclude even the

* Double decinormal $\mathrm{NaOH}$ or $\mathfrak{n} / 5 \mathrm{NaOH}=0.8$ per cent., or 8 grm. to the litre of distilled water, normal sodium hydrate being a 4 per cent. solution. 
smallest solid particle from getting into the vein, where it might cause either a pulmonary embolism or hemiplegia; $200.0 \mathrm{c} . \mathrm{cm}$. must be considered the maximum dose.

Two points must be observed concerning the saline: In the first place, the sodium chloride must be chemically pure; secondly, the solution must not be less than 0.8 per cent. or more than 1.0 per cent. A hypotonic solution is more dangerous than a hypertonic one, because the former causes hæmolysis-setting free the hæmoglobin from the red blood-corpuscles. Should this happen, the patient may collapse after the injection, and there may be hæmoglobinuria. Needless to say, every vessel used should be sterile and the sodium hydrate solution should be boiled before use.

Another vessel filled with saline is placed by the side of the one containing the "606." The patient comes to the side of the bed and hangs his arm over, then a tourniquet* is placed on the arm, and the limb made to rest on a table in as comfortable a position as possible. The bend of the elbow is then sterilised by first rubbing with acetone, and then with ordinary tincture of iodine.

When a vein cannot be seen it can often be felt, and should be marked out with a blue pencil to indicate its course. If this cannot be done, a vein should be exposed by an incision, either under a local or a general anæsthetic. There is no danger in a general anæsthetic, for the subsequent reaction is not in any way influenced. Hitting the bend of the elbow, or warming the arm with hot towels, will often make a vein prominent. An intravenous injection may be the simplest, or one of the most difficult operations possible. A common trouble is due to the vein slipping about when the needle tries to pierce it; extending the arm as much as possible, or pulling the skin taut to fix the vein, may prevent this.

The solution can either be injected or transfused, injection being far preferable as-

1. The needle is not so easily dislodged. If this should occur while the solution is flowing in, by transfusion, some must escape into the tissues before the flow can be stopped; with the syringe, merely a few drops need escape, as the tap can be turned off at once.

2. The operator has more control over the proceedings.

3. There is less danger of air or a solid particle gaining access to the vein. Air is easily seen in the syringe, and remains at the top, never coming over the centre of the outlet unless the piston is pushed right home. A solid particle is also seen; it falls to the bottom of the syringe and is not disturbed, provided that the solution is injected slowly and steadily, and the piston not rammed home.

4. The operation is pleasanter from the patient's point of view, because it is so much quicker.

* The simplest and best tourniquet is a piece of rubber tubing, which should be wound tightly around the arm, and the two ends fixed with pressure forceps, which can be removed without disturbing the limb. 
5. The operation can be performed without an assistant, and there is practically no apparatus to carry about.

A good syringe is one invented by Schreiber. The cannula is bayonetshaped, bent, and fixed to a three-way metal stopcock, so that the fluid can be sucked up from the vessel, and injected dircctly into the vein. The needle has also a plate at its base, upon which a finger can rest to keep it steady. The one disadvantage of this syringe is that the whole apparatus is rigid; therefore the slightest movement of the syringe may be sufficient to dislodge the needle.

To overcome this difficulty, Allen and Hanbury have constructed for me a needle, which is $1 \frac{1}{4}$ inches in length, behind which is a slightly concave metal

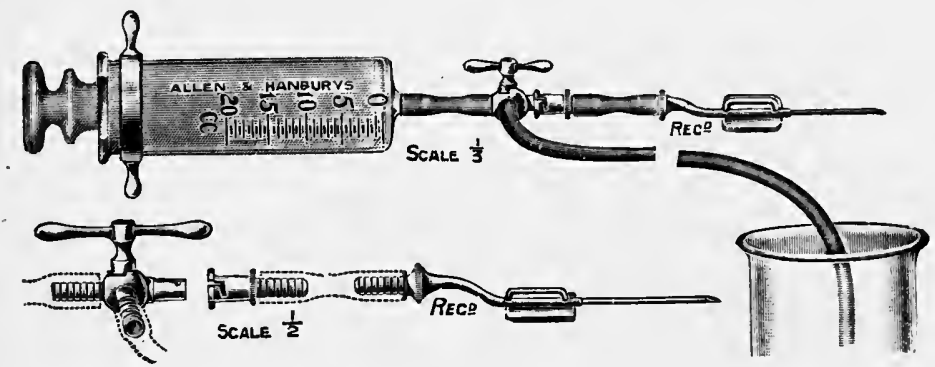

Author's Three-way Syringe.

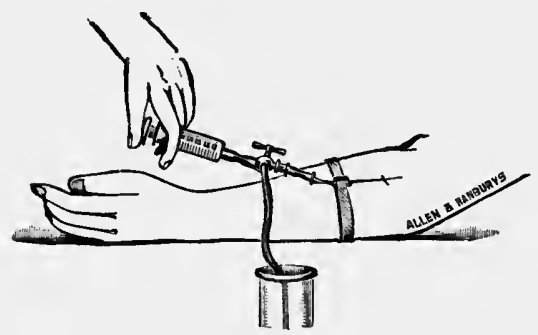

The Same IN USE

plate, which rests on the arm, and which may be fixed by a piece of tape which runs under a metal bridge and is tied under the arm should it be required, This needle is fixed by means of a bayonet-catch to the three-way stopcock; but connection between the needle and the bayonet-catch is made by a piece of thick rubber tubing, so that every movement of the stopcock or syringe behind is broken by this flexible connection, and so does not affect the needle,

The all-glass syringe, which should hold 20.0 or $30.0 \mathrm{c.cm}$., fits on to the stopcock by means of a piece of stout rubber tubing, instead of being inserted into a metal tube, which may not fit every syringe. The best syringes are Luer's; they are of French manufacture, but can be ordered in London through Allen and Hanbury. Both the English and the German makes are bad fits, and do not withstand frequent boilings. 
The syringe is first filled with saline solution, and all air is expressed both through the tubing and the needle; then the needle is inserted into the vein with the stopcock open, being guided and held by the metal bridge above it. If the vein has been pierced, and this can at once be told by touch, or by blood flowing back into the syringe, the tourniquet should be removed and some saline injected. If the cannula is not completely in the vein, the saline will produce infiltration; this being the case, the needle should be withdrawn and another vein chosen, as it is most important to prevent any of the solution getting under the skin, as considerable pain is caused thereby. If much escapes, there will be painful induration and œdema of the arm, and it takes weeks to disappear. When the solution has all been injected, some saline should finally be used to avoid leakage of a drop or two of arseno-benzene; this is done by transferring the tubing from the arseno-benzene vessel to the one containing saline. If, during the injection, the needle slips, and some of the solution escapes-the patient complaining at the same moment of a burning sensation-one should immediately take the needle out, apply a tourniquet to the arm, and allow the vein to bleed, which will often prevent infiltration. If the injection is skilfully done the patient has no pain.

Allen and Hanbury have recently made for me a double-barrelled syringe, which is very convenient for administering drugs intravenously (vide p. 204). By means of this syringe blood may be withdrawn for examination just before the drug is injected, thereby saving the patient a double puncture. Blood may be drawn into the second barrel and thoroughly mixed with the drug in the first barrel, a procedure which prevents shock or venous thrombosis, should the drug under ordinary circumstances be liable to cause these. The second barrel may be filled with saline or my calcium chloride-glucose-gelatine mixture to be injected after the drug has been administered, either to wash out the vein or to prevent shock should it be liable to occur. Another great advantage the syringe possesses is that the glass is separable from the metal fitting, enabling both to be boiled separately, and preventing the syringe from being useless should glass break, owing to its being easily replaceable.

Owing to the great convenience of injecting the solution of arseno-benzene in a concentrated form, the soluble salt is now very largely employed. The salt may be dissolved in the syringe itself in as little as $10.0 \mathrm{c.cm}$. of water, or better in a sterile vessel in $30.0 \mathrm{c.cm}$. of water, as then it can be filtered into the syringe. A Luer's $30.0 \mathrm{c.cm}$. all-glass syringe is the best. Air is excluded from the syringe, the needle attached, and inserted into the vein, from which, when pierced, blood flows into the syringe. Should some of the solution escape into the subcutaneous tissue, it never causes the pain or induration which are apt to follow the insoluble salt. Thrombosis is less frequent with the soluble than with the insoluble salt, and, on the whole, the patient is less prone to after-effects following an injection of the former.

Another method is to put the powder into the syringe, insert the needle, which is attached to the syringe, into the vein, and let the blood which flows 
back into the syringe dissolve the salt. When the powder is dissolved, the blood containing the neo-salvarsan in solution is reinjected into the vein. When the solution injected is in bulk, and is below the body temperature, the median basilic vein should be used in preference to the median cephalic, since the distension of the vein and the cool solution running along it irritates the circumflex nerve and causes considerable pain in the region of the shoulder.

A great advantage of the concentrated solution is that, after it has been injected, there is no need to keep the patient at rest; therefore it is eminently suited for out-patient work.

As the arseno-benzene compounds readily oxidise in the air to produce toxic compounds, each tube must be carefully examined before being filed to see that there has been no air inlet, a fact which can readily be proved by immersing the tube in alcohol or ether. In hospital work, a stock solution may be made to save time, but it should not be kept for more than one hour, and, when it is possible, a fresh solution should be made for each patient. After an injection has been given, both the syringe and needle should be boiled or cleaned with spirit, because a trace of blood from one patient may, if injected into another, be the cause of a disease being spread. I know of instances in which malaria was conveyed in this way. As a rule, after the first two intravenous injections the patient has a feeling as if he were going to get influenza-i.e., he complains of a headache, pains in the back, he may faint or have a rigor, etc. This reaction sets in about four hours after the injection, is most pronounced in the generalisation stage, and is relieved by acetyl-salicylic acid. The reactionary inflammation, which occurs practically only in the late stages of syphilis, especially when the patient has an intracranial lesion, sets in on the third day following the first, second, or third injection-usually following the second. It is therefore advisable to keep the patient in bed and watch him on this day. Shock following an intravenous injection is discussed in Chapter XVII.

\section{Intra-Arterial Injection of Arseno-Benzene.}

Intra-arterial infusion of arseno-benzene has been advocated by Sinclair ${ }^{2}$ in cases of nervous syphilis. In view of the relationship between certain branches of the internal carotid and the C.s.F., theoretically the intra-arterial route is one of the two most rational routes to take in treating syphilis of the nervous system, but, unfortunately, repeated exposure of the internal carotid is not a measure lightly to be undertaken, especially when no drug is definitely known to cure syphilis.

\section{Intrathecal Injection of Arseno-Benzenised Serum and Auxiliary Puncture.}

The method to be now described is that which has been elaborated by Homer Swift and Ellis. ${ }^{3}$ The patient is first given an intravenous injection of arseno-benzene. One to two hours later, about $50.0 \mathrm{c.cm}$. of blood are 
withdrawn from a vein into a sterile tube. The tube must be shaken occasionally to prevent the clot from sticking to its sides, which prevents the separation of the serum. When the serum has separated out, the tube is placed in an incubator at $57^{\circ} \mathrm{C}$. for one hour. The following day the serum is collected, mixed with an equal quantity of saline (about $25 \cdot 0 \mathrm{c.cm}$. of each), and, after an approximately corresponding amount of C.s.F. has been withdrawn, the diluted serum is injected into the canal. (For the details of performing a lumbar puncture, vide Chapter XII.)

The best needles for performing a lumbar puncture are Barker's; and.both Messrs. Maw, Son and Sons, and Allen and Hanbury have constructed a mount for my three-way syringe which makes the injection of arsenobenzenised serum a very simple matter.

After the operation, the patient is placed with his head low down, and the bottom of the bed is well raised, so as to allow the fluid to gravitate towards the brain. It has been advised to allow the prepared serum to wait for some days before being injected, but in my experience such a manœuvre decreases its therapeutic action and increases its toxicity. Much the same effect can be produced by adding formalin to the prepared serum owing to the effect both waiting and formalin have in increasing the size of the protein colloidal particles in the serum. Cases I treated with formalinised serum experienced twitching of the limbs and pain, the patients became excitable and restless, had delusions, and temporary retention of urine and fæces, almost exactly the symptoms met with when the arsenical product proves toxic.

Many observers have attempted to inject arseno-benzene directly into the spinal canal, but the general consensus of opinion is that the danger of toxic symptoms supervening contra-indicates such a measure being adopted.

The very small doses of the drug which can in this way be injected must, even by the enthusiasts, be considered insufficient to be curative, and the good results which have been obtained could have been equally well achieved with the arseno-benzenised serum. We have yet to learn the future of the cases which have been treated with arseno-benzenised serum, since it is mainly on theoretical grounds that its administration is based. There is no doubt that it does good in meningeal lesions, but whether it is only palliative or curative we have yet to learn. Recent experience has shown that better results may be obtained with intravenous injections and auxiliary puncture-e.g., draining the fluid within an hour of administering an intravenous injection, and within twelve hours of an intramuscular injection.

The intrathecal injections have, unfortunately, two very great disadvantages. One is, that the symptoms are usually aggravated by the first two injections-this is true only of those that accompany degenerative lesions -and the other is, that patients do so object to repeated lumbar puncture, that they often refuse to continue the treatment. If the treatment be discontinued, the patient's condition is very often permanently aggravated. I think this rule may be safely made in the case of nerve syphilis, that it is 
useless, and often harmful, to prescribe treatment unless it is intended to be drastic, or, in other words, sufficient to render the C.s.F. approximately normal.

I would here draw the reader's attention to the fact that the globulin test may fail after the first or second injection of arseno-benzenised serum. By the unwary this is assumed to show that one or two injections have cured the patient. Not only may the globulin disappear, but the C.F.T. may become negative. Exactly the same thing may happen with the serum of a late case of syphilis. Treatment by auxiliary puncture cannot in all cases be regulated by pathological examinations of the C.s.F., because one effect is to make the tests at first more positive, and later the C.s.F. has the property of laking red blood-corpuscles, which masks the C.F.T.*

\section{Other Methods of Administering Arsenic in Nervous Syphilis.}

(a) Prolonged Intraspinal Injection of Prepared Serum under High Pressure. -The technique practised by Ayer ${ }^{23}$ is as follows: The patient is placed on his stomach, with back arched by a pillow, and lumbar puncture performed. The C.s.F. is allowed to drain, then the arseno-benzenised serum is allowed to gravitate in, and quickly followed by $150.0 \mathrm{c.cm}$. of normal saline. The idea is based on experiments of Weed, ${ }^{24}$ who found it possible by means of prolonged spinal injections of potassium ferrocyanide and iron ammonium citrate, at pressures above the normal of the C.s.F., to precipitate Prussian blue in the cerebral perivascular spaces, and even in the capillaries and substance of the brain.

(b) Subdural and Intraventricular Injection of Prepared Serum.-As the same cranial operation is needed in both cases, and as injections by the latter route give the better results, a single description of the technique will suffice for both. A button of bone, $1.5 \mathrm{~cm}$. in diameter, is removed with a trephine a little in front of the bregma, and $2.5 \mathrm{~cm}$. from the sagittal sinus. The dura is then incised, and a blunt-pointed cannula is passed downwards and a little backwards into the lateral ventricle. The head of the table is then lowered, and about $20.0 \mathrm{c.cm}$. of C.s.F. allowed to escape, after which the head is again raised, and the arseno-benzenised serum allowed to gravitate very slowly into the ventricle. A lumbar puncture should be performed before the cranial operation is begun, and no general anæsthetic is required for the subsequent intraventricular injections. At present this route is to be employed only in eases of degenerative encephalitis, as the procedure is one not to be undertaken lightly. As some excellent results have been obtained by Campbell and Ballance ${ }^{25,26}$ and others, ${ }^{27,28,29}$ this method of treating degenerative encephalitis will have to be seriously considered in the future.

* In meningeal cases, supposing the first cell count to be 200 , the next, taken a week

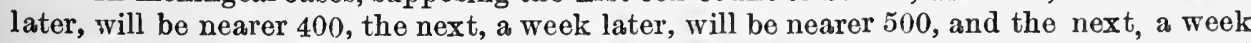
later, may have dropped to 10 . In degenerative cases the increase is less marked, and there is seldom a sudden fall to nearly normal. 


\section{Other Arsenical Compounds.}

As the French laws dealing with the patenting of drugs differ from the English ones, and as France is particularly renowned for her famous chemists, not only have salvarsan and neo-salvarsan been prepared for some considerable time in France, but also other similar products have seen daylight. The French equivalent to neo-salvarsan-namely, Nov-arseno-benzene (Billon)is by many thought to be the best soluble salt of arseno-benzene now obtainable. Mouneyrat has prepared what he calls "1116" (Galyl) and "1151" (Ludyl), and Danysz ${ }^{5,6}$ " 102 " (Luargol).

Galyl is soluble in a saturated solution of sodium bicarbonate, which was at first supplied in a separate ampoule. Now a preparation of galyl can be obtained which is soluble in water, and which can be injected in a concentrated form, like the soluble salt of arseno-benzene. The maximum dose of galyl is $0.4 \mathrm{grm}$. Normally, galyl is in the form of a yellow or greenishyellow powder, and the solution may have a light olive-green tint. The variation in colour is apt to frighten the user, but a tube need only be discarded in which the powder has turned brown or black. Galyl is a very convenient preparation to use; it seldom upsets the patient, but it is not quite so potent as the soluble salt of arseno-benzene, although perhaps a little less toxic. Galyl can also be injected intramuscularly. Ludyl never came into general use. Luargol is an excellent preparation, which contains silver and antimony in addition to arsenic. When first introduced, thrombosis at the site of injection was common, a disadvantage which has been overcome by the introduction of di-sodo-luargol, a dark brown powder, which being readily soluble in water, enables it to be injected in a concentrated form. As the drug is comparatively non-toxic, and more potent than any of the other preparations of arseno-benzene, it is probable that in time it will be more generally used. The dose varies from $0.1 \mathrm{grm}$. to $0.35 \mathrm{grm}$., and is best increased gradually, as the six or seven injections are given at about five days' interval between each. I always prefer to prescribe maximum doses from the start (Danysz).

Luargol was not the first arsenical preparation to have silver combined with it, as Ehrlich had produced a compound containing silver which he named $\mathrm{K}_{3}$, but it turned out to be too toxic for therapeutic use. Kolle, ${ }^{7}$ who has succeeded Ehrlich as Director of the Chemotherapeutic Institute in Frankfurt, has experimented with various new compounds of arsenobenzene since the latter's death, in 1915. Kolle divides the various compounds into four groups:

1. Salvarsan, neo-salvarsan, and hex-amino-salvarsan.

2. Arsalytes, with halogen substitution products $(\mathrm{Br}, \mathrm{Cl}, \mathrm{I})$.

3. Arseno-benzene compounds containing phosphorus (galyl) or metals. such as platinum, gold, copper, and silver.

4. New sulphoxylate compounds, especially No. 1495, the constitution of which is at present a secret. 
No. 1495 is said not to become more toxic when exposed to the air even up to forty-eight hours, a property which will, of course, prove most useful, other things being equal. The most potent, and at the same time the least toxic, preparation appears to be the silver salvarsan, which has been extensively used by Gennerich ${ }^{8}$ and Sellei. ${ }^{\theta}$ The constitution of the so-called silver salvarsan is not revealed, but it does not appear to be the same as $K_{3}$. It is probably an adsorption compound of arseno-benzene with colloidal silver. With the number of arsenical preparations now on the market, not to mention the increase there is bound to be when international commercial channels are once more open, the reader will naturally wonder which preparation he ought to use; therefore he will probably find the following remarks of some use to him.

When several modifications of a drug follow one another in quick succession, it proves that the object for which the parent drug was introduced has not been attained, and experience has always shown that the branches from the parent trunk have only carried us further into the air, to leave us more insecure, until we are obliged to descend to the trunk in order to regain our safety. No preparation of arseno-benzene has yet been prepared which is so potent as was the original salvarsan. It may be quite true that some of the later preparations, especially those which contain elements in addition to arsenic, may kill Spirilloe and Spirochoeto in animals more quickly than the original salvarsan did, but the knowledge to be gained from an animal experiment has no right to be judged as being applicable when translated to the human body. From my own research work ${ }^{10} \mathrm{I}$ have found that no organism is attacked directly in corpore by a drug, but only indirectly, by the action the drug has in increasing the parasiticidal.action of the host's resisting substance, which is the protein colloidal particles in the serum. The resisting substance kills the parasites by destroying the oxygen in a simple surface action of electrical neutralisation. Metals increase the oxidising action of the resisting substance, and non-metals the reducing action. Three injections of three different oxidising agents are more potent than three injections of the same oxidising agent, hence the explanation of the increased power of an arseno-benzene compound when it has other metals combined with it. But-and here we come to some important points-(1) the oxidising action of all metals is not the same; (2) the introduction of another metal to an arseno-benzene compound depresses the percentage of arsenic, thereby lessening the potency of the preparation if the attached metal has a feebler oxidising action than arsenic; (3) the additional metals are not attached directly to the benzene nucleus as is the arsenic, a fact which detracts from the physical action of the compound; $(4)$ the dissociation constant is all-important.

The metals which are the most toxic are those which have the greatest bactericidal action in vitro, but this does not mean that they have the same action in vivo. As the metals exerting the greatest bactericidal action in vitro are mercury, arsenic, antimony, and silver, the reason is now apparent 


\section{CLINICAL ASPECT AND TREATMENT OF VENEREAL DISEASES}

why the last two named have been incorporated with arseno-benzene. The oxidising action of none of these metals can compare with that of manganese and iron, suitable organic compounds of which attempts are being made to prepare, feeling sure that these two non-toxic metals will ultimately replace arsenic, antimony, and silver in the treatment of syphilis. As none of the arseno-benzene compounds stimulate the reducing action in corpore, I have introduced intramine $11,12,13,14,15,16,17$ (di-ortho-di-amino-thio-benzene), an organic preparation of sulphur, as non-metals regulate the reducing action as metals do the oxidising action. Since using metallic drugs exerts only half the beneficial action possible, the introduction of.intramine has made an enormous difference in the treatment of syphilis, as our therapeutic armamentarium is now complete.

\section{Antimony.}

As an alternative to arsenic, antimony by some is considered to be a useful drug to have in reserve. I prefer intravenous injections, as intramuscular injections are apt to be very painful. I have not tested all the antimony products which have from time to time been advocated; therefore I cannot say that any one preparation is better than any other. As no preparation of antimony is as powerful as arseno-benzene, and therefore is not one's sheetanchor, but is used only as an alternative, it does not matter very much which preparation is employed. For intramuscular injections I have used antiluetin (a double salt of antimony). The salt is readily soluble in water, and is best put up in ampoules containing $1.0 \mathrm{c.cm}$. according to the following formula:

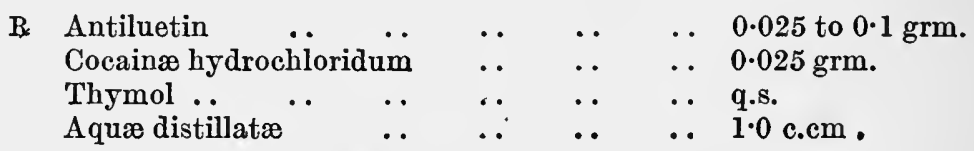

A course should consist of twelve injections, given daily, or every other day, beginning with $0.025 \mathrm{grm}$. and ending up with $0.2 \mathrm{grm}$.

For intravenous injections I employ either tartar emetic or antiluetin.

If the former, I have ampoules made up containing $1.0 \mathrm{c.cm}$. of distilled water and 1 to $1 \frac{1}{2}$ grains of tartar emetic. I place the contents of one ampoule in 5 ozs. of distilled water, and give ten injections in all, twice or three times a week.

Antiluetin is used in the same way, and for each injection 0.025 to $0.05 \mathrm{grm}$. is employed, without the cocaine.

Toxic symptoms occurring after antimony injections are much like those occurring after intravenous injections of mercury, but even slight symptoms. are very rare.

Although not a venereal disease, I should like to mention here that I have had great success in treating cases of bilharzia with intravenous injections of antimony. In my experience, arseno-benzene is of no use in this complaint. 
Colloidal (collosol) antimony is also an excellent preparation which can be injected both intravenously and intramuscularly. It is an 0.04 per cent. emulsion, of which $1.0 \mathrm{c.cm}$. to $10.0 \mathrm{c.cm}$. can be injected by the former route, and $1.0 \mathrm{c.cm}$. to $4.0 \mathrm{c.cm}$. by the latter route. Larger doses injected intramuscularly are not advisable, as much pain is caused.

\section{Mercury.}

\section{A. Intravenous Injection.}

A very good way of prescribing mercury is intravenously, and the two best salts are the cyanide and the bichloride.

If the cyanide is going to be used, a stock solution of 1 in 100 should be mâde, and $0.5 \mathrm{c} . \mathrm{cm}$. to $1.5 \mathrm{c.cm}$. of this solution may be injected daily or every other day. A course usually consists of ten to twelve injections.

If the bichloride of mercury is preferred, ampoules of $1.0 \mathrm{c.cm}$. should be made up, each containing $0.4 \mathrm{grm}$. of the salt. A course should consist of ten to twelve injections, given twice or three times a week. For the first three doses only $0.5 \mathrm{c.cm}$. should be used, and for the remaining doses $1.0 \mathrm{c.cm}$.

Instead of using the solutions concentrated, I think it is best to put the contents of an ampoule into a syringe holding $30.0 \mathrm{c.cm}$. of pure distilled water. Diluting the solution diminishes the likelihood of producing local thrombosis, and also of toxic symptoms arising. Toxic symptoms may follow intravenous injections of mercury, but they are of little note; the commonest are abdominal pain and diarrhœa. A peculiar sensation in the throat is sometimes complained of, and momentary congestion of the face and difficulty of breathing may now and again occur almost immediately after the injection.

Colloidal (collosol) mercury in the form of a 1.0 per cent. emulsion is now the best preparation for intravenous work. It can be injected as supplied in doses of $5 \cdot 0 \mathrm{c.cm}$. to $15 \cdot 0 \mathrm{c.cm}$.

\section{B. Intramuscular Injection.}

There is a legion of preparations of mercury for intramuscular use, and they can be divided into two classes: $(a)$ soluble, and $(b)$ insoluble. There is absolutely no doubt but that the insoluble salts are far more efficacious than the soluble ones. So far as the insoluble salts are concerned, one's choice lies between three. One of these-and that is calomel-can, from my experience, be deleted, since, although it is perhaps more potent than grey oil, its administration is, unfortunately, frequently so very painful that patients get frightened away from all kinds of intramuscular injections. Moreover, however careful one may be, abscess formation is more liable to follow intramuscular injections of calomel than injections of any other preparation I know.

That leaves us with two preparations, and the choice between them will rest upon whether a quicker and a less potent action, or a slower and a more potent action is required. 


\section{CLINICAL ASPECT AND TREATMENT OF VENEREAL DISEASES}

Before the arseno-benzene era, it was often necessary to get rid of the lesion as quickly as possible; consequently, one preferred to use a preparation which was very quickly absorbed to one which was slowly absorbed, although the potency of the latter was greater than that of the former. With the exception of the insoluble salts, which are naturally rapidly absorbed, but whose action, even in the maximum doses, does not amount to very much, no preparation is so suitable as the salicylate of mercury, because not only is it quickly absorbed, but its action exceeds the action of the maximum doses of the insoluble salts. The best preparation for injection is $1.0 \mathrm{c.cm}$. of a 10.0 per cent. emulsion of mercury salicylate in liquid paraffin. Either $1.0 \mathrm{c.cm}$. of this emulsion may be injected weekly, or $0.5 \mathrm{c.cm}$. may be prescribed twice a week.

As arseno-benzene has rendered the question of the rate of absorption of the mercurial preparation of no account, one need only consider the question of the efficacy.

In my opinion, the best mercurial preparation, and the one I now always use myself, is Adam's cream. It is a grey oil preparation, which is liquid at the ordinary temperature, and therefore does not need to be heated; its bulk is small, and therefore its use is practically painless.

The grey oils in previous use have been unsatisfactory because they required heating. Now the action of heat is to cause the globules of mercury to agglomerate, so that, in time, most of the metal is at the bottom of the bottle, with the result that the first injections contain less mercury than the last. Their bulk was always great, consequently the patients frequently complained of pain.

The following is the prescription of Adam's cream, and it is prepared for me by Squire and Sons, 413, Oxford Street, London, W. 1 :

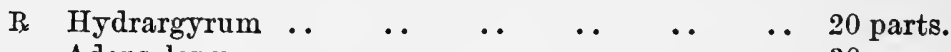

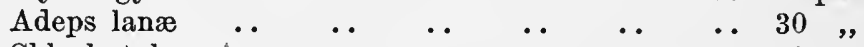

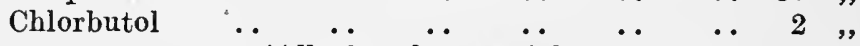

$$
\begin{aligned}
& \text { (All of each by weight.) }
\end{aligned}
$$

The injections should be made into the gluteal muscles, the scapular muscles, or into the latissimus dorsi muscle below the ribs or the angle of the scapula. If the gluteal region is chosen, the injection should be made round about the upper and outer margin of the main fleshy mass. In the scapular region, the needle should be inserted from above downwards, deep into the muscles, about midway between the posterior border of the scapular and the spinous processes of the vertebræ. The best syringe to use is the Record syringe for intramuscular injections of mercury supplied by the Holborn Surgical Instrument Company, 26, Thavies Inn, London, E.C. 1.

The laws of asepsis having been strictly complied with, plunge the needle in quickly for $2 \frac{1}{2}$ to 3 inches, but avoid touching the bone. Remove the syringe 
from the needle to see if any blood flows from it. If so, withdraw and plunge again, as the presence of blood shows that a vein has been entered with obvious danger of embolism. Inject very slowly; then withdraw quickly, while pressing the skin around the puncture to prevent bleeding. Rub the part well for a few minutes, and send your patient for a brisk walk.

As the soluble preparations are very seldom called for nowadays, it is not necessary to mention all that have from time to time been used. If I ever use a soluble salt, I most frequently prescribe enesol.

Enesol is a useful mercurial preparation, especially in those cases in which arseno-benzene cannot be given, and in which it is wished to get the patient under the influence of mercury as quickly as possible. Enesol is a salicylarsenate of mercury, readily soluble in water, and is put up in ampoules of $1.0 \mathrm{c.cm}$. In each ampoule there is $0.0115 \mathrm{grm}$. of mercury and $0.0043 \mathrm{grm}$. of arsenic. From 1.0 to $3.0 \mathrm{c.cm}$. may be injected intramuscularly daily, or every other day, until a marked improvement has taken place; or the salt may be injected intravenously. The latter route being the better of the two, $1.0 \mathrm{c.cm}$. to $4.0 \mathrm{c.cm}$. in $30.0 \mathrm{c.cm}$. of distilled water may be injected intravenously every second day, or twice a week, until twelve injections have been given. Other soluble preparations in frequent use are the benzoate and succinimide of mercury.

A mercurial preparation which is sometimes useful in checking the lightning pains of degenerative myelitis when other preparations have failed is the so-called Hirsch's injection. The solution contains 1 per cent. oxycyanide of mercury and 0.4 per cent. acoine; $1.0 \mathrm{c.cm}$. to $3.0 \mathrm{c.cm}$. are injected intramuscularly daily, or every other day, until $30.0 \mathrm{c.cm}$. have been used. This preparation does undoubtedly in some cases give relief; but, in my experience, the pains soon recur when the action of the drug has worn off. Acoine is a white crystalline powder, and is the name applied to the local anæsthetic di-para-anisyl-monophenetyl-guanidine hydrochloride, for which the British Pharmaceutical Codex, 1911, gives the short name of guanicaine.

A mercurial preparation for intramuscular administration which is very efficacious, although painful, is colloidal (collosol) hydrargyrum, a $5 \cdot 0$ per cent. emulsion, supplied by Crookes' Laboratories, 22, Chenies Street, London, W.C. 1.

\section{Intraspinal Injection of Mercurialised Serum.}

The mercurialised serum is prepared in two strengths; No. 1 contains the equivalent of $1.3 \mathrm{mgr}$. of mercuric chloride in normal serum diluted with normal saline to a total volume of $30.0 \mathrm{c.cm}$. No. 2 contains the equivalent of $2.6 \mathrm{mgr}$. of mercuric chloride in normal serum diluted with normal saline to a total volume of $30.0 \mathrm{c.cm}$. Both solutions are dispensed in sealed ampoules, ready for use, and supplied by the Mulford Co., Philadelphia, U.S.A. Byrnes, ${ }^{18}$ who originated the idea of giving mercury in this way, advises that No. 1 should be first used, and No. 2 proceeded with only should the former be well borne. The method of giving the injection does not differ frcm that, 
already described for arseno-benzenised serum. As an alternative to using arseno-benzenised serum, mercurialised serum is useful, but, per se, the drug in neither so active nor so efficacious. As auxiliary tapping has proved more beneficial than direct intraspinal medication, there is no further need for this preparation.

\section{Inunction.}

There can be no doubt that the best way of administering mercury is by inunction, but unfortunately this method is valueless unless it is carried out by a trained rubber.

Inunction is one of the oldest methods of administration. It does not upset digestion, it gets the patient under the influence of mercury more quickly than oral administration, and it is easily regulated. It is best to use either unguent. cinereum, which consists of one part $\mathrm{Hg}$ with two of lanoline and unguent. simplex; or mercury resorbin, 1 to 2 . The adult dose of ointment is from 1 to 7 drachms; children take from 1 to 1 drachm. The following is a good order of inunction, one hour a day being given to the work:

\begin{tabular}{|c|c|c|c|c|c|}
\hline First day & $\cdot$ & & & .. & Both thighs. \\
\hline Second day & .. & - & .. & . & Both legs. \\
\hline Third day & $\cdots$ & .. & . & $\cdots$ & Both arms. \\
\hline Fourth day & . & . & . & .. & Chest and abdomen. \\
\hline Fiftl day & . & .. & .. & .. & Back. \\
\hline Sixth day & . & . & . & .. & Hot bath. \\
\hline Seventh day & . & .. & . & 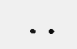 & Recommence as on first day. \\
\hline
\end{tabular}

The inguinal region, nipples, and all hairy parts must be shunned, as acute pustular eczema may arise. The flat part of the palm should be used for rubbing, and a fair even pressure maintained. Inunction is contraindicated in cases of eczema, prurigo, and psoriasis. If the patient has rubbed himself, or has been rubbed, effectively, his skin-when all superfluous ointment has been removed with a towel-should show a number of black dots where the mercury has penetrated the pores. Mercury is in part absorbed through the pores of the skin, and in part evaporated by the heat of friction. The vapour so given off is inhaled, and inhaled mercurial vapour speedily gives rise to stomatitis. In a ward where syphilitic patients are using inunctions, it is not rare for non-syphilitic ones to get mercurial stomatitis from evaporation and inhalation. It is, therefore, well that inunction be done in the early morning, in a well-ventilated room, in which the patient is not going to remain, and that the mouth be rinsed out several times with potassium chlorate while rubbing. A patient should not keep to his room except in cold and windy weather, and his clothes should not be too warm. Menstruation is no bar to continuing inunction. A course of inunctions should consist of thirty to forty rubbings. 


\section{E. Baths.}

Baths are indicated in cases of ulcerative skin lesions which make inunctions impossible. They serve two purposes, for the sublimate is absorbed from the raw surfaces, and also acts as a local treatment to them; 1.0 to 2.0 grm. of sublimate are dissolved in a hot bath, in which the patient remains for half an hour, temperature being kept at about $95^{\circ} \mathrm{F}$. by the addition of hot water or by a warming apparatus. A bath is given daily, and the patient is kept in bed for one hour after it.

\section{F. Fumigation.}

Fumigation is only mentioned to be deprecated, since mercurialism is almost unavoidable.

\section{G. Impregnation.}

Impregnating the underclothing with mercury, or spreading emplast. cinereum over a large surface of the body, is quite a good method of treating infantile cases.

\section{H. Suppositories.}

Mercurial suppositories can sometimes be employed with advantage if an alternative method of prescribing the drug is required.

A suppository should be made up as follows:

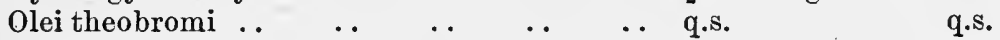

Metric. $0 \cdot 03-0 \cdot 2$ grm.

One should be inserted every night just before going to bed, until either the rectum begins to get sore or the gums begin to get tender. If the suppositories cause much local pain, this may be obviated by adding a little cocaine to each.

In tropical countries a little white wax has to be added, owing to the low melting-point of the oil of theobroma.

\section{Internal.}

Internal treatment has the great disadvantage that, by irritation of the mucous membrane, digestion is upset. We also know not how much mercury is being assimilated, because we know not how much is being passed per viam naturalem. The dose, therefore, has to be somewhat greater so as to be on the safe side. It is useful in late stages of the disease, where it is not necessary to get the patient quickly under the influence of mercury, and as a change from inunctions and injections. Its great sphere of usefulness is in sucklings, in whom inunctions are generally followed by eczema, but who stand $\frac{1}{2}$ gr. of hydr. $\bar{c}$ cret. twice a day well. For adults, Hutchinson's pill is largely used in England:

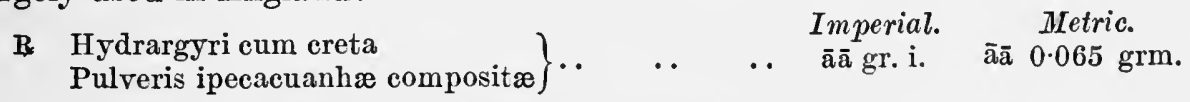
One pill to be taken three times a day after meals. 
Of greater utility is Ricord's pill, which contains iodine:

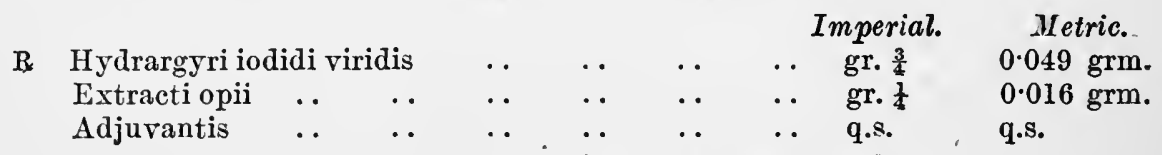

One pill to be taken twice a day. after meals.

The following formula is commendable, in that the mercury is in a form less likely to injure the mucous membrane, so upsetting digestion:

\begin{tabular}{|c|c|c|c|c|c|c|c|c|}
\hline \multirow[t]{3}{*}{ R } & \multirow{2}{*}{\multicolumn{4}{|c|}{$\begin{array}{l}\text { Hydrargyri tannici oxydulatis } \\
\text { Extracti opii .. }\end{array}$}} & & \multirow{2}{*}{\multicolumn{2}{|c|}{$\begin{array}{c}\text { Imperial. } \\
\text { gr i. } \\
\text { gr. } \frac{1}{4}\end{array}$}} & \multirow[t]{2}{*}{$\begin{array}{c}\text { Metric. } \\
0.065 \text { grm. } \\
0.016 \text { grm. }\end{array}$} \\
\hline & & & & & & & & \\
\hline & Adjuvantis & .. & . & .. & $\ldots$ & .. & q.s. & q.s. \\
\hline
\end{tabular}

Mercurial stomatitis is the complication which most usually besets us. It is also the most valuable guide by which to regulate the dose of mercury. Stomatitis must be solely dependent upon the teeth, for the toothless at both ends of life's ladder are never affected. If the teeth be well looked to-: cavities filled, tartar scraped, and stumps removed-before commencing treatment, and well looked after during treatment, stomatitis will seldom become severe. The care of the teeth during treatment consists in absolute abstinence from both alcohol and tobacco, and the brisk use of the toothbrush after each and every meal, followed by an astringent mouth-wash, as potassium chlorate gr. $x$. ad zi.; or-

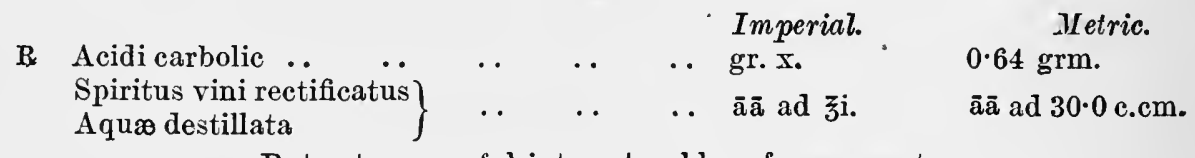

Put a teaspoonful into a tumbler of warm water.

Should the gums bleed, the following should be painted on:

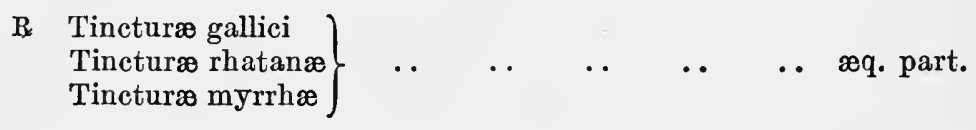

Or simply glycerinum acid. tannici. Should pus or ulceration be present, paint twice daily with tinct. iod. or hydrogen peroxide.

A slight stomatitis is a valuable guide to the degree of mercurialism attained. It should be our aim to press mercurial treatment to the exact point at which slight stomatitis-a trace of salivation, with slight swelling of the gums, a diminution of the papillæ till the free edge of the gums forms a nearly straight line-is reached and maintained.

Mercurial treatment must be intermittent, because of the danger of mercurialism. In whatever form mercury enters the system, it is eliminated as a sulphide viâ the bowels, kidneys, and saliva. It is excreted very slowly, and therefore accumulates and becomes fixed in the system. It is only the excreted portion which has had any action on the virus. The fixed portion is harmful, and is the cause of the chain of symptoms known as mercurialism. 
These generally begin with fœtor of breath and soreness of gums, followed by a metallic taste in the mouth, swelling, softness, bleeding, and ulceration of gums. The salivary glands become enlarged and tender, causing increased salivation; the tongue swells, teeth become loose, and ulceration of the mucous membrane of the mouth, even necrosis of the jaw, may follow; anæmia becomes marked, the blood becomes watery, and fails to coagulate, thereby increasing the liability to hæmorrhage. Now, patients do not like these symptoms, and it is best to avoid them by intermitting the doses of mercury, and by varying the manner of getting that metal into the system, by changing from mouth administration to inunction through the skin. Treatment does not cease because the taking of mercury is given up for a period, since excretion goes on for some time after the last dose.

It must not be forgotten that there are some patients who exhibit a marked idiosyncrasy to mercury, and there are several whom it makes very depressed. Depression, and all the symptoms which constitute what we call mercurialism, are far more likely to follow the internal than any other form of administration, and this is not because more of the drug circulates in the system when it is prescribed per os; on the contrary, symptoms of mercurialism may become manifest long before the therapeutic action is making itself felt. I have no doubt in my own mind that the reason why we see so much chronic superficial glossitis and carcinoma of the tongue in this country is largely due to our routine treatment of syphilis by means of pills. In countries where it has been the rule for many years to treat syphilis with mercurial inunctions and injections, chronic superficial glossitis is a very rare condition. When I first became attached to the Lock Hospital, and took over the cases of my predecessor, who had always used pills, I was obliged to examine the mouth of nearly every patient. Now that all my patients are treated with injections, it is very seldom that a mouth has to be examined, and patients, while under treatment, are almost unknown to complain of sore tongues, sore throats, etc. Different surgeons have different views on the matter, and some still maintain that pill treatment is the best. My best answer to this is that several of their patients ask if they can come upon a day when mercurial injections are given, as they have noticed how much fitter the patients look who are having them. Out-patients in a large hospital, while waiting for their turn to be examined, have ample opportunities to discuss affairs with those around them, and as the average patient's main idea in attending a hospital is to get well as quickly as possible, the matter of treatment is uppermost in their minds; consequently, their opinion of what they consider to be the best is usually the correct one.

Because of the great disadvantages of the oral administration of mercury, I always use mercurial injections or mercurial inunctions when the patient can afford the time and inconvenience caused by the latter. For reasons already stated, I prefer the grey oil to any other preparation. If the patient cannot have mercurial injections because he is travelling, or something of that sort, then he must take mercury internally or in the form of suppositories. 


\section{Sulphur.}

The preparation of sulphur now in frequent use is intramine, an aminocompound of sulphur, di-ortho-di-amino-thio-benzene. Sulphur regulates the reducing action in the body and produces oxygen indirectly by preparing the ground, so to speak, for the metals to work upon. As overdoses of metals lead to their own oxidation, and so destroy the medium in which they act, it can be readily seen, in virtue of the power intramine has in restoring the medium, how useful this drug must be, not only in aiding the action of metals; but in overcoming the toxic effects produced by their over-use. As the resisting substance in the body produces its oxygen indirectly, when its production by direct means has failed to vanquish the parasite-in other words, when the disease becomes chronic-it is necessary still further to stimulate this action, which is done by means of intramine before prescribing metals. From this, three facts stand out: (1) in the acute stages of a disease metals should be prescribed up to that point where they just fall short of being themselves oxidised, when intramine should be administered before their use is continued; (2) intramine is a specific for metallic poisoning; (3) intramine should precede arseno-benzene and mercury in the late stages of the disease. As the reducing action of the host's resisting substance is the same in all diseases, intramine is useful in any chronic infection, and has not the limited use of arseno-benzene and mercury.

Intramine is absolutely non-toxic, mainly because the element it contains (sulphur) is a normal constituent of the human body. The introduction of intramine into the treatment of syphilis has practically revolutionised it, because it not only increases the action of arseno-benzene and mercury, but it also prevents toxic symptoms following the use of the last two named drugs and the recurrent chancres and nervous symptoms which have become so common of recent years. Intramine has the effect of reducing the induration of chancres and of healing late syphilitic lesions, which are often so rebellious to arseno-benzene: gummata, glossitis, etc. For arsenical and mercurial poisoning, intramine is absolutely a specific. Intramine can be injected intramuscularly, intravenously, and taken internally.

\section{A. Intramuscular.}

For this purpose intramine is now supplied by the British Drug Houses, Graham Street, City Road, London, N. 1, in two solutions, which are best mixed immediately before use in the proportion of one part of the No. 1 solution with two parts of the No. 2 solution. The resulting colloidal emulsion contains 1.0 per cent. of intramine, and will pass through the finest bore needle. The dose of the emulsion is from $1.0 \mathrm{c.cm}$. to $5.0 \mathrm{c.cm}$., and it may be repeated as often as is desired. The injections cause a little pain, but leave no induration. 


$$
\text { . }
$$




\section{PLATE L}

No. 96.-An Iodide Rash with a Recurrent Syphilitic Lesion oN THE Foreilead

Note that the centres of some of the iodide lesions resemble those of Molluscum contagiosum, and that the further stage is haemorrhagic, when the lesions tend to eoalesee and become grouped. The lesions are hard and typical, in that the smallest are to be seen in the periphery and vice versấ 


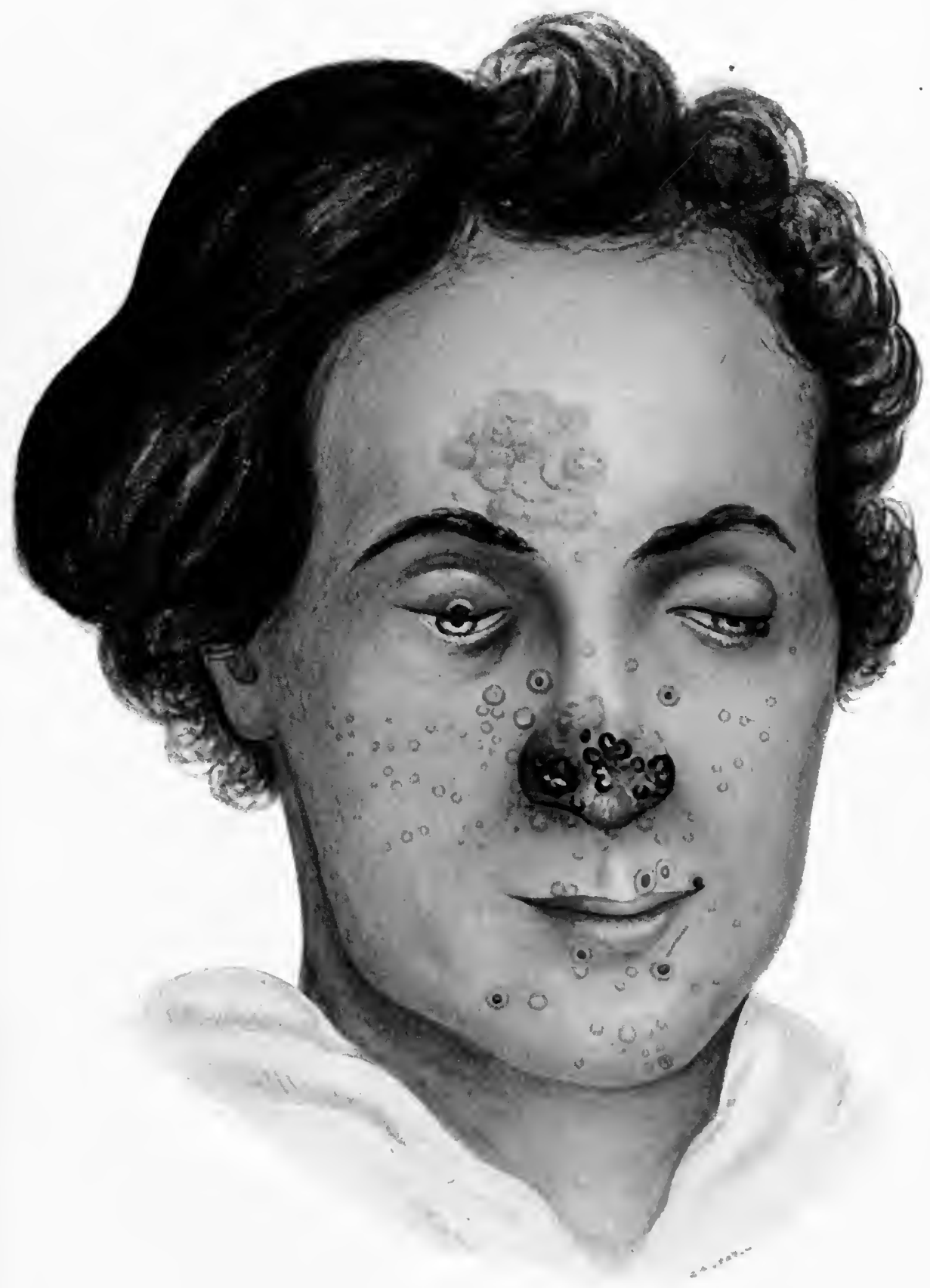

No. 96

Plate L 


.




\section{B. Intravenous.}

For this purpose intramine is supplied in the form of a $0 \cdot 1 \cdot$ per cent. colloidal emulsion, which is ready for injection after it has been filtered through a thin layer of cotton-wool. The usual dose is $50.0 \mathrm{c.cm}$., but much larger doses may be safely given. Intramine can be injected intravenously into out-patients; and it does not cause venous thrombosis, or upset the patient in any way. When large doses are used the patient sometimes has a fit of coughing, but this only lasts for a minute or two, and it can always be avoided if the emulsion is made alkaline with sodium hydroxide just before it is injected. The fit of coughing is more pronounced when collosol iodine instead of collosol mercury precedes the intramine. If intramine is being injected the day after collosol iodine, to every $50.0 \mathrm{c.cm}$. of intramine $3.5 \mathrm{c.cm}$. of a 4.0 per cent. solution of sodium hydroxide should be added, but after collosol mercury $2.5 \mathrm{c.cm}$. of the sodium hydroxide solution is ample. If the emulsion is made too alkaline, no harm other than venous thrombosis will be caused. The rapidity with which the gingivitis, diarrhœa, feeling of malaise, etc., caused by colloidal mercury disappear under intramine is remarkable. Taking injection for injection, an intramuseular injection has a greater therapeutic action than an intravenous injection, because in the latter case the intramine is excreted more quickly, and it has to be so highly protected.

\section{Internal.}

For this purpose intramine is supplied in keratinised gelatine capsules, each of which is equivalent to $0.5 \mathrm{c} . \mathrm{cm}$. of a 1.0 per cent. colloidal emulsion of intramine. One to three eapsules can be taken three times a day after meals. Intramine taken in this form is useful in cases of intestinal intoxication, especially Acne rosacea, as well as in recurrent and late cases of syphilis.

\section{Iodine.}

The most potent iodine salt which we have is undoubtedly potassium iodide, but unfortunately there is a large number of patients who cannot take it, partly owing to its depressing action, and partly because of its readiness to produce iodism. The depressing action may often be overcome by using the ammonium and sodium salts, but those patients who readily exhibit the symptoms of iodism will usually do so with any inorganic salt. Sometimes the symptoms of iodism may be avoided by the administration of calcium lactate or sodium bicarbonate. Owing to the frequency of iodism with the inorganic salts, a legion of organic compounds have been put upon the market, all of which are stated to be equally potent as the inorganic preparations and absolutely innocuous.

With many, iodism does not supervene, but I have yet to find one which will be entirely innocuous when given to a patient who exhibits a marked idiosyncrasy to any preparation of iodine, and such patients are not at all 
infrequently to be met with. No organic preparation appears to be as powerful as potassium iodide. The best organic preparations are iodoglidine. sajodin, and tiodine.

Since the above was written, colloidal (collosol) iodine, 1 in 500 , has been introduced, which is not only more potent than any other preparation of iodine known, but it practically never produces symptoms of iodism. Colloidal iodine can be taken internally in doses of 1 to 3 drachms, ter in die, post cibos, or injected intravenously in doses of $50.0 \mathrm{c.cm}$. to $300.0 \mathrm{c.cm}$. Large intravenous injections occasionally cause thrombosis; but they do not upset the patient in any way, and can be safely employed in out-patient work. Iodine has an action akin to sulphur, and is particularly valuable in increasing the effect of intramine. In cases where time is of no value, it is a good plan to get the patient under the influence of iodine before prescribing intramine; but in those cases where no time must be lost, such as in bad cases of arsenical and mercurial poisoning, it is a good plan to inject colloidal iodine intravenously at the same time as intramine is being injected intramuscularly.

\section{Nucleinate of Soda.}

There is no doubt that nucleinate of soda will sometimes produce a period of quiescence in a case of degenerative encephalitis when every other kind of treatment has proved unavailing. It does not cure the disease, but a patient may be brought from the imbecile state into the normal state, and may remain in the latter for some months. This drug has been used on a fairly extensive scale by Fischer in Prague, and Donath in Budapest. In this country, Gordon-Lane has used it in several cases with very satisfactory results. As the drug is not widely known, and as the dose varies with different observers, I will mention the methods of the three men whose names I have above referred to.

Fischer ${ }^{19,}{ }^{20}$ uses 0.5 to $3.0 \mathrm{grm}$. dissolved in water, and injected intramuscularly every three to five days. Donath ${ }^{21,22}$ first recommended intramuscular injections of $50.0 \mathrm{c.cm}$. to $100.0 \mathrm{c.cm}$. of a 2.5 to 3.0 per cent. aqueous solution, and he gave eight injections at intervals of five to seven days. Donath $^{21}$ now employs a $10 \cdot 0$ per cent. solution dissolved in normal saline, and injects from $10.0 \mathrm{c.cm}$. to $50.0 \mathrm{c.cm}$. every four or five days, until six to twelve injections have been given.

Gordon-Lane tells me that he always employs a $2 \cdot 0$ per cent. solution, and injects $50.0 \mathrm{c.cm}$. to $100.0 \mathrm{c.cm}$. once a week, for about eight weeks.

Some hours after the injection the patient gets a rise of temperature to about $104^{\circ} \mathrm{F}$. The next day it falls, to rise slightly again, and, as a rule, the following day it falls and remains normal until the next injection is given. The rises of temperature may be higher, and may be maintained over a longer period than this.

The injections also produce a leucocytosis. 


\section{Vanadium.}

Vanadium, a metal belonging to the fifth group of the periodic system, which includes nitrogen, phosphorus, arsenic, antimony, and bismuth, has from time to time been used in the treatment of syphilis. Owing to its strong oxidising properties, it might a priori be thought to be a most useful metal, but unfortunately the compounds are unstable; it appears to be impossible to prepare a strongly adsorbed organic compound, and only the higher oxides are available. Proescher, Seil, and Stillians, ${ }^{30}$ published an interesting paper on vanadium, in which they state that sodium hexa-vanadate, which is a soluble neutral salt, readily sterilised in solution, and therefore capable of being put up in ampoules, has a specific effect on early syphilitic lesions, and is capable of favourably affecting the C.F.T. Working with Mr. Patterson, he prepared a stable colloidal preparation of vanadium oxide, but it had to be used in too big doses to be practicable and safe, and the results obtainable were not as good as could be procured with manganese and iron.

\section{Mode of Action.}

Metals act as oxidising agents and non-metals as reducing agents. The metal which regulates oxidation in the human body is iron, and the nonmetal regulating reduction is sulphur. The action of iron can be shown diagrammatically in the following formulæ, in which $R$. stands for the radicle or protein colloidal particles (protective substance):

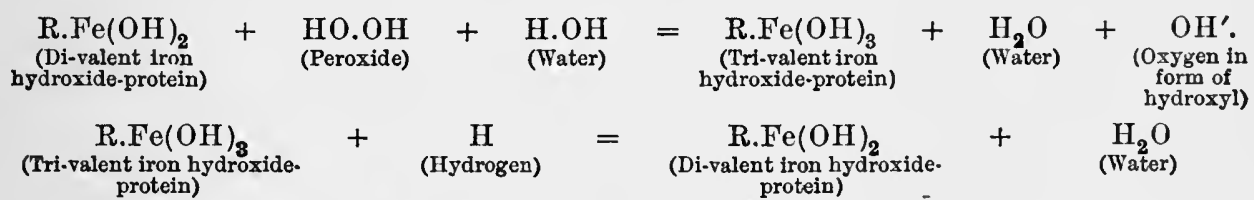

From the above formulæ it will be seen that the metal acts as a catalyst, and is indestructible provided hydrogen is present. The medium upon which the metal acts to produce oxygen is peroxide, which is destructible, unless its manufacture is kept going by the action of sulphur. Arsenic acts as an oxidising agent like iron, but not so efficiently, as it does not exist in two hydroxide forms, which are readily reversible by hydrogen, hence the reason why I say that a suitable compound of iron or manganese would soon replace arsenic, because manganese in this respect resembles iron. Mercury acts partly as an oxidising agent, as it has more than one valency, but its main action is a physical one, owing to its high atomic weight. It is not specific, as other multi-valent metals with as high an atomic weight are as efficacious as mercury. The action of sulphur can be shown diagrammatically in the following formula:

$\underset{\text { (Di-sulphide-protein) }}{\text { R.S.S.R. }}+\underset{\text { !(Water) }}{2 \mathrm{H} . \mathrm{OH}}=\underset{\substack{\text { (Sulphur. } \\ \text { protein) }}}{\text { R.S. }}+\underset{\substack{\text { (Mercaptan. } \\ \text { protein) }}}{\mathrm{R} . \mathrm{SH}}+\underset{\text { (Peroxide) }}{\mathrm{HO.OH}}+\underset{\text { (Hydrogen) }}{\mathrm{H} \cdot}$ 
TABULATED LIST OF THE MOST IMPORTANT DRUGS.

\begin{tabular}{|c|c|c|c|}
\hline Name. & Formula and Strength. & How used. & $\begin{array}{l}\text { Average Dose } \\
\text { for Adult. }\end{array}$ \\
\hline $\begin{array}{l}\text { Salvarsan } \\
\text { Kharsivan (Eng.) } \\
\text { Diarsenol (Can.) } \\
\text { Arsphenamine (U.S.A.) } \\
\text { Arseno-benzene, Billon (Fr.) }\end{array}$ & $\begin{array}{c}\mathrm{OH} \quad \text { OH } \\
\text { As } 31 \cdot 56 \text { per cent. }\end{array}$ & $\begin{array}{l}\text { Intramuscular } \\
\text { and intra- } \\
\text { venous }\end{array}$ & $0.40 \mathrm{grm}$ \\
\hline $\begin{array}{l}\text { Neo-salvarsan } \\
\text { Neo-kharsivan (Eng.) } \\
\text { Neo-diarsenol (Can.) } \\
\text { Neo-arsphenamine (U.S.A.) } \\
\text { Nov - arseno - benzene, Billon } \\
\quad \text { (Fr.) }\end{array}$ & $\underbrace{\mathrm{OH}}_{\mathrm{OH}} \mathrm{SO}_{2} \mathrm{Na}$ & $\begin{array}{l}\text { Intramuscular } \\
\text { and intra- } \\
\text { venous }\end{array}$ & $0.50 \mathrm{grm}$ \\
\hline Galyl & $\overbrace{1}^{\text {As }}$ & $\begin{array}{l}\text { Intramuscular } \\
\text { and intra- } \\
\text { venous }\end{array}$ & $0.35 \mathrm{grm}$ \\
\hline Luargol & $\begin{array}{l}\left(\mathrm{C}_{12} \mathrm{H}_{12} \mathrm{O}_{2} \mathrm{~N}_{2} \mathrm{As}_{2}\right)_{2} \\
\mathrm{AgBr}, \mathrm{SbO}\left(\mathrm{H}_{2} \mathrm{SO}_{4}\right)_{2} \\
\mathrm{As} 20 \cdot 60 \text { per cent. } \\
\mathrm{Ag} \quad 7 \cdot 40 \text { per cent. } \\
\mathrm{Br} \quad 5 \cdot 52 \text { per cent. } \\
\mathrm{Sb} \quad 8 \cdot 19 \text { per cent. }\end{array}$ & Intravenous & 0.2 grm. \\
\hline \multicolumn{4}{|c|}{ MERCURY. } \\
\hline $\begin{array}{l}\text { Colloidal mercury } \\
\text { Grey oil (Adam's cream; }\end{array}$ & 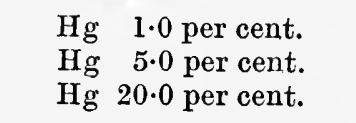 & $\begin{array}{l}\text { Intravenous } \\
\text { Intramuscular } \\
\text { Intramuscular }\end{array}$ & $\begin{array}{l}7 \cdot 0 \text { c.cm. } \\
1.0 \text { c.cm. } \\
0.5 \text { c.cm. }\end{array}$ \\
\hline \multicolumn{4}{|c|}{ SULPHUR. } \\
\hline Intramine & S & \begin{tabular}{|} 
Intravenous, \\
0.1 per cent. \\
Intramuscular, \\
1.0 per cent.
\end{tabular} & $\begin{array}{l}50.0 \mathrm{c.cm} \\
3.0 \mathrm{c.cm}\end{array}$ \\
\hline Colloidal iodine & $\begin{array}{l}\text { Iodine. } \\
1 \text { in } 500\end{array}$ & Intravenous & $100.0 \mathrm{c.cm}$. \\
\hline
\end{tabular}


It will be seen from the formula that sulphur not only forms the peroxide or medium necessary for the metal, but hydrogen as well, which converts the metal into that state in which it is able to act.

The action of iodine, which converts mercaptan-protein back again into di-sulphide-protein, the catalytic form of sulphur, can be shown diagrammatically in the following formula, where it will be seen to have in the reducing reaction an action analogous to hydrogen in the oxidising reaction:

$$
\underset{\text { (Mercaptan-protein) }}{2 \mathrm{R} . \mathrm{SH}}+\underset{\text { (Iodine) }}{2 \mathrm{I}}=\underset{\text { (Di-sulphide-protein) }}{\text { R.S.S.R. }}+\underset{\text { (Hydriodic acid) }}{2 \mathrm{HI}}
$$

1. Harrison (1918): Ven. Dis. in General Practice. Oxford Med. Publication, London.

2. SincLaIr (1917): Medical Times, xlv., 89.

3. HoMer SwIFT AND EluIs (1914): Studies from Rockefeller Inst. for Med. Res., xix., $471,492,573$.

4. Mouneyrat (1915): Eng. P., 3087, 9234.

5. DANYSZ (1913): Compt. rend. Acad. des Sciences., clvii., 644.

6. Danysz (1914): Compt. rend Acad. des Sciences., clviii., 199.

7. Kolue (1918): Deutsche med. Woch., xliv., 1177, 1211.

8. GENNerich (1918): Deutsche med. Woch., xliv., 1243.

9. Seller (1918): Deutsche med. Woch., xliv., 1247.

10. MCDonagh (1916): Links in a Chain of Research on Syphilis. Harrison and Sons, Pall Mall, London.

11. McDonagi (1916): Practitioner, xevii., 501.

12. McDonagh (1917): Practitioner, xcix., 17.

13. McDonagh (1918): Practitioner, c. 41, 416.

14. McDonagh (1919): Practitioner, cii., 129.

15. McDonagh (1918): Brit. Journ. of Dermatology, xxx., 67.

16. McDonagh (1918): Archives Médicales Belges, lxxi., 263.

17. McDonagh (1918): La Presśe Médicale, xxvi., 612.

18. Brrnes (1914): Journ. Amer. Med. Assoc., lxiii., 2182.

19. Fischer (1909): Prag. mediz. Woch., xxxiv., 401.

20. Fischer (1911): Zeitsch. f. d. Ges. Neur. u. Psych., iv., (orig.), 482.

21. DoNatr (1909): Wien. klin. Woch., xxii., 1291.

22. Donatru (1910): Berl. klin. Woch., xlvii., 1057.

23. A YER (1916): Boston Med. and Surg. Journ., clxxiv., 614.

24. WEed (1914): Journ. Med. Research, xxvi., 108.

25. CAMPBeLL (1914): Lancet, i., 1529.

26. Campbeli and Ballance (1919): Lancet, i., 608.

27. RIGGS (1915): Journ. Amer. Med. Assoc., IXv., 840.

28. Hammónd aNd Sirarpe (1915): Journ. Amer. Med. Assoc., lxv., 2147.

29. Hammond, Sharpe and Smith (1917): Journ. Amer. Med. Assoc., lxix., 23.

30. Proescher, SeIl and Strulians (1917): Amer. Journ. of Syphilis, i., 347. 


\section{CHAPTER XVII}

\section{TOXIC SYMPTOMS OF ARSENIC AND MEROURY}

\section{General Considerations.}

THE arseno-benzene used when the drug first came upon the market was less potent and less toxic than the samples used for experimental purposes. Before the profession was able to obtain salvarsan, there were two varieties, "Ideal" and "Hyperideal." Both of these were less soluble than salvarsan, and, although they were more potent and more toxic, it was partly on account of the insolubility that salvarsan superseded the "Ideal " and "Hyperideal."

As we all know, salvarsan had not been in use long before neo-salvarsan saw daylight. The reason which prompted the discovery of neo-salvarsan was the desire to obtain an easily soluble and neutral salt, since some of the unpleasant symptoms following the use of salvarsan were undoubtedly to be attributed to an excess of sodium hydrate, which it was necessary to add to neutralise the solution, as salvarsan had an acid reaction.

From 1912 till the outbreak of war may be regarded as the quiescent period so far as toxic symptoms following arsenic were concerned. From 1914 onwards there was a rise in the incidence of toxic symptoms, owing to the substitution products for salvarsan being more toxic than the parent compound. When the rule was made to prescribe mercury synchronously with arseno-benzene, the rise was particularly marked. When two toxic metals are used simultaneously, each increases the toxic action of the other. Although toxic symptoms are more apt to occur with one preparation than another, I know of no compound of arseno-benzene with an absolutely clean sheet. In my experience, neo-kharsivan was the most toxic substitution product; then came kharsivan, nov-arseno-benzene (Billon), and, finally, galyl. Unfortunately I can give no first-hand information concerning diarsenol or arsphenamine. Luargol has always served me well; but I have not given a sufficient number of injections to warrant me in saying that it is the least toxic of the arseno-benzene compounds.

There is no doubt that some patients are more likely to develop toxic symptoms than others, but since we cannot pick them out beforehand, personal idiosyncrasy plays little or no part in the problem. We have to distinguish between true toxic symptoms and what may be described as pseudo-toxic symptoms-that is to say, trouble arising either from insufficient use of the remedy or dependent upon certain lesions being present. The water used 
for dissolving the arseno-benzene at one time played a rôle in the ætiology of toxic symptoms. Wechselmann ${ }^{8}$ was the first to point out this, and showed that it was due to the presence of dead bodies of bacteria and fungi or their toxines, which quickly contaminate distilled water that has been allowed to stand. In this connection it is interesting to note that the toxicity of arsenobenzene is increased by the presence of organisms other than those which it is destined to destroy. For instance, if a patient with influenza be injected, the reaction may be violent; or if a patient with septic tonsillitis be injected, he may run a temperature for some days, and the tonsillitis may become very much aggravated. Bronchitis has likewise occurred after injection, due to a lighting up of some dormant pathogenic organism. It is possible that some cases of encephalitis may be caused in the same way, and that their onset may be stimulated by the endotoxines in the distilled water. Patients liable to acne or boils are particularly apt to get a crop while under treatment with arseno-benzene. I have also seen pleurisy arise in many cases.

Most important work on the action of bacterial endotoxines in increasing the toxicity of salvarsan has been undertaken by Yakimoff., 6

The first endotoxine experimented with was that obtained by a twentyfour hours' broth-culture of Bacillus coli communis. The tolerant dose of both this and salvarsan being ascertained, such doses were given intravenously to animals, mixed and separately, at varying intervals, with the result that a dose of salvarsan which was ordinarily non-toxic became, on the addition of a harmless dose of endotoxine, lethal. Toxicity was, in fact, increased two and a half times. If the animals were infected with protozoa, the toxicity was still further increased. In mice, for instance, slightly infected with trypanosomes, the result of injecting endotoxine with salvarsan was to increase the toxicity of the latter eight times, and, if markedly infected, sixteen times. The same results were produced by giving the endotoxine intravenously and the salvarsan subcutaneously. The endotoxine of Bacillus pyocyaneus increased the toxicity of salvarsan three and a half times, and still higher if the animals had trypanosomiasis. Other bacteria, such as Staphylococcus aureus, Bacillus subtilis, etc., were also found to increase toxicity in varying degrees; while, on the contrary, Bacillus tetragenus was inert.

These experiments suffice to show that Wechselmann's statement, that the reaction following injection is due to endotoxines in the distilled water, stands correct. They also corroborate the view that the reaction depends somewhat upon the number of protozoa present.

The water problem has lost much of its significance of late, because administering arseno-benzene in the concentrated form has become prevalent. The rôle that intramine has played, furthermore, in reducing the incidence of toxic symptoms has been so great that special reference will be made to it later. 


\section{Immediate Effects following Intravenous Injections of Arseno-Benzene.}

Some patients vomit after each injection. Alterations in blood-pressure now seldom occur with concentrated solutions. When $250.0 \mathrm{c} . \mathrm{cm}$. and more were injected, an immediate rise, to be followed by a fall in blood-pressure amounting to total collapse, not infrequently occurred. The occasions on which a pronounced fall in blood-pressure occurs nowadays happen when the preparation is incompletely protected, and causes what is known as shock. Owing to the importance of shock, the subject will be dealt with under a separate heading. As a rule, after the first and second injections, less pronounced after the latter than the former, setting in about four hours later, the patient, especially if he is in the generalisation stage, experiences a feeling of malaise, much as if he was in for an attack of influenza. He complains of headache, muscular rheumatism, especially in the small of the back; he may have a rise of temperature, perspire profusely, and experience a rigor, to feel perfectly fit the next day. The muscular rheumatism, with the addition of pains in the bones and joints, may persist and become aggravated by the subsequent injections, only to disappear when intramine is prescribed. Patients who complain of the above suffer from insomnia as well-a chain of symptoms met with so commonly in old syphilitics, whose protein colloidal particles are particularly large and deficient in oxidising powers.

This chain of symptoms, persisting after several injections of arsenobenzene have been administered, should be a warning that the oxidising action of the drug has reached its maximum. If the warning is disregarded, neurasthenia, and even organic nervous trouble, may supervene. I have also seen periostitis occur, and polyarticular arthritis result from the introduction of too much arseno-benzene and too little intramine. Thrombosis resulting from intravenous injections is due to the solution being too acid or too alkaline, or to an injury caused by the needle. Colloidal mercury and luargol are especially apt to cause thrombosis on the lining of the vein. This can be obviated by using my double-barrelled syringe, and either allowing blood to come back and mix with the drug before it is injected, or by injecting some normal saline afterwards to wash the drug away. Thrombosis may extend from the elbow right up to the axilla, and although I have seen it occur many times, I have never known it once to cause any harm. The vein may either become patent again, or become a solid fibrous band. A clinical observation of some value is that a patient who experiences the greatest reaction after an injection is one who gets rid of his symptoms quickest.

\section{Shock.}

There is probably no condition which has more diverse names than this, simply because the rationale is not understood, and observers have thought they were dealing with different phenomena, when all the time it was the same. Hence we read of anaphylaxis, allergy, hypersensitiveness, and nitritoid 
crisis. $^{9} \quad$ Although the following case is not absolutely germare to the subject in hand, it is particularly illustrative of the above, and describes the pulmonary form of shock which is apt to follow an injection of arseno-benzene, when some of the metal happens to be in an unprotected colloidal form, or when the protein particles in the serum are particularly liable to have their colloidal character changed.

CASE 104.-The patient, a medical man, aged 34, had his first bad attack of hay fever in 1904. From then onwards he had frequent attacks. After some years he ultimately discovered it was due to horses. If he approaches a horse, rides one, or even drives in a carriage, he always develops a violent attack of hay fever. He has found that nothing else, except contact with horses, will bring on an attack. This always begins in the same way; the eyes become bloodshot, watery, and itch; then a cough sets in; mucus pours from his mouth and nose, and sometimes asthma occurs. In March, 1916, the patient had a sore throat, for which he had one injection of diphtheria antitoxine $(6,000$ units). In a minute or two the eyes became red and itched, his tongue and lips swelled, and he quickly found that he could not breathe. A few minutes later he went black in the face from asphyxia. In other words, the patient had a typical attack of the pulmonary form of shock, owing to the horse serum content of the antitoxine. This attack of pulmonary shock was only an attack of hay fever accentuated. After a time the patient recovered, although those around him thought he was dead. In April, 1916, I gave the patient an intravenous injection of intramine which had not been sufficiently protected. After $5.0 \mathrm{c.cm}$. had been injected the patient suddenly developed the peculiar condition of the eyes above described; a violent fit of coughing ensued, and the patient said he felt exactly as he did after the antitoxine injection. Although the symptoms were mild, they were typical of the pulmonary form of shock.

As the proteins used for protecting intramine do not come from the horse, and the shock produced by an insufficiently protected colloid is the same as that produced by antitoxine, it is clear that shock and anaphylaxis are synonymous. In my experience, shock occurs more often in the early than in the late stages of syphilis, more frequently after the fourth and the subsequent injections than after the first three, if the interval between the injections is more than ten days, with the English and French substitution products for salvarsan, and if the patient has had a meal just before an injection. Overpreparation of a patient before an injection may predispose to shock, just as over-preparation before an operation may lessen the resistance to shock due to the anæsthetic.

A few minutes after the injection the patient suddenly becomes faint, then vomits, and becomes unconscious; the heart stops beating, and the patient may die in spite of all efforts to save him. On the other hand, he may become deeply cyanosed, the lips and tongue may swell up, and the patient appears to be at death's door. A true picture of asphyxia is produced, due to a tetanic contraction of the smooth muscle fibres of the small bronchi; when the tetanic contraction ceases, the patient regains consciousness. In the first case the attack is primarily cardiac, and in the second case primarily 


\section{CLINICAL ASPECT AND TREATMENT OF VENEREAL DISEASES}

pulmonary. In the first case, as soon as the patient regains consciousness, he begins to complain of excruciating pains in the legs and feet, but gets perfectly well in a short time. After some days, a toxic erythema may or may not supervene. Some cases are followed by typical arsenical dermatitis. Why in one case the attack should be pulmonary and in the other cardiac depends, I suppose, upon the varied concentration of the salts of the serum in different parts of the body.

The cardiac attack is usually accompanied by a very decided fall in the temperature, and, besides the vomiting, the patient may pass his fæces and urine. When the motions are passed in this way they are generally fluid, showing that an irritation of the intestines has been produced by the fall in the blood-pressure.

If death occurs during a pulmonary attack, the lungs post-mortem exhibit the picture of emphysema, with, sometimes, considerable œdema and hæmorrhages, or only the results of venous congestion. The post-mortem appearances of the organs in the cardiac form are very important, because the cardiac attack is more frequently met with than the pulmonary, and there always appears to be such a mystery about these cases when they come before a coroner's court. There may be ecchymoses in the abdominal organs, the gallbladder is often decidedly distended, and there is almost invariably some degeneration of the renal cells.

The renal changes are those which lead to a wrong diagnosis. The pathologist's report usually states that the patient was an unhealthy individual, and that if his kidneys had been normal presumably he would not have died. Shock can occur in any individual; indeed, the healthier his condition, I will not say that the greater are his chances of being affected, but they are greater than those of chronic alcoholics, whose kidneys may show some sign of disease before the injection is given. For all practical purposes, one may say that in most cases the kidneys are perfectly normal, and that the degeneration noticed post-mortem is entirely the result of shock. To prove this, it is only necessary to examine the kidneys of rabbits which have been poisoned with a toxic drug like neo-kharsivan. The degeneration can be so rapid that calcification of the vessels sets in before the animal dies.

The following case is one typical of the cardiac form of shock:

CASE 105.-The patient complains of pain at the site of injection, the vein for about an inch of its course appears swollen, and becomes distinctly blue in colour. The patient then gets red in the face, and the eyes look as if they were bloodshot. A buzzing sound in the ears and a whirling sensation in the head are the next symptoms to ensue. The patient then experiences difficulty in breathing, and finds he cannot talk; at this stage he thinks he is going to choke and die. Pain over the heart, especially in the pit of the stomach, sets in, and the patient becomes bathed in perspiration. As the other symptoms are disappearing, and the patient's voice returns, pallor follows the redness, and the pain complained of at the lower end of the sternum increases in intensity. The symptoms then pass off one by one, and about half an hour later the patient feels all right. He may vomit and pass urine and fæces. 
The pulse-rate at first becomes very rapid, then feeble, until it can scarcely be felt. As the symptoms disappear, the pulse gradually becomes normal. In the pulmonary form of shock the pulse-rate may not be affected, and in animals shock may produce death long before the heart stops beating.

With the arsenical colloidal preparations now in use shock is not common, because the drugs are injected in solution, and if they are properly prepared the metal is sufficiently protected by the amino-groups. As there is only one perfect amino-group in neo-salvarsan, and the drug is readily soluble and neutral in reaction, it is less likely to be followed by early shock, but more likely to be followed by late shock. Should shock follow an intravenous injection of an arsenical compound, it is, unfortunately, liable to be fatal, and I have known of eight such cases since the beginning of the war. In these fatal cases it is probable that some of the arsenic was in the suspensoid. state, and not firmly attached to the benzene ring. Under such conditions, the arsenic would immediately act as an ion on reaching the blood-stream, and increase the hydrogen-ion concentration; therefore, the action would be instantaneous, and it usually is so. Unfortunately, it is impossible to gauge beforehand whether a certain sample is liable to cause shock or not.

The treatment of the case will naturally depend upon whether the patient has a pulmonary or a cardiac attack.

In a pulmonary case, atropine must be injected; giving oxygen to inhale or the usual methods for producing artificial respiration may be tried, but, as a rule, they are useless. In a cardiac case, the patient should immediately have his head and thorax lowered; injections of ether, camphor, and strychnine should be given, oxygen should be inhaled, and artificial respiration instituted. Heat to the heart region should be applied, and brandy should be taken by the patient if possible.

In both the cardiac and the pulmonary cases, pituitary extract or adrenalin should be used. The former is better than the latter, but neither of these drugs will be of great service unless they are injected before the administration of the drug which caused the shock. Injecting pituitrin as a routine procedure is impracticable. The action of pituitrin and adrcnalin is to cause vaso-constriction of the capillaries, and thus they prevent the vaso-dilatation which is an accompaniment of shock, although not the main feature. Their action is, therefore, only an indirect one. One of the most powerful drugs capable of raising the blood-pressure, even when adrenalin has failed to do so, is barium chloride. Barium chloride causes a contraction of unstriped muscular tissue, and was used in experimental work on shock first by Boehm. ${ }^{10}$ Unfortunately, this drug has always been regarded as extremely toxic, and, so far as I know, has not been used in cases of shock in man. In animals it can be used with impunity; therefore, attempts should be madc to define its limitations in man. As the direct cause of shock is an upset of the normal hydrogen-ion concentration, which changes the colloidal character of the protein particles, and results in the patient getting insufficient oxygen, the obvious 


\section{4}

procedure to adopt is to increase the stability of the permeability of the protein particles, and thus prevent the oxidase-reducase system from becoming deranged. Calcium chloride will do this; therefore, in every case of shock, $10.0 \mathrm{c.cm}$. of a 2.5 per cent. solution of calcium chloride should be injected intravenously, and the patient's life will be saved if the injection be administered quickly enough. It is a good plan to make the solution isotonic with glucose, and to add gelatin; consequently, I now use $10.0 \mathrm{c.cm}$. ampoules, containing calcium chloride, $5 \cdot 0$ per cent.; glucose, $15 \cdot 0$ per cent.; and gelatin, 3.0 per cent. As this mixture forms a jelly, it must be heated and diluted up to $50.0 \mathrm{c.cm}$. or $100.0 \mathrm{c.cm}$. before it is injected. Ampoules of $10.0 \mathrm{c.cm}$. are handy, and the jelly is easily melted. If the occurrence of shock is delayed the course it runs is not so rapid, and premonitory symptoms usher it in. In these cases, iodine and intramine, provided they are used sufficiently early, the patient's life will invariably be saved. In such cases I inject either $100.0 \mathrm{c.cm}$. of intramine intravenously, or $100.0 \mathrm{c} . \mathrm{cm}$. of colloidal iodine intravenously, and $5.0 \mathrm{c.cm}$. of intramine intramuscularly. It is simply astonishing to see the patient's condition instantaneously improve the moment these drugs reach the circulation. If there is a toxic erythema it will vanish as the injections are being given. Having done some experimental work $\mathrm{k}^{11,12}$ on the rationale of shock caused by drugs, I may be excused for dwelling a little longer on the subject, to explain my reasons for using iodine and sulphur. I found by experimenting on rabbits that a shock-producing drug had to be a colloid, that the phenomenon was the same whether the colloid was negatively or positively charged, but that it did not occur if the negative-charged colloid was injected into one eal while the positive-charged colloid was injected into the other ear. This proved shock to be produced by an upset of the hydrogen-ion concentration of the serum. As the so-called complement in the serum diminished or disappeared in shock, it suggested that complement was nothing more nor less than the standard alkalinity of the serum, which further experiments proved it to be. Therefore, that mystic substance is not a substance at all, but a state. As metals have a positive electric charge and non-metals a negative, it becomes at once clear why I should have chosen iodine and sulphur to counteract metallic poisoning. Finding, further, that calcium chloride injected intravenously just before the shock-producing substance was used, or the day before if injected intramuscularly, prevented the occurrence of the phenomenon, owing to the influence the drug has in increasing the stability of the permeability of the protein colloidal particles, it is clear why calcium chloride should have been advised in the acute cases. As the upset of the hydrogen-ion concentration needs to be only minimal to alter the physical state of the colloidal particles in the serum, the reader will see why the change may be undetectable owing to want of fine enough instruments to measure it, and why the so-called theory of acidosis as the cause of shock has been wrongly given up. ${ }^{13}$ There is also no need to suggest as a cause of shock the formation of broken-down products of protein metabolism, ${ }^{14}$ 
because, in cases of drug shock, the phenomenon can occur and kill a patient long before any such products could be formed. Summed up, shock is nothing more nor less than an altered physical state of the protein colloidal particles in the serum produced by a suspensoid or unprotected colloid, and which gives rise to precipitation, vaso-dilatation, etc., symptoms which may be overcome by stabilising the permeability of the particles, or by injecting a drug possessing an opposite electrical charge to the one producing the shock.

\section{Pseudo-Toxic Symptoms which usually appear on the Third Day.}

These are symptoms which are not directly due to arsenic, but only indirectly, owing to the nature of the syphilitic lesion present. A better term is "reactionary inflammation," although it is often known as the Herxheimer-Jarisch reaction. The symptoms almost invariably set in, or, rather, are capable of being diagnosed for the first time, on the third day following the second intravenous injection. If the case is one of late syphilis, the symptoms may not set in until after the third injection. In every case the patient has or has had generalised syphilis; by this I mean that what is to be described cannot occur in a patient who is in the primary stage. Special emphasis is laid upon this point, because many of the cases of acute yellow atrophy of the liver-which is directly due to arsenic, and may follow treatment in the primary stage-are described wrongly as being cases of reactionary inflammation. The symptoms fall into two main classes: (1) intracranial; (2) visceral. The cutaneous reactionary inflammation, which amounts to nothing more than to an exacerbation of the generalised rash, which occasionally takes on an almost urticarial character, or, on the other hand, may become more diffuse to resemble scarlet fever or measles, is too obvious to require further mention. Furthermore, the cutaneous reactionary inflammation may occur with the first injection.

Intracranial Reactionary Inflammation.-The lesion in the generalisation stage is a hæmorrhagic encephalitis, which, unless specially treated, invariably ends fatally. The patient first of all complains of headache; then has twitching of certain muscles, enters into a condition of Status epilepticus, and dics. The following is an illustrative case:

CASE 106.-On the third day after the second injection of salvarsan the patient, after having previously complained of bad headaches, developed Jacksonian epilepsy, which rapidly merged into Status epilepticus, in which condition the patient died.

The patient was in the generalisation stage. He had a severe papular rash, and an iritis of the right eye. The first injection of salvarsan $(0.6 \mathrm{grm}$.) had been given a week previously, without any symptoms following. The second injection was also $0.6 \mathrm{grm}$.

Post-mortem, the patient had a sub-arachnoid cyst over the Rolandic area on the side opposite to that in which the Jacksonian epilepsy started. This was of old duration, and was probably produced by an accident some years before; nevertheless, it would appear to have been a locus minoris resistentice. A microscopic examination showed a degeneration of the nerve cells around and underneath the cyst, but nothing else. In this case, as in ali, the vascular dilatation had vanished post-mortem. 
The condition is due to the battle which goes on between the protein colloidal particles and the active phases of the organism. The remedy intensifies the engagement, in which the host assists by a dilatation of the capillaries in the area involved, in order that more protein particles may be brought upon the field. The dilatation results in raising the intracranial pressure, with the sequence that certain nerve cells get pressed upon, and the patient becomes comatosed and dies. Little or no good results from trephining or lumbar puncture. The only thing to do is to inject adrenalin repeatedly the moment anything appears to be wrong. The best way to obviate the trouble is to allow as short an interval as possible to elapse between the first three or four injections of arseno-benzene, and always to use maximum doses. Although many might fear giving another injection the moment the condition was diagnosed, it is, nevertheless, a wise procedure to adopt. I have seen five such cases, three of which ended fatally before anything could be done; in one of the other cases, life was saved by adrenalin, and in another by the simultaneous use of adrenalin and arseno-benzene.

In later cases of syphilis, reactionary inflammation is most apt to occur in cases of widespread pachymeningitis, especially when some nerve tissue is involved as well. I have had several instances of this class of case, and in almost all the patient became suddenly unconscious on the third day after the third injection. In these cases, lumbar puncture is very useful. Adrenalin should also be used; but the main thing to do is to push the treatment, as one has time, because death is not so sudden as in cases of hæmorrhagic encephalitis. Colloidal iodine and intramine are also very useful, not only in combating the condition when present, but also in preventing it, provided they precede the injections of arseno-benzene.

The following case will show the importance of continuing the treatment:

CASE 107.-Patient, a man, aged 29, had no history of a sore, but had had a bad throat in 1911, which was diagnosed as syphilis. The patient was treated with mercury internally until January, 1914, when he developed a rash (? nature). At this time he was also said to have had a left hemiplegia, which came on quite suddenly, but the attack had not been preceded by headaches. As far as I could gather from the patient, when I saw him in September, 1914, he completely recovered from the hemiplegia in a few days, and had had no treatment since. On examination, I found that the pupils were unequal, right $>$ left. The reflexes of the right pupil were not as brisk as those of the left pupil, and in both eyes the reflexes were diminished. All the other reflexes were very brisk indeed, but not more so on the right side than on the left. There was bilateral ankle-clonus, and an extensor response on both sides. Sensations were unaltered. Patient answered questions correctly and quickly, and beyond feeling, as he called it, "nervous," he had no symptoms to complain of.

Examination of the Blood.-C.F.T. positive.

Examination of the C.s.F.-Pressure not raised; fluid clear; strong positive lymphocytosis; strong positive Nonne-Apelt reaction; excess of albumin and globulin; C.F.T. 100.0 per cent. positive.

Patient was admitted into the Lock Hospital, and received two intravenous injections of " 606 ." On the third day after the second injection the patient went off 
his head-he talked the most utter nonsense, took his discharge, and found his way home. Two days later the patient was brought to see me. He stood staring, took several minutes to answer a question, and then could only answer it correctly if it were a simple one, such as asking him his name, age, etc. His reflexes had become brisker than ever. I then gave him another intravenous injection of salvarsan, and an intrathecal injection of salvarsanised serum the next day, with the result that in three days' time the patient was normal again. Further intrathecal treatment was given.

Although death may rarely result in cases of degenerative encephalitis after the first and after the second injection of salvarsan, it is more usual for death to occur much later, and for the patient to die with symptoms of aggravation of the trouble. Arseno-benzene cannot be said to cause death directly in cases of degenerative encephalitis, but it is liable, in the majority of cases, to make the patient worse, so that he dies sooner than he would have done if he had been left alone.

Although I have never had a sudden death in a case of degenerative encephalitis myself, I have had six cases in which the patients were made very much worse, and died sooner than they otherwise would have done. I know of four other cases, in which I was personally interested, which came to an untimely end. In two of these the patients committed suicide-one on the third day after the first injection, and the other one week after the second. Oddly enough, both of these cases were in the quiescent period when arsenobenzene was prescribed. In one case the man had been "perfectly well" for two years, and in the other case for nine months only. Both had had only one attack before.

Visceral Reactionary Inflammation.-The lesion may be in the liver or in the kidneys, and occur in either early or late syphilis. In early syphilis, it occurs only in those cases in which the organ is pervaded throughout with organisms, the causation, pathology, and treatment being identical with that described in hæmorrhagic encephalitis. If the liver is affected, severe jaundice occurs, which in my experience does not go on to acute yellow atrophy. If the patient in the generalisation stage develops jaundice on the third day following the second injection of arseno-benzene, adrenalin should be prescribed at once, and two maximum intravenous injections of arseno-benzene given at not more than five days' interval between the two. It is useless to inject intramine, this drug being called for in cases of toxic jaundice only; when jaundice occurs as the result of reactionary inflammation in a case of gummata of the liver, the onset is not so regular as just described, and intramine may with good effect be given, although it is wiser to continue with arseno-benzene, provided the correct diagnosis has been made. As gummata of the liver may be elinically undiagnosed, it is sometimes impossible to differentiate icterus due to arsenic from icterus due to reactionary inflammation.

What has just been said concerning the liver applies equally to the kidney, and the reader cannot be too strongly urged to recognise pronounced pro- 


\section{CLINICAL ASPECT AND TREATMENT OF VENEREAL DISEASES}

teinuria occurring on the third day following the second injection as a symptom of reactionary inflammation, and a symptom which calls for immediate continuation of the treatment. If this advice is not followed, the patient runs the risk of having his kidneys damaged.

It is so necessary that reactionary inflammation should be distinguished from toxic symptoms, as the treatment of each of the two is diametrically opposite; and if the right treatment be given, such excellent results may be obtained.

In cases of reactionary inflammation, continue arseno-benzene. In cases of arsenical intoxication, prescribe colloidal iodine and intramine.

\section{True Toxic Manifestations of Arsenic.}

These will be considered from a clinical point of view, although I should point out that the pathology is not the same in all cases. In some cases, the vessels alone are attacked; in others, the arsenic causes a parenchymatous degeneration which may extend to complete necrosis, while in others the interstitial tissue appears to be mainly involved, leading to fibrosis. Before describing the various symptoms met with, it will be well to point out that most of the symptoms occur when the patient has finished treatment, and has forgotten all about it. This results in his falling into other hands, and in his running the risk of having his condition wrongly diagnosed. The toxic manifestations are more apt to occur between the sixth and twelfth week after the last injection of arseno-benzene; they may set in after the first injection, or as long as eighteen months after the last; they are more liable to result from a second course of arseno-benzene than from the first, and from the first when too long an interval is allowed to elapse between the injections. The simultaneous administration of mercury with arseno-benzene increases their incidence.

I have seen a generalised arsenical dermatitis follow one suppository of arseno-benzene, and occur eighteen months after one intramuscular injection.

An odd feature of arsenical intoxication is that an implication of one organ usually means freedom for the rest. A fatal case of severe dermatitis seldom shows marked pathological changes in the liver or kidneys, and death is usually due to a septic broncho-pneumonia. Fatal cases of acute yellow atrophy of the liver or of uræmia, due to necrotic changes in the liver and kidney respectively, are practically never accompanied by arsenical dermatitis, although they may be preceded by a toxic erythema, of which the most common type is Erythema multiforme.

The following is an exceedingly instructive case:

CASE 108.-A patient had had nine injections of galyl, and was receiving weekly intramuscular injections of grey oil, when he came to see me complaining of a sore mouth and a rash. Three months had elapsed since the last injection of galyl, and the patient had had in all fourteen intramuscular injections of mercury. The rash, which affected the hands, elbows, knees, and ankles, was a severe form of Erythema multi- 



\section{PLATE LI}

No. 98.-The Tongue From the Same Case, Showing TIIE Combination of Metalide Stomatitis and Recurrent Sypinilitic Mucous Papules

Wires arsenic and mercury produce toxic symptoms they frequently precipitate a recurrence of the disease.

\section{No. 97.-A Bullous Erythema Multiforme winci AT-FIRST w.s of the Inis Type caused by Arsficic}

Tne patient had had nine injeetions of arseno-benzene and eleven injections of mercury. The patient presented himself with the rash two and a half months later. The rash affected the hands, forearms, both knees, ankles, and feet. The urine went solid on boiling, from containing so much protein, and the patient was beginning to present signs of acute yellow atrophy of the liver. Under three intramuscular injections of intramine the patient completely recovered, and the urine contained no albumin. This is not a true arsenical dermatitis, but an exudative erythema which, when it occurs some time after the last injection, is a sign of metallic nephritis, hepatitis, or both.

No, 99.-Two NaIls Frou ANother but TyPICAL Case of Arsenical DerIATITIS AFTER TIE RASI HAD DISAPPEARED 


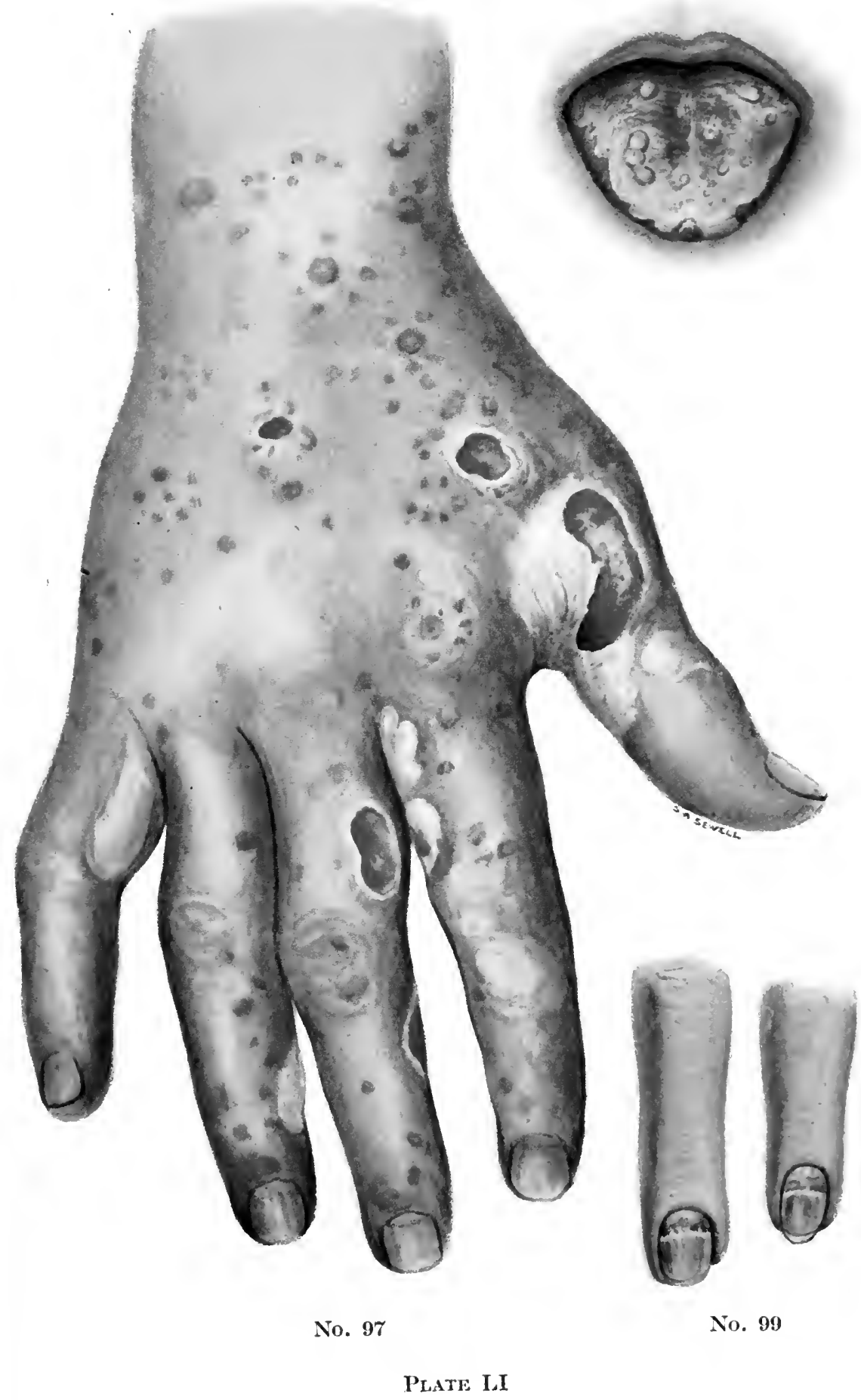


forme. Besides the metallic stomatitis, mucous papules were evident on the tongue. The urine contained a large quantity of protein, and jaundice was just appearing. The patient had a sort of ashen grey look behind it all, and was desperately ill. After three intramuscular injections of intramine, given in a space of ten days, every lesion mentioned above completely disappeared, and the patient was, and felt, extremely well. I am confident that this case would have ended fatally but for the intramine, as the worst cases of metallic poisoning are, in my experience, those which begin with a toxic erythema, and present the complexion of a mild asphyxia.

Arsenical Dermatitis.- The true arsenical dermatitis usually occurs de novo, and resembles from the start either a Dermatitis scarlatiniforme or a cheiropompholyx. When fully developed, the rash may resemble either a Dermatitis exfoliativa, a seborrhoic eczema, or a Pityriasis rubra. On the other hand, the rash may begin and remain patchy, when the lesions resemble chronic patches of seborrhœic eczema, or even the pre-mycotic condition of Mycosis fungoides. Arsenic may produce no more than a hyperkeratosis of the palms or soles, with atrophic lesions of the nails. In such cases, toxic alopecia of the areata type is liable to occur. Although not generally known, arsenic is not an uncommon cause of gingivitis, and recently I have seen quite a number of cases of Vincent's angina accompany it. Apart from Herpes zoster, which is due to inflammation of the posterior root ganglia, and which may be caused by arsenic, although more frequently due to reactionary inflammation in the meninges covering the ganglia, arsenic is not an uncommon cause of Herpes febrilis, the penis and the mouth being the parts most frequently affected. Herpes febrilis is most probably due to metallic irritation of the peripheral ends of certain sensory nerves. A patch occurring on the site of a chancre is not uncommon. Severe arsenical dermatitis is a most distressing condition, as, apart from the rash itself, multiple abscesses so frequently form, and conjunctivitis, with actual implication of the cornea, is by no means unknown. If intramine were only universally employed in conjunction with arsenobenzene, not only would cases of arsenical dermatitis be never seen, but all the other toxic manifestations would become rarities, as a five years' experience with the drug has shown me. Next to prevention is cure, and, fortunately, in intramine we possess a drug which will certainly cure most cases of arsenical intoxication, if prescribed early enough, as the following cases will show:

CASE 109.-Patient developed a universal dry, itching, and scaly dermatitis one week after the fifth intravenous injection of nov-arseno-benzene (Billon). I saw him three days later, when he presented the following features: A universal dermatitis, which was beginning to get crusted and to weep, especially about the ears and on the face, which was œdematous. Many of the hair follicles on different parts of the body were developing pustules. I immediately injected $100.0 \mathrm{c} . \mathrm{cm}$. of colloidal iodine intravenously, $2.5 \mathrm{c} . \mathrm{cm}$. of intramine into each buttock, and the next day $100.0 \mathrm{c.cm}$. of intramine intravenously. A week later the patient was walking about and the skin was perfectly healthy. If left alone, this patient would probably have died, as they generally do when sepsis sets in so early as the third day. Even if he had 
recovered with his life, he would have most probably been ill for months, and then left with complete alopecia, arsenical pigmentation, or both.

CASE 110.-A woman, suffering from cerebro-spinal syphilis, had six injections of galyl, three being intraspinal, and then was given mercury internally for nine months. The last galyl injection produced an arsenical dermatitis, which, in spite of treatment, persisted for nine months, when the cerebro-spinal symptoms reappeared. Within three days after an intravenous injection of colloidal iodine and an intramuscular injection of intramine, the dermatitis had practically vanished.

CASE 111.-This patient had syphilis, for which he received seven injections of " 606 " and seven injections of mercury, in May. In August of the same year he was circumcised under chloroform anæsthesia. A few days later, an eruption appeared on the hands, like cheiropompholyx, and quickly spread all over the body, when it assumed the appearance of an acute seborrhœic eczema. The eruption became very severe, and typical of arsenical dermatitis. In September the patient had one intravenous injection of colloidal iodine $(100.0 \mathrm{c.cm}$.) and two intravenous injections of intramine (100.0 c.cm.), at two days' interval between each. Within a few hours after the first injection the patient said his skin felt better, and particularly remarked upon the improvement after the intramine. Under local treatment, by the end of the month the eruption had completely disappeared, and the patient was able to leave hospital with his skin perfectly clear, with no loss of hair, and no pigmentation.

This last case presents more than the usual interest, because the onset of the dermatitis was delayed, which is not infrequently the case; because it occurred after the patient had had chloroform, a drug which, like arsenic, when acting as a toxic agent, increases the hydrogen-ion concentration; and because the eruption began as a cheiropompholyx.

The following case of arsenical dermatitis is interesting, showing that the condition can recur without more arsenic having been given:

CASE 112.-Five weeks after the fourth injection of neo-salvarsan, the patient developed a universal arsenical dermatitis, which lasted for about a month. Two months later, apparently without rhyme or reason, the patient developed an acute exfoliative dermatitis, which took about nine weeks to disappear. One year later the dermatitis recurred, with acute peripheral neuritis, and kept the patient bedridden for two months. Although the trouble cleared up, the paticnt continued getting patches of dermatitis resembling dry scaly seborrhœic eczema, which never entirely disappeared until intramine was prescribed.

Acne vulgaris, furunculosis, and even carbuncles, may be started, or, if present, aggravated by arseno-benzene. It is interesting to mention in this connection that trimine exerts just as beneficial an action in these staphylococcic lesions as it would have done had no arseno-benzene been administered. In several cases I have given injections of arseno-benzene and trimine on the same day, with no ill effect. Although the case about to be reported is a little foreign to the subject in hand, its interest will doubtless compensate for its irregular appearance.

CASE 113.-A woman, aged 31, had been a martyr to a widespread skin eruption since the age of 5 . Although the rash had not always been as bad as when I first saw it, the patient had never been absolutely free from it. The patient was brought to 
me because she was pregnant, and because it was thought she had contracted syphilis, which, unfortunately, turned out to be the case. The patient was almost covered from head to foot with a seborrhœic dermatitis. The scalp was crusted, the flexures were weeping, and on the trunk the rash was in patches, dry and scaly. I felt obliged to prescribe arseno-benzene, as the patient was pregnant, although I felt certain the dermatitis would be aggravated, which it was to such an extent that the patient's existence became unbearable. I then prescribed three intramuscular injections of intramine, with the result that the rash completely disappeared for the first time for twenty-six years.

Arsenical Hepatitis.-Simple catarrhal jaundice may appear early or late after any injection, but when it sets in some weeks after a full course of intravenous injections, coupled with a series of intramuscular injections of mercury, caution must be observed, as such cases are apt, almost without warning, to develop into acute yellow atrophy, a condition which is almost invariably fatal. The clinical history and pathology of the cases of acute yellow atrophy due to arsenic are so typical that the following case will suffice for a description:

CASE 114. - A man, aged 23, was admitted with a primary sore, for which he received eight intravenous injections of arseno-benzene and nine intramuscular injections of mercury. As far as could be ascertained, the disease had not become generalised, which rules out syphilis as being the cause of the icterus, which set in seven weeks after the treatment had been completed: The patient was admitted into hospital having been jaundiced for five days. Two days later the icterus became intensified, the patient was restless, and had several attacks of hæmatemesis. Two days following the patient became comatosed, and died. The urine was deeply bile-stained, and revealed both tyrosin and leucin. Post-mortem, the liver was found to be very much diminished in size, and histologically there were found to be areas of necrosis which predominated, although in some parts the cells had not passed the stage of fatty degeneration. Fatty changes were to be particularly noticed in the kidneys. The other organs showed no more than hæmorrhagic infiltration.

Having had the opportunity of studying the livers from seven different cases, the following points may be of interest: The liver in all was smaller and weighed less than the normal; and those which were smallest were the most necrosed. In others the liver was patchy, being bright green in some parts and red in others, the difference being due to the degree of the parenchymatous degeneration which had taken place being less advanced in the former than in the latter. There appears to be at first a simple degeneration of the liver cells, which, advancing to fatty degeneration, ends in complete necrosis. Most of the cases showed some cirrhosis, which appears to be typical of metallic poisoning, as will be noted in the section on arsenical nephritis. There were signs of extravasation of blood all over the organ, as if the walls of the capillaries had degenerated. All cases are not so acute as the one described, and in those less acute, tyrosin and leucin may be missed in the urine, a point which requires attention, because many refrain from making a diagnosis of acute yellow atrophy unless these two bodies are present. The points to 
remember are, that icterus follows a course of metallic treatment for syphilis, and that, should the icterus at any time become suddenly aggravated, making the urine almost black, a strong suspicion of acute yellow atrophy supervening should be entertained. In some cases arsenic is demonstrable in the liver post-mortem, and in others not. Jaundice is not at all a common syphilitic symptom, and acute yellow atrophy is extremely rare; therefore, when within a space of twelve months fifteen cases of acute yellow atrophy are reported, 1, 2, 3 occurring in patients who have received a course of ars̉eno-benzene and mercury, it would appear reasonable to assume, with no other evidence forthcoming, that the condition was due to metallic poisoning. The histology of the affected liver is so similar to that met with in the kidneys of animals which have succumbed to arsenic that the evidence, to my mind, appears to be complete. Between 1910 and 1919 I saw at the Lock Hospital in cases of untreated syphilis only one case of mild jaundice, and not a single case of Icterus gravis. From an analysis of 15,799 cases of early syphilis in Engel-Reimer's clinic, Werner ${ }^{4}$ found that 57 , or 0.37 per cent., were jaundiced. Harrison, writing to Fenwick, ${ }^{2}$ states that jaundice occurs during treatment of syphilis with arsenical compounds in $\mathbf{0 . 6}$ per cent. of cases. From this it may be concluded that arsenic and mercury, especially when coupled together, are a potent cause of toxic hepatitis. Special emphasis is being laid on this subject, because since I have used intramine in conjunction with metals I have had only one case of jaundice, and because intramine will rapidly clear up a case of simple toxic jaundice and prevent acute yellow atrophy from supervening, if it is prescribed in time, but not if time is wasted by prescribing ichthyol first.

Arsenical Nephritis.-If a patient has protein in the urine before treatment is commenced, arseno-benzene will at first increase it, and then cause it to disappear. If protein in the urine occurs, for the first time, on the second or third day following the second injection of arseno-benzene, treatment should be pushed, as the proteinuria is a sign of reactionary inflammation. If protein appears for the first time after the completion of the course, it may be within a few days, or not for a few weeks, then the case can be regarded as one of arsenical nephritis. As a rule, arsenical nephritis is only diagnosed by accident, as many patients may pass large quantities of protein without complaining of being ill. These cases, as a rule, get well in time, provided no more mercury or arsenic are prescribed; but if intramine is injected intramuscularly and colloidal iodine given internally, the protein may be made to completely disappear in a few days. The cases which are really ill, and in which a fatal termination is not unlikely, are those in which the amount of urine passed is small. In such cases the urine contains less protein than might be imagined, but blood in many cases, and cells. Post-mortem, macroscopically, the kidneys may appear unaltered, or hæmorrhagic, or smaller than normal. Microscopically, apart from the degeneration of the capillaries, which accounts for the extravasation of blood, the two main changes to be noted are those affecting the tubular epithelium and the fibrous tissue. The tubular epithelium 
undergoes the same changes as are met with in the hepatic parenchymae.g., beginning with simple degeneration, which advances to fatty degeneration and complete necrosis. The interstitial tissue shows the ordinary signs of inflammation, plus a pronounced new formation of fibrous tissue. Having made a somewhat detailed examination of the kidneys of rabbits which had succumbed to overdoses of arsenic, mercury; and bismuth respectively, an outline of the changes found may be given here, as they coincide so closely with those found in the liver and kidneys of human beings who have died after a course of arseno-benzene and mercury. Except in points of degree as regards the main tissue affected and the extent of the involvement, which was largely dependent upon how long the animal lived with the nephritis, the type of nephritis was practically the same, whether caused by arsenic, mercury, or bismuth. The kidneys were always hyperæmic and hæmorrhagic, which suggested primary involvement of the walls of the capillaries. In those kidneys where regenerative changes had taken place, new perivascular fibrous tissue had formed, which in one case had gone so far as to produce calcification of the vascular coat. The epithelium involved was always the tubular, which showed up the glomeruli in sharp contrast. The tubular epithelium showed simple and fatty degeneration. In some kidneys the interstitial tissue was more affected than the epithelial. 'It exhibited the usual small round-celled infiltration, with the formation of numerous fibroblastic cells, which in some cases had formed new fibrous tissue. Pierce and Brown ${ }^{5}$ investigated the nephritis produced in guinea-pigs by various arsenical drugs, and found the same changes as above described, but that a relationship existed between the tissue involved and the chemical constitution of the drug which caused it.

Arsenical Arteritis.-It has been noted that patients under the influence of arseno-benzene who had been recently operated upon were liable to have capillary oozing in the wound. Far more serious, though, are those cases of hemiplegia and paraplegia which occur after the completion of the metallic course. I have seen three cases of hemiplegia and two of paraplegia in which the symptoms set in between two and fifteen days after the last injection of arseno-benzene had been given. All the cases were young men who had only recently contracted syphilis, and who had been put under treatment before the disease itself could have had time to produce a similar change in the vessels. Although it is usual for these cases to recover, occasionally extensive nerve degeneration may result, and kill the patient. In these severe cases huge bed-sores may form quite early in the trouble.

We have now clinical evidence of the three changes which are so characteristic of metallic poisoning-evidence which is sufficiently complete to warrant the conviction of arsenic in certain cases. Arsenic is more likely to produce such symptoms when coupled with mercury. There is no doubt that in some cases the disease itself acts as a contributory ætiological factor, and one cannot help being struck with the resemblance of certain of the changes produced by chronic metallic poisoning with those produced by syphilis itself 


\section{CLINICAL ASPECT AND TREATMENT OF VENEREAL DISEASES}

(vide the tendency to fibrosis, arterial sclerosis, and raised arterial bloodpressure, associated with nephritis, etc.).

Arsenical Intoxication of Nerve Tissue.-The connection between arsenical poisoning and the so-called neuro-recurrences is fully.dealt with in Chapter XIII., and requires no further mention here. That arsenic is a nerve poison is well known, and all of us are familiar with the optic atrophy which followed atoxyl and arsacetin. Although it is possible that the too free use of metals may predispose nerve tissue to be involved ị a syphilitic process, I have not met a case in which arseno-benzene has produced a primary neuritis in a cranial nerve, but I have seen cases where both mercury and arsenic have caused a peripheral polyneuritis. The polyneuritis may be of both the sensory-motor and the pseudo-tabetic type. In the sensory-motor type, the sensory symptoms, as a rule, predominate; in fact, when motor symptoms are present, the sensory are always slight, and likely to be overlooked. The main sensory symptoms are numbness, hyperæsthesia, and sensations like electric shocks in the hands and feet. On examination, patches of anæsthesia are discernible, and, as a rule, the reflexes are abolished. In two cases of the sensory-motor type the patients had mental disturbance, which suggested a Korsakow's psychosis. In both cases the patients became very forgetful, had peculiar hallucinations, and suffered terribly from insomnia, but ultimately recovered. Arsenical polyneuritis is not infrequently associated with arsenical dermatitis, the nervous symptoms becoming most evident as the rash fades. Fortunately, outstanding cases of arsenical polyneuritis are rare; but if every observer would thoroughly examine every patient he had treated with arseno-benzene, he would be surprised at the number of cases he would come across which presented only one symptom. Sleeplessness is, perhaps, the commonest symptom; neurasthenia is not at all rare; the same may be said of paræsthesiæ of the fingers and toes. I have also seen cases in which a patch of anæsthesia was discernible here and there, although the reflexes were normal; likewise, cases exhibiting transitory bladder disturbances. Vaso-motor trouble may be the only sign present, such as 'œdema of a hand, alteration of the circulation in certain fingers or toes, etc. Such symptoms as these are nearly always missed unless specially looked for, but, fortunately, they disappear in time. Insomnia seems the most persistent symptom, possibly because it worries the patient more than any other; in one of my own cases it lasted for about two years. Neurasthenia is perhaps the most troublesome symptom, as it may lead to suicide. The symptoms of arsenical neuritis may be intermittent, although no more of the metal has been prescribed. Chronic pharyngitis, muscular rheumatism, and polyuria, with or without loss of sexual desire, are very common symptoms of mild metallic intoxication.

\section{Miscellaneous.}

I have seen a few rather odd cases which might with advantage be mentioned. A patient had two intravenous injections of salvarsan, one in each arm, and, as the operator had had a difficulty in getting into the vein, both 


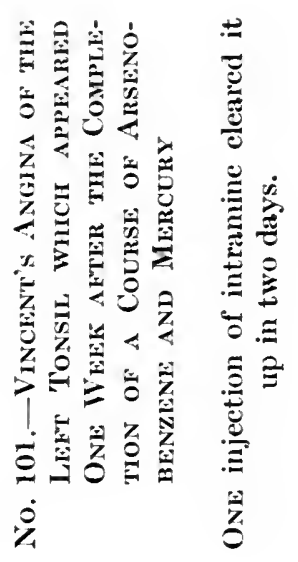

ב

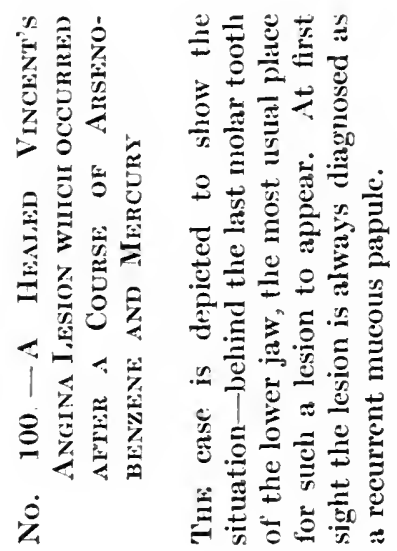




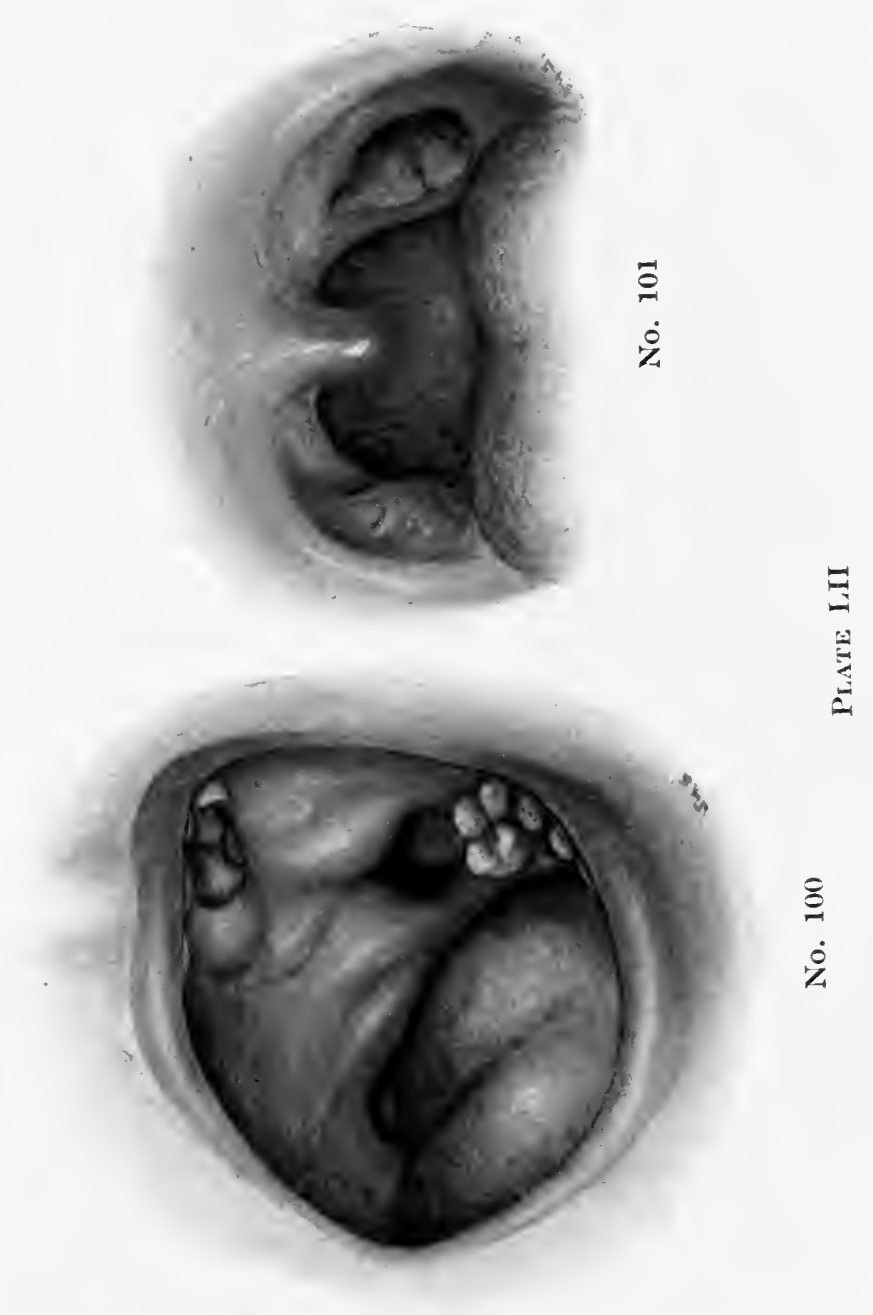


had been exposed through a small incision. Some weeks later the patient consulted me about her arms, and where the incisions had been made were large Condylomata lata. It appeared that the wounds never healed by first intention, and, owing to the injury caused, syphilitic lesions had developed locally; and, owing to the continuous oozing from places which had not healed properly, the papules had developed into condylomata.

In another case, also in a woman, wherever I punctured a vein, a crop of Lichen planus lesions appeared some time afterwards. I once had a patient with syphilitic palmar papular hyperkeratosis, the most resistant syphilide to treatment that I know, and he invariably developed a typical syphilitic lesion wherever he injured his hand, and a lesion always resulted in the spot where I pricked his finger for blood. This patient had seventeen injections of salvarsan, and thorough mercurial treatment, and yet the papules still persisted in coming out, and his blood was always positive. The patient married, and has already had two children, who are both clinically and serologically sound, in spite of the fact that the father still gives a positive C.F.T.

Syphilitic patients, whether in the early or late stages of the disease, may get general enlargement of the lymphatic glands after arseno-benzene, doubtless due to the production of lymphocytes, which the drug stimulates.

Patients with peripheral nerve lesions, not of syphilitic origin, and of syphilitic origin if late, often have their symptoms very much aggravated by arseno-benzene.

Peripheral neuritis, such as sciatica, if aggravated, does not very much matter, as the trouble will in time disappear; but when it is the eighth nerve that is affected, irreparable damage may be done. I have had two cases of old syphilitics with nerve deafness become absolutely stone deaf after salvarsan. I have also known of patients over 50 years of age, in whom the deafness was certainly not of syphilitic origin, have their deafness permanently increased by salvarsan. I had a case of congenital syphilitic deafness, in which the ear trouble did not commence until the patient was 32 years of age, and he went stone deaf after the second injection of salvarsan. I have seen so much trouble caused by arseno-benzene in aural lesions that, unless the lesion is syphilitic and acute, or unless I am compelled to give arseno-benzene for other reasons, I always avoid using it.

Some other interesting cases are the following: Severe nose bleeding after each injection of arseno-benzene in two patients with a high arterial bloodpressure. I have had four cases of bad sexual neurasthenia after arsenobenzene, but, of course, it is very difficult to say whether the arseno-benzene was in any way the cause, or whether the neurasthenia simply developed because the patient had had syphilis. The reason why I think arseno-benzene had something to do with it is that some patients do become very much depressed after it, and all the four cases referred to never had any other symptoms except the chancre.

From the above the reader will observe that symptoms of arsenical intoxi- 


\section{CLINICAL ASPECT AND TREATMENT OF VENEREAL DISEASES}

cation either appear before the patient has left the injection table, or not until the third day; therefore, nothing is to be gained by putting the patient into a nursing-home for a night. There are many objections to a nursinghome, and there is no risk in treating all cases as out-patients, and letting them go and do what they want after the injection. This has been my practice since 1912, and I have had no reason to alter it.

\section{Mercurial Intoxication.}

Very much of what has already been described under arsenic is equally applicable to mercury, because all metals appear to produce the same symptoms of intoxication, although some produce one symptom more often than another. With mercury stomatitis is most frequently associated, and with lead musculo-spiral paralysis (wrist-drop); but lead is not an uncommon cause of stomatitis, and I have known mercury produce wrist-drop. The cases of wrist-drop due to mercury followed intramuscular injections of mercury succinimide for gonorrhœa. That the symptoms of metallic poisoning should be the same, whatever the metal, is only to be expected when it is realised that metallic poisoning is nothing more nor less than acid intoxication; hence the reason why colloidal iodine and intramine are just as useful in cases of mercurialism and plumbism as they are in cases of arsenical poisoning. If these drugs are not immediately handy, and the case is a matter of urgency, much may be done by prescribing sodium bicarbonate internally $10 \cdot 0 \mathrm{grm}$. per diem, or by injecting $100 \cdot 0$ to $500 \cdot 0 \mathrm{c.cm}$. of a $5 \cdot 0$ per cent. solution intravenously. One of the most frequent symptoms of acid intoxication is the reduction in the output of urine, which may go on to complete anuria, a state which invariably occurs in very acute mercurialism, and one which is almost always to be found in the reports of fatal cases of arsenical poisoning. In this respect the following case will prove of interest:

CASE 115.-The patient had a primary sore on the skin of the penis, general adenitis, and was more or less covered with a follicular syphilitic eruption. I injected intravenously $10.0 \mathrm{c.cm}$. of 5.0 per cent. emulsion of colloidal mercury. In the quantity injected there was $0.5 \mathrm{grm}$. of colloidal mercury, the action of which would be very much greater than 0.5 grm. of ordinary mercury. On the following day the patient had severe abdominal pain and diarrhœa. The diarrhœa increased, and blood was passed per rectum. This continued for a week, during which time not a drop of urine was passed. On the second day severe stomatitis set in, which became gangrenous a few days later. The patient also had hæmorrhagic conjunctivitis, Herpes oris, looked, and was, desperately ill, and I had fears that he might die. This was a typical case of acute mercurial poisoning, and the mercury was acting as a poison in virtue of its oxidising power. This being the case, it struck me that the administration of an equally powerful reducing agent might cure the condition. I injected $100.0 \mathrm{c.cm}$. of colloidal iodine intravenously, and $5.0 \mathrm{c.cm}$. of intramine intramuscularly. Within forty-eight hours the diarrhœa ceased, and the patient passed nearly his full amount of urine. The condition improved so much from day 



\section{PLATE LIII}

\section{Nos. 102 and 103.-A Severe Case of Gangrenous Stomatitis produced by Mercury}

T'ne patient had haemorrhagie diarrhoea, anuria, and aseites. Under eolloidal iodine and intramine the patient travelled from death's door to perfect healtl within a week. Note the Herpes oris at the left angle of the mouth in the upper figure. 'This patient had no resulting albuminuria. 
No. 102
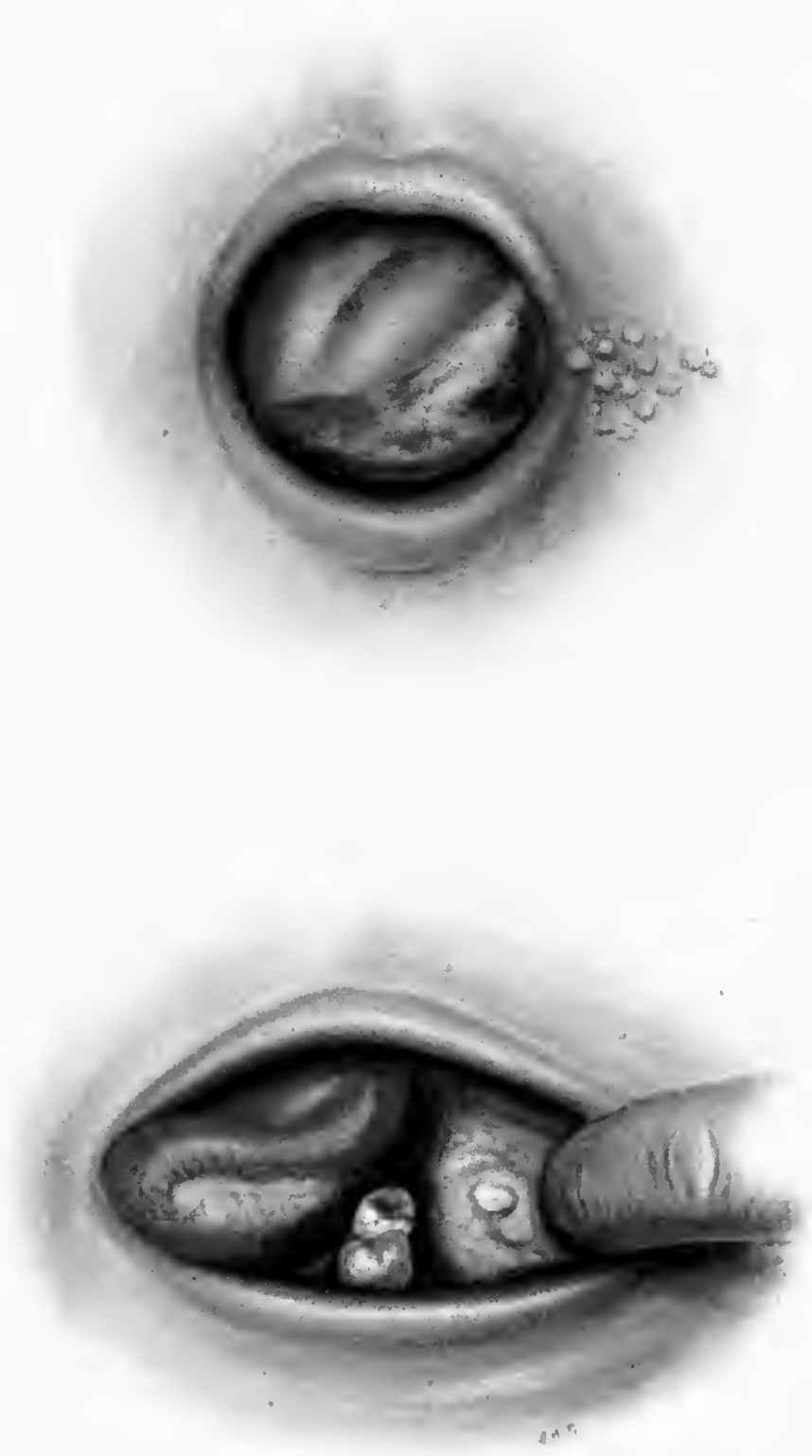

No. 103

Plate LiII 
. 
to day that, with no other treatment whatever, he was perfectly well eighteen days later, when there was no albumin in the urine, and every syphilitic symptom had vanished.

Another patient received 10.0 c.cm. of the same preparation intravenously the same day, having already had $10.0 \mathrm{c.cm}$. injected intramuscularly three days previously. Therefore the patient had $1.0 \mathrm{grm}$. of colloidal mercury in his system. This patient had exactly the same symptoms as above described, and recovered in the same rapid way after an intravenous injection of colloidal iodine, and an intramuscular injection of intramine. I have had three other cases in which the symptoms were not so marked, smaller doses having been prescribed, and the symptoms improved at once with one injection of intramine. In all these five cases, the syphilitic manifestations disappeared in a few days, and some of the lesions were such as would not have vanished with six or more injections of arseno-benzene. The urine in these cases has been tested several times since, and has always been free from albumin.

Both arsenic and mercury may cause alopecia and trophic conditions of the nails, as well as be responsible for loss of sexual desire.

When we realise that the symptoms of metallic poisoning are identical, whatever be the metal, and that many of these symptoms are the same as those produced in certain tissues in the chronic stage of a disease such as syphilis, for instance, we must surely begin to wonder whether it is justifiable to fence round certain areas in medicine to make them distinct from others. To be more specific: Are we right in regarding mercury as a specific for syphilis? In my opinion we are not. Mercury is useful in syphilis, in virtue of its high atomic weight, which has a physical action on the protein particles in the serum, which happen to be increased in number and size in syphilis more than in any other disease. Mercury has no direct action upon the parasite, and doubtless could be as efficaciously supplanted by other metals with as high, or a higher, atomic weight still. Most metals, especially the heavy metals, produce acld intoxication, because they break up the colloidal protein particles into such small ones till that stage is reached in which they go into true solution and cease to be colloidal, in which state they cannot be carriers of oxygen, the main function they have to perform. Their carrying capacity of oxygen is also diminished when they increase in size, which is what happens in the chronic stages of a disease; hence the reason why some of the symptoms met with in late syphilis are indistinguishable from those of mild acid intoxication. The fibrosis of chronic syphilis is the same as that of chronic acid intoxication, and the reason why iodine and sulphur are so useful to prevent and to overcome it is simply because they have the action of altering the colloidal protein particles in such a way as to enable them to be carriers of oxygen again. A point of some interest is that most, if not all, cases which present symptoms of metallic poisoning give a negative C.F.T. This, of course, is only to be expected, because the metal would not have produced 
toxic symptoms if there had been sufficient protein colloidal particles present to adsorb and so protect it, and the C.F.T. is a colloidal reaction, which obviously cannot come off if the particles are in true solution and no longer colloidal. It is interesting to note, in this connection, that if intramine is prescribed, the moment the blood becomes negative after a series of injections of arseno-benzene and mercury, it has the effect of rendering it positive againan observation which goes far to prove the efficacy of this sulphur compound.

\section{Sulphur Intoxication.}

Except for an occasional occurrence of neuritis, muscular rheumatism, and a transient dermatitis, I have never known intramine to exhibit harmful effects; presumably because sulphur is the element which regulates the reduction process in corpore. When muscular rheumatism occurs it is probably due to the mercaptan (SH) group which is formed in the process leading to the production of the peroxide, because the symptoms immediately disappear the moment iodine is prescribed, owing to the influence iodine has in converting mercaptan into a di-sulphide-the form sulphur appears to take when acting as a catalyst. Although intramine was painful injected intramuscularly when first supplied owing to the fact that the best vehicle for it had not been found, it is now practically painless, leaves no induration behind, and never causes an abscess.

1. Stuart McDonald (1918): Brit. Med. Journ., i., 76.

2. Fenwick, Sweet and Lowe (1918): Brit. Med. Journ., i., 448.

3. Wile and Karshner (1917): Journ. Amer. Med. Assoc., lxviii., 1311

4. Werner (1897): Münch. med. Woch., xliv., 736.

5. Pierce axd Brown (1916): Journ. Experim. M€d., xxiii., 443.

6. Yakimoff (1911): Münch. med. Woch., lviii., 2601.

7. YакIMOFF (1912): Münch. med. Woch., lix., 124.

8. Wechselmaxn (1911): Deutsch. med. Woch., xxxvii., 778.

9. Milian (1919): Paris Médical, ix., 261.

10. Seligmaxy (Boenm) (1913): Handbuch der Biochemie Ergänzungsband, 284. G. Fischer, Jena.

11. McDonagh (1916): Links in a Cliain of Research on Syphilis. Harrison and Sons, London.

12. McDonagh (1917): Practitioner.

13. Bayliss (1919): Proc. Roy. Soc. of Med., xeix., 17; xii., 1.

14. Dale: Ibid. 


\section{CHAPTER XVIII}

\section{THE TREATMENT OF SYPHILIS}

For the sake of lucidity, syphilis will be divided into the following stages, and the treatment of each will be considered separately: $(a)$ stage of the initial lesion; $(b)$ stage of the generalisation of the virus; (c) recurrent stage; $(d)$ latent stage; $(e)$ syphilis in women; $(f)$ congenital syphilis; $(g)$ syphilis of the nervous system.

The treatment about to be described is that I have found to be most useful in the majority of cases. I do not wish the reader to regard it as stereotyped, as every case must be treated individually.

\section{Primary Sore.}

Excision of the primary sore should be practised when possible. When impossible, owing to. the site affected, it should be cauterised, or, failing cauterisation, intramine-colloidal mercury ointment should be rubbed in until every trace of the induration has vanished.

It is most necessary to get rid of the induration of a chancre, as spores are liable to get locked up in it, to awaken later and produce a recurrent chancre, or even to give rise to a general infection. It is for this purpose that intramine is so beneficial, applied locally and injected intramuscularly. As intramine is a reducing agent, it should not be prescribed until the maximum effect to be produced from the use of oxidising agents (metals) has been reached; then the introduction of intramine will, apart from reducing the fibrosis, also form the peroxide necessary for the further administration of arseno-benzene and mercury.

The question as to the advisability of removing the lymphatic glands draining a primary sore has frequently arisen, and their excision is even practised by some.

Those in favour of removal state that it is only necessary to excise the lymphatic glands which are enlarged.

Those cases in which the lymphatic glands are most enlarged are usually those in which the infection is slight and histological examination reveals the smallest number of parasites. Those cases in which the lymphatic glands are smallest and hardest are usually those in which the infection is severe, and a histological examination reveals the largest number of parasites. Therefore a ratio exists between the size of the lymphatic glands and the protective capacity of the host against the disease. 
Since the lymphatic glands are responsible for some of the protective substances with which the host attacks the parasite, it would appear wiser to leave them alone.

Having treated the sore locally, I give from five to seven intravenous injections af arseno-benzene, with four or five days' interval between each, with or without an intravenous injection of colloidal iodine forty-eight hours before the first injection of arseno-benzene. The advantage of the iodine is that it increases the action of the arseno-benzene by increasing the receptivity of the colloidal protein particles in the serum for the latter drug. In other words, it breaks up the colloidal particles into smaller ones, thereby increasing the area of their action. If $0.6 \mathrm{grm}$. of arseno-benzene will kill the spirochætæ in a chancre in twenty-four hours, a previous injection of colloidal iodine will enable the same result to be produced with $0.3 \mathrm{grm}$. Intramine should be injected after the fourth or fifth injection of arseno-benzene. The treatment should be closed with mercury, iodine, and intramine, given in small doses, and in three courses extending over twelve months-mixed intermittent treatment. Intramuscular injections of mercury may be given simultaneously with the arseno-benzene, if desired; but as mercury is not a particularly good oxidising agent, and as the combination of two toxic poisonous metals increases the toxicity of each, I think it best to keep the mercury for the intermittent treatment, unless it is used to supplant an injection of arseno-benzene. If used for this purpose, I advise an intravenous injection of colloidal mercury $(10.0 \mathrm{c.cm}$. of a 1.0 per cent. emulsion).

It is unnecessary to examine the blood until six months have elapsed after the completion of the intermittent treatment.

Tabulated, the treatment appears as follows:

1. Local treatment, with or without an intravenous injection of colloidal iodine, $100 \cdot 0$ c.cm.

2. Intravenous injection of arseno-benzene, maximum dose.

3. Five days later, second injection of arseno-benzene, maximum dose.

4. Five days later, third injection of arseno-benzene, maximum dose.

5. Five days later, fourth injection of arseno-benzene, maximum dose.

6. Five days later, fifth injection of arseno-benzene, maximum dose, or intravenous injection of colloidal mercury ( $1 \cdot 0$ per cent. emulsion), $10 \cdot 0 \mathrm{c.cm}$.

7. Five days later, intramuscular injection of intramine, $3.0 \mathrm{c.cm}$.

8. Five days later, sixth injection of arseno-benzene, maximum dose.

9. A week later, begin intermittent treatment.

\section{Intermittent Treatment.}

One course consists of the following (10-12):

10. Weekly intramuscular injections of grey oil (1 grain of $\mathrm{Hg}$ ) for eight weeks. Every other week inject into other buttock intramine, $1 \cdot 0$ c.cm., to prevent mercurialism and to aid the action of the mercury. 
11. Colloidal iodine internally for one month ( 3 ii. ter in die, post cibos).

12. No treatment for four weeks.

If the induration is very pronounced, it is best to prescribe two injections of intramine instead of one, and to follow the second by two or three injections of arseno-benzene.

\section{Stage of Generalisation.}

In this stage it is necessary to give more maximum injections; six courses of the intermittent treatment should be prescribed, and about the twelfth week after the last injection of arseno-benzene the patient should be thoroughly examined to see if his nervous system has been attacked. If thought necessary, the C.s.F. can be examined as well. Six months after the completion of the intermittent treatment the blood should be examined, and, if found positive, three more courses should be advised.

Tabulated, the treatment appears as follows:

1. 2 to 8 as above.

2. Five days later, seventh injection of arseno-benzene, maximum dose.

3. Five days later, second injection of intramine, $3 \cdot 0 \mathrm{c.cm}$.

4. Five days later, eighth injection of arseno-benzene, maximum dose.

5. Five days later, ninth injection of arseno-benzene, maximum dose.

6. Six courses of intermittent treatment.

If on examining the patient about the twelfth week his nervous system is found to be involved, two intramuscular injections of intramine, followed by five intravenous injections of arseno-benzene, should be prescribed. Roughly, four or five days should be allowed to elapse between the injections, and one hour after each alternate injection the C.s.F. should be drained (auxiliary tapping).

\section{Recurrent Stage.}

If the recurrence is early, and if the previous treatment has been poor, then the case should be treated as belonging to the stage just described. If, on the other hand, the recurrence is late, and the previous treatment has been good or poor, three facts should be considered before any line of treatment is adopted-site of recurrence, marriage, age of patient.

If the site is in or near a vital organ, and if any spread or future recurrence would endanger the life or blight the happiness of the patient, he should receive the same treatment as previously mentioned. A man who is going to marry should be treated likewise, but marriage need not necessarily be delayed for two years. If the site is not an important one, and if the patient is not going to marry, and if he is of a certain age, his treatment should be symptomatic-i.e., three injections of arseno-benzene, with one or two injections of intramine, and two courses of the intermittent treatment. If the recurrent lesion is early and widespread, two injections of arseno-benzene 


\section{CLINICAL ASPECT AND TREATMENT OF VENEREAL DISEASES}

should precede the intramine, but vice versâ if the lesion is late and local. After the treatment it is not necessary to test the blood.

\section{Latent Stage.}

When one relied solely upon the C.F.T. in this stage, treating those who gave a positive reaction, and leaving those who gave a negative reaction, the course was clear; but now that I no longer attach the importance to the reaction which $I$ once did, I have had perforce to alter my routine. In this stage I now begin by examining the C.s.F., should a clinical sign or symptom draw attention to the nervous system, and if I cannot make up my mind as to the nature of the nervous lesion. If the lesion is non-degenerative I prescribe two intramuscular injections of intramine, five to seven intravenous injections of arseno-benzene at five days' interval between each, and tap the spinal canal one hour after every alternate injection. After this I prescribe six courses of the intermittent treatment. If the lesion is degenerative, I try to ascertain whether it is active or quiescent. If quiescent, I simply prescribe colloidal iodine internally for one month every fourth month for the next two or three years, and advise the patient to be clinically overhauled once or twice a year. If active, I take into consideration the treatment the patient has had before. If the treatment has been good and recent, it is best to leave the patient alone, and only order him colloidal iodine internally. If the treatment has been inadequate, injections and auxiliary tapping should be prescribed, but stopped immediately should the patient not appear to improve. In other cases of degenerative lesions, and in those which do not improve, intraventricular injections should be discussed.

\section{Syphilis in Women.}

Since syphilis in women is the worst of the evils caused by syphilis, I must repeat a few lines that have already appeared in Chapter XIV:

Syphilis in women may be looked upon as the greatest curse of the disease, since a woman who has once conceived a syphilitic infant may infect, in utero, all her subsequent offspring, although the father of the latter may be a different husband, who has never had the disease.

To make matters worse, conceptional syphilis is not recognised until the infant has been seen to settle the diagnosis, owing to the fact that many mothers show no evidence of the disease until after the child-bearing period is over, and, as often as not, the C.F.T. during this period is negative.

A general rule may be formulated- viz., that if a woman contracts syphilis after she has conceived, the C.F.T. will be positive, because the disease becomes generalised and behaves in the ordinary way; that if a woman contracts syphilis at the time of conception, the C.F.T. will often be negative, because the disease does not become generalised, at any rate, not until some later date. 
Herein we have the explanation why such patients develop manifestations only after the child-bearing period is over, and why it so frequently happens that the first and last pregnancies result disastrously, while one or more healthy children may be born in the interim. It is interesting to inquire into the rationale of conceptional syphilis.

The germ must, in the first instance, he conveyed by the semen. But does the germ, which is with the embryo in the uterus, develop after a time into the gamete forms described by me-which I regard as responsible for the symptoms-at the expense of the embryo, with, maybe, its death, and leave some of the spores behind after its expulsion, to be already there to develop at the expense of the next embryo ? Or does the mother get infected directly, but the symptoms are prevented from recurring, owing to the formation of some chemical substance, possibly in the form of a lipoidglobulin complex from the embryo, which prevents the gametes from being developed?

When the question was discussed after the Spirochoeta pallida had been discovered, when the Spirochota pallida was held to be responsible for everything syphilitic, only confusion resulted. If my discovery of the Leucocytozoon syphilidis be accepted, and the views accepted that the spore is the infective agent, and that the gametes are responsible for the symptoms, the alternative need not appear in the above illustration, as both, in part, may turn out to be correct.

It may be considered that the spores, themselves inert, travel in the semen, reach the uterus, and find themselves in both the maternal and fotal portions of the embryo. Those in the fœtal portion, after a period of some weeks, develop into gametes, which may or may not kill the embryo.

Those in the maternal portion find themselves unable to develop, owing to a chemical substance from the chorionic cells which circulates in the mother's blood, but not in the fœtal blood, and so they remain dormant for a time. Herein lies the solution of the phenomenon that a mother may give birth to an actively syphilitic infant without herself giving even so much as a positive C.F.T.

The theory above put forward will also explain the fact that a woman who has once given birth to a syphilitic child is always liable to do so again, although the father of her later children may be another busband, who has himself never suffered from the disease.

Hence the necessity for treating such a case throughout the whole period of each succeeding pregnancy.

To women who are syphilitic I give, as soon after they have conceived as possible, six intravenous injections of arseno-benzene, one intramuscular injection of intramine, and I continue the treatment with mercury, iodine, and intramine till as near the time the child is to be born as can be done. 


\section{CLINICAL ASPECT AND TREATMENT OF VENEREAL DISEASES}

Tabulated, the treatment appears as follows:

1. Intravenous injection of arseno-benzene, maximum dose.

2. Five days later, second injection of arseno-benzene, maximum dose.

3. Five days later, third injection of arseno-benzene, maximum dose.

4. Five days later, fourth injection of arseno-benzene, maximum dose.

5. Five days later, intramuscular injection of intramine, $3 \cdot 0 \mathrm{c.cm}$.

6. Five days later, fifth injection of arseno-benzene, maximum dose.

7. Five days later, sixth injection of arseno-benzene, maximum dose.

8. Two courses of the intermittent treatment.

\section{Congenital Syphilis.}

Before considering the specific treatment, a few words must be said about the feeding of the patient, because one of the most important factors which determines the welfare of the child is the general nutrition. If the mother is known to be syphilitic and the infant is clinically and serologically sound, which is very likely to be the case if the former has been adequately treated throughout her pregnancy, under no circumstances should the infant be suckled by its mother.

If an infant is known to be syphilitic, every endeavour should be made for the mother to suckle it, as breast feeding is such a useful adjunct to the specific treatment. In this country wet nurses are not much used, which is contrary to the case on the Continent, where good results are obtained. If a wet nurse is employed, only a syphilitic one should suckle a syphilitic child, and vice versâ.

In former years an effort was made to treat infants indirectly through the milk of mothers, cows, goats, etc., which had been subjected to mercurial medication. Later, Teage, Duhot, and others, tried the same method with salvarsan through the mother's milk, while Jesionek used goats for the purpose. The results were variable and inconstant, and to-day the treatment may be said for the most part to have been given up. Should the reader wish to try this method, he should only prescribe it for infants which are not very ill, and in which the syphilitic lesions are not widespread. Marasmic infants, with florid widespread syphilis, are too apt to succumb, as the small amount of arsenic which comes over through the milk primarily excites and does not destroy the active phases of the syphilitic organisms. This action can be obviated by injecting, in addition, arseno-benzene intramuscularly or intravenously through the anterior fontanelle or viâ the external jugular; but as the intramuscular route almost invariably causes necrosis, and the intravenous route is considered too heroic to find many adherents, mercury is still the favoured drug for syphilis affecting infants.

Even with improved technique, and in older children, intramuscular injections of neither arseno-benzene nor mercury find favour; consequently, to-day the former drug is not widely used except where it can be given intra- 
venously. In infants, then, we are practically limited to the use of mercury, which, administered by inunctions, is undoubtedly the best method. For infants with open syphilitic cutaneous lesions, sublimate baths are very useful, as inunctions are impracticable. For this purpose 20 grains of perchloride of mercury are dissolved in 3 gallons of warm water, and a daily bath is given until the sores have healed.

When inunctions are used, 10 to 20 grains of unguentum cinereum should be rubbed in gently for about half an hour every, or every other, night, according to the age of the patient, a fresh site being chosen for each application. The rubbing should be performed either in the early morning or in the evening; the part should then be well covered with a flannel binder, and the ointment washed off at the next bath time. An occasional colloidal sulphur bath, say once or twice a week, is very useful in aiding the elimination of the mercury and in preventing its causing dermatitis. Infants are especially liable to dermatitis; therefore it is importani never to allow any of the ointment to get near the groins, where the urine acts as an additional irritating factor.

Mercury prescribed internally is useful in congenital syphilis, and infants are particularly tolerant of it, because they are toothless, and consequently run no risk of stomatitis. Half a grain of grey powder should be given in milk twice or three times a day. If there is any diarrhœa, 3 grains of pulv. creta aromat. may be added. If mercury is not well borne, and whenever it begins to show signs of producing gastro-intestinal trouble, recourse should be had to iodine, prescribed either in the form of colloidal iodine, 3ss. to 3i., twice or three times a day, or in the form of syrupus ferri iodidi, $m v$. to $m \times$., three times a day.

The application of emplastrum cinereum, or wearing next to the skin clothes impregnated with mercury, is a useful adjunct in treatment if the other forms of mercury cannot be continued.

In the treatment of congenital syphilis it is a good plan to vary the treatment from time to time, and in every case I consider it wise to keep the patient intermittently under treatment for three years. In my opinion, it is useless to gauge the amount of treatment required by the C.F.T. Cases of Syphilis hereditaria tarda should be treated symptomatically, much in the same way as described under the head "Recurrent Stage" in the acquired form. It is in the late cases of congenital syphilis that intramine is so useful (vide Chapter XV.).

Taking a type case, I should tabulate the treatment as follows:

\section{First Year.}

1. Inunctions for one month.

2. Mercury internally for two months.

3. Iodine for one month.

4. Repeat 1 to 3 twice. 


\section{Second and Third Years.}

1. Mercury internally for three months.

2. Iodine for one month.

3. Rest from treatment for one month.

4. Mercury internally for four months.

5. Iodine for one month.

6. Rest from treatment for two months.

Reverting to arseno-benzene: in infant syphilis I only use the drug when I an of the opinion that, unless something drastic is done, death will undoubtedly occur, and then I inject into the longitudinal sinus $0.01 \mathrm{grm}$. of a soluble salt dissolved in $5.0 \mathrm{c.cm}$. of water, repeating the injection every fourth or fifth day for five or six times, according to how the case progresses.

From the age of seven onwards arseno-benzene can generally be injected intravenously at the bend of the elbow, in which case I never exceed $0.3 \mathrm{grm}$. until the child is fourteen years of age. From this age onwards full doses of arseno-benzene may be given with impunity. If the lesion is acute, it is best to begin with three or four injections of arseno-benzene before prescribing intramine, while if the lesion is chronic, or the patient has already had a course of oxidising agents (metals), two injections of intramine, preferably intramuscular, should precede the arseno-benzene. In all cases in which intramine is used, the last one or two injections given should be of arseno-benzene.

\section{Syphilis of the Nervous System.}

Before we consider the treatment of syphilis of the nervous system, a word or two must be said about prevention, because many of the cases are avoidable. Syphilis of the nervous system has been steadily on the increase since the advent of arseno-benzene. In my opinion, it is progressively on the increase, and I believe I can foresee the time when syphilis will be looked upon as a nervous disease. The increase has been, and is, due to four causes: (1) sterilising the systemic and not the nervous part of the body; (2) too free use of oxidising agents (metals); (3) insufficient use of reducing agents (nonmetals); (4) failing to supplement the maximum course by intermittent courses for one to two years.

Since I have introduced colloidal iodine and intramine into the treatment of syphilis, and invariably followed up the maximum course with mercury, iodine, and intramine intermittently for one to two years, I have seen very little nervous syphilis develop in my early cases. The precaution of examining the patient and testing the C.s.F. about the twelfth week after the completion of the maximum course, and giving the treatment necessary, should the result be positive, still further lessens the occurrence of nervous syphilis. The cases of early syphilis of the nervous system following treatment can be divided into two classes: (1) meningeal; (2) arterial. 
In the meningeal cases, diagnosed clinically or by pathological findings in the C.s.F., the following treatment should be given after the fluid has been drained by a lumbar puncture.

1. Colloidal iodine internally for one month, 3iii. ter in die, post cibos.

2. Same day as colloidal iodine is ordered, inject intramine intramuscularly, $3.0 \mathrm{c.cm}$.

3. Five days later, inject intramine intramuscularly, $5 \cdot 0 \mathrm{c.cm}$.

4. Five days later, inject arseno-benzene intravenously, maximum dose, and tap spinal canal within an hour and a half.

5. Repeat arseno-benzene three times, at five days' interval between each injection.

6. Repeat intramine $(5 \cdot 0 \mathrm{c.cm}$.$) , and then two more injections of arseno-$ benzene, at approximately five days' interval between each injection. An auxiliary tapping should be made after every alternate injection.

7. Intermittent treatment for two years.

-In my opinion, it is most necessary to withdraw the C.s.F. before treatment is commenced in all cases in which the meninges are involved. The drugs reach the meninges, and doubtless percolate through them to reach the intrameningeal spaces and the nerve tissue itself. In all cases of meningitis, the C.s.F. is under an increased pressure, which must obviously limit the filtering properties of the meninges. If the fluid is withdrawn the opposite occurs, which allows the maximum quantity of the drug to find its way through. I feel confident that constant tapping of the C.s.F. is as efficacious as prescribing intraspinal injections; but still, innocuous drugs like colloidal iodine and intramine can be directly administered into the subdural space.

In the arterial cases, such as hemiplegia, paraplegia, etc., the same treatment as above should be prescribed, without the auxiliary tapping. Arterial lesions occurring early in syphilis, when no or only mercurial treatment has been given, should be treated in the same way as described under the heading "Generalisation Stage." The same treatment is also indicated in acute cerebro-spinal syphilitic meningitis, occurring independently of treatment, with the addition of auxiliary tapping, approximately after every alternate injection.

When cerebro-spinal syphilitic meningitis occurs as a recurrent syphilitic manifestation, as it does from the fourth year and onwards after infection, one may be dealing with a purely meningeal lesion, or more often with a meningo-encephalitis and meningo-myelitis. Once nerve tissue has been involved, great care must be exercised in the line of treatment to be chosen; too much arsenic and mercury should be avoided, as, being metals, they are toxic to nerve tissue, and apt to aggravate the lesion, as the resisting power of the nerve tissue has already been reduced by the disease, and a guarded prognosis should invariably be given. The following is the treatment I usually advise for such cases, after having first tapped the spinal canal: 


\section{CLINICAL ASPECT AND TREATMENT OF VENEREAL DISEASES}

1. Colloidal iodine internally for three months, 3iii. ter in die, post cibos.

2. Immediately an intramuscular injection of intramine, $3 \cdot 0 \mathrm{c.cm}$.

3. Five days later, intramuscular injection of intramine, $5 \cdot 0 \mathrm{c.cm}$.

4. Five days later, inject intravenously arseno-benzene, maximum dose, and tap the canal within an hour and a half.

5. Five days later, repeat arseno-benzene.

6. Five days later, repeat arseno-benzene and tap the canal.

7. Five days later, repeat intramine, $5 \cdot 0 \mathrm{c.cm}$.

8. Five days later, repeat arseno-benzene and auxiliary tapping.

9. Five days later, repeat arseno-benzene.

10. Five days later, repeat arseno-benzene and auxiliary tapping.

11. Intermittent treatment for two years.

It is not of much value to gauge the treatment by examinations of the C.s.F., because an effect of auxiliary tapping is at first to increase the constituents of the C.s.F., such as cells, protein, etc.

One of the great advantages of commencing the treatment, after first tapping the canal, with colloidal iodine and intramine is that all risk of a severe reactionary inflammation ensuing is avoided. Arseno-benzene is particularly apt to cause reactionary inflammation, and if it occurs in a closed space like the skull, the increased pressure produced renders the patient unconscious, and occasionally kills him. Although reactionary inflammation is practically a thing of the past, it deserves further mention now, as anyone may be called upon suddenly to deal with a case.

Reactionary inflammation occurs mostly in cases of meningitis when the patient suddenly becomes unconscious on the third day following the second or third injection of arseno-benzene. Subcutaneous injections of adrenalin should be prescribed at once, $1.0 \mathrm{c} . \mathrm{cm}$. of 1 in 1,000 solution every four hours. A lumbar puncture should be performed, and an intraspinal injection of colloidal iodine be made. At the same time, an intravenous injection of colloidal iodine (100.0 c.cm.) may be administered, with an intramuscular injection of intramine, $5.0 \mathrm{c.cm}$., or even another intravenous injection of arseno-benzene will do almost as well.

In those cases of meningo-encephalitis and myelitis where the signs and symptoms point to the chief mischief being in the nerve tissue proper, the same treatment as just described may be given, although, in my opinion, however beneficial the immediate results appear to be, sooner or later the lesion shows signs of progression, and then it is practically always degenerative in nature. If the clinical signs disappear as the treatment is being undertaken, I complete the course. If, on the other hand, only a very slight improvement results, and a stationary period is reached, which strongly suggests the degenerative character of the mischief, I stop the maximum course and resort to the intermittent courses, because I am decidedly of the opinion that too much treatment is apt to accelerate the disease. In pure cases of degenerative encephalitis and myelitis, I think it is better not to prescribe anti- 
syphilitic treatment unless the lesion is undoubtedly active, and even then great care has to be exercised. Many cases which I have treated in the past have, I feel certain, relapşed more quickly than they would have done had no specific treatment been undertaken, and many of the relapses have proved fatal.

In degencrative encephalitis, no antisyphilitic treatment short of auxiliary tappings or intraventricular injections should really be considered.

If the patient has already had one attack, and presents himself with a recurrent one, intraventricular injections may be considered, but $I$ am not at all sure that the future will not teach us to leave such cases alone.

In early and active cases of degenerative myelitis, the treatment above outlined for cerebro-spinal meningitis should be undertaken. In old and quiescent cases, antisyphilitic treatment is contra-indicated.

Reference must now be made to what may be called the indirect methods of treatment-methods which are adopted in the degenerative cases, especially in degenerative myelitis. Nothing is more troublesome to alleviate than the pains of which a tabetic complains. Having tried all the antisyphilitic drugs, which do little or no good, we begin to go through the pharmacopœia. Some say salicyl-arsenate of mercury (enesol) is useful, which has not been my experience. Others believe in Hirsch's injection, which is a mixture of oxycyanide of mercury and acoine. I have found these to produce a temporary relief only. Salts of aluminium and gold have proved of extremely little value. Intramuscular injections of lymphoid extract (British Organotherapy Company) - they may also be taken internally in the form of capsules -have been useful now and again. Acetyl-salicylic acid (aspirin) is in most cases the patient's stand-by, in spite of the indigestion to which it gives rise. In many-cases I have tried the calcium or soluble salt of this drug, but its action is less potent. Of Förster's operation-division of the posterior nerve roots-I have no first-hand experience. Maloney is a great believer in the psychotherapeutic treatment. When a patient has ataxia, and he sees himself getting better from his exercises, psychotherapy is a useful adjunct to the exercises in the treatment of the pains; but when pain is the only symptom complained of, psychotherapy is not so trustworthy. Maloney treats cases of gastric crises by reducing vagal activity with intramuscular injections of atropine and hyoscine, reinforcing their power through stimulation of the sympathetic system by adrenalin administered per rectum. Naturally, careful dieting is necessary, and rest essential. Much can be done for tabetics with bladder trouble by teaching the patient to pass his water at regular intervals. Washing the bladder out occasionally with colloidal silver or colloidal iodine is useful, and dilatation of a stricture, if present, is essential. No fluid should be taken for at least two hours before the patient goes to bed. Maloney lays emphasis on the psychotherapeutic treatment in cases of optic atrophy, while perception of light still remains, and in cases of deafness. The psychotherapeutic treatment originated from observations which have frequently 
been recorded that ataxia has been produced when the patient became first aware that he had Locomotor ataxia, and that ataxia has disappeared when optic atrophy supervened. It is the greatest mistake to let a patient know he has Locomotor ataxia, a term well known to laymen; hence one of the reasons why I prefer to call it "degenerative myelitis." There is no doubt that the attitude of the tabetic is an expression and a cause of the tension in which he lives, and the removal of that expression relieves his mental tension. To remove it Maloney devised the following rest exercises: The tabetic is instructed to breathe deeply, and to pause at the end of inspiration and of expiration. Every joint is passively moved in turn. The muscles are first stretched slowly and steadily, and next are similarly relaxed, and the patient is instructed $r e$ diet, baths, etc. Properly instituted movements, well practised, will enable a confirmed ataxic to walk again, and the same may be said when diplopia is present. For fuller details the reader is referred to Maloney's most interesting and instructive work (Maloney, Locomotor ataxia, 1918. D. Appleton and Co., New York and London). In bad gastric and intestinal crises I have known injections of colloidal palladium (pallamine) and inhalations of amyl nitrite do good. In pure vascular cases the treatment varies according as to whether the lesion is an early or late one.

Hemiplegia and paraplegia occurring in early syphilis should be treated as a case in the generalisation stage. A vascular lesion occurring in late syphilis, which often shows itself as an ocular nerve palsy, or even as a hemiplegia, should receive two injections of intramine (half doses) and three of arseno-benzene (half doses), and the patient should take colloidal iodine internally for one month every quarter for the rest of his lifetime. Whether treatment is given or not, the chances are that a recurrent lesion will set in, and will kill the patient, usually within a space of three years. 


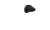




\section{PLATE I.IV}

No. 104.-A Single Ulcus Molle on the Skin of the Penis

THe sore is sharply circunscribed. The edge is raised and undermined, the base is covered with pus, and the sore is surrounded by acute inflammation, which has produced a Paraphimosis interna and acute inflammatory oedema of the prepuce. In all the single soft sores it is difficult to find Ducrey's bacillus, and in many of them its morphology is different from that of the organism usually met with.

No. 105.-A Single Ulcus Molle on the Skin of the Penis

NOTE the irregularity of the base, the heaped-up edge, which was undermined, and the circumferential inflammation. Ducrey's bacillus is difficult to find in this type of sore. 
No. 104
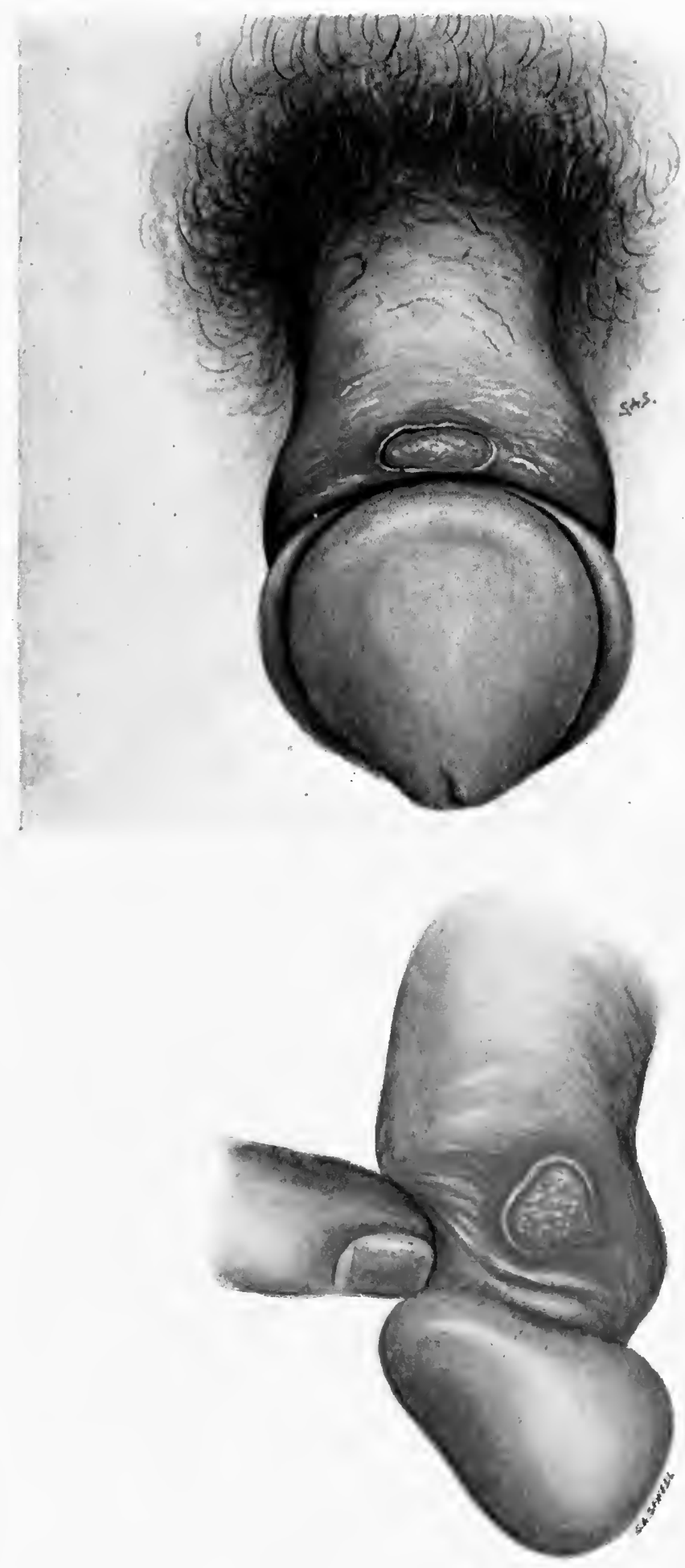

No. 105

Piate LIV 



\section{CHAPTER XIX \\ ULCUS MOLLE (SOFT SORE)}

\section{History.}

IT is practically certain that what we now call soft sores were known to the ancient Egyptians, Indians, Greeks, and Romans, but little of interest has been handed down from them to us. The connection between the bubo and soft sore does not appear to have been understood until explained by Pietro di Argelata, who died in 1423. This observer warned against the treatment of the sores with caustics, as predisposing to formation of buboes. The word bubo is derived from the Greek $\beta o v \beta \omega \nu$, groin. When syphilis invaded Europe utter confusion arose between the two diseases, and it was not dispelled until 1852, when Bassereau, a pupil of Ricord, showed that the two sores were distinct. Bassereau's work was likely to be upset, because, two years later, Clerc showed that the secretion from a hard sore inoculated on the carrier produced a soft sore, to which the name chancroid was given; but, fortunately, Rollet, a few years later, proved that Bassereau's contention was correct. The cause of Ulcus molle was discovered by Ducrey in 1889; but I think it has yet to be proved that every sore we label to-day as soft sore is caused by the same specific organism.

It is interesting to note that Paracelsus did not consider a bubo to be syphilitic in origin, and Van Swieten evidently observed that the pus was non-infectious. In days gone by some of the buboes must have been particularly severe, because Celsus refers to them as reaching bones, and causing death by leading to a fistulous opening of the intestines. Both John Andree (1779) and John Hunter (1786) were familiar with the lymphangitis, but William Nisbet (1787) made the important observation that the lymphangitis on the dorsum of the penis might remain localised and ulcerate to form a "lymphatic chancre," which was known for long afterwards as Nisbet's chancre or bubonulus. Needless to say, all kinds of treatment for bubo were employed, from simple incision to complete extirpation of the lymphatic glands, extremes which have their adherents to-day, in spite of the fact that Cullerier oncle, so long ago as 1815 , stated that buboes which spontaneously burst left no trace behind.

\section{General.}

A soft sore, or Ulcus molle, is a specific sore caused by Ducrey's bacillus. The sore, or sores, as they are most frequently multiple, occur usually on the genitals. They may occur on any part of the body, as they are inoculable, 261 
and are also auto-inoculable. I have seen five cases of a soft sore infection on the finger followed by suppuration in the epitrochlear lymphatic gland. The usual incubation period is about five days, when a tiny ulcer appears, and spreads rapidly. The sore is a true ulcer, the base of which is uneven and covered with pus. The circumference of the ulcer is sharply circumscribed, but irregular in outline, because, when it spreads, it does not do so evenly; often one part of an ulcer will heal while the other end extends. The edge is slightly undermined. The ulcer is surrounded by a marked inflammatory ring, the inflammation being most marked in the periphery of the spreading end. The ulcers tend to heal spontaneously, but treatment materially hastens the process. A lymphangitis is often to be observed, running from the sore along the penis to the inguinal lymphatic glands on both sides, or only on one side, and not necessarily on that side on which the sore is situated.

One of the most interesting points about the soft sore infection is the fact that a bubo-i.e., suppuration in the lymphatic glands-may not appear for wecks, and even for months, after the sore has healed and has been forgotten.

A soft sore confers no immunity on the individual affected, in spite of the fact that the organisms often remain for a long time in the system, either in the site of the initial lesion, or more often in the lymphatic glands draining it. In this connection the following two cases will prove of interest:

CASE 116.-The patient had a soft sore in 1916; he was reinfected at the end of 1917. One week after the appearance of the second sore an ulcer developed between the nail and the skin, along the outer lateral border of the index finger of the right hand. This digital sore rapidly took on the characters of an Ulcus molle, and would not heal until two intramuscular injections of intramine and one of colloidal manganese had been given. Six months later a bubo made its appearance in the right axilla, and it rapidly healed under colloidal manganese.

CASE 117.-A patient had a soft sore in 1913, a bubo in 1916 , which was incised widely, and took three months to heal. In October, 1918, having had no connection for four months, the patient presented himself with a typical Ulcus molle on the site of the old lesion. The recurrent sore had more the character of an Ulcus molle serpiginosum than of a simple soft sore.

When a bubo results from a soft sore and heals, and then either syphilis or gonorrhœa is contracted later, the risk of a recurrent suppurative adenitis ensuing is great, due to the streptobacillus. The following is an illustrative case :

CASE 118.-A patient had a soft sore infection in 1893, and had buboes incised in both groins. In 1915 he contracted syphilis, and before the rash, etc., came out a multilocular bubo appeared again in both groins.

Ulcus molle, although a widespread disease, favours some countries more than others. I have noticed over and over again how much more common it is on the Continent than in England, and, oddly enough, it appears to be more common in London at some times than at others. Whether there is 
a seasonal variation or not I have been unable, as yet, to determine. In my experience, Ulcus molle is more frequently to be met with in men than in women. It has become more common since the war, especially affecting those soldiers who have seen foreign service.

Ulcus molle affects different individuals in various ways. In some, the sores heal spontaneously in a few days; in others, especially those who have tight foreskins and have to take much exercise, the ulcers spread rapidly, and they may even become phagedænic.

Exercise is more likely to set up or to increase any tendency there may be to lymphangitis and suppurative adenitis. A sore, after it has persisted for some time, may develop what may be called a pseudo-induration, and hence suggests to the unwary a primary sore. Such a difficulty in diagnosis need never arise if the observer will always remember that, however long a soft sore persists, and however wide its dimensions become, it never loses its clinical characters-the undermined edge, with its surrounding area of inflammation, is practically always present.

Every student is taught that a soft sore may develop into a chancre, and that the change may be detected by the induration which supervenes. A soft sore may become indurated, and yet not become a chancre.

My own opinion is that a soft sore rarely, if ever, becomes a chancre. If a chancre develops on what was diagnosed as a soft sore, then the initial diagnosis was wrong. I have noticed with others, as well as with myself, that the riper one's diagnostic acumen becomes, the more rarely the double infection is met with, and vice versâ; so noticeable is this fact that $I$ am prompted to believe that the persistence in the literature of the occurrence of the dual infection is due to tenderness to the amour propre of the clinician.

When a soft sore is situated on the frænum, hæmorrhage from the frænal artery, owing to the spread of the ulceration, is a complication which should always be borne in mind. The hæmorrhage has never been in my experience severe, but it is usually quite severe enough to alarm the patient.

Although the main characteristics of a soft sore are practically always the same, there are some different clinical forms to be met with which require very careful mention, owing to the difficulty which they cause in differential diagnosis.

\section{Ulcus Molle Elevatum.}

This sore differs from the ordinary soft sore in being raised above the surface (Plate LVII.). It is, nevertheless, an ulcer, but the edge is not undermined. This type is often single, the surrounding inflammation is not so marked as it is in the ordinary sore, and it is extremely resistant to treatment. Pseudo-induration is liable to be met with in this type of sore; hence it is very apt to be mistaken for a chancre. Another peculiarity that this type of sore presents is its not infrequent long incubation period. 


\section{CLINICAL ASPECT AND TREATMENT OF VENEREAL DISEASES}

\section{Ulcus Molle Miliare.}

According to Tomasczewski, ${ }^{4}$ this type is more commonly to be met with in women than in men, and the sites of predilection in the former are the labia majora and the perineum. Each lesion is a raised papule, in the centre of which there is a crateriform ulcer, and it looks at first sight like a hair follicle infection. The lesions persist for some time, and there is usually a very great number of them. This type of soft sore is not very uncommon in men, when it usually affects the prepuce. The sores give rise to a great deal of discharge and generally are painful.

\section{Ulcus Molle Phagedcenicum.}

A soft sore, as a rulc, does not become phagedænic unless it is hidden beneath a tight foreskin, and the first appearance of the phagedæna is a perforation of the foreskin. In very severe cases, part or the whole of the penis may be destroyed. Phagedæna is a not common complication of a soft sore; it is certainly more often met with in syphilis, and I cannot help thinking that the free use of carbolic acid-a drug to which many individuals show a marked idiosyncrasy-has often been responsible for the complication. As is the case in syphilis, the specific organism is destroyed when a sore becomes phagedænic. In nearly all phagedænic sores, the fusiform bacilli and the spirochætæ which exist in symbiosis with them, organisms which appear to be the cause of Vincent's angina and Balanitis gangrenosa, are usually to be found. Indeed, they are really the cause of the phagedæna.

\section{Ulcus Molle Serpiginosum.}

This type of ulcer is as chronic as any known, lasting, as it did in one case I saw, for twenty-nine years.

The condition is much more common than is thought to be the case; it is almost invariably wrongly diagnosed, and unless exactly the right treatment is given, curative measures are of no avail.

The primary lesion is a furuncle, the edges of which become blue, bluishwhite, and then break down until a distinct ulcer is formed.

The base of the ulcer is fleshy, uneven, and secretes freely. The edges are ragged, look as if they had been gnawed, and are deeply undermined; the overhanging portion is œdematous, and bluish-white in colour; external to this the colour becomes purplish, and still further out, and spreading for some distance into the healthy tissue, one sees the red colour of inflammation. The inflammatory zone is most marked where the ulcer is spreading, as it invariably spreads in one part more than in another; in fact, one pole may heal while the other is steadily advancing. A very favourite route for one tongue of the ulcer to take is down the genito-crural fold. Occasionally such a process reaches as far back as the anus. The scar left when the alcer heals is absolutely typical of the condition, and is well portrayed in Plate LX. 

PI.ATE LVI

No. 108.-A Chronic Spreading Ulcus Molle easily diagnosed by tile Raised Undermined and Irregular Edge.

Is this ease the many parallel chains of the eommon type of streptobaeillus were found

No. 109.-An Clcus Molle Caused by the Boat-Shaped Form of Ducrey's Bacilius

The sore heals slowly and not infrequently has a long incubation period. Note that the two halves are similar, that the base is rough, that a red inflammatory ring marks the slowly spreading edge, and that one edge of one half is healing. 
No. 108
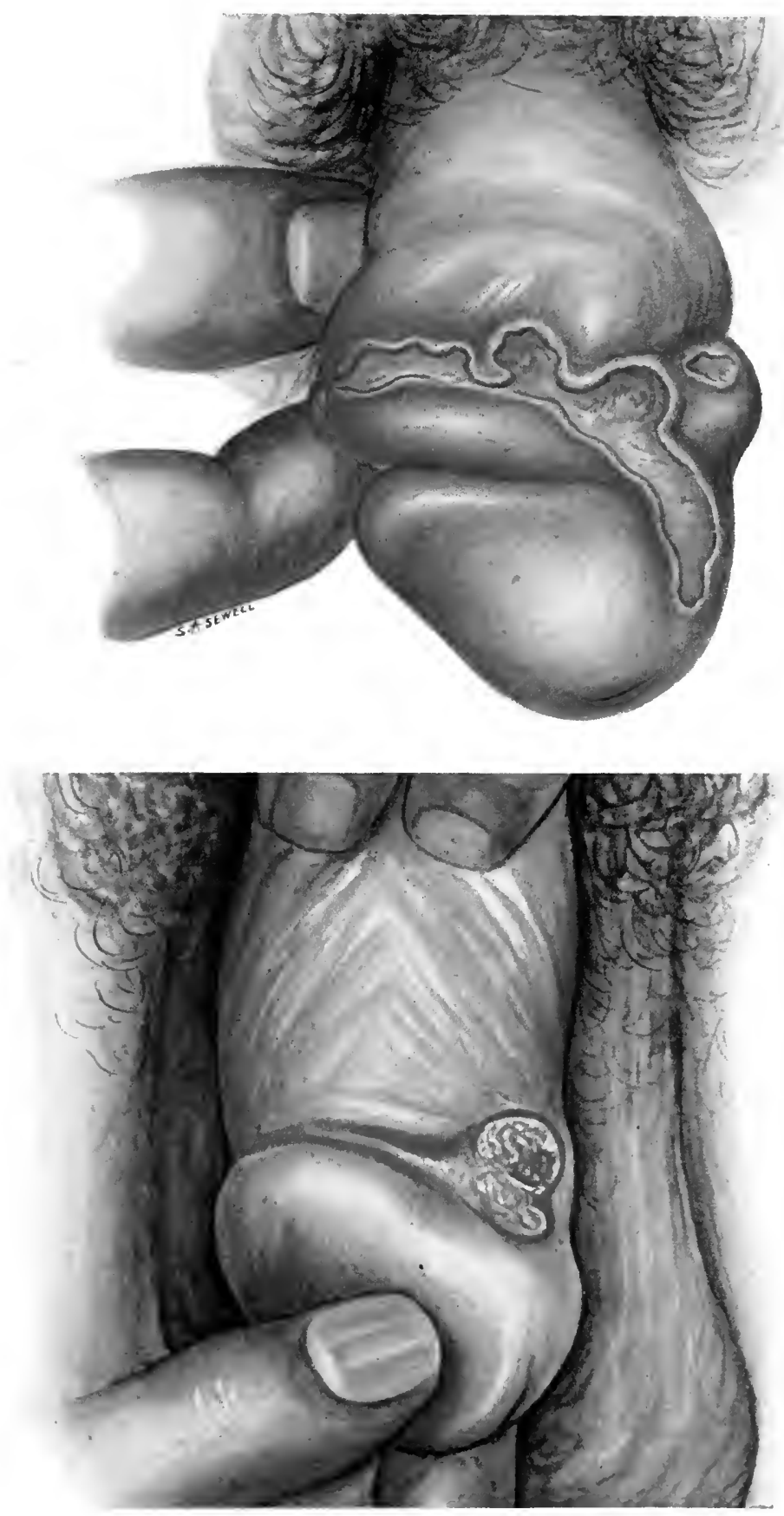

No. 109

Plate LVI 
CASE 119.-A man, aged 25 years, was shown by Mr. Shillitoe before the Dermatological Section of the Royal Society of Medicine, June 15th, 1911 (Plate LIX. is from this case), as a case of (?) Granuloma inguinale. The patient consulted Mr. Shillitoe first on March 16th, 1911, and gave the following history: "Just before last Christmas he developed multiple sores around the corona (soft sores), and a bubo, which was opened January 10th, and which had not healed on his arrival in England. He left Singapore on February 17th, having already developed the three ulcers still present in his left groin. He was told he had syphilis, and was advised to have salvarsan. During the voyage home he took 150 tablets of 3 minims each of iodine, together with local applications of pure carbo'ic, silver nitrate, etc., without any marked effect on the ulcerations. He had been four years in the East, and had been really ill with malaria during the first year only. Ten years ago he had albuminuria, following typhoid fever. The urine had been periodically examined since, without any fresh appearance of albumin, until early in February.

"When Mr. Shillitoe saw him on March 16th, the urine was acid, sp. gr. 1024, and contained a decided amount of albumin. He had three superficial ulcerations in the left groin, and an unhealed bubo. The bases of the ulcerations, which were not punched out, were glazed, devoid of granulations, and discharged a thin sanious fluid; the edges were rounded and firm. Mr. Shillitoe did not consider them to be specific, nor could he find other evidence of syphilis.

"March 23rd.-C.F.T. positive; no albumin, so he was put under mercury.

"March 31st.-The sores not healing, Mr. Shillitoe gave him in addition sanatogen internally and externally.

"A Aril 12th.-The sores were gradually closing, and granulations were appearing, which bled readily. He has increased one pound in weight-now 10 st. $12 \mathrm{lb}$.; there was a small trace of albumin and Herpes preputialis.

"April 21st, and again on $24 t h$. -0.59 grm. of salvarsan were injected intravenously.

"April 27th.-The sores were spreading at the edges."

The painting was done in June, after the excision of one ulcer had been attempted, without success. Later, $\mathrm{X}$-rays and also radium were tried, with no better results. Every conceivable drug was tried locally, but the extension could not be stopped. The only drug which seemed to do the least good was potassium iodide, given internally. When the salt was pushed up to 200 grains per diem, and the sores washed with perhydrol, and then dusted with iodoform, the ulcers, after severa! weeks, completely healed.

When the ulcers began to heal, zinc ionisation was tried, with a little success; iodine and copper ionisation were useless.

The C.F.T. was positive, presumably because the patient had had a very bad attack of malaria three weeks prior to the test being made.

Owing to the fact that the ulcers did not heal, the question of tubercle was raised, but when there was no reaction to tuberculin, and both von Pirquet's and Moro's reactions were negative, that disease was excluded.

Several films and cultures were made, with negative results; these were again repeated when the ulcers secreted more freely, and every time a pure culture of proteus resulted.

Vaccines made therefrom stopped the discharge, and removed the fearful odour to which it gave rise, without in any way causing the ulcers to heal. The proteus was Proteus vulgaris; it was Gram-negative, very motile, and the bacilli varied in length. It gave acid and gas in glucose and lactose, and it clotted peptonised milk. 
It rapidly liquefied gelatin. A rabbit which was injected died in forty-eight hours from acute septicæmia.

Pieces of tissue were removed and injected into a rabbit, a guinea-pig, and a mouse, intravenously, intraperitoneally, and subcutaneously respectively. Only the mouse died a fortnight later, and, in spite of a thorough examination of all its organs, nothing abnormal was discovered.

The bloods of the rabbit and guinea-pig were tested from time to time, with negative results.

The patient's own blood-count was as follows: Red blood-corpuscles, 4,500,000; white blood-corpuscles, 12,500; polymorphonuclear leucocytes, 51.56 per cent.; lymphocytes, $\mathbf{4 6} \cdot 35$ per cent.; cosinopliles, 1.99 per cent.; basophiles, $0 \cdot 207$ per cent.; lymphocytes-large, $26 \cdot 7$ per cent.; small, $73 \cdot 3$ per cent.

The lymphocytosis was doubtless due to the foregone attack of malaria, and had nothing to do with the complaint.

Just on the chance that a fungus might be the cause of the mischief, I tried some agglutination tests with a spore emulsion of sporotrichosis, with negative results.

Two pieces were removed and examined histologically.

The picture was typical of a granuloma. There were no giant cells, but a large number of amino-plasma cells. In places, the walls of the vessels were very much thickened, so that every pathologist who saw the sections diagnosed syphilis or tubercle at once.

In the most superficial layer of the undermined portion streptobacilli were to be found. The bacilli were Gram-negative, usually in pairs, and never in chains or more than five or six. No intracellular organisms were to be found.

Having demonstrated Ducrey's bacillus in section, I made several attempts to culture the organism on both rabbit's and human blood-agar, but failed.

CASE 120.-A man, aged 27 years, who had spent several years in the Malay States, came home to consult me for some chronic ulcers of the groin. In both groins were several ulcers, indistinguishable from those seen in the colouredillustration; they were extending above on to the abdomen, and below on to the thighs, and on both sides they had reached far down in the genito-crural folds. The ulceration began seven months before I saw the patient.

Five years before he had had some sores on the penis (Ulcera mollia), which healed up without any complications arising therefrom, such as bubo, etc. In November, 1911, the patient fell over a log of wood, with the result that, two days later, a swelling appeared in the skin in the inguino-scrotal folds on both sides. The swel!ings behaved like boils, so were lanced, and from that time onwards they became ulcers, which rapidly tended to increase in size. As no local application was of any use, the patient was put under an anæsthetic, and the ulcers were well scraped, with the result that they spread more quickly than ever.

When I first saw the patient he could not walk, owing to the pain caused by the ulcers; the ulcers discharged freely and had that peculiar indescribable odour which $I$ have noticed in most of the cases I have seen.

I made some films of the secretion from under the overhanging portion of skin in the region where an ulcer was spreading, and succeeded in obtaining Ducrey's bacillus. Separate streptobacilli were to be seen; also many in pairs, and some in chains; but, contrary to what one finds in ordinary soft sores, many of the leucocytes were crammed with a different form of Ducrey's bacillus. To prove that the ulcers were due to Ducrey's bacillus, I inoculated the patient's arm, and succeeded in obtain- 
. : 
PLATE LVII

No. 110.-A Typical Case of Ulcus Molle Elevatum

The patient has two sores, one on the penis and one on the scrotum. The penile sore is sharply circumscribed, irregular in outline, an ulcer which is slightly depressed beneath the surface, but the edge is not undermined and there is no circumferential inflammation. The scrotal sore is sharply circumscribed, more regular in outline, an ulcer which is well raised above the surrounding and healthy skin. The edge is red and inflamed, but there is no area of acute inflammation exterior to it. It will be noticed that both sores are red, and that they are not covered with pus. The sores were rough on the surface and bled easily on friction. 'The sore on the scrotum is a beautiful example of the so-called Ulcus molle elevatum. The incubation period of this type of sore is often a long one, it may be a matter of weeks, and it may be extremely resistant to treatment. It was the boat-shaped form of the streptobacillus which caused the sores in this case. The boat-shaped form of the bacillus is never found in such great numbers as the ordinary streptobacillus, nor does it occur in groups of long chains. The bacilli are fewer in number and more irregularly scattered about. 


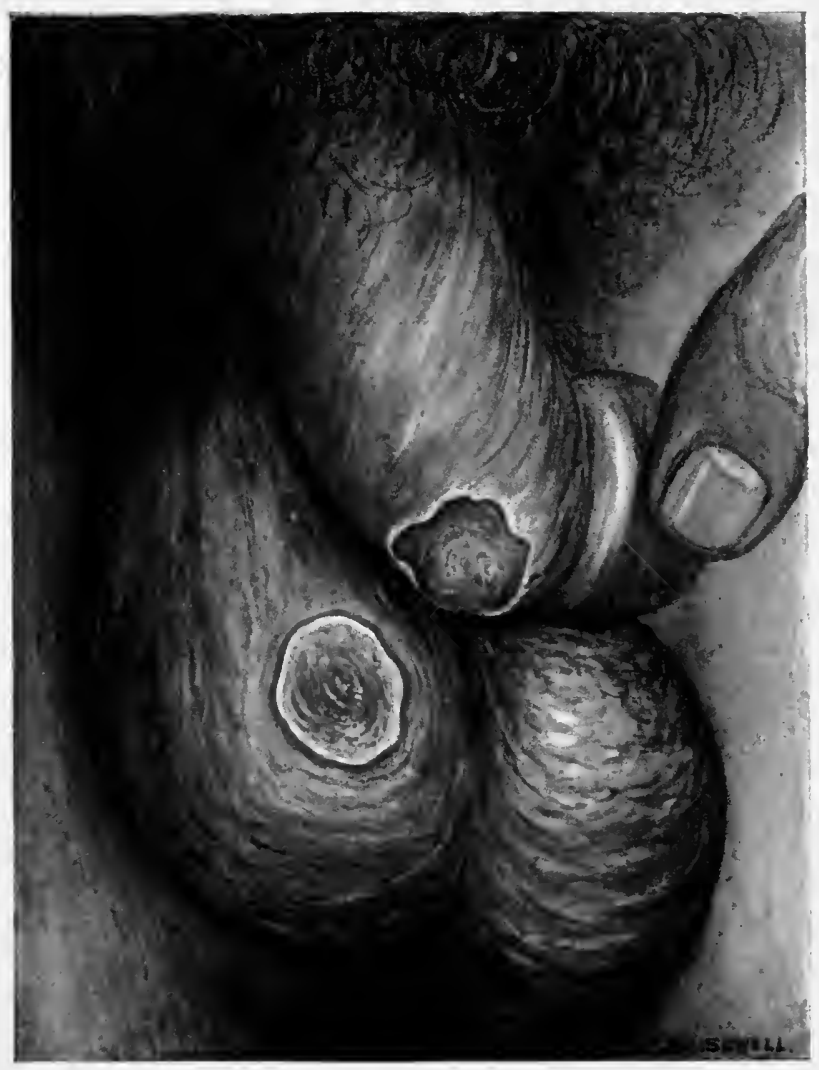

No. 110

Plate LVII 

ing, after the usual incubation period, a typical soft sore, from which I was able to isolate the ordinary soft sore bacillus.

Treatment consisted in gradually increasing doses of iodides, until the patient took 200 grains per diem; the maximum was maintained for one week, and then gradually decreasd, and so on until the ulcers had completely healed, which took place in three and a half months.

Locaily the ulcers were painted with camphphenol, and then dusted with iodoform.

CASE 121.-A man, aged 32 years, who had spent the last few years in Ceylon, consulted me for an ulceration he had in his groin and upper part of the thigh. The ulceration started in the groin as a little furuncle three years after he had had some sores on his penis (Ulcera mollia). He had never had a bubo. The furuncie became an ulcer, which spread down over the thigh, so that, when I saw the patient, practically the whole anterior surface of the upper half was one huge ulcer, although the superior part had commenced to heal. The ulcer had persisted for two and a half years, and, in spite of having had every kind of treatment imaginable, no good had resulted. From this ulcer I also found the intracellular form of Ducrey's bacillus. The treatment consisted in iodides internally, camphphenol and iodoform externally, and, in addition, the patient had five intravenous injections of tartar emetic every four days, $0.1 \mathrm{grm}$. in $100.0 \mathrm{c.cm}$. saline, with the result that, in three weeks, the ulcer completely healed.

CASE 122.-A man, aged 34 years, who had spent some years in the tropical part of Australia, consulted me for a chronic ulceration of one groin. The patient had had a soft sore, and a bubo resulting therefrom, which latter had to be incised. The soft sore healed rapidly, but the edges of the bubo became ulcerated, until a typical picture of Ulcus molle serpiginosum presented itself. This ulcer rapidly healed under potassium iodide internally, camphphenol and iodoform externally, and tartar emetic intravenously.

CASE 123.- The patient had a soft sore in 1900. Five weeks after the sore had healed a bubo appeared in the right groin, burst, then healed up, to break down again a few weeks later and form ulcers, which spread up over the abdomen and down the right leg to below the knee-joint. The ulceration was still spreading, in spite of various methods of treatment which had been employed, when the patient consulted me. Within fourteen days after an intramuscular injection of intramine $(10.0 \mathrm{c} . \mathrm{cm}$.$) ,$ preceded by an intravenous injection of colloidal iodine $(100.0 \mathrm{c.cm}$.$) , the ulceration,$ which had progressed without ceasing for seventeen years, had completely healed.

A patient who has, or who has had, a soft sore, should he contract another or infect himself in another part of his body, the second sore may take on the characters of an Ulcus molle serpiginosum, as evidenced in the following case:

CASE 124.-Two years ago patient had a soft sore and a bubo. At the same time he had scabies, and must have infected his leg from scratching with a finger which had come in contact with the bubo, for a typical Ulcus molle serpiginosum developed on the skin of the right leg, and went on spreading for two years. During most of this period the patient spent his time in various army venereal hospitals undergoing treatment for syphilis, which had no effect upon the ulcer. When the ulcer was dressed with intramine, and two intramuscular injections of the same drug had been prescribed, followed by one of trimine (colloidal mixture of manganese, iron, and zinc), the ulcer healed immediately. 


\section{CLINICAL ASPECT AND TREATMENT OF VENEREAL DISEASES}

Out of fourteen cases of Ulcus molle serpiginosum which $I$ have seen, in only two did the lesion appear and spread while the patients were in this country.

Summing up these cases, we find that we are dealing with a peculiarly chronic form of ulceration, which, at irregular periods, invariably follows a soft sore, appearing independently of a bubo, or after a bubo has been incised. It is further characterised by the fact that in most cases the patients had lived in the Tropics.

It is quite clear that any operative procedure makes matters very much worse, and that, unless exactly the specific treatment is prescribed, nothing is of any avail.

The best treatment is to prescribe colloidal iodine internally (3iii. ter in die, post cibos), to dress the ulcer with camphphenol and iodoform, or with intramine and a copper lotion or ointment as an alternative. Colloidal iodine should be injected intravenously $(100.0 \mathrm{c.cm}$.), followed two days later by an intramuscular injection of intramine $(3.0 \mathrm{c.cm}$.), which should be repeated $(5 \cdot 0 \mathrm{c.cm}$.) five days later. After this one to three injections of trimine or an antimony salt should be given. Lately, I have found the best results follow when trimine is injected intramuscularly and tartar emetic, antiluetin, or colloidal antimony is injected intravenously jointly, after the intramine has been given. Potter ${ }^{9}$ has reported recently some excellent results with intravenous injections of potassium antimony tartrate.

\section{Treatment.}

Great care must be taken if the sore is to be cauterised, since many cauterising agents may set up a phagedæna. The worst offender in this respect is diluted carbolic acid. The concentrated carbolic acid can be used with impunity, and a preparation I nearly always employ myself in camphphenol. If camphor and carbolic acid crystals are pounded together in a mortar, a syrupy liquid results, to which the name camphphenol is given. The sore should be dried as much as possible before this caustic is applied, as this renders its application painless. Cauterisation by means of zinc ionisation quickly causes soft sores to heal, but special apparatus is required, and it takes up some time.

A good apparatus for ionisation is a Morton's switchboard.* This apparatus has a rheostat, and can be worked from the main. A $2 \cdot 0$ per cent. solution of zinc sulphate should be applied to the sore by means of a piece of soaked lint attached to the negative electrode. The positive electrode should have a wide area, and it may be placed under one buttock. The strength of the current should be as great as the patient can stand, and the application should last about twenty minutes.

After cauterisation a powder should be dusted on, and undoubtedly iodo-

* Schall, New Cavendish Street, London, W. 1. 


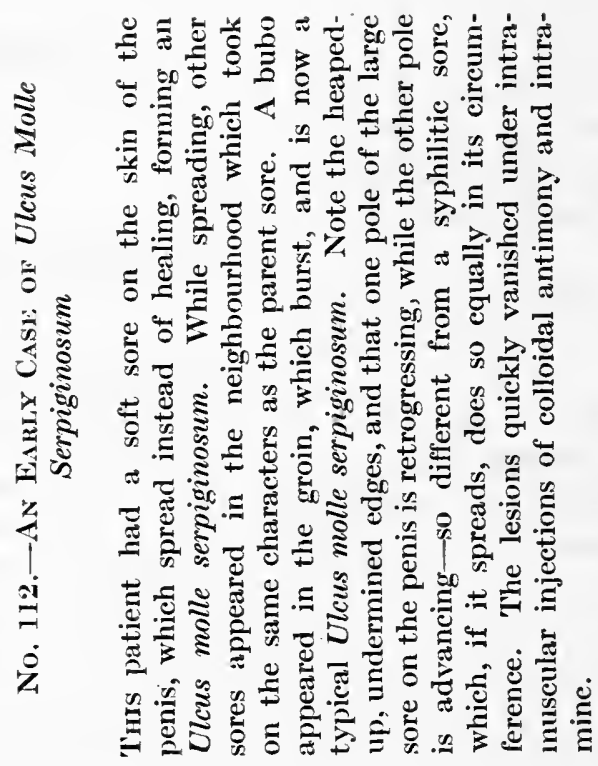

㝵

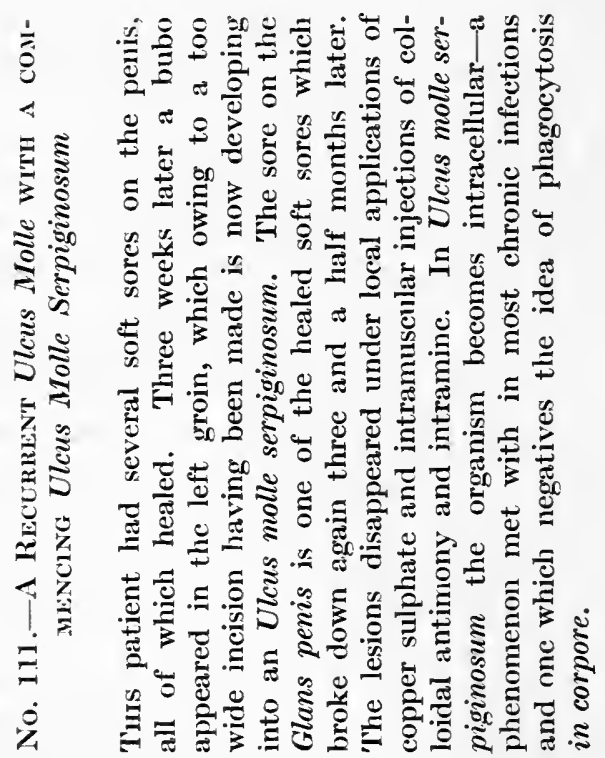




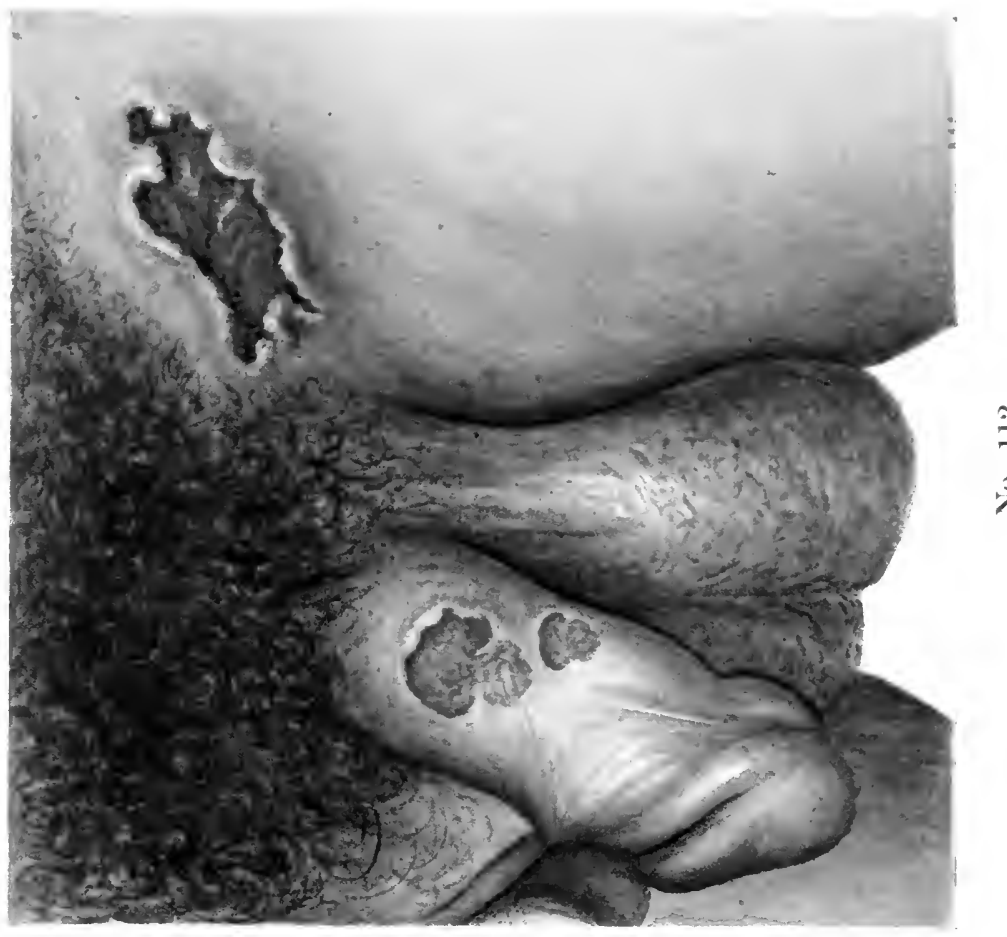

$\stackrel{3}{3}$
$\dot{3}$

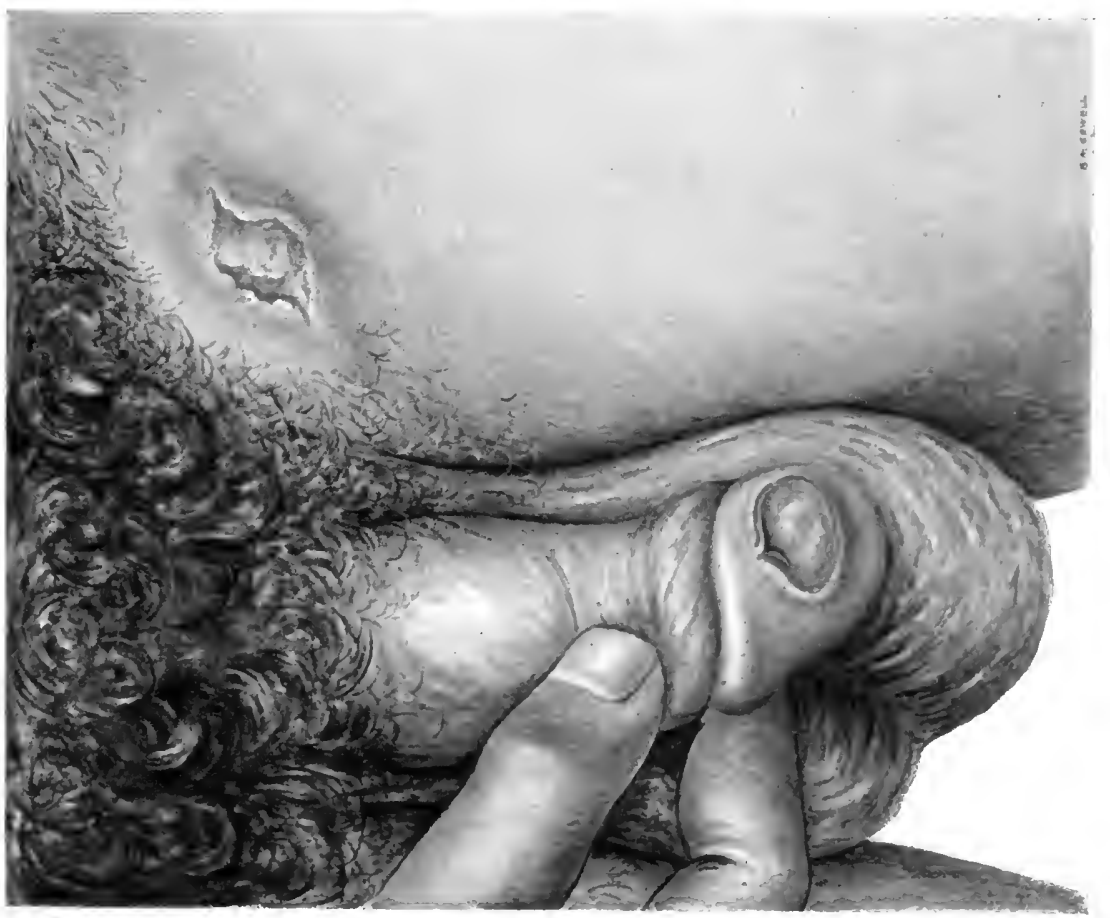

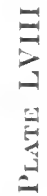

3
$\dot{8}$ 
form is the best. As the smell of iodoform often bars its use, I prefer to prescribe the following:

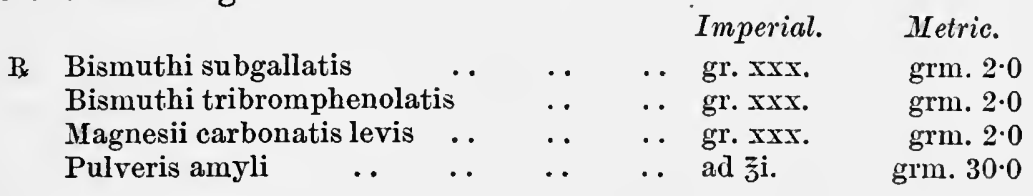

When the sores are chronic, intramine is an excellent dressing alternated with a copper lotion or ointment (1.0 to 10.0 per cent.). Healing is undoubtedly accelerated in these chronic sores by two intramuscular injections of intramine ( 1.0 and 1.5 c.cm. respectively), followed by one or two intramuscular injections of trimine $(1.0$ and $1.5 \mathrm{c.cm}$.). Previous treatment with arseno-benzene or mercury detracts from the value to be gained from the use of the drugs just mentioned; therefore it behoves the observer in all cases to make a correct diagnosis before submitting the patient to treatment.

As already stated, hot applications are extremely useful, hot sitz baths, hot air baths, etc. The sores should be exposed to the air as much as possible, and not firmly bound up in air-tight dressings.

If the patient has a tight foreskin, and the sores cannot be reached, and there is ever the risk present of phagedæna ensuing, the foreskin should be slit up, or even a circumcision should be performed. Under no other circumstances should any operative procedure be undertaken. The wounds invariably become infected, and phagedæna often sets in. I once removed, widely, and under the strictest antiseptic precautions, a soft sore which had barely been present for forty-eight hours, and the whole of the foreskin ultimately became infected and sloughed.

\section{Lymphangitis.}

Lymphangitis is most often seen on the dorsum of the penis, but it may also occur along the sides. It frequently gives rise to strands, which are to be so commonly felt in syphilis. Lymphangitis ex ulcere molli is never so hard as it is in syphilis; it is, moreover, painful and inflamed-i.e., the skin over it is red. While the lymphangitis still persists, and, more rarely, after it has vanished, a little swelling may appear anywhere along its course. This swelling is inflammatory, and soon breaks through the skin covering it; an abscess or an ulcer forms, and quickly assumes the character of a soft sore. Such a lesion is usually called a bubonulus. A bubonulus may heal spontaneously as quickly as it appeared, or it may spread and give rise to a lesion like Ulcus molle serpiginosum.

\section{Lymphadenitis.}

The organisms reach the lymphatic glands along the lymphatics. They may cause merely an enlargement of the glands, or suppuration in them. The term "bubo" is usually applied to the suppurative Lympladenitis ex 
ulcere molli, and one of the most interesting points about it is the fact that a bubo may not appear for weeks or months after the site of infection has healed and has been forgotten. Such a late bubo is very apt, in tropical countries, to be the starting-point of an Ulcus molle serpiginosum. The retardation is very probably due to certain of the organisms having taken up an intracellular habitat. As is the case with most abscesses, the causative organism is not to be found in the pus; hence a negative search for the streptobacilli, in the discharge from an abscess in the groin, is not against the lesion being a bubo. If one wishes to demonstrate the streptobacilli, it is necessary to take a scraping from the inner wall of the abscess cavity. In a large number of cases, the pus from a bubo is sterile; in the remainder, there is a mixed infection.

The pathology of a bubo is very simple, but in order that the best means of treating it may be adopted it is necessary to understand it thoroughly.

At first, several glands become infected with the streptobacilli. In one gland only does the inflammation proceed to suppuration. While the process is maturing, the gland approaches the skin, and becomes adherent to it. Either one of two courses is now open. The abscess bursts through the skin, or, laterally, into the inter-glandular tissue. If the route is the latter, then the suppurative process is almost bound to reach to, and to implicate, the neighbouring lymphatic glands.

If the abscess bursts through the skin, the lateral walls are usually strong, and they prevent infection of the inter-glandular tissue. Hence it can be readily seen that the employment of operative measures, before the bubo has pointed, or, in other words, before the lateral walls have been perfected, may easily allow the infection to become inter-glandular.

Incision should be delayed as long as possible, and recourse should be had to cold applications, in particular to evaporating lotions-

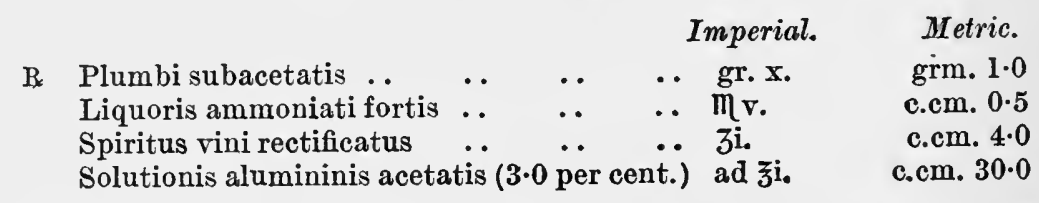

-and the observer will be astonished to find how many resolve. The overlying skin becomes pink and tender before any pus has formed in the gland.

Owing to the fact that the pus originates in the substance of one gland only, it follows that the quantity evacuated on incision will fall considerably short of the quantity expected, and it is likewise equally obvious that only a tiny incision is required to drain the bubo.

A long incision will break through the barriers formed by the lateral walls, with the result that the inter-glandular tissue is bound to become infected. A wide incision also exposes other glands, which are nearly always removed by the surgeon, for reasons unknown. 



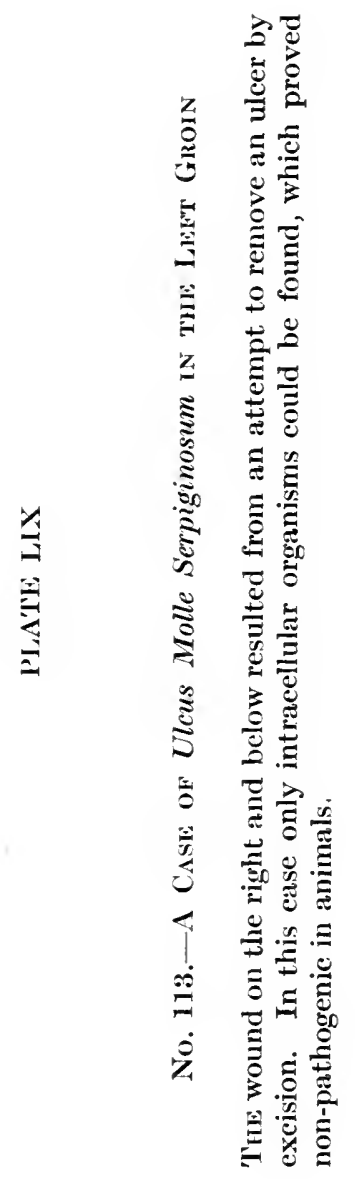




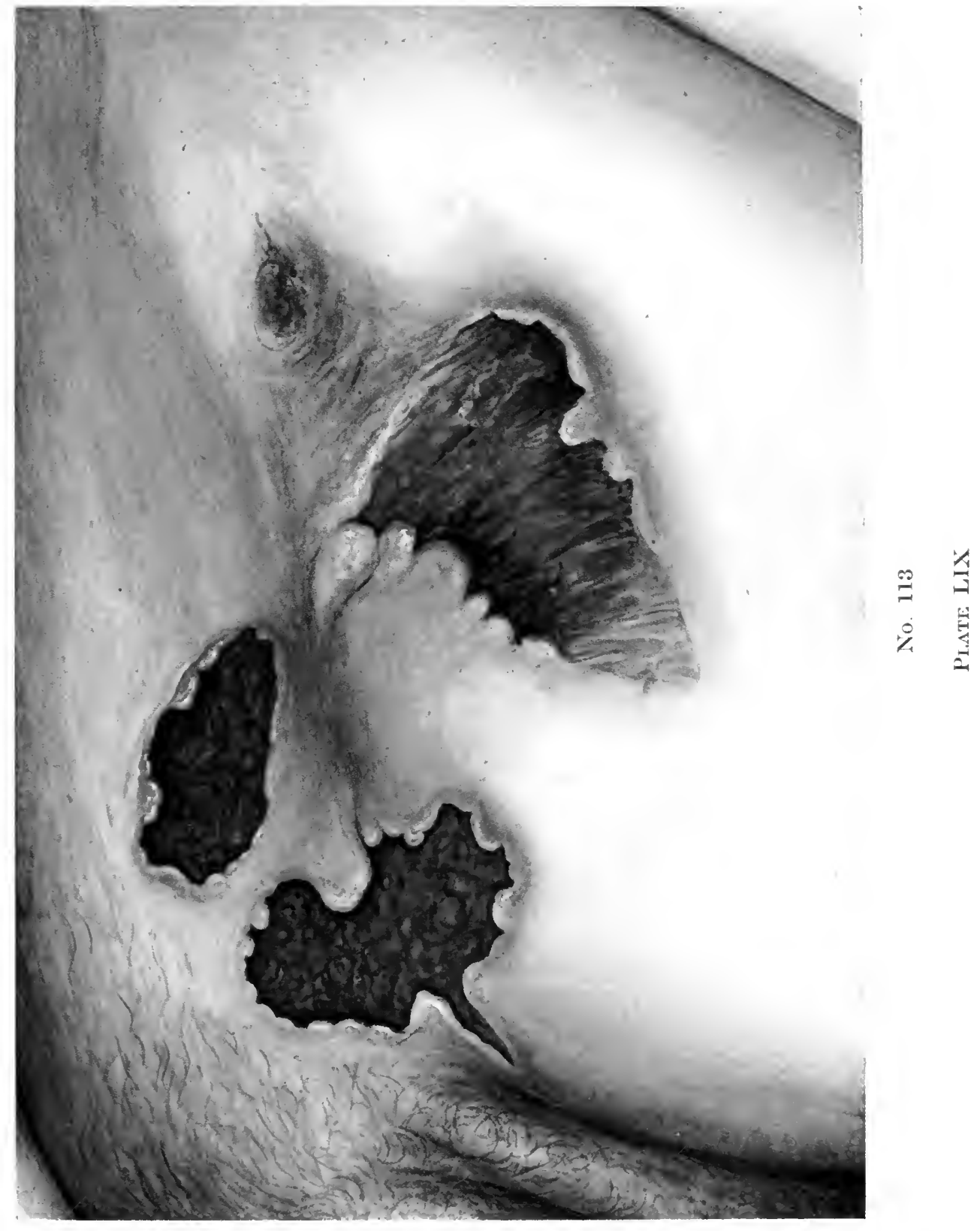




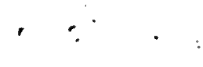

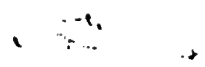





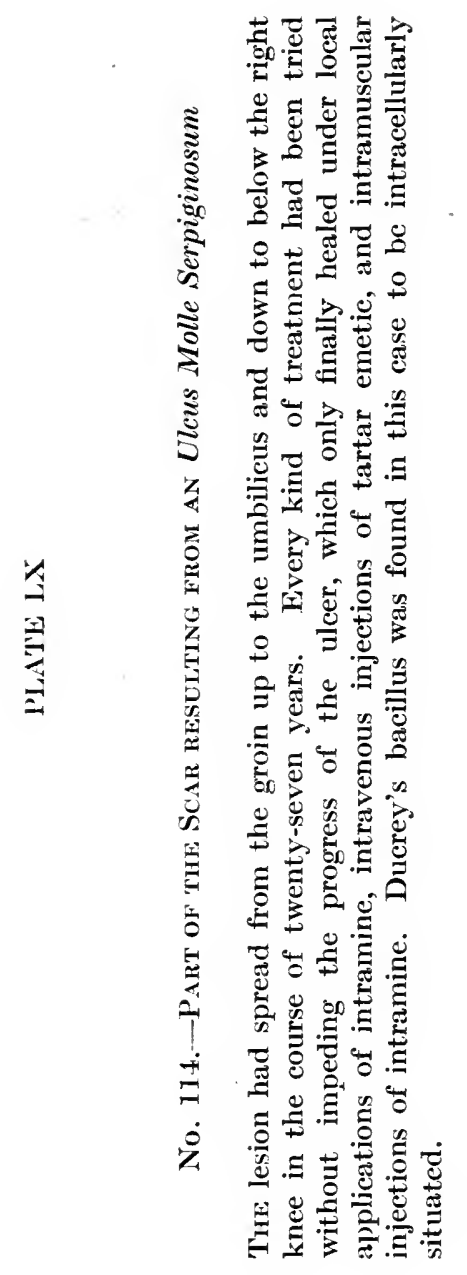




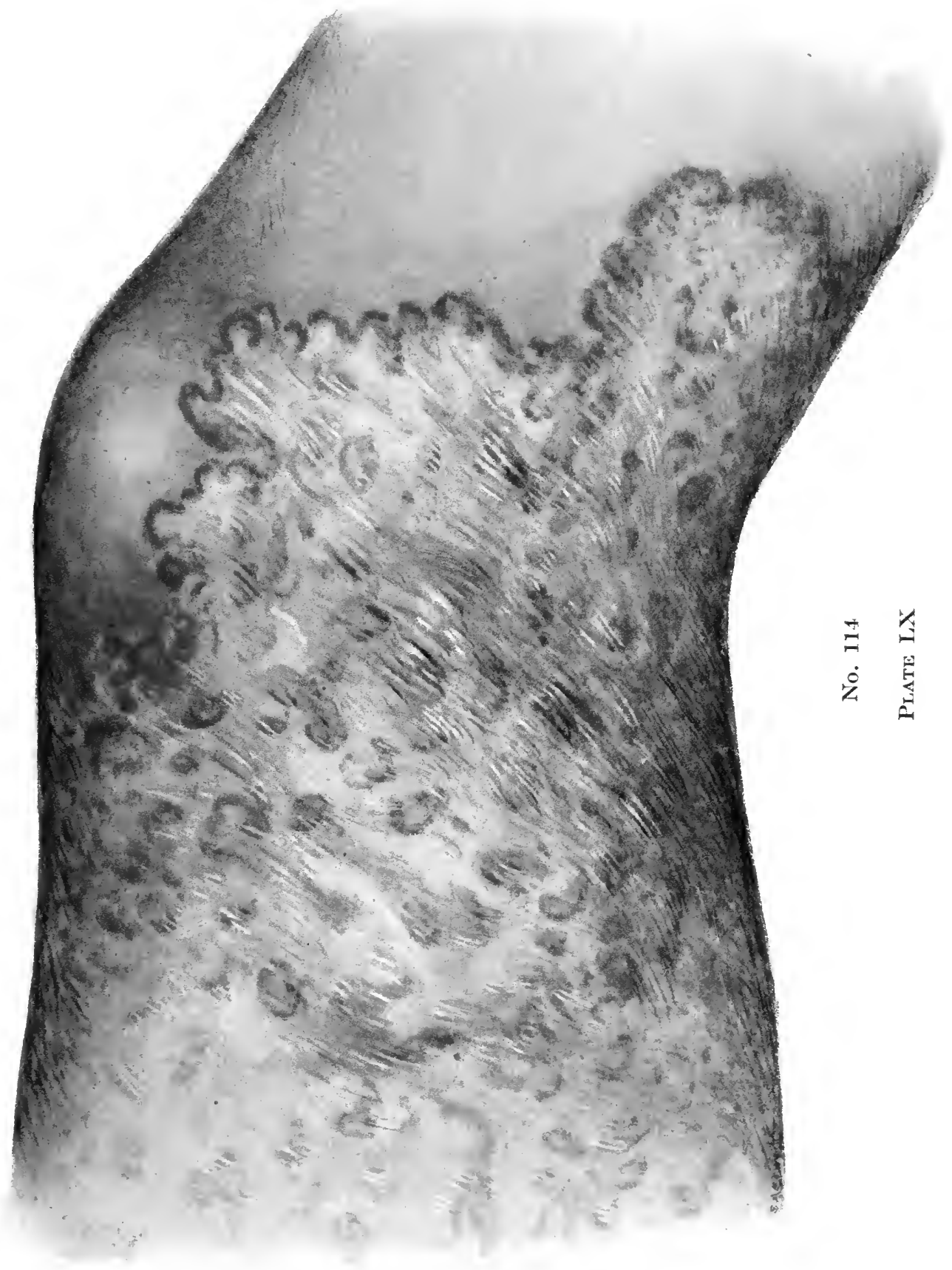



In the first place, removal of all the lymphatic glands in one inguinal region is not a simple matter, and permanent œdema of the corresponding half of the scrotum is a complication quite liable to result.

Moreover, the bigger the incision, the bigger the wound which has to granulate up from the bottom, with the result that a patient may be obliged to be absent from his work for six months or more.

As the vitality of the skin over a bubo is often very much impaired, any other procedure than a nick with a bistoury may destroy it altogether, and then the complicating factor of phagedæna has to be dealt with.

A nick of 0.5 to $1.0 \mathrm{~cm}$. should be made where the abscess is pointing, or where the skin is softest. The pus should then be expressed, and the cavity lightly washed out with saline. No powerful antiseptic should be used, owing to its tendency to set up gangrene in the edge. A fine piece of gauze should be left in the opening, the powder above mentioned should be dusted on, and the wound dressed daily until it is healed, which is usually a matter of days only.

My oxidising (trimine) and reducing (intramine) agents have proved most successful in the abortion and treatment of bubo. If a patient complains of pain in the groin, and shows signs of developing a bubo, an intramuscular injection of trimine $(1.0 \mathrm{c.cm}$.$) , repeated (1.5 \mathrm{c.cm}$.) in five days' time, may stop the process from extending. If pus has already formed, the injections will bring it to the surface, where it can be evacuated, and healing will rapidly follow. An incised bubo which heals slowly will mend quickly if two doses of intramine, followed by one of trimine, are injected. It must not be thought that every suppurative inguinal adenitis is the result of a soft sore infection. Apart from syphilis and gonorrhœa, which may be a cause, scabies and echthymatous impetigo have during the world war been a fruitful source for buboes, especially among the coloured troops. In several West Indians I saw buboes following scabies, and on incision they developed chronic ulcers, which closely resembled Ulcus molle serpiginosum. Intramine and colloidal manganese worked like a charm in these cases. In my opinion chronic soft sores are increasing in frequency, and they are more often single than used to be the case. Taking the last hundred cases of Ulcus molle, $19 \cdot 0$ per cent. were single.

1. Ducrey (1889): Giorn. Ital. d. mal. Ven. e. d. Pelle, xxx., 377.

2. DuCREY (1889): Monatsh. f. prakt. Derm., ix., 387.

3. DuCRey (1895): Monatsh. f. prakt. Derm., xxi., 57.

4. ToMasczewski (1912): Handbuch d. Geschlechtskrankheiten, ii., 613.

5. UNNA (1895): Monatsh. f. prakt. Derm., xxi., 61.

6. Nicolle (1906): Presse Méd., xiv., 265.

7. Dobreuilh (1893): Arch. Clin. de Bordeaux, ii., 500, 513.

8. McDonagh (1914): Brit. Journ. of Derm., xxvi., 1.

9. Potter (1918): Medical Journ. of Australia, ii., 65. 


\section{CHAPTER XX \\ UNCOMPLICATED GONORRHEA}

History.

JUST as the history of syphilis is vague, so is that of gonorrhœa clear. There appears to be no doubt but that the Israelites were sufferers therefrom, as the disease is referred to in Leviticus. According to the famous Egyptologist, Ebers, in his so-called "Papyrus Ebers," which was published in 1874, the ancient Egyptians were familiar with the disease at a period prior to Israelitish history. The first detailed description of the discharge hails from Aretæus, a Greek physician, born in Cappadocia in the first century A.D. Aretæus described the discharge continuing day and night, and the depressing and lowering effect it had upon the sufferer, and regarded the discharge as unfruitful semen, an opinion held later by Galen, who was born about A.D. 130. The word gonorrhoea means flow of semen, and comes from the Greek yóvos,

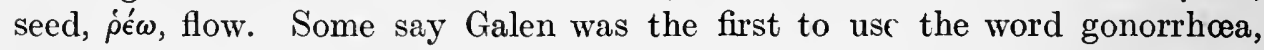
but as Cleopatra was familiar with the disease, and probably herself a sufferer therefrom, and refers to it in the words, "Aliquando etiam spermatis spontanei et importuni fluxu fomince fatigantur quod et Grceci gonorrhoeam appelant," it is more probable that Hippocrates invented the word, as Cleopatra was dead before Galen was born. There is evidence that Hippocrates, in the fifth century before Christ, was cognisant of the disease, both in men and women. Ætius, a Greek, who was born in Mesopotamia about A.D. 500, described ulcers in the urethra as resulting from gonorrhoea, and strongly advised against injections being used. For, roughly, the next 600 years we have no records of writings on gonorrhœa except from the two well-known Arabian physicians, Avicenna and Albucasis, who added little or nothing to the knowledge which had emanated from their Greek masters and forerunners. In fact, it was not until Bernard Gordon, in the thirteenth century, and Valescus von Taranta, in the fourteenth century, published their writings that we are able to pick up the trail again. Both these authors drew attention to the social evil of the disease, the latter saying: "Gomorream non solum infert damnum individuo, imo et toti speciei humani generis. Quod si homines omnes paterantur gomorream, sic cito humanum genus deperiret." Benedetti, a century later, said: "Veluti enim pestilentia plurimos afflixit." The next historical period is one of confusion between gonorrhœa and syphilis, a fact 
which certainly lends colour to the view that syphilis had not invaded Europe at any rate prior to the fifteenth century. Paracelsus, the well-known GermanSwiss pseudo-physician and alchemist, regarded gonorrhœa as an initial symptom of syphilis, an error which many of his followers continued. It is not clear who was the first to recognise that gonorrhœa was not a discharge of semen; but the first attempt to change the name, to avoid the mistake surviving, was made by Turquet de Mayerne, who called the disease muóp’ora-a flow of pus. According to Proksch, ${ }^{1}$ from whose admirable historical account many of these notes are taken, gonorrhœa became recognised as an inflammation of the urethra and the lacunæ, which were discovered by Terraneo, in 1709, as a result of six post-mortem examinations. These observations were finally confirmed by Cockburn in 1715, and by Morgagni in 1719. Although Cockburn and others described the genital complications of gonorrhøea, and pointed out that the lesions did not disappear with mercury, as did those of syphilis, clearly showing that many held that the two diseases were distinct, the unity view again came into prominence through Hunter's unfortunate experiment in 1767. Benjamin Bell, in 1793, James Tongue, in 1801, and Jean Hernandez, in 1812, all inoculated themselves with gonorrhœal pus and developed gonorrhœa only; but Hunter's name was held in such esteem that it was not until Ricord, in 1838, as a result of several experiments, proved that gonorrhcea was a distinct disease that the dualists were finally proved to be correct. The history of the treatment of gonorrhœa is not particularly interesting, and consists mainly in the antiphlogistic therapy which was practised for all diseases alike for many hundreds of years. Avicenna advised the introduction of a louse into the patient's urethra, and for retention Bernard Gordon advised hot-water baths, combined with the application of living or crushed bugs to the penis.

For many years the favourite injection was woman's milk. Balsams appear to have been introduced by Franz de la Boë Sylvius, a German physician, in 1674; and in this country by Thomas Sydenham, in 1680. According to Renaud, ${ }^{2}$ the first preventive measure recommended was micturition immediately after coitus, a teaching which emanated from the school of Salerno. In the thirteenth century William of Salicet advised washing out with water after suspicious intercourse, and his pupil, Lanfranc, used ablutions with equal parts of water and vinegar, to which the patient's urine was sometimes added. John of Gaddesden is credited with invention of the suspensory bandage, and at the end of the fourteenth century the same device was used by Guy de Chauliac, a French physician, who practised at Lyons and Avignon. The causative organism was discovered by Neisser, ${ }^{3}$ in 1879.

\section{Incubation Period.}

The usual incubation period varies from two to eight days. Occasionally it may be very much longer. The longest I have ever known was just over six weeks. In all infectious diseases-especially is this the case with gonor- 


\section{CLINICAL ASPECT AND TREATMENT OF VENEREAL DISEASES}

rhœa-several factors are at work which influence the infection. There is the resistance of the patient to be considered, the strain of the organism with which he is infected, the quantity of infected material which is implanted on the patient, and the area over which it is spread. Any one of these may cause a variation in the length of the incubation period. In a patient who has had the disease before, and has been cured, the incubation period is more likely to be shorter than usual, and in a patient who has had the disease before, and who is now suffering from a recurrence, the incubation period may be only twelve or twenty-four hours. Since the introduction of the preventive measures, which are now in wide use in this country, it is not uncommon to meet with cases in which the incubation period has been long, and in which the disease, to use an Irishism, runs a chronic course from the start.

The gonococcus is not necessarily a pus-producing organism; note how comparatively infrequently gonorrhœal complications are associated with pus formation. When pus does develop, it is nearly always due to the supervention of a secondary infection; therefore it is probable that the main result of the preventive treatment is to cut out this factor in the disease. The gonococcus is an organism which calls for production of fibrous tissue on the part of the host; note the readiness with which adhesions form in a gonococcal arthritis. It is well to bear this in mind, because the "mild" infections which appear to be so common now are more apt to be followed by stricture than infections which are ushered in with an acute discharge.

\section{The Manner of Spread of the Organisms.}

When gonococci first are implanted upon the urethral mucous membrane, they multiply extracellularly, and produce no pus. In a few days, polymorphonuclear leucocytes reach the lumen of the urethra, viô the spaces between the epithelial cells, from the bloodvessels in the sub-epithelial tissue. Then the organisms become intracellular, and, in my opinion, live and multiply at the expense of the polymorphonuclear leucocytes. From this time onwards pus is formed, and the gonococci wander in between the epithelial cells, possibly in search of the polymorphonuelear leucocytes as they are on their way to the surface. As time progresses, the number of gonocccei diminishes, and their destruction is brought about in three ways: (1) by antibodies circulating in the blood-stream; (2) by chemical substances which emanate from the epithelial cells; (3) by the growth of other organisms.

From almost the very start, the gonococci may reach the sub-epithelial tissue, and may even enter the blood-stream and give rise to metastases. I once had a case which developed acute polyarthritis and teno-synovitis on the seventh day of the infection, while the infection was still limited to the anterior portion of the urethra. It is highly probable that the production of antibodies occurs very early in the disease, since the administration from the very start of potent vaccines, which increase the antibody content of 
the serum, does undoubtedly influence the future course of the infection. It cannot be proved when antibodies are first formed, as we have no test which can be applied in gonorrhœa to detect infinitesimal quantities. The C.F.T. is not nearly delicate enough.

The reason why I think the epithelial cells can destroy gonococci is because some individuals seem more or less immune to gonorrhœa, because the vaginal mucous membrane of adults is practically never affected, while that of young girls is especially prone to be affected, and because both the nasal mucous membrane and the buccal mucous membrane are very seldom involved. The secretion of the epithelial cells in the different localities must have varying anti-gonococcal properties. Considering that we are obliged to use strong antiseptics in the treatment of gonorrhœa, it would be well worth while to pay some attention to the chemistry of the secretion from the nasal and buccal mucous membranes, with the hope of finding a non-irritative substance which would destroy the gonococci.

The advent of other organisms often means the destruction of the gonococci. Although gonococci may be found in urethral abscesses, prostatic abscesses, in the pus from a suppurative epididymitis and adenitis, it is far more common to fail to find the gonococcus, or even to culture it. In probably over $90 \mathrm{per}$ cent. of the suppurative lesions primarily caused by the gonococcus, only extraneous organisms are to be found, and, in very many of these cases, the secondary infection often results in the spontaneous cure of the gonorrhœa.

After the organisms have been multiplying for some time in the anterior part of the urethra, they gradually extend backwards, and, usually while the condition is still acute, reach the posterior part of the urethra. Occasionally the inflammation may be chronic before the posterior part of the urethra is affected, and the patient may have signs of a chronic prostatcurethritis, although the posterior infection has never been acute.

Once the posterior part of the urethra is affected, there is more likelihood of the organisms getting into the blood-stream, and of their giving rise to metastases; still more likely is this the case when the prostate and seminal vesicles are implicated.

The organisms spread up the prostatic ducts and infect the prostate in the majority of the cases in which the disease spreads to the posterior part of the urethra. The organisms may also spread up the seminal ducts, reach the vesiculoe seminales, which they frequently infect. From here they spread along one or both rasa deferentia, which usually escape infection, for a reason not yet known, and reach one or both testicles, which quickly succumb. Hence it follows that it is almost invariably the lower pole of the epididymis which is involved.

Although arthritis is most apt to occur in those cases with vesiculitis, it is odd, in view of the fact that the same is the case with epididymitis, that arthritis and epididymitis very rarely accompany one another. 
Although the organisms may reach the bladder by direct extension backwards of the pus, from the prostatic portion of the urethra, the bladder very rarely is infected.

The gonococcus, or its toxine, may produce lesions in almost any part of the body. Lesions produced by the gonococcus itself are metastatic, and those produced by its toxine are toxic, and it is by no means always easy to say whether such and such a lesion is a metastatic or a toxic one. Observers are still very much divided in their opinions as to whether the so-called rheumatic iritis is due to the gonococcus or to the gonotoxine. These various metastatic and toxic lesions will be dealt with in a separate chapter, after the local infection has been described, and, before the local infection is discussed, it would be as well to make a few remarks upon the anatomy of the part.

\section{Anatomy.}

The male urethra is divided into pendulous, bulbous, membranous, and prostatic portions. Of these, the two anterior-which lie in front of the triangular ligament-are surrounded by cavernous tissue; the two latterwhich lie behind that ligament-by muscular tissue. The membranous portion is surrounded by muscle fibres, contraction of which compress its. lumen, and so cause that retention and straining which is typical of a posterior infection. Since the two anterior portions of the urethra are surrounded by cavernous, and the two posterior by muscular tissue, we can make a clinicoanatomical distinction, and assert that an anterior urethritis is an inflammation of cavernous tissue, while a posterior urethritis is an inflammation of muscular tissue, the triangular ligament being the anatomical demarcation between the two clinical types. It is probably owing to this difference of tissue in the two parts of the canal that strictures are most apt to develop in the posterior part of the urethra, where fibrous tissue formation and contraction can more easily take place.

\section{Symptoms.}

The first signs noted are that a few hours after passing water the urethra contains a greyish-white viscid fluid, its meatus is reddened and swollen, but the urine is clear. A few days later the secretion thickens, and is stained yellow, or green, or blackish, from hæmorrhage of the inflamed mucous membrane. It forms a "bead" over the orifice in the morning. The passage of urine causes that burning sensation described by a coster as "like passing red-hot pins and needles." At night erections are frequent, and excruciatingly painful.

The inflammation, which begins at the orifice, now extends backwards along the penile portion; the danger and severity of the attack depend upon the extent of spreading. The patient now runs an evening temperature, and cannot sleep on account of painful erections. 
By the end of the second week the infection has travelled backwards as far as the bulbo-membranous junction. The bulbous portion being inflamed, pressure on the perinæum causes pain. The tenderness or non-tenderness of the perinæum gives a useful key to the extent of the inflammatory process.

At this stage two courses are possible. Either the inflammation is localised to the penile and bulbous portions in front of the triangular ligament, and constitutes an anterior urethritis only, or it may spread back to the membranous and prostatic portions, constituting a posterior urethritis.

In anterior urethritis the symptoms begin to subside at the end of the third week, and have, with the exception of a slight tingling sensation on micturition, disappeared by the end of the fifth. The discharge is less in amount, thinner, and whiter than that of a posterior urethritis. So long as there is discharge, the urine appears turbid when passed, which forms a deposit on standing. The discharge consists of epithelial and pus cells, which take the form of threads and flakes on account of the acidity of the urine. About 75 per cent. of gonorrhœa patients suffer from a posterior urethritis. The symptoms which distinguish a posterior from an anterior infection are frequency of micturition during working hours, but not necessarily at night. This frequency is accompanied by strangury-a spasm of the sphincterwhich causes either complete retention, or the passage of urine in drops after long straining. Hæmaturia is common, the blood appearing with the last drops of urine. Generally the patient complains of a burning or tickling sensation about the rectum and anus.

The discharge both stiffens and stains linen; but so does the pus from a balanitis, so that, if the glans penis be inflamed, the patient must draw back the foreskin and wipe the meatus before micturition, or the usual "twoglass test" will be useless. The two-glass test should be carried out in every case. The patient, whose bladder must be nearly full, is made to micturate into two test glasses, and the contents are compared. Into the first he passes a few ounces; into the second, the remainder. If the contents of the first glass be thick, he has an acute anterior urethritis; if the contents of the second be also thick, then he has a posterior urethritis.

The pus of anterior "cavernous" urethritis, having nothing to keep it back, discharges freely at the meatus, while any that remains in the canal will be flushed out by the first flow of urine. That first portion of urine must, therefore, be clouded, while the remainder may be clear. On the contrary, the pus of a posterior "muscular" urethritis is shut in between the sphincter prostatce, on the one hand, and the compressor urethroe, on the other.

When urine has not been pased for some hours, the amount of pus secreted becomes greater than the space can contain. It must then go in the direction of least resistance, which is through the prostatic sphincter into the bladder, clouding the urine therein. If water be now passed into two glasses, both will be thick, but the former the more so, as it contains also that pus which was left in the urethra. Should the quantity of pus formed be not greater 
than the urethra can accommodate, there will be no regurgitation, and the second glass will be quite clear.

The morning urine is the best on which to try this test, as, owing to the longer retention of urine, more pus is formed, and therefore regurgitation is more likely. Some points about the morning urine should be noted. Pus dissolves in urine owing to a trace of a proteolysin. Hence, should the morning urine be first examined in the afternoon, a wrong idea may be formed. The presence of this proteolysin also accounts for the occasional loss of an albumin reaction in cases of slight nephritis, being present one day and absent the next, and also for the disappearance of casts. Pus dissolves more quickly in morning than day, warm than cold, acid than alkaline, urines. Bacillus coli communis is also said to have the power of dissolving albumin.

A very simple method of telling whether the posterior part of the urethra is affected, should the test already mentioned fail, is to pass a catheter as far as the triangular ligament, and wash out the anterior part of the urethra with a weak solution of boracic acid. This is collected into a glass, and examined. Such a procedure washes out all the threads which are in the anterior part of the urethra. The patient is then made to pass his water into another glass. Should any threads be seen, they must have come from the posterior part of the urethra.

In cystitis, both urines are thick, but the second thicker than the first, because the pus produced in the bladder settles to the bottom, and is passed last. Cystitis may be diagnosed from posterior urethritis, when both do not occur together, for the urine is usually alkaline in cystitis, acid in urethritis. Microscopically, one finds the typical transitional bladder epithelial cells in cystitis, in which, too, pain in the small of the back and in the region of the symphysis is common.

Two pitfalls wait for him who would diagnose posterior urethritis:

(a) The thickness of the urine may be due to soluble phosphates, which clear on adding dilute acetic acid, or to oxalates and insoluble phosphates.

Patients with sexual neurasthenia are very prone to have phosphaturia and oxaluria. During the fruit season phosphaturia is very common, and patients who have had a meal devoid of meat, and who have partaken freely of green vegetables, and who have drunk large quantities of effervescent lemonade, are almost certain to have phosphaturia for some hours afterwards.

Vaccines, and the chemotherapeutic agents now used in the treatment of gonorrhœa, produce phosphaturia, as a result of the reaction they cause.

(b) It is difficult to get patients to do the two-glass test carefully. If you suspect the patient of carelessness, ask him how often he gets up at night to pass water. Nocturnal frequency is usual in posterior urethritis and cystitis, not so in anterior urethritis.

Chronic Urethritis.-The characteristic picture of chronic urethritis is morning discharge, sticking together of the lips of the urethral orifice, cloudiness of, or threads in, the urine; but one must remember that there are patients 
who show all these features, and yet they have not got gonorrhœa, but only its after-effects. After chronic urethritis there occurs a collection of flat epithelial cells in the recently diseased areas, and these cells desquamate to such an extent as to produce a milky discharge and threads. The differential diagnosis lies in the fact that, microscopically, these threads show mainly flat epithelial cells, and only a few pus cells.

There is one great difference between acute and chronic gonorrhœa. In acute gonorrhœa the inflammation is diffuse, and spread over the greater part of the urethral mucous membrane. In chronic gonorrhœa it affects limited areas. There are two stages in chronic gonorrhœa: one, in which there is a connective-tissue hyperplasia with hyperæmia, swelling of mucous membrane, catarrhal desquamation of epithelium, especially of Littré's glands; the other, in which connective tissue is covered by an overgrowth of flat epithelial cells.

The first stage might be ealled subacute urethritis, and is not limited to definite areas. The second stage-may be called the chronic stage. The two stages admit of differentiation, since in the former the catarrh of the mucous membrane clouds the urine, whereas in the chronic for $\mathrm{m}$ the urine is clear and contains threads only.

Inflammation may spread under the mucous membrane, and produce, if it spreads forward, peri-urethritis or cavernitis; or, if it spreads backward, prostatitis. The diagnosis between superficial anterior chronic urethritis and the deep form must be made with Otis's urethrometer, with a bougie à boule, or with a Kollmann's dilator. Superficial inflammation causes no stricture; but the deep form, which affects cavernous tissue, quickly reduces elasticity and dilatability, and so causes broad strictures.

The diagncsis of urethritis is not a difficult matter. In the acute and subacute forms it is very easy to tell, either by the two-glass test or by the irrigation test, whether the infection has reached the distal side of the triangular ligament or not. In the chronic form it is not so easy, as both the tests just mentioned may fail to give an accurate result.

Much may be learned by watching the patient pass his water. If he is unable to stop the flow when asked, then it is probable that he cannot use his compressor urethra, because fibrous tissue has taken the place of the muscular. If this has occurred, or a stricture has formed, then the patient will shake his penis to rid the urethra of the last few drops of urine. Shaking may sometimes fail to produce the desired effect; then the patient notices that he passes some urine as soon as he has tidied himself up after the act of micturition.

One can ascertain for certain whether one is dealing with a chronic anterior or a chronic posterior urethritis by an endoscopic examination of the urethra. There are two forms of endoscope: one with which you can see the anterior portion of the urethra only, and the other which allows a view of the whole urethra.

An endoscopic examination of the anterior part of the urethra is, to all 


\section{CLINICAL ASPECT AND TREATMENT OF VENEREAL DISEASES}

intents and purposes, never required. If the endoscope is needed at all, it is for the examination of the posterior part of the urethra, as other tests used for diagnosis of a chronic posterior urethritis may fail. An endoscopic examination is needed, then, only in cases of chronic posterior urethritis. Many cases of chronic posterior urethritis are complicated by a narrowing of the urethra, which prevents the instrument from being passed, and, in the majority of cases of chronic posterior urethritis, the prostate is implicated, and the prostatitis is a more serious condition than the chronic urethritis. Prostatitis cannot be diagnosed by an endoscopic examination, only by a digital examination per rectum.

Assuming that an endoscopic examination informs the observer that the patient is suffering from a chronic posterior urethritis, the observer is no better off, as the treatment of chronic urethritis is the same, whether the inflammatory process is limited to the anterior or to the posterior part of the urethra. As a chronic anterior urethritis is a very rare condition, one will not be far wrong in diagnosing every case of chronic urethritis as one of chronic posterior urethritis-indeed, the word "posterior" becomes superfluous, and the mere mention of chronic urethritis will convey the idea that the posterior part of the urethra is affected, and, in nine cases out of ten, the prostate is also affected. Posterior endoscopy is far from being a simple manœurre; it is often a very dangerous one, as time after time $I$ have seen the patient's condition very much aggravated by its use. Posterior endoscopy has its adherents, and Wossidlo's new pattern urethroscope, through which treatment can be applied to the spot affected, has in certain quarters met with a great réclame.

I can honestly say that I have never yet met a case which has been cured, although the operator may have pronounced him to be so, by endoscopic treatment, and I have seen many made very much worse. Many postgraduate courses are given in urethroscopy on the Continent, and $I$ have frequently noticed that many of the instructors never use the urethroscope on their private patients, unless it be to impress them.

Summing up, I am of the opinion that urethroscopy is only an infinitesimal aid to diagnosis, and no help in the treatment, and some of the most chronic cases of gonococcal urethritis I have ever seen have been kept going by use of the urethroscope.

From some years' experience with catheters $a$ boule (Teevan's catheters), and the sensation the passage of the same imparts to the fingers, I am sure as much knowledge can be gained of the condition of the urethra by the passage of one of these catheters as may be achieved with an urethroscopic examination, without exposing the patient to the risk of injury. Furthermore, drugs may be instilled into the urethra viâ a Teevan's catheter at the spot they are most required, as the holes are in the base of the acorn (vide figure on page 281). 
The more cases of chronic urethritis I see, the more I am beginning to realise that, not only have many of them been caused by too much treatment, but that many are kept in being by too much treatment, or by too drastic an administration of it.

Why is chronic urethritis so difficult to cure? For the simple reason that the gonococci have invaded the sub-epithelial tissue. Broadly speaking, local treatment is not going to have much influence upon these sub-epithelial gonococci; cauterisation of the surface does not reach them, nor does cauterisation of a follicle, should there be proof that the gonococci had obtained

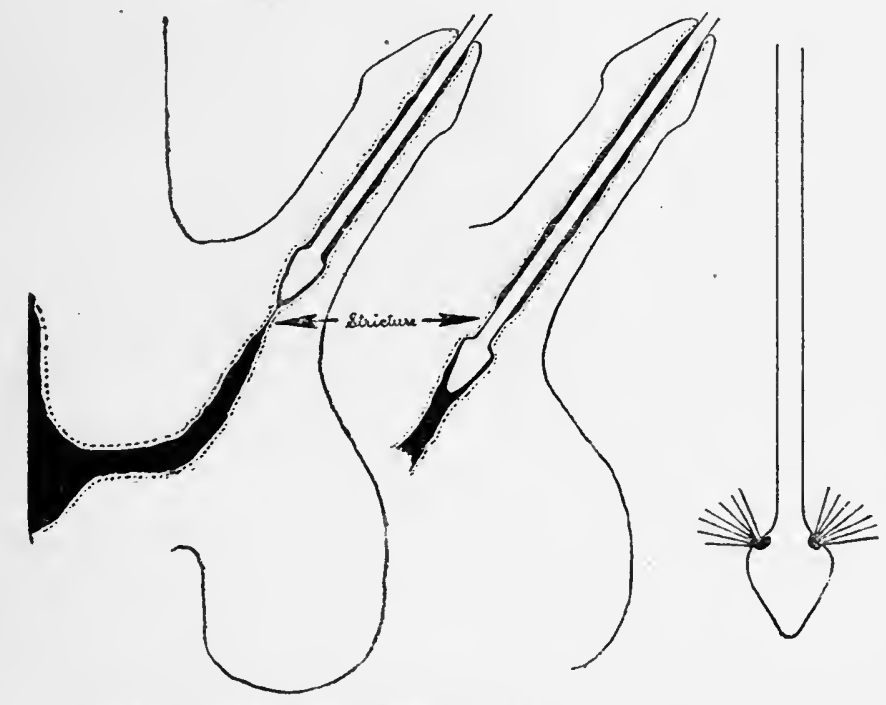

Bovgle a Boule in Urethra, Demonstrating Stricture, and showing Mode of Irrigation. (After Petersen.)

entrance to the connective tissue viâ a follicle. Too much treatment and too drastic treatment-i.e., the use of too strong solutions of antisepticscause inflammation. If the gonococci are not killed directly, and in most cases they are not, the effect of inflammation thus caused is to destroy the host's local protective power, which will enable the parasites to develop at their host's expense, and it will also lay open a path to a secondary infection. If most of the instruments invented for the diagnosis and treatment of urethritis were burnt, chronic urethritis would become a rare condition. I shall never forget having to examine 190 cases of gonorrhœa, which were sent home from Egypt as incurable, the average stay in hospital per man in Egypt having been five months. Although all had an acute discharge when put on board, and received no treatment on the voyage, only twenty-three had to be detained for further treatment when examined. 


\section{Influence of Secondary Infection.}

We do not yet realise in full the significance which the secondary infection has in cases of chronic urethritis, so it would be as well to go into the question somewhat fully; but, before doing so, I should like to draw the reader's attention to the similarity which exists between gonococci situated in sub-epithelial tissue and a piece of buried septic silk after an operation. In the former, the gonococci have to be killed; and in the latter, the silk has to be got rid of, or the fistula caused by it will nerer close. No surgeon would dream of cauterising the opening, or even the canal of the fistula, and yet it is recommended to adopt this treatment in the gonococcal case. One method of getting the piece of silk is to enlarge the opening; dilating the urethra will also often allow one to reach the gonococci with a mild antiseptic. In some cases the silk comes away of its own accord, and in some cases the gonococci die out. Fortunately, in the gonococcal case we can attack the parasites from behind by increasing the protective power of the host with vaccines and chemotherapeutic agents - a method which, in the case of the silk, is not applicable.

Returning now to the influence of the secondary infection in cases of chronic urethritis.

The limits of the rôle played by a secondary infection in chronic prostatourethritis are far from being ascertained, but one fact is certain, and that is that many of the cases of supposed chronic gonococcal urethritis which resist all treatment are due to a secondary infection, and they will get well if treatment is suspended.

The point that now arises is how a chronic urethritis of gonococcal origin can be distinguished from that caused by a secondary infection.

If the patient seeks advice, and you learn that he has had little or no treatment, the chances are that the chronic urethritis is still due to gonococci.

If, on the other hand, he has been well treated, and the condition seems to get worse the more treatment he has, and is especially aggravated if too strong solutions are used, the chances are that the chronic urethritis is now due to a secondary infection.

One must next ascertain that there is no active prostatitis, and no stricture of the urethra.

The patient should then be asked if the morning drop or the early discharge are more than is just sufficient to stick the lips of the meatal orifice together, and whether the threads in the urine are decreased or increased in the morning following a sexual connection, a nocturnal emission, or the taking of alcohol.

All three are almost certain to aggravate the condition if gonococci are still present. The first two may improve the condition if the inflammation is due to a secondary infection, and the taking of alcohol may make little or no difference. 
The patient should then be given a big provocative injection of a potent non-sensitised gonococcal vaccine. If the condition during the first fortyeight hours after the injection is either improved or aggravated, gonococci are still present. If the condition remains in statu quo ante, the inflammation is more probably due to a secondary infection.

It is practically useless to make a microscopic examination of the discharge or threads, because, if gonococci are present, they will not be in the discharge or threads, but only in the sub-epithelial tissue. In all cases, within a few days of the infection, a microscopic examination of the discharge reveals myriads of other organisms. The presence of polymorphonuclears does not assist one at all in making a diagnosis. It is most important to bear in mind the possibility of secondary infection, since it is usually caused by inflammation produced by too much instrumentation and too strong antiseptic solutions; therefore, any treatment which increases this inflammation will favour the invasion of the sub-epithelial tissue by the secondary infection.

A urethritis due to a secondary infection is not, as a rule, infectious.

The secondary infection is usually due to diphtheroids, streptococci, and staphylococci.

The presence of a secondary infection can be positively ascertained by a provocative injection of an autogenous vaccine made from the organisms cultured from the expressed prostatic secretion.

A urethritis due to a secondary infection is best left alone so far as local treatment is concerned, and recourse had only to injections of raccines or chemotherapeutic agents.

\section{Treatment.}

Prophylaxis.-Malthusian appliances, washing with soap and water, and micturating immediately after coitus, are the most useful.

Irrigation of the urethra is likely to prevent an attack, provided the solution used for the purpose is not too strong, and the process is carried out as soon as possible after intercourse. Bougies, in my experience, are to be avoided. Speaking generally, prophylactic measures can be said to be successful only when they are instituted within an hour following connection.

Treatment of acute urethritis may be considered under five heads:

1. Hygienic.-First of all, rest, both to the part-by wearing a suspensory bandage-and to the person. If there is much inflammation or œdema of the penis, and the discharge is very thick or blood-stained, relief may be obtained by carrying the penis upwards over the abdomen. Bed is seldom necessary, but active exercise must be forbidden. Alcohol must be avoided, and milk and water substituted. If the patient has been used to much alcohol, it is unwise to kncck it off suddenly, but one should gradually get hin accustomed to further dilution with water. No hot focds and condiments-mustard, pepper, sauces-must be taken. The diet should be as free of meat as possible, and tasty articles of food, as savouries, hors d'œuvres, shell-fish, celery, 
asparagus, etc., should be avoided. The most important matter of all is to see that the bowels are well regulated.

2. Symptomatic.-Pain can generally be diminished by diluting the urine by giving more milk and water; barley-water is excellent. Decreasing the acidity of urine by drinking lime-water often affords relief. For the acute pain caused by spasm of the compressor urethrce muscle, nothing is better than a warm hip-bath, since this often relieves the retention. If narcotics be required, use suppositories of belladonna and opium. To prevent erections, the bromides are useful. In hæmaturia, ergot or liq. ferri perchlor. should be given internally, with morphia subcutaneously, which, by stopping spasm, acts as a styptic. Owing to the introduction of pallamine and trimine, which immediately relieve the patient of such symptoms, the above-mentioned measures are now no longer needed.

3. Local.-Treatment aims at the destruction of the coccus. Two routes have been tried: giving, by mouth, such drugs as are excreted through the urethra, and direct application of drugs to the urethra itself. The drugs usually given internally are resins and balsams, such as cubebs, copaiba, turpentine, and sandal-wood oil. Of these, sandal-wood oil is the best, but all of them have the disadvantage of upsetting digestion and causing rashes. To get over these defects, sandal-wood oil has been put up in capsules (savaresses), the membrane of which is not digested until the pancreatic juice is reached, so that no oil gets loose in the stomach, eructation and vomiting being thus avoided. Balsams, if they irritate the kidneys, must be avoided when there is any suspicion of nephritis. Sodium salicylate is always a useful adjunct. The innumerable patent drugs-gonorrhol, gonosan, salosantal, etc.-have no advantage over those already mentioned. Really the only medicines required are alkalis and aperients.

For direct application to the urethra, two groups of drugs have to be considered: the pure antiseptics, like potassium permanganate and argyrol; and the antiseptic astringents, such as zinc permanganate and silver nitrate. Pure antiseptics should be used in the early, astringents in the later, stages of the disease.

Injection should not be commenced so long as there is any swelling of the glans penis, œdema of the prepuce, phimosis, dorsal lymphangitis, blood in secretion, or painful erections. The only aim at this stage-that is to say, if lavage cannot be carried out-should be to allay the inflammation by such simple means as lotio plumbi $\bar{c}$ opio, and, internally, hyoscyamus and sandalwood oil.

When the inflammation has subsided, pure antiseptic injections, such as potassium permanganate, 1 in 10,000, or colloidal silver, 1 in 10 , should be commenced. The patient should inject himself three times in every twentyfour hours at, as nearly as possible, eight-hour intervals. He should, by holding his finger over the meatus, retain the injection about five minutes. The bulk of each injection should be about three teaspoonfuls, so that the 
whole of the diseased membrane shall be under its influence at the same time. The exact amount should always be given, and for this purpose a $12 \mathrm{c.cm}$. syringe with a conical end, over which the orifice of the urethra passes, should be used. Inject very slowly, and use but slight pressure, for, if injected too quickly, the muscles come into action, and the whole is ejected.

In acute posterior urethritis, no local treatment must be started until the subjective symptoms have disappeared. Hip-baths, sodium salicylate, and sandal-wood oil must be employed until subjective symptoms are over, then lavage should be started as above by the patient, and the doctor, when the symptoms have become subacute or chronic, can employ either Diday's irrigation or instillation by Guyon's catheter. The difference between the two lies in the fact that, in the former, diluted solutions in large quantity, and, in the latter, concentrated solutions in small quantity, are employed. The former is the milder and better to start with. The patient must have a full bladder, but should pass a little urine to clean the urethra. In Diday's method, a soft catheter is passed until urine just begins to come out; its eye must then be in the bladder. The moment urine appears, draw the catheter back, say, half an inch, until no more comes; its eye must then be in the prostatic portion. Then inject very slowly and gently, withdrawing the catheter as you inject. The solutions most useful are, in the acute and subacute cases, colloidal silver, and in the chronic cases, colloidal iodine or intramine.

In acute and subacute cases the best treatment is lavage, carried out by the patient himself; but before discussing the technique of lavage, the abortive treatment must be considered. If the patient can be put under treatment within twenty-four hours of his first noticing a discharge, which is usually on the third day of his infection, the abortive treatment may be successful. The abortive treatment should not be attempted after this time, as it may make the patient worse, and on no account should it be tried if the subjective symptoms are at all severe.

The abortive treatment as usually employed should not be continued for more than three days, and, if it has been successful, no gonococci should be found in smears of the secretion examined at the end of the third day.

Three drugs are commonly used-hegonon, protargol, and argyrol. They are employed in solution, and injected into the urethra by a syringe in the following strengths respectively: 3.0 per cent., $5 \cdot 0$ per cent., $25 \cdot 0$ per cent.; or the anterior part of the urethra is washed out with solutions of a weaker strength : 1.0 per cent., 1.0 per cent., $5 \cdot 0$ per cent. When the fluid is inserted by means of a syringe or by lavage, it should be done twice a day, and after each application it is a good plan to wash out the urethra with a 1.0 per cent. solution of aluminium acetate to overcome the hyperæmia caused by such strong solutions, and partly to lessen the pain. It is seldom that the abortive treatment is successful, and it is always painful, so that it is usually difficult to get the patient to consent to having treatment twice a day.

Fortunately, we have another method of abortive treatment, which is 
not only generally successful, but also it is painless; and, furthermore, if it is applied too late, the patient's condition will not be aggravated by it. Indeed, whenever it is started, although it will not abort the attack, it will improve the local condition. The method, which originated with Ballenger, ${ }^{4}$ has been used by him with very great success, and I can substantiate what he has said. Ballenger's method is called the "sealing-in " abortive treatment.

A 5.0 per cent. solution of argyrol is sealed into the anterior part of the urethra, once daily, for five days. The solution is sealed in with non-contractile collodion, and for at least four hours at a time. The technique is simple, but, unless it is done exactly, the attempt will fail. The patient first empties his bladder, the penis is then well cleaned and dried, and a syringe is obtained which just holds 20 to 25 minims, and no more. This quantity of a $5 \cdot 0$ per cent. solution of argyrol is injected, the syringe removed quickly, the lips of the meatus are closed together, wiped, and then brushed over with collodion. The lips of the meatus should be kept pressed together until the collodion has dried. The usual fault is to inject too much argyrol, and the collodion will not hold it in. Acetone will remove the collodion when required. When the solution is allowed to escape, the patient should drink freely of lime-water or barley-water, so as to flush out the kidneys well, and to overcome the hyperæmia caused by the argyrol. A very important point to remember is that the argyrol solution must be freshly prepared each day. All the organic preparations of silver oxidise very quickly in solution, and the more they are oxidised the less their bactericidal action.

Another point which medical men never seem to realise is that great care has to be taken in making up the solutions of these organic silver salts. The measured quantity of water should be first obtained, and then the powder or powdered tablet should be slowly dissolved by degrees in it. The water should never be poured on to the powder, as the protein which many of these preparations contain is immediately precipitated, and none of the silver goes into solution.

I have found that undiluted colloidal silver $(1$ in 2,000$)$, which is ready for use, is just as efficacious as argyrol, and less likely on its own account to aggravate the hyperæmia.

In all ordinary cases of acute anterior urethritis, and in those cases in which the abortive treatment has failed, the patient should either be treated by lavage or by injections, and only by the latter when the former cannot be undertaken.

The lavage may either be undertaken by the doctor or by the patient; if by the latter, the following is the best procedure: A glass receptacle, holding from a pint to two pints, is hung up on the wall, and from it hangs about five feet of rubber tubing, to the end of which a single or a two-way cannula is attached. The patient lies in a bath of warm water, and washes out his urethra twice a day, night and morning, with either a solution of potassium permanganate, the colour of which is not deeper than that of ordinary red blotting-paper-i.e., 1 in 10,000 to 1 in 4,000 -or with a $0 \cdot 05$ per cent. to $0 \cdot 10$ per cent. solution of hegonon. If weak antiseptic solutions are always employed 
no harm whatever will result, and the patient will never get a stricture. A patient only gets a stricture if he is not treated at all, or if he is treated with too strong solutions. The fear of driving the discharge back into the posterior part of the urethra, and of setting up an acute prostato-urethritis and epididymitis, is theory, and does not occur in practice.

If the lavage is carried out as just mentioned, and is continued for fourteen clear days after the patient last saw anything whatever in the morning urine, in a very large percentage of cases the patient will be cured, and he will never develop a posterior urethritis.

If the lavage is undertaken by the doctor, there is a danger of starting the anti-peristaltic contractions which lead to epididymitis. If done by the patient himself, there is not this risk, since a patient is unable to wash out the whole length of the urethra until he has lost his self-consciousness and the compressor urethroe ceases to contract against any fluid that is injected. As a patient does not become au fait at washing out his own urethra until he has been at it for ten days or a fortnight, and if he does not commence the treatment until all the subjective symptoms of the posterior urethritis have vanished, and as, moreover, the fluid will only reach the posterior part of the urethra slowly, naturally, and never by force, the risk of starting the anti-peristaltic movements is nil.

In subacute urethritis, lavage should also be employed, internal medication should be persisted in, and more energetic local treatment at the hands of the physician may be undertaken.

The following prescription is the one I find most useful in acute and subacute urethritis:

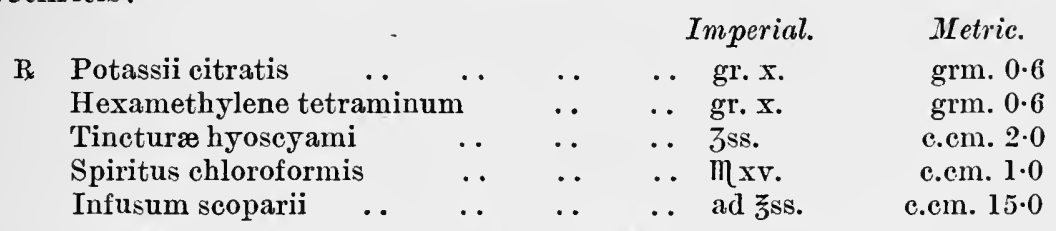

M. f. mist. S.: 亏̌ss. ex aq. sod. effervesc. Žii. ter p.c.

If the patient has much pain, the addition of sodium salicylate is advisable, and if there is any tendency to the formation of a narrowing of the urethra, colloidal iodine should be added in increasing doses.

In all subacute cases it must be ascertained whether the patient has a narrowing of the urethra or not. If he has, Kollmann's dilator should be used for stretching it, and the wrethra should be well washed out through the instrument with colloidal silver one day and colloidal iodine or intramine another, and so on alternately, first with catheters à boule, then with gradually increasing sizes of French gum elastic catheters, and, finally, if there is no narrowing, lavage done by the patient himself is usually sufficient.

The best drug to use for lavage in these cases is zinc permanganate ( 1 in 10,000). The lavage should be undertaken, preferably, only once a day, and then at night-time. 


\section{CLINICAL ASPECT AND TREATMENT OF VENEREAL DISEASES}

In chronic urethritis the prostate is usually affected, therefore the treatment is discussed in Chapter XXI.

In uncomplicated chronic urethritis there is usually a narrowing of the urethra, or a stricture, which is the cause of the trouble. If only a narrowing, dilatation with a Kollmann's dilator should be undertaken. At each sitting, either undiluted colloidal iodine or intramine should be instilled into the urethra. Intramine is extremely useful injected intra-urethram in this way; but great care must be exercised in preventing any of the emulsion reaching the skin of the penis or the scrotum, as, although intramine does not damage a mucous membrane, it is very liable to set up a dermatitis when it comes in contact with the skin. If there is a stricture, it must naturally be attended to.

4. General.-The general treatment is treatment vi $\hat{a}$ the blood-stream, a treatment which is carried out with vaccines and chemotherapeutic agents, and which aims at killing the gonococcus by increasing the protective action of the host. For fuller details as to the exact mode of action, the reader is referred to the various articles I have published on the subject, 5, 6, 7, 8, 9, 11 as Chapter XXVII., which is devoted to this subject, gives only the outline. The vaccine and chemotherapeutic treatment are only adjuncts to the treatment already described; therefore, whatever intramuscular injections are prescribed, the "sealing-in" method and irrigations with potassium permanganate in the acute stages, and zinc permanganate in the late subacute and chronic stages, or injections of colloidal silver in the former and colloidal iodine or intramine in the latter, the treatment $I$ have found to be most useful, must be strictly adhered to. In cases of acute urethritis, whether anterior or posterior, provided it is the patient's first attack, the following intramuscular injections should be given: First day, $0.5 \mathrm{c.cm}$. colloidal palladium (pallamine); fifth day, 0.5 c.cm. pallamine; ninth day, 0.5 c.cm. trimine or pallamine; thirteenth day, if necessary, $0.5 \mathrm{c} . \mathrm{cm}$. or $1.0 \mathrm{c.cm}$. trimine. Whenever pallamine is used, methylene blue and potassium nitrate may be given internally, the former in 3-grain pills three times a day, and the latter in the following mixture:

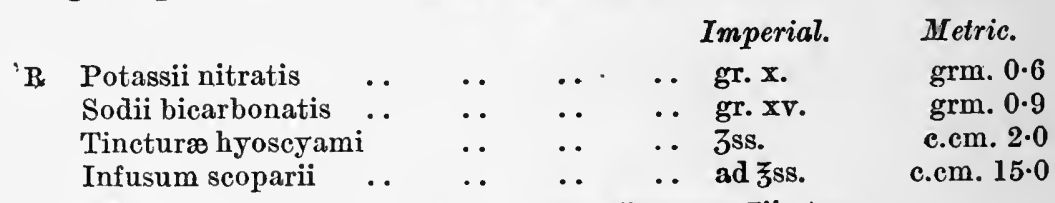

M. f. mist. S.: گ̌ss. ex. aq. sod. effervesc. そ̌ii. ter p.c.

Instead of injecting two or three doses of pallamine of $0.5 \mathrm{c} . \mathrm{cm}$.'each, one dose of $1.0 \mathrm{c} . \mathrm{cm}$. may be employed, which should be followed by $0.5 \mathrm{c} . \mathrm{cm}$. trimine on the fifth, sixth, or seventh day. There are no other cases of gonorrhœa in which it is advisable to use pallamine. In recurrent gonococcal urethritis pallamine is apt to precipitate a prostatitis or vesiculitis, which in their turn may give rise to an arthritis. If pallamine is not used, the following is the best plan of procedure in cases of acute urethritis, whether anterior or posterior, primary or recurrent; First day, $0.5 \mathrm{c.cm}$. trimine; fifth day, $0.5 \mathrm{c.cm}$. trimine; 
twelfth day, $0.5 \mathrm{c.cm}$. trimine; twentieth day, if necessary, $0.5 \mathrm{c} . \mathrm{cm}$. or $1.0 \mathrm{c} . \mathrm{cm}$. trimine. Trimine is a colloidal mixture of manganese, iron, and zinc in certain proportions. When trimine is used, an alkaline mixture with a balsam should be prescribed internally, but the urine should not be allowed to get too alkaline, an action which trimine is liable to have. These colloidal preparations, besides making the patient himself feel better in every way, almost immediately begin to stop the discharge, so that painful micturition, chordee, etc., are now things of the past, and prevent complications from ensuing. In the last 1,000 cases I treated in the army, only 31 developed epididymitis, 6 vesiculitis, and 0 arthritis. Some of these cases were cases of acute posterior urethritis which were already on the point of developing epididymitis, because in 22 of the cases the epididymitis set in after the first or second injection. There were 73 relapses of which I was cognisant; how many relapsed and went elsewhere I cannot say; but when, in the ordinary course of events, over 150 would be relapses, 150 cases of epididymitis, 70 cases of vesiculitis, and 20 cases of arthritis, I think it may be said, at least, that the chemotherapeutic treatment, which is as yet in its infancy, is a success. In the last 100 cases (first attack) treated with pallamine at the Lock Hospital, 2 cases developed epididymitis, 1 case acute prostatitis, 1 case acute Cowperitis, and 1 case recurred. The number of visits, including rest from treatment and final rectal and urethral examination for each patient, a veraged 7.9-a very different state of affairs to that which had existed in the past. The greatest care must be taken not to give too many injections, or to give them at too short intervals, as exactly the contrary effect to that desired may be produced.

In subacute cases of gonorrhœa it is best to inject $0.5 \mathrm{c} . \mathrm{cm}$. trimine on the first day, and 1.0 c.cm. trimine a week later. If the discharge has not entirely stopped, $1.0 \mathrm{c.cm}$. or $1.5 \mathrm{c.cm}$. trimine should be injected after an interval of a week. If the discharge has stopped, two injections of intramine, $1.0 \mathrm{c.cm}$. and $1.5 \mathrm{c.cm}$. respectively, should be injected at five days' interval, followed a week later by either a third injection of intramine, $1.5 \mathrm{c.cm}$., or by an injection of trimine, $1.0 \mathrm{c} . \mathrm{cm}$. In chronic urethritis, colloidal iodine should be given internally, and the following injections prescribed immediately if time is precious, or, better, in ten days or a fortnight if time is of no object: intramine, $1.0 \mathrm{c.cm}$., $1.0 \mathrm{c.cm}$., and $1.5 \mathrm{c.cm}$., with five days' interval between each, to be completed with $1.0 \mathrm{c} . \mathrm{cm}$. trimine a week later. Colloidal iodine and intramine have a surprising action in softening chronic and fibrotic patches of perifolliculitis, rendering in many cases dilatation a simple procedure, when under ordinary circumstances it would have needed an operation. If success has not followed the chemotherapeutic treatment, which is possible if the rationale upon which it is based is not understood, or each case is not treated individually, it is best to leave the injections alone, because an indiscriminate and haphazard use thereof is just as apt to render the disease immune to all kinds of treatment, as too many injections of arseno-benzene and mercury do in syphilis. It is well to have the maxim, when treating gonorrhœa, that more harm can be caused by over- than by under-treatment. 


\section{CLINICAL ASPECT AND TREATMENT OF VENEREAL DISEASES}

5. Miscellaneous.-A brief reference must be made to other methods of treatment which have from time to time been advocated.

Mention will be first made of Russ's ${ }^{10}$ treatment by electrolysis. A platinised catheter which has numerous perforations is passed intra-urethram. A 2.0 per cent. solution of sodium iodide is injected into the catheter, inside of which is also passed a piece of platinum wire, the other end of which is connected to the positive pole of the battery. The negative electrode, which is a lint-covered pad, lined by a flexible metallic core, soaked and wrung out in warm saline, is applied to the perinæum. A current of 1 to 3 milliampères is passed for ten minutes, and then reversed for a further ten minutes, and the catheter is kept full of the solution from time to time by means of a syringe. In my experience, the treatment is most useful in subacute cases of folliculitis and perifolliculitis which have resisted other methods. In acute cases it is too apt to cause an extension and aggravation of the disease, and it takes up too much time to become generally utilised. If a weaker current were used and a non-irritative solution employed, it is possible that better results might be obtained. Various forms of suction apparatus have been employed for emptying follicles, the two in most general use being Cambell's bougie and Mills's negative pressure catheters. The bougie is connected to a manometer and hand-pump, while the catheter consists of a framework of four arms, the suction being carried out by means of a rubber ball attached to the handle. Having no personal experience of this method of treatment, I can only say that the authors mentioned claim to have had good results. The treatment by heated bougies and by medicated gauze plugs have only to be mentioned as a warning not to use them. Vaccines in uncomplicated urethritis are alone not of much value, but, combined with chemotherapeutic agents, are most useful in cases of long standing and relapsed cases of subacute urethritis. The best vaccines and the mode of administration will be described later.

Proflavine has recently been advocated for urethral irrigation, but the more drugs I try the more I am convinced that no preparations can compare with the potassium and zinc salts of manganese. In some cases of secondary infection, or when a change is required, the most suitable preparation is a 1 in 10,000 solution of mercury oxycyanide.

1. Proksch (1910): Handbuch der Geschlechtskrankheiten. A. Hölder, Wien u. Leipzig.

2. Renaud (1918): Quoted from Brit. Med. Journ., i., 513.

3. NeIsSER (1897): Zentralbl. f. d. Med. Wissenschaft, xvii., 497.

4. Ballenger (1914): Genito-Urinary Dis. and Syphilis. Butterworth and Co., London.

5. McDonagh (1918): Practitioner, c., 41.

6. McDonagh (1918): Practitioner, c., 416.

7. McDonagir (1919): Practitioner, cii., 129.

8. McDonagh (1918): Brit. Med. Journ., ii., 31.

9. McDonagh (1918): La Presse Médicale, xxvi., 612.

10. Russ (1916): A New Treatment for Gonorrhœa. H. K. Lewis and Co., London.

11. McDonagh (1920): Practitioner, civ., 14. 
ADDENDUM.

"ELECTROLYSIS TREATMENT FOR GONORRHCEA,"

Pag 290.

Since the publication of this book the Author has been Since the publication of the method by which improved
notified of a change in the
results are claimed. A description by Dr. Russ, of the new method, will be found in the British Medical Journal, May I2th, I917.

\section{CHAPTER XXI}

\section{COMPLICATIONS OF URETHRITIS GONORRHOICA, DUE TO DIRECT EXTENSION OF THE ORGANISM}

\section{Cowperitis Gonorrhoica.}

CowPER's glands were discovered in 1702, although it had been known for twenty years that their locus was a favourite seat for gonorrhœa. Morgagni, in 1761, was the first to give a description of gonorrhœa of these glands, from two post-mortem examinations he made in recent cases, but the most comprehensive early account hailed from Benjamin Bell, in 1793.

The ducts of Cowper's glands open into the penile portion of the urethra, hence an extension of the organisms into these glands takes origin from an anterior urethritis. Under ordinary circumstances, Cowper's glands cannot be felt, and even when they become enlarged they are missed, unless the observer knows exactly how to locate them. The forefinger of one hand is inserted per rectum, and the thumb of the same hand is pressed against the triangular ligament. The forefinger feels for the region of the symphysisi.e., for the middle line-and then gradually works outwards on either side, remembering always to keep pressing anteriorly, and, in the lateral course, the gland will be felt between the forefinger and the thumb. In my experience, abscess formation in Cowper's glands is more common than in any of the other organs affected by the gonococcus. Acute inflammatory Cowperitis is not at all uncommon, but chronic Cowperitis is relatively rare.

The subjective symptoms of Cowperitis are the same as those experienced in inflammation of the bulb, and they are, moreover, characteristic. The first thing a patient complains of is pain in the perinæum, but the pain is felt only in the acts of sitting down and getting up. Later, the pain becomes more acute, and extends to the rectum, scrotum, and inner surfaces of the thighs. Most of the cases of acute Cowperitis with abscess formation which I have seen have burst spontaneously in the perinæum. Owing to the proximity of the abscess cavity to the rectum, rectal gonorrhœa may complicate the case; but, generally speaking, abscesses in Cowper's glands heal very quickly, and without any complication. The abscess may also burst into the urethra, and may, in the process of healing, lead to a urethral stricture.

I have seen two cases in which a urinary fistula followed, and in one of these the patient had extravasation of urine. Unless the patient comes up 
for treatment early, and unless the condition heals quickly, a fistula is very liable to result. Urinary fistulæ, following Cowperitis, do not, as a rule, close spontaneously, so it is usually necessary to dissect out both the fistula and the gland. Retention cysts may follow a Cowperitis, in the same way as a labial cyst may follow a Bartholinitis. In both cases the only means of curing the condition is extirpation.

The treatment of ordinary Cowperitis rests in local applications of cold, in not incising an abscess until it points, and in treating the urethral condition as if a Cowperitis did not exist.

Provided no pus has formed, chemotherapeutic treatment will abort the trouble. If pus has formed, chemotherapeutic treatment will localise it, and bring it to the surface, where it can be evacuated through a small nick made with a bistoury, when subsequent healing will be rapid. In any acute case, $0.5 \mathrm{c} . \mathrm{cm}$. trimine should be injected on the first day, and $1.0 \mathrm{c.cm}$. on the third day, followed by 1.0 c.cm. or 1.5 c.cm. on the eighth day, if necessary, In a chronic case, where a fistula has already formed, two injections of trimine, $1.0 \mathrm{c.cm}$. and $1.5 \mathrm{c.cm}$. respectively, should be given at four days' interval followed by two injections of intramine, $1.5 \mathrm{c.cm}$. and $2.0 \mathrm{c.cm}$. respectively, at the same interval, and, finally, another injection of trimine, $1.5 \mathrm{c.cm}$. This manœuvre has closed fistulæ which have been present for months. The vaccine treatment of this, as well as the other complications, should be undertaken as follows: 7,500 million of Thomson's detoxicated vaccine for the first week in two doses of 2,500 and 5,000 million respectively. In the second and third weeks bi-weekly injections of 5,000 million should be prescribed, and the same during the fourth week if necessary. The vaccine should not be mixed with the chemotherapeutic agent unless it be intramine, so it is best injected into the other buttock, or another day chosen.

\section{Folliculitis, Perifolliculitis, and Cavernitis Gonorrhoica.}

A folliculitis is very common. It is probably partly responsible for the chronicity of the disease, and it arises from an infection of the Littre's glands in the mucous membrane of the urethra. A plain folliculitis is indistinguishable from a plain urethritis, and the only way in which to tell whether the glands are infected or not is to examine the urethra endoscopically, or to adopt the following procedure: The urethra is first well washed out; then a bougie a boule is passed, and the urethra massaged from the outside over it. This presses out the secretion from the follicles, which sticks to the bougie, and then the secretion can be examined later for gonococci. Fibrous tissue formation is common to all gonococcal lesions, especially in those lesions which do not suppurate. As suppuration in the follicles leads to a perifolliculitis, when reference is made to folliculitis proper, it may be assumed at once that the lesion or lesions are never suppurative. This being the case, fibrous tissue formation is a common sequel. A fibrotic folliculitis may lead either to the formation of hard nodules, or even to a stricture. This is probably 
the pathology of the narrowing or narrowings, as there are often two or even three to be met with right in the fore part of the urethra, after a chronic attack of gonorrhœa. The fossa navicularis is a favourite site for folliculitis. This being so, it will follow that perifolliculitis will frequently have its origin here.

A perifolliculitis arising from the fossa navicularis first appears as a hard swelling on the under-surface of the penis. The swelling at this stage is not particularly painful to the touch, nor markedly inflammatory; hence the diagnosis of intra-urethral chancre is often made. Such a mistake in diagnosis need not arise if the reader will bear in mind that almost all intra-urethral chancres can be seen at the urethral orifice, and that one part of the chancre has usually eroded a portion of the glans penis. Further, micturition is very painful in cases of perifolliculitis. The swelling may bulge in the middle line, and may either burst or spontaneously disappear, or it may point to one, or more often to both, sides of the frænum in the corona. If the abscess bursts on one or both sides of the frænum, pus is exuded for a few days, and then the opening closes, and the swelling spontaneously disappears. Occasionally a penile fistula results. In my experience, it is wisest to leave these fistulæ alone, as I have seldom met with a case in which they did not ultimately close of their own accord, and it is a difficult and painful operation to close such a big opening by electrolysis; moreover, trimine and intramine, combined as above described, will rapidly produce the result desired. A perifolliculitis may burst anywhere along the raphe of the penis; it may also point laterally, or even on the dorsum of the penis. The region of the bulb is a very common situation for an abscess.

Treatment by rest and cold applications, or by painting on ichthyol, will often cause these abscesses to abort, while a timely intramuscular injection of trimine will always do so. If not, they should be allowed to point, and should then be incised at the apex, or allowed to burst. If left alone, a penile fistula practically never results, except when the abscess arises from the bulb, and even then it soon closes. In most of the cases, a folliculitis does not become a perifolliculitis until the urethral opening of the former becomes blocked. The more the abscess advances to the skin, the thicker the fibrous tissue wall it leaves behind it. Therefore, under ordinary circumstances, a penile fistula is rare. If the abscess be incised before it points, or if too big an incision be madc, a false passage may easily be produced, and a penile fistula is very apt to follow. When penile fistulæ arise from too energetic operative treatment, they are extremely difficult to close, while those which arise from Nature's cure heal quickly; thercfore, in most cases, no harm is done in letting a perifolliculitis burst of its own accord. Fortunately, the chemotherapeutic treatment has altered the outlook of these cases, as evidenced by the following two cases:

CASE 125.-A patient had a periurethral abscess just behind the corona, which had to be incised. A few days later another appeared two inches behind, and another in the perineum. The day following the first injection of colloidal manganese 


\section{CLINICAL ASPECT AND TREATMENT OF VENEREAL DISEASES}

$(2.0$ c.cm.) the patient was able to get up, and, after two further injections of 3.0 and $4.0 \mathrm{c} . \mathrm{cm}$. respectively, the three abscesses disappeared, except for a small sinus, which followed the incision of the foremost swelling. This sinus healed up after one injection of intramine and another of colloidal manganese.

CASE 126.-A patient had had a perineal periurethral abscess, which had left a urinary fistula, through which pus still discharged. The patient had been under treatment for the best part of two years without much improvement. After four injections of colloidal manganese and two of colloidal antimony the discharge stopped, but the fistula did not close. I then gave the patient two injections of intramine, and a final one of colloidal manganese, with the result that within ten days the fistula had completely closed, and the patient was discharged.

It is very seldom that a perifolliculitis bursts into the urethra.

A perifolliculitis may become a cavernitis-that is, the gonococci may: spread peripherally into the corpus cavernosum. All that results may be a dense fibrous nodule or band, which may be so bad as to render erection almost impossible, in any case acutely painful. A cavernitis, on the other hand, may be suppurative, and may lead at first to a perpetual erection. Some of these cases end fatally from pyæmia; but, as a rule, they resolve, and, owing to the amount of fibrous tissue formed, and to the contraction of the same, sexual connection is for ever made a difficult and usually an unpleasant proceeding.

Something can be done in these cases by prescribing large doses of colloidal iodine internally, by rubbing in iodex ointment, or colloidal iodine oil externally, and by injecting intramine intramuscularly. Thiosinamine or fibrolysin has been strongly recommended, but I can only say that I have never seen it do any good. Perifolliculitis with abscess formation is very liable to recur years afterwards, and lead to a chronic œdematous and almost elephantiasic condition of the penis, and even of the scrotum. I had one case which remained latent for twenty-five years.

In cases of folliculitis, the urethra should be stretched with a Kollmann's dilator, and well washed out with some antiseptic. Treating each follicle by cauterisation through a urethroscope is usually futile.

The name periurethritis is often given to the three conditions which have just been discussed, and this must not be confounded with the term paraurethritis, a condition first described by Ricord in 1846. It is interesting to note that relapses of anterior urethritis after chemotherapeutic treatment are due to a localised folliculitis, which frequently disappears under a single injection of trimine, 1.5 or $2.0 \mathrm{c} . \mathrm{cm}$.

\section{Paraurethritis.}

This is an inflammation of either accessory urethræ or canals which branch from the urethra, and it is usually produced by the gonococcus. These canals may be single or multiple; they may open externally anywhere along the median raphe of the penis, or in the glans penis on either side of the meatal 
orifice. The meatal paraurethral canals may open in the meatus itself. I have seen paraurethral canals situated wholly in the skin in the median raphe -i.e., neither end being in communication with the urethra. I have just had a case under observation in which the only gonococcal lesion the patient had was a paraurethritis. The canal was blind posteriorly, but open anteriorly; it was two and a half inches in length, and in several places the gonococci had ulcerated through, producing ulcers not unlike soft sores. A paraurethral canal may be part of the normal urethra in some of its length, becoming only paraurethral about the level of the bulb. These cases may be very baffling, as, when passing an instrument, it may follow the paraurethral canal instead of going into the urethra proper. In such cases the instrument suddenly stops dead, and the observer diagnoses a stricture.

I have had quite recently under my care four cases in which the patients with such an abnormality had been treated for stricture for several months.

The point is not generally settled whether these canals are always congenital, or whether they are sometimes produced during the course of the gonorrhœa, but as they are much more frequently met with in patients with other congenital defects, my own opinion is that they are always congenital. Ricord, in his original description, noted that they were more common in patients who had hypospadias. They vary very much in length, but are usually about $2 \cdot 0 \mathrm{~cm}$. long. Meatal paraurethritis is a frequent cause of recurrent gonorrhœa, reinfection, and infection.

Any pressure from behind forces the discharge out of the canals, and the discharge is usually full of gonococci. It is most necessary, as soon as the acute stage of the gonorrhœa is over, to close these canals as soon as possible. Closure is best effected by electrolysis. A very convenient apparatus is Morton's switchboard. To the negative pole is attached a needle-holder containing a platinum needle, or a fine piece of platinum wire. The needle or wire is then passed down the canal after all the discharge has been pressed out. A large pad is attached to the positive pole, and is placed under one of the buttocks. A current of 1 to 4 milliampères is then run through for one to two minutes. More than one application may be necessary. If several applications fail to close the canal, it will mean that the needle has not passed down it. It is often impossible to guide the needle down the lumen of the canal, in which case the canal can be attacked from the side, in its centre, instead of through its opening. Those canals along the median raphe of the penis are especially difficult to treat, so that it is always advisable to dissect them out. To avoid confusing an intra-urethral paraurethral canal for a stricture, in all cases in which an instrument is being passed for the first time, as large a one as possible should be used, as the end will in most cases be too big to enter the adventitious passage. 


\section{Prostatitis.}

Considering the prostate becomes implicated in about 75 per cent. of all cases of gonorrhœa, it is an odd fact that the older authors paid little attention to it. Ambroise Paré, in 1575, was the first to mention a prostatic abscess, which he observed in a corpse of a man who had suffered from "strangury" for ten years. Morgagni was the first to describe prostatitis from the pathologico-anatomical point of view. Stoll (1788) appears to have examined the prostate in all his cases of gonorrhœa; but most of our presentday knowledge is founded on the work done by Sir Henry Thompson (1857).

There are two ways of examining a prostate to see if it is diseased- $(a)$ palpation per rectum; $(b)$ microscopic examination of expressed secretion. In palpating the prostate gland, it must be remembered that a great variation normally exists in the relative sizes of the two lobes. If the gland is enormously enlarged, painful, and the two lobes cannot be differentiated, the patient, if under 50 years of age, has a prostatic abscess. With the exception of the increase in size, due to an abscess, it is better to discountenance the size, and to pay attention to the consistency and the state of the surface.

In most cases of gonococcal prostatitis, only one portion of one lobe is affected, or several distinct portions of one or both lobes may be involved.

The affected area is first swollen, painful, and soft; but, as the condition becomes chronic, and fibrous tissue takes the place of the cellular infiltration, the area becomes hard, and eventually indented, owing to the drawing in of the surface by the contraction of the fibrous tissue. Consequently, the mere examination of the surface of both lobes will tell the observer at once whether the gland has been affected or no. Unevenness of the surface, or irregularity in the consistence of the different areas, signify an active or a healed gonococcal infection. One can only be certain whether the prostatitis is active or healed by making a microscopic examination of the expressed secretion. Experience, gained by the palpation of several hundreds of cases, will usually enable a trained observer to say at once whether the condition is active or healed. If the surface or the consistence of the prostate alters on massaging the gland, it denotes active trouble, as the alteration is due to pressing out the cellular débris from one of the soft and swollen areas. A lobe which is shrunken and uneven on the surface, due to the contraction of fibrous bands in various parts, is usually indicative of a healed condition. Broadly speaking, the right lobe is more frequently affected than the left, and the most common place to get a soft area is in the centre of the medio-lateral margin. The soft area in this situation is usually crescentic in shape.

Gonococci should be looked for when a microscopic examination is being undertaken, and, if none be found, attention should be paid to the number and nature of the leucocytes present. The presence of polymorphonuclear leucocytes alone does not necessarily signify an active gonococcal infection, because soon after gonococci reach the prostate a secondary infection follows 
in their wake, and a secondary infection may persist, and so give rise to the production of polymorphonuclear leucocytes long after all gonococci have disappeared. If there is a great number of polymorphonuclear leucocytes, it is suggestive of an active gonococcal infection, and if there are several large mononuclear leucocytes and eosinophile cells, it is practically certain that there are gonococci in the prostate gland. Trying to grow the gonococcus from the secretion may give positive evidence in cases where other methods have failed.

The presence of polymorphonuclear leucocytes and absence of bacteria is in favour of gonorrhœa. The presence of polymorphonuclear leucocytes and presence of bacteria other than gonococci is in favour of a secondary infection. The presence of epithelial cells in abundance is suggestive of over-treatmenttoo much massage.

In many cases of prostatitis, if the urine be carefully examined, and the three-glass test used, in the last portion passed are to be found either what looks like a granular precipitate or bodies which are bigger, but not quite so opaque. The size and density depend upon the amount of mucin present in the particles. The particles consist of pus cells, epithelial cells, salts, and mucin, and they are pressed out of the acini and ducts in the act of expelling the last few drops of urine. Their presence usually denotes that the patient is either neurasthenic or has been over-treated. These particles must not be confused with much bigger clear bodies, which also find exit with the last few drops of urine, and which arise quite independently of a prostatitis. These bodies are about the size of, and look very much like, boiled sago grains. They often go by the name of Lallemand-Trousseau bodies, and they come from the vesiculoe seminales, and consist merely of coagulated mucin, which has included in its meshes a few epithelial cells and leucocytes.

A naked eye examination of the threads in the urine will often give a clue as to whether the prostate gland is implicated or not. Long threads are usually urethral; if they are dense, and fall quickly to the bottom, they usually consist of myriads of leucocytes, which suggests that the gonococcal process is still active. If the long threads float for some time, and appear more transparent, they contain mostly mucin, which indicates that the treatment is being overdone. Punctate threads usually come from the prostate, and their presence generally means that the gonococcal process in the gland is active. Threads like scales and threads coloured by the solution used for irrigation almost invariably indicate over-treatment.

Prostatic Abscess.-The prostate often swells to a considerable size before giving rise to subjective symptoms. A prostatic abscess may even burst without the patient being aware that he had had an abscess. The bursting of a prostatic abscess may result in the spontaneous cure of the disease.

When a prostatic abscess gives rise to subjective symptoms, those usually complained of are a feeling as if there were a foreign body in the rectum, acute pain on defæcation, or on contraction of the abdominal muscles. 


\section{CLINICAL ASPECT AND TREATMENT OF VENEREAL DISEASES}

Priapism and painful pollutions are common. These prevent the patient from sleeping. Consequently, he soon begins to wear a haggard expression, and looks very ill. Oddly enough, fever is far from being a constant symptom. Naturally, micturition is extremely painful, and the urine may be quite clear, just as it often is in the early stage of epididymitis.

Many cases resolve of their own accord, but recurrences are very liable to set in from time to time.

If a case is caught early, rest in bed, the internal administration of urinary antiseptics, and intramuscular injections of trimine and vaccine, will usually quickly bring about resolution, but in any case give instant relief.

Acute inflammation of the middle lobe of the prostate is one of the most troublesome events in gonorrhœa, as it invariably leads to retention, and necessitates the passage of a catheter. Once a catheter has been passed, owing to the increase of inflammation produced thereby, it becomes necessary to pass it once or twice a day for, maybe, several weeks. This vicious circle is liable to cause complications, which may render the patient a sufferer from gonorrhœa for months, and even years. In no condition is the effect of colloidal manganese more marked, because one injection will rid the patient of the subjective symptoms and enable him to pass his urine without assistance. Prostatic abscesses and purulent vesiculitis are apt to occur if the chemotherapeutic agents are injudiciously used in cases of acute urethritis. Palladium may do so if used in cases other than when it is the patient's first attack. Manganese only does so if too big doses are used, and the injections are prescribed too quickly after one another. Therefore, until one has had considerable experience with the use of these drugs, it is wisest to refrain from employing them in acute cases of urethritis if the patient is run down or if no improvement appears to follow the first or second injection. Prostatitis and vesiculitis never follow when these drugs are prescribed for the complications of gonorrhœa, which suggest, that such precipitation is due to over-treatment. This opinion is probably correct, because nothing affords such instant relief to such cases as vaccines, which act by increasing the patient's immunity against the infection.

In the early stage of practically any acute inflammatory condition, the application of cold may be very beneficial.

In the case of acute parenchymatous prostatitis, cold may be applied to the gland by inserting a psychrophore into the rectum, and passing a continuous current of cold water through it. Some patients prefer heat to cold, and it is certainly easier to apply, being most convenient in the form of hipbaths. Suppositories containing morphia, belladonna, and ichthyol sometimes give relief.

Many prostatic abscesses burst spontaneously, and usually into the urethra. The majority of the cases heal very rapidly, and they are best left alone. It is not wise to employ any local treatment for fear of breaking down part of the abscess wall. Breaking down the abscess wall may end in an extravasa- 
tion of urine, a complication which is extremely rare under ordinary circumstances. Spontaneous rupture into the rectum may occur. Should the observer have reason to think that the abscess is going to burst into the rectum, he should forestall it, and make a small incision at the place where the abscess is pointing. Cases which have been treated by incision run a better course than those in which the abscess bursts spontaneously-for one reason, a gonococcal catarrhal inflammation of the rectal mucous membrane is less likely to supervene.

A rupture of a prostatic abscess into situations other than those just mentioned is a pathological curiosity, and requires no special mention.

Chronic Prostatitis.-A chronic inflammation of the prostate gland is the lesion with which one mostly has to deal. As a rule, the subjective symptoms are nil. Chronic prostatitis undoubtedly plays a rôle in the retiology of sexual neurasthenia, but this is a subject which will be considered by itself later. The objective signs have already been dealt with, but, nevertheless, a point should be emphasised-i.e., that in the two-glass test, should the second glass be clear, it is no sure proof that the prostate is not affected. In many cases of chronic prostatitis the inflammation is so slight that only a very little cellular débris finds exit into the urethra during twenty-four hours. The little that is in the urethra may be washed away with the first few drops of urine passed, and this would leave the second glass clear. The three-glass test is a little more accurate, as the abdominal muscles are made to contract and press out secretion from the prostate in an effort to pass the last drops of urine in the third glass; but this test may be fallacious. The irrigation test in such a case does not help one, hence it is necessary to palpate the gland, or to examine its expressed secretion.

An increase in the discharge, or in the number of threads, or a decrease of the same within forty-eight hours of a provocative injection of vaccine, is strongly suggestive of chronic prostatitis, since on plain urethritis vaccines exert little influence.

When a provocative injection of vaccine causes a nocturnal emission on the same night or the next night, it is almost certain that the patient has a chronic prostatitis.

That a patient develops, on the one hand, an increase of discharge after a provocative injection of vaccine, and, on the other hand, a decrease, is entirely due to the state in which the antibody was when the injection was given. If the antibody was stale, it will be destroyed when a vaccine is given, and therefore the discharge will be increased; while, if the antibody is fresh, the vaccine will stimulate it, and therefore the discharge will be decreased.

In the cases which have subjective symptoms, the following are those of which the patient most frequently complains: Pain in the rectum, perinæum, and small of the back. The pain may radiate down the groins, the scrotum, and the legs; it is often aggravated by nocturnal emissions and sexual con- 
nection. Itching sensations around the anus and behind the scrotum may be very troublesome symptoms.

Chronic prostatitis is frequently said to be a cause of sterility, and a cause of enlarged prostate in later life.

In most cases of chronic prostatitis only a portion of one lobe is affected; therefore the diminution in the prostatic secretion is negligible. Even if the normal secretion is reduced to practically nil, it does not affect the vitality of the spermatozoa, and there is no proof whatever that chronic prostatitis causes sterility. Even while the condition is still active, provided there are no gonococci present, it is perfectly safe to let the patient marry. In my opinion, the danger of infection is very much over-estimated, and if the case has been well treated the risk is practically non-existent.

As to whether chronic prostatitis is a cause of prostatic hypertrophy is merely a matter of opinion, as no proofs can be adduced for or against the suggestion. I am strongly of the opinion myself that there is no causal relationship between the two conditions.

So far as getting rid of the gonococci from cases of chronic prostatitis is concerned, the prognosis is good; but as regards ridding the gland of leucocytes, the prognosis is bad. As the presence of leucocytes means nothing more than that there is a secondary infection, and as a secondary infection is harmless, and as it may give rise to symptoms indistinguishable from a gonococcal infection, it is most important to differentiate between the two conditions.

If a patient has been well treated, and if neither a provocative injection of a potent vaccine, nor sexual connection, nor a nocturnal emission, nor the consumption of alcohol, alters the proportion of the threads in the urine, I think the patient may be informed that he is cured and fit to marry. It must always be remembered that the mere presence of threads in the urine does not mean that the patient still has gonococci lurking about somewhere. Patients who have undergone drastic treatment may have threads for the rest of their existence, and yet be quite cured of their gonorrhœe. Still another very important point has always to be boine in mind. Patients who have had chronic prostato-urethritis, especially if the treatment has been largely instrumental, and if strong antiseptic solutions have been injected, are very liable to have a glycerine-like discharge. The glycerine-like discharge is mucin, which continues to be excreted in large quantities from the mucous membrane of the ducts and acini of the prostate and urethra for months, and even for years, after all treatment has been suspended.

A point of some importance is that this discharge becomes more marked the more continent the patient is; therefore, if it temporarily disappears after a nocturnal emission or sexual connection, the patient can be told that he has no infection, and that the discharge is merely due to hypersecretion of the epithelial cells of the urethra and prostate.

It is very difficult to convince neurasthenics that such a discharge is not gonococcal; consequently, such patients run from one doctor to another until 
one has been found who will treat the case. As the mucin discharge is due to irritation, it will naturally follow that any further injections will only tend to aggravate the condition. A very large percentage of the cases I see of patients who have had gonococcal prostato-urethritis are suffering from irritation due to over-treatment. I think it is highly probable that more cases of so-called gleet are suffering from over- than from under-treatment, and the less we use instruments, and the weaker our injection solutions, the more likelihood there is of curing the disease.

The treatment in chronic prostato-urethritis is quite simple. The first point that must be ascertained is whether the patient has a stricture or narrowing of the urethra. If so, then this must be dilated before any special treatment is begun. If there is no narrowing, or if the constricted urethra has been reduced to its normal calibre, the patient should have his prostate massaged twice a week for not longer than three weeks. The prostate is massaged with the forefinger inserted per rectum; the finger is preferable to any instrument. An Ultzmann's syringe or Teevan's catheter is then passed down the urethra, and $3.0 \mathrm{c} . \mathrm{cm}$. of either colloidal silver, colloidal iodine, or intramine is injected. It is best to use an oxidising agent at one sitting and a reducing at the next, and so on alternately.

An Ultzmann's syringe is a narrow curved metal catheter, which reaches only as far as the prostatic portion of the urethra, and it has a fine bore.

The patient should wash himself out once a day with a pint of a 1 in 10,000 solution of zinc permanganate for fourteen clear days after the last trace of discharge has vanished. Intramine and trimine are especially valuable in these cases, and should be used as detailed in description of chronic urethritis. The treatment may also be supplemented with vaccines if thought desirable.

For the last two or three times that an Ultzmann's syringe has to be passed it is a good plan to use a Kollmann's posterior dilator. With this instrument one can both dilate the urethra and inject the drug as well.

One knows only too well how common recurrences of prostato-urethritis are, but it is not so well known that a fair percentage of these recurrences are not due to a re-awakening of a gonococcus colony, but are merely catarrhal conditions. Observers who are used to seeing gonococcal cases will, no doubt, have noticed that a patient will never get a recurrence if he has connection with his wife, but only if he has connection with another woman. A patient who has had a gonococcal arthritis, which has been cured for years past, is very liable to get a slight catarrhal synovitis after any undue exertion of that joint, or changes in the weather may even influence it; but such cases are not regarded as outbreaks of the old gonococcal infection; the same with the prostate.

There are cases, on the other hand, in which it is absolutely impossible to rid the prostate of gonococci, and a cure for these cases is yet to be discovered. I think the number of such sad instances would be considerably diminished if the rule were made not to pass more instruments, and not to 
use stronger solutions, than were absolutely necessary in all cases of gonorrhœa. I am certain that too much and too drastic treatment are the curse of the present-day treatment of gonorrhœa. We have no absolute proof that the gonococci in such cases are invariably pathogenic; indeed, we may have nonpathogenic gonococci as we have non-pathogenic meningococci, two organisms which are particularly closely related. Here I should like to mention that it is practically futile to rely upon a microscopical examination as a means of being able to inform the patient when it is safe for him to resume marital intercourse.

For the prostatorrhœa which may follow a case of chronic prostatitis practically nothing can be done. The most important thing is to impress upon the patient that it is a natural sequence of the treatment. It should be explained to him how it is produced, and that it has nothing to do with spermatorrhœa. As a rule this suffices, and the patient worries no more about it; but if he is still concerned, the secretion of mucin can be diminished a little by using a urethral psychrophore and running through cold water, and by giving belladonna internally. Finger ${ }^{1}$ is in favour of ergotin in these cases, and Sellei ${ }^{2}$ states that extractum hydrastis liquidum has a beneficial action. Two or three intramuscular injections of intramine will sometimes stop it.

The treatment of chronic prostatitis is long, and it is frequently complicated by sexual neurasthenia, so that the physician is often consulted on the question of having sexual connection. In the present day of faddists the patient may stumble across a physician who may be led by his moral convictions rather than by the desire to do the best for his patient.

Provided the prostatic discharge is free from gonococci, sexual connection once or twice a month often has a very beneficial effect upon a case of prostatourethritis, and this may be especially noticeable if the condition is further complicated by sexual neurasthenia. One cannot be too careful in the treatment of a neurasthenic. In bad cases, a sexual neurasthenic may lose all sexual power, and may be unable to get an erection in a short space of time. Such a state of affairs aggravates his condition, and may lead to suicide. Allowing the patient to have occasional sexual connection at the beginning is often the only means of saving him. It is exactly the same with an alcoholic and with a patient addicted to drugs. Sudden stoppage may lead to disaster, while gradual stoppage may result in one's being able to cure the patient.

\section{Cystitis Gonorrhoica.}

Considering the fact that the urethra and the bladder are continuous, it might, a priori, be assumed that gonococcal cystitis would be as common as acute posterior urethritis. Although the statement has been copied from one textbook to the other that gonococcal cystitis is a common complication of gonorrhœa, it is, nevertheless, the fact that cystitis is one of the complications most rarely to be met with. 
Of all the complications of gonorrhœa, I should say cystitis, pyelitis, and nephritis, were the rarest, yet in the ancient literature frequent mention is made of them. This probably accounts for the fact that many are still of the opinion that they are frequent complications, and it suggests that gonorrhœa was possibly a more severe disease at one time than is now the case. Hippocrates was familiar with cystitis, and Sydenham regarded it as a fairly common complication. Both Morgagni and Hunter describe all three as being by no means rare, but since the latter's time good descriptions cease, which is suggestive that gonorrhœa became a more localised disease at the beginning of the nineteenth century. I have not seen more than three cases of gonococcal cystitis, and only one of pyelitis and nephritis, during the last fourteen years.

Another reason why gonococcal cystitis is said to be common is due to the fact that many cases of acute posterior urethritis are wrongly diagnosed as cases of cystitis. The mucous membrane of the bladder must be peculiarly insensitive to the gonococcus, since, in all cases of posterior urethritis, pus containing myriads of gonococci gains entrance to the bladder, in which organisms may remain alive for hours. The bladder mucous membrane appears to be not only insensitive to the gonococcus, but also to most other bacilli, as evidenced by the rarity of cystitis in cases of bacilluria due to the Bacillus coli, etc.

In true cases of gonococcal cystitis gonococci cannot be found in the urine for more than two or three days, as they quickly give place to a secondary infection, by which they are exterminated. It is not very easy to distinguish a case of gonococcal cystitis from a case of very severe posterior urethritis, but in the former the patient almost always complains of a very dull aching pain over the symphysis. Further, however frequent be the act of micturition, the second glass test in cases of cystitis is always thick-indeed, it may be thicker than the first, and it always contains more gonococci. Bleeding at the end of micturition is common in acute cases of posterior urethritis. In the case of cystitis all the urine passed is blood-stained, and there is no more blood in the last portion than in the first. In very severe cases the cystitis may become gangrenous, and the lining of the bladder come away as a slough.

In all cases of cystitis the patient looks extremely ill, has fever, and sweats profusely. So long as the cystitis remains gonococcal, the urine is acid; when the secondary infection supervenes, the urine becomes alkaline. If, instead of the two-glass test, three glasses be used, the urine passed into the third will be the thickest in cases of cystitis, and in cases of posterior urethritis there will be no difference in the opacity of the three.

The organism which most frequently takes the place of the gonococcus is the Bacillus coli. Every case of cystitis should be treated as quickly as possible, since the prognosis of true gonococcal cystitis is good; but the moment a secondary infection takes its place, the course of the disease may be very 


\section{CLINICAL ASPECT AND TREATMENT OF VENEREAL DISEASES}

long. On no account should an instrument be passed, as it is certain to hasten the advent of the secondary infection.

The patient should be put to bed, and cold compresses applied to the lower part of the abdomen and perineum. The following medicine should be given internally:

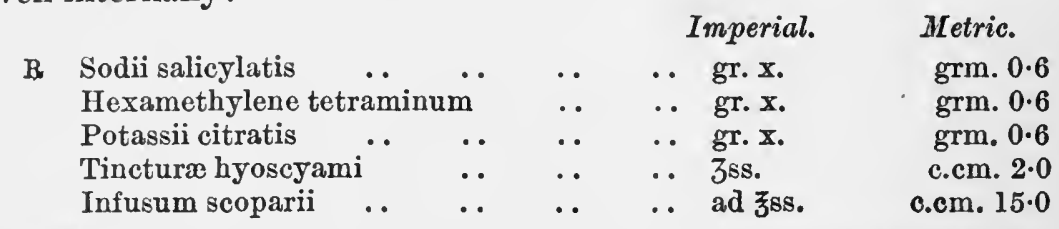

M. f. mist. S.: そss. ex aq. sodæ effervesc. そii. omni quartis horis.

The bladder should be washed out twice a day with a 1 in 10,000 solution of colloidal silver from the tip of the urethra (Janet's wash-out). A sensitised vaccine should be injected intravenously, if handy, or Thomson's detoxicated vaccine intramuscularly. As early as possible $1.0 \mathrm{c} . \mathrm{cm}$. of trimine should be injected intramuscularly, and $1.5 \mathrm{c.cm}$. on the fourth day. I had one case of cystitis where two injections thus administered appeared to cure the patient.

\section{Vesiculitis.}

Gonococcal vesiculitis was first described by Morgagni, and it is interesting to note that van Swieten regarded it as the worst complication of the disease. Hunter declared he had never seen a case of genuine vesiculitis-i.e., in which the prostate and bladder were not implicated as well. It was Ricord who pointed out the connection between resiculitis and epididymitis.

The seminal vesicles are not very easy to palpate. When an examination is being undertaken, the knee-elbow position is the best, and the bladder should be full. Examination of the secretion is often as necessary as mere palpation. To collect the seminal secretion, the following procedure is advised: The patient should have a full bladder; the prostate is then massaged, and, if the prostatic secretion shows at the urethral orifice, the bladder should not be quite emptied. Then the seminal vesicles can be massaged, and the secretion from them washed out with the remaining fluid in the bladder. If, after massaging the prostate, the prostatic secretion does not show at the urethral orifice, the chances are that it has passed back into the bladder. When such is the case, it is, of course, necessary to empty the bladder before examining the vesiculae seminales. As the expressed seminal secretion usually falls back into the bladder, it can be washed out with a little sterile water. Unfortunately, even this manœurre may fail in enabling one to diagnose vesiculitis, and clinical experience renders it unnecessary, because in almost every case of persistent urethritis in which the last few drops of urine passed are thick and muddy a diagnosis of vesiculitis may be made.

Spermatocystitis gonorrhoica occurs more frequently than is supposed to be the case-in roughly $7 \cdot 0$ per cent. of cases-but the subjective symptoms 
are few, and in many cases it is impossible to palpate the seminal vesicles; consequently, they are not often examined. The vesiculoe seminales are infected vi $\hat{a}$ the ejaculatory ducts. Inflammation without abscess formation is the usual trouble, an abscess of the seminal vesicles being comparatively rare. The same holds good in the case of the prostate and the epididymis.

I once had a case of a vesicular abscess in which a regular bag could be felt per rectum overlapping the upper pole of the prostate, and it emptied itself of its own accord when the patient assumed the knee-elbow position. Massage of the seminal vesicles, daily washing out, and vaccines, resulted in a complete cure of the case.

The usual subjective symptoms are painful micturition, acute pain on ejaculation of semen, and peculiar sensations in the perineal and perianal regions. Sexual desire is frequently stimulated, and the patient may suffer from extended erections, or even from priapism. If the ejaculatory ducts are closed by the inflammation, the patient is unable to express the semen during sexual connection, with the result that, instead of having an orgasm, he experiences the most excruciating pains. The pains begin in the perinæum, spread to the rectum, scrotum, and even down the legs. The type of sensation described is absolutely typical. A useful point to remember is that in every early case of vesiculitis the patient complains of pains or heaviness in the region of the kidneys.

A microscopic examination of the seminal secretion should be undertaken with the view of seeing if there are any gonococci and pus cells, and if the spermatozoa are destroyed or not.

Chronic spermatocystitis may give rise to no symptoms, but it should be remembered that it is a very frequent cause of sexual neurasthenia. The patient's sexual desire is often increased, but the pleasure derived therefrom is often nil; the quantity of semen may be very small, and occasionally bloodstained-all symptoms which are very liable to lead to sexual neurasthenia, or to aggravate it if the patient is already suffering from it. Many patients ccmplain of a heavy feeling in the perinæum and of pain in the end of the penis. Pain in the latter situation causes the sufferers much anxiety, and is generally present when there is an inflammatory condition anywhere along the genito-urinary tract. As too energetic instrumentation and the use of too strong solutions may lead to prostatorrhœa, so may they lead to spermatorrhœa, and the occurrence of the Lallemand-Trousseau bodies is, in my experience, usually a sign of the increased secretion which emanates from mucous membranes, both after a chronic inflammatory lesion has been got rid of and when the treatment has been too drastic. In very acute cases the application of cold per rectum should be tried, but immediately discontinued if the symptoms show signs of being aggravated. Narcotics, in the form of suppositories, should be inserted per rectum. In chronic cases massage of the vesiculce seminales should be undertaken, but the process should not be prolonged nor be too frequent-not more than once every other day for 
about twelve times. After massage, colloidal silver and colloidal iodine or intramine should be injected alternately into the posterior part of the urethra by means of an Ultzmann's syringe or Teevan's catheter. The patient should wash himself out daily with a 1 in 10,000 solution of zinc permanganate, and vaccines should be employed. The best results are to be obtained by prescribing colloidal iodine internally and by injecting intramine and trimine intramuscularly, as described in the other chronic gonococcal lesions.

Massage of the seminal vesicles, plus the treatment which I have already outlined, is often the only means of curing a case of spermatorrhœa and sexual neurasthenia, but in both these conditions success sometimes follows the cold water treatment by means of the urethral psychrophore.

Massage, etc., will usually result in closed ejaculatory ducts becoming patent again, but this is not always the case. When ordinary treatment appears to be unavailing, it is advised to probe the ducts viâ a urethroscope, but $I$ have yet to see a case in which such a procedure has been successful. Vasostomy for injecting directly into the vesicles has recently been practised, but I have never seen any benefit result. To-day vesiculotomy and vesiculectomy are being advised, but it has yet to be determined whether one is justified in resorting to such drastic measures. An almost pathognomonic symptom-complex of long standing vesiculitis is general lassitude, absolute lack of energy and pains in the small of the back, which are worst when the patient is at rest.

\section{Deferentitis.}

According to Oppenheim and Löw, ${ }^{3}$ the organisms reach the epididymis by means of an antiperistaltic movement of the vas deferens which commences at the colliculus seminalis. Although the condition is rare, the gonococci may, on their passage along the vas, give rise to a true deferentitis. Deferentitis rarely occurs without an epididymitis, while an epididymitis without a deferentitis is the rule. Therefore, the symptoms of deferentitis are practically the same as those to be now described under the heading of "Epididymitis." The vas can usually be felt along its course, as the cellular tissue around it is usually inflamed in sympathy; consequently, one is really dealing with a funiculitis. The pain in the inguinal region is very severe, and it usually radiates down the inner side of the thigh. I have seen the condition mistaken for appendicitis, and on one occasion the patient was operated on for the latter. Chronic cases are very apt to be mistaken for tubercular disease.

\section{Epididymitis.}

Epididymitis was known as a complication of gonorrhœa by the ancient Greeks and Romans. Boerhaave was the first to point out that it was the epididymis and not the testis which was implicated. Deferentitis, as distinct from epididymitis, was described by Andree in 1777; but both Hunter 
and Ricord denied its occurrence. On the other hand, Benjamin Bell published several cases of genuine deferentitis.

Epididymitis results from a direct extension of the gonococci from the urethra. Owing to the stagnation of the stream, the gonococci settle in the caput minor, hence it is usually the lower pole of the epididymis which is affected. Owing to the more dependent position of the left testicle, the epididymis on this side is more frequently affected than on the other. Unwise instrumentation in the acute stage of urethritis, and massaging a prostate gland in the acute or subacute stages of prostatitis, often results in the onset of an epididymitis. Washing out the urethra from the tip in acute cases of both anterior and posterior urethritis will not provoke an epididymitis unless the solutions used be too strong, or the pressure at which they are injected be too great. By far the greater number of cases of epididymitis arise in those individuals who have been treated by internal medicamentation only.

As a rule, before an epididymitis gives rise to objective symptoms the patient complains of drawing pains in the inguinal region, and of neuralgic pains in the testicle and upper part of the thigh on the affected side. The testicle often feels unusually heavy. If the patient is made to rest at once, to have ice or cold compresses applied, to be injected with a sensitised vaccine and trimine, and to suspend urethral treatment, the majority of cases, if diagnosed thus early, never develop objective symptoms.

As a rule, it is the objective symptoms which compel the patient to seek advice. Such symptoms are usually ushered in by high fever, rigor, etc., and within a few hours the affected testicle is about three or four times its natural size. The skin of the scrotum becomes œdematous, fluid forms in the sack, and the inflammation, which commences at the caput minor, spreads to the body and caput major of the epididymis. The testis itself is very seldom affected. A transient peritonitis is also a rare complication.

The acute inflammation in time subsides, and leaves a hard and swollen nodule in the lower pole of the epididymis. Only rarely does the epididymis become adherent to the skin. "When this happens, there is always an abscess in the epididymis, and, in the majority of the cases, the abscess bursts through the skin. Bursting of an abscess through the skin usually results in the spontaneous cure of the epididymitis.

Owing to the density of the fibrous tissue which forms in the tail of the epididymis, and which may be sufficient to constrict all the tubules, it is necessary, especially if both sides are affected, to put the patient under treatment at the earliest possible moment. 'Dense fibrous tissue for mation, following bilateral epididymitis, is certain to cause sterility. The patient should be put to bed, and ice or cold evaporating lotions should be applied. Personally, I prefer cold to hot applications, but the former are more difficult to manage. Should the application of cold quickly aggravate the symptoms, it means that the inflammation has overstepped that stage in which cold applications can do any good. Then hot fomentations are useful, and painting 
the organ daily with ichthyol is a good substitute. Cold will often abort acute inflammation, heat never will. Heat is mainly useful in bringing the inflammation to a head.

Cold applications will fail in their purpose if they are allowed to get warm and clammy, hence they are difficult to use. Cold applications which become clammy quickly lead to venous congestion, and this may readily cause the inflammatory process to extend. Ice is the best cold application, and, failing that, an evaporating lotion. When an evaporating lotion is used, the dressing should be changed several times a day, and it should always be lightly covered over.

The following is a good evaporating lction:

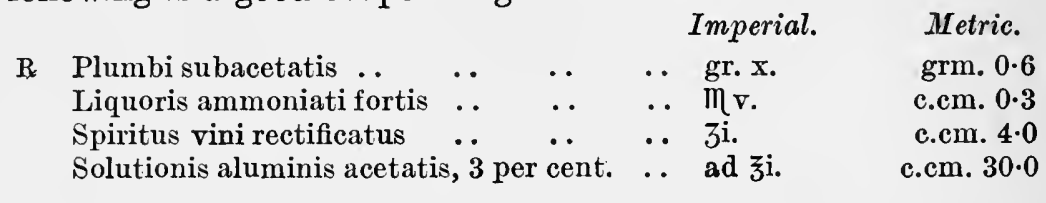

When the patient gets up, a suspensory bandage should be worn until he has been completely cured of his gonorrhœa. While in bed, it is a good plan to suspend the testicles by a broad bandage from the shoulders. The diet should be light, and the following medicine should be taken internally:

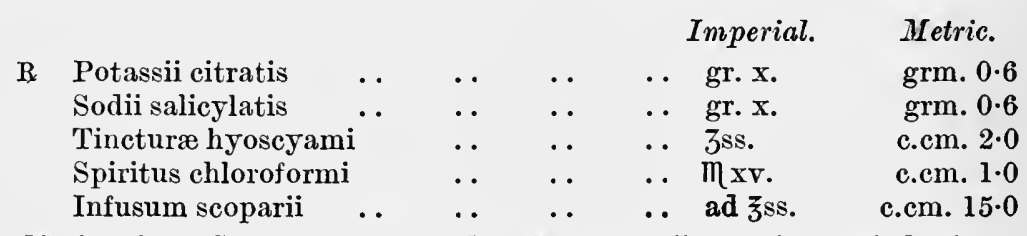

M. f. mist. S.: そss. ex aq. sodæ effervesc. そ̇i. omni quartis horis.

If the pain is very bad, morphia suppositories should be used. In every case great attention should be paid to the bowels, and trimine and vaccines should be injected without delay. A characteristic of all gonococcal foci is that they recur, even after they have been apparently cured, and gonococcal epididymitis is no exception to this rule; therefore, monthly injections of a potent vaccine should be prescribed for about six months after the epididymitis has vanished. I once treated a patient who had had fifteen attacks of epididymitis in the course of twenty-one years.

Provided the urethra is washed out gently, and very weak solutions are used, there is no reason to su'spend the treatment of this part. If both testicles are affected, or the only sound one, and provided the case is seen early enough, radical local treatment may save the epididymis from becoming injured or disorganised. Either of the two following methods of treatment may be employed - the first is simpler and more efficacious:

1. The scrotum is taken in the left hand, and the skin is made taut over the affected epididymis. A sharp-pointed bistoury is then plunged into the 
epididymis at two or three points parallel to the tubules. The pain is momentary, and the relief afforded is almost instantaneous.

2. 1.0 to $2.0 \mathrm{c.cm}$. of colloidal silver are injected into the epididymis. The epididymis reaches almost its normal size in one to three days; but, as a rule, a second injection is necessary to produce complete resolution. The rapid administration of the chemotherapeutic agents, combined with vaccines, have practically made both of these methods superfluous.

Mention must now be made of the chemotherapeutic treatment of epididymitis. The colloidal preparations have done more to prevent epididymitis than to cure it. Luys puts the percentage of epididymitis as high as $25^{\circ} 0$ per cent. In my experience it is about 15.0 per cent., but since the introduction. of colloidal manganese it is only 3.0 per cent. If $1.0 \mathrm{c.cm}$. trimine be injected the moment the signal shows an epididymitis is about to supervene, and $1.5 \mathrm{c.cm}$. on the fourth day, the lesion may be aborted. If already pronounced before treatment has been commenced, the injections should be administered as follows: First day, $1.0 \mathrm{c.cm}$. trimine; sixth day, $1.5 \mathrm{c.cm}$. trimine; tenth day, 1.0 c.cm. intramine; fifteenth day, $1.5 \mathrm{c.cm}$. intramine; twentieth day, 1.5 c.cm. trimine. The intramine is particularly valuable in getting rid of, or preventing, the formation of the fibrous tissue nodule in the epididymis. In chronic cases two or three injections of intramine should be prescribed before the trimine. Should an epididymitis supervene during a course of injections of trimine, either the chemotherapeutic treatment should be stopped, or an injection of intramine, $1.5 \mathrm{c.cm}$., administered as quickly as possible. Such an occurrence means that the oxidising capacity of the host's protective substance has been over-taxed, and that the metal has become itself oxidised, two conditions which can at once be rectified with intramine. The advantage of the chemotherapeutic treatment is that urethral irrigations need not be suspended. Vaccines can be combined with trimine and intramine, as it is always another arrow in the quiver.

The following case of a suppurative epididymitis which led to hernia testis illustrates well what can be achieved with oxidising and reducing agents:

CASE 127.-Patient was admitted with a swollen testicle, which he had had for three weeks. Under further treatment for a fortnight an abscess developed in the epididymis, which had to be incised. This led to a hernia testis, which failed to improve under local remedies. Every second day the patient received an intramuscular injection of colloidal manganese and colloidal antimony $(0.04$ per cent. emulsion) in each buttock as follows: First day, $1.5 \mathrm{c.cm}$. of each; third day, $1.5 \mathrm{c.cm}$. of each; fifth day, $1.5 \mathrm{c} . \mathrm{cm}$. of each; seventh day, $2.0 \mathrm{c} . \mathrm{cm}$. of each; ninth day, $3.0 \mathrm{c.cm}$. of each. By this time all the swel'ing had disappeared, but a sinus still persisted. A week later $2.5 \mathrm{c} . \mathrm{cm}$. of intramine were injected, which cured the sinus, and caused all the induration to disappear.

I had another case, in which a sinus leading to the epididymis persisted for three months without healing. Under two injections of intramine and one of colloidal manganese, within ten days the sinus had completely closed. 


\section{Ureteritis, Pyelitis, and Pyelonephritis Gonorrhoica.}

There are three ways in which the urinary tract above the bladder may be involved: $(a)$ direct extension from the bladder; $(b)$ vi $\hat{a}$ the blood-stream; (c) viâ the lymphatic stream. Neither ureteritis, nor pyelitis, nor pyelonephritis are common complications; consequently, it is not easy to say by which route the infection usually reaches these structures. If ureteritis, etc., follow a gonococcal cystitis, the chances are that the infection has spread per continuitatem, but should a pyelitis or a pyelonephritis occur independently of a cystitis, presumably the infection is a blood or lymphatic one. That the gonococcus can reach the kidney by the blood and the lymphatic stream is proved by the cases of perinephritic abscesses which sometimes follow gonorrhœa. Whether a perinephritic abscess is hæmic or lymphatic in origin it is impossible to say.

Ureteritis, at any rate, is due to a direct extension of the gonococcus from the bladder. Ureteritis can only be diagnosed by a cystoscopic examination of the bladder. A patient suffering from pyelitis or pyelonephritis is always extremely ill; the complexion is white, fever is constant, and there is nearly always profuse sweating. There is pain and tenderness over the kidney region; the pain may run down to the penis and scrotum, and even to the leg, and colic attacks may be experienced, as if the patient had a stone. A swelling is often to be felt in the kidney region, and there is usually marked polyuria, and, of course, pyuria. Owing to the fact that there is cystitis in most of these cases, a certain diagnosis cannot be made except by catheterising the ureters, a procedure one does not care to undertake in such an acute condition. A diagnosis may sometimes be made between cystitis and pyelitis by comparing the quantity of pus with the amount of protein. In the severest cases of cystitis, according to Rosenfeld, ${ }^{4}$ the amount of protein in the urine which has been allowed to settle never exceeds 0.15 per cent.; while the protein, in cases of pyelitis, in which the urine is no thicker than that from cases of cystitis, is always two to three times as much.

Finding broken up red blood-corpuscles in the sediment is highly suggestive of pyelitis. Great attention is usually paid to the kind of epithelial cell found in the urinary deposit, but it is very easy to confuse the different varieties.

Gonorrhœa may cause nephritis, but, as a rule, parenchymatous nephritis occurs only in cases suffering from gonococcal endocarditis.

Balsamic nephritis is frequently talked about, but seldom seen, and it is very doubtful whether albuminuria often follows the administration of sandalwood oil, copaiba, etc. Some observers are of the opinion that a gonococcal cystitis and pyelitis open the door for a tubercular affection of these organs, in the same way as gonococcal epididymitis is supposed to predispose the organ to an infection with the tubercle bacillus. Tuberculosis of the urinary. 
tract is so common in patients who have never had gonorrhœea, and gonorrhœea is so common in patients who never develop tuberculosis, that it is quite impossible to say whether gonorrhœa is a predisposing factor or not.

\section{Proctitis Gonorrhoica.}

Gonococcal inflammation of the rectum may be a primary lesion or a secondary one, due to an extension of the organisms from the urogenital tract. Owing to the proximity of the vagina and the anus, gonococcal proctitis is much more common in women than in men. Actual figures as to the frequency of the rectal complication vary enormously, but it may safely be said that it occurs in a much higher percentage of cases than is generally thought to be the case, and there can be no doubt whatever but that it is a frequent source of infection to a second party.

The symptoms are far from marked, and they often consist of Pruritus ani only. Occasionally there is pain on defæcation, and rarely a little bloodstained discharge. Eczema and Pruritus ani in a woman should always make the observer at least think of a rectal gonococcal infection. A naked eye examination of the rectum usually suffices one in making a diagnosis, as a microscopic examination of the secretion can so easily fail to reveal the gonococcus. The mucous membrane is red, swollen, and granular; it bleeds easily if injured, and there are usually several erosions, some of which may be covered with a sort of membrane. Only rarely does true ulceration occur, a condition which is sometimes followed by stricture, or, more generally, by a diffuse fibrous pelvic cellulitis. Only the lower part of the rectum is affected. The process is slow in disappearing, and is not very easy to cure. The rectum should be treated. in exactly the same way as the male urethra is, and no instrument passed until the acute and subacute stages have ended. Then a proctoscope can be inserted, and the erosions cauterised with a 3.0 per cent. solution of silver nitrate. In the severe cases, especially those complicated with pelvic cellulitis, intramine produces excellent results.

1. Finger (1908): Die Geschlechtskrankheiten. II. Teil. F. Deutiche, Leipzig u. Wien.

2. SeLLeI (1907): Orvosi Hetilap, li., 43.

3. Oppenteim U. Löw (1905): Virchow's Archiv, clxxxii., 39.

4. Rosenfeld (1898): Berl. klin. Woch., xxxv., 661. 


\section{CHAPTER XXII}

\section{COMPLICATIONS OF GONORRHEA DUE TO A SPREAD BY METASTASIS OF THE ORGANISM}

\section{Tenosynovitis and Bursitis.}

BeNJAMIN BRODIE, in 1818, was the first to describe gonococcal tenosynovitis, a complication which appeared to be very little understood for many years, becanse no cases are recorded till 1861, when Rollet described three. It was really not till Fournier's work appeared that one's knowledge of the condition was at all complete.

A tenosynovitis may occur alone, or in company with an arthritis, in which case the tendon sheaths around the affected joint are those most frequently affected. Extensor tendon sheaths are more often affected than flexor ones, and, of the former, the extensor tendons of the hands and feet are most often involved. The secreted fluid is usually serous, rarely purulent, and, as is the rule in all gonococcal lesions, fibrous tissue formation is pronounced, and unless this fact be borne in mind in all cases of tenosynovitis, most troublesome adhesions may form. A tenosynovitis may set in very quickly after the onset of the urethritis. I once had a case with bilateral tenosynovitis of the extensor communis digitorum which was well marked on the fifth day after infection.

Tenosynovitis may complicate gonococcal conjunctivitis of infants, and it is not at all uncommon in cases of vulvo-vaginitis of young girls.

In the treatment of all metastatic complications, attention should first be paid to the site of inoculation, which is usually the urethra. Operative procedure should always be the last consideration, and in the case of tenosynovitis should be employed only when there is suppuration. In all ordinary cases, firm pressure should be applied to the affected part when the acute inflammatory stage has vanished under cold applications or ichthyol; but exercises should be commenced as soon as possible, so as to prevent any contraction from adhesions.

Tenosynovitis is apt to occur if too big doses or too frequent doses of an oxidising agent are prescribed when a reducing agent should have been instituted. Fortunately, in these cases, as indeed in all cases of tenosynovitis, however acute the lesion, intramine works like a charm, as the following interesting case shows: 
CASE 128. - A patient was treated for a recurrent prostato-urethritis with colloidal manganese. Four injections had been prescribed, with forty-eight hours' interval between each dose, and the last dose used was 2.5 c.cm. The day after the third injection an acute tenosynovitis of the tensor vagina femoris of the right thigh set in. The day following the fourth injection an acute tenosynovitis of the whole of the right palmar sheath occurred. Within twenty-four hours of an injection of intramine these complications had practically vanished.

Gonococcal bursitis is rare, except in those cases in which neighbouring bursæ of a joint become infected by direct extension. The patellar and subcrural bursæ may be affected alone, and I have also seen cases of trechanteric and ischial bursitis. In one case of ischial bursitis under my care suppuration set in, and left a sinus from the skin of the buttock, which took a long time to heal.

The pain in the Achilles tendon, of which complaint is so frequently made in gonorrhœa, may be due to an inflammation of the bursæ in this region, but it is more often due to inflammation of the tendon itself. The pain complained of in the heel and foot is usually due to inflanmation of the os calcis, of the bursa underneath this bone, and of the plantar fascia.

Inflammation of the plantar fascia is the cause of flat foot, which is so commonly met with in patients suffering from gonorrhœa. It has been especially noticeable in the present war, owing to the marching which has had to be done. Exostoses of the os calcis may be found in some of the intractable cases.

\section{Gonococcal Arthritis.}

Just as most of the cases of gonococcal arthritis are wrongly diagnosed to-day, and are treated for any cause but the right one, so was it always the case. Practically all the earlier writers ascribed the condition, when complicating gonorrhœa, as being due to syphilis, and even Hunter thought the arthritis was gout, a disease which was also responsible for the urethral discharge which co-existed.

Monteggia, who was the first to describe genuine cases of gonococcal arthritis in 1794, was careful to note that none of the cases had suffered from gout. In 1830, William Lawrence not only drew attention to the true nature of gonococcal arthritis, but also pointed out that in twenty-four cases of eye trouble due to gonorrhœa, ten of the patients suffered from rheumatism, observations which were six years later confirmed by Astley Cooper. In spite of these observations, even so short a time as twenty years ago we find authors disbelieving in the actual existence of gonococcal arthritis.

No complication of gonorrhœa has increased more of recent years than has arthritis, due in part to the stress of war, soldicrs being chiefly affected, and in part to over-treatment of the disease. In spite of the increased attention drawn to the complication, as, roughly, 2:0 per cent. of soldicrs who contracted gonorrhœa and were admitted to venereal hospitals developed it, 


\section{CLINICAL ASPECT AND TREATMENT OF VENEREAL DISEASES}

quite another $2 \cdot 0$ per cent. were wrongly diagnosed, and admitted to general hospitals. Many, if not most of the described cases of dysenteric arthritis are gonococcal in origin in my opinion.

For years past there has been continued and acute discussion as to whether gonococcal arthritis was a metastatic lesion or a lesion due to the gonotoxine. The reason why several observers held the latter view was because they failed to find the gonococcus in the fluid which they drew off. I have examined the fluid from nine cases of gonococcal arthritis; in every one it was sterile. In those cases tested for the presence of antibody and antigen, the former was found, but not the latter. The reason why the gonococcus is not generally found in the fluid is that it remains limited to the synovial membrane. It is just the same with the tubercle bacillus in a cold abscess. The organism is not found in the pus, but in the wall of the abscess. It is now almost universally agreed that a gonococcal arthritis is a metastatic lesion.

No case of gonococcal arthritis should be treated lightly, because a joint affected from any specific cause is always liable to become secondarily infected. If anyone has seen a pyogenic infection of a gonococcal joint, the picture is not likely to be forgotten. Within a few days of the joint becoming purulent the patient may die, and should the case not terminate fatally, bony ankylosis is all that can be hoped for.

Gonococcal arthritis can generally be prevented if all gonorrhœal patients are strictly forbidden taking any exercise while the arthritis is acute, and if all medical men will follow the rule never to massage the prostate or to pass an instrument while the patient has a discharge, points which have been proved by the enormous numbers of soldiers who have been invalided home during this present war with joint trouble.

Before a true arthritis becomes manifest, the patient usually experiences fleeting sharp pains in the joint which is to become affected. If rest is ordered, and if vaccines, or, better, intramine, are injected without delay, the progress of the inflammation may be checked.

The statement has frequently crept into writings that women are immune from gonococcal arthritis. Such is far from being the case, as both men and women are equally affected.

A man who contracts arthritis is practically certain to have a prostatourethritis or a vesiculitis, and a woman is certain, at least, to have a cervicitis. More often she has an endometritis, and in many cases she has a salpingitis. Therefore, no case of arthritis should be treated without the main attention being paid to the site from which the organisms entered the blood-stream. It must be remembered that one reason why gonococcal arthritis is not frequently diagnosed is because the arthritis complicates a recurrent attack more frequently than the initial attack, and because its onset may either occur after the discharge has stopped or before it comes on.

It must not be forgotten that, although we generally refer to adults when speaking of gonococcal arthritis, the complication may also occur in cases 
of infantile gonococcal conjunctivitis, and it is not at all uncommon in cases of vulvo-vaginitis affecting young girls.

An arthritis may complicate the first attack of gonorrhœa, but it more often starts during the first or second recurrence of the uncured original attack. Should the patient have repeated recurrences of his urethritis, and an arthritis of one knee-joint, which complicated his first recurrence, this same knee-joint is apt to light up again in each future recurrence. This condition of affairs is quite pathognomonic of gonococcal arthritis. The types of gonococcal arthritis allow themselves to be conveniently divided into four classes: (1) Hydrops articuli ; (2) sero-fibrinous arthritis; (3) purulent arthritis; (4) phlegmonous arthritis.

Hydrops Articuli.-Usually without any warning, a joint, which is most commonly the knee, becomes suddenly distended with fluid. The joint is not even painful. The fluid may disappear as quickly as it came, recurrence is common, and, as the amount of fluid may be considerable, repeated sudden distension of a joint is liable to lead to a destruction of its ligaments. More rarely the fluid takes a long time to disappear. In these cases, if the distension is very marked, it is imperative to tap the joint.

A joint should never be tapped except under the strictest aseptic precautions. The knee is practically the only joint for which the operation has to be undertaken. The limb is extended, and a Barker's lumbar puncture needle is inserted between the external condyle of the femur and the external tuberosity of the tibia. The fluid should be allowed to escape only slowly, and after it has been removed a Martin's rubber bandage should be applied from below upwards, and the patient should be kept at rest. If the knee is not bandaged at once, it may fill up again with fluid in an hour or two. Subcutaneous injections of the withdrawn fluid in the region of the affected joint have never, in my experience, been of any benefit.

The synovial membrane and capsule remain thin in cases of Hydrops articuli.

Arthritis Sero-Fibrinosa.-This is by far the most common form of gonococcal arthritis. Both the synovial membrane and capsule are usually thickened. The withdrawn fluid looks not unlike serum, and it is called fibrinous owing to the amount of contained fibrin, which frequently causes the fluid to clot.

With this form of arthritis the patient usually looks very pale and ill. The muscles around the joint soon become atrophied, and any of the focal complications which have been described may accompany this form.

This form of arthritis is very liable to recur, and at each recurrence the capsule becomes still more thickened. Owing to the amount of fibrin which is formed, adhesions are liable to follow the subsidence of the inflammation.

The purulent arthritis usually arises from the sero-fibrinous form becoming infected with pyogenic cocci.

Phlegmonous arthritis, which was so christened by König, is an arthritis 
in which the capsule and the periarticular tissues are the structures most affected. The joint is often markedly swollen, but the amount of fluid in it is very small. This form of arthritis has frequently received the name of pseudo-membranous. As the inflammation is most acute in the periarticular tissue, the subcutaneous tissue is cdematous, and the skin over it is very red and painful. The inflammation quickly spreads to the interior of the joint, and destroys all the ligaments; hence it is in the phlegmonous arthritis that subluxation of the tibia and other orthopædic deformities of joints are most likely to occur.

In phlegmonous arthritis absolute rest should be enforced, since, owing to the acuteness of the inflammation, a pyogenic infection is apt to find its way into the joint. Vaccines or intramine should be injected as quickly as possible. All antiphlogistic measures should be employed, and the limb fixed in that position in which bony ankylosis would least impair its usefulness, since bony ankylosis is the result to be hoped for.

In the mild cases of phlegmonous arthritis, and in the chronic cases of Arthritis sero-fibrinosa, changes in the joint may be produced which become absolutely indistinguishable from the condition that is called osteo-arthritis. The cartilage is worn away, the bony surfaces become eburnated, and the edges devclop osteophytic growths. I have notes of three cases of gonococcal osteo-arthritis of the hip-joint, in one of which the head of the femur had already been dislodged from the acetabulum; and of two cases of a similar condition affecting the knee-joint. Naturally, treatment in such cases is unavailing.

As a rule, not more than two or three joints are affected at a time, although several may be affected at different intervals.

Owing to the small size of the finger and toe joints, periarticular changes are practically constant; and as these almost invariably lead to a chronic thickening of these tissues, the patients usually have a permanent broadening of their fingers and toes at the metatarso- and metacarpo-phalangeal joints.

Any patient who has had a gonococcal arthritis is very liable to suffer for years afterwards with what may be called arthralgia. Such patients are apt to notice changes in the weather and changes in temperature. It is well to bear this arthralgia in mind, as it is often mistaken for a recurrence of the arthritis.

The local application of unguentum iodex, or colloidal iodine oil, or unguentum guaiacol $(10 \cdot 0$ to 40.0 per cent.), quickly disperses the arthralgic pains.

The knee is far and away the most frequent joint to be affected-indeed, Hydrops articuli is rare in any other. I have seen a case in which the elbow was affected. After the knee come the ankle and wrist joints. The metacarpo- and metatarso-phalangeal joints are frequently affected, also the other small joints of the foot.

Arthritis of the joints of the upper extremities other than those already 
mentioned is not very common. The elbow is affected more often than the shoulder; in many cases it is the only joint affected, and there is usually a marked wasting of the muscles around the joint.

When the hip-joint is affected, it is usually the only joint that is involved. Any joint may be affected, but arthritis in other than those already mentioned is rare; but there is one which requires special mention-i.e., Spondylitis deformans.

I have had altogether seven cases of Spondylitis deformans under my care. Spondylitis deformans is an uncommon condition, and is said to be of varied ætiology. It starts as an inflammation of the ligaments of the vertebræ, which leads to contraction of the former, and, finally, to ankylosis of the latter. A section or sections of the spine, or even the whole vertebral column, may be affected, in which case the movements of the ribs may be stopped entirely, with the risk of subsequent lung trouble.

Unfortunately, nothing is certain in medicine, and if a statement is made, something will be bound to crop up the next moment to contradict it, but I cannot help feeling that gonorrhoea is the commonest cause of Spondylitis deformans. In all my cases the trouble started during a recurrent attack of a bygone gonorrhœa; in every case one or more of the joints in the body were, or had been, affected, and all seven were men. Unless the case is obtained early, treatment is unavailing.

CASE 129.-Patient, a man, aged 27, contracted gonorrhœa seven years ago, which disappeared without complications. First recurrence four years later, which likewise vanished without trouble. The second recurrence began in January, 1913, and fourteen days after the onset of the discharge the patient developed an arthritis of the left tarso-metatarsal joints, which spread in the following order to other joints: right knee, left temporo-maxillary, both shoulder and cervico-vertebral joints.

Under local treatment and injections of an autogenous vaccine the patient improved, except that pain down the whole spine set in and increased. A mixed vaccine was made of the organisms obtained from the prostatic secretion, with the result that a severe epididymitis followed, and the patient became worse than he had ever been before.

I saw the patient for the first time in August, 1913, by which time he had lost over three stone in weight; he walked slowly with the aid of a stick, had a pronounced stoop, head was thrown forwards, and shoulders were raised to avoid moving spine. On examination, he was found to have a chronic prostato-urethritis and vesiculitis, an acute tenovaginitis of the extensor shealth of the left wrist, the spine was tender, but quite movable. Under the usual local treatment, coupled with the internal administration of potassium iodide, and daily injections of a sensitised gonococcal vaccine up to $1,000,000,000$, with a further weekly injection of the maximum dose, the patient completely recovered, and within a few weeks the movements of the spine were natural and elicited no pain.

Treatment.-The one word which should be in every physician's mind when he is called upon to treat a case of gonococcal arthritis is-adhesion. The thought of adhesions suggests exercises, and as to when exercises should 


\section{CLINICAL ASPECT AND TREATMENT OF VENEREAL DISEASES}

be commenced hardly any two observers are of the same mind. Too early movements are very apt to cause a recurrence, and the formation of adhesions is always more marked in the recurrent attacks than in the first attack of arthritis. I think it wiser myself to keep the joint at rest until the acute stage has passed. Then it is safe to commence massage and exercises. In all joint cases, sodium salicylate and colloidal iodine should be given internally.

When the arthritis is acute, cold applications should be used; and when the acute stage has passed, painting on tincture of iodine, or colloidal iodine oil, or oil of wintergreen is useful.

Other points concerning treatment have already been dealt with, but before closing this part of the subject mention must be made of Bier's treatment. Bier's treatment may be adopted in any stage, and, if properly used, it will quickly heal a joint in the acute and subacute stages, and will prevent adhesions from forming in the chronic stage.

In the acute stages, the bandage must remain applied for every twenty hours out of the twenty-four, while in the chronic stage it should not remain in contact for more than an hour at a time. The great advantage of Bier's treatment is that exercises can be started earlier.

The best bandage to use is an elastic one, about two and a half inches wide, and it should be commenced with two or three turns well to the proximal side of the affected joint, and then carried right down the limb. When the bandage is applied for as long as twenty hours œedema may result. That does not matter, but it is essential to avoid pain and to prevent the limb from becoming blue. The pain which is experienced when the bandage is first put on soon dies down if the bandage has been applied properly. The greatest advantage of Bier's treatment rests in the fact that it is not necessary to keep the joint absolutely at rest and stiff. The joint may also be moved when the bandage is changed, hence the risk of formation of adhesions becomes practically nil, and the muscles around the joint do not atrophy. Bier's treatment in gonococcal arthritis cannot be too warmly recommended. Atophan (phenyl-quinolin-carboxylic acid), given internally, is sometimes beneficial in cases of gonococcal arthritis.

Guaiacol carbonate is also a useful drug, and in all subacute and chronic cases increasing doses of colloidal iodine should be prescribed.

Since the advent of the chemotherapeutic treatment, much of what has been written is superfluous, as intramine has proved particularly valuable in gonococcal arthritis, provided it has been prescribed early enough. Intranine gets rid of the pain so quickly that I never find it necessary to keep a joint at rest. In all cases, colloidal iodine should be prescribed internally, 3iii. ter in die, post cibos. In very rare cases $100 \cdot 0 \mathrm{c.cm}$. colloidal iodine should be injected intravenously at the same time as $2.5 \mathrm{c.cm}$. intramine are injected intramuscularly. On the fifth day the intramine should be repeated $(3.0$ c.cm.), and five days later 1.5 c.cm. trimine injected, or a third injection of intramine should precede it. Such a course can be repeated once or twice, 
at monthly intervals, if necessary. If one does not have to act quickly, it is wiser to keep the patient under the influence of iodine for ten to fourteen days before injecting the intramine. In those mono-articular cases associated with pronounced wasting, and in which the patient has been allowed to get into a very low and anæmic state, his general condition must be improved before any chemotherapeutic agents are employed. In such cases intramine and trimine are only apt to aggravate the condition and start an iritis. In France, and at Rochester Row, intravenous injections of typhoid vaccine have been widely used in view of the observation which has been frequently made that the arthritis improves when the temperature is raised. Indeed, at the last-named place it was thought that intramine acted in virtue of the pyrexia it caused. As intramine is equally efficacious when it causes no rise of temperature, and is infinitely more useful than typhoid vaccine in all cases, but especially in the acute cases, for which one hopes to do most by treatment, there is no call for the latter.

The following cases are particularly illustrative of the above:

CASE 130.-A patient contracted gonorrhœa for the first time. Four days after the onset of the discharge-which, by the way, was never particularly profusean acute arthritis of both knees and the left ankle set in. Three days later the joints were much swollen and very painful, the superficial veins of the limbs stood out prominently, and the urethal discharge had become muco-purulent. The patient received $0.5 \mathrm{c.cm}$. colloidal manganese, and twenty-four hours later the pain had diminished so much as to allow the patient to sleep. On the third day the same dose was repeated, with the result that the day following the patient was able to move his joints himself, and the swelling was considerably diminished. On the sixth day $1.0 \mathrm{c} . \mathrm{cm}$. colloidal manganese was injected, and this was followed by $2.5 \mathrm{c.cm}$. intramine on the tenth and fourteenth days respectively. On the eighteenth day $1.5 \mathrm{c} . \mathrm{cm}$. colloidal manganese concluded the injection treatment. The following day the patient was able to walk about the ward. Subsequent examination of the urethra and prostate showed nothing abnormal, so the patient was discharged on the twenty-fifth day perfectly well.

CASE 131.-Patient contracted his first attack of gonorrhoea, and four weeks later developed arthritis of both ankles and right knee. He was in bed for five weeks, during which time he was treated with the ordinary remedies, and received, in addition, eight injections of a sensitised vaccine, which produced little or no improvement. Before the first injection of intramine was given the joints were swollen, and the patient was unable to move them owing to the intense pain caused. The day following the first injection of intramine the swelling diminished, and the movements were free. Three days after two injections of intramine and one of manganese the patient was able to walk about. An examination of the urethra and appendages revealed nothing abnormal, and ten days later, after a course of massage, the patient was able to return to duty.

CASE 132.-Patient was suffering from his first attack of gonorrhœa, which he had had for five weeks before he came under my care. When I first saw him he had arthritis of his cervical vertebræ (Spondylitis deformans), and was unable to move his head in any direction. He also had arthritis of both wrists, fingers, the right knee, both ankles, and some toe joints. The joint trouble had been present for a fortnight. 
After the first injection of intramine the patient was able to move his head in any direction. The day after the injection of manganese, which was prescribed on the third day following the second injection of intramine, the patient was able to walk about and look after himself, and a fortnight later he went to duty: This patient also had Keralodermia blennorrhagica, which was limited to his penis.

In long-standing and recurrent cases the combination of intramine with manganese will produce changes unobtainable with other methods of treatment, as the following case will show:

CASE 133.-Patient contracted his first attack of gonorrhœa, and within a week of its onset both lobes of the prostate and the seminal vesicles became infected. A week later he developed arthritis of both shoulders, both knees, and both ankles. Under local treatment and sensitised vaccines the patient did not improve to any great extent; in fact, he had been bedridden for four months before he was treated with intramine and manganese. Two days after the last injection the patient was able to walk about the ward for the first time, and a week later he required no further attention. After a fortnight's convalescence the patient was able to do his ordinary work.

It cannot be too frequently repeated that the whole success of intramine in the treatment of gonococcal arthritis is to get the patient under its influence at the earliest possible opportunity. Just as the judicious use of the chemotherapeutic agents will lessen the onset of tenosynovitis, so will they of gonorrhœal rheumatism. I am describing the present case not only to show the truth of this, but to emphasise the point that pallamine should not be used in any cases but those of acute urethritis.

CASE 134.-A patient consulted a friend of mine for a chronic urethral discharge. On the first day he received $1.0 \mathrm{c.cm}$. pallamine, and $1.0 \mathrm{c.cm}$. colloidal manganese on the fourth and eighth days respectively. Four days later the patient was brought to me complaining of being ill and of acute rheumatism. On examination, I found that he had Erythema multiforme, gonorrhœal rheumatism, and a fresh right vesiculitis. With two injections of intramine and a final one of manganese the patient got perfectly well.

If intramine had only been given in the first place, as I have advised, none of the unpleasant sequelæ would have resulted, and the treatment would not have been blamed for having been inefficient.

\section{Gonorrhœa of the Muscles.}

There is no doubt that a true gonococcal myositis may arise by a direct extension of the mischief from a joint. But the muscular wasting which so rapidly occurs around an affected joint is more often due to the gonotoxine, which causes a trophoneuritis. Gonotoxic trophoneuritis may be of central or peripheral origin. If the former, a lymphocytosis in the C.s.F. can be demonstrated. One of the most interesting points in connection with this condition is the very frequent occurrence of anæsthesia over and around the affected joint. The presence of cutaneous anæsthesia is almost pathognomonic of gonococcal arthritis. 
The so-called muscular rheumatism or gonococcal myalgia may be diffuse, or limited to one group of muscles, or even to one muscle. It is not a true gonococcal myositis, but a gonotoxic lesion, which, as a rule, produces no changes in the muscle or muscles involved.

A true gonococcal myositis may pick out any muscle. It gives rise to a localised inflammatory swelling, which is acutely painful. After the inflammation has vanished, atrophy may result.

\section{Gonorrhœa of the Bones.}

The periosteum is the part most frequently affected, and a secondary involvement is far more common than a primary one. A tenosynovitis of the tibialis posticus is almost certain to lead to a periostitis. A periostitis usually results from a trochanteric bursitis, and the periosteum is always liable to become affected in severe cases of arthritis. A primary periostitis may occur anywhere. Osteophytes from the os calcis are by no means uncommon, and may be the cause of pain in the heel and sole of the foot, especially when the plantar arch has gone.

\section{Gonorrhœal Diseases of the Nervous System.}

Peripheral neuritis is not at all an uncommon complication, but a gonococcal infection of the central nervous system is very rare. A peripheral neuritis may be the only symptom of a metastatic infection, but, as is the case in the majority of the metastatic lesions, the lesions usually accompany an arthritis, succeeding more often than preceding the joint trouble.

Although peripheral neuritis is here being described as a metastatic lesion, no actual proof has yet been brought forward that it is due to the direct presence of the gonococcus. It may be due to the gonotoxine, but, as it behaves clinically like all other metastatic lesions, I am most inclined to ascribe its origin to the organism itself. The neuritis may affect a single nerve or many nerves, and in nearly every case it is one or more of the nerves of the lower extremity that are affected. In my experience, the most common gonococcal neuritis is a neuritis of the trunk of the sciatic nerve, and I have only seen a few cases in which the sciatica was not accompanied by an arthritis of the knee or ankle on the same or on both sides. Sciatica accompanying an arthritis of the hip is generally secondary in nature, and, as a rule, becomes marked only when osteo-arthritic changes have set in. A very troublesome form of neuritis is that which affects the genito-crural nerve. It appears to me to be more persistent and less influenced by treatment than neuritis of the sciatic nerve, and, owing to the course run by the nerve, an inflammation of it may be the start of a sexual neurasthenia. Only once have I seen a case of gonococcal polyneuritis. The upper extremities were more affected than the lower; there was marked muscular wasting of the arms. The patient was also suffering from a pyelo-nephritis.

So far as the central nervous system is concerned, a few cases of myelitis 


\section{CLINICAL ASPECT AND TREATMENT OF VENEREAL DISEASES}

have been described. Gonococcal meningitis appears to be very rare, but cases of hemiplegia have from time to time been reported.

The following is one of the most interesting cases I have seen:

CASE 135.-Patient had first attack of gonorrhœa sixteen years ago. The first recurrence occurred two years later, and was complicated by an epididymitis. The second recurrence occurred three years later, and passed off quickly. Iritis set in for the first time during the second recurrence; it recurred three times, and necessitated an iridectomy. Seven days after the first attack of iritis the patient had weakness in the right arm, defective speech, facial paresis, and ptosis on the left side, all of which came on suddenly. The trouble vanished after some weeks in bed, but recurred when the second recurrence of urethritis supervened, to disappear again after a few weeks' rest.

\section{Gonorrhœa of the Heart and Bloodvessels.}

The name of Ricord should stand pre-eminent in the roll of honour of those who have added to our knowledge of venereal disease. He lived at a time when pathology and bacteriology had not opened for research the paths which exist to-day. He tore aside the veil of fallacies with which Hunter's work had dimmed the true view of this branch of medical science. Ricord was the first to recognise that gonorrhœe could cause an endocarditis and pericarditis.

Endocarditis is a rare complication of gonorrhœa, and one of the reasons for this is that, in many cases, the gonococcus has failed to be found. The gonococcus has, no doubt, been the predisposing cause, but the destruction of the cardiac valves has been produced by a secondary infection. These cases come under the care of the general physician, rather than under that of the venereal specialist. I have seen five cases of gonococcal endocarditis. In two, a pure culture of gonococci was obtained during life from the peripheral blood-stream, and, post-mortem, from the heart's blood. In a third case the cultures grew streptococci as well.

I was able to make a microscopic examination of the cardiac valves in one of the two former cases, and the vegetations contained practically nothing else but gonococci.

In many of the reported cases of gonococcal endocarditis, especially in the earlier ones, the cultures remained sterile. This is undoubtedly due to the fact that an appropriate medium was not employed; therefore it must not be assumed that they were not true cases of gonococcal endocarditis.

I have mentioned these various points in order to draw more attention to this complication, which I am certain is more common than is thought to be the case. Cases do not run an invariably fatal course, and, if caught early, a great deal can be done by treatment. Many patients suffering from gonorrhoea have a transient cardiac lesion-stabbing pain in the chest, increased cardiac action, and perhaps a faint pericardial rub.

If every observer carefully examined the hearts of all his patients suffering from gonococcal arthritis, he would be surprised at the comparatively large percentage which showed a disturbance of some kind or other. 
Benign gonococcal endocarditis is more common than malignant gonococcal endocarditis. The former is almost invariably associated with either rheumatism or arthritis of gonococcal origin, and it is very difficult, unless the urethra be examined, to distinguish this form of endocarditis from the common so-called rheumatic endocarditis.

The malignant gonococcal endocarditis usually causes death before even the diagnosis of gonorrhœa has been made. Whether it is the rule or not I cannot say, but anyhow, in most of the cases which I have seen, the patients have had no other complication, and in the case in which I was able to examine the cardiac valves I made microscopic sections of the prostate and seminal vesicles, but both were normal.

According to Schlagenhaufer, the valves most frequently involved are, first, the aortic, then the mitral, and only very occasionally the tricuspid and pulmonary.

The case I had the chance of examining thoroughly had vegetations on the aortic and tricuspid valves, with commencing atheroma of the aortic valves and of the aorta in the neighbourhood of the valves.

The spleen is always swollen, and there may be an acute toxic nephritis or renal infarction.

What has already been -said about endocarditis applies equally well to pericarditis, but it should be remembered that, although a patient who has a gonococcal pericarditis is almost certain to have an endocarditis, the pericardial lesion may be the only one that can be diagnosed clinically.

A gonococcal phlebitis is very rare; the vein which is most commonly affected is the internal saphenous vein. The dorsal veins of the penis may be affected in severe cases of gonorrhœa, and in most cases of gonorrhœa a lymphangitis of the penis occurs, and in almost every case the inguinal lymphatic glands are enlarged and painful.

A microscopic section of lymphatic glands removed from the inguinal region during an acute attack of urethritis always reveals a typical inflammatory adenitis. Rarely any mention is made of the fact that inguinal adenitis accompanies practically every case of acute gonorrhœa, with the result that the diagnosis of syphilis is made almost every day from not realising this fact.

A possible explanation for the occurrence of malaise with slight cardiac trouble is probably to be found in the fact that the gonococcus is the organism $\kappa a \tau$ ' $\epsilon^{\prime} \xi o \chi \eta^{\prime} \nu$ for increasing the "acidness" of the blood. To my mind this is undoubtedly the reason why the gonococcus causes arthritis and the production of fibrous tissue wherever it is to be found. In this observation lies also the partial explanation for the benefit which follows the judicious employment of chemotherapeutic agents in this disease, such as colloidal iodine and intramine. 


\section{CHAPTER XXIII}

\section{NON-GONOCOCCAL URETHRITIS}

THIS is one of the most interesting, most difficult, and least understood subjects with which one has to deal. Several conditions may cause a urethritis, but a non-gonococcal urethritis is always rare. The discharge from a balanitis, from a soft sore infection, and from a syphilitic sore, especially if the patient has a tight foreskin, may cause a urethritis. An intra-urethral chancre and a recurrent urethral syphiloma may produce a discharge acute enough to be mistaken at first sight for gonorrhœa. In these cases the ætiology is obvious, but it is not so in the majority of cases. Non-specific urethritis must have always caused considerable dispute, because Swediaur, in 1784, injected his own urethra with ammonia, and produced a discharge which took six weeks to clear up, and infected the posterior part of the canal, although the material had only been injected into the anterior.

Phosphaturia and oxaluria may give rise to a urethritis, but if the crystals irritate the mucous membrane of the urethra, they are certain to have irritated the epithelium of the bladder, hence these cases usually complain of frequency of micturition. The urine in both glasses is generally thick, so that an unwary observer may diagnose an acute posterior gonococcal urethritis. Frequently the urine does not become clear on adding acetic acid, a point which may further add to his difficulty.

Phosphaturia is a very important condition, as it not infrequently complicates an old-standing gonococcal infection which is most rebellious to treatment. Such a state of affairs is always met with in a certain type of individual. The patient is very neurotic; he takes his gonococcal infection very much to heart, and severe cases may even have suicidal notions. Whether the phosphaturia is post hoc or propter hoc I cannot tell; all I know is that the phosphaturia and the psychic condition are associated.

The thickness of the urine in phosphaturia is due to the precipitation of insoluble phosphates, which is dependent upon an increase of the alkalinity, or a decrease of the acidity of the urine. The insoluble phosphates are mainly the calcium salts $\mathrm{Ca}_{2}\left(\mathrm{PO}_{4}\right)_{3}$, and these are mixed with magnesium salts. The carbonates are especially abundant after a fruit meal, or after drinking large quantities of lemonade.

Since motor cycling has become so popular, one occasionally sees cases of urethritis caused by it. Ordinary cycling, and even horse riding, may very rarely produce an attack. Strong antiseptics are a more common cause of 
urethritis than is generally supposed. The urethritis of gonococcal origin is often kept going by using too strong solutions. A test which is often practised to see whether a gonococcal attack is cured or not is to inject a strong solution of an antiseptic. If a discharge is produced or increased, the paticnt still has gonorrhœa; if not, he is cured.

There are many men who have never had a urethritis who would speedily develop one after such a test; therefore, how much more likely must this be the case in a patient who has been already over-treated. Many men make a rule of injecting themselves with Condy's fluid directly after having had connection, and I have seen several cases in which an acute discharge has been produced by such a measure. Either the solution was undiluted, or the erection persisted while the injection was being carried out. Metschnikoff's 33.0 per cent. calomel ointment, which is commonly used as a protection against syphilis, may likewise set up a transient urethritis, and, in passing, I may state that it is not so good a protection as it is thought to be.

Coitus interruptus and chronic constipation may be the cause of a nongonococcal urethritis, especially if the patient has had a previous urethritis.

These cases of what may be called traumatic urethritis can usually be diagnosed by the facts that there is no incubation period, that the acme is reached between the second and the third day, and that it then begins to abate without treatment.

The increased secretion of mucin which is so common after a gonococcal infection, and about which most patients are alarmed, is an occurrence which requires very special mention.

If a patient has been cured of his gonorrhœa by frequent douchings with strong antiseptics, by the passage of medicated bougies, and by somewhat severe instrumentation, he is very liable to have a discharge of mucin which may persist for months, and even years.

The mucin discharge is clear and sticky. It quickly forms clouds in the urine, which are increased by the addition of acetic acid. Sometimes the secretion contains a mucinoid substance which is soluble in acetic acid. This mucinoid substance has strong reducing properties, and is more commonly met with in cystitis than in urethritis. The clear discharge is most marked in the morning on rising, but I have seen cases in which it has persisted throughout the day. The mucin comes partly from the prostate and partly from the urethra. Frequent erections, onanism, active exercise, and alcohol are very apt to increase it. The importance of this mucinous urethritis lies in the fact that the sufferer still thinks his gonorrhœa is not cured; consequently, he consults one doctor after another, probably receiving treatment from each. The result is that, as the prime cause is irritation, further treatment only tends to aggravate the condition.

Urethral injections of bismuth sometimes allay the irritation, but they should not be persisted in for long. Keeping the bowels well open checks the secretion, and this can be still further aided by giving the potassium citrate and hyoscyamus mixture internally. A urethritis, and even an epi- 


\section{CLINICAL ASPECT AND TREATMENT OF VENEREAL DISEASES}

didymitis, may occur in infectious fevers, and this brings me on to describe those cases produced by local bacillary infections. Almost any organism may produce a urethritis, but, in my experience, the most common infections are those produced by the Bacillus coli, by a diphtheroid, streptococcus, and staphylococcus, and Micrococcus catarrhalis. Many of these infections result from sexual intercourse.

It must be remembered that the Bacillus coli is not at all an uncommon cause of abscesses in Bartholin's glands.

Silver salts, in my experience, are apt to aggravate these non-gonococcal cases of bacillary urethritis, and I always prefer to use injections of boracic acid, quinine, or very dilute solutions of the perchloride or biniodide of mercury.

The rôle the so-called inclusion bodies play in causing a urethritis has not yet been settled.

Halberstädter and v. Prowazek ${ }^{1,2}$ have described certain inclusion bodies in the epithelial cells from cases of chronic urethritis which they consider to be parasites. These observers, moreover, state that the bodies seen in the epithelial cells from the urethra are the same as those seen in the epithelial cells of the conjunctiva from cases of trachoma. These parasites are thought to be chlamydozoa.

Several observers have confirmed Halberstädter and v. Prowazek's work, especially so far as trachoma is concerned.

These inclusion bodies have been found in the conjunctiva of newly born infants, and the infection doubtless was conveyed during the birth of the child, in the same manner as the gonococcal infection.

Apes have been infected on the conjunctiva with material obtained from a human vagina. ${ }^{3,4}$ Therefore it is highly suggestive that these inclusion bodies are parasites, and that they can cause a urethritis as well as a conjunctivitis. Fritsch ${ }^{5}$ has succeeded in inoculating a monkey's conjunctiva with the secretion from a urethra which was infected with these inclusion bodies; and Heymann ${ }^{6}$ has further proved that the genitals of apes can also be successfully inoculated from human material.

The special staining properties of these inclusion bodies have yet to be worked out, as up to the present only Giemsa's stain has been used. The chemistry of Giemsa's stain is so complicated and so little understood, and its staining action is so uneven, that it is impossible to draw any conclusions from the results obtained. According to Halberstädter and v. Prowazek, the inclusion bodies stain red with Giemsa ; and according to Lindner, blue. Microchemical means would soon settle the point as to whether these inclusion bodies are parasitic or not.

1. Halberstädter U. v. Prowazek (1909): Deutsche med. Woch., xxxv., 764.

2. Halberstädter U. v. Prowazek (1909): Berl. klin. Woch., xlvi., 1839.

3. LINDNER (1910): Wien. klin. Woch., xxiii., 283.

4. Lindner (1911): v. Graefes Arch. f. Ophthalm., Ixxviii.. 345.

5. Fritscir (1910): v. Graefes Arch. f. Ophthalm., Ixxvi., 547.

6. Heymanx (1910): Berl. klin. Woch., slvii., 663. 


\section{CHAPTER XXIV}

\section{GONORRHGAL DISEASES OF THE EYES AND OTHER SENSE ORGANS}

\section{History.}

THere is no doubt that Hippocrates was familiar with gonococcal conjunctivitis, and both Plato and Aristotle knew that the infection could be conveyed to the eyes. Prior to Hunter's time gonococcal ophthalmia was a pretty well recognised condition, but much of the knowledge gained was lost because this observer negatived its occurrence. Sir William Lawrence added much to our knowledge, but even to as late as 1872 a well-known syphilologist looked upon gonorrhœal iritis as a condition to be found in books only. Although Credé's name will always be familiarised with the prevention of infantile gonorrhœal ophthalmia, the precautionary measure was first advocated by Eisenmann in 1830. Credé, as is well known, in 1881 advised silver nitrate, while Eisenmann had advised chlorine water.

\section{Conjunctivitis.}

Gonococcal conjunctivitis should be divided into two classes- $(a)$ adult; (b) infantile.

Adult Gonococcal Conjunctivitis.-Conjunctivitis in the adult may be due either to the gonotoxine, or to the gonococcus itself. If due to the former, the condition is bilateral; and if due to the latter, the condition is unilateral. The gonotoxic conjunctivitis gradually disappears under appropriate treatment of the urethra, and within a few days of the administration of a vaccine. A toxic dose of a vaccine may, on the other hand, give rise to the condition. Bathing the eyes with cold water, or with a weak boracic solution, is all that is necessary. Gonotoxic conjunctivitis is most frequently associated with gonococcal arthritis-indeed, it often ushers in the latter. The conjunctivitis due to the gonococcus may be a very serious affair; therefore it should be diagnosed and treated as early as possible. At first the symptoms are those of gonotoxic conjunctivitis, but in a few hours the inflammation becomes much more acute, and the photophobia becomes intense. About twenty-four hours later the conjunctiva is markedly swollen, and a discharge becomes evident, at first serous in nature, and then purulent. Owing to the swelling of the conjunctiva the lids do not meet, and the patient can scarcely open them. The conjunctiva of the lids becomes covered with a membrane, and bleeds 
easily when the latter is brushed away. By this time the skin surrounding the eye is red and œdematous, and the pre-auricular lymphatic gland is swollen and painful. If allowed to continue, the purulent discharge quickly causes a destruction of the conjunctiva, the cartilage of the upper lid becomes soft, there is a marked formation of new bloodvessels, and the cornea now becomes attacked. If the cartilage of the upper lid is affected, there is always the danger, when the fibrous tissue formation begins, after resolution sets in, that the upper lid may remain permanently thickened, and this leads to a condition of what is usually called false ptosis. The ptosis is probably due to the damage and destruction of some of the tendinous fibres of the levator palpebra superioris. The swelling of the lower lid may cause an ectropion, but it is very seldom that it remains permanent.

One of the most remarkable points about gonococcal conjunctivitis is that the lachrymal sac never becomes affected. There must be some peculiarity in the secretion from the mucous membrane of the sac, the knowledge of which might reveal an absolutely complete cure for gonorrhœa.

The danger of gonococcal conjunctivitis is not the damage done to the conjunctiva, but the damage which is certain to result if the cornea becomes involved. Within a few hours of the commencement of a keratitis the cornea may be ulcerated right through, and the iris may be prolapsed.

Even in mild cases of keratitis a leucoma is bound to result, and this often blocks the vision of that eye.

If the iris prolapses and a staphyloma forms, there is always the danger of the supervention of a secondary glaucoma. In very bad cases a panophthalmitis may occur.

Gonococcal conjunctivitis in the adult ought always to be prevented, hence every patient who contracts gonorrhœa ought to be warned against touching his eyes with his fingers, or with any dirty handkerchief, piece of rag, or towel. If conjunctivitis has begun, the first consideration should be given to the unaffected eye, and the healthy eye is best protected by what is called a Buller's shield, which is simply a watch-glass fixed peripherally with strapping.

In the affected eye, the lids should be prevented from sticking together by applying a little boric ointment to them. The whole of the conjunctiva should be washed out very gently, at intervals of a quarter of an hour to an hour, night and day, with a weak solution of potassium permanganate. The colour of the solution should never be deeper than that of ordinary red blottingpaper. The conjunctiva should never be touched or wiped with wool or any other absorbent, for fear of breaking its surface, and thereby causing an ulcer. Hourly irrigations of a $10^{\circ} 0$ per cent. solution of argyrol are also very efficacious, but there is no preparation of silver which may not be followed by argyria. Although cases of generalised argyria have been known to result from the local use of argyrol, protargol, etc., the lesion generally affects the treated area only. Argyria is most likely to occur if there is any abrasion of the skin; therefore in such cases potassium permanganate should be used for 
preference. Argyria tends to disappear spontaneously, but hexa-methylenetetramine, prescribed internally, is stated to hasten its disappearance.

As the continual passage of antiseptics over the inflamed skin may readily lead to a dermatitis, from which a secondary infection may arise, and may ultimately reach the eye, the inflamed area should be anointed with simple boric ointment.

Although patients of the present day object to leeches, there is no other means so satisfactory for reducing the inflammation as the application of one or two to the temple on the affected side. Failing leeches, ice must be used. The patient should be instructed to open his eye as widely as possible and to move his lids occasionally, so as to prevent the constant pressure of a lid at one point breaking through the conjunctiva.

In cases which are complicated by keratitis, the treatment will depend upon whether there is a simple iritis, or upon whether there is a likelihood of Descemet's membrane giving way. In the former, when the iris is practically bound to become attached to the cornea, the pupil should be made as large as possible; therefore atropine should be used.

In the latter, where there is the danger of the iris prolapsing and of the lens becoming dislocated, the wider the pupil is, the more atropine is naturally contra-indicated, and eserine is called for. Moreover, on the eye should be maintained a gentle pressure, which should only be removed when the eye is hourly washed out. Intramuscular injections of trimine have proved invaluable in cases of gonorrhœal ophthalmia, as I am aware of five cases in which the eye was saved from removal and the patients recovered with full vision. Three injections are best prescribed, as follows: first day, $1.0 \mathrm{c.cm}$.; fourth day, 1.0 c.cm.; eighth day, $1.5 \mathrm{c.cm}$.

Gonococcal Conjunctivitis of the New-Born.-The eyes are affected during delivery, and the incubation period is two to three days. Infantile conjunctivitis does not run such a severe course as the adult form, and the cornea is not so frequently implicated. Provided treatment is commenced early, the prognosis is good, but if delayed there is always the risk that the infant may be permanently blind. The eyes should be washed out every half-hour with a weak solution of potassium permanganate, and once or twice a day with a 1.0 per cent. solution of silver nitrate, or, better, a 25.0 per cent. solution of argyrol. If argyrol only is used, the strength should not exceed 10.0 per cent.

Prophylaxis should play a greater rôle here than almost anywhere else, since attention to the eyes of any new-born infant will prevent its developing gonococcal conjunctivitis, provided, of course, the mother is instructed re towels, etc. Directly after birth, the eyes should first be wiped with dry wool, and then with wool dipped in a weak solution of potassium permanganate. The eyes are then opened, and two or three drops of a 1.0 per cent. solution of silver nitrate are dropped in, or a 25.0 per cent. solution of argyrol. Having recently had such excellent results in gonorrhœal ophthalmia with colloidal silver $(1: 2,000)$, which causes neither pain nor argyria, I have given up the use of the protein compounds of silver and now rely solely on the former. 


\section{Iritis.}

As to whether the so-called rheumatic iritis, which so often accompanies pains in the muscles and joints during an attack of gonorrhœa, is due to the gonococcus or to its toxine no one is yet absolutely certain. In no case of iritis or irido-cyclitis have gonococci ever been found in the anterior chamber. That an examination has not so far yielded positive results is far from being conclusive proof that the iritis is not of metastatic origin, since, even in undoubted cases of streptococcic and pneumococcic iritis, it is not always possible to find the organism in the aqueous humour.

In favour of gonococcal iritis being of toxic origin is the fact that the iritis is usually bilateral, and that injudicious use of vaccines may produce it. Moreover, the iritis is more often accompanied by rheumatic pains-which are of toxic origin-than by metastatic arthritis. The iritis is very liable to recur, and it usually runs an innocent course.

Although gonorrhœal iritis is almost always bilateral, both eyes are not always affected at the same time, and recurrences frequently affect each eye alternately. In nearly all cases the patient has a chronic prostato-urethritis, although all signs of a focus in this region may have vanished years ago. Therefore, in treating gonorrhœal iritis, it is imperative to pay particular attention to the prostate gland, if it be wished to avoid a recurrence. So far as the eye itself is concerned, atropine must be used to prevent synechiæ, which very readily develop. Vaccines are also of value, but must be used with caution, since their toxic action may at first materially aggravate the eye condition. This fact again suggests a gonotoxic origin for this form of iritis; therefore, if vaccines are going to be used, sensitised ones should be injected for preference, since the endotoxines therein are neutralised beforehand.

In cases in which a hypopyon results it is sometimes necessary to tap the anterior chamber. I have known intramine to start an iritis, when used in cases of arthritis, where the patient had been very much pulled down. In some cases of particularly frequent recurring iritis, when on each occasion new synechiæ form and contract the pupil more and more till it is almost pin-point, the only remedy is to perform an iridectomy.

Other forms of gonorrhœal eye lesions are simply pathological curiosities, and it is extremely doubtful whether any others really exist.

\section{Gonorrhœa of the Mouth.}

The mouth is very rarely affected, but having seen an undoubted case in which the mucous membrane of the lips was infected by direct contact, a description would not be out of place. The patient came to me with both lips swollen, red, and extremely painful; the mucous membrane was granulated, and covered by a thin membrane in parts, from which pus exuded. An examination of the pus revealed numerous gonococci, and the patient admitted having performed a perverse sexual act six days prior to the onset of the 
trouble. In reading Proksch's book I note that Ricord was familiar with the

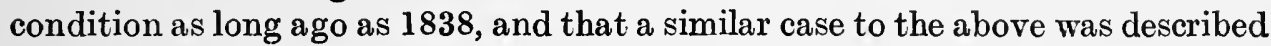
by Baumès two years later. Another case was reported by Petrasi, in 1850, and quite recently one by Mayhew, in 1918.

I have frequently noticed that patients with gonorrhœa have painful and swollen gums, especially on the inner surface of the upper central incisors, but whether this is a case of post or propter hoc I am unable to determine.

\section{Gonorrhcea of the Nose.}

Duncan, in 1778, was the first to describe a case of gonorrhœal rhinitis, and since then various observers have noted that some patients with gonorrhœa developed "colds" which proved intractable. Edwards, in 1857, reported a case where a woman infected her nose through using a handkerchief belonging to her son, who was suffering from gonorrhœa. Von Sigmund had a case of a libertine who infected himself direct per vulvam.

\section{Gonorrhœa of the Ears.}

It is conceivable that by means of a metastasis the disease might affect the ears, but as far as I know no genuine case has been described. Deafness and so-called Otitis blennorrhagica have been described, but as mercury in most of the cases cleared the trouble up it is probable that syphilis was really the cause of the ear lesions. 


\section{CHAPTER XXV}

\section{GONOCOCCAL RASHES}

THe history of gonococcal rashes is nothing but confusion. There is, first of all, the confusion with syphilitic rashes, and then the confusion with balsamic rashes. The first gonococcal rash to be thoroughly described was the Keratodermia blennorrhagica, by Vidal, ${ }^{1}$ in 1893 , since when several cases have been exhibited and reported in France. The first case to be described in this country was one by Sequeira. ${ }^{2}$

The known gonococcal rashes are five in number; only one is due to the organism itself, the other four being due to its toxine. The rashes are: (1) gonococcal ulcer and abscess; (2) toxic erythema; (3) toxic urticaria; (4) toxic hæmorrhagic and bullous dermatitis; (5) toxic hyperkeratosis.

\section{Ulcus Blennorrhagicum.}

Although many of the ulcers which appear during a gonococcal infection are doubtless of a secondary nature, some are certainly due to the gonococcus itself. To prove this, it is not only necessary to demonstrate the gonococci in films, since they might only have been implanted upon the surface, but also, on cleaning the surface, it is necessary to grow pure colonies of the organism therefrom. Clinically, it is impossible to differentiate the ulcers caused by a secondary infection from those caused by the gonococcus, and these ulcers are commonly mistaken for soft sores.

Ulcera blennorrhagica are very much more common in women than in men. They may affect any part of the genitals, the perinæum, the skin around the anus, and the adductor region of the thighs. Big ulcers, varying from 1 to $3 \mathrm{~cm}$. in diameter, are very rare, and so are serpiginous ulcers, but they do occur, and they are very difficult to cure. The commonest ulcers are small, and vary from the size of a pin's head to that of a pea. The lesion is either superficial, more like an erosion, or crateriform-i.e., the edges are raised, heaped up, and swollen. The edges are not undermined, and the surrounding inflammation is not so marked as it is in Ulcera mollia. The sores may be indurated, and in the male produce a phimosis.

The gonococcal abscesses are usually the terminal ends of paraurethral canals which have not perforated the surface, but true gonococcal abscesses may be met with, and these are always secondary to a lymphangitis, and they occur most frequently on the dorsum of the penis. 


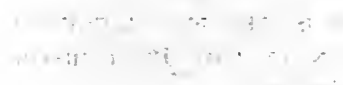

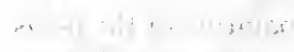

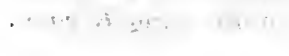

$$
\begin{aligned}
& -
\end{aligned}
$$$$
31+19
$$ 
PLATE LXI

No. 115.-A Case of Balanitis Circinata, Which is OFTEN ASsociated with Goxorriofal Rheumatism and is frequently the Precunsor of Keratodermia Blennorrhagica

Tus patient had his arthritis with his first attack of gonorrhoea. The rheumatism began one week after the infection. The painting of the penis was made five months later, when the patient had a commencing Keratodermia blennorrhagica affecting both big toes.

No. 116. - A CASE of Keratodermia Blennorrhagica Which AFFected the Penis Only

Thus was preceded by a Balanitis circinata. The patient was suffering at the time from his first attack of gonorrhoea and had arthritis of both knees and both ankles. The two cases depicted on this plate showed no improvement under vaccines, but recovered at once with colloidal iodine and intramine. This painting was made from a case under the care of Mr. E. Wragg. 
No. 115
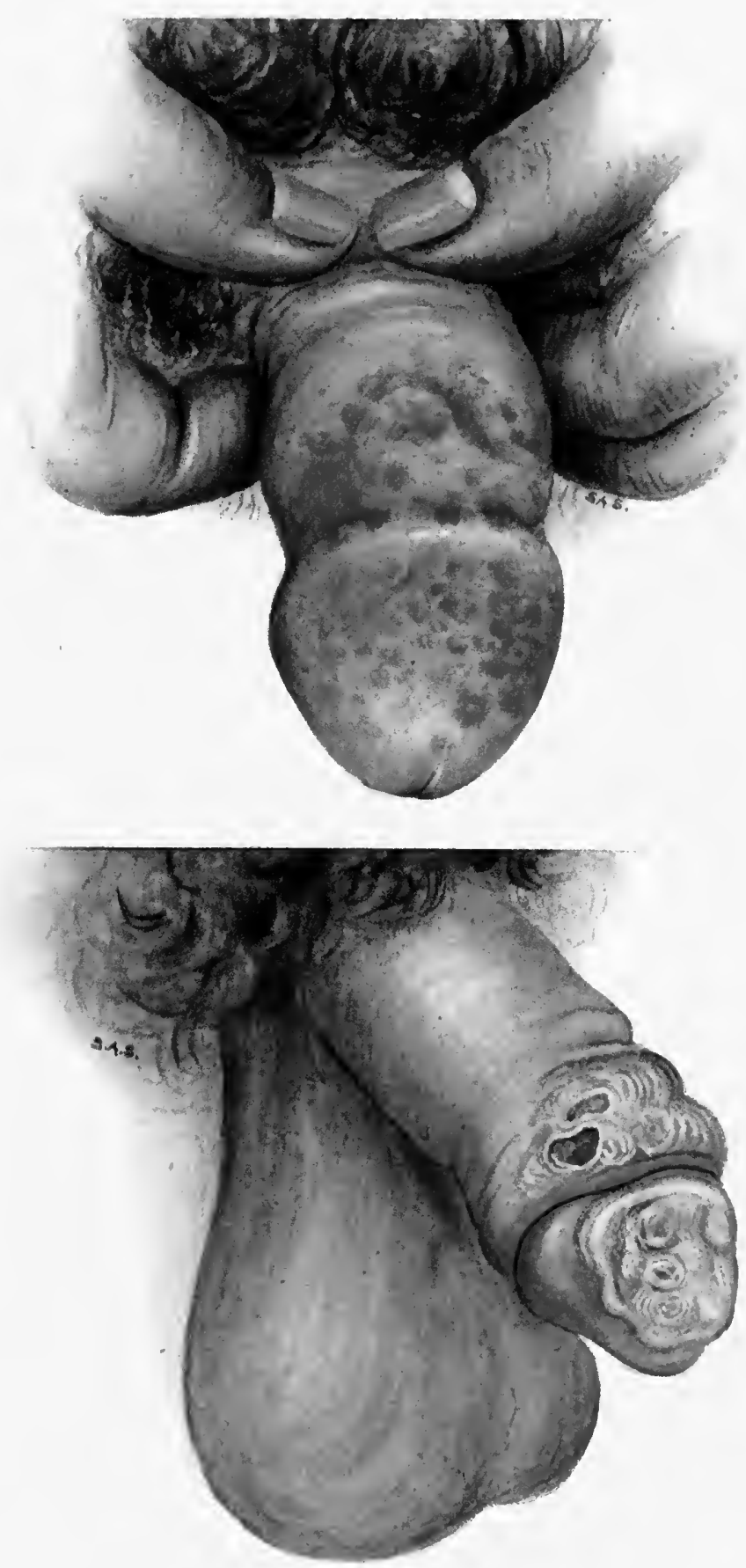

No. 116

Plate LyI 

A few cases of metastatic abscesses have been described, but lesions so rare are nothing more nor less than pathological curiosities. Bartholinitis not infrequently causes ulceration of the walls of the gland.

\section{Toxic Rashes.}

Toxic rashes, though seldom described, are quite frequently to be met with if looked for. Winkelried Williams, ${ }^{3}$ in a recent article on Keratodermia blennorrhagica, stated that, on searching the literature, he could find only fifteen recorded cases. At the London Lock Hospital we get about three cases per annum, and the numbers would doubtless be very much greater if we examined the feet of every patient suffering from gonorrhœeal rheumatism and arthritis. The toxic erythemata are usually brushed aside at once as being copaiba rashes, even if it be known that the patient has not taken any medicine.

I have seen gonotoxic erythemata which could not be distinguished from a copaiba rash, and the similarity of some cases to scarlet fever and measles was very close. Conjunctivitis is not at all an uncommon gonotoxic symptom, and when it accompanies a discrete macular rash, a hasty diagnosis of measles may be very easily made. Some of the scarlatiniform rashes may be accompanied by a sore throat. Many of the rashes are ushered in with fever, and joint pains are not at all uncommon. True Erythema nodosum may undoubtedly be met with as a gonotoxic rash. I remember one case very well, as the patient suffered also from polyarticular arthritis, and died of gonococeal endocarditis. Microscopic sections of the cardiac valves revealed masses of Gram-negative diplococci, and pure cultures of gonococci were obtained postmortem from the heart's blood and, during life, from a venepuncture. I have seen two cases of Erythema Multiforme associated with gonococcal arthritis.

Urticarial, hæmorrhagic, and bullous exanthemata are rare, and only a very few cases have been described. I have seen purpura develop in severe cases of polyarticular arthritis, but I have never seen a case of true urticaria or pemphigus.

Perhaps the most interesting, because the most distinctive, gonotoxic rash is the toxic hyperkeratosis, or, as it is more commonly called, Keratodermia blennorrhagica (Plate LXII.). The patient from whom this picture was made was suffering from his first attack of polyarthritis. The rash had taken only four months to develop. The other leg was in a similar condition; he also had lesions on both hands and arms, and the typical Balanitis circinata.

The lesions are primarily vesicles; these quickly become pustules, and then the wall of the pustule becomes keratinised. Several lesions may coalesce, or they may develop singly. Half a dozen lesions may appear on the big toes, and the condition may not develop further; hence it can be readily understood that several cases are overlooked, as a slight eruption of this sort is by far the most common. After attacking the dorsum of the big toe, the lesions extend inwardly, and ultimately reach the sole. If the lesions increase 


\section{CLINICAL ASPECT AND TREATMENT OF VENEREAL DISEASES}

in size, they do so by adding on successive layers of keratin, so that it ultimately resembles a limpet shell.

In the severe cases, any part of the body may be affected, and I may say that in practically every case I have seen, mild or severe, the patient has had a balanitis, and frequently a conjunctivitis.

Discrete circular lesions first make their appearance on the glans penis, corona, and under surface of the prepuce. As the surfaces continually rub together, the epithelium in between the areas soon becomes white, heaped up, and then entirely denuded, so that the true circinate areas can only be seen at the periphery. These circinate patches only rarely become keratinised, true keratodermia more often affecting the skin of the penis. I have seen cases in which the penile lesion was the only one present. When the horny process is removed, no ulcer is exposed, but simply a denuded epithelium. Although the patient just referred to, from whom the painting illustrating this chapter was made, was suffering from his first attack of polyarthritis, it is more usual for the keratodermia to develop during the second or some subsequent attack. Another interesting feature of this condition is that the patients usually have marked hyperidrosis, and this probably plays a large part in the causation of the horny excrescences. The lesions have been very carefully examined for gonococci, but always without success; therefore, I think it may be assumed that Keratodermia blennorrhagica is a toxic manifestation.

Treatment consists in merely removing the scales and in giving vaccines, by which means the condition is very rapidly cured.

The case from which the painting was made ran a course which I had never scen or heard described before. Localised blue congested patches developed in the skin at the base of all the finger and toe nails. The congestion gradually spread backwards as far as the metacarpo- and metatarsophalangeal joints respectively; the swelling then subsided and gave way to horny bands, which appeared to constrict the affected areas. To the proximal side of the congested digits a skin lesion developed, which was absolutely indistinguishable from Psoriasis vulgaris. The psoriasiform rash on the feet spread as far as the ankles, and on the hands to above the wrists. Oddly enough, both elbows and knees became covered with psoriasiform lesions, and even the nails developed the ridges and punctate depressions which are so commonly to be seen in cases of psoriasis.

For the opportunity of studying this unique case I am indebted to $\mathrm{Mr}$. Lane and Mr. Gibbs, under whose care the case was.

Since the above was written I have seen twenty-one cases of Keratodermia blennorrhagica, as the condition was not at all an infrequent one in the field hospitals. In some cases the lesion was limited to the penis, in two cases there was no arthritis, and in five cases the whole of the skin, with the nails, peeled off both feet and hands. Wasting and anæmia were common in all the cases. Having noticed that vitiligo and Dermatitis herpetiformis had 


\section{PLATE LXII}

No. 117.-A CASE of Keratodermia Blennorrhagica UnDer THE CARE OF Mr. E. Lane and Mr. C. Gibbs 


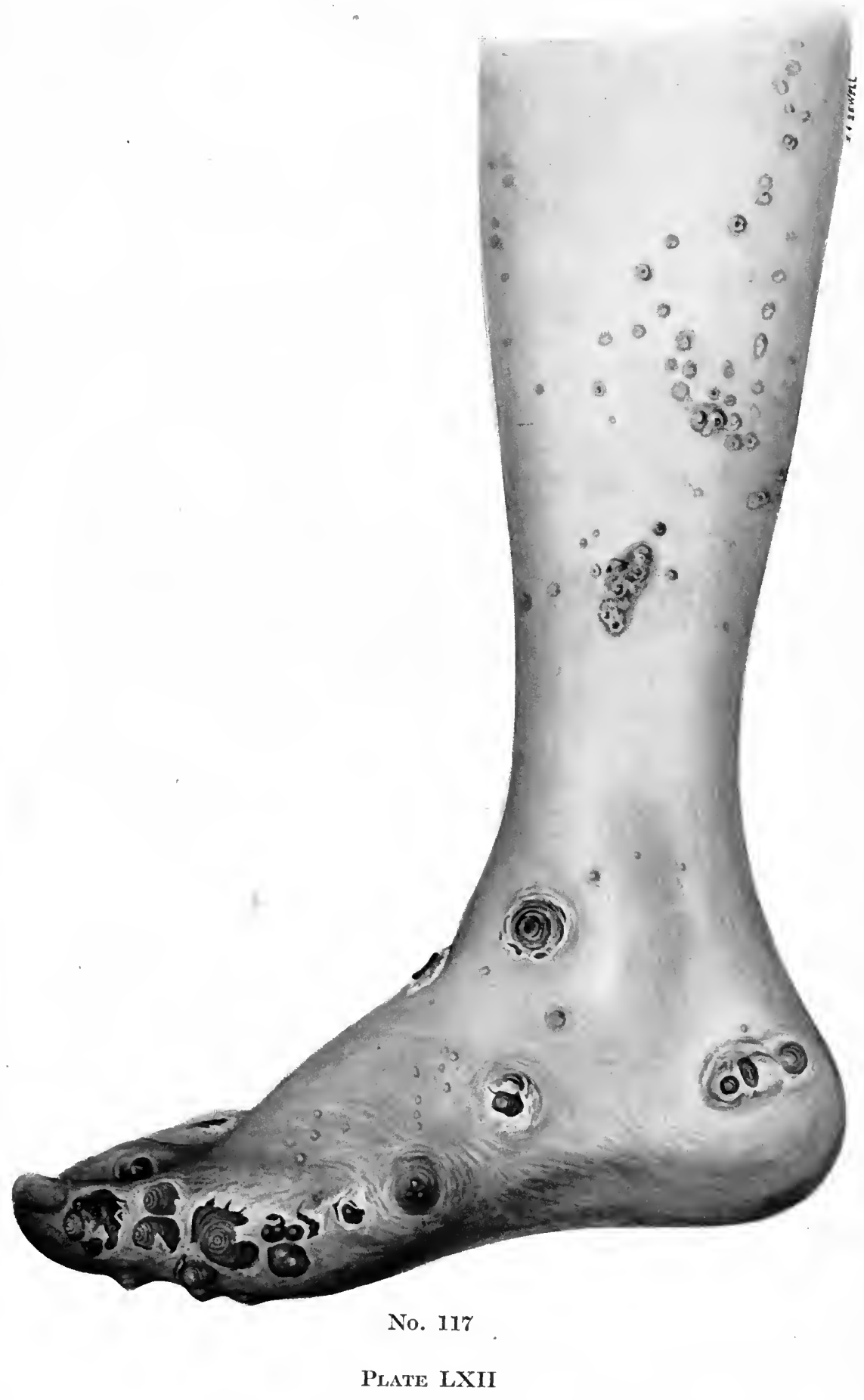




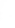





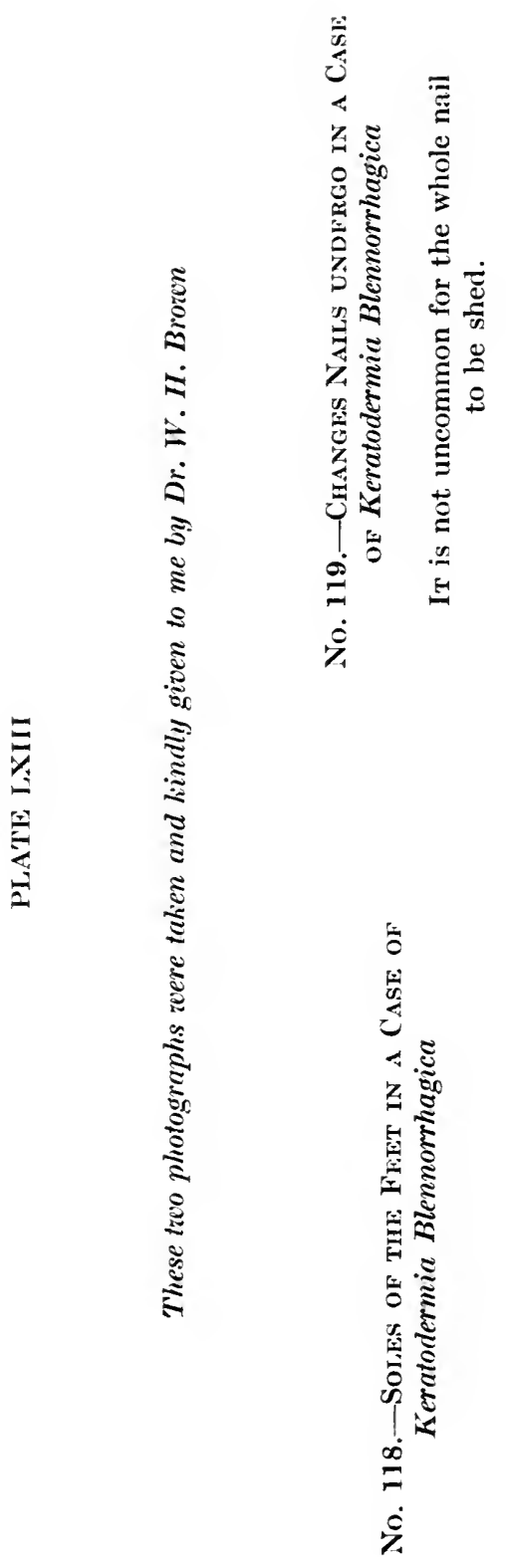



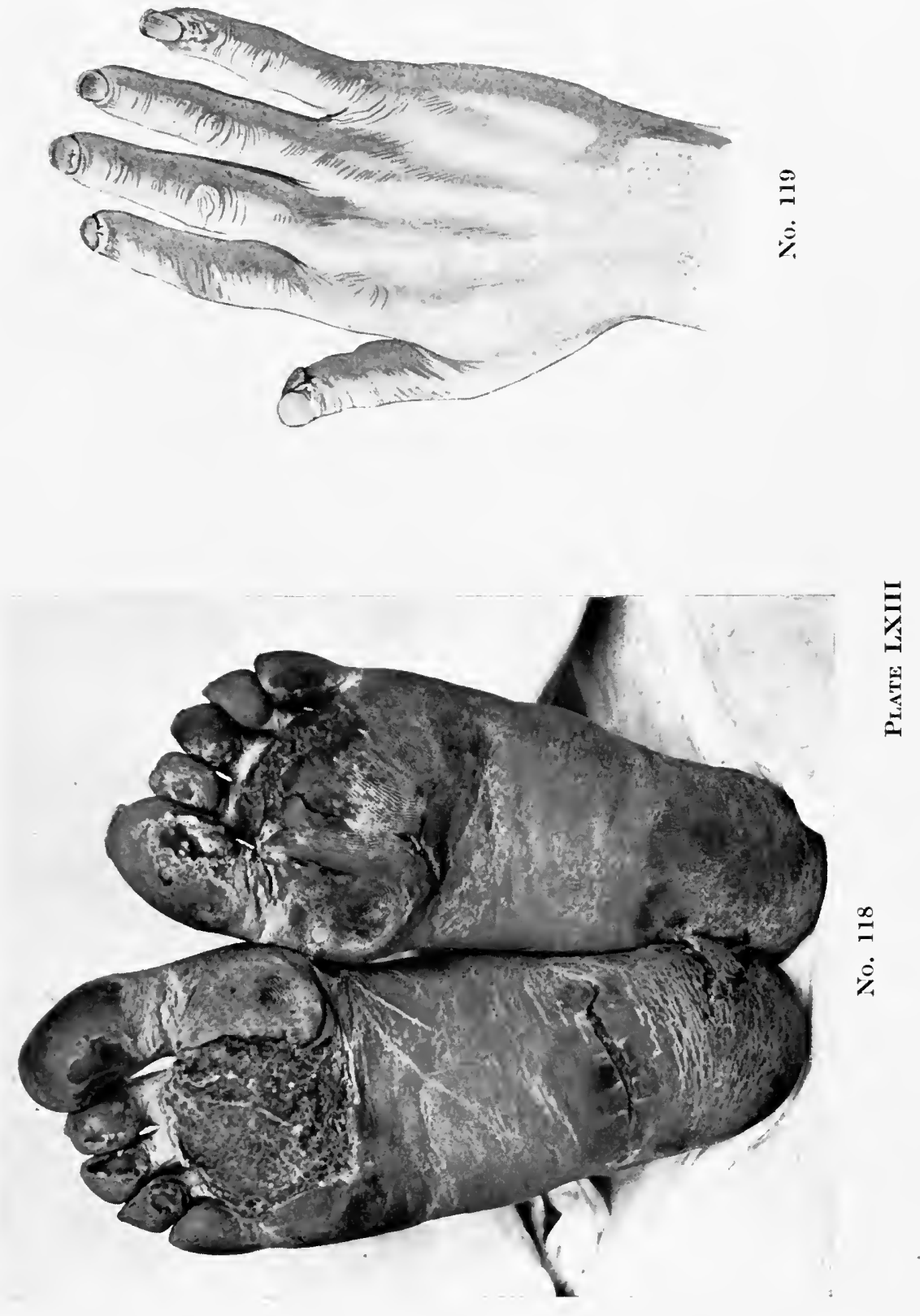

also increased during the war, affecting mostly combatant soldiers, and having seen cases in which vitiligo was a prodromal symptom of syphilitic degenerative myelitis, I examined the C.s.F. of a few cases, and found that in the worst cases of Keratodermia blennorrhagica there was a lymphocytosis amounting to 17 cells per c.mm. As Dermatitis herpetiformis is in many respects a widespread condition of Herpes zoster, which latter is due to inflammation of the posterior root ganglion or of the meninges covering it, and as the lesion in degenerative myelitis is sometimes primarily meningeal, there seems to be good evidence for assuming that Keratodermia blennorrhagica is due to meningeal irritation of the trophic fibres in the posterior nerve roots caused by the toxine of the gonococcus. It is in view of this meningeal irritation that hexamethylene-tetramine should be prescribed. The urethral lesion should always be attended to, and a combination of intramine and trimine is in all cases indicated.

1 VIDal (1893): Annales de dermat. et de syphiligraphie, iv., 3.

2 Sequeira (1910): Brit. Journ. of Dermatology, xxii., 139.

3 Winkelreid Williams (1914): Brit. Med. Journ., ii., 627. 


\section{CHAPTER XXVI \\ GONORRHEAA IN WOMEN}

THE history of gonorrhœa in women is interesting, apart from the allusion already made to Cleopatra. There appears to be no doubt but that Hippocrates was familiar with, and actually used, a vaginal speculum, since distinct reference is made in the "Collectio Hippocratica" to ulcers on the cervix and discharge from the womb. Soranus from Ephesus in the second century A.D., Antyllus in the third century A.D., and Paulus Æginæ, undoubtedly used the speculum for therapeutic purposes. Three- and four-bladed specula were later found in the ruins of Pompeii and Herculaneum. The use of the speculum was widespread and general until the end of the eighteenth century, when it fell into disuse owing to the teaching of Girtanner and Theden. Its use was re-introduced by Ricord. For many years in ancient times the uterus was considered to be the main seat of the trouble, which was thought by many to be due to the stagnation of semen there. It was not till many years had passed that it was recognised that the vulva and urethra were sometimes the seat of disease. Boerhaave was the first to draw attention to the implication of Bartholin's glands, and denied that gonorrhœa attacked the uterus. Van Swieten taught the same, in spite of the affirmative evidence Morgagni had produced in the meantime. At one time the female urethra was considered to be immune, an opinion which prevailed until Ricord conclusively proved it to be otherwise. Although Morgagni was the first to draw attention to gonorrhœa of the female pelvic organs, most of our knowledge of salpingitis, oöphoritis, etc., was founded by Ricord and Noeggerath. The first mention of vulvo-vaginitis in children, a name given to the condition by Behrend in 1848, was not till 1750. Almost from the time the lesion was first recognised it was regarded as being due to syphilis, and to Sir Astley Cooper belongs the credit of proving that it was due to gonorrhœa.

Gonorrhœa in women is, as is also the case with syphilis, the greatest curse of the disease. A man always contracts a urethritis, and knows at once when he is infected, and it is his own fault if he does not seek instant advice. Not so with a woman. The genital organs may be the first attacked, or, if the disease begins in the urethra, the symptoms may be so slight, and the trouble may spread to the genital tract so quickly, and, even having affected that, the symptoms may not be sufficiently severe to necessitate her taking advice. As a rule, a woman knows when she is infected; at least, she knows there is 
something wrong, although she may be in ignorance of the cause, so that she is usually to blame for not seeking medical opinion. Considering how widespread gonorrhœa and syphilis must be in women, it is odd what a very small percentage of medical practice outside the hospitals, and even in hospital practice, is taken up by women.

Where do women go for their treatment? The answer is that they have none-at any rate, not until the setting in of a complication demands surgical interference. The importance of the fact that women do not, generally speaking, seek advice during the acute and the most infectious period cannot be over-estimated, and it is a point which should be most seriously considered by any committee formed to deal with the extirpation of venereal diseases. It is highly probable that druggists and charlatans are more frequently consulted by women than by men. The former could very easily be dealt with, but the latter, unfortunately, not so. Women who are the subjects of gonorrhœa are further placed at a greater disadvantage than men, in that menstruation stops the treatment and aggravates the disease. Moreover, once the cervix has become affected, the disease is far more difficult to cure than when the prostate in men is involved. Therefore, in no disease is an early diagnosis more to be wished for than in gonorrhœa in women. The gonococcus is also a greater cause of sterility in women than it is in men; but, oddly enough, it practically never causes sexual neurasthenia in women. Men can contract gonorrhoea from sexual connection only, and this is the most frequent source of infection in women; but it must be remembered that, in the latter, there is another source of infection which is too frequently overlooked. Many women have the habit of wiping themselves after micturition with any towel that may be handy. I have known of four cases in which virgins contracted gonorrhœa in this way at ladies' clubs in London, and I also know of a family in which the mother and three daughters all contracted gonorrhœa from presumably using the same bath-towel.

In most cases of gonorrhœa in women the urethra is the initial site of infection. The disease then spreads to Bartholin's glands, and from here, via the vagina, to the cervix. The disease may remain localised in the cervix, or it may spread into the uterus, along the Fallopian tubes, into the general peritoneal cavity.

Although a true gonococcal vaginitis is common in young girls, it is not often met with in adults. In adults it is more likely to occur in those patients who have had least sexual connection.

Complications of the urinary tract are rarer in women than in men. Gonococcal proctitis is very much more common, and other complications, such as arthritis, etc., affect both sexes alike. In virgins, and in recently married women, in whom the disease is more likely to remain for some time in the vulva without spreading, great care should be taken not to insert any instrument into the vagina, as such a procedure is sometimes a source of infection of a previously healthy cervix. In women who have bcen married some 


\section{8

time the cervix is apt to be the initial site of the infection. In them, vaginal douching can be started straight away.

There are several reasons why colpitis should be less common in the sexually matured than in virgins. In the former, the mucous membrane is not so delicate, as the superficial layers of the epithelium make a more or less satisfactory attempt to form a horny layer. The adult vagina contains organisms which are not met with in the vagina of young maidens, and the gonococcus, as has been frequently pointed out before, will not live in symbiosis with other bacteria. Finally, the mucous membrane of the adult vagina secretes a mucinous substance in which the gonococcus cannot flourish, while the vaginal mucous membrane of young girls has no secretion.

Urethritis in the female tends to heal very quickly by itself, hence it can be easily reinfected. It is important to remember this, since, if a woman has symptoms of a urethritis, one is apt to assume that she has just been infected, while it is by no means a rare occurrence for the cervix to be affected first and the urethra afterwards by a spread of the organisms from the cervix to the urethra viâ the vagina.

Bartholinitis is an extraordinarily chronic complication, and it may not only give rise to an auto-reinfection of the urethra and cervix, but also it is a frequent source of infection of a second party.

The vulvo-vaginitis of children is caused most frequently by the gonococcus, and it is usually contracted from dirty sponges and linen. The condition is very infectious, and may become almost epidemic-i.e., in a children's ward, if there is one case of gonccoccal vulvo-vaginitis, unless the very greatest care be taken, every female child in the ward may be infected.

The inflammatory process always involves the urethra and lower portion of the vagina.

Vulvo-vaginitis may also affect new-born infants and maidens. The former infection can occur during birth, or a few weeks later. The latter infection is of importance from the forensic point of view.

Vulvo-vaginitis of children is extremely painful, and the secretion of pus is usually profuse. The pus stains the linen and makes it stiff, and this is usually the first indication that anything is wrong; then the pain on passing water, etc., is noticed.

On examination, the inflammation is found to have involved the labia, the clitoris, the hymen, the vestibule, the urethra, and the vagina. These structures are red, swollen, and covered with pus; and, owing to the irritation of the pus, an intertrigo in the genito-crural folds is the rule. The vestibule is often covered with small ulcers. The hymen is pushed forwards, owing to the quantity of pus that collects behind it. The inflammation of the labia and the intertrigo are caused, not by the gonococcus, but by the irritative nature of the pus which comes from the vagina. The acute stage of vulvovaginitis is rather a long one, and the condition is rather obstinate to treatment. The acute stage ultimately passes into the chronic stage, and it is in 
this stage that spontaneous cure ultimately ensues. Spontaneous cure will take place whether the case is treated or not, but the disease may persist for a very long time before this happens. Fortunately, cervicitis and Bartholinitis do not complicate vulvo-vaginitis.

In adults one does not speak of vulvo-vaginitis, owing to the fact that the vagina is so rarely affected, but otherwise there is practically no difference between the two conditions. Vulvitis occurs in adults; it is an inflammation of the vestibulum and of Bartholin's glands, with sometimes ulceration. The ulcers are multiple, and apt to be mistaken for soft sores.

The gonococcal urethritis in women is of quite minor importance compared with that in men, but it may be complicated by a paraurethritis. There may be one or more canals, and the openings are just external to the urethral orifice. A paraurethritis does not give rise to symptoms, and it is, as a rule, only diagnosed by accident, when the secretion is pressed out of the urethra by the finger in the vagina. These paraurethral canals are undoubtedly congenital, and possibly the embryonic remains of Skene's tubules. As a rule, they are easily closed by electrolysis.

When Bartholin's gland becomes affected, and not infrequently both are involved at the same time, the patient complains of acute pain on movement. On examination in the early cases, if pus has formed in the glands, it can usually be pressed out, but later the duct and orifice become closed. So long as the pus can find exit, it gets expressed at almost every movement on the part of the patient, and the swelling does not attain to a great size. The pain is naturally acutest when the swelling is greatest; therefore, when the only bearable position for the patient is to lie on her back with the legs wide apart, the diagnosis of an abscess in Bartholin's gland may be made. Once an abscess has formed, the chances are that the lining membrane of the gland has been destroyed. Consequently, when the pus has been evacuated and the abscess healed, a recurrence is unlikely to ensue.

When Bartholin's gland merely becomes inflamed, the inflammation passes in time from the acute to the chronic form; and, once it is chronically inflamed, frequently recurring retention cysts are liable to arise, or suppuration may at any time occur, due to a secondary infection. Suppuration may cause an ulceration of one or both walls of the gland, which ultimately leads to perforation. When such an ulcer first occurs it is apt to be mistaken for a chancre.

Owing to there being gonococci ever present in the chronically inflamed gland, it can be easily understood what a great source of infection a woman is likely to be; and as a chronic Bartholinitis runs a symptomless course, except when retention cysts form, a woman may be quite unaware that she is infectious. As already stated, a true gonococcal vaginitis is most often seen in children, but it should not be forgotten that a gonococcal vaginitis is far from being uncommon in pregnant women. This is due to the fact that the mucous membrane is swollen and softer, and possibly its secretion is less acid. 


\section{0}

Anyhow, the gonococci can flourish in it. Gonococcal vaginitis is also met with in women who have had the uterus and ovaries removed. Castration causes shrinkage of the vaginal mucous membrane, reduces the number of layers of epithelial cells, diminishes the secretion, and in this way the gonococci are more readily able to penetrate into the connective tissue beneath.

When the gonococci reach the cervix in women who have had children, they readily gain access to the uterus; but in all cases of cervicitis, whether in women who have had children or in those who have not, the organisms quickly spread into the sub-epithelial tissue, and this is the reason why cervicitis is so difficult to cure. The affected cervix is usually somewhat œdematous, and there is always a purulent or a mucous discharge from the external os. As a rule, a cervicitis gives rise to no symptoms, but sometimes, during the menstrual periods, owing to the constriction of the internal os and the canal which may be caused by chronic inflammation, a patient may complain of dysmenorrhœa.

The constant discharge from the external os is very liable to cause an erosion.

It is practically impossible to say when a cervicitis becomes an endometritis, but, from the extraordinary resistance to treatment of almost every case of so-called cervicitis, one would probably not be far wrong in stating that endometritis more often accompanies a cervicitis than that a cervicitis exists alone. Once the organisms have gained entrance to the uterus, they rapidly spread over the surface and under the mucous membrane. The ease with which the gonococci invade the sub-epithelial tissue is doubtless partly due to the changes the epithelium undergoes at each menstrual period, and to the increased vascularisation of the organ at this time.

The uterus in acute endometritis is very painful on pressure, and the patient cannot endure sexual connection. General symptoms are usually severe, and the patient nearly always has to take to her bed.

The acute stage ultimately runs into the chronic, and it is in this stage that the main symptoms are complained of. There are three regular symptoms: (1) dysmenorrhœa ; (2) menorrhagia; (3) metrorrhagia.

Unfortunately, those symptoms may persist, even after all the gonococci have vanished, probably because a secondary infection takes the place of the gonococci, and maintains the chronic inflammatory process.

Gonococcal endometritis is a frequent cause of abortion, and naturally complicates and aggravates the after-results thereof. From the uterus, the gonococci travel to the Fallopian tubes, and as the organisms in most cases come from all over the uterus, a bilateral salpingitis is much more commonly met with than a unilateral one. The onset of an acute salpingitis can frequently be diagnosed as early as can the onset of an epididymitis, because in both cases the discharge suddenly stops owing to a reversed peristaltic action carrying the pus in the opposite direction-in fact, this is how both these complications arise. In salpingitis, as well as in epididymitis, the 
moment the acme of inflammation has been passed, the pus follows its normal direction, and the discharge reappears. This diagnostic sign is of the utmost importance, because the complication may be aborted by the timely use of trimine.

When salpingitis has set in the tubes swell, become odematous, and often the lumen is obliterated. The pus formed at first flows into the uterus, but it may easily get pent up, and so produce an abscess. As in all gonococcal affections, abscess formation is by no means the usual sequence of events, it being far more common for the acute inflammation to subside gradually into chronic inflammation. A chronic salpingitis runs a very similar course to a chronic Bartholinitis; that is to say, the openings become periodically blocked, the secretion can find no exit, and a retention cyst forms-a hydrosalpinx. A patient with salpingitis usually has endometritis as well, hence the symptoms are very much the same, but in the former pain is complained of in the lateral portions of the abdomen. When occurring on the right side only, the pain is frequently mistaken for appendicitis.

A certain diagnosis of salpingitis can only be made by a thorough digital and bimanual examination.

Owing to the adhesions to which a chronically inflamed Fallopian tube is liable to give rise, patients frequently have varied abdominal and pelvic pains, some of which suggest bowel trouble, others bladder trouble, and so forth.

Owing to the patent external ostium of the Fallopian tube, the gonococci can reach both the ovary and the general peritoneal cavity. A point worthy of mention is that a woman who develops gonococcal peritonitis, either following curettage or not, provided she recovers, finds she is cured of the disease. A corpus luteum abscess is not infrequently of gonococcal origin. Pelvic gonorrhœa, a name which may well be given to a gonococcal infection of the organs just mentioned, almost invariably produces chronic invalidism. The patient has no regular symptoms, perhaps never has had them, but complains of vague pains, and always feels ill; she is often anæmic, and is usually constipated. Unfortunately, it is in such a condition that the patient for the first time seeks advice, and although one knows that the gonococcus is at the bottom of the whole trouble, it is difficult to explain the situation to the patient, as in many cases the infection occurred many years back. Hence pelvic gonorrhœa is better treated by a gynæcologist than by a venereal specialist, and, moreover, an operation may at any future time be required.

Pelvic gonorrhœa is a frequent cause of functional neuroses in women; but, oddly enough, one seldom sees a condition exactly analogous to the sexual neurasthenia so common nowadays in men.

Treatment.-The general treatment should be the same as that advised for men. In the acute stage, the patient should be kept in bed when possible. The greatest attention must be paid to the bowels, and urinary antiseptics internally may be prescribed, such as sandal-wood oil, urotropin, salicylic acid, or cystopurin. 


\section{CLINICAL ASPECT AND TREATMENT OF VENEREAL DISEASES}

Vaccines from the very commencement are useful, as they increase the patient's natural protective power, and they no doubt check the spread of the disease. The chemotherapeutic agents are invaluable, especially in the acute inflammatory complications.

In chronic gonorrhœa, especially in pelvic gonorrhœa, vaccines combined with intramine may be the only treatment that exerts any influence on the disease-indeed, in some cases of cervicitis and endometritis I have seen such treatment cure the patient. Intramine is particularly valuable in cases where adhesions have formed and in cases of pelvic cellulitis, provided it is prescribed before the fibrous tissue becomes completely organised.

In acute urethritis, the patient should wash out the urethra twice a day with a 1 in 10,000 solution of potassium permanganate. In chronic urethritis a 1 in 10,000 solution of zinc permanganate should be used.

In cases of acute vulvitis, the vulva should be frequently washed with an antiseptic solution, then well dried, and a dusting powder used, because, the drier the region, the less able is the gonococcus to live and multiply. The dusting powder should contain light magnesium carbonate, as this salt absorbs more than two and a half times its own weight of water.

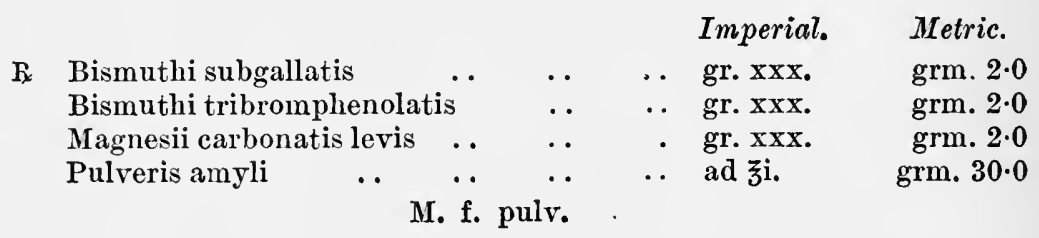

On no account should the vagina be douched, for fear of carrying gonococci on the tip of the nozzle of the syringe from the vulva to the cervix. In cases of vaginitis, naturally the vagina must be douched, but the patient should be warned against using too strong a solution, douching too often, or employing an instrument which sprays with any great force.

In the acute stage, a 1 in 10,000 solution of potassium permanganate is the best solution to be employed. In the subacute or chronic, zinc permanganate, albargin, formalin, and lysol are the most suitable.

Under no condition should the cervix be dilated in a case of cervicitis or endometritis. Small superficial injuries are bound to result, and if the gonococci have not already entered the sub-epithelial tissue they are certain to do so now. Instrumentation of the cervix or uterus, in my opinion, aggravates every case of gonorrhœa of these organs, whether the case is subacute or chronic. Curetting for endometritis I have never yet seen do any good.

Furthermore, instrumentation of the cervix or the uterus, even in the chronic stage, is always liable to start up an acute salpingitis, with the additional risk of a peritonitis following. Applications of antiseptics can only kill those gonococci with which the solution comes in contact; therefore dilating the cervix and painting the surface, or the interior of the uterus 
is never going to affect those organisms which have already penetrated deeply into the connective and muscular tissue. These can only be reached by the blood-stream; therefore, sole reliance must be placed upon the chemotherapeutic agents and vaccines we have at present, hoping that in the future still more active ones will be produced. Some observers are in favour of cauterising the cervix and uterus in chronic cervicitis and endometritis with formalin, iodine, tri-chloracetic acid, or silver nitrate, but I am, personally, very sceptical as to the amount of good it does.

Menge lays a great deal of value on the treatment of chronic cervicitis and endometritis by cauterisation with formalin. In his hands it appears to have been successful; therefore, in those cases which have failed to respond to trimine, intramine, and vaccines, one may fall back upon this as a reserve treatment.

Menge uses formalin either undiluted, or in a 50.0 per cent. solution. $\mathrm{He}$ runs wool round a hard gum elastic uterine sound, soaks the wool with the solution, and swabs out the cavity.

In Bartholinitis, with abscess formation, the pus should be evacuated through a small incision, and the abscess cavity washed out with normal saline or a weak antiseptic solution, and then the trouble usually cures itself. In chronic Bartholinitis, in which cysts are continually forming, the only thing to do is to remove the sac.

The treatment of vulvo-vaginitis in children merely consists in washing out the vagina with a $2 \cdot 0$ per cent. solution of silver nitrate, and keeping the genitals and genito-crural folds dry, to prevent the spread of the dermatitis. If the skin be already inflamed, it is a good plan to apply liquid paraffin, to let this dry, and then to put on a weak Lassar's paste, which contains no salicylic acid.

As the child usually struggles when being treated, and as the vaginal orifice is small, the best way to wash out the vagina is through a narrow rubber catheter. 


\section{CHAPTER XXVII}

\section{THE CHEMOTHERAPEUTIC AND VACCINE TREATMENT OF GONORRHEA}

\section{General.}

So far as gonorrhœa is concerned, I think the opinion is unanimous that a cure is only likely to be obtained viâ the blood-stream. Up to the present there are two main methods of carrying this out: (1) by the introduction of drugs; (2) by the introduction of bacteria in an altered form. The former originally aimed at producing a drug which would show a special affinity for the parasite attacked, and cause its death without injuring the cells of the host-chemotherapy. The latter aims at increasing the host's immunity against the parasite so as to prevent the invader flourishing and ultimately to lead to its destruction-vaccine-therapy. Although the application of these two methods have resulted in great advances being made, we cannot say the success achieved has been as great as had been anticipated. The reason chemotherapy has fallen short of its expectations is because the foundation upon which it was built was unsound.

As there is little or no difference between the chemical composition of a parasite and certain cells of the host, it is illogical to suppose that a chemical agent could be prepared which would attack one and leave the other. As there is no limit to the number of organisms capable of becoming parasites, while there is to the number of the chemical elements available, it is unreasonable to suppose that a specificity could exist between a certain element and a certain parasite. Chemotherapy has failed because the physical action and the way the host naturally protects itself against invasion were left out of account. The introduction of the former is the link between chemo- and vaccine-therapy, to which reference will be made below, it being necessary first to discuss the natural protection, forming, as it does, the basis of both. The host is always on guard against the attack of any parasite, be it an old or a new comer, which allows one to say that the protective substance has a common action. The protective substances are the protein colloidal particles in the serum. Each particle has Brownian movement, and consists of several chemical groups which are arranged, and capable of being re-arranged, so as to give the particle certain and varied physical properties which exhibit their action on the surface. The action, then, between the protective substance and the parasite is at first a common and a surface one.

As oxygen is necessary for all life, it is only reasonable to suppose that the aim of the protective substance is to deprive the parasite of the oxygen necessary for its existence. At first this is done by a process of electrical 
neutralisation, which requires a little explanation. The chief chemical groups on the surfaces of both the parasite and the protective substance are hydroxyl $\left(\mathrm{OH}^{\prime}\right)$ and hydrogen $\left(\mathrm{H}^{\circ}\right)$, the former of which is negatively and the latter positively charged. It is probable that the oxygen above referred to is a specialised hydroxyl-group. When a negative meets a positive charge, the charge is annulled-electrical neutralisation. Normally, the protective substance has a prevailing negative charge due to the excess of $\mathrm{OH}$-groups over H-groups. Under abnormal circumstances this balance is upset so as to lead to a decrease of the $\mathrm{OH}$-groups and an increase of the H-groups, a condition which, if excess is reached, is known as acid intoxication. Slight changes at present cannot be registered, but presumably occur when a patient is ill, run down, or under the influence of alcohol. We all know that gonorrhœa is especially liable to be contracted, or a relapse to occur, under such circumstances; therefore, the gonococcus finds it easier to implant itself on the host when the $\mathrm{OH}$-groups on the surface of the protective substance are diminished. In any case, the parasite is at a disadvantage; consequently, it is obliged to protect its oxygen, which it does by exhibiting an excess of H-groups on its surface. It is now clear that the interaction between the parasite and the protective substance is a neutralisation of the H-groups on the former by the OH-groups on the latter. From this it may be assumed that one ideal to aim at is to increase the number of these specialised $\mathrm{OH}$-groups on the surface of the protective substance.

It is necessary now to inquire as to how these specialised $\mathrm{OH}$-groups are normally formed. In my opinion, they are formed by metals-by manganese in plant life, by copper in certain invertebrates, and by iron in vertebrates. The introduction of metals then increases the action of the protective substance, and two metals which have this action more than others are manganese and iron; hence the reason why I introduced colloidal manganese, and later trimine, which contains colloidal iron and a trace of colloidal zinc in addition. My reason for combining metals was owing to the fact that three injections of three different metals are more active than three injections of any one. Iron was added for obvious reasons, and zinc because in traces it activates both iron and manganese.

When the parasite has become implanted in the host, the protective substance has to adopt methods of its own to increase the action of its specialised $\mathrm{OH}$-groups on the surface, which it does by re-arranging the chemical groups of the particles or substratum. The re-arrangement approximates as near as possible to that existing in the substratum of the parasite, and this imparts to the protective substance certain physical properties which enables it to dovetail, so to speak, into the parasite, thereby giving greater force to the specialised $\mathrm{OH}$-groups on its surface. There are two main chemical groups of the substratum, amino $\left(\mathrm{NH}_{2}\right)$ and carboxyl $(\mathrm{COOH})$, of which the former is by far the more important. From this the reader will be able to see at once that the action of a metal in increasing the action of the protective 
substance will depend upon its substratum-in other words, upon its chemicophysical constitution. Further, that if the substratum contains aminogroups, the metal will be better able to dovetail or to become attached to the particles of the protective substance. It is now obvious why di-ortho-diamino-arseno-benzene (salvarsan) should be more efficacious than colloidal arsenic. Similarly, why colloidal manganese should be more active than a salt of the same metal.

Just as a simple thing leads to a more complex, and a complex to a still more complex, so may the various parasites be graded. The simplest parasites are the staphylococci and streptococci, and going up the scale we pass the following in this order: gonococci, fungi, protozoa, and the cancer cell, derived from the host himself. The simpler the parasite the less need there is for the protective substance to exert itself, and therefore the less complex the metallic chemotherapeutic drug used to stimulate it need be. This explains why plain colloidal manganese and trimine have done even more for staphylococcic and streptococcic infections than arseno-benzene, even at its best, did for syphilis, and why neither of these preparations is as efficacious in gonorrhœa, still less so in syphilis, a protozoal disease, and useless in cancer. That manganese and iron are the metals for choice in any chemotherapeutic research there is no doubt, but that the substratum has to be more complex than it is at present to vanquish the gonococcus, and still more complex to conquer the Leucocytozoon syphilidis, and still more complex to overcome the cancer cell. There comes a time, if the parasite has not already been vanquished, when the protective substance has to resort to indirect methods to keep up its supply of specialised OH-groups on the surface, and this is normally done by the action of sulphur--a non-metal. The time the protective substance has to do this coincides with the chronic stage of the disease, hence the reason why I have advocated sulphur and iodine, which increases its action in chronic gleet and gonorrhœal rheumatism. Fortunately, I have been successful in preparing a good substratum for the sulphur, which has enabled mast excellent results to be obtained in the conditions mentioned, and explains why di-ortho-di-amino-thio-benzene or intramine should be vastly superior to plain colloidal sulphur.

With all that has been said it is legitimate to forecast the excellent results which would be obtained in gonorrhœa if only an amino-compound of manganese and iron could be produced. It has been suggested that intramine and the colloidal metallic preparations act in the same manner as intravenous injections of peptone, a therapy which has been used on a large scale in infectious diseases by Nolf, ${ }^{8}$ and called the pyrogenic therapy by Auld. ${ }^{9}$ That this is not the case is proved by the antagonistic action between intramine and the colloidal metals, and by the fact that the colloidal metals act differently, in spite of the protective substance added being the same in all. Colloidal copper is not so efficacious in coccogenic infections as colloidal manganese, and colloidal vanadium in gonorrhœa is practically useless.

In vaccines we have the substratum, but not the ideal surface, hence the 
reason why they have not reached the goal set out for. In my opinion, vaccines will always fall short of complete success, because the particles undergo physical changes when the vaccine is prepared, which extend to those of the protective substance when introduced into the body, and diminish its action-toxic effect. When Besredka, 2,3 lessened the toxic action of vaccines by sensitising them, Klein and $\mathrm{I}^{4}$ carried the principle to gonorrhœa, prepared some sensitised gonococcal vaccines, and injected them intravenously, with better results than had been obtained heretofore. Owing to the difficulty of preparing sensitised vaccines, and to the fact that they quickly become desensitised on keeping, as a routine method of treatment it became impracticable.

About this time various commercial vaccines were used, such as arthigon, gonargin, phylacogen, serobacterin, etc., all of which were sometimes useful, but deteriorated on keeping. The results being unsuccessful, taken as a whole led to vaccines falling into disuse, till they were recently resurrected by Thomson ${ }^{5}$ with his detoxicated vaccine. Thomson's vaccine contains the active principle of several strains of gonococci, the toxic portion having been removed. Although this vaccine is easier to prepare, keeps longer, and is therefore more useful than sensitised vaccine, no better therapeutic results are obtained with the former than with the latter, and, employed alone, it is not so efficacious as are the chemotherapeutic agents. As the gonococcus, when cultured, develops a substratum different from the one it forms when growing in corpore, it may happen that the immunity produced by vaccination is an artificial one, and not identical with the immunity occurring in the body during the infection. This explains why the results of vaccinetherapy are so uncertain. Owing to the fact that the host has more need to increase his protective substance in the cases with complications than in those in which the urethra only is involved, it stands to reason that the clinical improvement following treatment by the blood-stream is more striking in the former than in the latter.

Recently I have treated a large series of cases by the combined chemoand vaccine-therapy, and, on the whole, have obtained better results than were obtainable with the former alone; but before relating my clinical experience, it is necessary to refer to some broad principles first. Any treatment which aims at stimulating and accelerating the action of the protective substance will, if continued long enough, lead to its destruction. As there is a minimum of protective substance formed in cases of plain urethritis, the reader will understand what care has to be exercised to avoid over-dosing the patient. Over-dosing aggravates the lesion because it alters the normal relationship between the hydroxyl- and hydrogen-groups, and brings about a state of affairs which does not differ from those which exist when the patient is ill, run down, or under the influence of alcohol. We now have the explanation as to why a recurrent attack of urethritis is more difficult to handle than any other gonococcal lesion. The urethritis would not have recurred had the relationship between the two important groups not been disturbed, and, being disturbed, the use of blood-stream therapy is more or less precluded. 
Over-dosing occurs more readily with chemo- than with vaccine-therapy, but occurring, as it can, with both, the importance of the doses and intervals which elapse between them cannot be over-estimated.

This brings us back to the point once more-namely, that every case requires to be treated individually, and that the way not to find out the value of a drug is to order this or that drug for the next two hundred admissions. of the disease in question. This is the sole cause for clinical reports being so much at variance. Wright, ${ }^{6}$ the founder of vaccine-therapy, was fully alive to this, and began early to devise a method by which the dosage could be regulated. Unfortunately, the method, which was known as the opsonic index, was unwieldy, and, so far as gonorrhœa was concerned, not applicable.

Contrary to the general opinion, I hold that the gonococcus lives at the expense of the polymorphonuclear leucocyte, and that phagocytosis as a protective mechanism plays a very subordinate position to the protein colloidal particles, which emanate in the main from the lymphocytes.

The polymorphonuclear lencocyte is a degenerate cell. The polymorphism of the nucleus is a sign of degeneracy, so is the fact that the nucleus contains. no nucleolus, and added to this are the feeble staining properties of the protoplasm. Another sign of degeneracy is the fact, which no one seems to have observed, that a polymorphonuclear leucocyte cannot change; in other words, it cannot be the starting-point of a new growth, innocent or malignant, as a lymphocyte or an endothelial cell can be. Therefore, one could not expect it to be more than a scavenger of dead bacteria, and to be itself sometimes. preyed upon.

As the opsonic index is the phagocytic index, and does not measure the protective capacity of the protein colloidal particles, Klein and I, in 1912, tried to regulate the treatment of complicated cases by means of the C.F.T. At that time I was under the impression that the goal to aim for was a negative C.F.T., which I have since found to be incorrect. When vaccines are first given the C.F.T. becomes negative (negative phase), to become positive again if the injections are repeated. If several injections are given, either the C.F.T. remains lastingly positive or becomes negative. The former occurs in cases which show the greatest clinical improvement. The latter remains negative for an indefinite period, but ultimately becomes positive again. Clinical recurrences are most apt to occur when the C.F.T. is negative. Therefore, the aim is not to produce a negative C.F.T., which signifies destruction of the protective substance, but to keep it positive, because a positive reaction signifies the presence of a protective substance. Owing to the fact that an artificial immunity produced by vaccine-therapy can cause a positive C.F.T., which does not become negative for several weeks after the last injection, and as there may be no relationship between such an immunity and the natural or specific immunity, it becomes extremely difficult to interpret the results of the tests, and chief reliance again has to be placed on the clinical results.

When working at the rationale of the C.F.T. I found that negative sera, if artificially prepared to give a positive reaction, could be made, by still 
further increasing the positivity, to give a negative reaction. Therefore, there is as close a relationship between a negative and a slightly positive reaction as there is between a negative and a very strongly positive reaction; consequently, the C.F.T. can be diagrammatically represented as below. It will thus be seen that the test is useless as a gauge of cure or as a means by which to regulate treatment.

As there are no reliable tests by which chemotherapy can be regulated, we come back to where we started, and find that only by clinical experience can we estimate as to how much treatment is required. As the kind and amount of treatment varies in each individual, I can do no more than mention here what I have in the generality of cases found most useful. The principles are (1) in chemotherapy not to give too much metal, which leads to its own oxida-

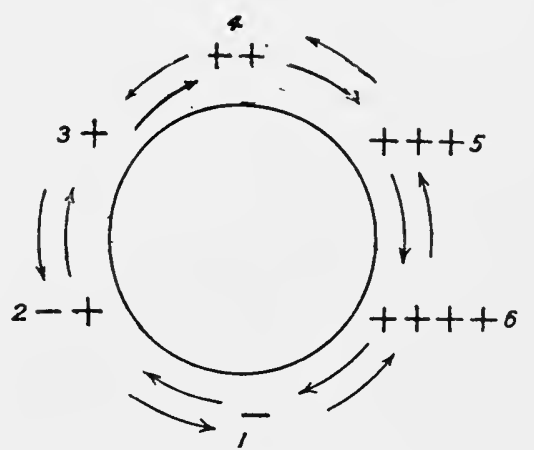

Self-fixation of complement occurs between the stages 6 and 1 . tion, and so deprives the patient of the oxygen of which he is most in need; and (2) in vaccine-therapy not to give too big doses, as they cause a negative phase, or, in other words, destroy the patient's prot ective substance.

Acute and Subacute Urethritis (first attack).-As there is so little protective substance, and metals like manganese and iron are so readily oxidised, I introduced colloidal palladium (pallamine), with a view to lessen the number of manganese injections required, and because it readily dissociates $\mathrm{OH}$ from H.OH, as in the following diagrammatic formula:

$$
\mathrm{Pd}+\mathrm{H} . \mathrm{OH}=\mathrm{Pd} . \mathrm{H}+\mathrm{OH} \text {. }
$$

Since palladium only temporarily fixes the hydrogen-group, the time has to come when the latter is freed, and then is liable to raise the hydrogen-ion concentration. This is the reason why $I$ never prescribe more than three injections of $0.5 \mathrm{c.cm}$. each, why the drug should never be used in a recurrent case of urethritis, and why it is theoretically advisable to order potassium nitrate and methylene blue internally, because hydrogen converts the former into a nitrite and the latter into its leuco-base, two innocuous substances. Owing to the freedom from painful micturition and chordee which pallamine brings about, King-Turner's idea that it also acts by relaxing contractile and unstriped muscular tissue is probably correct. Pallamine keeps well; but, unfortunately, colloidal manganese, colloidal iron, and trimine, lose some of their therapeutic action on standing. Another unfortunate thing about some of the colloidal preparations is that no two samples are exactly alike, although manufactured in the same way. It is, in any case, advisable to keep the preparations away from light and air as much as possible. Intramine only becomes more painful on standing, especially if some of No. 1 gets into No. 11. When vaccine- is combined with chemo-therapy, the two should 


\section{CLINICAL ASPECT AND TREATMENT OF VENEREAL DISEASES}

be injected into different parts, and preferably on different days. When mixed in the same syringe, the pain is increased, except in the case of intramine. Coming back to the treatment, the following is the routine most generally applicable for this stage:

First day: An intramuscular injection of $0.5 \mathrm{c.cm}$. pallamine.

Sixth day: The same.

Twelfth day: The same or an intramuscular injection of $0.5 \mathrm{c.cm}$. trimine. If a case has been caught early, and the infection has not invaded the posterior part of the urethra, two or three injections of pallamine will usually suffice.

Eighteenth day: The same.

Twenty-fourth day: If necessary, $1.0 \mathrm{c.cm}$. trimine.

If by this time the discharge has not vanished, it is worse than useless to continue the injections. Pallamine is useless if the discharge has been present for more than a month, in which case trimine should be used from the start. An excellent procedure in this, as in all cases of urethritis, is to prescribe three injections of vaccine, 2,500, 2,500, and 5,000 million respectively, on three successive days before beginning the chemotherapeutic agents, or to keep the vaccines to fall back upon if the chemotherapeutic agents have failed. Experience has shown that the latter is the better procedure.

\section{Recurrent Urethritis.}

In acute or subacute recurrent urethritis chemotherapy is best omitted until the discharge has ceased, sole reliance being placed on local treatment and vaccine-therapy. If the patient is very run down, mild local treatment only should be employed, and every step taken to make the patient fit again. More harm than good may be done by blood-stream treatment in these cases, as has unfortunately been proved to me on several occasions. There is no doubt more knowledge is to be gained by one's failures than by one's successes, and it is in this stage that the former are apt to be frequent. If I prescribe vaccines in this stage $I$ inject three doses of 2,500, 2,500, and 5,000 million respectively on three successive days, or with two or three days' interval between each, should the first cause much reaction, gastritis, etc. When all the discharge has stopped, I prescribe colloidal iodine internally, and an intramuscular injection of intramine $(1.5 \mathrm{c.cm}$.). If the discharge increases, then trimine is indicated, and, if not, more intramine is required, either being injected five days later.

\section{Discharge Increased.}

Fifth day: 0.5 c.cm. trimine.

Twelfth day: 0.5 c.cm. trimine.

Nineteenth day: 1.0 c.cm. trimine.

Twenty-sixth day: 1.5 c.cm. trimine

(if necessary).
Discharge not Increased.

1.5 c.cm. intramine.

1.5 c.cm. intramine.

1.0 c.cm. trimine. 
If the recurrence is an acute one after treatment with pallamine, due to a localised folliculitis, an injection of trimine $(1 \cdot 0 \mathrm{c.cm}$.) will stop it immediately.

If the recurrence is chronic from the start, then three injections of intramine, with or without an injection of trimine, will suffice, no vaccines being required. These cases do extraordinarily well, and all the subjective symptoms, such as heaviness underneath, etc., which mean so much to the patient, vanish after the first injection.

If the occurrence is an extremely obstinate one, due probably to the gonococci being ensconced in the polymorphonuclear leucocytes, then colloidal antimony will often heal the lesion, as it does in cases of kala-azar, and in those cases of Ulcus molle in which the bacilli are intracellularly situated. During the first week, two intramuscular injections each of $0.5 \mathrm{c.cm}$. should be prescribed; during the second week, one injection of $0.5 \mathrm{c.cm}$. and one of $1.0 \mathrm{c.cm}$; and during the third week, one injection of $1.0 \mathrm{c.cm}$. and one of $1.5 \mathrm{c.cm}$.

\section{Complications by Extension.}

Vaccines are only necessary in the acute and subacute stages, when they should be given as advised in cases of recurrent urethritis. In the acute and subacute stages, the chemotherapeutic agents should be prescribed as follows:

First day: 0.5 c.cm. trimine.

Fourth day: $1.0 \mathrm{c.cm}$. trimine.

Tenth day: 1.5 c.cm. trimine.

Fifteenth day: $1.5 \mathrm{c.cm}$. intramine.

Twenty-first day: $1.5 \mathrm{c.cm}$. intramine or $1.5 \mathrm{c.cm}$. trimine. Former if a fibrous nodule is still present, as in lower pole of epididymitis, when a final injection of trimine must be given; and latter when the fibrous tissue formation has disappeared, or is minimal.

In the chronic stage - that is to say, when there is no tenderness or painthe drugs are best injected in this order:

First day: $1 \cdot 5 \mathrm{c.cm}$. intramine.

Fifth day: $1.5 \mathrm{c.cm}$. intramine.

Eleventh day: $2 \cdot 0 \mathrm{c.cm}$. intramine.

Seventeenth day: 1.0 c.cm. trimine.

In bad cases of gonorrhœal ophthalmia, when not a day can be lost, $1.0 \mathrm{c.cm}$. trimine should be injected on the first day, and repeated three days later, and $1.5 \mathrm{c.cm}$. three days later still. This is the best plan to adopt in cases of acute salpingitis and peritonitis.

\section{Complications by Metastasis.}

With the exception of gonococcal septicæmia, which is fortunately very rare, the only metastatic complication of note is rheumatism. In cases of gonococcal septicæmia, trimine should be injected as advised for ophthalmia, and either colloidal silver or mercuric chloride should be injected intravenously 
in addition. If colloidal silver is chosen, 1.0 to $5.0 \mathrm{c.cm}$. should be injected every other day, and if mercuric chloride, 1.0 to $3.0 \mathrm{c.cm}$. of a 1 in 1,000 solution. Towards the end of the first week it will be known if the patient is going to recover or not. I treated one case successfully in this way with colloidal manganese intramuscularly and colloidal silver intravenously. In gonorrhœal rheumatism, including under this head tenosynovitis, arthritis, and neuritis, however acute the lesion may be, it is best to prescribe intramine at once. Intramine is so valuable in these cases, provided the patient is put under treatment early enough, that vaccines are not called for. It is worse than useless to prescribe intramine in those cases which have been allowed to waste to skin and bone, which may happen quickly to some of the mono-articular cases, as it will do no good, and may start an iritis. These cases must first be built up before any specific treatment is advised. In the chronic cases, where the bony changes met with in osteo-arthritis have already set in, intramine will naturally do no more than relieve some of the subjective symptoms. As iodine increases the action of intramine, in those cases where no time can be lost it is wise to inject colloidal iodine intravenously, but where there is time to spare it may be given internally for ten to fourteen days before the first injection of intramine. However acute or sudden in onset the trouble may be, the following course is the best to adopt:

First day : Intravenous injection of colloidal iodine $(100.0 \mathrm{c.cm}$.$) and$ intramuscular injection of intramine, $2 \cdot 0 \mathrm{c.cm}$.

Fifth day: Intramuscular injection of intramine, $2.5 \mathrm{c.cm}$.

Eleventh day: Intramuscular injection of intramine, $3 \cdot 0 \mathrm{c.cm}$.

Seventeenth day: Intramuscular injection of trimine, $1.5 \mathrm{c.cm}$. In long-standing and recurrent cases better results still can be obtained by injecting a vaccine the day following each injection of intramine.

Sometimes it is necessary to repeat this course, but about a month should be allowed to elapse prior thereto. Cases of gonococcal iritis should be treated with intramine, but smaller doses given than advised when an arthritis accompanies it. Simple cases of relapsing iritis also do well with vaccines.

In my experience anti-gonococcal serum is never called for.

1. Besredka (1902): Ann. de l'Instit. Past., xvi., 918.

2. Besredka (1902): Compt. rend. de l'Acad. des Sci., cxxxiv., 1330.

3. Besredka (1910): Bull. de l'Instit. Past., viii., 241.

4. McDonagh and Klein (1913): Journ. of Path. and Bact., xvii., 599.

5. Thourson (1919): Science Progress, xiv., 326.

6. Wright (1909): Studies in Immunisation. Constable and Sons, London.

7 McDonagh (1915): Biology and Treatment of Venereal Diseases. Harrison and Sons, London.

8. Nolf (1919): La Presse Médicale, xxvii., 93.

9. Auld (1918): Brit. Med. Journ., i., 195. 


\section{CHAPTER XXVIII \\ PHIMOSIS AND PARAPHIMOSIS}

\section{History.}

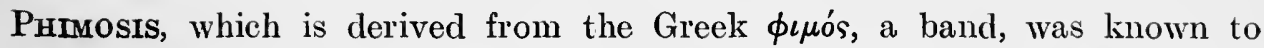
Celsus, who was the first to differentiate between the congenital and the acquired forms. For the congenital form Celsus advised splitting the prepuce or removing a triangular piece from it. Acquired phimosis, which was merely inflammatory, Celsus treated by syringing with warm water, advising circumcision only for those cases in which there was ulceration. Later, various operative procedures were advised at one time or another, but simple slitting the prepuce was early condemned, owing to the unsightly appearance it gave the member. About the causation and separation of paraphimosis there was little Celsus did not know; indeed, the operative measures employed by him are those in use to-day, such as cutting through the band on the dorsum of the penis.

\section{Phimosis.}

Phimosis may be congenital or acquired. Only the acquired form need be discussed, because the only thing to say about the congenital form is that the patient should be circumcised. Acquired phimosis is due to inflammation, and it may be caused by any venereal disease. There are, clinically, two kinds of phimosis, and if this point be borne in mind, it is usually possible to make a diagnosis of the underlying condition at sight. If a man with phimosis consult you, first notice if the prepuce is very red and swollen; if so, then it is an acute inflammatory phimosis, and is probably caused by gonorrhœa, a soft sore, or Balanitis erosiva et gangrenosa. If the prepuce is red and swollen, the swelling is uniform, painful to the touch, and there is always a purulent discharge. A phimosis caused by Balanitis erosiva et gangrenosa is the most painful, then that caused by a soft sore, while a gonococcal phimosis is not, as a rule, very painful. If the prepuce is not red, and is swollen in only one part, or is only red in that part which is swollen, and if the discharge is thin, you may be quite sure the patient has a primary sore in the corona, and that that part of the foreskin which is the most swollen marks its position. A syphilitic phimosis is generally painless.

In the acute inflammatory phimosis the foreskin should be slit up at once, but circumcision should not be done. In the, so to speak, non-inflammatory 
phimosis-i.e., the syphilitic phimosis, non-inflammatory, since the phimosis is really due to a diffuse syphilitic lymphangitis of the prepuce-circumcision can be delayed until the lymphangitis has subsided somewhat under salvarsan. Circumcision should be done later, so that mercurial ointment may be rubbed into the chancre.

Another clinical point in the soft sore phimosis is that, in several cases, there are some sores on the tip of the foreskin. When a phimosis is very red, shiny, and stony hard, the diagnosis of a malignant epithelioma should be considered.

\section{Paraphimosis.}

Paraphimosis may occur mechanically by a patient's retracting his foreskin and being unable to pull it forward again, but it is more often due to some inflammatory condition. There are two kinds of paraphimosis-the Paraphimosis interna and the Paraphimosis externa, the former being the rarer of the two.

Paraphimosis externa can only occur if the limbus of the prepuce is particularly narrow. The limbus is formed by circular fibres of connective tissue in the fore part of the prepuce. If the limbus is narrow, it will naturally follow that retraction of the foreskin may be possible, but that the corona will make reposition difficult. This, then, leads to considerable swelling of the external lamella of the prepuce.

Owing to the narrowness of the limbus, and to the fact that the inner lamella of the prepuce is turned in, it will follow that all the pus that was in the corona will now be in between the two lamellæ of the foreskin. Therefore, unless the condition be relieved quickly, the increased inflammation will further aggravate the condition, so that sloughing of the foreskin may easily occur.

Provided gangrene has not set in, attempts at reposition should be made. As the corona is the hindrance to natural reposition, the first thing to do is to reduce the size of the glans penis. Cold applications, or tying round with indiarubber, will usually effect reduction; then reposition becomes simple. As Paraphimosa interna is not so noticeable as Paraphimosis externa, most of the cases seek advice when it is too late. Under these circumstances, the best procedure is to cut the limbus, wash out all the discharge with hydrogen peroxide, and then keep the area as dry as possible by using iodoform powder. Owing to the pent-up pus between the two lamellæ of the prepuce, a dorsal lymphangitis of the penis, and bilateral inguinal adenitis, with abscess formation in either condition, is a complication which often has to be feared.

In the Paraphimosa externa the limbus is usually wide enough, the condition being produced by an œdema of the inner lamella of the prepuce. If a case of Paraphimosis externa be examined, it will be noted that a band usually exists only on the dorsum, and, according to its severity, on the lateral walls of the penis posterior to the prepuce. The base is, as a rule, not constricted, 
but it is very œdematous. Unless the condition be quickly relieved, a fissure is very likely to occur in the dorsal constriction, and this is often the beginning of gangrene.

In trying to replace the prepuce, the œdematous foreskin should be held with the fingers of both hands and pushed forwards over the glans, which should at the same time be pressed backwards with both thumbs. If there is a fissure already, or if there is a good chance of producing one by prolonged efforts at reposition, it is best to resort to another method.

The chief cause which prevents reposition is the œdema of the dorsal part of the foreskin; therefore, if this can be reduced, replacing the foreskin is an easy matter. A good plan is to press out the œdema, either backwards under the dorsal constriction, or downwards into the preputial swelling underneath.

If both these manœurres fail, it is best to employ the débridement method, or to remove the incarcerated portion totally.

The former can be done, however fissured the dorsal constriction may be, and even if gangrene has set in. It is best performed by retracting the skin of the penis, and then dividing the dorsal constriction with a bistoury in the middle line. The incision should be from $1.0 \mathrm{~cm}$. to $1.5 \mathrm{~cm}$. long, and the depth can be ascertained by the feel, as one knows at once when the fibres have been covered.

Total removal should be performed only when there is no fissure, and when the area is moderately clean. The two œdematous preputial swellings are removed betwen two parallel incisions, and then the edges are sewn together. This operation produces a good cosmetic effect, as it obviates a chronic fibrous swelling, which often results from the œdema of the basal portion of the prepuce. 


\section{CHAPTER XXIX}

\section{SUBSIDIARY VENEREAL DISEASES : BALANITIS, CONDYLOMA ACUMINATUM, MOLLUSCUM CONTAGIOSUM, HERPES GENITALIS, GRANULOMA INGUINALE, INDURATIO PENIS PLASTICA, AND PEDICULOSIS PUBIS}

\section{Balanitis.}

History.-Balanitis has been known since the beginning of history, but Thomas Sydenham (1680) was the first to point out that it might be primary and not necessarily secondary to some other infection, such as gonorrhœa, etc. For many years after Sydenham's time the condition was called "spurious gonorrhøa," and not christened "balanitis" till the beginning of the nineteenth century. Even up to Ricord's time, balanitis was considered by many to be the initial stage of a syphilitic infection.

Simple Balanitis.-Simple balanitis is a very common condition, and is one for which patients frequently seek advice. It is most common in patients with long foreskins. It often gives rise to considerable local irritation, and may, in consequence, be the cause of subsequent self-abuse. The secretion may be even sufficient to give rise to a discharge, and this may, in its turn, set up a urethritis, or, more often, may cause a phimosis. On withdrawal of the foreskin, white or yellowish-white material will be seen, which can be brushed away easily, and which has a peculiar odour. This white material usually goes by the name of smegma, and is supposed to be secreted by special glands, which are called Tyson's glands. It is extremely doubtful whether such glands really exist. It is far more likely that it is merely the sodden and desquamating epithelium of the surface of the glans and the under surface of the prepuce. In this sodden epithelium all manner of organisms flourish; urine collects in the corona glandis, and supplies the pabulum with moisture. The prodigious growth of organisms soon gives rise to inflammation, and to the symptoms arising therefrom. Organisms which, under ordinary circumstances, are saprophytic may become pathogenic, as such a condition can be conveyed to a woman, in whom it may set up vulvitis and urethritis. An analogous condition may occur in the female, and it commences around the clitoris. Cleanliness is at once the cure and the prevention. There can be no doubt that Moses was right in making circumcision compulsory, and it was doubtless his knowledge of the multitude of evils for which a foreskin may be responsible that led him to make the law, which only later developed a religious signifi- 
cance. Most of the Jewish laws were primarily preventive measures against disease. It is highly probable that pork was forbidden because of the prevalence of trichinosis in Palestine, and so on. Failing circumcision, the foreskin should be withdrawn daily, and the glans penis, sulcus coronarius, and the under surface of the prepuce should be well washed. Then the foreskin should not be replaced until the surfaces have been well dried.

In acute cases of simple balanitis a mild antiseptic lotion should be employed, and, after bathing the surfaces with it, an antiseptic powder should be used.

Balanitis erosiva et circinata was first described by Bataille and Berdal,4, 5 in 1889. The lesion commences as a small, circular, greyish-white patch, which is merely the erosion of surface epithelium. As more epithelium becomes eroded, the surface of the lesion becomes red, moist, and shiny; then all the lesions begin to coalesce. The desquamated epithelium collects in the sulcus coronarius, and quickly becomes transformed into pus, which has a characteristic odour. The discharge is infectious. The organisms which cause this condition appear to be able to flourish only under anaerobic conditions, since it occurs only in patients with foreskins, and, if the prepuce is withdrawn, the lesion does not advance. Hence the best treatment is to bathe the area with nascent oxygen, as may be done by employing hydrogen peroxide and by leaving the wounds uncovered, exposed to the air.

In severe cases in which the foreskin is tight, and the discharge is fairly abundant, the prepuce itself may become eroded, and an inflammatory œdema of the prepuce, leading to almost complete phimosis, may ensue. In such cases there may be a dorsal lymphangitis of the penis, with marked pain and swelling of the inguinal lymphatic glands; but, oddly enough, the lymphatic glands never suppurate.

Occasionally the erosions may become ulcers, and this transformation is most likely to be met with in the sulcus coronarius, and in the neighbourhood of the frænum. The ulcers are deep, the edges are regular, and the base is covered with a diphtheritic-like membrane, which is adherent.

In every case in which ulcers form there is always a bad phimosis. The ulcers may sometimes be found on the under surface of the prepuce, and, at first sight, resemble soft sores. The ulcers in question are more or less funnelshaped, and the edges are smooth and regular-not irregular, like the edges of soft sores.

In a few cases the ulcers may extend so rapidly as to perforate the prepuce, and even the whole prepuce may be destroyed.

In most of the cases the local pain is more or less severe, and the patient usually has a rise of temperature.

The Balanitis gangrenosa is really the same condition as the Balanitis erosiva, with the difference that, in the former, the ulceration is not preceded by erosions. In Balanitis gangrenosa the discharge is, as a rule, profuse, and often blood-stained, the pain is very acute, and the ulcers are, as the name 
implies, gangrenous-i.e., the ulcers are surrounded by a dusky-red zone, the edges are raised and acutely inflamed, and the base is covered with a yellow, brown, greenish, or almost black diphtheritic membrane, which cannot be removed. The organisms responsible for this condition may also cause a soft sore or chancre to become gangrenous.

The gangrene may become so extensive as to destroy the whole of the penis, and such a state of affairs is often brought about by the use of strong disinfectants, such as carbolic acid and potassium permanganate crystals. Almost any lotion other than the one specific, hydrogen peroxide, may aggravate the condition. I have seen fatal results in two cases in which carbolic acid had been used. In both, the gangrene extended so rapidly as to involve the whole of the scrotum, and it spread up over the abdominal wall before any measure could be taken to check it.

An analogous condition to Balanitis erosiva et gangrenosa cannot be reproduced elsewhere unless the skin at the site of inoculation has been badly injured and in part rendered necrotic. Therefore, strictly speaking, Balanitis gangrenosa is not auto-inoculable, and this point serves to distinguish it from the soft sore infection.

Balanitis erosiva et gangrenosa usually results from coitus, and sets in about two to fourteen days afterwards. The condition is due to two organisms existing in a state of symbiosis. One is a Gram-positive vibrio-shaped organism, which varies from $2 \cdot 0 \mu$ to $3.25 \mu$ in length; one end is often thicker than the other, and the organism is usually curved. The other organism is a Gram-negative spirochæta. The spirochæta varies in length from $4 \mu$ to $16 \mu$, it is much thinner than the vibrio, it stains violet with Giemsa, and the coils are very irregular-in fact, there may be only one or two. Occasionally, quite regularly coiled spirochætæ are to be seen, but the coils are large, and never more than five in number. Both the spirochæta and the vibrio are actively motile, and are indistinguishable from the organisms which cause Vincent's angina.

Clinically, Balanitis erosiva et gangrenosa resembles the lesions of Vincent's angina, and both conditions are readily cured by arseno-benzene. In my experience, the condition is much more common on the Continent than in England.

In any inflammation of a mucous membrane vibrio-shaped organisms and spirochætæ can be demonstrated, and, owing to the numerous folds and crypts which exist in the vulva, vagina, and tonsil, these organisms can flourish anaerobically. A woman need not necessarily have an acute vulvitis or vaginitis to be infectious; the mere implantation of a few of these organisms on a glans penis which is covered with a tight foreskin will be quite sufficient for the few implanted to flourish on the desquamated epithelium, and to reach the corona, where the most favourable anaerobic conditions prevail.

I should think it is highly probable that a man with a tight foreskin may even develop this condition without the infection coming from the female, 



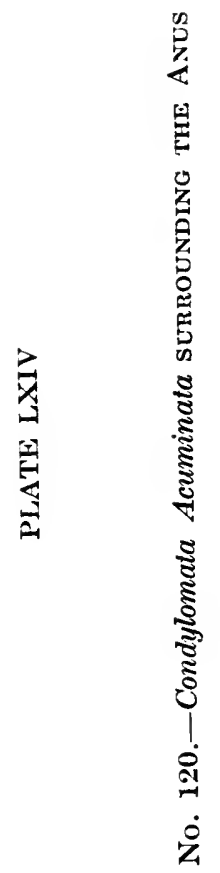




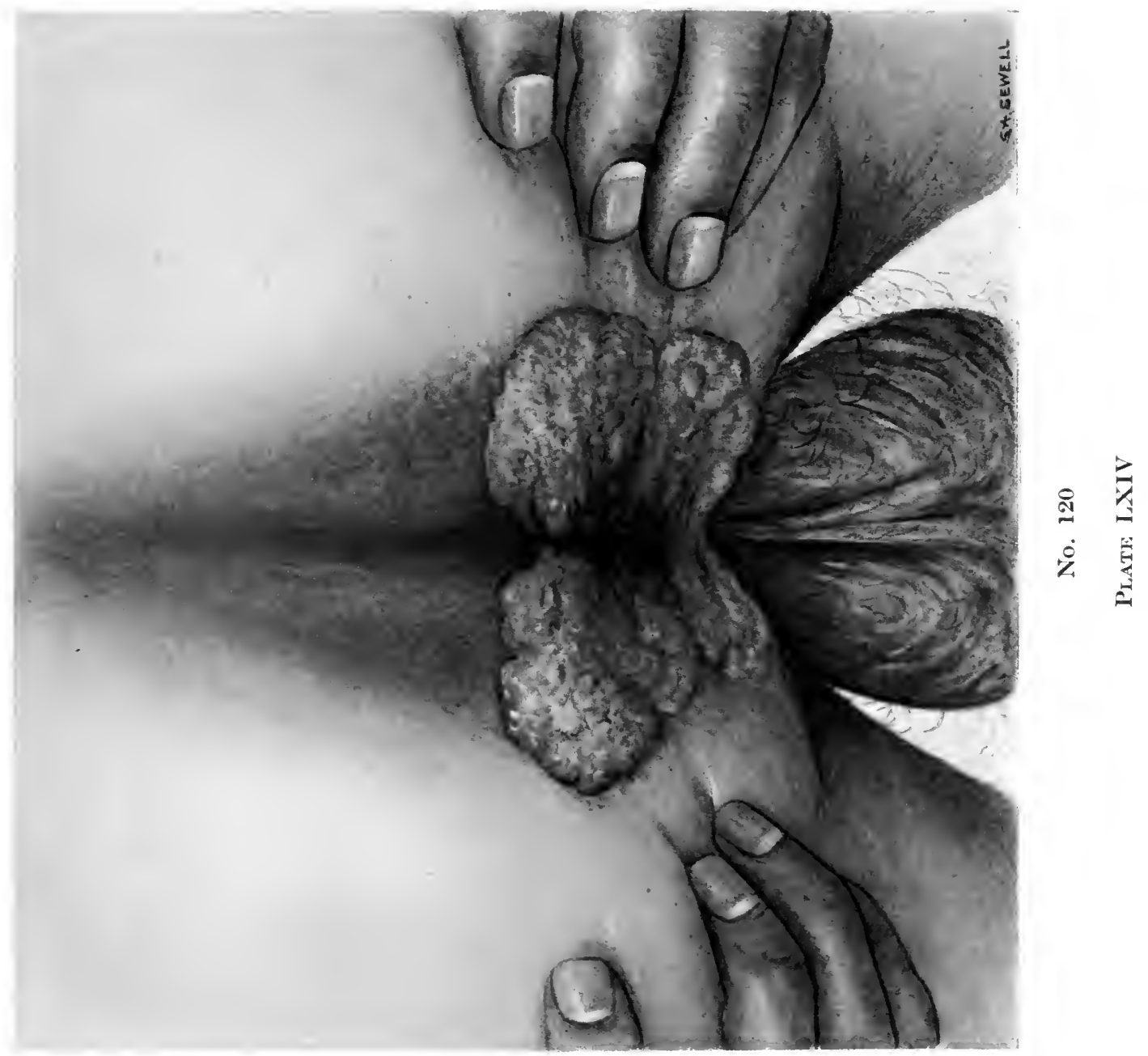



the starting-point being the irritation and slight inflammation caused by the coitus.

In the severe cases the question of operation often arises. Let me warn the reader that, with the exception of slitting up a tight foreskin, operative interference may often do more harm than good. The main object must be to keep the gangrenous parts as dry as possible; and therefore, after bathing them with hydrogen peroxide, some powder should be applied, and the part exposed to the air or covered up as lightly as possible. The best powder in my experience is one containing bismuth subgallate and bismuth tribromphenol, the prescription for which is given on page 342 .

In closing my remarks on balanitis, I should like to draw attention to one or two other points, which, although not venereal, may easily be mistaken for such.

Unless the diagnosis is absolutely obvious, it is wise in cases of balanitis to test the urine, as balanitis is not an uncommon complication of certain general disorders-for instance, diabetes. Diffuse lymphogenic syphilitic infiltration of the glans penis may be mistaken for a simple balanitis, but it may readily be distinguished by the violaceous tint of the organ. A syphilitic balanitis may be confounded with Lichen planus or psoriasis, two diseases which not only not uncommonly affect the glans penis, but also arc occasionally limited to this organ. Erythema multiforme may cause a balanitis very difficult to distinguish from a case of Balanitis erosiva, and, in gonorrhœea, a circinate form of balanitis is not at all uncommon. The gonococcal balanitis may or may not be a specific balanitis. If specific, it is due to the gonotoxine; if not, it is merely produced by the irritative discharge.

\section{Condyloma Acuminatum.}

History.-Condylomata acuminata must have been very well known to the Greeks and Romans, as evidenced by the diverse names given to the condition, such as $\kappa o \nu \delta u ́ \lambda \omega \mu a, \theta \dot{u} \mu \iota \nu, \sigma v \kappa \dot{\eta}, \dot{a} \kappa \rho \circ \chi \circ \rho \delta \dot{\omega} \nu$, verruca, ficus, marisca, etc. Hippocrates advised powdering with alum and excision, and some of the earliest observers noticed that the warts might spontaneously vanish. When syphilis appeared and produced lesions (condylomata lata) which simulated the "verrucæ," as warts were then called, great confusion arose, and it appears that Fallopius was the first to describe the difference between Verrucoe gallicee and non-gallicae. For many years the warts were considered by some to be due to syphilis and others to gonorrhœa, and even to-day they are not infrequently called gonorrhoal warts. Hunter, Nisbet, Bell, and Ricord were firm that mercury had no influence upon the warts, therefore they had no connection with syphilis.

The terms venereal warts and gonococcal warts are often applied to this condition. The lesions do not differ from ordinary warts, except that they spread and grow more rapidly, and this is simply due to the moisture and warmth of the regions in which they occur. A wart is nothing more nor less 


\section{CLINICAL ASPECT AND TREATMENT OF VENEREAL DISEASES}

than a hypertrophy of epithelium surmounting an inflamed area of corium, the blood-supply of which is considerably increased. Condylomata acuminata may be caused by any infective agent except, perhaps, the gonococcus. The reason why venereal warts are more common in patients who are or have been suffering from gonorrhoa is simply due to the fact that, owing to the discharge caused thereby, saprophytic organisms can flourish, and it is these saprophytic bacteria which are responsible. Moisture is absolutely necessary for the growth of Condylomata acuminata, and it is owing to this fact that they may sometimes be met with in the umbilicus and the axillæ, and the avoidance of moisture is the main factor to be borne in mind when treatment is considered. If the area is kept absolutely dry, the warts may disappear spontaneously. If there are only one or two, they are best removed with a Volkmann's spoon, after being frozen with ethyl chloride, and the base should then be cauterised with silver nitrate stick to stop the bleeding. Condylomata in the urethra must always be removed, as it is impossible to keep the urethra dry. Large masses are best bathed twice a day with either a $4 \cdot 0$ per cent. solution of formalin, or a $2 \cdot 0$ per cent. solution of lactic acid, then dried, and the combined bismuth powder dusted on.

\section{Molluscum Contagiosum.}

History.-Thomas Bateman, in 1817, was the first to describe Molluscum contagiosum, and gave it the name we know it by to-day. Bateman only came across two cases, and even ten years later the best French dermatologists stated that no case had been observed in France, therefore it is probable that the disease is a recent one.

Under this name we understand papular lesions which vary from the size of a pin's head to that of a pea. The lesions are usually discrete; non-inflammatory, unless secondarily infected; they are whitish and waxen in appearance; they are depressed in the centre, in which there is a little plug capable of being pressed out. The matter, which can be pressed out, can be examined without staining under the microscope, and the typical even sized and shaped degenerated epithelial cells characteristic of the condition may be recognised.

In men, they are most commonly found on the skin of the penis and scrotum; in women, on the labia; and in children, on the face.

Occasionally several lesions may coalesce and cover quite a wide area with a whitish waxen mass, which is raised above the surface of the skin, is flat on the surface, and has little or no inflammation surrounding it. Such a condition is most commonly seen somewhere on the trunk. Histologically, Molluscum contagiosum gives one of the prettiest and most characteristic of pictures, and it is only necessary to be seen once never to be forgotten, and to see it without knowing it is one of the greatest puzzles.

The epithelial cells are the cells affected; they increase in size, the nucleus swells and disappears, the nucleoli first increase in number and then vanish, so that the cell, which retains its outline almost to the end, is filled with 
$$
\text { . }
$$ 
PLATE LXV

No. 121.-Molluscum Contagiosum

Note central depression from which a soft core can be pressed out.

No. 122.-Molluscum Contagiosum

The lesion has become secondarily infected, which is not uncommon with some of the older ones. As this lesion was single it was mistaken by most for a chancre. 
No. 121
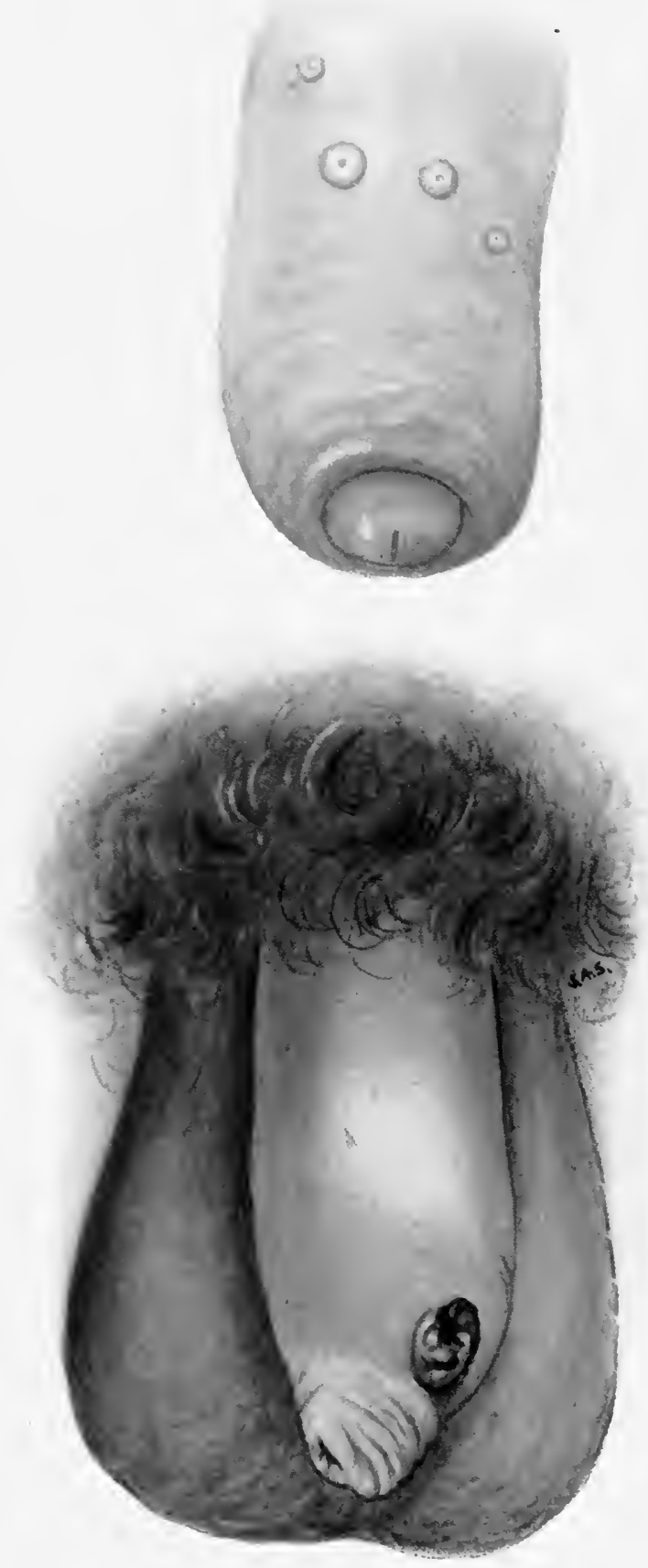

No. 122

Plate LXV 

homogeneous material or débris. The epithelial cells exhibit all the changes which are to be met with in the hydrolysis of the protein, down to and including the amino-acid stage, according, naturally, to the extent to which they have degenerated. The different analytic products of protein have different actions; some are basic, others are acidic; some have a reducing action, others have none; hence the reason why, with almost any dye that is used, the picture of the epithelial cells reminds one of Joseph's coat of many colours.

It is highly probable that the cause of Molluscum contagiosum is an ultramicroscopic organism, but little is known about it at present. I have seen similar bodies to those described by Borrel $^{6}$ and Lipschütz, ${ }^{7,8}$ but it is practically impossible to distinguish them from the débris in the cells, and no reliance can be placed upon the way in which they stain, owing to the fact that some of the amino-acids present stain in the same way.

The best treatment is to remove the lesions with a sharp spoon, and to cauterise the base with silver nitrate.

If there are several, and if many have coalesced, it is best to cover them with a strong salicylic acid plaster. Unna's salicylic acid and creosote, or salicylic acid and soap plaster-mulls, are very efficacious.

\section{Herpes Genitalis.}

History.-From the beginning of the seventeenth century till well into the nineteenth century Herpes genitalis was known by the name of "Crystallina." Although many considered the condition as harmless, others confused it with the primary sores of syphilis. Hanmann, in 1840 , considered it to be an after-effect of gonorrhœa, and noticed that it was particularly frequent among prostitutes. William Lawrence, in 1843, showed that genital herpes had nothing to do with venereal diseases, and Ricord proved it was not an infectious disease by inoculating four people, with negative results.

Although most common in patients who have suffered from a venereal disease, especially from gonorrhoea, it may occur in those who have not. The clinical course of the condition is nearly always unvarying. The patient first complains of itching, examines the region, and finds a group of tiny discrete vesicles on an inflamed area. When the vesicles are well pronounced, as a rule, the subjective symptoms vanish, and the condition may spontaneously disappear, to recur and recur, at varying intervals, later. On the other hand, the vesicles may become pustules, and these in turn become ulcers. Several of the lesions may coalesce, and the loss of surface caused by them may have an irregular outline, and may closely simulate a soft sorc. Many patients periodically have local pain and itching, and all that is to be seen is a red urticarial-like lesion, which vanishes before any vesicles appear. Some women have an attack of herpes every time that they are unwell.

In men, the most common positions to find herpes are on the skin of the penis, on the under surface of the prepuce, and on the glans. In women, 
herpes is most frequently seen on both the immer and outer surfaces of the labia majora and minora.

Herpes genitalis is frequently accompanied by aphthous ulcers, which are very apt to appear on a mucous membrane where there is any irritation; indeed, the two conditions are probably identical. Irritation is the prime cause of Herpes genitalis, and the toxine which gives rise to the irritation no doubt affects the sensory nerves peripherally in some way or other. Once the latter are affected, the original cause of the irritation need not necessarily be repeated, as the patient's being below par is sufficient to cause a recurrence, and even a dream often starts it; so, too, will an injection of a gonococcal vaccine, or of arseno-benzene.

The recurrences will often occur in the same area, and the site of a chancre is not at all an uncommon spot.

Herpes genitalis is frequently a very troublesome condition, as it may cause sexual neurasthenia (vide Chapter XXX.).

Every case of herpes in which it is the patient's initial attack must be watched, as it is sometimes the premonitory sign of a chancre. Be herpes destined to be followed by a chancre or not, under no circumstance whatever must any irritative application be used. Any form of irritation only aggravates the condition, and it may set up an induration which is often mistaken by the unwary for a syphilitic lesion. Herpes is certainly more common in Gentiles than in Jews, so we have again another indication for circumcision. The only treatment necessary is an antiseptic evaporating lotion, after the application of which a non-irritating dusting powder should be used. Resinol ointment is also a very useful remedy. Herpes is not uncommonly associated with a transient non-gonococcal urethritis, a condition which is frequently called "husband's clap." It is most likely to result when connection is had just before a menstrual period, or when the wife first becomes pregnant.

\section{Granuloma Inguinale.}

This is a tropical disease, and it is found most frequently in British Guiana, Brazil, West Africa, South China, and Australia. It is occasionally seen in Ceylon, the Malay Peninsula, and Central Africa. It is highly probable that the condition is to be met with in every tropical country. The Italians came across it in Tripoli. ${ }^{9}$ As the disease more commonly affects women than men, it is often called Granuloma pudendi.

In the female, the labia majora and vagina are the parts first affected, then the granulomatous masses spread to the mons veneris, to the genitocrural folds, and backwards to the anus, which they not infrequently surround.

In the male, the groins, prepuce, glans penis, and the anus are usually involved. The penis is affected first of all. In both sexes the inguinal lymphatic glands are enlarged. The granulomatous masses are often accompanied by ulcers, and it is on this account that the condition is sometimes 



\section{PLATE LXVI}

No. 123.-Herpes Genitalis, SHowing TIF VESICUlaR AND GroupED LESIONS

No. 124.-A Single Herpetic Lesion which ias passen the Vesicu-

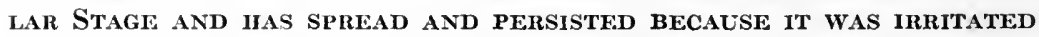
by Local Applications in the Belief tuat it was a Chancre 
No. 123
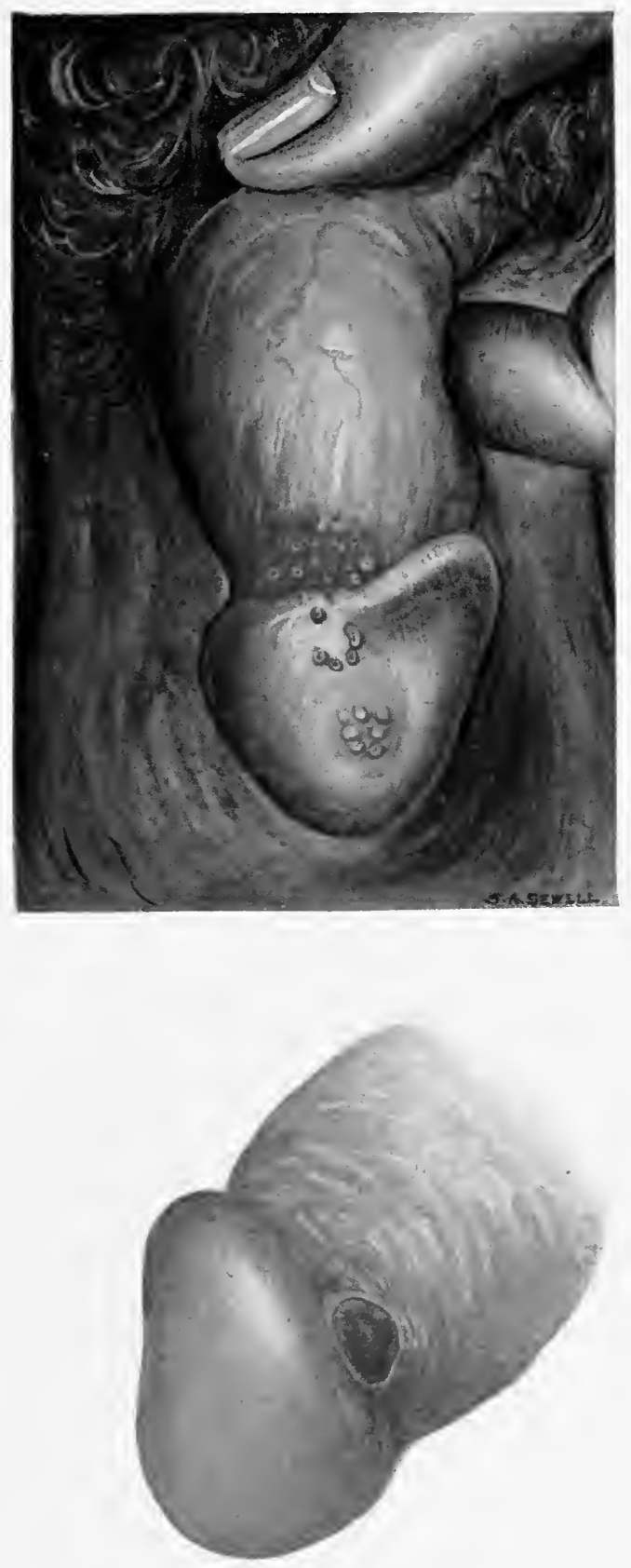

No. 124

Plate IdXVI 
confused with the Ulcus molle serpiginosum. It is quite possible that the two diseases are caused by the same organism. The growth and rate of spread are extremely slow; often a matter of several years.

The disease has a natural tendency to cure itself, but the scar tissue formed is so dense that the cicatrisation may be very disfiguring.

The widest diversity of opinion prevailed as to the nature of the condition, $11,12,13,14,15,16$ but it is finally settled now to be due to an intracellular capsulated bacillus, which is possibly allied to the intracellular form of Ducrey's bacillus. The disease is certainly infectious and auto-inoculable, and no ordinary remedial treatment appears to be of much avail, although the cases described in Tripoli $^{\theta}$ healed up with arseno-benzene are the only-instances of their kind. The best method of curing the disease is by surgical removal. Dr. Wise has written to me several times from British Guiana, and he has informed me that since he has made it a rule to operate upon every case the disease is becoming rare in the country. MacLeod ${ }^{10}$ reports a case which he cured with $\mathrm{X}$-rays. Araujo, ${ }^{22}$ in his recent book on Granuloma venereum, states that antimony is the drug which has given the best results in his hands. The lesions are dressed daily with compresses soaked in a 1.0 per cent. solution of tartar emetic dissolved in normal saline, and this local treatment is combined with intravenous injections, $5.0 \mathrm{c.cm}$., of the 1.0 per cent. solution in normal saline given daily, or on alternate days. The quantity of the injection can be increased to $6 \cdot 0,7 \cdot 0$, or $10 \cdot 0 \mathrm{c.cm}$. Low and Newham ${ }^{23}$ also report good results from the use of antimony.

\section{Induratio Penis Plastica.}

Induratio penis plastica, or, as it is sometimes called, van Buren's disease, although not a venereal disease, usually comes under the observation of venereal and genito-urinary specialists. Therefore, I feel that I ought to devote some space to it.

Induratio penis plastica is a disease of unknown origin, and it appears to be independent of any local trouble. It begins as a very gradual and painless thickening of the tunica albuginea of the corpora cavernosa. It may occasionally begin in the septum, between the two corpora cavernosa, but, in either case, it is almost invariably on the dorsum of the penis that the process takes place.

The condition is called van Buren's ${ }^{17}$ disease, after an observer of that name. He called attention to the disease in America, in 1874, but it had been well known in Great Britain and on the Continent for several years previously. ${ }^{18,19,20,21}$

Morgagni was familiar with the condition, describing it as contractures of the penis. Peyrilhe described it fully in 1786 as Duretés des corps caverneux and John Hunter was under the impression that the trouble was due to adhesions produced by chronic chordee in gonorrhœa. For many years the condition was known as Chorda venerea chronica. Ricord thought some 
of the cases were caused by syphilis, and others by gonorrhœa, and it was not until the present day that the lesion was considered not to be venereal in origin.

Induratio penis plastica is not a cavernitis, and, therefore, it must not be confused with those hard nodules resulting from a cavernitis of gonococcal origin. Syphilis, trauma, and, very rarely, leucæmia, may give rise to a dense cavernitis, but the condition under discussion has nothing to do with the diseases mentioned, and it may be stated here and now that Induratio penis plastica is never of venereal origin, nor is it secondary to any local lesion.

Generally speaking, the ætiology is quite unknown, but in a few cases the condition is obviously a symptom of a known and general disease or diathesis.

A large number of the cases definitely suffer from gout and rheumatism, others have sugar in the urine, and in nearly all the patient is over forty years of age. One of the most extraordinary points in the disease is the fact that the patient may also be suffering from a Dupuytren's contraction, and, if he is not suffering from it himself, a male relative of his often is a sufferer. The association with Dupuytren's contraction is so frequent that it certainly looks as if Induratio penis plastica was due to what we loosely call a uric acid diathesis or arthritism. The close resemblance between this condition and thromboangiitis suggests the cause is due to some error in the carbohydrate metabolism. Hyperglycæmia appears to lead to thrombosis, and so to fibrous tissue formation and contraction.

Whatever be the cause, the question naturally arises as to why the penis, in the first place, is involved, and, second, why only a special portion of the connective tissue is affected. One is tempted to invoke the aid of atavism in order to find a solution, and it will be remembered that the so-called septum pectiniforme-i.e., the septum between the two corpora cavernosa-is ossified in many mammals; in otters for instance, the bone goes by the name of "baculum."

The lesion is situated on the dorsum of the penis by the symphysis. In its early stage it feels like a nodule; then this spreads superficially-i.e., lengthwise and anteriorly-until it becomes ribbon-shaped. The ribbon is thickest in the centre, because of the implication of the septum pectiniforme, and its edges usually thin. It does not become attached to the skin above.

The ribbon, though usually flat on the surface, may occasionally be uneven, owing to there being denser masses of fibrous tissue in some parts than in others. The densest part of the ribbon is the end by the symphysis, where the growth starts. As a rule, the patient is unaware of his condition until he finds that the penis has a peculiar shape when erected. The kinking caused may prevent sexual connection, and, very often, from the start, coitus is painful, especially during the act of ejaculation, owing to the swelling causing a mechanical narrowing of the urethra. The act of micturition is never interfered with.

There is nothing characteristic in the pathological anatomy of the induration. The induration is composed of fibrous tissue, and the vessels in it do 
not show any morbid changes, hence the view that Induratio penis plastica is only a sign of a general arterio-sclerosis cannot be held. Occasionally, typical bone cells may be found in the tissue. Treatment is, unfortunately, almost hopeless. Some observers state that they have had success with the various methods which have, from time to time, been advocated; but most of these I have tried religiously on five patients, and all without success.

Surgical removal is almost invariably followed by a recurrence soon after the wound has healed, and there is often a risk of making the last state worse than the first.

Other local measures, such as massage, electric treatment, and ionisation, are useless.

Some improvement appears to have followed vigorous local inunction. Shillitoe tells me of a case which he benefited with unguentum iodex.

Iodides internally appear to be given by all observers, and there is one drug which is specially in favour-namely, tiodine, of which 3 to 6 pills are to be taken daily. Tiodine is an ethyl-iodide compound of thiosinamine. Intramuscular injections of thiosinamine and fibrolysin, if the manufacturer's reports are correct, should certainly be prescribed. Personally, I have had no success with these drugs, and on two occasions I have observed severe toxic symptoms to supervene. Fibrolysin is merely a sodium salicylate compound of thiosinamine. I have seen benefit in an early case from the use of colloidal iodine internally and intramine intramuscularly. I have just had two early cases in which the lesion entirely disappeared under intramine.

Recently I have observed three cases in which the lesion was situated in the fore part of the penis, and in all the shape was that of a signet-ring. One of the cases is of sufficient interest to report in some detail.

CASE 136.-A man, aged 47, contracted syphilis in October, 1916, for which he received three injections of arseno-benzene and mercury for six months. Five months after the last injection of arseno-benzene he had an attack of what was diagnosed as influenza, but which was really acute syphilitic cerebro-spinal meningitis, as it left him with optic neuritis and facial and auditory paresis on the left side. When sent to me early in 1919 to see if anything could be done for the nerve trouble, I noticed that the patient had an anterior signet-ring Induratio penis plastica, and a commencing Dupuytren's contraction. There was no sugar in the urine, but the blood was hyperglycæmic. The patient's wife died of diabetes, and so did the eldest son, when aged 8. There are two daughters alive, and apparently healthy. The patient had paid no attention to the penile or palmar troubles, and therefore could not say when they commenced; but, although occurring in a syphilitic subject, I am certain they were not caused by that disease.

\section{Pediculosis Pubis.}

The insect, whose chief haunt is the pubic hair, is often called the crab louse, or Phthirius inguinalis, the first word of which is merely the Greek word for a louse. 


\section{CLINICAL ASPECT AND TREATMENT OF VENEREAL DISEASES}

The Pediculus pubis is much broader and flatter in proportion than the other pediculi-indeed, its body is more or less square-shaped. The male is about $0.6 \mathrm{~mm}$. to $1.0 \mathrm{~mm}$. in length, and the female $1.1 \mathrm{~mm}$. to $1.4 \mathrm{~mm}$. The terminal segment of the abdomen is rounded in the male and notched in the female. The ova are about ten to fifteen in number; they are fixed to the hair by a chitinous substance; they hatch out in a week, and the young are sexually mature in about a fortnight.

The pediculi first affect the pubic hair, from which they spread up the lateral walls of the abdomen and thorax to the axillæ; they may affect the whiskers and beard, and in children they may be found only in the eyelashes and eyebrows. The scalp is very rarely affected, but the lanugo hairs on the body may be, in which case the most common sites are the sternal region, the sacral region, and the thighs and legs.

As the pediculus is small, lies flat on the skin, and looks like a little brown spot when casually noticed, it is usually mistaken for a small mole, hence its wide distribution is not generally recognised.

As a rule, the pediculus causes itching, but the pruritus is by no means constant. If the itching is intense, a pyogenic dermatitis usually results from the continued scratching. Although it is only in the minority of cases that the blue spots are seen, the so-called Macula corulea are absolutely pathognomonic of the condition.

The Maculae corulece are blue, or rather steel-grey, spots, which appear to be slightly depressed; they vary from the size of a pin's head to that of a sixpenny-piece, and they may be circular or irregular in outline. The lesions are, as a rule, grouped, and are generally to be found on the antero-lateral walls of the abdomen and the sides of the thorax, or, in other words, along the course of the journey made by the pediculi from the pubis to the axillæ.

The Maculce corrulece disappear in a week or two after the destruction of the pediculi. They are merely stains in the skin, and the connection between them and Phthirius inguinalis was first noticed by Mourson. ${ }^{2}$ Duguet, $^{1}$ by rubbing into the skin an extract of the pediculi, was able to produce the lesions.

Oppenheim $^{3}$ demonstrated a pigment in the bodies of the pediculi, and he considers that the blue spots are produced by a bite from the insect. The bite results in an excretion of this colouring-matter; this forms a compound with the hæmoglobin, which results in the appearance of the blue spots.

The lice themselves are easy to kill, but the ova are more difficult to exterminate. The pubis should not be shaved, as the discomfort following the growth of the new hair is out of all proportion to the benefit to be derived from such a measure.

A good method of treatment is to spray the affected parts with 96.0 per cent. alcohol, and then to rub in the unguentum hydrarg. ammon. or the plain 



\section{PLATE LXVII}

No. 125.-Maculae Coeruleae on the Flank, Causfed by the Bite of the Pediculus Pubis on its way From the Gentals to the AxIli.ae 
No. 125

Plate LAViI 



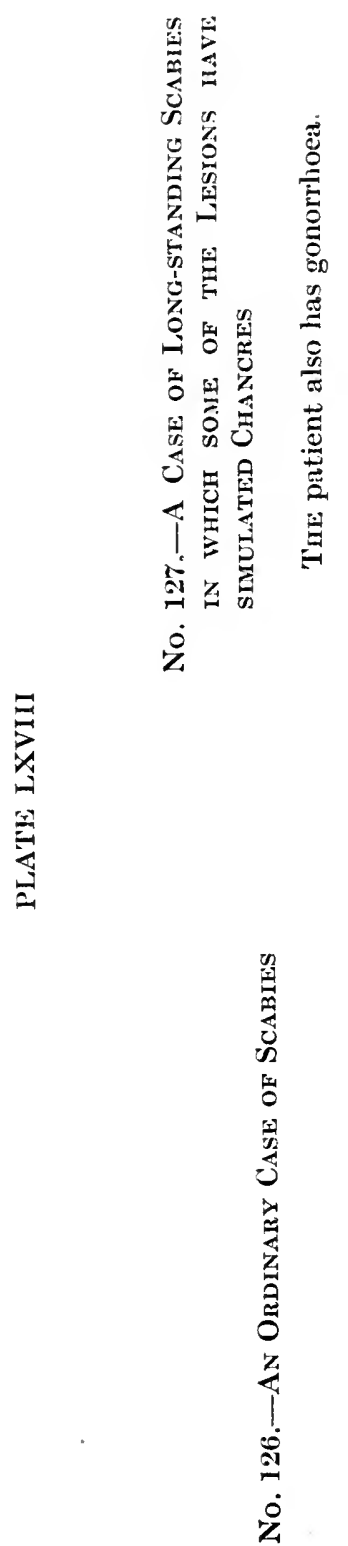

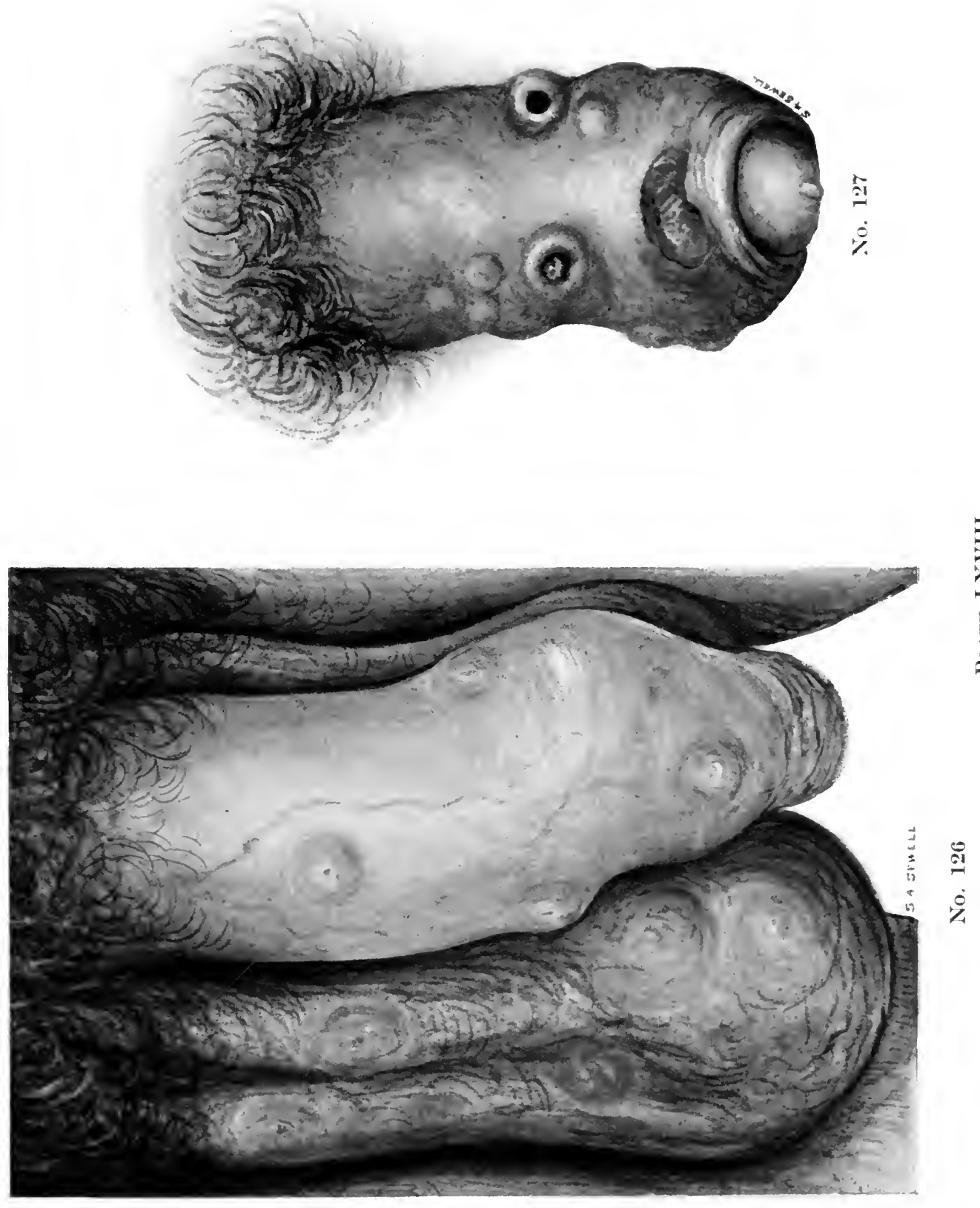
unguentum hydrarg. of the British Pharmacopœia. An excellent ointment to use is that suggested and tried by Bory: ${ }^{24}$

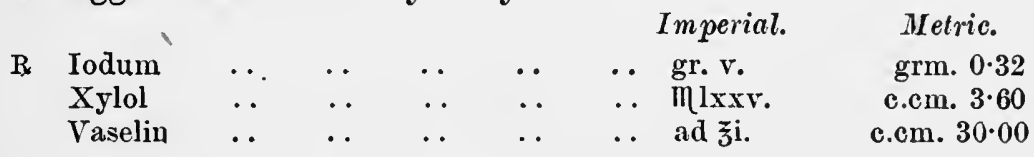

A peculiarity of this form of phthiriasis is that some individuals are much more prone to be affected than others, and no prophylactic measures appear to save them. Hospital students sometimes get an attack whenever they return to hospital, after being away for some time. I know of one case of a medical student who was obliged to give up his work, because, whenever he was at hospital, he used to get these lice all over his body-even constant shaving of the pubis and axillæ did not save him.

1. Duguex (1880): Gaz. des Hôpit., liii., 362.

2. Mourson (1878): Annales de Derm. et Syph., ix., 198.

3. Oppenheim (1901): Archiv f. Derm. u. Syph., lvii., 235.

4. Bataille et Berdal (1889): Compt. rend de la Soc. de Biol., xli., 689.

5. Berdal (1897): Traité des Malades Vén. A. Maloine, Paris.

6. Borrel (1904): Compt. rend. de la Soc. de Biol., lvii., 642.

7. LIPSchü Tz (1907): Wien. klin. Woch., xx., 253.

8. LiPSCrütz (1908): Zentralblatt f. Bakteriol., xlvi. (orig.), 609.

9. Sabella (1913): Giorn. Ital. d. Malattie ven. ed. Pelle, liv., 306.

10. MACLEOD (1913): Brit. Journ. of Dermat., xxv., 66.

11. Wise (1914): Brit. Guiana Med. Annual. Garden City Press, Letchworth.

12. Siebert (1908): Archiv f. Schiffs- u. Tropenhyg., xii., 291.

13. Fud (1911): Archiv f. Schiffs- u. Tropenhyg. (Beiheft 9), xv., 481.

14. Dovovan (1905): Ind. Med. Gaz., xl., 414.

15. Carter (1910): Lancet, i., 1128.

16. Wise (1906): Brit. Med. Journ., i., 1274.

17. Van Buren and Keyes (1874): New York Med. Journ., xix., 390.

18. Cullerier (1866): Maladies Vénériennes, G. Masson, Paris.

19. Föster (1863): Handb. d. spez. path. Anatomie, 2 Aufl., $372 . \quad$ L. Voss, Leipzig.

20. KIrby (1849): Dublin Med. Press, xxii., 210.

21. MacClellan (1828): Journ. Univ. des Scienc. Méd., xlix., 340.

22. De Souza Araujo (1917): Trabalho do Instituto Oswaldo Gruz. Co Lith, F. Pinto, Rio de Janeiro.

23. Low and Newham (1916): Brit. Med. Journ., ii., 387.

24. Bory (1919): La Presse Médicale, xxviii., 87. 


\section{CHAPTER XXX \\ SEXUAL NEURASTHENIA}

THERE are three kinds of sexual neurasthenia: (1) the form that occurs in patients who have never had a sexual disease; (2) the form that occurs in patients who have had gonorrhœa; (3) the form that occurs in patients who have had syphilis.

The form of sexual neurasthenia which is sometimes met with in patients who are continually suffering from recurrences of Herpes genitalis is usually the same as that form which follows gonorrhœa. Herpes genitalis cannot strictly be called a sexual disease, in the light in which we regard gonorrhoea and syphilis; but as most of the cases have had gonorrhœa, it is always wise thoroughly to examine every neurasthenic who complains of frequent attacks of Herpes genitalis, so as to see if he has a chronic prostatitis or spermatocystitis. Patients who suffer from sexual neurasthenia, but who have never had a sexual disease, are, as a rule, patients particularly deficient in intellect, effeminate, or those who have especially given way to self-abuse in their early youth. Such patients either complain of constant nocturnal emissions, or that they cannot perform the sexual act.

Those who complain of the former are generally men between 20 and 30 years of age, men who have to work hard in a stuffy office, who can get but little exercise, and who have their meals irregularly, and not good ones at that. They come home brain-fagged, but not bodily fagged, and, having had little or no exercise, they fecl the cold very much, with the result that they load their beds with heaps of clothes. A too warm bed is a very frequent cause of nocturnal emissions. These patients will not infrequently tell you that they suffer from nocturnal emissions much more frequently when they lie on their backs than on their sides, and when the head is not well raised above the rest of the body.

These patients are almost invariably very constipated, and not infrequently they have a varicocele. A great deal can be done by hygienic treatment for this class of case. If the patient has a varicocele, he should wear a suspensory bandage, the bowels should be put in order, meals should be taken at regular intervals, and a carbohydrate diet should be avoided. A little red wine is beneficial, but malt liquors should not be taken. Tonics containing strychnine, iron, arsenic, and phosphoric acid should be prescribed. The patient should be advised to take a certain amount of exercise every day; 
he should not go to bed for three or four hours after his dinner, nor have a nightcap of whisky and soda, nor have too many clothes on his bed; he should have a pillow and a bolster, or two pillows, and his windows should be wide open, winter and summer alike. A great deal can be done by an occasional talk with the patient, and it is a good plan to urge him to take up a hobby; and, in all cases, the literature he reads should be considered by the physician in charge. Even medical men are inclined to ridicule the condition. Those who do, little know what a serious condition it is; it. is a disease, and should be regarded as such. There can be little doubt that there is a lesion somewhere which we are not advanced enough to detect; but the fact that it is hidden does not warrant us in assuming that there is nothing wrong. I mention this particularly, because the sweating and mode of living of a large proportion of the population, in big cities, is very conducive to this form of sexual neurasthenia. The disease is undoubtedly on the increase, and those who are accustomed to see cases know well what havoc it can play with a man's life.

Those patients who complain that they cannot perform the sexual act, if they have had no venereal disease, are generally men who may be said to have passed the sexual period, or men who have an enlarged prostate, or men who have lived in tropical climates. Other causes are alcohol, and more or less sudden adiposity. In spite of the literature, and of the manifold "tips" which are widely circulated in all Continental cities, there is no cure whatever for this condition. Aphrodisiacs, such as damiana, muiracethin, yohimbin, etc., and the various forms of electrical treatment and appliances which are advocated, are useless. It is the duty of the physician to explain to the patient that the condition is a physiological one, and that he must make the best of what, perhaps, may be to the patient a bad job. I had one very interesting case in which a man, aged 39, consulted me on the question of marriage. For the last two years he had been unable to have sexual connection, as he could not get an erection. Three years ago the patient had a bad attack of blackwater fever on the Gold Coast, and ever since his convalescence he had been gradually putting on weight, so that he weighed, when I first saw him, 19 stone, his previous weight having been only 12 stone. In spite of reducing his weight somewhat with careful exercise, massage, and the administration of organic extracts, the patient has never been able to get an erection. Since then I have seen other cases of adiposity which were accompanied by a loss of sexual function.

If the patient has had syphilis, and is on the right side of 45 , a loss of sexual function generally signifies that he has a degenerative myelitis. As in some cases of degenerative myelitis there is no history of syphilis, it is always wise to bear this trouble in mind if a patient seeks advice for loss of sexual power. Another very common cause of sexual neurasthenia is coitus interruptus, a continued practice of which may even lead to dementia.

The gonorrhœal form of sexual neurasthenia is different from the syphilitic 
form. In the former, the patient usually has a gonococcal lesion, and the neurasthenia is doubtless a toxic manifestation of the disease. In the latter, the patient generally is either cured of his syphilis or he has never had syphilis; but imagines that he has had it. Putting aside for a moment the neurastheria which patients are liable to get when they first get syphilis, and know that it is syphilis that they have got, true syphilitic neurasthenia is undoubtedly rare.

Syphilitic neurasthenia is not due to the toxine of the syphilitic organism, because toxines do not play a marked rôle in protozoal diseases, because the type of neurasthenia is seen in patients who have never had syphilis, because $I$ have seen very bad cases in which the patient never had more than the primary sore, as the treatment was begun early, and because extraneous catastrophes often bring it on or aggravate it. Syphilitic neurasthenia is very apt to affect patients who have had an attack of neurasthenia beforepatients, one can say, who have a mental family history.

The world war has been a potent cause of syphilitic neurasthenia, as well as every other form of neurasthenia-indeed, it has caused a neurasthenia of its own.

None of these factors affect gonorrhœal neurasthenia; therefore, in my opinion, gonorrhœal neurasthenia is a true toxic manifestation of the disease, since it often disappears when the patient is cured, while syphilitic neurasthenia, as a general rule, does not differ from an ordinary neurasthenia, except in so much as it is the idea of the syphilis which has started the ball rolling in a patient who is normally unstable or mentally weak. Syphilitic neurasthenia does not differ from war neurasthenia, hence neither the cure of the syphilis nor the cessation of the war would cure the patient. The few rare cases of syphilitic neurasthenia which are benefited by treatment have been considered elsewhere (Chapter XIII.).

We will, first of all, discuss the gonorrhœal neurasthenia. The symptoms complained of vary, but they may all be described as disturbances of the sexual function. The sexual desire may be abnormally augmented, or the patient may be impotent. Patients frequently complain of what they commonly call sexual weakness-that is, inability to get full erection-ejaculatio proccox, loss of sensation, or even extreme pain, just prior to and during the ejaculation. Patients frequently note that the amount of seminal fluid passed is very small. Some patients complain bitterly of prostatorrhœa, which they always mistake for spermatorrhœa; and the constant passage of what they think to be their semen, which they usually seem to look upon as part of their spinal cord, has a most baneful influence on their mental condition. The symptoms just mentioned, which are typical of gonorrhœal neurasthenia, are also the symptoms of a chronic inflammation of the colliculus, the prostate, and the vesiculce seminales. The great pain sometimes complained of prior to and during the seminal ejaculation is due to a narrowing or closure of the ejaculatory ducts, or of some of the ducts of the prostate; hence in these cases the amount of seminal fluid passed is often far below the normal. 
Once a patient begins to worry about these symptoms, he quickly loses weight, is usually constipated, and complains of headaches, vague pains over the body, indigestion, and inability to work or sleep.

Some patients who have been under treatment for some time, and who look upon gonorrhœe as an incurable disease, or with as much awe as syphilis is commonly regarded, are naturally apt, if their mental balance is not properly adjusted, to develop a form of neurasthenia indistinguishable from that met with in syphilis. These are patients who would have developed neurasthenia on any very strong provocation, therefore the only way to treat them is by suggestion.

Because a patient has gonorrhœal neurasthenia, it must not be hastily assumed that he has an active gonococcal lesion, since the prostatitis, or whatever organ it is that is affected, may be kept up by a secondary infection, and this may keep the neurasthenia going. That gonorrhœal neurasthenia may be, and is, often met with long after all gonococci have vanished might throw doubt upon the gonotoxic origin of it.

Not at all. Herpes genitalis is a very frequent gonotoxic symptom, but, nevertheless, the condition may go on recurring and recurring long after the gonorrhœa has been cured and forgotten. Take, again, a syphilitic neuritis. Once the sensory fibres have been affected, in spite of treatment and the disappearance of the syphilitic process, pains and other symptoms may still persist.

In cases of gonorrhœal neurasthenia it is necessary to find out, first of all, if the lesion of the prostate or seminal vesicles is still due to the gonococcus, or to a secondary infection.

If due to the gonococcus, the patient should be treated according to the trouble found. If, on the other hand, no gonococci are found, and it is tolerably certain that a secondary infection persists, the patient should be treated on hygienic lines, and by suggestion. Cold applications to the prostate by means of the psychrophore often do a great deal of good, and, in very obstinate cases, it might be advisable to treat the patient with a mixed autogenous vaccine made from his prostatic secretion: In these cases, instrumentation of the urethra and the injection of antiseptics should be avoided as far as possible.

It is a very odd fact that many patients with gonorrhœal neurasthenia develop-when the neurasthenic symptoms appear-phosphaturia, oxaluria, or uraturia. The thickness of the urine caused by these salts is often a very great source of worry to the patient, and, of course, an excess of crystals in the urine is apt to aggravate any chronic inflammation of the prostate. As so many of the other symptoms of gonorrhœal neurasthenia-such as the neuralgic pains, errors of digestion, chronic constipation, cardiac neurosesare suggestive of an affection of the sympathetic nerves, it is possible that the polyuria, etc., is also a sympathetic nerve disturbance. Although the hypochondria which sometimes accompanies gonorrhœeal neurasthenia is not, as 
a rule, so severe as that which follows syphilitic neurasthenia, it should not be forgotten that several cases have been known to commit suicide. Owing to the injudicious handling of gonorrhœal patients in venereal hospitals, and the damage done by over-instrumentation, gonorrhœal neurasthenia is on such an increase as to be alarming; therefore, in all cases in which it is tolerably certain the patient is not infectious, he should be treated generally, with the greatest care, and by suggestion, if there are any indications of his becoming a profound neurasthenic.

\section{Syphilitic Neurasthenia.}

The type of person usually affected with this form is mentally weak, conscientious in his work and habits, and one who has always had an awful dread of catching syphilis. Consequently, such patients come for advice very early, and therefore they can generally be cured before they develop any further manifestations of the disease. In spite of being cured, and in spite of what you tell them, this class of patient cannot be convinced. They may even realise that they are foolish, and they may be impressed for the moment by the rosy picture painted for them; but, on leaving the source of comfort, they recede to their melancholic state. The worst cases commit suicide. I have been unfortunate enough to have had two such cases. Both these cases, and milder ones which I have had, I have sent on to physicians who practise psychotherapy, but I have found that if the case is not sent early enough either no good can be done or the relief is only temporary. Patients with a bad family history, in my experience, never recover. Not all these cases have the same ideas. Some imagine that they cannot be cured, and that they will ultimately become mad through the syphilis. Others, on the other hand, imagine that they are a continual source of infection to others. One of my cases-who terminated his own existence-first imagined that he had infected all his relations. To please him, I saw his relations in turn, and tried to convince him that his idea was foolish. He next said that anyone who passed him in the street became infected, and he would often cross over on to the other side to avoid them. He was so perturbed at thinking he had infected so many people that he never went about without a pistol in his pocket, as he expected any moment that one of his victims would shoot him in the back. When I suggested to him that he did not appear to mind whether his frequent visits to me infected me or not, he imagined that I could protect myself and all those around me. He, moreover, accused me of not protecting all those whom he had already infected. This man ended his life by cutting his throat.

Syphilitic neurasthenia is on the increase. Life being more strenuous and more of a bustle, especially in densely populated cities, than it was, is one of the causes. The more ready access which patients have to medical literature, and the intelligent interest that a very large proportion of the lay public take in medical matters, is another cause. The lay Press has recently allowed articles concerning the disease to appear in its columns. The way the words 
are veiled, the peculiar heading that is attached to them- "Hidden Plague" - defeats its purpose, and is enough to put the fear of God into anyone.

The type of man who used to commit suicide when he knew that he had contracted syphilis, fortunately, is not met with nowadays. The man was not a neurasthenic, but simply committed suicide to save himself the horror and misery of being an invalid for the rest of his life, and then ending it in a madhouse. This type of man is open to reason, and, once he is told that he can be cured, he no longer worries. A strong, healthy-looking man, with early generalised syphilis, once consulted me. His first question was, Have I syphilis? His second question was, Can I be cured? He afterwards informed me that if $I$ had not answered his second question in the affirmative he would have done away with himself. What may be called true syphilitic neurasthenia is either a premonitory sign or symptom of a degenerative nerve lesion, or is simply due to the lack of oxidation of the nerve cells, produced by the large increase in size of the colloidal protein particles in the bloodserum. As treatment, especially with oxidising agents (metals), breaks down these large particles into several smaller ones, thereby increasing their surface for oxidation, benefit naturally results. Owing to the fact that too much arsenic destroys oxidation, neurasthenia may be a symptom of arsenical poisoning. I have known two such cases where the patients committed suicide.

Every venereal patient should be looked upon as a special sort of individual, and the physician in charge should always adopt a most optimistic tone. The knowledge which many patients have of medical science is often much greater than that with which they are credited, so that, in any suspicious patient, I have always found it to be a good plan to let him follow my line of argument, and to tell him the reason why so and so many injections are given, and why the treatment is continued for so and so long. When the position is laid before them, they naturally ask questions, the answers of which can always be true, and yet be quite optimistically painted. Answers to questions put to you by a patient penetrate far deeper into the patient's mind than all the talk in the world from the doctor. The present-day patient is flattered when the physician takes him into his confidence and explains to him the rationale of every step he takes. For the bad cases of syphilitic neurasthenia one certainly can do nothing. Mild cases can often be cured in time, and any number of susceptible patients can be prevented from developing neurasthenia if they are dealt with as I have described above. Considering that neurasthenia is such a common disease of the female sex, it is an odd fact that one seldom sees a case of sexual neurasthenia-i.e., neurasthenia caused by either gonorrhœa or syphilis-in a woman. The following case is typical of the kind of sexual neurasthenia met with nowadays, and shows to what harm pathological tests may lead:

CASE 137.-A man, aged 35, married, with no mental history, consulted a doctor for a supposed urethral discharge, which was said to follow a motor-bicycle accident. As a result of much instrumentation the urethritis was aggravated to an extent which 


\section{CLINICAL ASPECT AND TREATMENT OF VENEREAL DISEASES}

caused the patient to be sent to a venereal hospital, although there had never been any evidence of gonorrhœa. The patient was unaware he had ever had a venereal disease. In hospital, owing to further instrumentation and vigorous prostatic massage, he developed cystitis. At this time he complained of his "blood jumping," so a C.F.T. for syphilis was made which came out positive; consequently, he was given an official course, although there was no history or clinical signs to warrant it. The patient ultimately left the hospital, having lost 3 stone, in weight. He then came home, and consulted first one doctor and then another. Some said he was all right, others said he had strictures and a poisonous staphylococcus in his prostate; others wanted to treat him for syphilis, and others to remove all his teeth, as he had a slight pyorrhœea. When I saw him he was a wreck and practically demented, and at times he failed to recognise his wife or his relations; he would sit for hours writing the utmost gibberish about his poisonous staphylococci, etc.; he could not sleep, and was incapable of getting an erection. He had no sign of syphilis; his urethra was fibrosed in places, owing to the damage done by the instruments which had been passed; there was practically no prostatic substance discernible, and his blood-pressure was abnormally low, which it usually is in these cases. All measures to improve him, including psychotherapy, were unavailing, and the patient is now an inmate of a lunatic asylum. 


\section{CHAPTER XXXI}

\section{VENEREAL DISEASE AND MARRIAGE}

\section{Syphilis.}

ALL authors, in discussing the question of syphilis and marriage, have hitherto pinned themselves down to a time limit. For instance, Hutchinson ${ }^{1}$ said that a man might marry with safety if he had continued treatment for two years from the date of his chancre, Fournier ${ }^{2}$ said that four or five years should elapse, and most other authors more or less echo Fournier's views. When either the cause of syphilis was unknown, or was thought to be only the Spiroshoeta pallida, theoretically it would have been justifiable to fix a time limit, and still greater would this justification be now that arseno-benzene has seen daylight, for the simple reason that we' are told that all the spirochætæ in the body are killed by this. drug. I cannot help thinking that all authors who have advocated a time limit have done so on theory alone, and have not allowed their clinical experience to influence them. My own clinical experience is not so great as that of Hutchinson, ${ }^{1}$ Fournier, ${ }^{2}$ Gougerot, ${ }^{3}$ and some of the other writers on this subject whom I have in mind, but yet I have learnt that a time limit is futile and untrustworthy.

The reader will see later on, from the cases I am bringing forward and from the previous pages, that the Spirochceta pallida cannot be the actual cause of the disease itself, but that some other phase or phases must exist, which can lie dormant for an indefinite period, and then re-awaken and cause symptoms again.

The spore is the dormant phase; it is the actual cause of the disease, and it may lie dormant in any part of the body without causing any disturbance of the host's cells in the locality in which it is situated.

Although it may lie dormant, it may re-awaken and give rise to other phases which will cause symptoms, or it may never re-awaken until it gains entrance to a new host; hence, if a patient is harbouring spores, he is harbouring a potentially harmful infective agent.

If the spores remain dormant for a sufficiently long time, the host will cease to form protective bodies; but the fact that the C.F.T. is negative is no proof that the patient is not harbouring the infective agent.

Many individuals - and I am inclined to believe that the kind of infection plays a rôle-will, provided the organisms have been present for a certain 


\section{CLINICAL ASPECT AND TREATMENT OF VENEREAL DISEASES}

period (the period varies enormously in different persons), continue to form protective bodies long after the organisms have been killed. Therefore, because a patient gives a positive C.F.T., it does not necessarily follow that he has active syphilis, and so must not consider himself a candidate for marriage.

As far as we can see, then, at present, it would appear that neither by clinical nor by pathological means can we say for certain when a patient may marry without risk. If we go more carefully into the matter, and bring every point into consideration, we can be more definite than this. Syphilis in the man will be dealt with first.

If a man has been put under treatment before he has reached the generalisation stage, and provided that treatment has been adequate, he can marry at any time.

I should consider four to five injections of arseno-benzene, with one injection of intramine and mercury and iodine for a year, to be adequate treatment.

In such an early case as this a C.F.T. reaction would be of value, since, if a positive result were obtained, it would obviously mean that the patient had entered the generalisation stage while under treatment. Such a thing is possible, but fortunately it very seldom occurs.

CASE 138. - I was consulted by a man with a primary sore on the corona, and the sore could not be removed. The C.F.T. was negative, both before and directly after treatment was inaugurated. I gave him five injections of neo-salvarsan, allowing four days to elapse between each pair of injections. As he had to leave England almost immediately, he was obliged to take mercury internally, and so could not have intramuscular injections. I saw him again nine months later, when he presented mucous papules in his mouth, a general adenitis, and a positive C.F.T. He also informed me that the site of his primary sore had swollen up, and had become very red a month or two previously.

To make more sure still that a case is cured, a provocative injection of arseno-benzene may be given, and the patient may be passed if the bloodtest is negative before, and forty-eight hours after, the injection. If the provocative injection is to be given, it must be ascertained that the patient has had no treatment for six months.

Speaking broadly, then, if a patient has been put under treatment before he has reached the generalisation stage, he may marry without risk.

If treatment is not begun until the generalisation stage has been reached, provided the patient has had about nine injections of arseno-benzene and two of intramine, and thorough mercurial and iodine treatment for two years, the risks of his infecting his wife are small, but they exist theoretically. I have actually seen such mishaps occur. The following is a case in which infection of another party occurred:

CASE 139.-Syphilis contracted in May, 1912; treatment commenced in June; and consisted of nine injections of neo-salvarsan, followed by a two years' course of mercury injections and iodides. Patient married in January, 1917, having on 
three different occasions given a negative C.F.T. The wife, when four months pregnant, developed condylomata, but gave birth to a child at full time, which died of syphilis two months later, in spite of the treatment the mother underwent.

If only two or three injections of arseno-benzene have been given, and even if mercury is prescribed for two years, the risks are certainly much greater, as I have already seen ten cases in which a wife has become infected. These cases had all been treated by other men, and they came to me because of what had occurred. It is, therefore, possible that my failures have also gone elsewhere. The treatment which I have advocated for the past eight years was generally considered in this country to be unnecessarily severe. I am quite positive that recurrences are far more frequent when only two or three injections are given at first than when nine are prescribed; therefore I think I am probably right in assuming that a man who has been treated in the latter way is a better candidate for marriage than one who has undergone the former treatment.

Once a patient has reached the generalisation stage, spores may have settled in any corner of the body, and, being only potentially harmful, neither a C.F.T. nor a provocative injection at a later date will give results from which an absolutely trustworthy statement of a cure can be made. Here, again, we can be more exact, since it will naturally depend upon how long the patient has been in the generalisation stage. The shorter the time, the greater the value of a C.F.T. and a provocative injection later, and vice versâ. - In actual practice we know that, provided the patient has been treatedand I am now thinking of those who never had arseno-benzene-and that, provided four or five years have elapsed before marriage, the percentage of those who infect their wives is so ridiculously small that, when a patient comes up for advice on this point, one almost feels inclined to tell him that there is no risk. Then the cases in which infection has been conveyed pass through one's mind, and as such instances are often such sad ones, it requires many hundreds of successful cases to set off against one unsuccessful one.

I will now mention two cases. In one the husband had no arseno-benzene before marriage, and in the other he had.

CASE 140.-The patient contracted syphilis five years before he married, and for the first three of those years he was treated with mercury. He had never developed a recurrence, and when his wife became infected his C.F.T. was negativc. They had been married for twelve years, and, with the exception of the occasion upon which the infection was conveyed the husband had always worn a preventative. The wife developed a very severe syphilis. Within a few weeks of the appearance of the rash she developed a unilateral optic neuritis. She quickly became blind in the affected eye, in spite of treatment, and ultimately the blind eye had to be removed.

CASE 141.- The husband contracted syphilis in 1906. He was treated with mercury for three years, and took mercury again for one year before he married, and as an extra precaution he had three injections of salvarsan. There had never been a recurrence. The patient married in June, 1912, and in October, 1913, he brought his wife to see me, and she had well-marked generalised syphilis. 


\section{CLINICAL ASPECT AND TREATMENT OF VENEREAL DISEASES}

I could cite other cases, but these two will suffice to show how difficult it is to advise a patient, and what a slender reed to lean upon is the time limit. Every case should be considered individually, and, when I am consulted upon this point, I first of all find out whether the treatment has been adequate or inadequate. I then attempt to gain as much knowledge of the "man" as I can, and, finally, I try to ascertain the kind of sore which the patient had, and the amount of resistance he brought up to combat the infection.

The question of treatment we have already considered, and we have now the "man" himself to discuss. If the patient is an intelligent man, and if there is reason to think that he will be able to follow the line of argument which is passing through one's own brain, the whole matter should be laid before him, and he should be left to choose whether he will run the infinitesimal risk or not.

If the patient is nervous, and if there is reason to think that his future life would be rendered miserable by being told that a risk existed, provided other things are equal, it is best to take the risk upon one's own shoulders, and to tell him that he may marry without entertaining any qualms.

Since we do not at present discriminate between our cases of syphilis, but regard all cases as being alike, and prescribe the same treatment for all, we always must be indefinite when we are requested to advise on the matter of marriage. I have no doubt in my own mind that cases of syphilis vary enormously, and that a relationship exists between the kind of sore, the degree of the enlargement of the lymphatic glands, and the future course of the disease, hence, indirectly, its degree of infectivity.

I am unable at present to lay down any hard-and-fast rules, since the matter can only be solved by clinical methods, and these have not yet been sufficiently long in force.

The plan is to note very carefully the kind of sore which the patient has. the phases of the Leucocytozoon syphilidis which it reveals in section, and the degree of the enlargement of the lymphatie glands. Then to watch the career of the patient, and to note what happens when he marries.

Although I can only speak in general terms at present, I can say that the risks of a man infecting his wife are greater when the sore is of the papuloindurative-erosive type than when the sore is of the papulo-non-indurativeulcerative type. The prognosis is distinctly better in those cases in which the lymphatic glands are markedly enlarged than in those in which they are scarcely enlarged, but are abnormally hard. It must be remembered that, in the papulo-ulcerative chancre, the lymphatic glands may remain unaltered, and that in many of the cases in which they become enlarged the enlargement is due to a secondary infection.

If a man has had a recurrence, it does not follow that the risks of his marrying are any greater than those of a man who has had no recurrence, since the fact that a man gets an orbicular syphilide of his arm does not mean that he is any more likely to have dormant spores in his sexual organs than 
a man who has never had a recurrence. In actual practice, we think that a man who has had a recurrence runs greater risks. As our knowledge is so incomplete at present, it is as well to think that this is true, as it is well to err on the side of caution.

Another factor which has to be taken into account is the part of the body which has been attacked by the syphilis. This aspect of the question has nothing to do with the risk of infection, but only concerns the future of the man and wife.

If the patient has an organic lesion caused by the syphilis, and there is any reason to fear that the lesion is likely to cut short his life, new points are presented, and they require very careful consideration before marriage is advised or not.

Again, if the patient has a lesion of his central nervous system, and it is feared that he may later develop a degenerative lesion, the doctor must carefully consider whether a few years of conjugal happiness are compensated by the, maybe, many years of chronic invalidism of the husband.

For some reason or other, recent authorities have imagined that there is a special breed of spirochæta which will give rise to nervous lesions, and another breed which will give rise to systemic lesions, and so on.

Hence, if a man who develops a generative nervous lesion infects his wife or his children, it is assumed that the wife or the children are more likely to develop nervous syphilis than systemic syphilis.

I mention this because, if such a view were true, the advice one would give to a patient who, one feared, might develop a degenerative nervous lesion, would on this score alone, be certainly not to marry.

I have had the opportunity of seeing and examining many families in which the husband had a degenerative nervous lesion. I have notes of five in which the wife, or the wife and children, were syphilitic; but I have never seen a case in which the wife or children had a nervous lesion. Cases have been reported in books, and the same cases have been copied from one book into another, so that, at first sight, a reader might imagine that these commonly occurred. I feel certain that they are only coincidences, because experimental work negatives the idea that there are special breeds of organisms that, on the one hand, produce nervous syphilis, and, on the other hand, systemic syphilis.

If a woman has had syphilis, and has been well treated, the chances of her infecting her husband are nil; but, however drastic the treatment has been, there is always a risk that her children will be syphilitic. Therefore, as has been already stated, once a woman has had syphilis, in spite of the treatment she has already had, she must be treated throughout the whole period of each succeeding pregnancy. 


\section{Gonorrhœa.}

Although gonorrhœa is a less serious disease than syphilis, when it becomes chronic it is certainly more difficult by treatment to render the patient theoretically non-infectious. The man and the woman will be considered separately, but, before going deeply into the subject, I would like to give the warning that a negative bacteriological diagnosis has absolutely no significance whatsoever. When the question of infectivity is concerned, it is a waste of time and money to examine bacteriologically the urethral and prostatic secretions of the man and the cervical secretion of the woman. The advice to be given in the case of gonorr hœa, as in syphilis, depends mainly upon clinical experience.

When a man seeks advice on the question of marriage, the first point to be ascertained is as to whether he has ever had a recurrence or a metastatic lesion. If not, the prospects are good from the start, while if he has had a recurrence or a metastatic lesion, the risks of his infecting his wife are somewhat greater.

We will, first of all, consider the patient who has never had a recurrence or a metastatic lesion.

From such a patient it is important to ascertain when he became infected, the period that has elapsed since he last had any signs of a discharge, and whether the infection reached the posterior part of his urethra and prostate, or not. He should then be asked whether sexual connection, nocturnal emissions, and alcohol have any effect upon the condition. If not, the chances are that he is cured, while if the status quo is not maintained, the chances are that he is not cured, and therefore is infectious.

The urethra and prostate should then be very carefully examined, and great attention should be paid to the dilatability of the urethra; this can be gauged by Kollmann's anterior and posterior dilators. If the dilatability is below the normal, the chances are that there are gonococci hidden in the follicles or in the sub-epithelial tissue. Dilatation will probably wake them up, and a big injection of a potent non-sensitised vaccine will also help to do this. If the dilatability is normal, and if a vaccine does not alter the status quo, the patient may be passed as a fit candidate for marriage.

If the patient has had a recurrence or a metastatic lesion, the same tests should be applied, but the additional factor of a secondary infection comes in. In other words, the tests employed above are not quite so conclusive in a recurrent case. A peculiarity of some of these cases is that an occasional sexual connection may not alter the status quo, but, when marital connection is indulged in, it may set up a copious discharge, which will, in its turn, be certain to infect the wife. Occasionally the discharge may not be copious; indeed, it may not even be increased, and yet the wife becomes infected. The infection runs its usual course-that is, it is first acute, then subacute, and finally chronic, or the wife's infection may be-to use an Irishismchronic from the start. It is odd how frequently gonococcal lesions are chronic 
from the start, and yet the point appears never to have received any recognition. I have seen cases of gonococcal epididymitis in which the whole of the caput minor has become stone-like, without the patient's ever being a ware that he had had an infection there. I mention the epididymis because it occurs in such a tender organ that an acute or a subacute infection of it could scarcely be overlooked. An analogy exists between a man who has entered the generalisation stage of syphilis and the man who has a recurrent urethritis or a metastatic gonococcal lesion. That is to say, in both cases one may make a mistake in advising a patient to marry.

All the tests just mentioned should be tried, and, if the patient is an intelligent man, the position should be discussed openly with him; if not, then the doctor must run the risk of making a mistake, and being blamed afterwards by the patient for it.

Speaking generally, it is not difficult to tell when a man is cured. In the case of a woman, it is well-nigh impossible.

In a woman there may be gonococci hidden in Bartholin's glands, or in the cervix, for years. They may be the source of infection, without necessarily producing any signs or symptoms in the person who harbours them. The provocative vaccine test is difficult to interpret in a woman, and even if Bartholin's glands and the cervix are harbouring gonococci in a chronic infection, a bacteriological examination will, in nine cases out of ten, fail to reveal them. If the patient has never had sexual connection, the chances are that the cervix has never been infected. In such a case, provided the treatment has been good, marriage may be advised. In a patient who has had sexual connection, the chances are that she has had cervicitis. The long presence of gonococci in the cervix leads to fibrous tissue formation, and destroys the dilatability, as is the case with the urethra. If the dilatability of the cervix is destroyed, the patient will almost certainly suffer from dysmenorrhoa, hence some information may be gained by going into this question. If the patient suffers from menorrhagia or metrorrhagia, the chances are that she still has an active gonococcal infection.

It is extremely seldom that the true position of affairs can be pointed out to a woman; therefore, in advising women on marriage, we must all expect to make mistakes.

In revising this chapter, after four years, I am even more emphatic on the point that a pathological report is practically useless, and that a clinical opinion is the only reliable means upon which to base an advice on marriage. Especially is this the case with gonorrhœa, and here I would like to warn the reader that threads in the urine are not necessarily indicative of active or latent disease. Many men who have had gonorrhœa pass threads for the rest of their lives without being infectious. A point $I$ forgot to mention before is that a man with latent gonorrhœa runs a greater risk of having this disease re-awakened and of being infectious by extra-marital than by 


\section{2}

marital intercourse. I have known men infect other women, while their wives went free. I have also come across cases in which men have had recurrent attacks of urethritis, epididymitis, etc., during marriage, while their wives have had children and escaped the disease, in spite of the risks they have run. After several years' experience I have come to the conclusion that the infectivity of a chronic venereal disease is very much over-estimated. I have even known men to present gonococci in their smears and their wives to escape the disease. The present methods advised for estimating cure would in many healthy patients produce a urethritis; therefore, what must be the result in those who have recently had gonorrhœa, and in those who have been overtreated for this complaint? The result is that the gonococci are prevented from sleeping, the patient is rendered more infectious, and the incidence of the disease is increased. Our fathers and grandfathers had gonorrhœa and were never treated with the methods in rogue to-day nor underwent the present-day tests for cure, in spite of which we are still here to tell the tale. The more I see of venereal diseases the more convinced $I$ become that an infection is rarely conveyed to another party except during the acute stage.

1. Hutchinson (1899 and 1902): Internat. Congress for the Prophylaxis of Venereal Diseases. Brussels.

2. Fournier (1890): Syphilis et Mariage. G. Masson, Paris.

3. Godgerot (1914): Le Traitement de la Syph. en Clientèle. A. Maloine, Paris. 


\section{CHAPTER XXXII}

\section{VENEREAL DISEASES AND PUBLIC HEALTH}

ExcEPT for the Salernitian teaching, the history of this subject is nil. The Regimen sanitatis Salerni, which emanated from the pen of Johannes de Meditano, was a compendium of suggestions on health, amongst which was the advice to micturate and wash immediately after sexual intercourse. Salerno was at one time the medical centre of Europe, and between the years 1484 and 1884 the work above referred to passed through 240 editions; yet the simple advice given on the prevention of venereal diseases appears to have been little known, and probably seldom taken.

With the present-day glamour for strong antiseptics, which are liable to damage the tissues of the host without necessarily killing the organisms attacking them, we are covering exactly the same ground which the surgeons covered between the antiseptic period of Lister and the aseptic period of to-day without realising the re-iteration. The time will probably come-as the present-day measures for the prevention of venereal diseases will be found to have been inadequate, and the statistics published inaccurate-when we shall return to take the advice, nearly 500 years old, which emanated from the wonderful medical school of Salerno, founded by the Romans in the second century B.c., and now no more than a name. The first stage in the presentday prevention was the legalisation of prostitution in brothels. The women were examined twice a week, and compelled to undergo treatment if found to be infected. As a.woman might become infected just after the last examination, and have sexual intercourse with several men before the next examination-as she could also be a carrier of disease without necessarily being infected -brothels were not so safe as was usually thought. Legalising prostitution increased clandestine prostitution, which proved to be a rapid source for disseminating venereal disease, and, moreover, it was unfair to make a law which was only applicable to one sex. In this country, prostitution was legalised only for a short time in certain towns, the C.D. Act being repealed in 1886. On the Continent, where it has been widely legalised for many years, the opinion was fast growing that the measure was not a success. In some countries the once stringent rules were relaxed, and it is very probable that if the world war had not occurred, and so diverted the enterprise needed to abolish the system altogether, by now legal prostitution would have been a thing of the past. In out-of-the-way places in foreign parts, where the same women remain in the same place, and where troops are quartered, compulsory 
and periodical examination of both sexes may be permissible and advisable; but elsewhere any measure of a similar nature, especially if it is only applied to one sex, is foredoomed to failure. An attempt was made during the war to institute a one-sided legislation, and the law was known as $40 \mathrm{D}$. A soldier was enabled to denounce a woman whom he thought had infected him. Even if the woman had a venereal disease which could be said to be active both on clinical and pathological grounds, it would be difficult to prove which party infected the other. Since a woman may be infectious by harbouring a venereal disease, which cannot be satisfactorily proved in more than 15.0 per cent. of the cases, it meant that in over three-quarters of the women examined sufficient evidence could not be produced upon which to warrant a conviction. The measure was so full of loopholes, and so unsound, that, in November, 1918 , it was rapidly dropped as being a war-time measure only.

The next step was the formation of societies for the prevention of venereal diseases, which were beginning to do useful work by propaganda just as the war broke out. In certain Continental cities centres were established for the free treatment of venereal diseases, but they have not been in action suffciently long to enable an opinion to be formed as to whether patients are more likely to attend where treatment is free than where they have to pay. Free treatment is now obtainable in most parts of the United Kingdom, and, so far as my own hospital is concerned, I am convinced that patients are more regular in their attendance, and more careful of their treatment when they pay, be it ever so little, than when they get treatment free. It is human nature to regard as of little account things obtained for nothing, and vice versâ. The establishment of a treatment centre in every town which has a population of not less than 10,000 will do much to benefit those affected, and will do a little to stop the dissemination of the disease, because it will be one of the ways of apprising the public of the dangers of venereal disease, and it will stimulate medical men to become proficient in a branch of medicine which has always been sadly neglected. It will be of interest to trace how these centres were established in this country.

On several occasions in the past, one or more medical men attempted to bring the venereal problem before the public, and on each occasion the Government acquiesced in the scheme, but failed to act when the moment for putting it into execution arrived. All attempts failed until a letter, signed by many medical men, appeared in the columns of the Morning Post, in 1912. This gave a lever to Sir Malcolm Morris, the chief instigator of the move, who was able to further it at the International Medical Congress in 1913, the result being the formation of the Royal Commission. Although the outcome of this Commission has been for the good, because the curtain has been raised, and the venereal problem is now in the glare of the footlights, it was perhaps a mistake to issue a report so full of pathological textbook matter while neglecting the clinical aspect. 
The result has been such a widespread public faith in laboratory tests that the time appears to be approaching when every patient will be a testtube, and every doctor an automatic machine. Such a state of affairs is deeply to be deprecated, because laboratory tests are always capable of being improved and refined; their rationale is often an enigma, and too much reliance placed upon them is bound to make the medical man neglect the human side of his patient. The sudden emergence of the venereal problem from darkness to light has had such an effect upon the psychology of the victims that it has become more than ever necessary to rely upon clinical knowledge, and to treat and advise each patient accordingly.

When the Commission ceased its work, the National Council for Combating Venereal Diseases was formed, and has done, since its inception, most valuable work.

Before the formation of this Council, the Eugenic Society had been responsible for propaganda work devised to appeal to the moral sensc, which doubtless has done much good. It is right to teach chastity, although the result obtained may be minimal. It is advisable to warn those who run the risk of the dangers they incur, and the parents are those from whom this advice should first come. Much has been done by lessening the consumption of alcohol, and advocating outdoor exercises and games, but these are only indirect measures, and will not materially assist in diminishing the incidence of venereal disease. The National Council was responsible for lectures being given to soldiers, for the establishment of venereal centres, with the assistance of the Local Government Board, and for the E.T. (early treatment), which is really a preventive measure. A discussion as to whether it is morally sound to issue preventive tubes, etc., may, I think, be quickly dismissed on the ground that by prevention many innocent persons are saved from infection, and I think our first duty is to those not infected, and then to those who are. What has prevention by drugs taught us, and has it been a success? If it is made compulsory to attend an ablution centre within one hour of exposure, almost a guarantee can be given that infection will not ensue. If the use of ointment and the injection of solution is undertaken by the individual himself within an hour of exposure, the success is good, but not so good as that just mentioned. From one hour onwards the chances of preventing infection diminish. Such preventive measures are applicable in the Army, but not in the civil community. As the success to be obtained from their use has not been so great as that anticipated, and has, in the case of gonorrhoea, led to many unrecognised cases of infection, because the pus-producing organisms have been killed and not the gonococcus, I feel sure that if the advice given at the opening of this chapter were acted upon immediately after exposure, as much disease would be prevented in the civil community as is prevented by the use of drugs in the Army.

A burning question of the day is that relating to notification. Apart from the fact that it is a debatable point whether notification diminishes the 
incidence of a disease, before such a measure was advocated a great deal of evidence would require to be brought forward to show that treatment prevented a disease from spreading. If venereal diseases became notifiable, a patient could be no more than compelled to continue treatment, and then only that treatment which the doctor considered adequate. At the best, treatment is only shutting the stable door after the horse has been stolen, and will never be an important factor in preventing venereal disêase. Notification would be useless unless every doctor was compelled to treat every case in such and such a way, and have laborious laboratory tests done before he discharged his patient as cured, and unnecessary if every doctor had a good clinical knowledge of the subject. Furthermore, patients could not be compelled to become inmates of a hospital until thought cured; and as outpatients, no check could be placed upon them should they be desirous of having sexual intercourse.

Another committee has recently been formed, which styles itself the Society for the Prevention of Venereal Diseases, apparently in opposition to the National Council for Combating Venereal Diseases. This has led to the division of opinion into (1) fighting the problem by moral means, (2) by preventive means. This is most unfortunate at a time when the medical profession need the whole support of the lay community, because the man in the street is already beginning to ask if the profession really understand the problem they are tackling. As committees are apt to achieve little unless their efforts are crowned with success early, the main point at issue becomes neglected, and their powers run the risk of becoming inadvertently misused. My own opinion is that no measure short of one akin to vaccination against smallpox will be really successful in ridding the world of venereal diseases. 


\section{CHAPTER XXXIII}

\section{SYPHILIS AS A POISON AND ITS RELATIONSHIP TO MALIGNANT DISEASE}

OwING to the pigeon-holing of symptoms, which is unavoidable when syphilis of this or that organ is described, one is apt to lose sight of the background and of the connecting-links between it and other diseases. It is my object, therefore, in this chapter to attempt to show the relationship between syphilis and other diseases, but particularly between the symptoms produced by it and well-known poisons, and the rôle it plays in the ætiology of malignant disease. At first sight it might be imagined that the more minutely one probed into the subject the further it would take one away from the general outline, but such is not the case, as reference to the histological aspect will show. Histology has been a favourite hobby of mine for many years, and I should imagine I have prepared and examined as many sections of the various syphilitic lesions as anyone, and the more I examine the more convinced I become that, unless the causative organism be found, there is no difference between a syphilitic granuloma and other granulomata caused by chronic sepsis, tuberculosis, sporotrichosis, etc. The so-called characteristic vascular changes to be met with in syphilis may be absent in a given section, and present in other granulomata. I have sections of syphilitic adenitis which were pronounced as typically tubercular by well-known pathologists, and which would certainly have caused the downfall of a student up for an examination if he had failed to make the same diagnosis. Giant cells are nothing more nor less than fused endothelial cells, and are to be found in any chronic infection. Further, the lymphocyte and its offspring, the plasma cell, form the local resistance in all chronic infections. Indeed, the only difference between these cells in the varied diseases is the altered physico-chemical molecular configuration possessed by the colloidal protein particles enclosed within the cell membrane. Later, these colloidal particles become free, and tackle the parasite in the same manner as do the particles which circulate in the blood-stream. As such changes cannot be detected histologically, it is scarcely to be wondered at that the gross microscopic picture of all granulomata or plasmomata is similar. We may now note, then, that the visible response of the host against such parasitic infections as syphilis, tuberculosis, sporotrichosis, leishmanniosis, etc., is the same. Syphilis and tuberculosis, the former to a greater degree than the latter, make a severer and more pro- 


\section{CLINICAL ASPECT AND TREATMENT OF VENEREAL DISEASES}

tracted call upon the lymphocyte than most other diseases. In other words, the lymphocyte, including its parent, the endothelial cell, and its offspring, the plasma cell, are more hard pressed in syphilis than in any other parasitic discase. When the call for protection is unduly prolonged, the colloidal particles in the serum become larger, richer in carboxyl-groups $(\mathrm{COOH})$, poorer in certain adsorbed salts, and more permeable. These changes depress the oxidising power of such particles, and increase their proclivity towards autolysis. In fact, the colloidal protein particles may become so changed as to lead to their behaving as foreign substances, because, under such conditions, a patient's own protein may be poisonous to himself. This I was able to prove in the following way: If serum is formolised, the protein particles become increased in size, the amino-groups are converted into imino-groups, and all trace of formaldehyde vanishes. If such serum be injected into the patient's own subdural space by lumbar puncture, symptoms of intoxication are produced, which do not differ from those caused by intrathecal injections of a toxic arseno-benzenised serum. This explains, then, why in cases of late syphilis the patient may present symptoms typical of chronic shock, or anaphylaxis, and of chronic metallic poisoning. In such cases, and we know it to occur in chronic shock, eosinophilia is the rule, an observation which supports my view that the granules or colloidal particles of the eosinophile cells are charged with oxidising properties to take the place of those which the circulating colloidal particles have lost in the change. The function of the basophiles is the same. The changes above described affect cells as well, and no mischief is wrought provided they stop at the colloidal protein particles, which form what we call the protoplasm. Should they extend beyond, the nucleus shows activity, and divides and subdivides, doubtless with the hope of forming more cells, the protoplasm of which can be thrown into the attack. If the call for resisting substance is still further made, the nucleus vanishes like the protoplasm did, and the nucleolus, which contains both nuclear and protoplasmic elements, by a process of multiple subdivision and budding, attempts to form cells in response to that call. By this time the changes in the colloidal particles have become so pronounced that the nucleolar cells or matter act as foreign bodies, and cause the host whom they were trying to assist to protect himself against them. As the nucleolar cells are of the host's own kith and kin, protection against them is impossible, with the result that they become even worse than any parasite, and produce what we call malignant disease. If the call affects in the main the plasma cell, then the malignant growth will be a plasma-celled sarcoma, which I have seen commence as an ulcer on the thigh in a syphilitic subject. The lymphocyte itself may be attacked, or even its parent endothelial cell, and give rise to typical malignant growths; but, as a rule, the call for protection is not pushed to this extreme; consequently, the lesion met with is usually of a semi-malignant nature. If the call is made in the skin, we have the clinical condition of Mycosis fungoides, which I am sure is not rarely caused by syphilis. If the 
call is made in the lymphatic glands, we have the clinical condition of Hodglkin's disease, which may also be caused by syphilis. If the call is made in the spleen, we have the clinical condition of parenchymatous splenomegaly, which may be caused by syphilis. If the call is made in other organs, where lymphocytic nests are normally found, we have the clinical condition of Miculicz's disease, which again may be caused by syphilis. There is every reason to believe that Hodgkin's disease and parenchymatous splenomegaly may be caused by tuberculosis. As to whether leishmanniosis may give rise to any of these conditions I have no first-hand knowledge. Anyhow, I have seen several instances of syphilis causing intermediary lymphocytomata, the name I have given to this class of case. Syphilis in the early stages may act as a direct protoplasmic poison, producing first cloudy swelling, then fatty degeneration, and finally necrosis. The organs most affected are the liver and kidneys. Cloudy swelling of the liver cells causes biliary obstruction, and this is the way I believe jaundice to be caused in early syphilis. Fatty degeneration and necrosis of the liver parenchyma give rise to acute yellow atrophy. The same changes occurring in the kidney are not so readily discernible as proteinuria, may exist without causing symptoms, and the final stage of uræmia may be reached before the seriousness of the condition is recognised. Substitute the word metal for syphilis in the above, and the rest stands as it is; therefore, syphilis may act as a poison in identically the same way as may a metal. It is interesting to note that the metals which are most liable to act in this way are those which have a high atomic weight, such as mercury $(200 \cdot 6)$, bismuth $(208 \cdot 0)$, and thorium $(232 \cdot 4)$. The only way in which metals with a lower atomic weight can produce the same effects is when they are firmly attached to a substratum which has the power of readily fixing itself to the protein colloidal particles, such as arsenic $(74 \cdot 0)$ in the form of arseno-benzene. This proves, then, a relationship as regards common properties between adsorption, high atomic weight, and syphilis. In other words, it shows that arseno-benzene acts primarily in virtue of its aminogroups; that mercury cannot strictly be called a specific; that the metal in neither kills the parasite directly; and why the C.F.T. should be positive in syphilis. Going deeper into the subject, we can see why intramine should be more powerful than plain colloidal sulphur, and, as we know from clinical experience that it is not toxic, we can safely forecast the beneficial effects which would follow the introduction of amino-compounds of iron and manganese. If the action of the amino-groups is capable of bringing arsenic up to the level of mercury, then for syphilis to be on the same level it is natural to expect the parasite to be of a particularly high nature (to be explained below) and the protective substance of the host to be particularly rich in amino-groups. We find that the protective substance is particularly rich in amino-groups, because there are more protein colloidal particles in the serum of a syphilitic than in the serum from any other disease. Since the C.F.T. is nothing more nor less than an adsorption test, is it to be wondered 


\section{CLINICAL ASPECT AND TREATMENT OF VENEREAL DISEASES}

at when a syphilitic serum fixes anything? Just as one amino-compound is better than another, according to how the amino-groups are arranged, so does the adsorptive capacity of the protein particles vary according to the arrangement of the amino-groups therein. In my opinion, the sole difference between albumin, globulin, and lipoid-globulin is the way in which the aminogroups are arranged. It is interesting to note here that a C.s.F. which contains no globulin, and therefore gives no ring with ammonium sulphate, will give a beautiful ring if it has been previously shaken with toluene and filtered. If this be true, then there is no hard-and-fast line between the various kinds of protein, each being really a further stage of the other. Globulin is higher in the scale than albumin, and lipoid-globulin than globulin. As, then, the highest kind of protein is met with in syphilisindeed, it is met with in malignant disease as well as the principal protein in pseudo-chylous ascites-it is logical to suppose that the syphilitic parasite is of a particularly high order, and one which more closely than any other approximates to the cancer cell, which is of human origin. If this is so, it is hardly likely that the Spirochaeta pallida is the sole phase of the syphilitic 'organism, and practically certain that it is not a vegetable parasite. Malignant disease is the hardest of all to cure, protozoal infections come next, and then diseases caused by vegetable parasites. It is probable that there has been an evolution in diseases, which allows us to grade some of the pathogenic parasites in the following order: (1) staphylococci; (2) streptococci; (3) streptobacilli; (4) gonococci; (5) streptothrices (tubercle bacillus); (6) fungi (sporotrichium); (7) Leishmann-Donovan bodies, the cause of kala-azar, and possibly a stage in the life-history of the trypanosome; (8) Leucocytozoon syphilidis ; (9) cancer cell. It is interesting here to note that the higher the grade of the parasite, the higher the scale of animal life it prefers to make its host. Plants are immune to the parasites mentioned; the lower forms of animal life less so, and so on until we come to syphilis, which affects the highest vertebrates only; and, finally, cancer is more common in man than in animals, and appears to become more so the further civilisation advances. I think it is permissible to state that the most highly organised cells in the human body are the nerve cells - at any rate, they are the most delicate. The more highly organised the parasite the greater the liking it has for nerve cells, as evidenced by the degeneration which is so typical in sleeping sickness and syphilis. The next most common cause of nerve degeneration is metallic poisoning; so once again we find an homology between the action of the syphilitic parasite and the higher metals. Going down to the lowly parasites, we find that they are combated in part by the polymorphonuclear leucocytes, but that, should the lesion they cause become chronic, it becomes a typical plasmoma. Polymorphonuclear leucocytes are met with in greater abundance in staphylococcic than in streptococcic infections, and in streptococcic than in gonococcic infections, and so on until we reach the orders of the protozoa and cancer cell, and find that this leucocyte plays no rôle in the protection of the host against 
the disease. When syphilis is first contracted and the generalisation stage is reached, there occurs a polymorphonuclear. leucocytosis, which I cannot help but regard as an atavistic phenomenon, because it seems reasonable to suppose that at one period this leucocyte did form the chief arm of protection of a host against a parasite. When we get to gonococcic and certain bacterial infections we find the tables are turned, or, in other words, the organisms living at the expense of the polymorphonuclear leucocytes-a phenomenon, when it first occurred, which must have been the starting-point of this leucocyte taking a subordinate rôle in protection. In view of these few words, it seems conclusive that phagocytosis is not that wonderful weapon of defence which it is still generally considered to be. In the soft sore infection, the moment the streptobacillus takes on an intracellular habitat lymphocytes and plasma cells take the place of the polymorphonuclear leucocytes, and the parasite itself alters. Ulcus molle serpiginosum is the clinical condition met with when the above change takes place, and is probably the same disease as Granuloma pudendi. As we advance up the parasitic scale, we find that it is natural for parasites to be intracellularly situated, and, further, that gross alterations in the organism take place which we now call phases of a lifecycle. It would seem that there has been an evolution of protection, in that the more powerful it became, the more strenuous the efforts the parasite would have to exercise to overcome it, and no effort could have been better designed than that of living in the cells of the host. The host, to protect itself against such an effrontery, would have to give up simple engulfing (phagocytosis), and take to attacking in another way. Attacking by means of tiny particles possessing an immeasurable area, which would besiege the parasites and starve them into submission, would be the expected mode of procedure, taking warfare as an analogy. Consequently, we find that the host mainly protects himself by besieging the parasites with myriads of protein colloidal particles, which starve the parasites into submission by depriving them of the oxygen, without which they cannot exist. The parasite's response to this is encapsulation, spore formation, etc., methods adopted to protect their oxygen. This brings us on to attack by reduction in contradistinction to oxidation, and explains why in the chronic stages of infections sulphur and iodine should prove so useful. Fibrous tissue formation is the host's response, and the reason is now clear why fibrosis is dissolved by intramine and iodine, and why fibrosis is more marked the higher we ascend the parasitic ladder. Gonococci cause the host to form fibrous tissue wherever they occur. Fibrous tissue formation is much more pronounced in Ulcus molle serpiginosum than in the plain soft sore. Induration is more marked in syphilis than any bacterial or other protozoal disease, while in malignant disease it is far greater in excess of what it is in syphilis. The type of syphilis in which there is most induration is in the type in which the intracellular phases of the Leucocytozoon syphilidis are in greater abundance than the extracellular. The significance of mesenchymatous syphilis is now clear, and we can understand why cirrhosis of the 


\section{CLINICAL ASPECT AND TREATMENT OF VENEREAL DISEASES}

liver, mesenchymatous splenitis, interstitial nephritis, etc., should frequently be met with in syphilis. This triad is also found in metallic poisoning, especially in lead poisoning; but it can be produced in animals with mercury and bismuth and arsenic in an organic compound; so once again we find syphilis acting as a poison in exactly the same way as the metals which have a high atomic weight. At one time I wrongly thought the function of plasma cells was to form fibrous tissue, but I now hold that fibrous tissue is formed from connective-tissue cells, and that the main function of these cells is to hedge the parasites in. Just as syphilis may cause lymphocytomata, so may it cause fibromata. I have seen sarcomata arise from what was previously syphilitic periostitis, and I have seen three cases of glioma in syphilitic subjects which revealed at autopsy pachymeningitis as well, which might possibly have been of specific origin. In both cutaneous tuberculosis and syphilis the lesions cause an epithelial hypertrophy, which is probably of a protective nature like fibrosis. In both these diseases we find benign and malignant epitheliomata arising on lesions formed by them, and most of us have seen malignant epitheliomata occurring on Lupus vulgaris. In syphilis, malignant epitheliomata are particularly common, affecting especially the tongue and the penis. I have seen a primary sore and gummata become malignant, and malignant epitheliomata arise on the sites of chancres, without being preceded by a gumma. Once again we find the same associated with metals-vide arsenical cancer, radium epitheliomata, etc. Stimulation of epithelial cells by syphilis and metals is still further shown in the increased pigmentation which may be caused by these two poisons. It is interesting just to recall the ephelides and Xeroderma pigmentosum caused by the sun's rays, and the malignant epitheliomata caused by the $\mathrm{X}$-rays, to complete the picture of syphilis as a poison, and of its relationship to malignant disease. 


\section{CHAPTER XXXIV}

\section{THE BRIDGE BETWEEN THE CLINICAL AND PATHOLOGICAL PARTS OF THE VENEREAL DOMAIN}

\section{General.}

As the trend of knowledge has been of late mainly along pathological lines, there has been a tendency towards the formation of a gulf between the clinical and pathological sides of the huge venereal domain, and this makes it necessary to bridge the two before closing this, the clinical volume, and opening the pathological volume.

The advances in pathology have been of inestimable value to the student of venereal diseases, but the reliance to be placed upon them has been exaggerated. Pathology is at the best only a means to an end-not the end itself-and an instrument liable to be worked by a hand independently of the brain. Pathological diagnosis is open to greater error than clinical diagnosis, since only a positive finding is evidence. Pathology is capable of being standardised, a fact which tends to make medical men automatons, and incapable of being moved by ever-changing conditions, which must be borne in mind when no two persons behave in exactly the same way to the same disease. It is true that good clinical knowledge takes longer to acquire than pathological; it has, nevertheless, the advantage, in that the greater the knowledge possessed, the more conscious is the possessor of his own shortcomings. To my mind, there is no greater attainment in medicine than clinical knowledge, because clinical knowledge compels the doctor to treat his patient as a human being and not as a machine.

A clinician owes his success not so much to the drugs he uses as to the influence he has over his patient. Such influence is more valuable and potent than the most useful drugs, and is really the essence of the success of suggestion. A clinician often knows at once by instinct-or, better, perhaps, call it experience-what pathology could never teach him in years. A patient gets infinitely more relief from having a heart-to-heart talk with the doctor who knows his case than he ever can, say, from a blood examination, the result of which may frequently be incapable of interpretation.

One must not forget the enormous influence the state of the mind has on the course run by the disease, a connection which is a close one in venereal diseases, owing to the sudden way in which they have emerged from darkness to the limelight.

The best clinician is he who does his own pathological work, but subordinates or merely regards it as an adjunct to his clinical work. 


\section{Syphilis.}

There are three rules generally laid down for the diagnosis and treatment of syphilis: (1) examine a sore for the Spirochceta pallida; $(2)$ treat if the C.F.T. is positive; (3) in the early stages, at any rate, continue treatment till the C.F.T. becomes negative. Before advising a routine examination of a sore for the Spirochceta pallida, the examiner should clearly have in his mind, (1) what to do in an undoubted case (clinical diagnosis) of syphilis when no spirochæta is found; (2) which examination is open to the greater error, $(a)$ clinical, $(b)$ microscopical. To avoid any bias, I had 100 consecutive cases of primary sores examined by a trained pathologist, and in 23 he failed to find the Spirochota pallida.

On another occasion I examined myself 100 consecutive cases of primary sores, making only one examination in each case, and in 41 failed to find the Spirochocta pallida. In the last article I have read on the subject, the author examined 115 cases, and only found the Spirochoeta pallida in $57 \cdot 3$ per cent.

I feel positive that a man who knew his clinical work, and relied on a naked eye examination, would not make so many mistakes. If the error is always as great as this, it means, from taking the best results above obtained, that over 20.0 per cent. of the patients are allowed to enter the generalisation stage, when a cure can never be guaranteed, before treatment is begun. To cite a case:

CASE 142.-A patient was admitted with a typical primary sore. On five different occasions examinations were made for the Spirochala pallida, and all were negative. Six weeks later a rash appeared, but before it was diagnosed two C.F.T's. were done, the first being negative and the second positive. After forty-five days had been wasted the patient was put under treatment, and after three intravenous injections of " 606 ," three intramuscular injections of " 606 ," and five of mercury, the patient developed severe arsenical dermatitis, and is not fit yet, although more than a year has elapsed.

In the early period of the primary stage the C.F.T. is valueless, as the reaction does not become positive until the disease has become generalised, and it is only positive in a sufficient number of cases to be reliable when the infection has existed for at least thirty-five days.

In the generalisation stage, provided there are clinical manifestations, no test is necessary, because no symptoms are more readily capable of being diagnosed clinically; therefore, a C.F.T. is only required in this stage when a questionable history exists and no signs or symptoms are present.

In the recurrent and late stages of syphilis clinical knowledge renders a test unnecessary, especially when the C.F.T. is only positive in $75 \cdot 0$ per cent. of the cases. Furthermore, it must be remembered that a positive C.F.T. only means that the patient presumably has had syphilis, and that it does not mean the symptom presented is necessarily syphilitic.

In the latent stage the C.F.T. is not of very much value, as so many factors 
come into play, each of which must be considered before a given result can be rightly interpreted. The main factors are: (1) the kind of infection; (2) the amount of treatment the patient has had; (3) the stage in which the patient was when the treatment was commenced; (4) the date when the patient last had treatment; and (5) whether he has had a recurrence or not.

Let us take each of these factors in turn, and assume that in every case the syphilis was contracted not less than four years prior to the date when the patient seeks advice, as this is about the period at which the latent stage can be said to have begun.

1. The Kind of Infection.-If the infection was mild, the check upon the production of the protective substance will have probably commenced about the fourth year, and therefore the C.F.T. will be negative. This does not necessarily mean that the patient is cured, as he may develop a degenerative nerve lesion, or even a systemic lesion, later. If the infection was severe, the production of "reagin," the name given to the protective substance, often continues throughout the patient's lifetime; hence, about the fourth year, the C.F.T. will be positive. This does not necessarily mean that the patient has active syphilis.

2. The Amount of Treatment.-The more treatment the patient has had, the greater the likelihood that the C.F.T., about the fourth year, will be negative, and vice versâ; but the results can convey no meaning, as a negative reaction is not definite proof that the patient is cured, and a positive reaction may onlybe an indication of the patient's protective capacity against the disease.

3. The Stage of the Disease at which Treatment was Commenced.--If the C.F.T. was negative before treatment was started, and negative again about the fourth year, the patient can almost certainly be assured that he is cured. If the C.F.T. was positive before treatment was begun, neither a positive nor a negative C.F.T. about the fourth year means anything.

4. The Date when the Patient last had Treatment. - The more recent the treatment, the greater the likelihood of the reaction being negative, and the less the reliance that can be placed upon the test.

5. Whether the Patient has had a Recurrence or not.-If the patient has had a recurrence, the chances are that the reaction will be positive, in spite of treatment. If negative, on the other hand, the chances are that the patient is cured, but there are several traps which await the physician who feels sure himself that the patient is cured. The chemico-physical properties of the reagin particles may be such as to prevent adsorption of complement in vitro, a condition which is very apt to occur in the sera of those patients who have had a recurrence, hence the explanation as to why the C.F.T. is negative in roughly 25 per cent. of the cases.

To treat a man who is, as far as one can say, clinically sound, merely because his C.F.T. is positive, is, in my opinion, not the best advice. A positive reaction tells you no more than that the patient presumably has had syphilis, and it certainly does not guarantee that he has active syphilis 
at the time; indeed, it may signify that his protective action against the disease is good. As the reaction will not discriminate, is one justified in running the risk of letting a sound man have his sheet-anchor destroyed-an action which, I have already explained, treatment has ?

In advising treatment to a sound man, it is logical to assume that the adviser not only regards a positive reaction as indicative of active disease, but also that treatment will prevent symptoms from arising in the future. Is this second assumption justified? Experience has shown me that it is not, and I am convinced that, if a patient is going to develop a degenerative or vascular lesion in the central nervous system, treatment before clinical signs are manifest will not only not prevent or retard their onset, but in many cases will actually precipitate them. I have had several instances, of which the following is a typical case:

CASE 143.-Patient had syphilis six years ago, for which he was fairly well treated. At the end of 1917, possibly to avoid a draft, he told his M.O. that he had had syphilis. Although he was perfectly fit and ailing nothing a C.F.T. was taken, and proved positive. He then had eight injections of arseno-benzene and nine injections of mercury. One week after the last injection he had an attack of hemiplegia, from which he partly recovered. He then developed degenerative encephalitis (G.P.I.), and is now dead.

We must now consider the relationship between treatment and the C.F.T. All treatment does is to cause such a fine subdivision of the protein particles forming the protective substance in the serum as to send many into true solution, in which form they cannot exhibit those chemico-physical properties upon which the C.F.T. depends. Because treatment has this action, it cannot be assumed that the same particles will not regain their colloidal form, or others will not be formed after its cessation; therefore, broadly speaking, as a guide to treatment the C.F.T. is valueless. In this connection it must be remembered the influence intramine has in rendering a serum positive which had previously been made negative by arseno-benzene and mercury. In $1911 \mathrm{I}$ conmenced a series of experiments with the hope of finding out how many injections of arseno-benzene were required in early syphilis to render the C.F.T. negative. In round numbers the figure was nine. Supposing no further treatment was given, I found the cases fell into two groups, (1) those which relapsed with clinical symptoms; (2) those which relapsed with a positive C.F.T. only. Most of the former group relapsed about three months after the completion of the course, and gave a negative C.F.T., showing that a positive C.F.T. may be a sign of the patient's protective action against the disease, and that the continuation of treatment for one to two years is necessary. I then took twenty-five cases, which were as nearly as possible in the same stage, both as regards the disease and treatment, and tested their blood monthly for one year, continuing treatment intermittently throughout this period. The most paradoxical results were obtained, as the following reports on one case will show: 
CASE 144.-The patient had an intra-urethral chancre, a positive C.F.T., but no generalised symptoms when the treatment was commenced. After nine intravenous injections of neo-salvarsan, which from first to last were completed in fifty-one days, the C.F.T. was negative, and remained so for three months. The sixth month the serum itself fixed complement. The seventh month C.F.T. was negative, eighth month positive, ninth month negative, tenth month negative, elcventh month positive, twelfth month negative.

As time went on, I found that it was best to give the arseno-benzenc injections necessary in one course, as toxic symptoms were too apt to follow if another course was instituted later; that intermittent treatment had to be continued from one to two years, according to whether the case was in the primary or generalisation stage when the treatment was commenced, and that, in spite of this, and irrespective of the C.F.T., a certain percentage of cases recurred. As many of the recurrences were in the nervous system, I examined the C.s.F. of a series of cases after the completion of treatment, and there again obtained paradoxical results. Some patients with a pathological fluid had no clinical symptoms, some of the cases with clinical symptoms had a normal fluid, and the majority of those who had clinical symptoms got well whether treatment was given or not. As a few of the cases have developed degenerative nerve lesions since, I have come to the conclusion that a person presenting any nervous symptoms within a year or two of being thoroughly treated should have more drastic treatment still, but that it is not necessary to make a routine examination of the C.s.F., because it is not always wise to advise treatment when the fluid only is pathological. If treatment is stopped when the C.F.T. becomes negative, a rule which has been followed in the Army for the past few years, nearly all the cases will be found to recur sooner or later. Furthermore, when symptoms recur, they do not readily respond to arseno-benzene, and more than 2 per cent. develop toxic symptoms, some of which, of course, are mild. Considering that the treatment has not been in vogue very long, and that many who have had it have since been killed, it was somewhat alarming, when I examined 1,000 consecutive admissions of syphilis into a venereal hospital, to find that 20.0 per cent. had already had a full course of what may be termed official treatment. The cases are most apt to relapse between the third and fifth months after the completion of the course. From all this I trust the reader will sec that tests must take a subordinate position to clinical knowledge. In this connection the following case will prove of interest:

CASE 145.-A man had a primary sore on the corona in 1910. He had no rash or other symptoms, and the C.F.T. was negative. He was treated in Berlin, and received seven intravenous injections of salvarsan, and an intramuscular injection of mercury weekly for two months. On six different occasions since the blood has been negative. In 1918 he came to me with a sore on the same site as the original. It was a typical chancre, and a recurrent one. The question of a second infection could be put out of court. 


\section{Syphilis in Women.}

A positive reaction in a woman, especially if she has never been pregnant, should be looked upon as evidence that the patient has had syphilis, because a woman who has had syphilis is liable to infect her child in utero, whether she has been wcll treated or not, and whether the father of the child has had the disease or not. This being the case, it is necessary for such a woman to be treated throughout the whole period of each and every pregnancy irrespective of the courses she has already undergone, as such advice given and acted upon will assure the birth of a healthy child. A positive reaction in a woman who has been pregnant is of less value, because many women, especially those who develop toxic symptoms of pregnancy, form a sufficient increase of globulin in their blood as to give a positive C.F.T. in spite of never having had syphilis. A negative reaction is of less value than in any other case, because such a result is not uncommon in cases where the infection was conceptional, and the influence of pregnancy may make a reaction negative, when it would have been under ordinary circumstances positive, because the more protein manufactured the greater its lipoidal character. This may result in the presence of an unsaturated fatty acid, which prevents an alteration of surface tension, or, in other words, a positive C.F.T. Syphilitic women may fail to give a positive reaction until after the child-bearing period is over.

\section{Congenital Syphilis.}

A positive reaction signifies that the patient has had syphilis, but in one who has been well treated and shows no signs or symptoms a positive reaction should not be regarded as an indication for treatment for several reasons: (1) treatment has little influence upon the reaction; (2) the reaction tends ultimately to become negative of its own accord, as the disease in the majority of cases appears to die out, especially in those cases which had active symptoms early in life; (3) there is no evidence to show that treatment will arrest Syphilis congenita tarda ; (4) a patient with Syphilis congenita tarda will give a positive reaction, irrespective of treatment, for the remainder of life.

A negative reaction in a child-syphilis suspect, born healthy, does not exclude syphilis, as the blood may first become positive just prior to the onset of clinical manifestations. As a rule, a child who is going to present symptoms will give a positive reaction before the sixth month, and usually before the second month. We do not know yet whether such a suspect giving a negative reaction is liable or not to develop Syphilis congenita tarda.

\section{Syphilis of the Nervous System.}

We must divide the subject into early and late syphilis, and the former into treated and untreated.

In early and untreated syphilis, any lesion in the central nervous system is almost certain to be accompanied by a positive C.F.T. in the blood, with 
either a positive or a negative C.s.F. If the spinal fluid is normal, then the nerve lesion is purely vascular (hemiplegia, paraplegia, etc.); if positive, then probably meningeal. In early and treated syphilis a vascular nerve lesion is most likely to be due to the arsenic, in which case the hemiplegia or paraplegia will most likely set in within a month after the last injection, and the pathological tests may be negative or positive. A nervous lesion setting in about the twelfth week after the completion of the course is nearly always accompanied by a negative C.F.T. in the blood, and a markedly pathological spinal fluid.

In late syphilis, a negative C.F.T. of the blood means nothing. A positive suggests either a vascular lesion or a degenerative lesion in the brain or cord in the order named.

In cases of syphilis of the nervous system, an examination of the C.s.F. is more valuable than one of the blood, because it will frequently indicate the nature of the lesion.

A positive spinal fluid in the majority of cases means active trouble, but does not in all cases call for treatment. In late cases of syphilis when the patient presents systemic gummata, pathological changes may be met with in the C.s.F., without there being any nervous manifestation. A negative spinal fluid in the majority of cases means that there is no active trouble, but it by no means precludes the possibility of its incidence at some future date. In some vascular lesions a negative fluid is not at all uncommon. Gummata in the substance of the brain, unconnected with the meninges, may not affect the fluid, and the fluid may be negative in severe cases of meningo-myelitis, when the dura and pia have become stuck together and adherent to the cord at a level above the place where the lumbar puncture has been made.

The main object to aim at when dealing with nervous syphilis is to determine whether the lesion is a degenerative one or not. This is important from the point of view of both treatment and prognosis. Clinically, it may be difficult to determine, and upon these cases an examination of the C.s.F. will often throw light. There are four tests usually employed, which will be considered separately.

1. Cytological Test.-A high cell count points to a meningeal lesion, and a low one to a degenerative lesion. In all cases the cells are mainly lymphocytes. The presence of polymorphonuclear and endothelial cells suggest meningeal trouble, while the presence of plasma cells and embryo lymphocytes suggest degenerative trouble. A cell count of over 2,000 per c.mm. occurs only in cases which have been treated recently with arseno-benzene and mercury.

2. Protein Test.-An excess of globulin points to a nervous lesion. When the excess of albumin is greater than that of globulin the lesion is probably meningeal, and when vice versâ it is almost certainly degenerative-in fact, in some cases of degenerative encephalitis the protein may be practically all globulin.

3. Complement Fixation Test.-A positive reaction in undiluted and low dilution only is in favour of a meningeal lesion, while if the reaction is positive 
in almost any dilution, then the lesion is undoubtedly degenerative. A low Dujardin's index is almost pathognomonic of a meningeal lesion, and vice versa

4. Gold-sol Test.-Broadly speaking, in cases of degenerative encephalitis and myelitis, the first one, two, or three tubes show complete precipitation of colloidal gold, and the rest are normal, while in meningeal affections the curve is irregular.

In order to enable the physician at a glance to tell whether he is dealing with a degenerative or a non-degenerative lesion, I append the following table:

\section{Degenerative.}

Globulin increased ont of proportion to the albumin. May be a diminution of albumin.

Cell count nearly always less than 100 cells per c.mm.

Cells are lymphocytes, plasma cells, and embryo lymphocytes.

Oxydase reaction may be present.

"Step-ladder" gold-sol curve obtained in over 90.0 per cent. of cases.

C.F.T. almost always positive in blood.

C.F.T. almost always positive in C.s.F., even when only a weak dilution of fluid is used.

Reducing action demonstrable at once.

\section{Meningeal.}

Albumin increased more than the globulin. In about $50 \cdot 0$ per cent. of cases there is an excess of globulin.

Cell count usually more than 100 cells per c.mm.

Cells are lymphocytes, polymorphonuclear leucocytes, and endothelial cells.

Oxydase reaction not present.

Curve, if present, is irregular; and it is often absent.

C.F.T. frequently absent in blood.

C.F.T. only positive in C.s.F. when strong dilutions of fluid are used.

Reducing action usually delayed, or may even be absent.

\section{Cuti-Reaction.}

The cuti-reaction is of very little diagnostic value. When negative, it means nothing; and when positive, the result must not be interpreted until various factors have been considered, and even then it only suggests that the patient has had syphilis. A positive reaction may be obtained in a normal patient who has had a similar test made before, either with luetin or even with tuberculin, and in one who has been taking iodine internally, etc.

\section{Test of Cure.}

Having read this, the reader will probably ask, How can you tell when a syphilitic patient is cured? The correct answer is that it is never possible to tell when any individual patient is cured; only generalities can be made and applied to this or that individual, according to the patient's outlook and the physician's experience. I thought at one time that the provocative injection of arseno-benzene, preceded and followed by C.F.T.'s, would prove of value, but the results obtained were not very accurate, which is only to be expected when we realise that nothing is known of the life of organisms when dormant, or of the resistance the host puts up during this period.

All we can say is that patients treated in the primary stage as outlined 
in Chapter XVIII. are practically certain to be cured, and that the likelihood is not nearly so great if they had entered the generalisation stage before treatment was commenced. In the recurrent stage the treatment should be symptomatic, as a cure in the strict sense of the word is out of the question, as being quite incapable of proof. In view of the widespread belief in bloodtests by the public, in whose minds a positive reaction means active syphilis, and a negative reaction means a cure, the physician must be doubly careful in interpreting the results, as I have known of suicides committed by sound men, who had been well treated, and required no more, merely because the blood was positive, and the physician had concurred with the lay interpretation thereof.

\section{Gonorrhœa.}

As the C.F.T. is fresh in our minds, we will consider this first, as I see a period ahead during which the test may be advised preceding and following a provocative injection of vaccine, before a patient is to be pronounced cured. In 1912, in association with Klein, I attempted to regulate the treatment with vaccines by the C.F.T., injecting the vaccines intravenously or intramuscularly until the test became negative. In all the cases I have been able to follow up the test has since become positive, and in most symptoms have recurred. It is true, most of the patients had arthritis; therefore the suggestion of the inability to gauge a cure by the C.F.T. might be said not to be applicable when the disease is limited to the genital organs. When the disease has not been metastatic, a negative C.F.T. is apt to be obtained, in spite of an active local lesion. If positive, it can be assumed that the patient has had gonorrhœa, and presumed that the lesion is either dormant or active; but not necessarily that the patient requires treatment. Although the test is more accurate and specific than the Wassermann reaction, it is not reliable as an absolute test of cure.

It must be remembered that the C.F.T., even when specific, when positive, only denotes the presence of a protective substance. The protective substance formed by the host when the disease is active probably exists beyond the time when it has ceased to be active. Moreover, once the host has had to form a protective substance, the manufacture of the same is probably continued, after the death of the parasite, for a period which is determined by the length of time the parasite was alive and active. These are problems which are required to be settled before a positive C.F.T. can be regarded as necessarily indicative of active disease. Clinical experience is a better guide by which to judge whether a patient requires more treatment or not. Owing to the ravages gunorrhœa has made, and to the appalling increase of complications which have occurred of late owing to too much treatment, coupled with this the fact that we have no treatment which has such a quietening effect on the lesions as arseno-benzene has in syphilis, I fear for the time when 
the C.F.T. is to be advised as test of cure. What is the physician to do to get the test negative, and what proof has he that it will not become positive again? Finally, a positive C.F.T. provoked by vaccines is not an indication of the patient's protective capacity, because the immunity so produced is not the same as that the body itself forms against the living bacteria therein.

Let us now consider the search for the organism. The pathologist says every case of gonorrhœa should receive treatment until the gonococcus avoids detection, but he will not tell us how many examinations we are to make, as the hundredth slide may be the first to reveal their presence. He has also not made it clear that in 80 per cent. of chronic cases the organism, although present, cannot be found. He has not realised that this advice in the hands of enthusiasts has led to the over-treatment of the disease, the manufacture of a flourishing nidus for the gonococcus, and the increase of a very large number of cases which never will be cured. What evidence is there that we ever exterminate the organisms causing a disease? What do we know of organisms in the latent stage, or of the host during the period of carrying them? In what other diseases is a similar advice given? Practically none; and yet we seem to get along all right. What a blessing it is that puncturing the gall-bladder is a major operation, for, if a simple procedure, doubtless it would have to be done before a sufferer from typhoid was regarded as cured. Even if such a measure were practicable, and typhoid bacilli were found, we have no treatment guaranteed to exterminate them; the same with gonorrhœa. From the experience I have had with gonorrhœa, I have come to the conclusion that a clinical examination is more trustworthy than a pathological one upon which to base an opinion re the safety of resuming marital intercourse. There is no certain test of a cure. Prolonged attempts to exterminate the gonococcus more often than not do more injury to the cells of the host, and drive the organisms deeper into the tissues; and, finally, is it not possible that the type of gonococcus found in some of the chronic cases is non-pathogenic? We have the non-pathogenic meningococcus; and clinical experience would lead to the view that the same occurs in gonorrhœa.

Although I have always done my own pathological work, and have striven roughly six hours a day for several years to unravel the mysteries of the various tests employed, I am more than ever convinced that the clinical part of the subject is the more important. Therefore, in closing this volume I do beg those who are dealing with venereal diseases to pay more and more attention to their clinical aspect. 
I wish heartily to thank my colleagues at the London Lock Hospital for the great help which they have given me in so many ways. My gratitude is also due to all those gentlemen from whose publications $I$ have derived so much inspiration and valuable information. I desire also to express my indebtedness to Mr. S. A. Sewell for the beautiful plates which he has drawn for me; to Mr. J. Patterson and to Mr. J. Ward for their work in the many chemical preparations which they carried out for me; to Mr. J. Patterson for so kindly reading through the proofs for me; and to my Publishers for the interest they have shown in the work and for the assistance they have so freely given me. 
. 


\section{INDEX}

A BDERHALDEN's reaction, 179

Abdominal viscera, syphilis 87

Abducens, syphilis of, 140

Abortion, gonococcal endometritis cause of, 340

Abortions, successive, as sign of syphilis, 181

Abscess, Dubois', of thymus, 194 formation of, after calomel injections, 215

after intramuscular injec. tions, 204, 205

gonococcal, 332

differential diagnosis from chancre, 25

of Bartholin's glands, 339

prostatic, 296,297

bursting of, 298

Acetyl-salicylic acid, 259

for relief of reactions after intravenous injections, 209

Achilles tendon, pain in, $\mathbf{3 1 3}$

Acne rosacea, 223 syphilis cause of, 38

vulgaris, aggravated by arsenobenzene, 240

Acolne, 217

Adam's cream, 216 prescription for, 216

Adenitis, inguinal, in acute gonorrhœa, 323

suppurative, diseases causing, 271

suppurative, recurrent, 262

Adenoids and congenital syphilis, 189

Adiposity and loss of sexual func. tion, 369

Adrenalin in treatment of reaction-

1 ary inflammation, 124,258

in treatment of shock, 233

Adrenals, syphilis of, 97

Atrican nations, syphilis among, 3

Alcohol, contra-indicated during mercurial treatment, 220

Aleucæmic cutaneous lymphocytomata, syphilis causing, 38

Alimentary galactosuria, 95

Allergy, 230

Alopecia, effect of arsenic or mercury, 247

syphilitic, 29
Amblyopia, atoxyl, 103

America, history of syphilis in, 1

Amnésie syphilitique, 152

Amyl nitrite, inhalations of, 260

Amyotrophic lateral sclerosis, 134

Amyotrophy, spinal remitting, 133

Anæmia and congenital syphilis, 193

pernicious, syphilis cause of, $\mathbf{5 5}$ secondary, in syphilis aggravated by mercury, 59

splenic, congenital syphilitic, 193

Anæsthesia in degenerative myelitis, 163

Anaphylaxis, 230

Aneurysms in congenital syphilis, 189

Animal extracts, use of, 202

Animals, syphilitic inoculation of, 7,8

Annular syphilide, 30

Antiluetin, 214, 268

Antlmony, colloidal, 268

in treatment of bilharzia, 214

in treatment of Granuloma inguinale, 363

in treatment of syphilis, 214

preparations of, antiluetin, 214 colloidal (collosol), 215 tartar emetic, 214

Anuria, symptom of acid intoxica. tion, 246

Anus, chancre of, 23

Aortitis, association of degenerative nerve lesions with, 48

Döhle-Malmsten's, 48 syphilitic, 47

characteristic features, 48

in degenerative myelitis, 164

Aphthous ulcers, differential diagnosis from chancre, 176

Arbor mirabilis, 202

Argyria, 328

Argyrol, 285

in treatment of gonorrhœal con. junctivitis, 328

Arsacetin, toxic action of, 203

Arsalytes, with halogen substitu tion products, 212

Arsenic in nervous syphilis, other methods of administering, 211
Arsenic in treatmentof syphilis, 202 poisoning by, neurasthenia symptom of, 373

preparations of, 203

arsphenamine, 203

diarsenol, 203

kharsivan, 203

neosalvarsan, 203

salvarsan, 203

pseudo-toxic symptoms of, 235

toxic symptoms of, 228

true toxic manifestations of, 238

Arsenical arteritis, 243

compounds (French), 212

galyl, 212

Kolle's division of, 212

luargol, 212

ludyl, 212

dermatitis, 239,244

conditions resembling, 239

hepatitis, 241

intoxication of nerve tissue, 244 nephritis, 242

Arseno-benzene, action on spirochætæ, 9

advantages of soluble over in. soluble salt, 208

and blindness, 103

compounds, 212

in treatment of syphilis, 203 varieties of, 203

concentrated form, 208

conditions aggravated by, 245

injections of, repeated, 10

intra-arterial injection of, 209

intramuscular injection of, technique for, 233

intravenous injection of, immediate effects of, 230

technique for, 205

in treatment of infantile syphilis, 256

shock following intravenous in. jections of, 230

Arseno-benzenised scrum, intra thecal injection of, 210

Arseno-phenyi-glycine, toxic action of, 203

Arsphenamine, 203

Arterial syphilis, 256

treatment of, 257

Arterles, a ffection \& of, in congenita l syphilis, 189 
Arteries, lesions of, in syphilis, 46

Arterltis, 46

arsenical, 243

arterio-sclerotic, 130

in congenital syphilis, 189

obliterans, syphilis cause of, 49

syphilitic, differential diagnosis of, 47

Arthralgia, 317

Arthritis, 275

deformans, 63

gonococcal, 313

Arthritis sero-fibrinosa, 315

Bier's treatment in, 318

classes of, 315

drugs in treatment of, 318

Hydrops articuli, 315

other conditions accompany. ing, 314

phlegmonous arthritis, 315

purulent arthritis, 315

Spondylitis deformans, 317

treatment of, 317

multiple, and congenital syphilis, 183

phlegmonous, 315

psendo-membranous, 316

purulent, 315

sero-fibrinosa, 315

syphilis as cause of, 188

syphilitic, 62

Arthropathy in degenerative myelitis, 164

Ascites and congenital syphilis, 191,193 pseudo-chylous, 98

Asia, history of syphilis in, 1

Aspirin, 259

Ataxia in degenerative myelitis, 161,163

Atophan in gonococcal arthritis, 318

Atoxyl, toxic action of, 203

Atrophy, acute yellow, of liver, 241

Atropine, 259

in treatment of shock, 233

Auditory nerve, syphilis of, 141 tests for, 142

Aural lesions, aggravated by arseno-benzene, 245

Auto-reinfectlon, 22

Bacillus coli causing urethritis, 326 in secondary infection of Cystitis gonorrhoica, 303

Ducrey's, 261

Bacteria causing urethritis, 326 toxicity of injections increased by, 229

Balanitis, 356

circinata, 333

differential diagnosis of, 359

erosiva et circinata, 357

erosiva et gangrenosa, cause of phimosis, 353

clinical features, 358

differential diagnosis from chancre, 25
Balanitis erosiva et gangrenosa, organisms causing, 358

treatment of, 359

gangrenosa, 264, 357

simple, 356

treatment of, 357

Ballenger's " sealing-in " abortive treatment of urethritis, 286

Balteum venereum, 201

Banti's disease and congenital syphilis, 192

Bárány's tests, 107

Barlum chloride in treatment of shock, 233

Barker's needle for lumbar puncture, 122

Bartholinitis, 338

chronic, 339

treatment of, 343

Bartholin's glands, abscess of, 339 infection of, 339

Baths in treatment of syphilis, 219 in treatment of Ulcus molle, 269

Bed-sores after metallic treatment, 243

Benda's syphilitic aortic sclerosis, 48

Benzoate of mercury, 217

Bier's treatment in gonococcal arthritis, 318

Bile ducts, catarrh of, in syphilis, 91

Bilharzia, antimony in treatment of, 214

Bismuth, urethral injections of, 325

Bladder, affections of, and nervous involvement, 85

infection in, in gonorrhœa, 276 syphilis of, 84

Blastomycosis, differential diagnosis from syphilis, 40

Blindness, arseno-benzene and, 103

Blood changes in syphilis, 53

count in syphilis, 54

pressure, effect of syphilis on, 51 effect on, of intravenous injections of arseno-benzene, 230

syphilis of, 41

Bloodvessels, gonorrhœa of, 322

Bone, lesions of, syphilitic, in fluence of irritation in origin, 61

Bones, affections of, in congenital syphilis, 186

gonorrhœa of, 321

gummata of, in congenital syph. ilis, 188

long, swellings of, in congenital syphilis, 187

rarefaction of, in congenital syphilis, 188

syphilis of, $1,2,58$ course in long and flat, 59 mercury and, 59

"Born half-baked," origin of term, 180

Bougie à boule, 279

Bowel,gummatous ulceration of, 92
Bowel, lesions of, more common in congenital than acquired syphi. lis, 191

Brachial plexus, syphilitic neuritis of, 136

Brain, defective development of, in congenital syphilis, 194

Breast feeding in congenital syphilis, 254

Bronchi, syphilis of, 74

Bronchial catarrh, syphilitic, 74

Bronchitis, syphilitic, 74

Brown-Séquard symptom conıplex, 154

Bubo, 269

deferred appearance of, 262

operative treatment of, 270

organisms found in, 270

pathology of, 270

treatment of, 270

Bubonulus, 269

Buller's shield, 328

Bursæ, syphilitic lesions of, 63

Bursitis, 312

Calcium chloride in treatment of shock, 234

Calomel, 215

abscess formations after, 215

ointment cause of transient urethritis, 325

Cambell's bougie, 290

Camphphenol, 268

Carbollc acid, dangers of, 268

Carcinoma of tongue follcwing use of mercury pills, 21

Cardiac syphilis, 52

Caries, syphilitic cervical, 128

Catarrh of mucous membrane of nose in congenital syphilis, 189

Catheter à boule, 280

Guyon's, 285

Teevan's, 280, 301

Cauterisation, 268

of Ulcus molle, dangers of phagedæna, 268

Cavernitis, 279

colloidal iodine in treatment of, 294

gonorrhoica, 292

perifolliculitis developing into, 294

pyæmia following, 294

Cavernous urethritis, 277

Cells entered by spores, 6,10

Cerebellum, syphilis of, 131

Cerebro-spinal fluid, effect of drugs upon, 125

flow of, 125

infectivity of, 124

tapping of, importance of, 257 withdrawal of, 122

Cervicitis, 340

and endometritis, 340

Cervix, chancre of, 175 differential diagnosis of,' 175

erosions of, differential diagnosis from chancre, 175 
Chancre, 12 ætiology of, 4

bacteriological examination ( 1 . 13

development of organism 15

diagnostic points, 12

differential diagnosis of, 24

digital, 23

ecthymatous, 19

of syphilis in women, 175

effect of moisture on, 15

erosive, 16

clinical features of, 17

of syphilis in women, 174

experimental, 7

extragenital, 23

hepatic, 19

hypertrophic, 19,65

of syphilis in women, 175

induration of, 6

intra-urethral, 19

lenticular, of syphilis in women, 175

lymphatic, 261

of anus, 23

of cervix, 175

of lips, 23

of syphilis in women, 174 of mouth, 65

of tonsil, 71

on mucous membrane, 23

papulo-erosive, 17

papulo-pustular, of syphilis in women, 174

papulo-ulcerative, 17 simple, 18

phagedænic, 9,19

protective response of host, 17

pseudo-membranous, 19 of syphilis in women, 175

recurrent, 20,249

auto-reinfection in, 22

effect of metals on, 22

grades of, 21

gummata in, 22

immunity in, 21

simple and syphilitic, 4

sites of, 19

super-infection, 22 *

types of, 16

ulcerative, of syphilis in women, 175

Charcot's joint in degenerative myelitis, 164

pachymeningitis, 154

Charles VIII., disease following his campaign in Italy, 2

Cheiro-pompholyx resembling arsenical dermatitis, 239

Chemotherapeutic agents in treat. ment of gonorrhœa in women, 342

treatment of folliculitis, 293

of gonorrhœa, 344

principles of, 349

rationale of, 344

Chiari's Mesaortitis productiva, 48
Children, syphilitic, appearance when born, 181

arrest of development of, 181

vulvo-vaginitis of, 338

n. China, history of syphilis in, 1

Chlorose syphilitique, 41

Chorda venerea chronica, 363

Chorea and congenital syphilis, 197

Chorionic ferments, 178

Choroiditls, symptoms of congenital syphilis, 183,197

syphilitic, 101

Cirrhosis of liver and congenital syphilis, 193

atrophic, 93

Clavicles, osteoperiostitis of, 61

Cleft-palate and syphilis, 181

Club-foot and syphilis, 181

Coitus interruptus, cause of sexual neurasthenia, 369

cause of urethritis, 325

Cold applications in epididymitis, 307

in treatment of bubo, 270

in acute inflammatory conditions, 298

Colles' law, 171

Colloidal antimony, 268

(collosol) antimony, 215

hydrargyrum, 217

iodine, 224

iodine in gonococcal arthritis, 318

in treatment of cavernitis, 294

preparations in treatment of epididymitis, 308, 309

Colpitis in gonorrhœa in women, 338

Columbus, history of syphilis from date of his voyage, 2

Complement fixation test, 5

Conception, syphilis and, 169 C.F.T. in, 272

Conceptional syphilis, rationale of, 253

Condyloma acuminatum, 359 treatment of, 360

latum, 28, 245

in syphilis of intestines, 91

Condylomata, distribution of, in congenital syphilis, 184

Condy's fluid, discharge through use of, 325

Congenital syphilis, 180

affections of bones and joints, 186

of central nervous system, 194

of digestive tract, 191

of ears, 198

of eyes, 197

of female sexual organs, 192

of kidneys, 192

of liver, 191

of lungs, 190

of lymphatic glands, 199

of mucous membranes, 189

of spinal cord, 194
Congenital syphilis, afiections of spleen, 192, 193

of suprarenal glands, 192

of teeth, 185

of testicles, 192

of thymus, 194

of vascular system, 189

age incidence of different manifestations, 183

and malignant disease, 183

and rickets, similarity of bone changes in, 188

and tubercle, differential diagnosis, 188

" born half-baked," origin of term, 180

clinical and pathological parts, 396

contrasted with acquired form, 180

diagnosis of, 200

errors of development caused by, 181

generalisation stage, symptoms of, 182

historical data, 180

immunity and, 180

incidence of, 181

late stage (Syphilis congenita tarda), symptoms of, 182

latent stage, 182

mortality from, 180

prognosis of, 200

results of, 180

skin lesions of, 183

stages of, 182

to third generation, 181

treatment of, 254

tabulated, 256

syphilitics propagating syphilis, 181

Conjunctivitis, gonorrhœal, adult, 326

prevention of, 328

gonococcal, of the new-born, 329

infantile, 327

Connective-tissue cells entered by spores, 6

Constipation after intramuscular injections, 204

chronic, and sexual neurasthenia, 368

cause of urethritis, 325

Corona, primary sore in, with phimosis, 20

Corymbose syphilide, 26

Coryza in congenital syphilis, 189

Cowperitis, complications follow. ing, 292

gonorrhoica, 291

rectal gonorrhœa complicating, 291

symptoms of, 291

treatment of, 292

trimine in treatment of, 292

vaccines in treatment of, 292

Cowper's glands, a bscess formation in, 291 
Cranial nerve lesions in degenera. tive myelitis, 161

palsies in congenital syphilis, 196

nerves in syphilis, involvement of, order of, 10 -

syphilis of, 108, 137

Craniotabes in congenital syphilis, 188

Crises in degenerative myelitis, 182

visceral, diagnosis of, $16 \%$

Crystallina, 361

Cutl-reaction in syphilis, 400

Cutis maculce ephelidibus similis, 11

Cystitis, differential diagnosis from urethritis, 278

gonorrhoica, 302

differential diagnosis of, 303

symptoms of, 303

treatment of, 303

urine test in, 303

syphilitic, 83

urine in, 278

Cysts, retention, following cowperitis, 292

Cytological test in syplilis of nerrous system, 399

Dacryo-cystitis, syphilitic, 101

Dactylitis syphilitica, 167

Deafness in congenital syphilis, 198 in syphilis, 105

Decoctions used in treatment of syphilis, 202

Deferentitis, 306

Defuvium capillitii in congenital syphilis, 185

Degenerative encephalitis, 155 myelitis, 160

nerrous lesions in congenital syphilis, 194

Dementia paralytica, 155 proceox, 16.5

Dermatitis, arsenical, 205, 239, 244 exfoliativa resembling arsenical dermatitis, 239

herpetiformis, $\mathbf{3 3 4}$

scarlatiniforme resembling ar senical dermatitis. 239

Development, errors of, in syphili. tic children, 181

Diabetes insipidus in congenital syphilis, 196

in syphilitic nerrous affec. tions, 153

Diagnostlc signs in congenital syphilis, $18 t$

Diarsenoi, 203

Diday's irrigation, 285

Difference between syphilis in men and women, 178

Differential diagnosis of congenital syphilis, 200

Digestive tract, affections of, in Eczema, differential diagnosis from congenital syphilis, 190

Digital chancre, 23
Dilator, Kollmann's, 279, 287, 301 Dl-ortho - dl - thio - amino-benzene, 214

Di - para - anisyi - mono - phenetylguanidine hydrochloride, 217

Dlphtheroid bacillus causing urethritis, 326

Diphtheroids in secondary infec. tion of urethritis, 283

Discharge in urethritis, $27 \%$

Döhie-Malmsten's a ortitis, 48

Dolores osteocopi, 58

Douching in acute vaginitis, 342

Drugs, effect of, in cerebro-spinal fluid, 125

influencing regetative nervous system, 144

in treatment of acute urethritis, 284

of gonococcal arthritis, 31

of non-gonococcal urethritis, 326

of syphilis, 201

antimony, 214

arsenic, 202

preparations of, 203

arseno-benzene compounds, 230

historical data, 201

intramine, $202,214,222$

iodine, 202, 223

mercurialised serum, 217

mercury, 201, 215

methods of administration, 201

mode of action of, 225

nucleinate of soda, 224

of nervous system, 259

vanadium. 2.25

of Clcas molle serpiginosum, 268

tabulated list of, with mode of action, 226

Dubois' abscesses of thymus, 193

Ducrey's bacillus, 261

discovery of, 4

Ductless glands, syphilis and, 97

Duodenum, catarrh of, in syphilis, 91

Dupuytren's contracture, Induratio penis plastica associated with, 364

Dystrophia adiposa genitalis in congenital syphilis, 196

Ears, affections of, in congenital syphilis, 198

gonorrhœe of, 331

syphilis of, 100,105

Ecthyma of vacciniform dermatitis, 183

Ecthymatous chancre, 19 of syphilis in women, 175

Ectropion, 328 squamons syphilide, 33

in gonococcal proctitis, 311 sulphur, 202, 2?
Eczema, seborrhœic, resembling arsenical dermatitis, $\mathbf{2 3 9}$

Ehrilch's discovery of salvarsan, 5

Ejaculatio prizcox, 370

Eiectrolysis in treatment of gonor. rhœa, 290

in treatment of para-urethritis, 295

Elephantiasis nostras, rôle of syphilis in ætiology of, 43

Embolus, pulmonary, formation of after, intramuscular injections, 204

Emplastrum cinereum in treat. ment of congenital syphilis, 255

Encephalitis, degenerative, 151 259

effects of treatment, 156 in congenital syphilis, 195 infantile, course of, 195 prodromata, 155 prognosis, 155 symptoms of activity, 155 hæmorrhagic, 124, 131

Endarteritis, 1:28

neural, 130

obliterative, 10

obliterans in congenital syphilis, 189

premonitory symptoms of, 129 syphilitic, 48

Endocarditis, gonococcal, 322

Endometritis and cerricitis, $\mathbf{3 4 0}$ chronic, srmptoms of, 340 gonococcal, $3 \pm 0$

Endoscope, 279

Endoscopic examination of urethra, 279

Endotheliai cells entered by spores, 6,10

Enesol, 217, 259

England, epidemic of syphilis in, 3

Enteralgia, paroxysmal, in con. genital syphilis, 191

Enuresis, paroxysmal, in congeni. tal syphilis, 191

Epidemics of syphilis, 3

Epididymis, fibrous tissue forma. tion in. 307

Epididymitis, 275. 306

causes of, 306,307

symptoms of, 307

syphilitic, 85

treatment of, 308, 309

Epilepsy, $15^{\circ}$

and congenital syphilis, 196

Epiphysis, changes in, due to congenital syphilis, 186

macroscopical a ppearance in congenital syphilis, 187

pathological changes causing separation of, 187

Epiphysltis, symptom of congenital syphilis, 183

Epitheliomata, syphilis, 39:2

Erb's syphilitic spinal paralysis, 153 
Ergotin, 302

Eroslon, nature of, 7

Erosions of cervix, differential diagnosis from chancre, 175

of mucous membrane in con. genital syphilis, 190

Erosive chancre, 16 of syphilis in women, 174

Erythema induratum, differential diagnosis from syphilis, 40

multiforme, differential diag. nosis for palmar sy philide, $\mathbf{3 3}$ syphilis cause of, 18

nodosum, 333 syphilis cause of, 38

Erythemata, non-syphilitic, characteristic appearance of, 183 toxic, $\mathbf{3 3 3}$ and syphilis, 37

Española, syphilis in, 3

Excision of primary sore, 249

Exercise in Ulcus molle, danger of, 263

Exercises in treatment of tabes, 260

Experimental syphilis, 7

Experiments to prove identity of syphilis and gonorrhœa, 4

Extractum hydrastis liquidum, 302

Extragenital chancres, 23

Eyes, affections of, in congenital syphilis, 197

gonorrhœal disease of, 326 history, 327

syphilis of, 100

Facial nerve, syphilis of, 141

Female sexual organs, syphilitic affections of (congenital), 192

Fever, tertiary syphilitic, $\mathbf{9 4}$

Fibrolysin, 294

Flbromata and syphilis, 392

Flbrositis, syphilitic, $\mathbf{6 3}$

Fibrous tissue, formation of, 6,391

Flstula, penile, 293

urinary, following abscess of Cowper's glands, 291

Flat-foot, gonorrhœa and, 313

Fœtus, effect of syphilitic infection on, 180,181

infection of, by syphilis, 172

Follicular syphilide, 26

Folliculitis, 292

ichthyol, 293

treatment of, 294

Formalln, 343

Formol index in gastric ulceration, 90

Förster's operation, 259

Fossa navicularis, site for folli. oulitis, 293

Fractures, multiple, in congenital syphilis, 188

spontaneous, in degenerative myelitis, 164

France, epidemic of syphilis in, 3

French arsenical compounds, 212

Fumigation, mercurialism from, 219
Fundus oculi, changes in, in con. genital syphilis, 197

Funiculitis, 306 syphilitic, 86

Furunculosls aggravated by arseno-benzene, 240

Galactosuria, alimentary, 95

Galyl, 212

$$
\text { "1116," } 212
$$

Gangrene, symunetrical, in congenital syphilis, 184

Gastric pains, significance of, 90

Gelatinous atrophy of skull-bones in congenital syphilis, 188

General paralysis of insane, 130. See also Encephalitis, degenerative

Generalised stage, treatment of, 251

Genito-crural nerve, neuritis of, 321

Genito-urinary tract, syphilis of, 79

Genu incurvatum, 163

German syphilitic trinity, 5

Germany, epidemic of syphilis in, 3

Gingivitis, arsenic cause of, 239

Glands, suprarenal, syphilis of, 97

Glandular enlargement following infection, 9

Glaucoma, secondary, 328

Globulin test, failure of, after in. jection of salvarsanised serum, 211

Glossitis, superficial, chronic, following use of mercury pills, 221 syphilitic, 70

in degenerative myelitis, 164

Glosso-pharyngeal nerve, syphilis of, 142

Glycophilia, 50

Glycosuria, intermittent, syphilitic, 96

Gold in treatment of syphilis, 202

Gold-sol test in syphilis of nervous system, 400

Gonococcal abscesses, 332 differential diagnosis from chancre, 25

arthritis, 313

bursitis, 313

conjunctivitis of the new-born, 329

cystitis, 303

endometritis, 340

iritis, 330

proctitis, 311,337

prostatitis, 296

rashes, 332

classes of, 332

ulcer, 332

ulcers in women, 177

urethritis in women, 339

Gonococcl in gonorrhœa, spread of, 274

Gonorrhœa, 272
Gonorrhcea, acute and chronic, difference between, 279

anatomy of, 276

and marriage, 380

O.F.T. in, 401

chemotherapeutic and vaccine treatment of, 345

chronic, stages of, 279

clinical and pathological parts, 401

complications by extension, chemotherapeutic treatment of, 351

complications by metastasis, chemotherapeutic treatment of, 352

complications of, bursitis, 312

due to spread by metastasis, 312

gonococcal arthritis, 313 myositis, 320

gonorrhœa of bones, 321

of heart and bloodvessels, 322

gonorrhoal disease of nervous system, $32 \mathrm{I}$

tenosynovitis, 312

electrolysis in treatment of, 290

historical data, 272

incubation period of, 273

infection of bladder in, 276

of urinary tract, routes of, 310

influence of secondary infection, 282

in women, complications in urinary tract, $\mathbf{3 3 7}$

historical survey, 336

methods of infection, 337

spread of infection, 337

treatment of, 34l

manner of spread of organisms, 274

metastasis in, 274

of bloodvessels, 322

of bones, 321

of heart, 322

of muscles, 320

of the ears, 331

of the mouth, 330

of the nose, 331

pelvic, 341

prophylactic measures, 2831

prostate implicated, frequency of, 296

pus in, 274

"spurious," 356

subacute, treatment of, 289

symptoms of, 276

syphilis believed to be identical with, 4

treatment of, historical survey, 273

uncomplicated, 275

Gonorrhoal diseases of the eyes and other sense organs, 327

neurasthenia, condition of urine in, 371

symptoms of, 370,371 
Gonorrhoal diseases of the eyes and other sense organs, treat. ment of, 371

pus, experiments with, 4

Gonorrhoica cavernitis, 292

Granuloma inguinale, 362

treatment of, 363

pudendi, 362, 391

Graves' disease, syphilis cause of, 78

Greece, epidemic of syphilis in, 3

Grey oil, 216

Guaiacum wood, 202

Guanicaine, 217

Gumma, formation of, 10,32

Gummata, 22

differential diagnosis from chancre, 176

in degenerative myelitis, 164

in nervous syphilis, 135

miliary, multiple, in stomach, etc., in congenital syphilis, 191

of bones in congenital syphilis, 188

of congenital syphilis, 184

of heart muscle in congenital syphilis, 189

symptoms of congenital syphilis, 183

Gummatous ulceration of rectum, 92

Guyon's catheter, 285

Hæmaturia, 277

Hæmopoietic systen, syphilis of, 41

Hæmorrhagic encephalitis, 131

Hair, affections of, in congenital syphilis, 185

Hare-lip and syphilis, 181

Hayti, syphilis in, 3

Headache sign of pachymeningitis, 151

Headaches after lumbar puncture, 123

influence of posture upon, 123

Heart, gonorrhœea of, 322

syphilis of, 52

Hegonon, 285

Hemiplegia, 128, 321

after metallic treatment, 243

incomplete, 148

treatment of, 257

with bulbar paralysis, 132

with hemianopia, 132

with third, fifth, sixth, and seventh nerve paralysis, 132 with third nerve paralysis, 132

Heparlobatum, 94

Hepatic changes in congenital syphilis, 191

Hepatitis, a rsenical, 241

interstitial or indurative, 93

interstitialis et gummosa, 192

Hernia testis, 86

Herpes febrilis, 239

genitalis, 361

clinical course of, 361
Herpes genitalis, differential diag. nosis from chancre, 25 sexual neurasthenia in, 368 treatment of, $36^{\circ}$

oris, 68

preputialis, 19

zoster, 335

arsenic cause of, 239

in degenerative myelitis, 164 sign of syphilitic meningitis, 38

Herpetic chancre, 19

Herxheimer-Jarisch reaction, 23厅

Hexamino-salvarsan, 212

Hirsch's injection, 217, 259

History of syphilis from ancient times, 1-5

Hodgkin's disease, syphilis and, 55, 389

Holland, epidemic of syphilis in, 3

“Homén's familiar disease," 195

Host, protective capacity of, 8

"Hot-cross-bun" appea rance of calvarium in congenital syphilis, 187

"Humpy molar" teeth in con. genital syhpilis, 186

Hungary, epidemic of syphilis in, 3

Hunter's experiments, 4

Hutchinson's pill, composition of, 219

teeth, in congenital syphilis, 185

Hydrarthrosis in congenital syphilis, 188

Hydrocele in congenital syphilis, 192

Hydrocephalus, 181

due to syphilitic arteritis, 194

in congenital syphilis, 187 development of, 194

Hydrops articuli, 62, 315

Hydrosalpinx, 341

Hyoscine, 259

Hyperglycæmia, 81

Hyperkeratosis, toxic, 333

Hyperleucocytosis from injection of nucleinate of soda, 224

Hypersensitiveness, 230 cervical pachymeningitis, 154

Hypertrophic chancre, 19 of syphilis in women, 175

Hypoglossal nerve, syphilis of, 142

Hypopituitarism in congenital syphilis, 196

Ichthyol in folliculitis. 293

Icterus following metallic treat. ment, 242

gravis syphiliticus, 92,93

Idlocy and syphilis, 195

Immunity and congenital syphilis, 180

in recurrent chancre, 21

Impregnation of clothing in treat. ment of syphilis, 219

Incluslon bodies, role of, in urethritis, 326
Incubation period of gonorrhœa, 273

of syphilis, 6

Indla, history of syphilis in, 1,3

Induratio penis plastica, 363 association with Dupuytren's contracture, 364 syphilis not a cause of, 86

Induration, explanation of, 6

in syphilis, 391

Infants, syphilitic, feeding of, 254 Infection greater in early lesions of syphilis, 9

secondary, influence on chronic urethritis, 282

Infectivity of cerebro-spinal fluid, 124

Inflammation of middle lobe of prostate, 298

" reactionary," 235, 237, 258

treatment of, 258

syphilitic, secondary infection of, 187

Injection, intra-arterial, of arseno. benzene, 209

intramuscular, of arseno.benzene, technique for, 203

intraspinal, of mercurialised serum, 217

intrathecal, of arseno-benzenised serum, 210

intravenous, of arseno-benzene, 205

subdural and intraventricular, of prepared serum in nervous syphilis, 211

versus transfusion, 206

Insomnia, 244

Intermittent treatment of syphilis, 270

Internal administration of intramine, 223

Intertrigo, in vulro-vaginitis, 338

Intestinal tract, ulceration of in congenital syphilis, 191

Intestines, syphilis of, 91

Intoxication, mercurial, 246

Intra-arterial injection of arseno. benzene, 209

Intracranial reactionary inflam. mation, 235

Intramine, 222, 268 effect on syphilitic lesions of tongue, $70 \mathrm{rr}$

effects of, 222

for Acne rosacea, 223

internal administration of, 223 intramuscular injection of, 222 intra renous injection of, 223

in treatment of bubo, 271 of gonococcal arthritis, 318 of gonorrhœea in women, 342

of oral sepsis, 67

of syphilis, 202,214

in children, 256

of tenosynovitis, 312

of $\mathrm{Clcus}$ malle, 269 
Intramuscular injection of arsenobenzene, technique of, 203 of intramine, 222

of mercury, 215

syringe for (the Record), 216

Intraspinal injection of mercurial. ised serum, 217

of prepared serum under high pressure, prolonged, 211

Intrathecal injection, disadvantages of, 240

of arseno-benzenised serum, 210

Intra-urethral chancre, 19

Intravenous injection of arsenobenzene, preparation of salt, 205

reaction after, 209

technique for, 205

of intramine, 223

of mercury, 215

Inunction of mercury, 218 contra-indications to, 218

method of administration, 218

Iodex ointment, 294

Iodide rash, differential diagnosis

from syphilis, 40

Iodine, colloidal, 268 (collosol), 224

in treatment, 9,10 of syphilis, 202,223

preparations of, 224

Iodism, symptoms of, 223

Iodoform, 268

Iodoglidine, 224

Iritis, gonococcal, 100,330 in congenital syphilis, 197 syphilitic, 100

Italy, history of syphilis in, 2,3

Janet's wash-out, 304

Japan, history of syphilis in, 1, 3

Jaundice, catarrhal, 241

in congenital syphilis, 191

in syphilis, 91, 92

Joints, affections of, in congenital syphilis, 186

changes in, due to arthritis, 316

incidence of affection of, in gonococcal arthritis, 316

syphilis of, 58,62

Kaposi's axiom in diagnosis, 183

\section{Keratitls, 328}

interstitial, and congenital syphilis, 183

characteristic of late syphilis, 197

double, in congenital syphilis, 197

in congenital syphilis, 102

Keratodermia blennorrhagica, 332, 333

Kharslvan, 203

Kidneys, affections of, after injections of arsenic and mercury, 242

caused by syphilis, 389
Kidneys, affections of, in congeni. tal syphilis, 192 .

gumma of, 83 syphilis of, 79

Knee, tapping of, in Hydrops articuli, 315

joint, syphilitic affections of, 62

Kollman's dilator, 279, 287, 301

Korsakow's syndrome produced by syphilis, 167$$
\text { ' }
$$

Labour, premature, sign of syphilis, 181

Labyrinthitis in congenital syphilis, 199

Lallemand-Trousseau bodies, 297

Landry's paralysis, syphilitic vas. cular disease causing, 134

Larynx, syphilis of, 73

Lassar's paste in treatment of vulvo-vaginitis, 343

Latent stage of syphilis, treatment of, 252।

Lavage in treatment of acute urethritis, $284,285,286$

Lead musculo-spiral paralysis, 246

Lenticular chancre of syphilis in women, 175|

Leprosy and syphilis, 1

Leptomeningitis in congenital syphilis, 194

Leucocyte count in syphilis, 54

Leucocytes, changes in, in syphilis, 54

Leucocytozoon syphilidis, 253

development of, 113

differences of, 17

in conceptional syphilis, 170

life-history of, $6,7,8$

not a pus-producing organism, 32

spore of, 13

Leucoderma syphiliticum, 29

Leucoma, 328

Leucoplakia, 68

Lichen planus, 245

differential diagnosis from syphilis, 39

syphiliticus, 32

Lightning pains and crises in degenerative myelitis, 162

Lignum sanctum, 202

Lips, chancre of, 23

Littre's glands, infection of, 292

Liver, acute yellow atrophy of, 241 syphilis and, 93

affections of, after injections of arsenic and mercury, 241

caused by syphilis, 389

in congenital syphilis, 191

atrophic cirrhosis of, 93

late syphilitic lesions of, 93

syphilis of, 92

Livido, 28

formation of, 32

Lobelia syphilitica, 202

Locomotor ataxia, 160
Lotlons, evaporating, in treatment of bubo, 270

prescription for, 308

Luargol (" 102 "), 212

Ludyl (" 1151 "), 212

Luer's syringes, 207

Lumbar puncture, 122 amount withdrawn, 123, 124

headaches after, 123

purposes of, 123

technique of, 122

Lungs, affections of, in congcnital syphilis, 189

syphilis of, 75

syphilitic and tubercular affec. tions, differential diagnosis, 76

Lupus erythematosus, 31

vulgaris, facial scars of, differential diagnosis from syphilis, 31

Lymphadenitls, 269

Lymphangitis, 4l, 269

ex ulcere molli, 269

Lymphatic chancre, 261

glands, affections of, in congenital syphilis, 199

enlarged and hard, 12

enlargement of, 245

inflammatory adenitis of, 323 removal of, question of desirability of, 249

spirochætæ found in, 8

syphilitic, changes in, 42 suppuration of, 271

system, syphilis of, 41

Lymphatics, organisms spread along, 9

Lymphocytomata, intermediary, syphilis cause of, 389

syphilis and, 392 syphilitic, 55

Lymphoid extract, intramuscular injections of, 259

Macula corulea, 366

Macular syphilide, 184

Macule, 26

Malformations, congenital, sy philis and, 182

Malignant disease, congenital syphilis and, 183

relationship of syphilis to, 387 syphilis, 66

Mania, syphilitic, 165

Marrlage and gonorrhœa, cases with no recurrence or meta static lesions, 380

cases with recurrence or metastatic lesions, 380

negative bacteriological diagnosis useless, 380

and syphilis, influence of kind of sore, 378

location of disease on body, 379

recurrence and, $\mathbf{3 7 8}$

risks to children, 379

temperament of man in, 378

chronic prostatitis and, 300 
Marriage, gonorrhœa and, 380 syphilis and, 375

time limit fallacious, 375

venereal disease and, 374

Massage of prostate, 301 of seminal resicles, 306

I.IcDonagh's syringe, 207

Meatal para-urethritis, 295

Media and adventitia, round-celled infiltration of, in congenital syphilis, 189

Ménière's symptom complex, 108

Meningeal syphilis, 256 treatment of, 257

Meningitis, 257

and optic neuritis, 103

causing Amnésie syphilitique, 152

epilepsy, 152

generalised, after arseno-benzene treatment, 146

gonococcal, 321

lozalised, results of, in brain and cord, 152

producing optic neuritis, $151^{\circ}$

syphilis of pituitary gland and, 152

svphilitic, 145

acute cerebro-spinal, 25

symptoms of, 145

treatment of, 150

Meningo-encephalitis, 257, 258

Meningo-myelitis, 257, 258

Mental deficiency and congenital syphilis, 182

Mercurial intoxication, 246

inunctions in treatment of syphilis, 201

suppositories in treatment of syphilis, 201

Mercurialised serum, intraspinal injection of, 217

Mercurialism, degree of, stomatitis as guide to, 220

signs of, 220

Mercurio-intramine ointment, 7

Mercury, action of, in treatment of syphilis, 202

on spirochætæ, 9

administration by inunction, 218

and affections of bones, 59

internal use of, 219

intramuscular injection of, 215

intravenous injection of, 215

in treatment of syphilis, 215 baths, 219

fumigation, 219

history of, 201

impregnation, 219

of syphilitic infants, 255

oral administration of, disad. vantages of, 221

oxycyanide, 290

preparation of, Adam's cream, 216

benzoate of mercury, 219

colloidal (collosol) hydrar. gyrum, 217
Mercury, proparation of, enesol, 217

Hirsch's injection, 217

Hutchinson's pill, 217

Ricord's pill, 220

succinimide, 217

pseudo-toxic symptoms of, 235 salicylate of, 216

toxic symptoms of, 228

treatment of syphilis in 1514 by, 4

Mesaortitis productiva of Chiari, 48

Metals cause of oral sepsis, 68, 69 causing psychosis, 167

poisoning by, similarity of symptoms to syphilis, 389

Metastasís in gonorrhœa, 274 complications due to, 313

clemotherapeutic treatment of, 352

Metritis, syphilitic, 177

Metrorrhajia nematorum caused by Endarteritis obliterans, 192

Micrococcus catarrhalis causing urethritis, 326

Microdentism in congenital syphilis, 186

Micturition, disorders of, in syph. ilitic cystitis, 84

painful, in folliculitis, 293

Miculicz's disease, syphilis and, 55 syphilis cause of, 389

Mills' negative pressure catheter, 290

Molluscum contagiosum, 360 treatment of, 361

Mongolian idiocy, congenital syphilis cause of, 196

Morton's switchboard for zine ionisation, 268

Mouth, gonorrhœa of, 330 syphilis of, 65 chancre of, 65

Mucin, increased secretion of, 325

Mucous membrane, chancre on, 23 membranes, affections of, in congenital syphilis, 189

Muscies, atrophy of, syphilitic, 134 gonorrhœa of, 320

Muscular urethritis, posterior, 277

Myalgia, gonococcal, 320

Mycosis fungoides, 388

resembling arsenical dermatitis, 239

syphilis and, $5 \tilde{5}$ cause of, 38

Myelitis, 321

degenerative, 160,259

anæsthesia in, 163

ataxia, 163

cases of, 164,165

with ataxia predominating 161

with cranial nerve lesions, predominating, 161

with lightning pains predominating, 161
Myelitis, case; of, caused by congenital syphilis, 194

palsies in, 163

reflexes in, 163

trophic disturbances in, 164

Myocarditis, diffuse interstitial, in congenital syphilis, 189

in congenital syphilis, 189

Myositis, gonococcal, 320

Nails, affections of, in congenital syphilis, 184

brittleness and pitting of, 34

lesions of, 29

papule on nail-bed, 35

syphilitic affections of, 34

trophic conditions of, effect of arsenic or mercury, 247

National Council for combating Venereal Disease, formation of, 385

Necrosis, 10

in syphilis of skin, 14

poison of syphilis causing, 389 production of, 7

Neisser's bacillus, 273

discovery of the gonococcus, 4

Neosaivarsan, 203,212

Nephritis, acuta syphilitica procox, 80

arsenical, 242

balsamic, 310

forms of, 82

gonorrhœa cause of, 310

interstitial, incongenitalsyphilis, 192

parenchymatous, and gonococcal endocarditis, 310

protein, 81

syphilitic, 79

parenchymatous, 81

Nerve degeneration, 243

tissue, arsenical intoxication of, 244

metals toxic to, 257

Nerves, cranial, syphilis of, 137

Nervou; diseases of congenital syphilis contrasted with those of acquired syphilis, 195

system, central, affections of, in congenital syphilis, 193 gonorrhœal diseases of, 321 syphilis of, affections of cord, 133

Amnésie syphilitique, 152 arterial, 256

biology of, 109

Brown-Séquard symptom complex, 154

clinical and pathological parts, 398

aspect of, 128

evidence before and after treatment, 114-116

complement fixation test, 399

cranial nerves, 137

C.s.F.in. 121 
Nervous syi tem, syphilis of, cytological test, 399

degenerative encephalitis, 154

Diabetes insipidus, 153

differential diagnosis from other lesions, 131

drugs in treatment of, 259

epilepsy, 152

Erb's syphilitic spinal paralysis, 153

evidence as to increase of, 110

gold-sol test in, 400

gummata, 135

hemiplegia with bulbar paralysis, 132

with hemianopia, 132

with third, fifth, sixth, and seventh nerve paralysis, 132

with third nerve paralysis, 132.

indirect methods of treat. ment, 259

influence of resistance of host, 119

influence of several injections of arseno-benzene, closely together, followed by mercury for two years, 118

influence of several injections iof arseno-benzene with intramine, followed by mercury and iodine, 119

influence of spasmodic or inadequate treatment by arseno-benzene, supple. mented or not by mercury, 118

influence of treatment by mercury alone, 118

influence of treatment upon the incidence of, 116

influence on, of stage when treatment commenced, 120

lateral sclerosis, 154

meningeal, 256

meningitis, 145

myelitis, 160

neurotropic action of organism and neuropathic disposition of patient, 111

parts first involved, 113

peripheral nerves, 135

pituitary gland, 152

protein test, 399

psychic changes, 132

psychotherapeutic treat. ment, 259

recognition of, 5

spontaneous cure in, 120

stage at which nervous system becomes involved, 112
Nervous system, syphilitic throm - Orbicular syphilide, 30 bosis, 133

treatment of, 256

vegetative system, syphilis of, 142

Neurasthenia as symptom of arsenical poisoning, 373

gonorrhœal, symptoms of, 370

in arsenical neuritis, 244

sexual, 368

syphilitic, 370,372

war, 370

Neuritis, gonococcal, 321

of genito-crural nerve, 321

optic, syphilitic, 102

peripheral, 321

syphilitic, 135

and neurasthenia, 371

Neurophlebitis, syphilitic, 134

Neurorecurrence, intramine preventive 148

Neurotrophic action of organism and neuropathic disposition of patient in nervous syphilis, 111

Neurovascular system, syphilis of, 128

New-born, gonococcal conjunctivitis of, 329

Nisbet's chancre or bubonulus, 261

Nitritoid crisis, 230

Non-gonococcal urethritis, 324

causes of, 324

treatment of, 326

Nose, bleeding from, after injec. tions of arseno-benzene, 245

bones of, syphilitic disease of, 60

gonorrhœa of, 331

perichondritis of, 72

syphilitic periostitis of, 72

Nov-arseno-benzene, 212

Nucieinate of soda in treatment of syphilis, 224

Oculo-cardiac reflex, absence of, 105

Oculomotor nerve, syphilis of, 139

Edema, toxic, after intramus. cular injections, 204

Esophagus, stenosis of, syphilitic, 88

syphilis of, 88

Oifactory nerve, syphilis of, 137

Onychia sicca syphilitica, 34 syphilitic, 184

Onyxis craquelé, 34

Ophthalmoplegia externa, 140

externa and interna, syphilis causing, 103

interna, 140

Optic atrophy in congenital syph lis, 194

nerve, syphilis of, 137

neuritis, meningitis and, 103 syphilitic, 102

Oral administration of mercury, disadvantage of, 221

sepsis, causes of, 67
Orchltis, symptoms of congenital syphilis, 183

Orientai sore, differential diagnosis from syphilis, 40

Osteitis, 59

Osteochondritis syphilitica, Is6, 188

incidence of, 186

Osteomyelitis, 59

in congenital syphilis, 199

syphilitic, 61

and tubercular, differential diagnosis, 61

spontaneous fracture follow. ing, 61

Osteoperiostitis, 59

gummatous, 60

in congenital syphilis, 187

syphilitic, differential diagnosis of, 60,61

Osteoporosis in congenital sypliilis, 187

Otis's urethrometer, 279

Otitis blennorrhagica, 331

media in congenital syphilis, 198

Otoscierosis in congenital syphilis, 199

Oxaluria, 278

causing urethritis, 324

in gonorrhœal neurasthenia, 371

oxygen, inhalations of, in treat ment of syphilis, 202

Pachymeningitis, cervical, 136 gummatous, in congenital syphilis, 194

lumbar puncture for, 124

reactionary inflammation in, 236

Paiate, hard, ulceration of, in congenital syphilis, 190

high-arched, in congenital syphilis, 189

perforation of, diagnostic of syphilis, 187

syphilitic periostitis of, 72

Pallamine, 260, 288

in treatment of acute urethritis, 284,349

Palms, syphilitic lesions on, 33

Palsies in degenerative myelitis. 164

Pancreas, affections of, in congenital syphilis, 192 syphilis of, 95

Panophthalmitis, 328

Panosteitis, 61

Papula carnosa in fronte, 11

Papular syphilide, 184

Papule, 26 of primary sore, 16

Papules of mucous membrane in congenital syphilis, 190

Papulo-erosive chancres, 17

Papulo-pustular chancre in women, 174

Papulo-ulcerative chancres, 17 


\section{CLINICAL ASPECT AND TREATMENT OF VENEREAL DISEASES}

Paralyses in congenital syphilis, 195

Paraiysis, spastic spinal, in con. genital syphilis, 195

Paraphimosis, 353, 354

externa, 354

interna, 354

treatment of, 354

Paraplegia, 128

after metallic treatment, 243

incomplete, 148

treatment of, 257

Parasites, pathogenic, grading of, 390

Paraurethral canals, 295

Paraurethritis, 294 electrolysis in treatment of, 295

in women, 339

meatal, 295

Paronychia, 35, 36

desquamativa, 36

papule on nail-bed, 35

Parrot's nodes, 187

Pediculus pubis, 365 treatment of, 366

Pelvic gonorrhœa, 341

Pemphigus neonatorum, strepto. coccal, differentiated from syphilitic pemphigus, 184

syphilitic, differentiated from streptococcal Pemphigus neonatorum, 184

syphiliticus neonatorum, 184

Pericarditis, gonococcal, 323

Pericranitis in congenital syphilis, 187

Perifolliculitis, 292

chemotherapeutic treatment of, 293

colloidal iodine and intramine in treatment of, 289 complications after, 294 developing into cavernitis, 294 with abscess formation, 294

Perinæum, tenderness of, in gonorrhœa, 277

Perionychia, 35, 36 papule on nail-bed, 35

Periosteum, syphilis of, $\mathbf{5 8}$ thickening of, in congenital - syphilis, 187

Periostitis, ossifying, in congenital syphilis, 187 syphilitic, 58

Peripheral nerves, syphilis of, 135 symptoms, 9

Periphlebitis in congenital syphilis, 189

Peripylephlebitis syphilitica, 192

Peritoneum, syphilis of, 98

Periurethritis, 279, 294

Phagedæna caused by cauterisa. tion, 268

Phagedænic chancre, 9, 19

sores, 7,264

ulcer, 14

Phagocytosls, 6, 391
Phalanges of fingers and toes, enlargement of, in congenital syphilis, 187

Pharynx, syphilis of, 73

Phimosis, causes of, $\mathbf{3 5 3}$

clinical features, 353

primary sore in corona with, 20

Phlebitis, gonococcal, 323 syphilitic, 45

Phlegmonous arthritis, 315

Phosphaturia, 278

causing urethritis, 324

in gonorrhoeal neurasthenia, 371 urine in, 324

Phthirius inguinalis, 365

Pills in internal treatment of syphilis, 220

Pillula barbarossa, 201

Pilocarpin, 143

Pituitary extract in treatment of shock, 233

gland, affections of, in congenital syphilis, 196

syphilis of, 152

Pityriasis rosea, differential diagnosis from syphilis, 39

rubra resembling arsenical dermatitis, 239

Pleura, syphilis of, 76

Pneumonia, interstitial, in congenital syphilis, 190

Poison, syphilis as, in relationship to malignant disease, 387

Poisoning, metallic, chronic, 243

Poliomyelitis, chronic anterior, 133

Polyneuritis, peripheral, caused by mercury and a rsenic, 244 syphilitic, 135, 137

Porcince carnis solitce color, 11

Potassium iodide as an antisyphilitic drug, 4

Pregnancy and syphilis, statistics, .171

effect of syphilis on, 181

gonococcal vaginitis in, 339

syphilitic infection during, effect on fœtus, 181

Primary sore, excision of, 249 in corona, with phimosis, 20 of syphilis in women, 174 treatment of, 249

stage of syphilis, 6

Proctitis, gonococcal, 337

gonorrhoica, 311

symptoms of, 311

treatment of, 311

Profeta's law, 172

Proflavine, 290

Prostate, abscess of, 296

acute inflammation of middle lobe of, 298

chronic inflammation of, 299

examination of, methods, 296

implication of, in gonorrhœa, frequency of, 296

infection of, in gonorrhcea, 275

massage of, 301

syphilis of, 86
Prostatic abscess, 297

Prostatitis, 279, 296

chronic, 299

subjective symptoms of, 299

treatment of, 302

urine test in, 299

vaccines in, 299

gonococcal, 296

syphilitic, 86

urine in, 297

Prostatorrhcea, 302

Prostato - urethritis, chronic, secondary infection in, 282 treatment of, 301

Prostitution, legal, and public health, 383

Protargol, 285

Protein test in syphilis of nervous system, 399

Proteinuria, syphilitic, classes of, 80

Pruritis ani, symptom of gonococcal proctitis, 311

Pseudo-membranous chancre, 19 of syphilis in women, 175

Pseudo-toxic symptoms of arsenio and mercury, 235

intracranial reactionary inflammation, 235

visceral reactionary in. flammation, 237

Psoriasis, differential diagnosis from syphilis, 39 vulgaris, 334

Psychic changes in nervous syphilis, 132

Psychoses, syphilitic, 165

Psychotherapeutic treatment in syphilis of nervous system, 259

Ptosís, false, 328

Public health, venereal disease and legal prostitution, 383 preventive measures, 385 question of notification, 385

Pupils, inequality of, after treatment by arseno-benzene, 147

Purpura, syphilis cause of, 38

Purulent arthritis, 315

Pus in anterior cavernous urethritis, 27:

in gonorrhœa, 274

in posterior " muscular" ure. thritis, 277

in urine, 278

Pustule, similarity to varicella, 27

Pyæmia following cavernitis, 294

Pyelitis gonorrhoica, 310

Pyelonephritis gonorrhoica, 310

Pyorrhoa alveolaris, 67

Rash of congenital syphilis, char. acteristic appearance of, 183

location of, 183

symptom of congenital syphilis, 182 
Rashes, gonococcal, 332 toxic, 333

"Reactionary inflammation," 235, 237

Reactlons after intravenous injec. tions of arseno-benzene, 209

"Reagin," 395

Record syringe for intramuscular injections, 216

Rectal gonorrhœa complicating cowperitis, 291

Rectum, gonococcal inflammation of, 311

syphilitic stricture of, 91

Recurrent chancres, 20

stage, treatment of, 251

syphilitic eruptions, 30

Reflexes in degenerative myelitis, 163

irregularity of, after treatment by arseno-benzene, 147

Renal changes following intra. venous injection of arseno-ben. zene, 232

Resinol ointment in treatment of Herpes genitalis, 362

Retention cysts following cowperitis, 292

Retinitis pigmentosa sine pigmento, 105

syphilitic, 102, 198

Rheumatic cutaneous lesions, differential diagnosis from syphilis, 40

Rheumatism, syphilitic, 63

Rhinitis, symptom of congenital syphilis, 182

Rickets and congenital syphilis, similarity of bone changes in, 188

Ricord's pill, 220

Ringworm, chancre of syphilis of 1 mouth simulating, 65 differential diagnosis from re. current circinate lesions, 33

Roentgenoscopic test in gastric ulceration, 90

Romans, sexual life of, 1

Roseola, syphilitic, congenital, 183

Ruplal syphilide, 28

Russia, epidemic of syphilis in, 3

Russ's treatment of gonorrhœa, 290

"Saddle-nose" in congenital syphilis, 189

Salerno, school of, 383

Salpingitis, acute and bilateral, 340

Salvarsan, 203, 212

-discovery of, 5

Saprophytlc infection, 7

Sarcoma and syphilis, connection between, 55

Sassafras, decoction of, 202

Scables, differential diagnosis from syphilis, 39

Scars of congenital syphilis, .184
Schaudinn and Hoffmann's dis. Skin, syphilis of, formation of covery, 5

Schreiber's syringe, 207

Sciatic nerves, gonococcal neuritis of, 321 syphilitic neuritis of, 136

Sciatica accompanying arthritis of hip, 321

Sclerosis, lateral, 154

Scotiand, epidemic of syphilis in, 3 Scrofuloderma, 190

Seborrhœic dermatitis, differential diagnosis from syphilitic rash, 183

syphilide, 28

Seminal ducts, infection of, in gonorrhœa, 275

vesicles, massage of, 306

Sense organs, gonorrhœal disease of, 327

Serpiginous syphilide, 31

Serum, arseno-benzenised, intrathecal iujection of, in treat. ment of syphilis, 210

mercurialised, intraspinal in. jection of, 217

prolonged intraspinal injection under high pressure, 211

subdural and intraventricular injection of, in nervous syphilis, 211

Sexual desire, loss of, caused by arsenic or mercury, 247 diagnostic value of, 84

intercourse, infection resulting from, 326

neurasthenia, 368

adiposity and loss of sexual function, 369

chronic constipation and, 368

Coitus interruptus and, 369

forms of, 368

gonorrhœal form different

from syphilitic form, 369

varicocele and, 368

power, loss of, 369

Shock following intravenous injection of arseno-ben. zene, 230

cardiac form, 232

pulmonary form, 231

Silver salvarsan, 213

Skin, differential diagnosis of syphilitic rashes of other origin, 37

diseases simulating syphilis, 38 dryness and itching of, in late syphilis, 38

eruptions in the generalisation stage, 25

corymbose syphilide, 26 follicular syphilide, 26 macule, 26

papule, 26

stages of, 26

lesions of congenital syphilis, 183 syphilis of, 11

chancre, 12 erosion and ulcer, 13

gummata, 22

listory of, 11

necrosis, 14

phagedænic ulcer, 14

primary sore, 12

recurrent eruptions, 30

Skull-bones, syphilitic disease of 60

"Snuffles" in congenital syphilis, 189

Soda, nucleinate of, in treatinent of syphilis, 224

Soft sore, 261

differential diagnosis from chancre, 24

See also Ulcus molle

Sore auto-inoculable up to eleventh day, 6

primary, 7

in corona, with phimosis, 20

of syphilis in women, 174

papule of, 16

stages of, 16

treatment of, 249 soft, 261

Spain, epidemic of syphilis in, 3

Spaniards with Columbus, syphilitic disease among, 2

Spermatocystitis, chronic, cause of sexual neurasthenia, 305 gonorrhoica, 304

Spermatorrhœa, 305

Spes hominum, 202

Sphincter paralysis in congenital syphilis, 194

Spina bifida and syphilis, 181

Spinal accessory nerve, syphilis and, 142

column, syphilis of, 128

cord, affections of, in congenital syphilis, 194

Spirochata balanitidis, 16

pallida, 253

adult male form of, 8

discovery of, 5

in primary sore, 13

Splrochætæ, action of arseno. benzene on, 9

action of mercury on, 9

from experimental chancres, 7

Spleen, affections of, in congenital syphilis, 192

enlargement of, 96 symptom of syphilis, 182

syphilis of, 96

Spienitis and congenital syphilis. 193

syphilitic parenchymatous, 96

Spienomegaly in congenitalsyphilis, 193

parenchymatous, syphilis cause of, 389

Spondylitis deformans, 317

Spores, development of, 6,8 
Sporotrichosis, differential diagnosis from syphilis, 40

" Spurlous gonorrhœa," 356

Staphylococcl in secondary infection of urethritis, 283

Staphylococcus causing urethritis, 326

Slaphyloma, 328

Status epilepticus, 235

effect of colloidal palladium on, 144

Stenosis, intestinal, in syphilis, 91

Sterility, chronic prostatitis and, 300

syphilis and, 173

Stigmata of Syphilis congenita tarda, 182

Stomach, pains in, significance of, 90

syphilis of, 88

differential diagnosis from other diseases, 69

formol index test, 90

ulceration of, Roentgenoscopic test, 70

syphilis cause of, 89

Stomatitis after mercurial treatment, 246

guide to degree of mercurialism, 220

mercurial, 218, 220

Streptococei in secondary infection of urethritis, 283

rôle of, in syphilis, 44

Streptococcus causing urethritis, 326

Strlcture of rectum, gummatous ulceration causing, 92

subdural and intraventricular injection of prepared serum in ner. vous syphilis, 211

Subsidiary venereal diseases, 356

Succinimide of mercury, 217

suckling in congenital syphilis, 254

Suggestion in treatment of gonorrhœal neurasthenia, 371

Sulphoxylate compounds, 212

Sulphur in treatment, 9,10 of syphilis, 202, 222

preparations of intramine, 222

Suppositories, mercurial, in treat. ment of syphilis, 201, 219

Suprarenal glands, affections of, in congenital syphilis, 192 syphilis of, 97

Switzerland, epidemic of syphilis in, 3

Synostosis of sutures of skull, premature, in congenital syphilis, 188

Syphilide, annular, 30

congenital, 183

macular, topical distribution of, 184

palmar, 33

papular, topical distribution of, 184

recurrent, 30
Syphilide, recurrent, usual sites of, 33

serpiginous, 31

squamous, differential diagnosis of, 33

verrucose, 33

Syphills and conception, C.F.T. and, 252

and marriage, 375

and sarcoma, connection between, 55

and sterility, 173

ante-natal, statistics of, 171

antimony in treatment of, 214

as a poison, and its relationship

to malignant disease, 387

baths in treatment of, 219

blood-changes in, 53

cardiac, 52

cause of Erythema multiforme, 38

nodosum, 38

clinical and pathological parts, 394

amount of treatment, $\mathbf{3 9 5}$ date when patient last had treatment, 395

kind of infection, 395

question of recurrence, 395

relationship between treatment and C.F.T., 396

stage of disease at which treatment was com. menced, 395

conceptional, Leucocytozoon syphilidis and, 170

rationalc of, 253

congenita tarda, stigmata of, 182 congenital, 180

clinical and pathological parts, 398

treatment of, 254

cuti-reaction in, 400

d'emblée, 18, 23

epidemics of, 3

experimental, ulceration in, 7

facial scars of Lupus vulgaris and, differential diagnosis of, 31

fibromata and, 392

fumigation for, mercurialism after, 219

genital and extragenital infection, 12

gonorrhca believed to be iden-

tical with, 4

hereditaria tarda, treatment of, 255

history of, from ancient times, 1.5

impregnation of clothing for, 219

influence on arterial hyper. tension, 51

internal mercurial treatment, 219

intramine in treatment of, 214
Syphilis, intraspinal injection of mercurialised serum, 217

intrathecal injection of arsenobenzenised serum in treat. ment of, 210

in women, 169

clinical and pathological parts, 398

Colles'law, 171

conception and, 169

differential diagnosis of chancre from other lesions, 175,176

ecthymatous chancre of, 175 hypertrophic chancre of, 175 lenticular chancre of, 175 papulo-pustular chancre, 174 primary sore of, 174

differential diagnosis of, 174

stage, 174

Profeta's law, 172

pseudo-membranous chancre, 175

statistics of effect of antenatal syphilis, 171

syphilitic sores of external genitalia, 176

treatment of, 252

ulcerative chancre, 175

value of C.F.T. in, 173

iodine in treatment of, 223

lesions of arteries, 46

of veins, 46

lymphocytomata and, 392

malignant, 66

epitheliomata in, 392

mercury in treatment of, 215

mesenchymatous, 391

mode of action of drugs in treat ment of, 225

national names for, at different periods, 2

nervous, other methods of administering arsenic in, 211

prolonged intraspinal injection of prepared serum under high pressure, 211

subdural and intraventricular injection of prepared serum for, 211

nucleinate of soda in treatment of, 224

of abdominal viscera, 87 history of, 87

of bladder, 83

of bones, 58

of bronchi, 74

of cerebellum, 131

of cranial nerves, 137

of ears, 100, 105

of eyes, 100

of genito-urinary ,tract, 179

of intestines, 91

of joints, 58

of kidneys, 79

of larynx, 73

of liver, 92 
Syphilis of lungs, 75

of lympho- and hæmopoietic systems, 41

of mouth, 65

of nervous system, biology of, 109

clinical and pathological parts, 398 aspect of, 128

complement fixation test in, 399

cytological test, 399

gold-sol test in, $\mathbf{4 0 0}$

protein test, 399

treatment of, 256

of neuro-vascular system, 128

of cesophagus, 88

of pancreas, 95

of periosteum, 58

of peripheral nerves, 135

of peritoneum, 98

of pharynx, 73

of pituitary gland, 152

of prostate, 86

of skin, 11

of spinal column, 128

of spleen, 96

of stomach, 88

differential diagnosis from other diseases, 89

of suprarenals, 97

of testicles, 85

of throat, 65

of thyroid, 77

of urethra, 86

of vegetative nervoussystem, 142

origin of the term, 3

primary stage of, 6

"raw ham colour of," 11

rôle of streptococci in, 44

secondary, contagiousness of, demonstrated, 4

skin eruptions in the generalisa. tion stage, 25

stage at which nervous system becomes involved, 112

stages of, 8

sulphur in treatment of, 222

superinfection, 22

suppositories of mercury in treatment of, 219

tabulated list of most important drugs and their mode of action, 226

test of cure, 400

treatment of, by arseno-benzene compounds, 203

by drugs, 201

latent stage, 252

primary sore, 249

recurrent stage, 251

stage of generalisation, 251

tabulated, 250

vanadium in treatment of, 225

vaso-motor affections, 50

Syphilitic aortic sclerosis of Benda, 48

arthritis, 62
Syphilitic fever, conditions simu. lating, 94

lymphocytomata, 55

neurasthenia, 370,372

osteoperiostitis, 59

psychoses, 165

"Syphilitic triad," 198

Syringe, double-barrelled, for intramuscular injection, 204

for intramuscular injections, 216

Luer's, 207

McDonagh's, 207

preparation of, for intravenous injection, 208

Schreiber's, 207

Ultzmann's, 301

Tabes, 130

dorsalis, 160

failure to diagnose, and operations, 162

Tartar emetic, 214, 268

Teeth, affections of, in congenital syphilis, 185

" humpy molar," 186

"Hutchinson's teeth," 185

care of, in mercurial treatment, 220

Teevan's catheters, 280,301

Tendons, syphilitic lesions of, 63

Tenosynovitis, 312

treatment of, 312

Tertiary syphilitic fever, 94

Testicies, a fiections of, in congenital syphilis, 192

infection of, in gonorrhœa, 275 syphilis of, 85

Theca, tapping of, 122

Thiosinamine, 294

Thomson's detoxicated vaccine, 292

Throat, syphilis of, 65

Thrombo-angiitis obliterans, 49

Thrombosis following intravenous injections of arseno-benzenc, 230

from iodine injections, 224

syphilitic, 133

Thymus, affections of, in congenital syphilis, 193

Thyroid, syphilis of, 77

Tic douloureux, 163

Tinnitus aurium, 142

Tiodine, 223

Tissue, spores contained in, 8

Tobacco contra-indicated during mercurial treatment, 220

Tongue, carcinoma of, following use of mercury pills, 221

changes in, in syphilis, 69

Tonsil, primary sore in, differential diagnosis of, 71

Tonsillitis and chancre, differential diagnosis, 71

Toxic manifestations of arsenic, arsenical dermatitis, 239
Toxic manifestations of arsenic, hepatitis, 241

true, 238

rashes, 333

symptoms after antimony injec. tions, 214

after intramuscular injections, 205

of arsenic and mercury, 228

arsenical arteritis, 243

intoxication of nerve tissue, 244

nephritis, 242

general considerations, 228

inmediate effects of in. travenous injection of arseno-benzene, 230

mercurial intoxication, 246

pseudo-toxic symptoms on third day, 235

relation of bacteria to, 229

shock, 230

Traumatic lesion, differential diagnosis from chancre, 24

Treatment, effect of, in various stages of congenital syphilis, 182

influence of, upon incidence of syphilis of nervous system, 116

of acute and subacute urethritis, 350

vulvitis, 342

of adult gonorrhceal conjunctivitis, 326

of Balanitis erosiva et gangrenosa, 359

of Bartholinitis, 343

of bubo, 270

of chronic prostatitis, 302

prostato-urethritis, 301

of Condylomata acuminata, 360

of congenital syphilis, 254

of cowperitis, 292

of epididymitis, 308, 309

of gonococeal arthritis, 317 cystitis, 303

of gonorrhœa, 283

chemotherapeutic, 344

historical survey, 273

in women, 341

vaccine, 344

of gonorrhœal ncurasthenia, 371

of Granuloma imguinale, 363

of Herpes genitalis, 362

of latent stage of syphilis, 252

of meningeal syphilis, 257

of Molluscum contagiosum, $36 \mathbf{1}$

of non-gonococcal urethritis, 326

of paraphimosis, 354

of paraurethritis, 295

of Pediculosis pubis, 366

of phimosis, 353

of primary sore, 249

of Proctitis gonorrhoica, 311 
Treatment of reactionary inflam. mation, 258

of recurrent stage of syphilis, 251 urethritis, 350

of shock after intravenous in. jections of arseno-benzene, 233 of simple balanitis, 357

of stage of generalisation of syphilis, 251

of syphilis, 249

drugs used in, 201

history of, 4

in women, 252

mercury in, 201

of nervous system, 256

of syphilitic meningitis, 150

of tenosynovitis, 312

of Ulcus molle, 268 serpiginosum, 268

of urethritis, 283

of vesiculitis, 305

of vulvo-vaginitis in children, 343

Trigeminal nerve, syphilis of, 140

Trimine, 268, 289

in gonorrhœal conjunctivitis, 329

in treatment of acute urethritis, 284

of bubo, 271

of cowperitis, 292

Trochlear nerve, syphilis of, 140

Trophic disturbances in degenerative myelitis, 164

Tubercle and congenital syphilis, differential diagnosis, 188

Tuberculldes, differential diagnosis from syphilis, 40

Tuberculosis, pulmonary, and congenital syphilis, 190

Tuberculous ulcers, differential diagnosis from chancre, 176

Tumor albus, 63

Ulcera mollia, 332

cervical chancre simulating, 175

differential diagnosis from chancre, 176

pseudo-venerea, 176

syplilitic sores mistaken for, 176

Ulceration in experimental syphilis, 7

of hard palate and jaw-bones in congenital syphilis, 190

of mucous membrane in congenital syphilis, 190

of stomach, syphilis cause of, 89

Ulcerative chancre of syphilis in women, 175

Uleers, aphthous, differential diagnosis from chancre, 25,176

gonococcal, 332

non-syphilitic, resembling gum. mata, 163

perforating, in degenerative myelitis, 164
Ulcers, phagedænic, 14

in Ulcus molle, 263

tuberculous, differential diagnosis from chancre, 176

Ulcus blennorrhagicum, 332 molle, 261

cause of, 261

clinical characters of, 263

elevatum, 263

exercise in, dangers of, 263

general characteristics of, 261

history, 260

lymphangitis in, 262

miliare, $26 \pm$

phagedanicum, 264

serpiginosum, 264, 269, 391

cases, 265-267

operations contra-indicated, 268

similarity to Granuloma inguinale, 362

treatment of, 268

topographical distribution of, 262

treatment of, 268

variation of effects of, 263

Ultzmann's syringe, 301

Unguentum cinereum, 255

Uraturia in gonorrhœal neuras. thenia, 371

Ureteritis gonorrhoica, 310

Urethra, endoscopic examination of, 279

gonococci in, method of spread of, 275

syphilis of, 86

Urethritis, acute, treatment of, 283 abortive, 285

Ballenger's "sealing-in," a bortive, 286

Diday's irrigation, 285

drugs in, 298

general, 288

hygienic, 283

injections, 284

instillation by Guyon's catheter, 285

local, 284

prescription for, 287

symptomatic, 284

and subacute, chemotherapeutic treatment of, 349

routine of treatment, 350

vaccine treatment of, 350

anterior, 277

cavernous, pus in, 277

symptoms of, 277

bacteria causing, 326

chronic, 278

differential diagnosis of, 278

gonococcal distinguished from secondary infection, 282

influence of secondary infection, 282

diagnosis of, 279

differential diagnosis from cystitis, 278

discharge in, 277
Urethritis, gonococcal, in women, 339 gonorrhoica, complications of, 291

Cavernitis gonorrhoica, 292

Cowperitis gonorrhoica, 291

Cystitis gonorrhoica, 302

epididymitis, 306

folliculitis, 292

paraurethritis, 294

perifolliculitis, 292

Proctitis gonorrhoica, 311

prostatitis, 296

pyelitis, 310

pyelonephritis, 310

ureteritis, 310

vesiculitis, 304

deferentitis, 306

inclusion bodies and, 326

in females, 338

mucinous, 325

muscular, posterior, pus in, 277

non-gonococcal, 324

causes of, 324

posterior, 277

symptoms of, 277

recurrent, treatment of, 350

secondary infection of, bacteria causing, 283

subacute, 279

tests for anterior and posterior, 277

traumatic, 325

" two-glass test," 277

Urethrometer, Otis's, 279

Urethroscope, dangers through use of, ${ }^{2} 280$

Wossidlo's, 280

Urinary tract, affections of, com. plicating gonorrhœa in women, 337

infection of, routes of, 310

Urine, condition of, in gonorrhœal neurasthenia, 371

in cystitis, 278

in phosphaturia, 324

in prostatitis, 297

in vesiculitis, 304

pus in, 278

tests for, in chronic prostatitis, 299

in Cystitis gonorrhoica, 303

in urethritis, 278

Urticaria pigmentosa, differential diagnosis from syphilis, 39

syphilis cause of, 38

Uterus in acute endometritis, 340

Vaccine treatment of gonorrhoea, 344 principles of, 349

Vaccines in treatment of acute urethritis, 288

of chronic prostatitis, 299

of cowperitis, 292

of gonorrhœa in women, 341

Vaginitis, gonococcal, 337

in pregnancy, $\mathbf{3 3 9}$ 
Van Buren's disease, 363

Vanadium in treatment of syphilis, 225

Varicella, similarity of pustular lesions to, 27

Varicocele and sexual neurasthenia, 368

Vasa deferentia, 275

Vascular lesions in syphilis, 10 system, affections of, in congenital syphilis, 189

Vasostomy, 306

Vegetative nervous system and ductless glands, relation between, and syphilis, 144

drugs influencing, 144 syphilis of, 142

Veins, affections of, in congenital syphilis, 189 .

lesions of, in syphilis, 45

syphilis of, 134

syphilitic lesions of, simulating Erythema nodosum and Erythema multiforme, 46

Venereai disease and marriage, 375 and public health, 383 preventive measures, 385 question of notification, 385
Venereal diseases, subsidiary, 356 clinical knowledge in, 393 pathology in, 393

Venus' girdle, 201

Verrucose yphilide, 33 tuberculide, 33

Vertebræ, syphilis of, neural complications, 62

Vesicula seminales, 275 infection of, 305

Vesiculectomy, $\cdot 306$

Vesiculitis, 275, 304

symptoms of, 305

treatment of, 305

urine in, 306

Vesiculotomy, 306

Vincent's angina, 264 differential diagnosis from syphilis, 72 rashes of, differential diagnosis from syphilis, 40

Viscera, abdominal, syphilis of, 87

Visceral disturbances, paroxysmal, in congenital syphilis, 191

reactional inflammation, 237

Vitiligo, 334

in degenerative myelitis, 164

Vomiting after intravenous injec. Zinc ionisation, 268 tions of arseno benzene, 230
Vomiting, paroxyemal, in congenital syphilis, 191

Vuivitis, acute, treatment of, 342 erosiva et gangrenosa, syphilitio sores mistaken for, 176 in adults, 339

Vulvo-vaginitis in children, 338 treatment of, 343

War neurasthenia, 370

Warts, venereal, 359

Wassermann's non-specific fixation test, discovery of, 5

"White pneumonia "in congenital syphilis, 190

Whitlows, painless, in degenerative myelitis, 164

Women, gonorrhœa in, historical survey, 336

treatment of, 341

syphilis in, 169

clinical and pathological parte, 398

treatment of, 272

WossidIo's urethroscope, 280

Wrist-drop after mercurial treatment, 246

Zittmann's decoction, 202 
(1)

$673-4$ 
、 



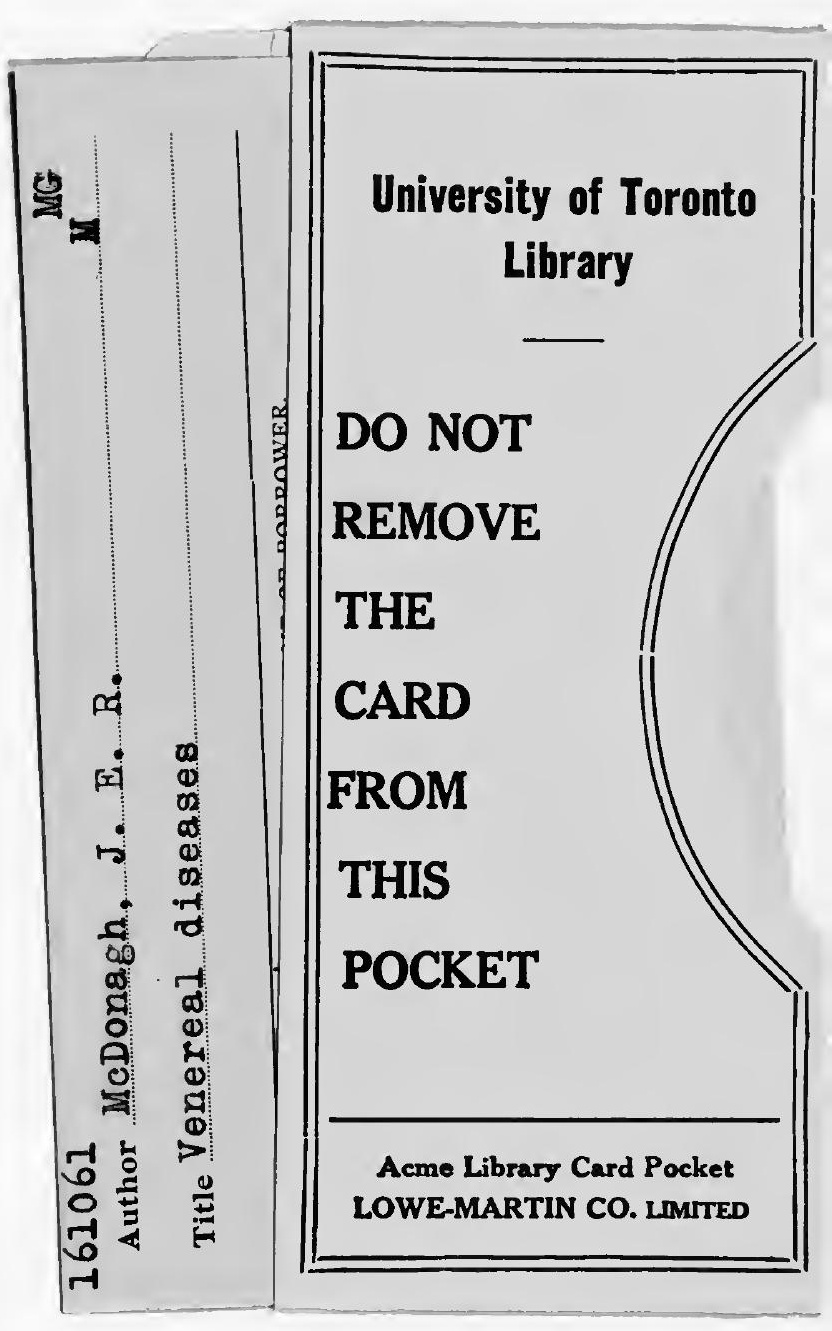


